\title{
DYNAMICS OF LARGE, WET VOLCANIC CLOUDS: THE 25.4 KA ORUANUI ERUPTION of Taupo volcano, New Zealand
}

by

Alexa R. Van Eaton

A thesis

submitted to Victoria University of Wellington in partial fulfilment of the requirements for the degree of Doctor of Philosophy in Geology

Victoria University of Wellington

Wellington, New Zealand 


\section{Thesis advisors}

Prof. Colin Wilson, Victoria University of Wellington, New Zealand Dr. James McGregor, Victoria University of Wellington, New Zealand

Prof. Stuart Bradley, University of Auckland, New Zealand

\section{Examination committee}

Prof. Lionel Carter, Victoria University of Wellington, New Zealand

Dr. Tony Hurst, Geological and Nuclear Sciences, New Zealand

Prof. Steven Self, Open University, United Kingdom

Submitted 30 April 2012

Defended 30 July 2012

(C) 2012

Alexa R. Van Eaton

All Rights Reserved 


\section{Abstract}

This work investigates the dynamics of large-scale, 'wet' volcanic eruption clouds generated by the interaction of silicic magma with external water. The primary case study draws from a detailed record of non-welded pyroclastic deposits from the $\sim 25.4$ ka Oruanui eruption of Taupo volcano, New Zealand, one of the largest phreatomagmatic eruptions documented worldwide. This research uses a three-pronged approach, integrating results from (i) field observations and textural data, (ii) mesoscale numerical modeling of volcanic plumes, and (iii) analogue laboratory experiments of volcanic ash aggregation. This interdisciplinary approach provides a new understanding of dynamic and microphysical interactions between collapsing and buoyant columns, and how this behavior controls the large-and small-scale nature of phreatoplinian eruption clouds.

Stratigraphic field studies examine the styles of dispersal and emplacement of deposits from several phases of the Oruanui eruption (primarily phases 2, 3, 5, 6, 7 and 8). Detailed stratigraphic observations and laser diffraction particle size analysis of ash aggregates in these deposits clarify the evolution of aggregation mechanisms with time through the relevant eruption phase, and with distance from vent. Deposits of the wettest phase (3) show the key role of turbulent lofting induced by pyroclastic density currents in forming aggregates, particularly those with ultrafine ash rims (30-40 vol.\% finer than $10 \mu \mathrm{m}$ ) which are uniquely formed in the ultrafine ash-dominated clouds above the currents. Drier deposits of phases 2 and 5, which also saw lower proportions of material emplaced by pyroclastic density currents, contain fewer aggregates that are related to low water contents in the medial to distal plume. Discovery and documentation of high concentrations of diatom flora in the Oruanui deposits indicates efficient fragmentation and incorporation of paleo-lake Taupo sediments during the eruption. This highlights the potential for incidental contamination of volcanic deposits with broader implications for correlation of distal tephras and possible contamination of paleoenvironmental records due to incorporation of diachronous populations of volcanically-dispersed diatoms.

The impact of extensive surface water interaction on large-scale volcanic eruptions $\left(>10^{8} \mathrm{~kg} \mathrm{~s}^{-1}\right.$ magma) is examined by employing the first 2-D large-eddy simulations of 'wet' volcanic plumes that incorporate the effects of microphysics. The cloud-resolving numerical model ATHAM was initialized with field-derived characteristics of the Oruanui case study. Surface water contents were varied from 0-40 wt.\% for eruptions with equivalent magma eruption rates of $c .1 .3 \times 10^{8}$ and $1.1 \times 10^{9} \mathrm{~kg} \mathrm{~s}^{-1}$. Results confirm that increased surface water has a pronounced impact on column stability, leading to unstable column behavior and hybrid clouds resulting from simultaneous ascent of material from stable columns and pyroclastic density currents (PDCs). Contrary to the suggestion of previous studies, however, abundant surface water does not systematically lower the spreading level or maximum height of volcanic clouds, owing to vigorous microphysics-assisted lofting of PDCs.

Key processes influencing the aggregation of volcanic ash and hydrometeors (airborne water phases) are examined with a simple and reproducible experimental method employing vibratory pan agglomeration. Aggregation processes in the presence of hail and graupel, liquid water $(<30 \mathrm{wt} . \%)$, and mixed water phases are investigated at 
temperatures from 18 to $-20{ }^{\circ} \mathrm{C}$. Observations from impregnated thin sections, SEM images and $\mathrm{x}$-ray computed microtomography of these experimental aggregates closely match natural examples from phreatomagmatic phases of the 25.4 ka Oruanui and Eyjafjallajökull (May 2010) eruptions. These experiments demonstrate that the formation of concentric, ultrafine rims comprising the outer layers of rim-type accretionary lapilli requires recycled exposure of moist, preexisting pellets to regions of volcanic clouds that are relatively dry and dominated by ultrafine $(<31 \mu \mathrm{m})$ ash. This work presents the first experimentally-derived aggregation coefficients that account for changing liquid water contents and sub-zero temperatures, and indicates that dry conditions $(<10 \mathrm{wt} . \%$ liquid) promote the strongly size-selective collection of sub-31 $\mu \mathrm{m}$ particles into aggregates (given by aggregation coefficients $>1$ ). These quantitative relationships may be used to predict the timescales and characteristics of aggregation, such as aggregate size spectra, densities and constituent particle size characteristics, when the initial size distribution and hydrometeor content of a volcanic cloud are known.

The integration of numerical modeling, laboratory experimentation and field data lead to several key conclusions. (1) The importance of the microphysics of ash-water interactions in governing the eruption cloud structure, boosting the dispersal power of the cloud and controlling aggregate formation in response to differing water contents and eruption rates. (2) Recognition of the contrasting roles of differential aggregation versus cloud grain size in controlling the formation and nature of aggregate particles, notably those with characteristic ultrafine outer rims. (3) The importance of pyroclastic density currents as triggers for convection and aggregation processes in the eruption cloud. 


\section{Acknowledgements}

First and foremost, I thank my primary supervisor Colin Wilson for taking a chance on a Floridian - and for giving generously of his time, patience and creativity, while encouraging independence and innovation. I thank my co-supervisors James McGregor and Stuart Bradley for encouraging my forays into atmospheric physics. Michael Herzog also deserves special thanks for supporting the numerical modeling aspects of this project, and facilitating my visit to the University of Cambridge. It has been a further, unexpected pleasure working with Margaret Harper on volcanic microfossil assemblages during the final phase of this project. A New Zealand International Doctoral Research Scholarship and grants from Victoria University and University of Auckland made this work possible. The Earthquake Commission of New Zealand is also gratefully acknowledged for a grant to attend the 2008 IAVCEI meeting in Iceland.

This $\mathrm{PhD}$ research has taken many surprising twists and turns, and eventually took on a life of its own. That spark I attribute to the vivacious Earth Science community in New Zealand, and particularly to my friends and colleagues at Victoria University of Wellington and the University of Auckland. I have thoroughly enjoyed working closely with Julie Vry on 'bunny time' with the undergrads, having benefitted from her sage advice on proposal writing and general academia. I am also indebted to Andrew Mellanby and Stacey Dravitzki for teaching me the evil ways of linux programming. Melissa Rotella and Simon Barker, you have been a sister and brother to me over the years. From celebrity volcanologizing in a chopper over White Island, to hauling each other's furniture up and down the gorges of Kelburn, we've been through a lot together, and I look forward to our future adventures in North America. I will especially miss my past officemates Evelien van de Ven, Ian Schipper, Netty Bolton, Sarah Martin, Kylie Christiansen, Jiao Ruohong, Lea Bello, Denise Tang, Aidan Allan, and Julene Marr.

To Ramona White and Katie Collins, our raucous outreach endeavors countless hours scouring the rock stores for workshop ideas and ushering hoards of preteen proto-scientists into the joys of geology - have been an absolute highlight of my time in New Zealand. Julene, Aidan, Mica and Eddie - I am so grateful for your friendship, and the exquisite surprise dinners in those little tupperware boxes with the 'keep trucking' truck, which will be forever synonymous with my hand-in time. All the people of SGEES at Victoria University - Katy Chamberlain, George 'k-shard' Cooper, Marc-Alban Millet, Chris Condor, Jenni Hopkins, Lucy McGee, Loretta Corcoran, Sarah Milicich, Kirsti Herbert, Matt Ryan, Shaun Eaves, Richard Jones, Nacho and the rest of the rowdy group - you have all been such a blast to live, work and play with. My family deserve special acknowledgement for supporting and encouraging my work from the very beginning, even though it meant that birthdays, Thanksgivings and Christmases together were few and far between. And to James. The ultimate field assistant, editor, superhero and teammate. Thank you for making this $\mathrm{PhD}$ the adventure of a lifetime. 


\section{Table of Contents}

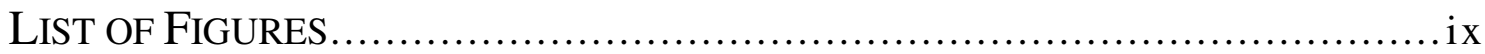

LIST OF TABLES ................................................... xii

PUBLICATIONS ARISING FROM THIS THESIS .................................. xiii

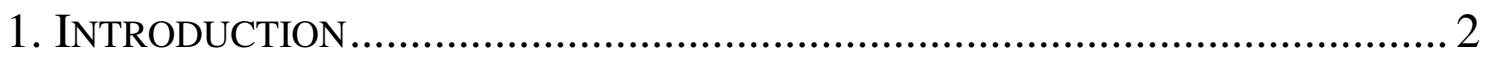

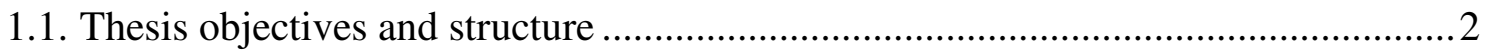

1.2. Motivation and broader impacts of the research......................................................

1.3. Processes and products of explosive wet volcanism................................................

1.4. Large-scale wet eruptions as 'dirty thunderstorms' ………..................................... 12

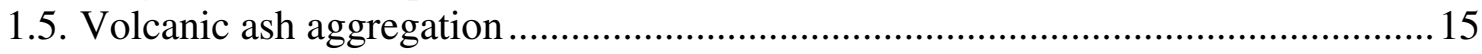

1.5.1. Key existing challenges regarding ash aggregation ....................................... 16

1.5.2. Volcanic hailstones: parallels and limitations ……………………………....... 19

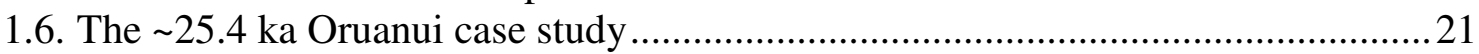

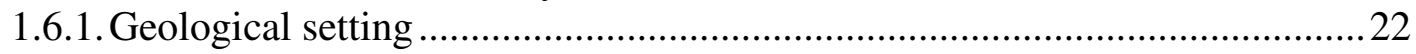

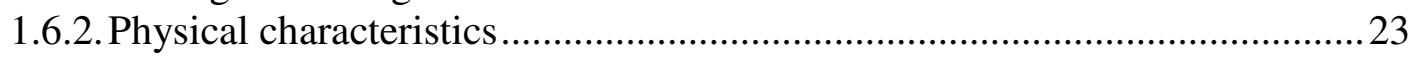

1.7. Models of explosive volcanic eruption plumes ………………………………......26

1.7.1. Categories of numerical models ..................................................................2

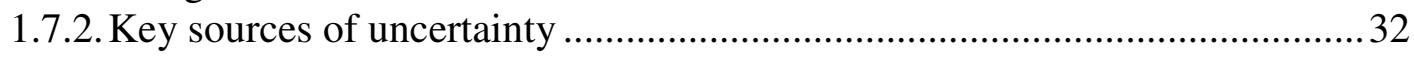

2. Ascent Dynamics of Large Phreatomagmatic ERuption Clouds:

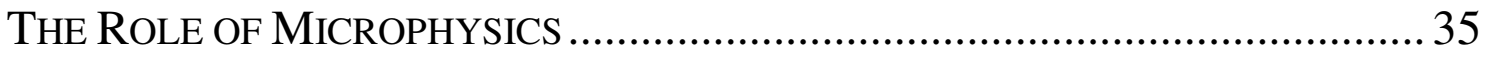

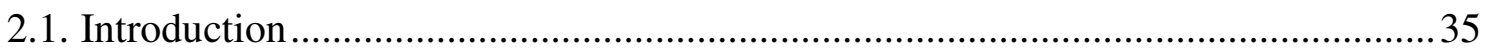

2.2. Dry Versus Wet Eruptive Styles ............................................................................ 37

2.2.1. The Dry to Wet Spectrum...............................................................................

2.2.2. 'Wet' Eruption Column Models .......................................................................... 38

2.2.3. Case study: The $~ 25.4$ ka Oruanui Eruption ..................................................... 41

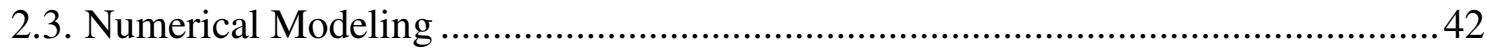

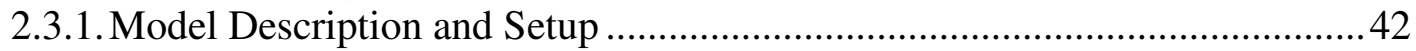

2.3.2. Volcanological Input ............................................................................. 44

2.3.3. Particle Characteristics ………………………………................................ 45

2.3.4. Thermodynamic Considerations...................................................................4

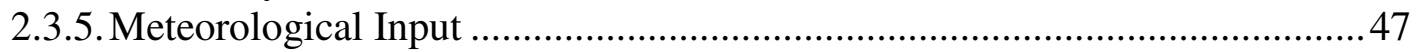

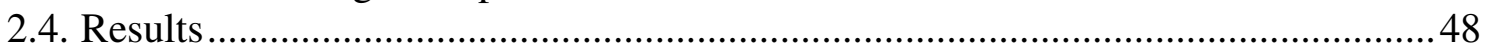

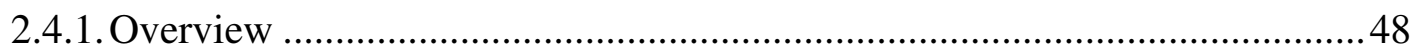

2.4.2. Reference Simulation: Saturated Eruption (24\% Surface Water) …………....50

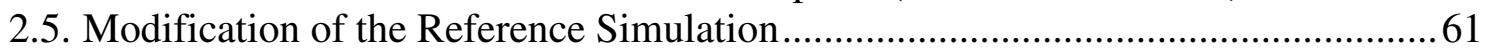

2.5.1. Dry Collapsing Column (0\% Surface Water) …………………………….......61

2.5.2. Unsaturated Plinian Column (10\% Surface Water)...........................................63

2.5.3. Saturated Transitional Column (40\% Surface Water) ........................................66

2.6. Sensitivity to Surface Water Content and Meteorological Conditions .......................68

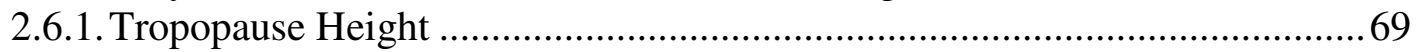

2.6.2. Background Humidity ................................................................................... 69

2.7. Implications for Formation of Hydrometeor-Ash Aggregates..................................70

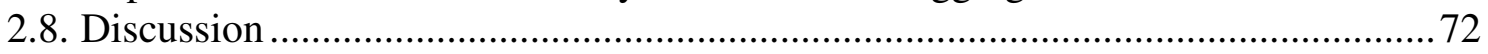


2.8.1. Dynamics of Column Instability: Comparison with Field Deposits ..............72

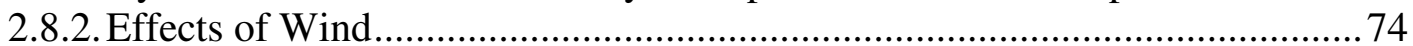

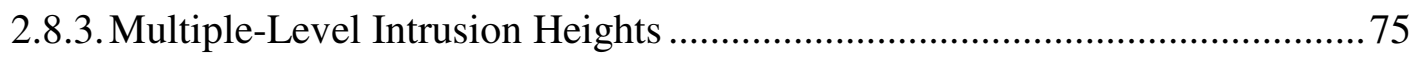

2.8.4. Defining Ash Cloud Heights: Maximum vs. Spreading Level........................75

2.8.5. Improving Parameterization of Wet, Unsteady Eruptions ............................ 76

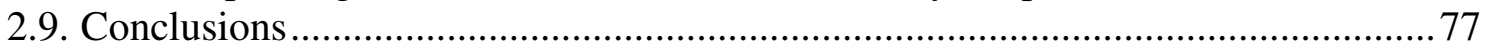

3. Methodology: Quantitative Size CHARACTERIZATION OF FINE

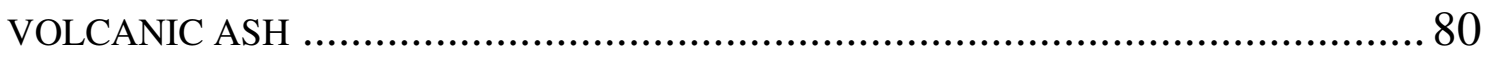

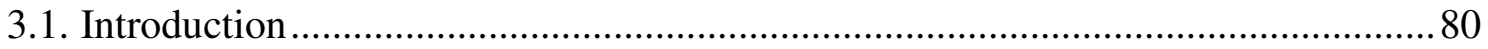

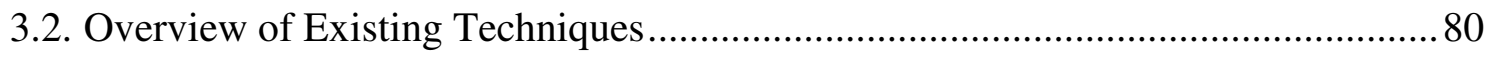

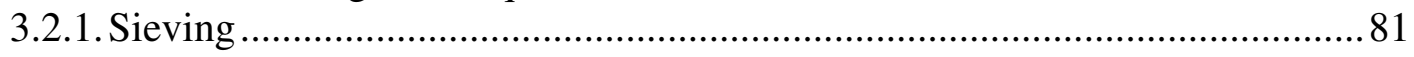

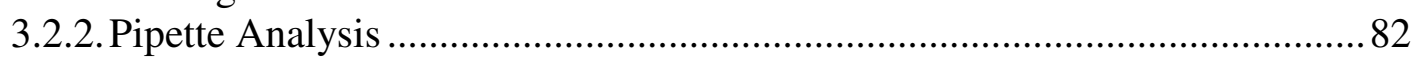

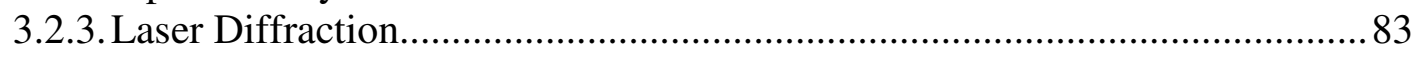

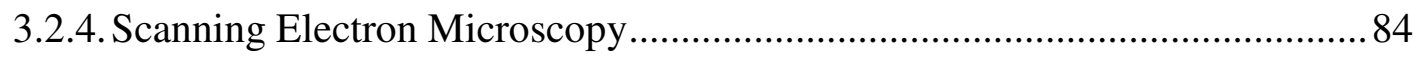

3.3. Laser Diffraction and Inter-comparison of Techniques ....................................... 84

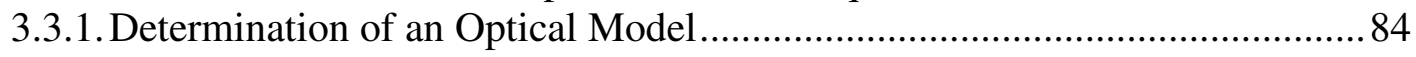

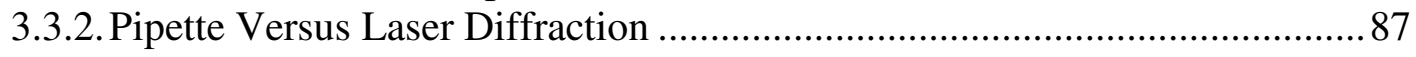

3.3.3. Sieving and SEM Counting Versus Laser Diffraction ................................... 88

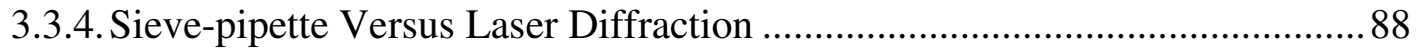

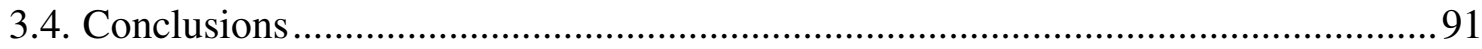

4. GROWTH OF VOLCANIC ASH AGGREGATES IN THE PRESENCE OF LIQUID WATER AND ICE: AN EXPERIMENTAL APPROACH .................................... 94

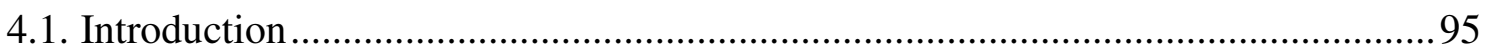

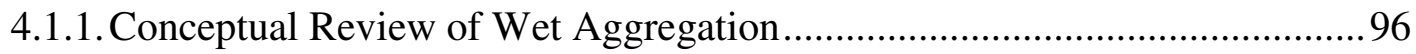

4.1.2. Geological Context for Experiments .......................................................... 98

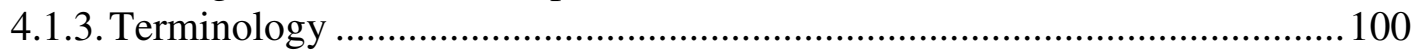

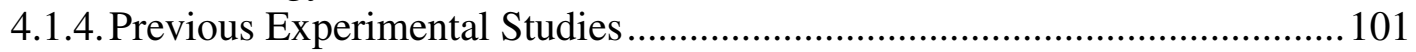

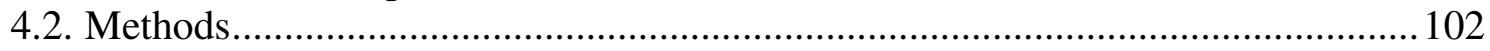

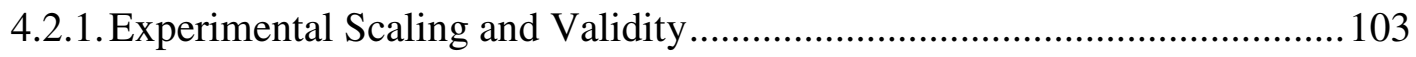

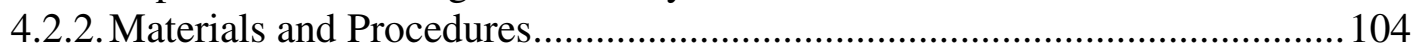

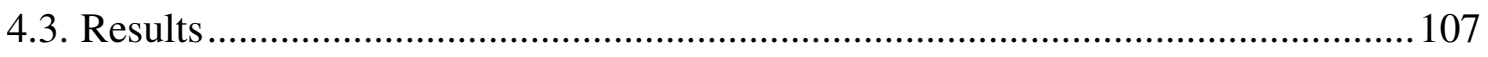

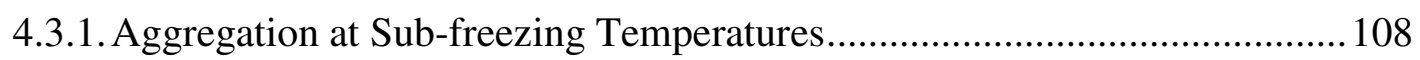

4.3.2. Aggregation of Ash and Melting Ice at Room Temperature (Mix-2) .......... 111

4.3.3. Wet Aggregation at Room Temperature ................................................. 114

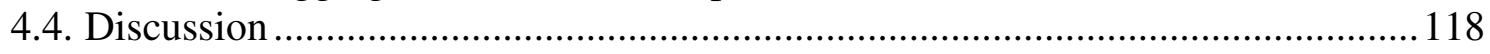

4.4.1. Comparison of Experimental Wet Aggregates with Natural Examples ....... 118

4.4.2. Analysis of Preferential Scavenging ..................................................... 120

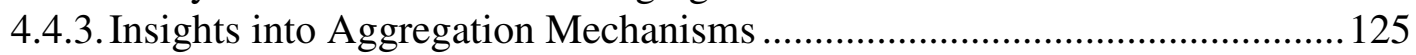

4.4.4. Origin of Aggregate Textures.............................................................. 128

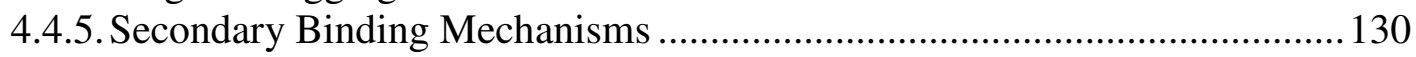

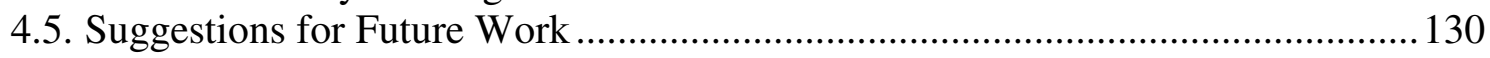

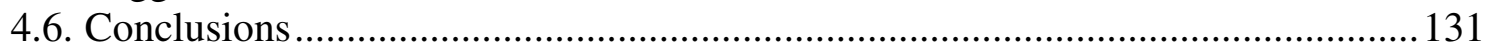


5. THE NATURE, ORIGINS AND DISTRIBUTION OF ASH AGGREGATES IN A LARGE-SCALE WET ERUPTION DEPOSIT: ORUANUI, NEW ZEALAND

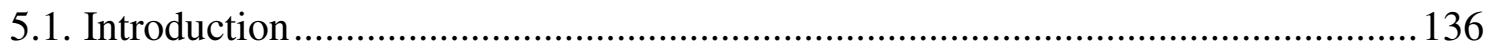

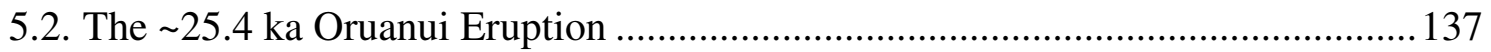

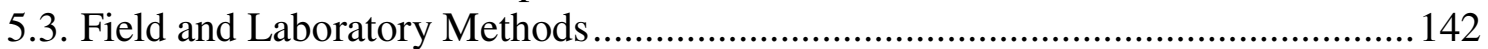

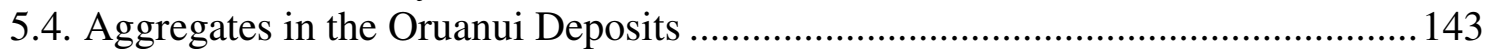

5.4.1. Aggregate Types - Classification and Terminology .................................. 143

5.4.2. Aggregate Types - Quantitative Classification.............................................. 152

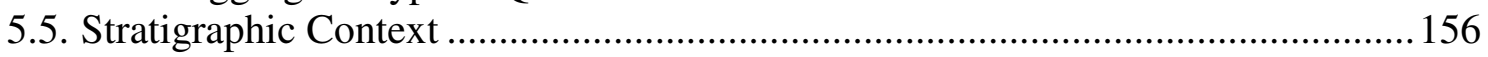

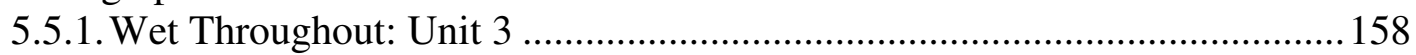

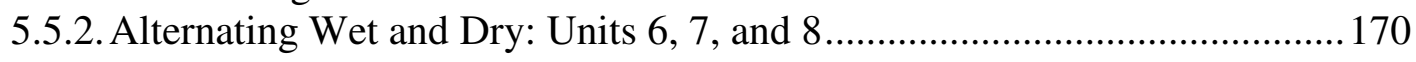

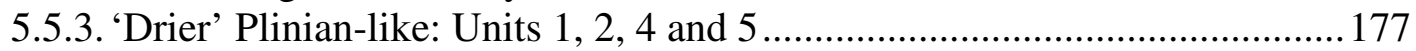

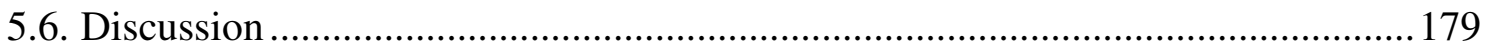

5.6.1. Summary Model: Impact of Aggregation on Cloud Development .............. 179

5.6.2. Do Ultrafine Rim-type Accretionary Lapilli Form in Pyroclastic Density

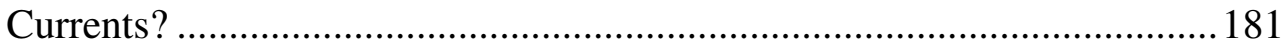

5.6.3. What is the Mechanism of Ultrafine Rim Formation? ................................. 182

5.6.4. How and Where do Ultrafine Rim Fragments Form? ................................ 184

5.6.5. How are $\mathrm{mm}$ to $\mathrm{cm}$-sized Aggregates Transported $>100-150 \mathrm{~km}$ from Vent?

5.6.6. How Does the Formation of Ice in the Volcanic Cloud Affect Aggregate

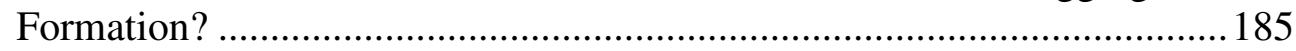

5.6.7. Comparison with Phreatoplinian Phases of the 1.8 ka Taupo Eruption ........ 186

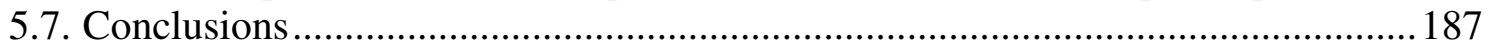

6. HIGH-FLYING DIATOMS: WIDESPREAD DISPERSAL OF MICROORGANISMS IN A VOLCANIC SUPER-ERUPTION ......................................................... 192

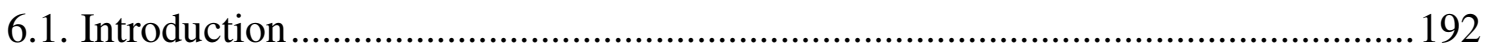

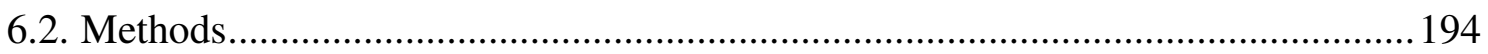

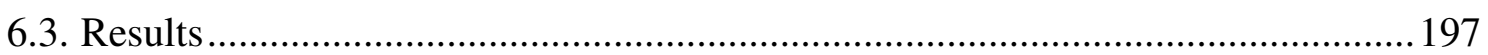

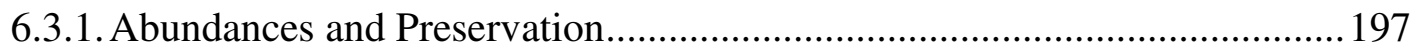

6.3.2. Volcanic Ash Assemblages and Comparison with Sediments .....................200

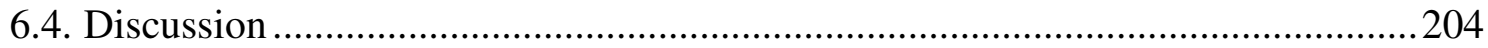

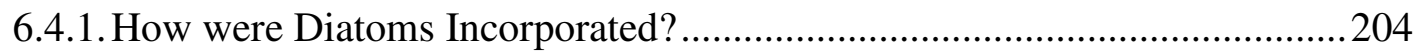

6.4.2. Could Some Microorganisms Survive the Eruption Process?.......................206

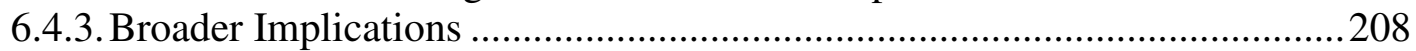

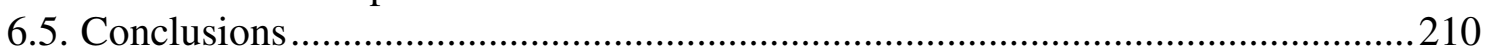

7. SYNTHESIS AND CONCLUSIONS .................................................... 212

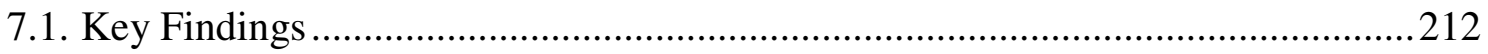

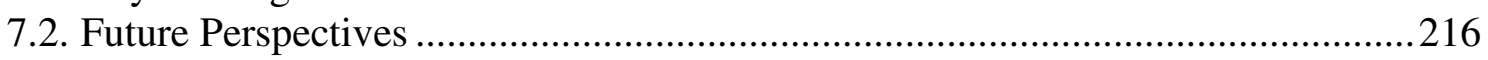

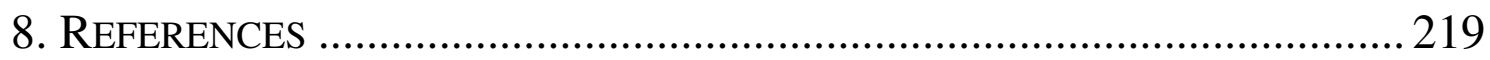

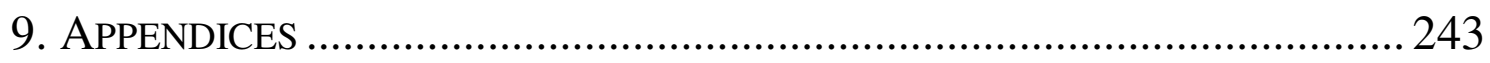




\section{List of Figures}

Figure 1.1 Schematic diagram of an explosive phreatomagmatic eruption.................... 9

Figure 1.2 Multi-level, coignimbrite column from Redoubt, Alaska ........................... 11

Figure 1.3 Chalazoidites of Berry [1928] from the 25.4 ka Oruanui deposits.............. 16

Figure 1.4 Parallels between hailstones and accretionary lapilli .................................20

Figure 1.5 Tectonic map of the North Island and modern Lake Taupo caldera .............23

Figure 1.6 Distribution and field characteristics of the Oruanui deposits .....................25

Figure 2.1 Cloud microphysical processes described by ATHAM................................44

Figure 2.2 Meteorological inputs used in sensitivity studies ......................................48

Figure 2.3 Summary of eruption cloud morphologies ..............................................51

Figure 2.4 Comparison between results of no-microphysics and microphysics-capable

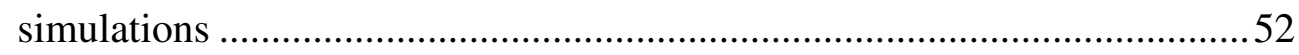

Figure 2.5 Time series of plume development in the reference simulation ....................53

Figure 2.6 Microphysical effects in the reference simulation.....................................54

Figure 2.7 Temporal evolution of ash cloud heights in the reference simulation...........57

Figure 2.8 Vertical ash concentrations with and without aggregation.........................59

Figure 2.9 Time series of plume development in a dry collapsing fountain...................62

Figure 2.10 Time series of plume development from the Plinian eruption involving $10 \%$

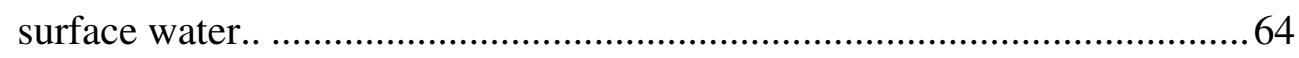

Figure 2.11 Vertical profiles of ash emissions for low MER eruptions.........................65

Figure 2.12 Horizontally integrated mass of airborne hydrometeors from low MER

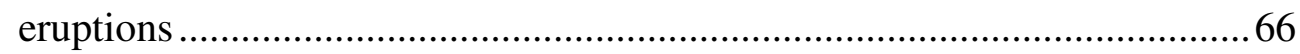

Figure 2.13 Time series of plume development in the saturated transitional column ....67

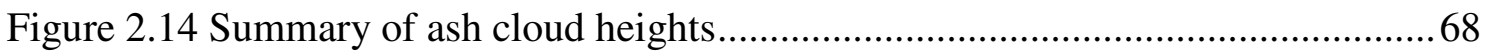

Figure 2.15 Sensitivity of volcanic cloud water content to changes in simulated

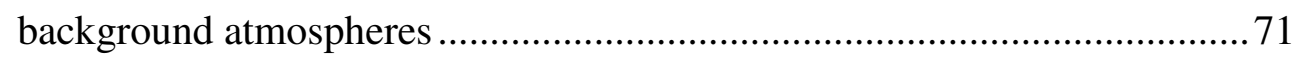

Figure 3.1 SEM images of ash morphologies and pumice textures from Oruanui unit 3 deposits.

Figure 3.2 Definition sketch of the principle diameters of irregularly shaped ash particles 
Figure 3.3 Sensitivity of grain size distributions to changes in the laser diffraction optical model

Figure 3.4 Comparison of grain size distributions from laser diffraction and pipette analysis

Figure 3.5 Comparison of sieving, laser diffraction and SEM counting tehniques 89

Figure 3.6 Comparison between sieve-pipette and laser diffraction data ..... .90

Figure 3.7 Correlation of sieve-pipette and laser diffraction data .91

Figure 4.1 Sketch of growth mechanisms in liquid-bound ash aggregates. .98

Figure 4.2 Examples of naturally occurring ash aggregates from Taupo volcano and Iceland 100

Figure 4.3 Particle size distributions of parent ash used in experiments ..... 105

Figure 4.4 Photograph of vibratory pan aggregation 106

Figure 4.5 Definition sketch of the key zones in the overlain particle size distributions of parent ash and aggregates 108

Figure 4.6 Results from the Hail-1 experiment (liquid-free hail) 109

Figure 4.7 Results from the Mix-1 experiments (freezing liquid drops) 111

Figure 4.8 Results from Mix-2 experiments (melting ice pellets) 113

Figure 4.9 Key aggregates textures from Liquid-1 and Liquid-2 experiments. 115

Figure 4.10 Sieve diameters versus water content for Liquid-1 aggregates 116

Figure 4.11 Example of thin outer films in clast-supported, massive ash pellets from the Oruanui deposits. 117

Figure 4.12 Comparison of representative particle size distributions from Liquid-1 aggregates and natural examples. 119

Figure 4.13 Equivalent spherical diameter vs. dry densities of natural and experimentally generated aggregates.

Figure 4.14 Liquid water added vs. mean dry density of Liquid-1 aggregates. 120

Figure 4.15 Liquid water added vs. maximum particle size in aggregates

Figure 4.16 Liquid water in aggregates vs. neutral zone in Liquid-1 and Hail-1 aggregates. 122

Figure 4.17 Aggregation coefficients at varying liquid water contents ....................... 124

Figure 5.1 Paleogeographic features relevant to the Oruanui eruption......................... 138

Figure 5.2 Definition sketch of an unstable, hybrid phreatoplinian eruption column .. 140

Figure 5.3 Field localities with respect to the lateral extent of Oruanui PDCs.............. 142

Figure 5.4 Thin sections of coalesced ash pellets in the Oruanui deposits ................... 146 
Figure 5.5 In situ examples of massive ash pellets

Figure 5.6 Examples of layered accretionary lapilli in Oruanui deposits .................... 148

Figure 5.7 Examples of ultrafine rim-type accretionary lapilli................................. 150

Figure 5.8 Examples of complexly layered accretionary lapilli ................................ 151

Figure 5.9 Particle size distributions representative of each aggregate type ................153

Figure $5.10 \mathrm{M}_{\mathrm{z}}$ vs. $\sigma_{\mathrm{I}}$ plot for Oruanui ash aggregates ............................................ 154

Figure 5.11 Ternary diagram for Oruanui ash aggregate grain size data..................... 155

Figure 5.12 Core-rim dimensional relationships in ultrafine rim-type and layered

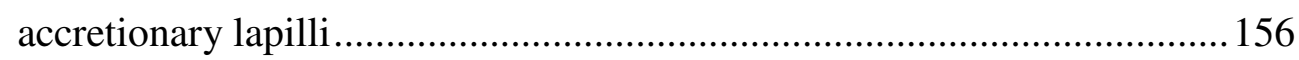

Figure 5.13 Morphological and dimensional data from all Oruanui aggregates........... 157

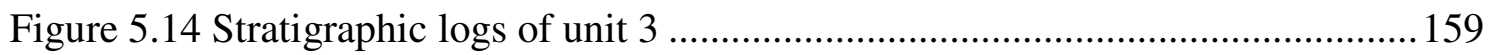

Figure 5.15 Model for the formation of unit 3 aggregate facies ................................ 161

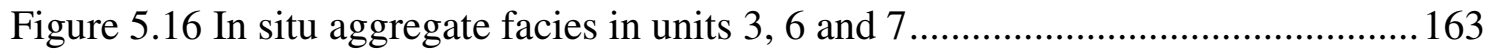

Figure 5.17 Proximal unit 3 stratigraphy and grain size distributions ......................... 164

Figure 5.18 Transitional unit 3 stratigraphy and grain size distributions ..................... 165

Figure 5.19 Distal unit 3 stratigraphy and grain size distributions ............................ 167

Figure 5.20 Coarsening signature in unit 3 and conceptual explanation ...................... 168

Figure 5.21 Stratigraphic logs of units 6, 7 and 8 with distance from source............... 171

Figure 5.22 Stratigraphy and grain size distributions of units 6,7 and 8 ................... 172

Figure 5.23 Maximum aggregate sizes with distance from source .............................. 174

Figure 5.24 Thin section and interpretation of the unit 8 vertical transition from fall deposition to laterally-emplaced material ............................................... 177

Figure 5.25 Schematic summary diagram of ash aggregation processes in the Oruanui

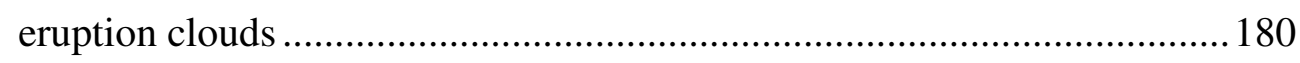

Figure 6.1 Map of sampling localities for primary Oruanui deposits, lacustrine lithic clasts and pre-Oruanui paleosol

Figure 6.2 Absolute abundances of diatom remains with distance from vent in primary Oruanui volcanic ash 199

Figure 6.3 Light microscope images of diatom specimens showing preserved organic matter. 201

Figure 6.4 SEM images of diatoms from the Oruanui volcanic ash and underlying paleosol. 202 
Figure 6.5 Comparison of diatom assemblages in the Huka Falls litic clasts, Oruanui deposits, and pre-eruptive paleosol .........................................................203

Figure 6.6 Sketch of the three hypotheses critically examined in this study................204

Figure 6.7 SEM images of a diatom after exposure to $800{ }^{\circ} \mathrm{C}$ for one hour..................207

Figure 6.8 Proposed model of syn-eruptive excavation and transport of diatoms during

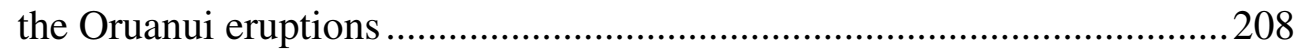

\section{List of Tables}

Table 2.1 Volcanic input parameters for simulations with different surface water

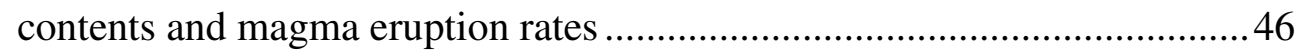

Table 2.2 List of numerical simulations used in this study....................................... 49

Table 4.1 Previous aggregation experiments using volcanic ash................................ 102

Table 4.2 List of experiments in this study using vibratory pan aggregation ............... 103

Table 5.1 Categories of aggregate types identified in the Oruanui deposits................. 144

Table 5.2 Aggregate facies in the Oruanui eruption deposits. ..................................... 158

Table 6.1 Locations and descriptions of samples used in this study............................196

Table 6.2 Quantitative abundances of diatom remains in the Oruanui volcanic ash.... 198 


\title{
Publications arising from this thesis
}

\author{
Journal articles
}

Van Eaton A.R., M. Herzog, C. J. N. Wilson and J. McGregor (2012). Ascent dynamics of large phreatomagmatic eruption clouds: The role of microphysics. Journal of Geophysical Research 117, B03203, doi 10.1029/2011JB008892

Van Eaton A.R., J. D. Muirhead, C. J. N. Wilson and C. Cimarelli, (In Press, 2012). Growth of volcanic ash aggregates in the presence of liquid water and ice: an experimental approach. Bulletin of Volcanology.

Van Eaton A.R. and C.J.N. Wilson (In Review). The nature, origins and distribution of ash aggregates in a large-scale wet eruption deposit: Oruanui, New Zealand Journal of Volcanology and Geothermal Research.

Van Eaton A.R., Harper M. and Wilson C.J.N. High flying diatoms: Long-distance transport of microfauna in volcanic eruption clouds. Manuscript in preparation.

\section{Conference abstracts \\ (*) indicates oral presentation}

Harper M. and Van Eaton A.R. (2011). High-flying diatoms: Widely dispersed microfossils from a New Zealand supereruption In: Litchfield, N.J., Clark, K. (eds). Abstract volume, Geosciences 2011 Conference, Nelson, New Zealand. Geoscience Society of New Zealand Miscellaneous Publication 130A: p. 45-46.

Van Eaton A.R. and Wilson C.J.N. (2011). Experimental aggregation and comparison with field deposits. IUGG General Assembly, Melbourne, Australia, 28 June - 7 July.*

Van Eaton A.R., Herzog M., Wilson C.J.N and McGregor J. (2010). Microphysical controls on ascent of water-rich ash clouds from supereruptions, EOS Transactions $f$ the American Geophysical Union 89(53), Fall Meeting Supplement, Abstract V13C-2375.

Van Eaton A.R., Wilson C.J.N, Herzog M. and McGregor J. (2010). Dynamical modeling of wet ash clouds: Insights from ATHAM simulations of the 27 ka Oruanui supereruption of Taupo volcano, New Zealand. In: Eccles, J.D., Grigor, M.R., Hoskin, P.W.O., Hikuroa, D.C.H. (eds). Abstract Volume, GeoNZ 2010 Conference, Auckland, New Zealand. Geoscience Society of New Zealand Miscellaneous Publication 129A: p. $301{ }^{*}$

Van Eaton A.R., Wilson C.J.N. and Bradley S. (2008). Processes in wet eruption plumes: insights from the Oruanui supereruption of Taupo volcano, New Zealand. New Zealand Geosciences Conference, Te Papa Tongarewa, Wellington, New Zealand, 2326 November.

Van Eaton A.R., Wilson C.J.N. and Bradley S. (2008). Processes in wet eruption plumes: insights from the Oruanui supereruption of Taupo volcano, New Zealand. IAVCEI General Assembly, Reykjavik, Iceland, 18-22 August.* 
Chapter 1 


\section{Introduction}

\subsection{Thesis objectives and structure}

This work focuses on the dynamics and transport of volcanic clouds from the largest scale examples of wet volcanism - phreatoplinian eruptions. This style of eruption, characterized by widespread dispersal of eruption products and arising from explosive interaction of magma and external water [Self and Sparks 1978, Houghton et al. 2000], presents fundamental challenges to the volcanological and meteorological communities. Despite the scarcity of phreatoplinian eruptions in historic times, the rich geologic archives of these enormous events lend insight into the behavior of recent explosive eruptions. For example, the generation of extremely fine ash, abundant ash aggregates and unsteady eruption styles that typify phreatoplinian eruptions provide somewhat amplified versions of the key challenges posed by volcanic events over the past few decades.

The central purpose of this thesis is to provide new perspectives on the behavior of water-rich explosive eruptions, both augmenting and challenging conventionally held views about these events. Using the $\sim 25.4 \mathrm{ka}$ Oruanui eruption from Taupo volcano (New Zealand) as a case study, my approach integrates field observations, textural data and analogue laboratory experiments with numerical modelling to reconstruct how ash was transported and deposited during selected phases of this super-eruption. Well preserved layers from units 3, 6, 7 and 8 of the Oruanui deposits form the backbone of this study. Building from the existing stratigraphic database of this eruptive sequence from Wilson [2001], I establish an integrated framework for characterizing the transport and dispersal of large-scale wet eruptions. The key aims of this work are to:

Examine the microphysical and dynamic impact of abundant magma-water interaction on eruption style and injection of volcanic ash into the atmosphere, providing a new conceptual model of explosive, water-rich eruptions; 
$>$ Investigate the mechanisms of volcanic ash aggregation, linking field observations of aggregate textures with the occurrence of specific processes and water phases in volcanic ash clouds;

$>$ Clarify the roles of magma-water interaction and ash aggregation in eruption, transport and depositional characteristics of large-scale wet eruptions;

Explore the conditions under which distal volcanic deposits may uniquely record evidence for magma-water interaction in a lake environment.

These specific aims are addressed within seven interrelated chapters, structured around the central theme of developing a coherent framework for characterizing wet eruption behavior. Although the chapters are written in a style suitable for publication, and are thus presented as self-contained studies, each builds progressively from the findings of the previous chapter. Published or accepted versions have been modified only slightly (stylistically and in terms of formatting, but not in content) for incorporation into a consistent overall thesis design.

Chapter 1: Places the Oruanui case study within its broader geologic context and provides a critical review of the tools and approaches used to characterize wet, explosive eruptions. An emphasis on the effects of aggregate formation in volcanic clouds and their parallels to meteorological storms is used to demonstrate how recent advances in observational and numerical techniques have provided new focus areas for interdisciplinary research. A number of advancements in the area of numerical eruption plume modeling have come about in the past decade or so, with implications for both real-time ash cloud forecasting and reconstruction of pre-historic eruption parameters from their deposits. Therefore, this chapter also reviews and evaluates the three types of volcanic plume models currently in use worldwide.

Chapter 2: Presents a field-based numerical modeling investigation, which challenges the traditional view that, because water-rich eruptions have cooler temperatures, they must be less powerful and less widely dispersed than their dry counterparts. Field data from the Oruanui case study are used to initialize a cutting-edge volcanic eruption column model that accounts for microphysics. Two-dimensional eruption column simulations using the Active Tracer High resolution Atmospheric Model (ATHAM) 
examine the fundamental redistribution of momentum and energy in phreatoplinian columns resulting from different amounts of magma-water interaction.

Chapter 3: Assesses the four primary methods of quantifying the size of volcanic ash, and evaluates their application to fine grained, phreatoplinian deposits. SEM studies are combined with image analysis, laser diffraction, sieving and pipette analyses to identify sources of variability between the techniques, and to establish a reproducible method of grain characterization by laser diffraction for the Oruanui deposits.

Chapter 4: Compares analogue laboratory experiments of volcanic ash aggregation with textures observed in the field. A new experimental technique is developed and utilized to assess the interaction of volcanic ash particles and films of liquid water, ice and electrostatic attraction at room temperature and below freezing. To clarify the role of and detailed mechanisms of aggregation in volcanic clouds, the interplay between electrostatic attraction, liquid tensional forces and ice sintering (in the absence of liquid) is examined with respect to the size of ash particles that can be preferentially scavenged into aggregates from a wider size population.

Chapter 5: Examines the nature, origins and impacts of volcanic ash aggregation in the Oruanui eruption deposits. Seven types of ash aggregates are categorized according to quantitative parameters, and documented with distance from vent, vertically in the deposits and in context with mode of emplacement (i.e. fall or flow) to establish the physical processes governing their formation.

Chapter 6: Pursues the unexpected finding that diatoms are present in primary volcanic deposits from the Oruanui eruption. Quantities and species of whole and fragmented diatoms in the Oruanui ash are documented at various sites in the North Island and Pitt Island (Chathams, New Zealand). Explanations for the presence of diatomaceous remains are tested with quantitative observations, and the implications for eruption mechanisms, vent processes and widespread dispersal of (possibly live) microorganisms are discussed. A new mechanism for airborne dispersal of recent and ancient microorganisms is proposed, indicating the potential for phreatomagmatic eruptions to contain a biogenic 'fingerprint' unique to their geographic source. 
Chapter 7: Integrates the primary findings of this doctoral work, and outlines new areas for targeted work in the future.

\subsection{Motivation and broader impacts of the research}

The presence of volcanic ash in the troposphere and lower stratosphere is now widely known to pose a serious threat to aircraft. However, it was not until thirty years ago that these dangers were first brought to the public and scientific eye. On 24 June 1982, a British Airways Boeing 747 unknowingly encountered airborne volcanic ash from Mount Galunggung, Indonesia [Hanstrum and Watson 1983]. The aircraft experienced failure of all four engines and plummeted more than $8 \mathrm{~km}$ before recovering with an emergency landing. This event, and subsequent ash encounters, made it clear that volcanic ash has disastrous effects on jet engines and that the damage depends on a number of volcanological factors including the composition and particle concentration of ingested ash [Casadevall 1994, Dunn and Wade 1994]. Nine Volcanic Ash Advisory Centers (VAACs) were thus established as part of an international warning system, the International Airways Volcano Watch, to predict and relay the movement of volcanic ash following the onset of an eruption [ICAO 2004]. These monitoring centers rely heavily on direct observations and remote sensing data (e.g. satellite imagery, radar) to characterize the eruption behavior and provide input for volcanic ash transport and dispersion models. However, the VAACs face formidable challenges in the form of conflicting and/or sparse field constraints during eruptions [Tupper et al. 2007]. Some key aspects of observed volcanic phenomena also remain poorly understood, particularly the influence of moist convection on cloud heights and the vertical distribution of ash in the atmosphere [Tupper et al. 2009].

These challenges were brought to light and to public scrutiny during the eruptions from Eyjafjallajökull, Iceland, in April-May of 2010. Without procedures in place at the VAACs to quantify 'safe' versus 'unsafe' ash concentrations in the volcanic clouds that drifted over Europe, a zero ash tolerance policy was enforced. Flights were grounded across the board for seven consecutive days, leading to approximately $€ 5$ billion in losses for industries affected by the unprecedented closures of European airspace [Langmann et al. 2012]. In an ironic twist of fate, I myself was stranded in Europe by this event for twelve days, attempting to return from the UK after having spent a month 
applying numerical modeling experiments to water-rich eruption scenarios. The event produced a fundamental shift in the context of my doctoral work, funnelling the more academic questions of my research toward the practical and pressing issues of plume dynamics that impact aviation and society. On a global scale, this event was similarly impactful to scientific communities around the world.

Following widespread abandonment of the zero ash tolerance policy by commercial airlines, came a call for efforts to improve our capacity to monitor and predict airborne ash concentrations from volcanic eruptions [European Commission 2010]. Less than one year later, the 2011 eruptions of Puyehue-Cordón Caulle in Chile triggered another, albeit smaller-scale 'ash crisis' by transporting a dilute volcanic cloud over Australia and New Zealand. This time, commercial airlines were given the choice of determining their own buffer zone around the ash-laden airspace, rather than being banned from flying altogether [Lahey 2012]. The increasing demand for greater accuracy and precision in ash forecasts is a considerable challenge to the scientific community, requiring a concerted effort on behalf of volcanologists and meteorologists to expand the current understanding of volcanic ash cloud behavior. Meanwhile, the demand for reliable airspace occupied by jet aircraft with more fuel-efficient (i.e. hotter burning and more ash-vulnerable) engines is increasing rapidly. This relatively recent conundrum of volcanic ash forecasting technology endeavoring to keep pace with public and industry demands has created a new context for studies of explosive volcanism.

Complementary to the efforts of near-real-time monitoring and forecasting of ash emissions, field-based studies of volcanic deposits provide crucial details of transport and dispersal. For example, characteristics such as erupted mass, total grain size distribution and the nature and extent of ash aggregation are key to numerical modeling outputs [Mastin et al. 2009, Folch et al. 2012], but are difficult to quantify during active eruptions. Geologic field observations also provide the historic context of eruptive styles and tempos characterizing a given volcanic complex. In turn, databases of these parameters provide a useful reference in the early stages of volcanic forecasting, when actual conditions of an eruption are not yet known [Mastin et al. 2009].

Over the past few decades, volcanic ash dispersal models have been tested extensively for relatively small examples of explosive eruptions that occur on the order of 20-30 
times a year globally. However, they are ill-prepared to handle the dynamics of eruptions resulting from large-scale interaction of magma and water [Wilson 1994, Houghton et al. 2000]. This owes as much to the complexity of ash transport dominated by water phase changes, unsteady columns and particle aggregation as to the infrequency of wet eruptions that have been observed historically (and therefore a scarcity of data for model validation). As such, our understanding of the full spectrum of plume behavior, including injection heights and atmospheric lifetime with respect to added surface water, is far from complete.

This project implements measurements and models of deposits from the world's youngest supereruption, the $\sim 25.4$ ka Oruanui eruption of Taupo volcano, New Zealand, by examining the field data in a context that is readily applicable to volcanic ash forecasting issues. The Oruanui case study is unique in that it records the explosive interaction of magma and water on the largest scale yet documented in detail, and provides an opportunity to address when and how the traits of wet volcanism begin to dominate over traditional 'dry' behavior.

\subsection{Processes and products of explosive wet volcanism}

This section addresses the plume structure and distinguishing features of wet versus dry eruptions in terms of fragmentation and plume development. Reconstructing the dynamics of large-scale, wet eruptions from their deposits is a fundamental challenge in physical volcanology. There are three sources of water which exert a transformative influence on the dynamics of explosive eruptions: (1) internal magmatic water in the form of exsolved volatiles, comprising up to $7 \mathrm{wt} \%$ of the magma [Carroll and Holloway 1994]; (2) ambient moisture entrained into the ascending volcanic plume from the surrounding atmosphere [Woods 1993]; and (3) surface water from reservoirs such as aquifers, oceans or lakes. As a general rule, an eruption is considered 'wet' if the magma interacts with the last of these sources of water [Houghton et al. 2000].

Diagnostic features of magma-water interaction in explosive eruptions typically include: (1) fine grained deposits, even close to source, due to efficient phreatomagmatic fragmentation at vent [Self and Sparks 1978, Walker 1981b, Sheridan and Wohletz 1983]; (2) direct evidence for ash aggregation in the form of preserved aggregate 
structures [Self 1983], and/or indirect evidence such as poorly sorted fall deposits [Carey and Sigurdsson 1982]; and (3) low-temperature pyroclastic density current (PDC) deposits inferred, for example, from a lack of welding even at the thickest nearvent exposures [Sparks and Wilson 1976].

However, for a wet eruption to qualify as 'phreatoplinian', the deposits must be both widely dispersed (Walker dispersal index $\geq 500 \mathrm{~km}^{2}$ ) and exceptionally fine grained (Walker fragmentation index >80\%) [Self and Sparks 1978]. The physical basis for distinguishing phreatoplinian eruptions from their drier counterparts is founded on distinctive behaviors arising from the interaction of magma and water. Whereas dry Plinian eruptions derive their initial momentum from the explosive expansion of magmatic volatiles [Sparks 1978], water-rich eruptions are additionally powered by the rapid expansion of water flashing to steam upon contact with magma [Wohletz 1986]. These effects have been observed to have a transformative effect on the behavior of volcanic eruptions. Well-documented historic events such as the 1965 eruptions of Taal volcano in the Philippines [Moore et al. 1966] and the 1983 eruptions of Miyakejima in Japan [Aramaki and Hayakawa 1984] illustrate that changes the water content of an eruptive mixture (e.g., from 5-40 wt.\%) govern whether the eruption cloud rises as a buoyant, convecting plume or collapses into a ground-hugging pyroclastic density current.

The overall dimensions and dynamics of eruption plumes thus alter fundamentally with addition of external water. The descriptive framework of this process has expanded in complexity since the pioneering work of Lionel Wilson and others in the 1970s [e.g. Wilson 1972, Wilson et al. 1978]. This section introduces the generalized anatomy of explosive eruptions (Figure 1.1), observing the tripartite structure of conventional volcanic column models [e.g. Sparks 1986], with additional consideration of the influence of water arising from more recent work [Woods 1993, Koyaguchi and Woods 1996, Glaze et al. 1997].

The process begins with silicic magma rising through a conduit. As the melt begins to decompress, dissolved volatiles expand as they come out of solution (exsolution surface of Figure 1.1), promoting ascent of an actively vesiculating magmatic foam [Sparks 1978]. Once vesicularity reaches a threshold value (70-80\%), the magma spontaneously 
and vigorously breaks itself apart (magmatic fragmentation) [Houghton and Wilson 1989, Cashman et al. 1994, Sparks et al. 1994, Gilbert and Sparks 1998]. If the rising magma meets external water from caldera lake, for example, the magma becomes quenched, and violent steam expansion shatters it into a fine-grained mixture of ash and vapor (phreatomagmatic fragmentation) [Self and Sparks 1978].

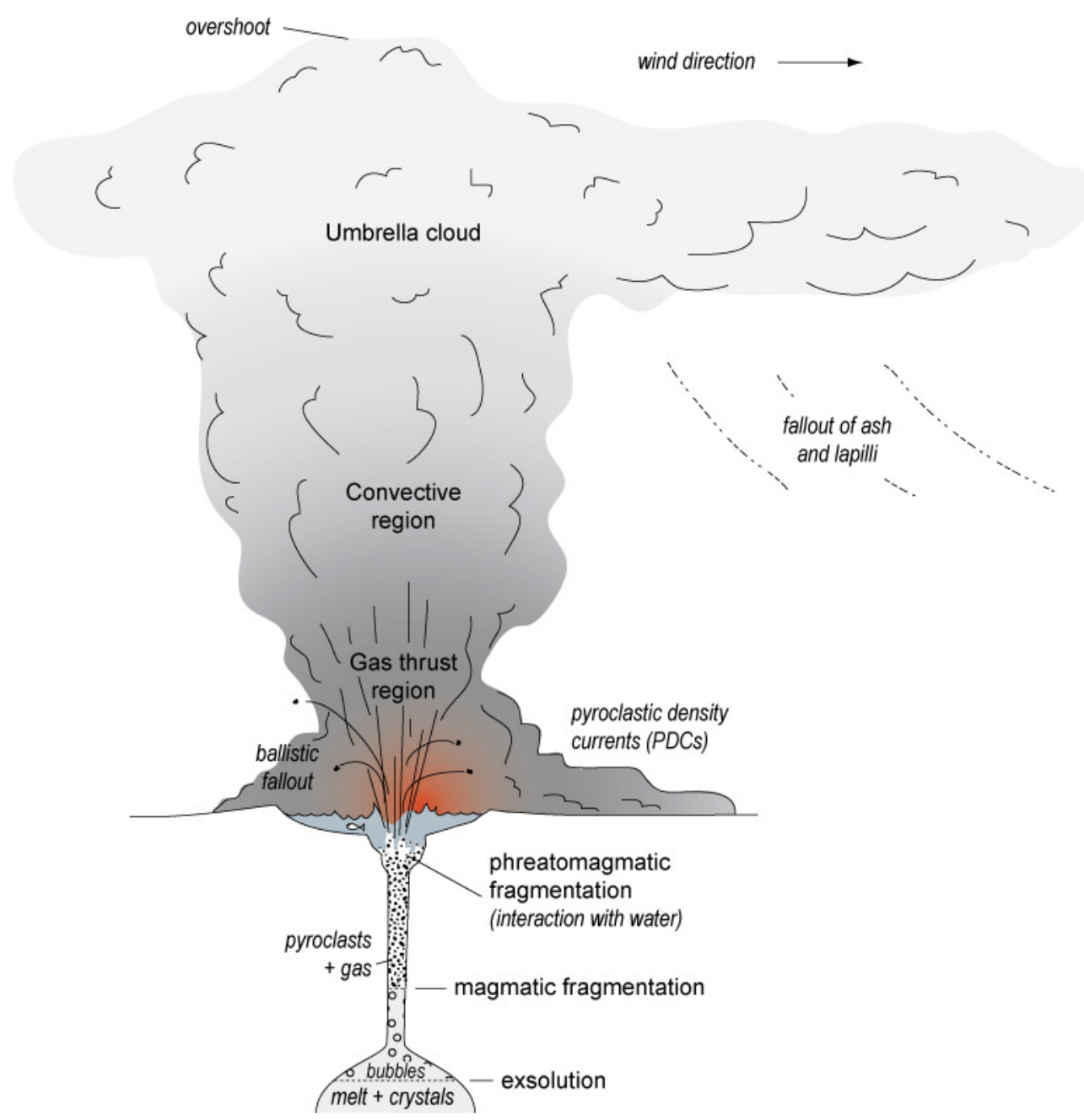

Figure 1.1 Schematic anatomy of an explosive phreatomagmatic eruption, illustrating key processes from the subsurface to the atmosphere. Levels of gas exsolution, fragmentation and interaction with non-magmatic (external) water are indicated (after Cashman et al. [2000]), followed by the three regions of the eruption column: gasthrust, convective region and umbrella after Sparks et al. [1997]).

These distinctive fragmentation processes are recorded in the pumice densities and grain size distributions of silicic volcanic deposits. 'Dry' fall deposits (i.e. lacking evidence for magma-water interaction) are typically well-sorted and dominated by highly vesicular (70-80\%) juvenile clasts [Houghton and Wilson 1989]. Morphologies of individual ash shards also dominantly reflect the presence of thinned bubble walls 
[Heiken and Wohletz 1991]. In contrast, phreatomagmatic fragmentation produces a broader range of vesicularities (15 to $>60 \%$ ), because variable proportions of magma are quenched early in the vesiculation process. Abundant magma-water interaction generates efficiently fragmented, finer grained particles, often with dense, blocky grain morphologies indicating that the particles did not necessarily break along bubble walls [Wohletz 1983]. However, a fundamental challenge in wet volcanism is determining how much water is 'abundant' enough to generate these kinds of changes in the eruption products. There is not yet a quantitative link between magma-water ratios and the grain size of resulting deposits [Zimanowski et al. 2003], which limits our ability to infer degrees of vent-derived water interaction from grain size characteristics alone.

Once magmatic and/or phreatomagmatic fragmentation has occurred, the pyroclastic mixture is ejected from the conduit, and may form a buoyant column of volcanic ash, gases and hydrometeors (i.e. airborne water phases such as liquid or ice particles), as described in three parts below. It is noted that the term plume is used to refer to the airborne ash that is linked or 'attached' to the source vent, whereas column refers more specifically to the vertical portion of the ascending plume. The terminology of Durant et al. [2008] is adopted in the use of volcanic cloud to describe the particle-laden suspension which may be associated with its vent or drifting freely.

Gas thrust region: As the fragmented mixture exits the vent at velocities on the order of tens to hundreds of meters per second, the basal region of the volcanic column is powered by its initial momentum, and buoyancy effects are comparatively negligible [Sparks 1986]. This gas thrust region (or jet) represents a relatively small fraction of the plume's total height, ranging from hundreds of metres to a few kilometers above the vent (Figure 1.1). The rising column decelerates as its initial kinetic energy is expended, while the bulk density of the mixture decreases from entrainment and heating of the surrounding air and, to a lesser degree, by fallout of ballistic clasts.

Convective (buoyancy-driven) region: As soon as the initial kinetic energy is expended and buoyancy effects begin to take over, the column is considered to be within the convective region (Figure 1.1). This is a pivotal stage of the ascent process because if the material becomes positively buoyant with respect to the ambient atmosphere it will continue upwards as a buoyant plume. Otherwise, partial to wholescale collapse occurs, 
sending the erupted material cascading downward into a gravity-controlled density current (as illustrated in Figure 1.1 [Woods 1988]). Coeval buoyant and collapsing behavior has been observed during historic eruptions, for example, in 1990 at Redoubt volcano, Alaska (Figure 1.2; Woods and Kienle [1994]), and is widely inferred to occur in prehistoric eruptions represented in the geologic record.

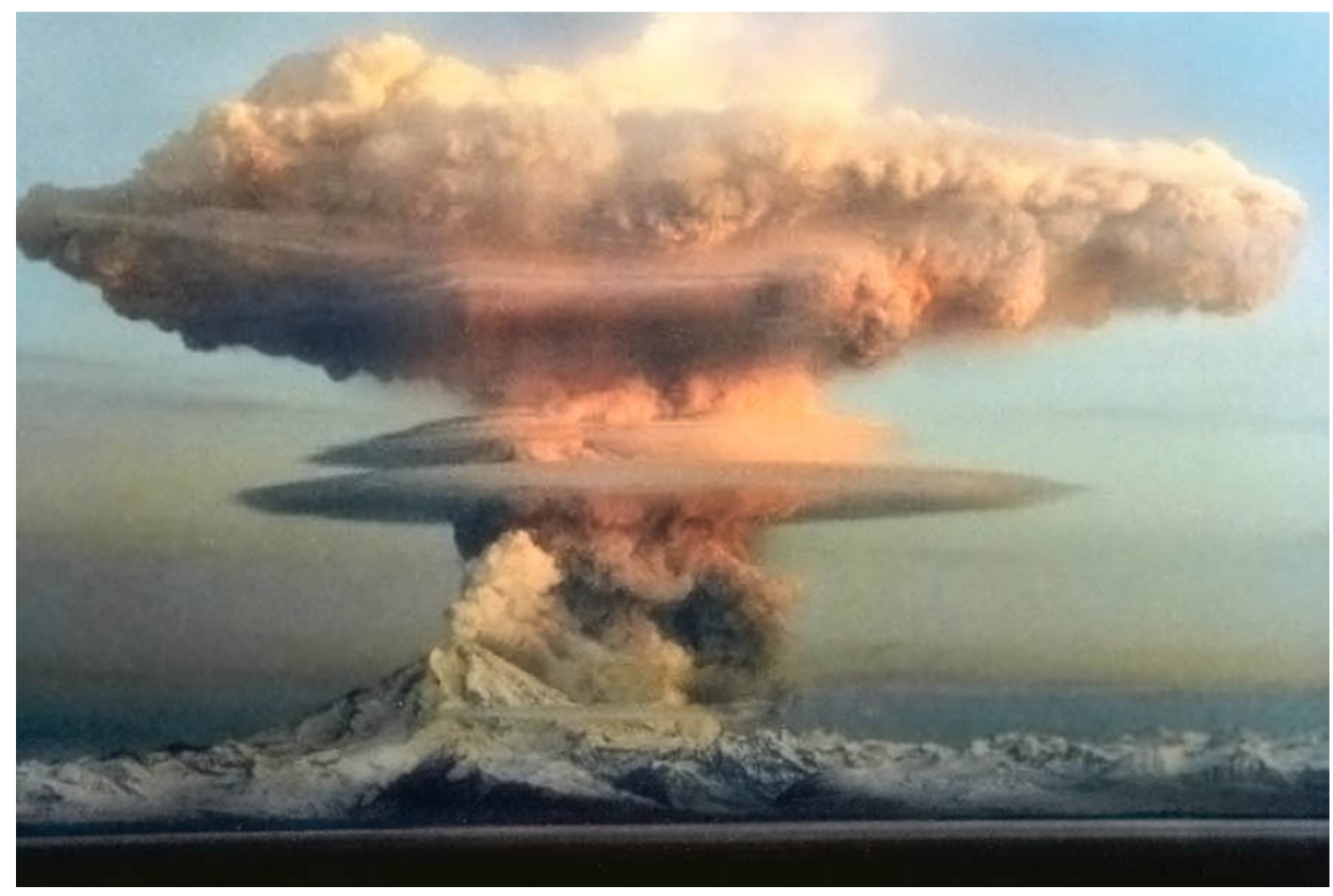

Figure 1.2 An example of a multi-level coignimbrite column lofted from PDCs during the eruption of Redoubt volcano, Alaska, on 21 April 1990. The column reached an altitude of approximately $12 \mathrm{~km}$ [Woods and Kienle 1994]. Photograph by R. Clucas from Neal et al. [2001]. Note the bent-over, collapsing eruption plume issuing from vent and simultaneously mixing with the buoyant ash rising from pyroclastic density currents. Mammatus clouds forming pouch-like protuberances on the underside of the plume are thought to reflect sublimation of ice particles in the cloud [Schultz et al. 2006].

It is conventionally assumed that the thermal energy of the pyroclasts drive buoyancy in this region by heating entrained air [Sparks et al. 1997]. An interesting effect of magma-water interaction is that the production of very fine grained material improves the efficacy of heat transfer to entrained air and promotes buoyancy [Wohletz 1983, Woods 1993]. If, however, the erupted pyroclasts (generally $700-850{ }^{\circ} \mathrm{C}$ for rhyolite) are cooled on contact with abundant external water at vent, the thermal energy initially available as sensible heat is locked up as latent heat in the water vapor. Therefore, the 
dynamics of convection are strongly influenced by the presence of water [Glaze and Baloga 1996, Graf et al. 1999]. This leads to some complex dynamics in water-rich eruptions, which are discussed in the following section on 'dirty thunderstorms'. However, on the assumption that the eruption column does become buoyant, this leads to the third region of plume development.

Umbrella region: The buoyant plume rises until its density equals that of the surrounding atmosphere (often at or near the local tropopause). Here the ash cloud has reached its level of neutral buoyancy and although it decelerates rapidly beyond this point, overshoot typically continues by $\sim 40 \%$ of the total plume height [Bonadonna and Phillips 2003]. The upward motion of the plume intruding into the neutral buoyancy level causes compensational downward motions known as Brunt-Väisälä oscillations, generating turbulence [Herzog et al. 1998, Doswell 2001] and gravity waves. The ash cloud is then injected within the boundaries of the equilibrium level as a gravity current [Sparks et al. 1997], expanding radially away from source. Atmospheric effects begin to take over as a combination of moist convection, wind advection and diffusion continue downwind along the dispersal axis. It is from this umbrella region where most particle fallout, and ultimately fallout, takes place.

\subsection{Large-scale wet eruptions as 'dirty thunderstorms'}

Although the dynamics of large-scale dry eruptions are reasonably well understood, their comparatively less common, water-rich counterparts challenge the standard approaches to plume dynamics. There is much to be gained from meteorological approaches in this regard, because the dynamic behaviors exhibited by large-scale wet eruptions operate on a spectrum between that of dry volcanism and severe thunderstorms.

As indicated in the previous section, the behavior of eruption clouds in the convective region of the plume is significantly impacted by the microphysics of added (nonmagmatic) water. There are two manners in which this occurs. The first is by magmawater interaction at the source, producing a relatively cooler mixture of ash and water vapor, with much of the energy stored as latent heat [Koyaguchi and Woods 1996, Herzog et al. 1998, Veitch and Woods 2001]. The second is by entrainment of abundant 
water vapor from the surrounding atmosphere during ascent of the volcanic cloud [Glaze et al. 1997]. In both cases, a significant 'boost' of energy is attained when the water vapor becomes cool enough to condense and release latent heat, triggering deep moist convection not unlike that in a severe storm [Kessler 1992, Doswell 2001]. This process has the potential to boost ash clouds much higher than by dry convection alone [Tupper et al. 2009].

In normal, meteorological ('clean') thunderstorms, the ascent dynamics are driven by moist convection, with much weaker ground-level vertical velocities than in a typical volcanic column. Arising from buoyant instability, convection is dominated by the microphysics of water phase changes [Cotton and Anthes 1989, Houze 1993]. In a standard meteorological scenario, solar energy heats the lower atmosphere, transporting sensible and latent heat through vertical updrafts [Kessler 1992]. If this energy accumulates at lower levels, parcels of air may acquire enough buoyancy to lift moist air beyond the condensation zone, where hydrometeors, such as airborne water droplets or ice, begin to form. In turn, regions of the cloud become negatively buoyant by the cooling effects of evaporation or sublimation and by the 'loading effect' of hydrometeors. These parcels are carried into downdrafts, initiating the vigorous internal circulation of severe storms.

One of the most striking examples of this microphysical process interacting with volcanism was observed after the 1991 activity of Mount Pinatubo in the Philippines. Oswalt et al. [1996] noted that post-eruptive activity related to secondary explosions associated with the hot volcanic deposits were frequently accompanied by formation of cumulus clouds, which developed into cumulonimbi. These 'volcanic thunderstorms', as they were called, generated multiple convective cells and produced abundant rainfall and ash-laden rain. A number of factors are thought to have locally enhanced convective instability in this case, including emissions of steam during the secondary explosions, ash particles providing condensation nuclei in the lower troposphere, an overall increase in ground-level heat from the volcanic deposits. These sources of instability persisted as long as 11 years after the climactic eruption [Tupper et al. 2005].

An important difference between the ascent of 'clean' versus 'dirty' (ash-laden) moist convection is the initial thermal energy and momentum provided from a volcanic 
eruption. The energy budget of a volcanic cloud is 5-6 orders of magnitude larger than that of a conventional storm. Furthermore, the abundance of ash particles provides abundant condensation nuclei [Lathem et al. 2011] and ice nuclei [Textor et al. 2006a, Durant et al. 2008]. This has two primary implications for plume dynamics: 1) atmospheric inversions such as the tropopause do not pose as significant a barrier to ascent, and 2) the condensation zone is much more readily attainable, meaning that deep moist convection occurs readily in large-scale 'wet' volcanic eruptions.

The dirty thunderstorm phenomenon induces some complicating factors into the behavior of volcanic eruptions, and the dynamics of the process are poorly understood. For volcanic ash forecasting, it means that both the volcanological and meteorological factors (e.g. convective available potential energy) need to be integrated into the predictions. Furthermore, it is difficult to distinguish if water-rich clouds are necessarily ash-laden. Are all cumulus or cumulonimbus associated with eruptions necessarily dangerous to aircraft, requiring incorporation into ash cloud forecasts? Another problem is that moist convection confounds predictions of the height of volcanic ash clouds (Tupper et al. 2007). Even a relatively small-scale eruption may be able to ascend to stratospheric heights purely by moist convection, and in the absence of a vigorous gas thrust region. The pioneering work of Woods [1993] first considered the thermodynamic impact of microphysics on volcanic cloud heights. This and subsequent work [Glaze et al. 1997, Veitch and Woods 2001] suggested that vapor entrainment would have important effects in smaller, weaker eruptions because they develop convection at lower altitudes, where significantly more moisture can be entrained. It was indicated that for these weaker eruptions, the entrained water vapor from the lower few kilometers of the atmosphere may be comparable to or even exceed the amount of particles in the volcanic column [Woods 1993, Veitch and Woods 2004]. In contrast, these early models suggest that larger, more sustained eruption columns maintain high velocities throughout the moist lower regions of the atmosphere, limiting entrainment of buoyancy-promoting air and water vapor. These observations suggest a role reversal in the ascent of volcanic plumes, in that smaller events may be capable of ascending higher than the most powerful examples.

Koyaguchi and Woods [1996] maintained that even large-scale phreatomagmatic eruptions may experience this limitation on ascent. Low water contents at vent $(\leq 25-35$ 
wt.\%) were modeled to lighten the erupted mixture enough to promote buoyancy. But with involvement of $>40-50$ wt.\% water, insufficient sensible heat was available to drive the plume upward, and cool damp pyroclastic flows shed from the modeled column. In the extreme instance, the column was expected to remain negatively buoyant, resulting in wholesale collapse and the formation of widespread pyroclastic density currents (PDCs) and coignimbrite ash clouds (which loft from PDCs) [Sparks et al. 1997, Gilbert and Sparks 1998].

\subsection{Volcanic ash aggregation}

A further distinguishing characteristic of wet eruptions is the abundance of volcanic ash aggregation. Ash aggregates, which are clusters of individual particles brought together by attractive forces, are known to be a significant factor in the transport and fallout of eruption clouds [Carey and Sigurdsson 1982]. They have been identified in some of the oldest rocks on Earth [Self and Sparks 1979, Raza et al. 2001], at meteorite impact sites [Salge et al. 2000, Pufahl et al. 2007, Branney and Brown 2011] and on Mars [Knauth et al. 2005, Wilson and Head 2007, Arvidson et al. 2008]. Despite a wide and varied range of occurrences, the mechanisms by which aggregates form in volcanic ash clouds are of particular interest, and the subject of ongoing debate, in studies of explosive volcanism.

Perhaps one of the most impressive early investigations of volcanic ash aggregates was undertaken in the 1920s by local New Zealander and physician, J. Allan Berry. Examining distal fall deposits of the $\sim 25.4$ ka Oruanui eruption preserved on the North Island's east coast, Berry [1928] documented the internal structure of the aggregates using an x-ray scanning technique (Figure 1.3) that has never been reproduced until the present work, 84 years later. Modern descriptions of ash aggregates now characterize a spectrum of different types [Sparks et al. 1997, Houghton et al. 2000]. Distinguished on the basis of textural and morphological features (not unlike the pioneering work of Berry [1928]), each type is inferred to be linked to the presence or absence of liquid water supplied to the forming cluster [Gilbert and Lane 1994]. The dry end-member types are fragile, irregularly-shaped chains or clusters of ash particles, inferred to have come together by electrostatic attraction [Schumacher 1994, James et al. 2002, 2003], mechanical entanglement of grains [Sorem 1982] or small amounts of liquid [Durant et 
al. 2009]. The wet end-member types are essentially ash-laden drops, or 'mud rain' [Walker 1981b], which splash upon impact with the ground and merge into a liquefied, vesicular deposit [Rosi 1992, Schumacher and Schmincke 1995]. Between the two endmembers are intermediate varieties most commonly known as accretionary lapilli [Moore and Peck 1962]. These are the varieties described and termed 'chalazoidites' by Berry [1928]. Owing to a more coherent structure, accretionary lapilli are the most frequently preserved aggregate types in the geologic record, and are lapilli-sized, spheroidal pellets (2-64 $\mathrm{mm}$ diameter, although some examples may be smaller or larger) composed of ash-sized particles $(<2 \mathrm{~mm})$. They may be internally massive or contain concentric laminations around a coarser-grained core [Schumacher and Schmincke 1991]. Some examples contain a single, larger clast at the center, known as armored or coated lapilli [Waters and Fisher 1971, Fisher and Schmincke 1984].
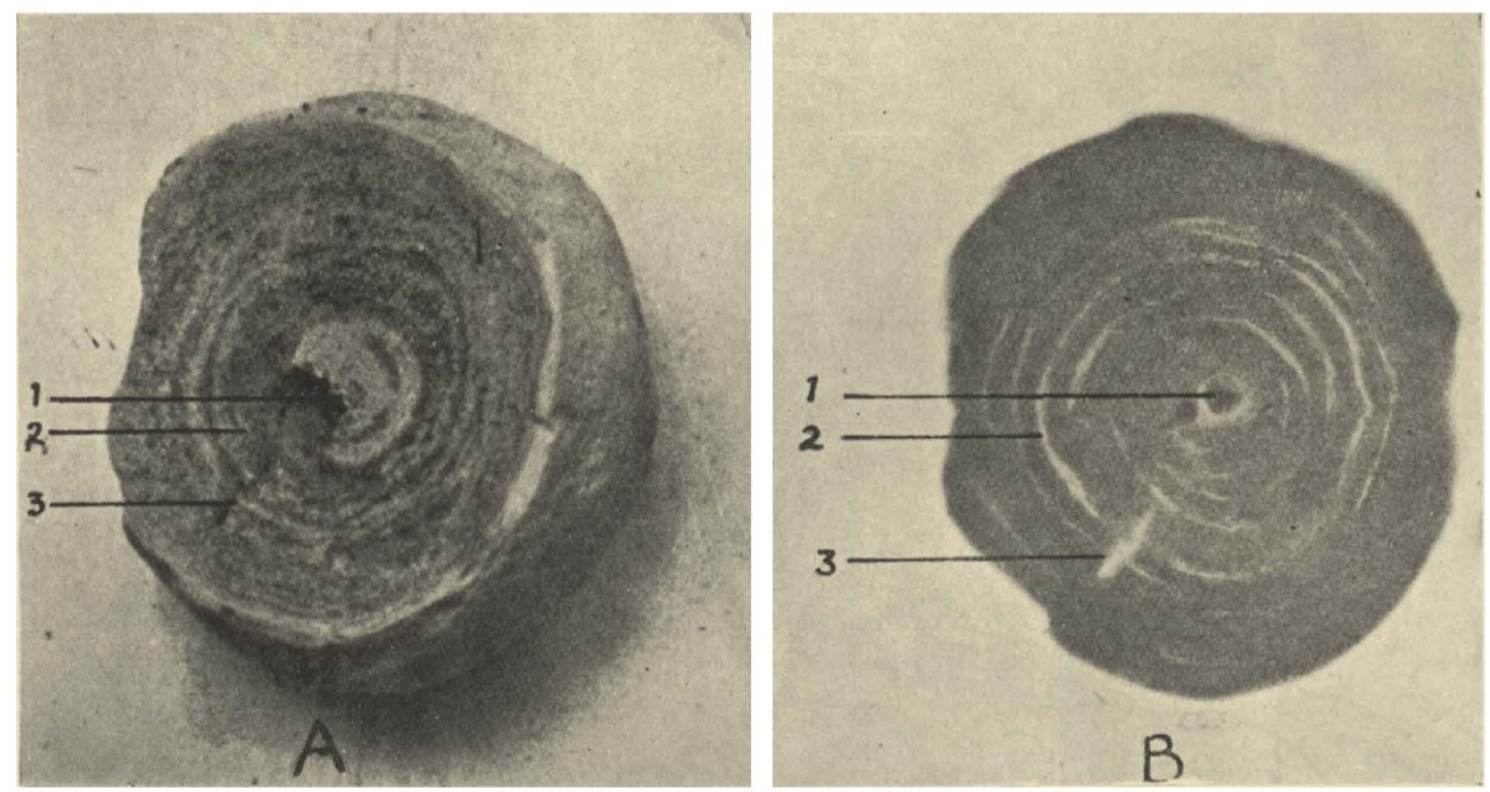

Figure 1.3 Detail of an accretionary lapillus (or 'chalazoidite') from the $25.4 \mathrm{ka}$ Oruanui eruption deposits. Diameter is approximately $2.5 \mathrm{~cm}$. The freshly broken crosssection in (a) exhibits multiple concentric layers around a central core. (b) Shows an Xray scan of the corresponding layers. Reproduced from Berry [1928].

\subsubsection{Key existing challenges regarding ash aggregation}

Although it is generally agreed that the formation of each aggregate type is influenced by the presence of liquid water and/or ice, electrical charging, and hygroscopic compounds in volcanic clouds, the wealth of information that may be recorded in the structure and distribution of ash aggregates remains largely untapped. The following 
addresses some of the key questions that target areas of uncertainty in this emerging sub-discipline of explosive volcanism.

Terminology: Even on the most basic level, the framework for detailed descriptions of ash aggregates is a matter of some controversy. Owing in part to the diversity of volcanic and depositional environments in which aggregates are found, the terminology has undergone piecemeal modifications over time to account for the unique assemblages documented in each separate field study [e.g. Reimer 1983, Schumacher and Schmincke 1991, Thordarson 2004, Brown et al. 2010]. Although this iterative process has expanded the variety of aggregate types recognized in volcanic clouds and their deposits, there are currently a number of (sometimes conflicting) classification schemes active in the volcanological literature and related disciplines. Recently, Brown et al. [2012] proposed to unify the existing terminology. They suggested 'accretionary lapilli' be abandoned altogether and replaced by the size-independent term 'accretionary pellets'. The accretionary pellets are further distinguished into massive versus concentrically layered types (termed AP1 and AP2, respectively), and mud rain (AP3) [Brown et al. 2012]. However, it remains to be seen if this framework is sufficiently flexible for more detailed field observations to be distinguished, quantified and compared between field areas.

Location and timing: The location of aggregate formation in eruption clouds and the timescales over which they develop are both areas of active investigation. Textor et al. [2006b] have demonstrated numerically that aggregation rates are much faster in cloud regions with higher particle concentrations and liquid content. However the relative abundance of aggregation processes in the column versus umbrella cloud, and accompanying timescales of formation, are yet to be extensively tested with field data. Although aggregates have been observed falling directly out of explosive eruption plumes [Brazier et al. 1982, Tomita et al. 1985, Gilbert and Lane 1994], observations from prehistoric volcanic deposits have recently challenged conventional views of aggregate development. Based on the abundant occurrence of accretionary lapilli in the deposits of pyroclastic density currents [e.g. Waters and Fisher 1971, Schumacher and Schmincke 1991, Brown et al. 2007], Brown et al. [2010] have proposed that some varieties may develop primarily in association with ground-hugging flows. This hypothesis stands in marked contrast to the classic view of accretionary lapilli forming 
solely in buoyant, high-level clouds [Moore and Peck 1962, Reimer 1983]. Can the identification of particular aggregate structures be used to infer vertical fallout versus laterally-emplaced flow? Detailed field studies of active eruptions [Bonadonna et al. 2011b, Taddeucci et al. 2011] and volcanic deposits, combined with further experimental [Gilbert and Lane 1994] and numerical modeling [Textor et al. 2006a, b] are required to test these concepts.

Binding forces: The dominant force in accretionary lapilli formation is inferred to be liquid-coated ash particles colliding and adhering by liquid tensional forces when their paths intersect [Gilbert and Lane 1994]. However, the respective roles of electrostatic attraction, ice formation and hygroscopic compounds are poorly defined on the spectrum of aggregate types. For example, are certain types or textures necessarily linked to the presence of ice in volcanic clouds (c.f. Brown et al. [2012])? Furthermore, it is widely accepted that volcanic ash aggregates require a more permanent source of cohesion (i.e. solid bridges) to survive impacts with the ground and to be preserved in the geologic record [Sparks et al. 1997]. Precipitation of secondary alteration minerals or salts, such as $\mathrm{CaSO}_{4}$ from acidic liquids, has been inferred to play a central role in keeping accretionary lapilli intact once water phases have been driven off [e.g. Tomita et al. 1985, Gilbert and Lane 1994, Scolamacchia et al. 2005]. However, ash aggregates from many volcanoes lack evidence for these 'secondary' films [e.g. Bonadonna et al. 2002], inviting alternative mechanisms to explain their structural integrity.

Impact on volcanic clouds: The aggregation process is highly size selective, preferentially collecting finer ash particles from a wider population by wet processes [Gilbert and Lane 1994, Schumacher and Schmincke 1995] and dry, electrostaticallydominated processes [Schumacher 1994, James et al. 2002]. This has important implications for airborne concentrations of volcanic ash, fallout patterns and predictions of ash loading at the ground. A classic observation is the 'Ritzville Bulge' in fall deposits from the 1980 eruptions of Mount St Helens [Sarna-Wojcicki et al. 1981a], a secondary maximum in deposit thickness hundreds of kms downwind of vent, attributed to the formation and fallout of particle clusters [Carey and Sigurdsson 1982, Sorem 1982, Brazier 1983]. This phenomenon has since been observed to occur in many eruption deposits generating fine ash [e.g. Scasso et al. 1994, Rose et al. 2001]. Another interesting impact on fallout patterns is that abundant near-vent deposition of fines has 
been observed to result in fall deposits that coarsen with distance from vent [Smith 1998, Wilson 2001]. This is a reversal of the typical pattern of fall deposits becoming finer and better sorted with distance from vent [Walker 1971]. The nature and style of aggregation occurring in volcanic clouds (particularly water-rich clouds) may thus have complex impacts on transport and depositional patterns.

\subsubsection{Volcanic hailstones: parallels and limitations}

The concept of aggregates forming like hailstones is not a new idea [e.g. Berry 1928, Kato 1986, Hayakawa 1990], but one that has seen renewed interest in recent years with the growing recognition of a strong microphysical influence on water-rich eruption plumes [Textor and Ernst 2004, Textor et al. 2006a, Durant et al. 2009]. The comparison is intuitive; for example, thin sections of accretionary lapilli and typical hailstones exhibit similar internal layering, with sharp to gradational contacts around a central core (Figure 1.4a-b). Comparative parallels are made even more attractive by the extensive meteorological literature dedicated to reconstructing cloud conditions from the microstructure of hail [e.g. Gokhale 1975, Pruppacher and Klett 1978]. Furthermore, the presence of ice in volcanic clouds is known to be abundant [Rose et al. 1995], and numerous accounts exist of ice or icy ash falling coevally with other volcanic pyroclasts [Brown et al. 2012]. During the 1969 Ruapehu eruption, for example, ash aggregates landed frozen and subsequently melted into a slurry (Figure 1.4c-d [Healy et al. 1978]). Some of the hailstone-derived approaches may thus lend insights into the temperature and velocity structure of volcanic clouds.

As in volcanic aggregation, the growth of hail operates along a continuum of dry and wet processes. The dry end member process, known as riming, occurs at temperatures below freezing by collection of supercooled water droplets [Pruppacher and Klett 1978]. Rime accretes as a low-density lattice of frozen drops, generating an opaque appearance (Figure 1.4a). If the hailstone is transported to warmer cloud regions, or alternatively, if the latent heat released during freezing is not adequately dissipated from the surface, wet growth occurs (typically at temperatures around $0{ }^{\circ} \mathrm{C}$ [Ludlam 1950]). In the wet growth regime, supercooled liquid drops are collected too rapidly to freeze completely, forming a 'spongy' texture of ice and liquid water [Knight 1968]. The freezing of liquid water forms dense, translucent ice (Figure 1.4a). This process of low- 

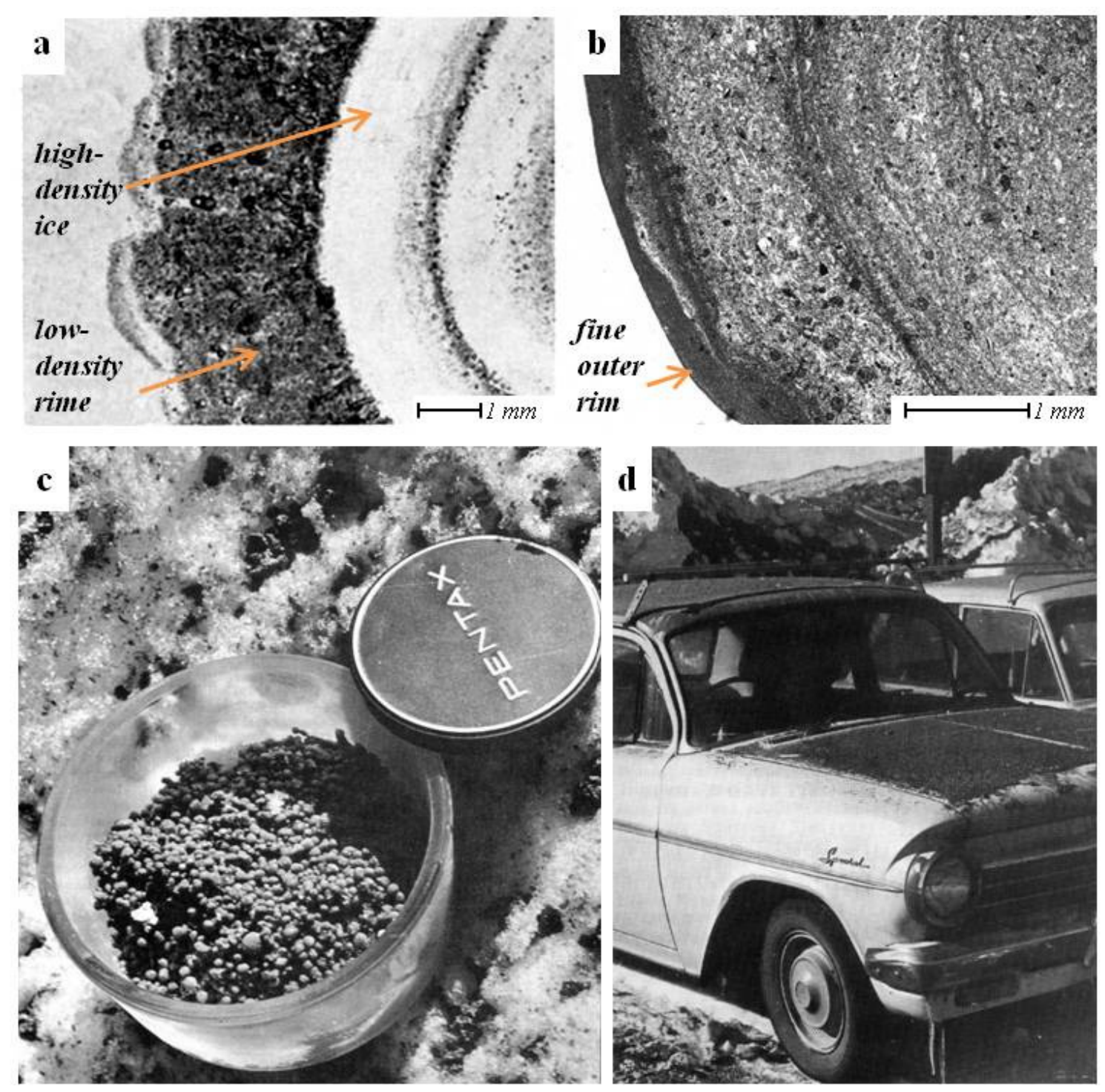

Figure 1.4 (a) Thin section photomicrograph in transmitted light of a typical hailstone, showing distinct layers of low-density rime and high-density ice. Reproduced from Knight and Knight [1968]. (b) Shows the internal layering of a thin-sectioned accretionary lapillus from the Oruanui eruption for comparison (unit 8 from locality 1622 of Appendix A), which also exhibits alternations between low-density layers of medium ash $(<300 \mu \mathrm{m})$ and high-density layers of ultrafine ash $(<10 \mu \mathrm{m})$. (c) Examples of frozen ash aggregates collected from volcanic fallout at Whakapapa Village during the 22 June 1969 eruption of Ruapehu, New Zealand. The aggregates range 1-6 mm diameter. (d) After landing, the 'frozen pellets of ash and ice' melted into a cohesive slurry. Photos by E.F. Lloyd, reproduced from Healy et al. [1978].

density riming followed by soaking and subsequent freezing comprises the standard two-stage growth model of hailstones [Prodi 1970]. Discrete layers, lobes and radial lines of bubbles in hail are interpreted to form this way [Knight and Knight 1968, Prodi et al. 1986]. Furthermore, the overall density and fall velocity of hailstones depend on the relative proportions of these low-density rime, high-density crystalline ice and liquid 
layers [Knight and Heymsfield 1983]. Hailstones therefore move upward or downward in deep convective clouds during various stages of growth, in a process termed microphysical recycling by Pflaum and Pruppacher [1978].

It is tempting to consider that the growth of discrete layers in volcanic ash aggregates may progress in a similar fashion, driven primarily by changes in temperature and the relative stability of liquid water versus ice. To simply translate the hail model, the lowdensity coarser grained core of volcanic ash aggregates would represent rime (dry growth), while the densely-packed fine rim would represent a liquid layer (wet growth) that subsequently froze. However, it has been demonstrated experimentally [Gilbert and Lane 1994, Schumacher and Schmincke 1995] that the textures of accretionary lapilli cores can be replicated in the presence of liquid water alone (i.e. without ice). Therefore it is difficult to ascertain the role of liquid-free, ice-initiated aggregation in volcanic clouds. The critical differences between 'clean' versus 'dirty' convective clouds, as mentioned earlier, impose further limitations on the applicability of the hailstone conceptual model. For example, how much supercooled liquid water can persist in an ash-laden volcanic cloud? Riming of supercooled liquid drops on pre-existing hail embryos is central to hailstone growth. However, the abundance of ice nuclei in the form of ash particles [Durant et al. 2008] in volcanic clouds suggests that hail formation may be suppressed due to overseeding. This situation would favor the formation of abundant ice-coated individual particles [Houze 1993], which may then adhere to one another via dry or wet mechanisms [Textor et al. 2006a, b]. Another limitation is the role of volcanic processes, such as strong thermal gradients, and abrasion due to turbulence in areas with dense particle concentrations that could induce breakage, all of which are poorly understood.

\subsection{The $\sim 25.4$ ka Oruanui case study}

A number of historic eruptions that have demonstrated the key features discussed so far in this chapter, including extensive interaction with external water, 'dirty thunderstorm' type behavior, and widespread occurrence of volcanic ash aggregates. For example, the 1980 eruptions of Mount St Helens, Washington [Lipman and Mullineaux 1981], and 1991 eruptions of Pinatubo volcano in the Philippines [Newhall and Punongbayan 1996], both illustrated aspects of these phenomena. However, no directly observed 
event has attained the level of complexity and dispersal power as those preserved in the geologic record. The archetypal examples of phreatoplinian behavior are thus inferred from physical characteristics of their deposits. These include the eruptions of (1) Layer C from Askja, Iceland, in 1875 [Sparks et al. 1981]; (2) Hatepe and Rotongaio units from the $\sim 1.8$ ka Taupo volcano [Walker 1981b, Smith and Houghton 1995a,b]; and (3) the 25.4 ka Oruanui eruption, also from Taupo volcano [Self 1983, Wilson 2001]. Numerous other localities worldwide also record the occurrence of phreatoplinian activity. Italy's 79 AD Vesuvius eruption [Cioni et al. 1992], Middle Peistocene Colli Albani [De Rita et al. 2002] and $\sim 12$ ka Neapolitan Yellow Tuff [Orsi et al. 1992], Greece's $~ 1640$ BC Minoan eruption [Bond and Sparks 1976] and 160 ka Kos Plateau Tuff [Allen and Cas 1998]; and the English Lake District's Ordovician Whorneyside Tuff [Branney 1991] are all documented examples.

Amongst the preserved deposits of phreatoplinian eruptions, the $\sim 25.4$ ka Oruanui eruption is unique in two respects. First, it represents one of the largest and most widespread examples of wet volcanism yet documented (exceeded only by the $1 \mathrm{Ma}$ Kidnappers-Rocky Hill deposits from Mangakino caldera, New Zealand [Wilson et al. 1995a, Cooper et al. 2012]). Second, it is exceptionally well preserved [Wilson 1993]. Preservation of even the finest vitric ash in most field exposures, an atypical feature of many prehistoric supereruption deposits, facilitates detailed grain size analysis on an enormous diversity of depositional features. The Oruanui eruption deposits thus provide a rich and varied catalogue of eruptive phenomena related to the most extreme example of magma-water interaction on Earth.

\subsubsection{Geological setting}

The North Island of New Zealand straddles a major crustal boundary (Figure 1.5a) where the Pacific plate subducts obliquely beneath the Australian plate at a rate of $\sim 45$

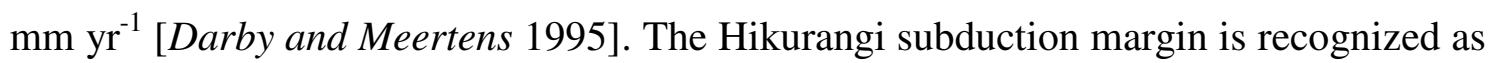
the continuation of oceanic backarc spreading southward into New Zealand's continental crust, producing a rifted volcanic arc known as the Taupo Volcanic Zone (TVZ) [Gamble et al. 1989, Smith et al. 1989]. Below the central TVZ, at depths of 8$15 \mathrm{~km}$, magnetotelluric studies have identified a mush zone of $<4 \%$ partial melt that likely contributes to delivery of magma to the thin, actively extending crust overhead 
[Heise et al. 2007]. Over the past ca. 2 Ma this activity has manifested on the surface as a $300 \mathrm{~km}$-long arc of volcanic vents and calderas, forming the locus of young volcanism in New Zealand and hosting the world's most productive silicic, arc-related activity [Wilson et al. 1995b]. Taupo volcano, in particular (Figure 1.5b), has produced three of the type-examples of phreatoplinian eruptions [Houghton et al. 2000].
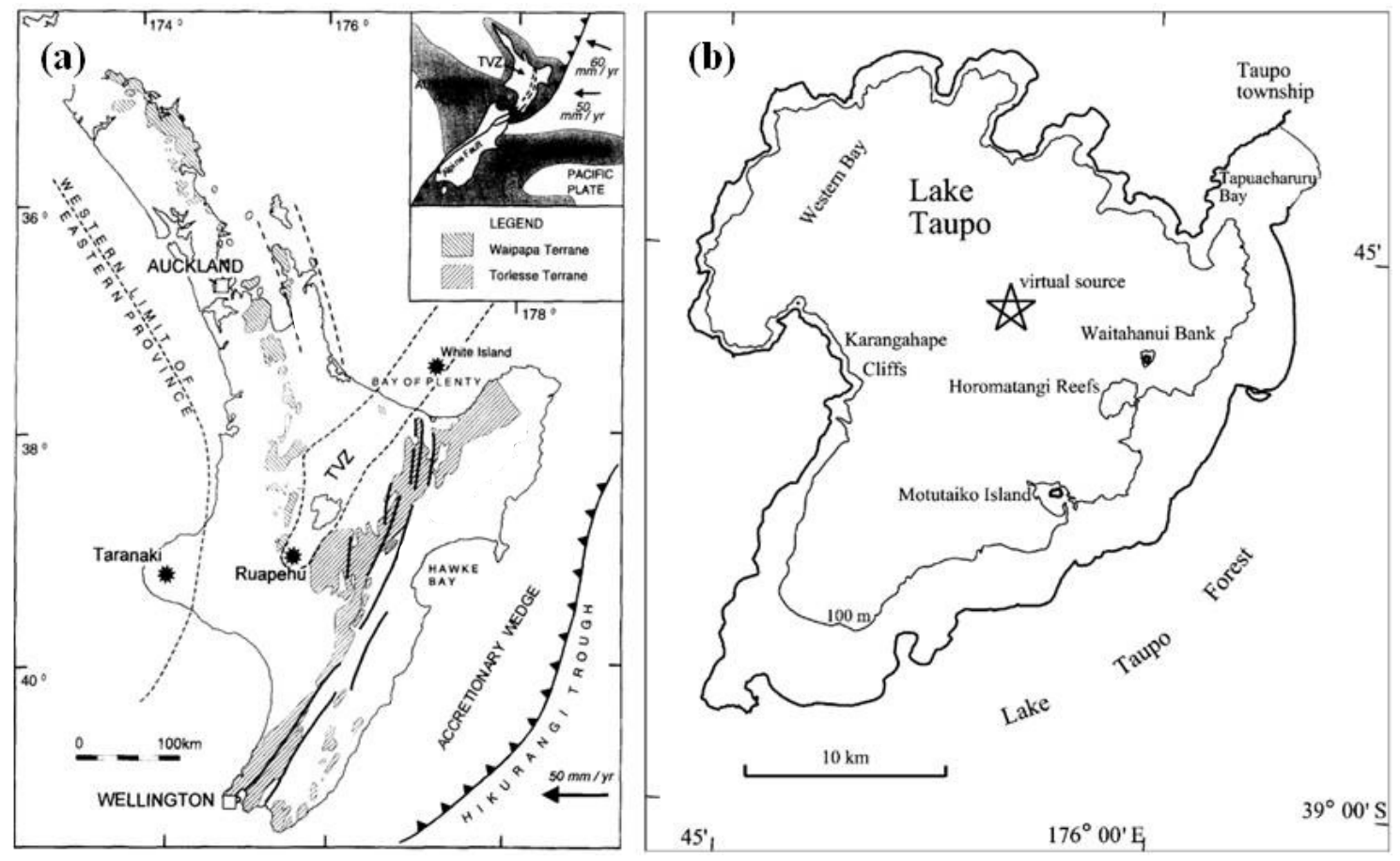

Figure 1.5 (a) Tectonic map of New Zealand's North Island, showing the location of the Taupo Volcanic Zone and Lake Taupo with respect to the active Hikurangi Trough (from Graham et al. [1995]). (b) Enlarged map of modern Lake Taupo, showing the post-Oruanui $140 \mathrm{~km}^{2}$ structural caldera (inner contour), and virtual source of Oruanui magmatism (star) represented by the geometric center of the collapse caldera (from Wilson [2001]).

\subsubsection{Physical characteristics}

Although the $\sim 25.4 \mathrm{ka}$ Oruanui eruption excavated the present day geometry of Lake Taupo caldera (Figure 1.5a) and produced some of the most widely dispersed volcanic deposits on the planet [Wilson 1994, 2001], the unusually fine grain size of the deposits and unremarkable appearance at outcrop scale belie its powerful emplacement history (Figure 1.6); more than $80 \%$ of the bulk eruption products could slip through the mesh of a tea strainer $(<500 \mu \mathrm{m})$. Only by mapping the occurrence of the Oruanui layers more than 1,000 km from source [Carter et al. 1995, Carter et al. 2004] does the incredible dispersal power of this eruption begin to come into view. It is a feature that has 
provided a crucial time marker for Last Glacial Maximum paleo-reconstructions throughout the Southwest Pacific [e.g. Pillans et al. 1993, Carter et al. 1995, Newnham et al. 2007].

Although the name of the eruption products has itself been a subject of debate (see Frogatt et al. [1988]), two identifiers are presently in use. The name Oruanui is used in studies recognizing the physical volcanology of the eruption deposits [Wilson 2001], which includes the present work, whereas tephrochronological studies use the name Kawakawa Tephra [Vucetich and Howorth 1976] or Kawakawa/Oruanui Tephra [Froggatt and Lowe 1990]. For the purposes of this thesis, the Oruanui eruption is taken to have occurred at approximately $\sim 25.4 \mathrm{ka}$ after Vandergoes et al. [2011], although the exact accepted age fluctuates by approximately $2 \mathrm{ka}$ with each recalibration of the radiocarbon calendar. Since charcoal fragments enclosed in the ignimbrite were dated to $22,590 \pm 230{ }^{14} \mathrm{C}$ yr B.P. by Wilson et al. [1988], the age of the eruption has varied from $26.5 \mathrm{ka}$ [Wilson 2001] to $27 \pm 1 \mathrm{ka}$ [Lowe et al. 2008] to the current $225.4 \mathrm{ka}$ [Vandergoes et al. 2011]. However, it is undisputed that the eruption coincided with the last glacial period in New Zealand [Alloway et al. 2007], during which the climate was a few degrees cooler on average, and stronger westerly winds swept across the North Island [Drost et al. 2007a, Lorrey et al. 2012].

During the Oruanui eruption, on a timescale of weeks to months, $\sim 530 \mathrm{~km}^{3}$ of highsilica rhyolite (dense rock equivalent) was erupted in ten major pulses identified from bedding in the individual fall deposits [Wilson 2001]. Multiple vents were active at various stages of the eruption, climaxing in large-scale caldera collapse in phase 10. For distances from vent are calculated with respect to a virtual source in the geometric center of the Oruanui collapse caldera [Wilson 2001]. Extensive evidence for magmawater interaction at the time of eruption has been inferred from: (1) the prevalence of vesiculated tuff and ash aggregates [Self and Sparks 1978], (2) characteristically 'blocky', poorly vesiculated ash shard morphologies [Heiken and Wohletz 1985], (3) stratigraphic evidence of a large paleo-lake preceding the eruption [Grindley 1965, Manville and Wilson 2004], and (4) entirely non-welded ignimbrite deposits, determined by paleomagnetic techniques to have been emplaced at relatively cool temperatures of 150 to $250{ }^{\circ} \mathrm{C}$ [Wilson and McClelland unpublished data]. 

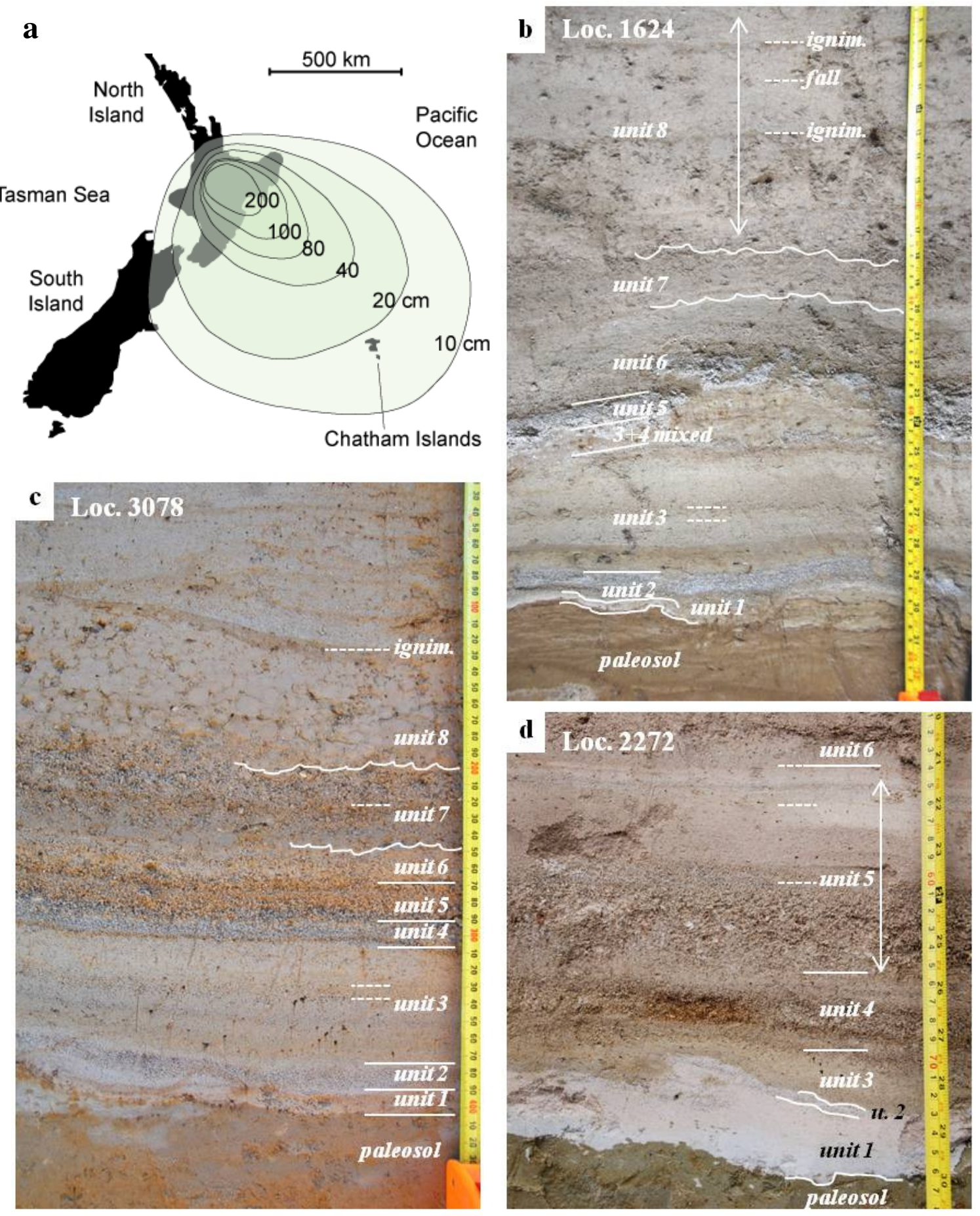

Figure 1.6 Distribution and field characteristics of the Oruanui eruption deposits. (a) Total fall deposit thickness isopachs (values in $\mathrm{cm}$ ) from Wilson [2001]. (b) Oruanui deposits at Locality $1624(\sim 81 \mathrm{~km}$ from virtual source). Note the coarse middle band in unit 3 and intercalated fall and laterally-emplaced material in unit 8. (c) Locality 3078 ( $\sim 85 \mathrm{~km}$ from source), showing the basal fall deposit sequence scoured by overlying ignimbrite. Note the coarse upper portion of unit 7 and coarse middle band in unit 3. (d) Locality 2272 ( 102 km from source), showing the fine top of unit 5. Note the upper 2$3 \mathrm{~cm}$ of unit 5 shows evidence for wind reworking before emplacement of unit 6 . 
The last of these lines of evidence is central to the behavior of the water-rich eruption plumes. For the purpose of illustration, assume that the Oruanui magma lost the majority of its sensible heat during mixing with water at the vent, cooling from an initial magmatic temperature of $770 \pm 30{ }^{\circ} \mathrm{C}$ [modified from Wilson et al. 2006] down to 250 ${ }^{\circ} \mathrm{C}$. This would require interaction with ca. $20 \mathrm{wt} . \%$ water. If the relationship held for the duration of Oruanui-related activity, approximately $100 \mathrm{~km}^{3}\left(10^{14} \mathrm{~kg}\right)$ of lake water would have been vaporized during the eruption and entrained into the atmosphere. Vaporization drains an enormous amount of energy from the erupting mixture, and is stored in the water vapor until a phase change can occur (e.g. condensation or deposition). In this case, the release of latent heat of condensation would be on the order of $10^{19} \mathrm{~J}$ based on the incorporation of lake water alone. That is 5-6 orders of magnitude larger than the energy released during a modest thunderstorm [Doswell 2001] and 4 orders of magnitude larger than a 1-megaton bomb - albeit, averaged over the ten phases of eruption. Such an extraordinary redistribution of energy in the volcanic column is a distinguishing feature of phreatoplinian eruptions, and would have certainly fundamentally redefined the 'dirty thunderstorm' dynamics of the Oruanui ash clouds.

One of the foremost conundrums in studies of wet volcanism hinges on this very observation [Wilson 1994, Houghton et al. 2000]. How do phreatoplinian eruptions attain such widespread dispersal if the pyroclasts are substantially cooled by magmawater mixing? Koyaguchi and Woods [1996] provided the first numerical description of the ascent of phreatoplinian eruptions. Their work integrated a simplified microphysics with a one-dimensional top-hat plume model, suggesting that volcanic columns from large-scale eruptions (i.e. with magma output rates $>10^{7} \mathrm{~kg} \mathrm{~s}^{-1}$ ) were unlikely to become buoyant. Although this was consistent with previous predictions that phreatoplinian eruption columns should collapse into cold, damp ground-hugging density currents [Wilson et al. 1978], field observations of extremely water-rich, extremely widespread deposits from the Oruanui eruption have provided scope for reassessment of the previous approaches.

\subsection{Models of explosive volcanic eruption plumes}

This section provides a critical assessment of the numerical models currently applied to problems in explosive volcanism. Plume modeling applications focus on diverse, but 
interrelated areas, including: (1) near-real time forecasting and predicting ash cloud movement and fallout from active eruptions, (2) sensitivity studies isolating governing factors in eruption dynamics, (3) reconstructing prehistoric or sparsely observed eruptions, and (4) initializing climate simulations to investigate long-term atmospheric impacts. Development of these numerical applications to recent and prehistoric eruptions has evolved rapidly over the past 10-15 years, roughly keeping pace with dramatic advances in computing power. While this section reviews some of the recent advances in model applications to ash cloud behavior, it specifically focuses on identifying the strengths and limitations of the various approaches as they apply to active eruptions and prehistoric events inferred from their deposits. First, the existing numerical approaches are categorized according to their targeted plume phenomena, setting out the conceptual basis and strengths and limitations of each. This is followed by an examination of volcanic processes that are primary sources of uncertainty in modelling efforts.

\subsubsection{Categories of numerical models}

Broadly speaking, volcanic plume modelling approaches fall into three general categories, each of which has traditionally focused on either the dynamics of the column or dispersal of the ash cloud.

\section{Averaged integral models}

Much of the theoretical basis of integral theory stems from early descriptions of turbulent fluid jets by Prandtl [1954] and Morton et al. [1956], which originally focused on the buoyant rise of smoke stack emissions. The field-based volcanological application of this theory began to take shape as George Walker's quantitative approach to pyroclast dispersal [Walker 1971, Walker et al. 1971, Walker 1973] was integrated with buoyant jet theory by L. Wilson [1976] and Wilson et al. [1978]. This framework was modified by Woods [1988] to include a more rigorous description of energy conservation [Valentine and Wohletz 1989].

The integral models focus primarily on one-dimensional, vertical changes in the evolution of a steady, sustained jet injected into an ambient cross-flow. The plume is modeled as a single-phase fluid with horizontally averaged properties (e.g. density, 
temperature, mass fraction of particles). This top-hat plume concept takes one averaged value for each horizontal slice (i.e. control volume) inside the plume, and one value for the ambient. Alternatively, a Gaussian distribution of these properties can be assumed to represent the decay of a plume 'core' around its periphery. Three sub-models treat the gas thrust, convective, and umbrella regions separately. Equations for conservation of mass, momentum and energy (or specific enthalpy flux) are integrated stepwise (e.g. every $5 \mathrm{~m}$ ) along the cross-section of the steady jet from the vent to the altitude of a specified minimum velocity (e.g. $0.1 \mathrm{~m} / \mathrm{s}$ ). For a thorough review of the governing equations, which will not be duplicated here, see Bursik [1998]. The primary simplifying assumptions underlying integral theory are summarized as follows:

Boussinesq approximation: This key assumption requires that the density of the jet equals that of the surrounding atmosphere, and that kinetic energy dominates over potential energy [Morton et al. 1956]. Moreover, the model is initialized by a volume flux at source, assuming that other variables (eruption temperature, grain size distribution, and initial eruption velocity) are insignificant. The simplifying term breaks down when there are strong temperature or density gradients in the column, and when the plume transitions into the convective or umbrella region (where most of the kinetic energy has been converted to potential energy). Due to the Boussinesq approximation, buoyant plume theory only reasonably approximates the behavior of the middle section of the gas thrust region after the establishment of steady flow in the plume, but before reaching the level of neutral buoyancy.

Empirically derived entrainment: A significant difference between integral theory and more complex models is the treatment of turbulence. Rather than applying the full time-dependent Navier-Stokes equations, an empirically derived entrainment factor is used to account for the development of turbulence at the plume boundaries. Entrainment velocity is usually taken to be proportional to the plume's centerline velocity [Turner 1979], and is typically changed for each region of the plume (e.g. gas-thrust region versus convective region).

Self similarity: Averaged, integral models resolve plume behavior on a single, defined scale (i.e. there are no nested grids). Therefore, turbulent motion governing the spreading of the plume (represented by the entrainment factor) is assumed to be self- 
similar, having the same effect on the flow at every length scale. This is a crude approximation that can represent some general properties of jet behavior - however the assumption breaks down for situations in which complex turbulent eddies arise (e.g. from interaction with wind or atmospheric instability).

The overwhelming strength of integral models is that they are simple to use, have fast run times (on the order of seconds), and provide a stripped-back exploratory tool for examining first-principle controls on eruption column behavior. The work of Bursik and Woods [1991] and Woods and Bower [1995], for example, significantly expanded the understanding of conditions related to column collapse. The use of buoyant plume theory also provides first-order approximations of relationships between eruption rate and column heights from sustained plumes in an operational forecasting setting [Bonadonna et al. 2011a]. However, the necessarily simple one-dimensional treatment of plume ascent neglects many of the dynamic feedbacks observed in nature. Fundamental behaviors of cloud movement in a spatiotemporally variable wind field, and interactions between buoyant and collapsing flows cannot be resolved using integral theory, and require more complex physical descriptions.

\section{Volcanic ash transport and dispersion models}

Volcanic ash transport and dispersion models (VATDM) focus on the airborne transport and fallout of ash (on length scales of tens to thousands of $\mathrm{km}$ from source) once a volcanic cloud has been emplaced into the atmosphere. The dynamics of the column are not resolved, and with few exceptions, the ash particles are treated as 'passive tracers', meaning they do not interact with each other or influence the dynamics of the cloud. VATDM are initialized with an ash cloud of specified dimensions in a specified wind field, and the particle trajectories are calculated [Suzuki 1983]. The advection-diffusionsedimentation equations are solved to account for transport by wind, turbulent dispersion, and gravitational fallout of particles in two- or three-dimensions [Searcy et al. 1998]. Two approaches to plume modeling have arisen using this basic concept: particle tracking models and tephra fallout models, although the distinction between them has begun to fade as computational efficiency increasingly allows similar treatment of issues from both approaches (using, for example, hybrid LagrangianEulerian frameworks [Bonadonna et al. 2011a]). 
Tephra fallout models are typically Eulerian formulations to solve for advection, diffusion and sedimentation of airborne volcanic ash on a plume-centered, fixed grid [Armienti et al. 1988, Macedonio et al. 1988, Glaze and Self 1991, Hurst and Turner 1999]. The Eulerian framework is advantageous for solving complex interactions at multiple points in gridspace. A highly simplified atmosphere is usually used, assuming horizontal layers with constant wind velocity. Tephra fallout models tackle the effects of lateral wind and atmospheric diffusion on particle behaviour by combining advection-diffusion equations with a sedimentation model. Examples include ASHFALL [Hurst and Turner 1999, Turner and Hurst 2001], FALL3D [Folch et al. 2012], and TEPHRA2 [Bonadonna et al. 2005]. These are frequently applied to reconstructions of field deposits using the inversion method [Connor and Connor 2006], but also used for forecasting ash loading at the ground during eruptions [Hurst and Turner 1999].

On the other hand, particle-tracking models are classically Lagrangian formulations of pollutant dispersion, in which the components are calculated at moving points [e.g. Tanaka 1994]. Lagrangian frameworks are well suited for handling emissions from a single point in space (point source), and for situations in which interactions are spatially limited. Each of the nine Volcanic Ash Advisory Centers worldwide rely on one (or a combination of) particle-tracking VATDM. Programs are thus designed to be fastrunning in an operational emergency context, and runtimes can be on the order of one to tens of minutes.

Required input or 'source term' characteristics of the cloud being modeled by VATDM can be very complex, specifying the particle size distribution, erupted mass and cloud dimensions. However, in the most simplified running mode, all that is required for particle-tracking models, such as PUFF [Webley and Mastin 2009], is a specified windfield and location and description of the cloud (defined by a number of tracer particles or height). For more detailed modeling, a non-point source of ash can be assumed, using a prescribed plume shape to describe the vertical distribution of ash mass in the atmosphere. Buoyant plume theory (as described in the section on integral models) is also used to initialized particle-tracking models without wind effects [Sparks et al. 1997] or with wind [Bursik 2001]. Generally there is no explicit particle-particle 
interaction (with the exception of an aggregation-capable version of FALL3D [Costa et al. 2010, Folch et al. 2010]), but aggregation can also be parameterized in highly simplified ways [e.g. Folch et al. 2012].

The ability to produce robust operational forecasts using VATDM depends on numerical weather prediction and reliable ash cloud updates during eruption, in addition to the physical description of volcanic processes encoded by the model. As the volcanic cloud is passively advected by a defined wind field, gridded meteorological data is a key aspect of particle tracking. The plume is treated passively in that it is swept by wind, but does not interact with the wind field (i.e. its internal velocity structure equals that of the wind). A key strength of VATDM is their ability to use detailed meteorological data that varies in time and space for forecasts of ash cloud movements.

\section{Eruption simulations}

Eruption simulations focus on resolving localized column dynamics on scales of meters to kilometers, rather than examining the long-distance wind transport and fallout. Simulations thus investigate the complex behavior of components in a multiphase flow (i.e. gas, particles, liquids), and turbulent interactions are solved in 2- or 3 spatial dimensions. The modeled fluid is compressible, so shock waves and gravity waves can be simulated. In the case of large eddy simulations, the smaller turbulent eddies are filtered out and parameterized using a sub-grid scale model, while the larger energycarrying eddies are directly solved using computationally expensive turbulence equations [Herzog et al. 2003]. A large eddy simulation requires much less computer power than a direct numerical simulation solution of the Navier-Stokes equations for viscous flow, which solves flow dynamics on all relevant scales of motion (e.g. the PDAC model of Valentine and Wohletz [1989]). An example of a large eddy simulation adapted to plume simulations is the Active Tracer High resolution Atmospheric Model (ATHAM) [Oberhuber et al. 1998]. This approach employs full solution of the NavierStokes equations of mass, momentum, and energy conservation using a finite difference method with an implicit time step scheme. The finite difference scheme makes use of embedded boundaries (nested grids) which feed back information to the coarser grid.

Unlike the volcanic ash transport and dispersion models, simulations do not require a plume 'height' to initialize the model. Rather, the volcanic forcing is initialized by 
eruption rate, conduit geometry and physical properties of the erupted mixture. By focusing on the dynamics of the column, simulations are capable of incorporating complex processes that cannot be accurately depicted by integral models or volcanic ash transport and dispersion models. These processes include column collapse [Neri and Macedonio 1996], turbulent entrainment [Herzog et al. 2003] and microphysical feedbacks [Herzog et al. 1998, Textor et al. 2003], which necessarily require two- or three-dimensional frameworks. However, there are two key weaknesses of the approach, both stemming from the computational expense of running such complex models. First, simulations currently employ a simplified description of the atmospheric wind field that is horizontally homogeneous and fixed in time. This becomes an important limiting factor in examining the long-distance dispersal of volcanic ash clouds, and generates high errors in model results far from source [e.g. Bonadonna et al. 2011a]. The second weakness is that, at present, computing power is not adequate to allow simulations to be applied in operational settings, where forecasting estimates of volcanic cloud positions are required within minutes. Simulations like those produced by ATHAM currently require run-times of hours to days, even on high-performance machines. This modeling framework is thus limited to research settings, to analyze past events or idealized scenarios in detail, and to calibrate the more simplified descriptions of plume dynamics used in volcanic ash transport and dispersion models.

\subsubsection{Key sources of uncertainty}

This section isolates key aspects of volcanic plume dynamics that are central to modeling efforts, but poorly constrained by field and theoretical observations. In particular, column instability and microphysical effects (including aggregation) are directly relevant to operational forecasting of ash concentrations, as well as to the interpretation of past eruptions from their volcanic deposits. An eruption is generally considered 'steady' if its duration exceeds the time required for ascent to the neutral buoyancy height (on the order of minutes to tens of minutes [Sparks et al. 1997]). If the eruption column does not become buoyant, or loses buoyancy due to changing conditions at vent, the flow regime shifts to that of a collapsing fountain, shedding voluminous ground-hugging density currents [Sparks and Wilson 1976]. These buoyant versus collapsing regimes are fundamental to the injection heights and masses of 
volcanic ash emplaced into different levels of the atmosphere, and by extension, on the dispersal dynamics of volcanic ash clouds.

Motivation for the early theoretical studies on column collapse drew from the eruptions of Mount St Helens, 1980 [Lipman and Mullineaux 1981], Redoubt, Alaska, 1991 [Woods and Kienle 1994] and Pinatubo, Philippines 1991 [Newhall and Punongbayan 1996]. These events share the trait that a significant component of their eruption clouds were supplied by lofted coignimbrite ash injecting into multiple levels of the atmosphere. The conceptual model of Woods [1988] attributes this transition between buoyant and collapsing behavior to competing processes within the rising plume: the buoyancy-promoting effects of decreasing density vs. the buoyancy-inhibiting effects of decreasing velocity. However, numerous physical factors can lead to column collapse, including excavation of a wider volcanic conduit, a decrease in magmatic volatiles, or significant increases in magma eruption rate [Wilson et al. 1980, Woods and Bower 1995]. Indeed, most large-scale eruptions with mass eruption rates $>10^{8} \mathrm{~kg} \mathrm{~s}^{-1}$ generally do show evidence for phases of significant column collapse [Carey and Sigurdsson 1989].

This shift from fall-type deposition to laterally moving flows is a crucial distinction for hazards assessment. However, the transition between these regimes is not always sharply defined in the resulting eruption column or deposits. For example, during the post-climactic 1980 Mount St Helens eruption, short-lived oscillations in the eruption rate invoked partial (dynamic) column collapse and emplacement of PDCs [Hoblitt 1986]. Field evidence for simultaneously emplaced fall and flow deposits has also been inferred from ancient deposits [e.g. Wilson 2001] and reproduced in numerical simulations [Di Muro et al. 2004]. Although it is clear that these dynamic systems produce a kind of hybrid cloud, fed simultaneously by the vent-derived column and lofted PDCs [Woods and Kienle 1994, Calder et al. 1997], there are few constraints on how this hybrid behavior affects the atmospheric injection of ash. Furthermore, the roles of moist convection and aggregation in this process, and their impacts on eruption behavior and dispersal are poorly defined. Given the pivotal role of vertical ash concentrations in initializing accurate volcanic ash transport and dispersion models, these topics require detailed investigation and form an integral component of this thesis. 
Chapter 2 


\title{
2. Ascent Dynamics of Large \\ Phreatomagmatic Eruption Clouds: The Role of Microphysics
}

\begin{abstract}
This study examines the impact of abundant surface water interaction on the development of volcanic clouds from large-scale phreatomagmatic eruptions $\left(>10^{8} \mathrm{~kg}\right.$ $\mathrm{s}^{-1}$ magma), presenting the first 2-D large-eddy simulations of 'wet' volcanic plumes that incorporate the effects of microphysics. The cloud-resolving model ATHAM was forced with field-derived inputs from the largest phreatomagmatic eruption yet documented: the $\sim 25.4$ ka Oruanui supereruption (Taupo volcano, New Zealand). Surface water contents were varied from 0-40 wt.\% for eruptions with equivalent magma eruption rates of $c .1 .3 \times 10^{8}$ and $1.1 \times 10^{9} \mathrm{~kg} \mathrm{~s}^{-1}$. The investigation confirms that increased surface water has a pronounced impact on column stability, leading to transitional column behavior and hybrid clouds resulting from simultaneous Plinian and coignimbrite ascent mechanisms. Contrary to the suggestion of previous workers, however, abundant surface water does not systematically lower the spreading level or maximum height of volcanic clouds, owing to vigorous microphysics-assisted lofting of pyroclastic density currents. The simulated behavior and ash cloud dimensions provide a strong match to field evidence from the Oruanui unit 3 case study. Cloud heights from the collapsing eruption columns also show a notable sensitivity to changes in the altitude of the tropopause, while ambient humidity primarily impacts the abundance of airborne hydrometeors (particularly ice) associated with the volcanic clouds. General relationships between eruption style, meteorological conditions and the resulting vertical profiles of volcanic emissions outlined here could also be adapted for use in operational volcanic ash forecasting and deposit reconstruction techniques already in existence.
\end{abstract}

\subsection{Introduction}

The explosive interaction of magma and external water in 'wet' eruptions has long been observed to have a transformative impact on eruptive processes and the dynamics of 
resulting ash clouds [Walker and Croasdale 1972, Sheridan and Wohletz 1983]. Clarifying how this interaction affects the injection of ash into the atmosphere is crucial for understanding the dynamics of water-rich volcanic plumes from past and present day eruptions. In particular, after the challenges of ash cloud forecasting were brought to international focus by the April-May 2010 eruptions of Eyjafjallajökull, new demands on dispersion models require increasingly sophisticated source terms [Stohl et al. 2011]. These models incorporate key assumptions about ash cloud characteristics, including fine ash content and the vertical distribution of mass in the atmosphere [Mastin et al. 2009], both of which are significantly influenced by magma-water interaction.

On a broader scale, the potential of explosive eruptions to affect local and global climate patterns also depends on the nature of ash dispersal and interaction of volcanic aerosols with the atmosphere [Textor et al. 2003, Self 2006, Timmreck and Graf 2006, Schneider et al. 2009]. Previous work has suggested that large scale magma-water interaction would result in significantly lowered injection heights associated with column collapse and generation of low-temperature ash flows [Wilson et al. 1978, Koyaguchi and Woods 1996]. However, given more recent evidence from field studies of eruption deposits and developments in numerical modeling techniques, these conclusions warrant reappraisal.

In this study, the effect of surface water interaction on plume dynamics is investigated using a natural analog to set up conditions specific to the largest phreatomagmatic eruption yet documented. The non-hydrostatic plume model ATHAM (Active Tracer High resolution Atmospheric Model) of Oberhuber et al. [1998] is coupled with inputs from deposits of the large-volume, wet Oruanui eruption, which occurred at $\sim 25.4 \mathrm{ka}$ from Taupo volcano in New Zealand [Wilson 2001, Vandergoes et al. 2011]. One fundamental aspect of the Oruanui eruption examined here is the apparent paradox of extremely widely dispersed fall deposits despite column instability and cool ignimbrite emplacement temperatures. Employing field constraints from the Oruanui case study, this work explores how water-rich eruptions are capable of producing such widespread deposits [Self and Sparks 1978], and provide a detailed numerical examination of their ascent and transport mechanisms. 


\subsection{Dry Versus Wet Eruptive Styles}

\subsubsection{The Dry to Wet Spectrum}

In contrast to dry explosive volcanism, which is driven primarily by decompressionexpansion of magmatic volatiles in the conduit, two sources of non-magmatic water influence ash transport and dispersal processes in 'wet' eruptions. These include: (1) surface water such as an ocean, aquifer, or overlying lake and (2) ambient atmospheric moisture entrained by the ascending ash cloud. The extent of interaction with these external water sources generates a spectrum of dry to wet eruption and deposition styles that behave in fundamentally distinct ways.

As an illustration of dominantly dry behavior, the 1996 eruption of Ruapehu, New Zealand, provides a relatively small-scale example $\left(3-4 \times 10^{-3} \mathrm{~km}^{3}\right.$ tephra). After drainage of the crater lake during earlier activity, the June $17^{\text {th }}$ eruptions saw only limited entrainment of surface water and atmospheric moisture; explosivity was driven primarily by rapid expansion of volatiles in the magma. The sub-plinian ash column reached tropospheric heights of c. 5-6 km above the volcano summit [Prata and Grant 2001], roughly consistent with the output of sensible heat from ejected pyroclasts [Sparks et al. 1997]. Ash was passively advected by the ambient wind field, and the cloud sedimented predictably finer grained and thinner deposits with distance from vent [Bonadonna et al. 2005]. Although weakly-bound ash clusters and pellets were observed depositing from the eastern edge of the plume (C.J.N. Wilson personal communication), general patterns of volcanic ash fallout could be reasonably reproduced with existing models of dry dispersal [Turner and Hurst 2001].

Compared to this end-member dry style, a number of examples, including the eruptions of Mount St Helens 1980 (Washington) and Pinatubo 1991 (Philippines), have exhibited a distinct transport style due to increased water influence. Eruption clouds from both climactic events were substantially fed by ground-hugging pyroclastic density currents (PDCs), which likely enhanced the entrainment of tropospheric moisture and minor vaporized surface water. In the case of Mount St Helens, loosely bound clusters of ash and hydrometeors that formed during atmospheric transport enhanced the sedimentation rate of ash [Carey and Sigurdsson 1982, Sorem 1982, Durant et al. 2009], producing a well-documented secondary thickness maximum some hundreds of kilometers from 
vent [Sarna-Wojcicki et al. 1981b]. At Pinatubo, vigorous updraft velocities and heat from the coignimbrite clouds triggered atmospheric instability and deep moist convection - factors that were likely compounded by the passage of tropical cyclone Yunya. This phenomenon formed precipitating, ashy cumulonimbus clouds coined 'volcanic thunderstorms' by Oswalt et al. [1996], which have also been observed in more recent eruptions [Tupper and Kinoshita 2003].

However, the most extreme end-member of this water-rich behavior is found in the geologic record. Phreatoplinian, or large-scale wet eruptions [Self and Sparks 1978], incorporate abundant surface water ( $>10-15 \%$ by mass) and generate extremely widely dispersed tephra deposits. Archetypal examples include Askja 1875 [unit C, Iceland: Sparks et al. 1981, Carey et al. 2010], Taupo 1.8 ka [New Zealand: Walker 1981b, Smith 1998], and Oruanui 25.4 ka [New Zealand: Self 1983, Wilson 2001]. A key distinction between phreatoplinian eruptions and their drier counterparts is that the energy budget of the ascending ash cloud is inferred to be dominated by latent heating and cooling from water phase changes (akin to conventional storm systems), rather than from the initial sensible heat and momentum of the ejected pyroclasts alone. Products of these eruptions are characterized by exceptionally fine particle sizes due to efficient magma-water fragmentation, and often contain abundant ash aggregates or their collapsed equivalents. There is also field evidence for transitions to unsteady, pulsating column behavior within individual phases of eruption, generating fall and PDC deposits that are synchronous and complexly interrelated. Column collapse in explosive eruptions has been variably linked to physical processes such as changes in surface water supply, erosional widening of the crater and diminishing volatile content in the magma, among other factors [Wilson et al. 1980]. However, the microphysical impact of external water interaction and column instability on the architecture of volcanic ash plumes intruding into the atmosphere, and consequent implications for formation of ash aggregates, has yet to be explored in detail.

\subsection{2. 'Wet' Eruption Column Models}

Available models for the theoretical description of volcanic plumes fall along a range of sophistication. At one end, 1-D, time-averaged models based on the buoyant plume theory of [Morton et al. 1956] simplify the processes of turbulent energy exchange into 
an empirically derived entrainment factor [Wilson 1976, Sparks 1986, Woods 1988]. Use of a top hat profile assumes that physical properties of the column (e.g. velocity and bulk density) can be taken as a single, averaged value at each height, and describes only the vertical motion of an ascending parcel. Early work on dynamics of volcanic plumes using these steady state models highlighted competing forces during ascent through the atmosphere, with gravitational forces working against the buoyancy gained from entrainment and heating of air [Sparks and Wilson 1976, Bursik and Woods 1991]. The role of latent heat heating and cooling from phase changes of water were explored by Woods and Wohletz [1991], Woods [1993] and Glaze and Baloga [1996]. However, Koyaguchi and Woods [1996] were the first to examine the critical conditions required for buoyant ascent of very water-rich columns. These calculations have since been adapted to a readily accessible web-based interface by Mastin [2007]. The previous theoretical work on phreatomagmatic eruptions has indicated that for large eruptions $\left(>10^{8} \mathrm{~kg} \mathrm{~s}^{-1}\right.$ magma), the sensible heat lost to abundant, cool surface water would not be sufficiently regained to form a buoyant cloud, leading to significantly lower ash cloud heights and diminished capacity for widespread dispersal [Wilson et al. 1978, Koyaguchi and Woods 1996]. However, these results are inconsistent with field evidence from phreatoplinian deposits, including the $\sim 25.4 \mathrm{ka}$ Oruanui eruption (Taupo volcano, New Zealand), which on the whole generated a voluminous $\left(320 \mathrm{~km}^{3}\right)$ lowtemperature PDC deposit that reach to tens of kilometers from vent, and extremely widely dispersed fall deposits [Wilson 1994, 2001]. From this contrast it is apparent that the mechanisms of ash emplacement and transport in large-scale wet plumes merit further investigation.

The other extreme of eruption column models in terms of spatiotemporal resolution focuses on small scale processes close to the vent. Included in this group are the multiphase large eddy simulations of Valentine and Wohletz [1989], Dobran et al. [1993] and Neri et al. [2003], which are ideal for resolving microscale phenomena $(<1 \mathrm{~km})$ near the vent during the initial tens of minutes of eruption. However, these models do not yet incorporate the effects of water phase changes, and simulated eruption durations on the order of hours are computationally prohibitive.

Providing a compromise between the simplified 1-D time-averaged and complex microscale frameworks, the Active Tracer High resolution Atmospheric Model 
(ATHAM) resolves mesoscale (1-100s of kilometers) cloud dynamics lasting on the order of minutes to hours. Though originally formulated to simulate volcanic plumes [Oberhuber et al. 1998], the code has been applied to diverse applications such as the convective dynamics of wildfires [Trentmann et al. 2006], cloud microphysics [Guo et al. 2007], and photochemical speciation and radiative effects of aerosols [Trentmann et al. 2003a, 2003b]. The key strength of ATHAM compared to volcanic ash transport and dispersion models (e.g. PUFF or HYSPLIT) is its ability to treat erupted particles as active tracers. That is, the pyroclasts influence the behavior of the volcanic cloud, rather than being passively transported by wind and gravitational fallout. This modeling approach is a particularly powerful tool for simulating column behavior when source conditions, such as erupted volume and grain size distribution, are better constrained than the final height and spatial distribution of the ash cloud (i.e. for prehistoric or sparsely observed eruptions). Given the problematic nature of reproducing phreatoplinian ash cloud heights from their ultra-fine grained deposits using the traditional Carey and Sparks [1986] isopleth inversion method [Houghton and Wilson 1998], the application of a physically based model like ATHAM provides an alternative approach to reconstructing eruption plume dynamics.

ATHAM has been previously employed to examine the sensitivity of Plinian and subPlinian cloud behavior to extrinsic factors such as atmospheric humidity, temperature and wind shear [Oberhuber et al. 1998, Graf et al. 1999, Tupper et al. 2009], and to intrinsic factors such as eruption rate [Tupper et al., 2009] and hydrometeor-ash aggregation [Textor et al. 2006a, b]. Herzog and Graf [2010] described the convective structure of coignimbrite ash clouds, building from the work of Woods and Wohletz [1991] to examine injection heights of coignimbrite columns. However, the impact of added surface water on phreatoplinian (and resulting wet coignimbrite) cloud dynamics has never been tested in more than one dimension, or with a model that considers all the phase changes of water.

In this numerical study linked to field data from the deposits of a large, phreatoplinian eruption, the following points are addressed:

1. Influences of surface water on maximum column height, level of neutral buoyancy, plume stability and availability of liquid water for wet aggregation; 
2. Sensitivity to background atmospheric properties;

3. New ways in which wet and/or unstable eruptions may be parameterized for eruption deposit reconstructions and operational ash cloud forecasting purposes.

\subsubsection{Case study: The 25.4 ka Oruanui Eruption}

The Oruanui eruption from Taupo volcano, New Zealand, $[27 \pm 1$ cal. ka: Lowe et al. 2008] is the largest phreatomagmatic eruption yet documented [c. $530 \mathrm{~km}^{3}$ magma: Wilson 2001]. A large, complexly bedded fall deposit of $430 \mathrm{~km}^{3}$ bulk volume was emplaced before, during and after $c .320 \mathrm{~km}^{3}$ of entirely non-welded PDC deposits. The eruption as a whole is recognized by the unusually fine grain size ( $>80 \%$ of the deposits are finer than $63 \mu \mathrm{m}$ ), abundance of ash aggregates, and evidence for simultaneous and overlapping emplacement of fall and PDC deposits [Self 1983; Wilson 2001]. The eruption column interacted to various extents with a large, pre-existing lake overlying what became the Oruanui collapse caldera [Lake Huka: Manville and Wilson 2004].

The Oruanui eruption is divided into 10 phases, delineated by bedding horizons in the fall deposits, with each phase lasting on the order of hours to days [Wilson 2001]. The eruptive products range from mostly dry to water-rich on deposition. For example, the drier fall units 5 and 7 are superficially similar to dry plinian fall deposits, although with exceptionally wide dispersal [beds A and B of Wilson 1994]. Wetter units (3, 6 \& 8) show evidence for liquid and frozen water being present on deposition, although water contents were never sufficient to cause large-scale remobilization like that observed in wet deposits of the 1.8 ka Taupo eruption [Walker 1981b, Smith 1998] .

Unit 3 of the Oruanui eruption is an example of a wet eruptive phase that is likely to have had a large, but not historically unprecedented, eruption rate [Self and Blake 2008, Wilson 2008]. Although it is challenging to obtain good constraints on eruption rate due to the lack of detailed information on exactly how long the eruption lasts, field relationships suggest a duration on the order of hours to days for unit 3 [Wilson 2001]. For the purposes of this work, a magma eruption rate (MER) on the order of $10^{8} \mathrm{~kg} \mathrm{~s}^{-1}$ is taken as a reference value, consistent with estimates from historical eruptions producing widespread fall deposits (e.g. Mount St Helens 1980, Askja 1875). This implies an eruption duration of $c$. 20-120 hrs to accumulate the volume estimated for this unit (5- 
$30 \mathrm{~km}^{3}$ tephra, depending on the extrapolation of deposit thicknesses over the area now beneath the modern Lake Taupo [Wilson, 2001]). For comparison, a higher MER on the order of $10^{9} \mathrm{~kg} \mathrm{~s}^{-1}$ was also tested. Initial magmatic temperatures of the high-silica rhyolite have been determined to be $770 \pm 30{ }^{\circ} \mathrm{C}$ from Fe-Ti oxides [modified after Wilson et al. 2006], with c. 5 wt.\% dissolved $\mathrm{H}_{2} \mathrm{O}$ [Liu et al. 2006]. The immediate post-emplacement temperature of near-vent ignimbrite (even where $>200 \mathrm{~m}$ thick) using paleomagnetic methods on incorporated lithic clasts is $150-250{ }^{\circ} \mathrm{C}$ [Barker 1991, E. McClelland and C.J.N. Wilson unpublished data] . Assuming that the cool emplacement temperatures can be primarily attributed to interaction with the overlying lake, c. 20 wt.\% water would have been required to mix with the erupted magma at vent [Wilson 1994]. This field information was used to initialize numerical simulations consistent with the observed eruption characteristics.

\subsection{Numerical Modeling}

\subsubsection{Model Description and Setup}

ATHAM is a non-steady Eulerian, cloud resolving model with a modular structure [Herzog et al. 1998, Oberhuber et al. 1998]. The dynamic core solves the full set of Navier-Stokes equations for volume mean quantities of a gas-particle mixture [Oberhuber et al. 1998]. ATHAM's turbulence scheme distinguishes between turbulent energy exchange in the horizontal and vertical directions, and parameterizes the subgrid eddies [Herzog et al. 2003]. Treatment of volcanic ash and hydrometeors as active tracers, including gravitational fallout, assumes an almost instantaneous exchange of energy and momentum between components. Previous work [e.g. Bursik and Woods 1991] has indicated that this assumption is justified for pyroclasts $<<1 \mathrm{~mm}$, which is reasonable for the Oruanui eruption because of its uniformly fine grain size. ATHAM has a number of running modes: fully 3-D, 2-D Cartesian for studying wind effects, and 2-D cylindrical mode. The latter geometrical configuration is used for this work because it captures entrainment processes and plume heights similarly to the 3-D results with minimal computational expense [Herzog et al. 2003]. In axisymmetric mode wind effects are not incorporated and, therefore, results provide an approximation of plume development in the crosswind range, which is primarily a function of column height rather than wind effects [Carey and Sparks 1986]. 
A key feature of the model is that it resolves microphysics processes such as latent energy exchange due to water phase changes. The bulk microphysical module used in this study is a Kessler-like description of the phase changes of water between five hydrometeor classes: vapor, liquid cloud water and rain, and frozen cloud ice and ice pellets (referred to as graupel: Figure 2.1). The microphysical parameterization is fully set out in Herzog et al. [1998]. Cloud water and ice crystals are prescribed a fixed diameter of $20 \mu \mathrm{m}$ and are assumed to have a negligible fall velocity relative to components of the flow. Larger rain drops and graupel grow by gravitational capture of other hydrometeors due to differences in fall velocity. Their sizes follow a MarshallPalmer distribution [Marshall and Palmer 1948] with fall velocity determined by mean diameter. Graupel is used here as a catchall reservoir for frozen aggregates, assuming a density of $700 \mathrm{~kg} \mathrm{~m}^{-3}$, which is intermediate between low density snow pellets and higher density hail. Energy transfer from the various phase changes influence the erupted mixture by releasing or consuming heat, as annotated in Figure 2.1. As it is possible to deactivate this module in ATHAM, this work compares simulations with and without microphysics to reveal the impact of water phase changes on ascent dynamics.

Other simplifications for these numerical experiments include assuming that there is no radiative heat loss from the plume and not explicitly solving for ash-hydrometeor aggregation. The latter is an indispensible factor in the ash dispersal and sedimentation from water-rich eruptions, so while the time-dependent interactions between hydrometeors and silicate particles in volcanic clouds [Textor et al. 2006a] are not explicitly solved in the version of the code used for this study, some simple aggregation tests are employed.

The model geometry for 2-D cylindrical (axisymmetric) simulations use a stretched grid with $259 \times 259$ grid points in a $300 \times 70 \mathrm{~km}$ domain in the horizontal and vertical dimensions, respectively. Grid resolution coarsens from $75 \mathrm{~m}$ at the centre to a few kilometers at the model boundaries. Unless stated otherwise, simulation runtimes were 90 minutes long, which includes 60 minutes of continuous eruption and 30 minutes of aftermath, minimizing computational expense while providing enough information to capture the main dynamical features. As this study does not focus on the effects of crater geometry and topography (in large part because these are unconstrained by 
available field evidence), all simulations begin after expansion and decompression of the erupted jet to atmospheric pressure $(1 \mathrm{~atm})$ at a topographically flat surface. For simplification, it is assumed that decompression occurs close to the volcanic conduit at sea level, and all heights are reported in kilometers above sea level (a.s.l.).

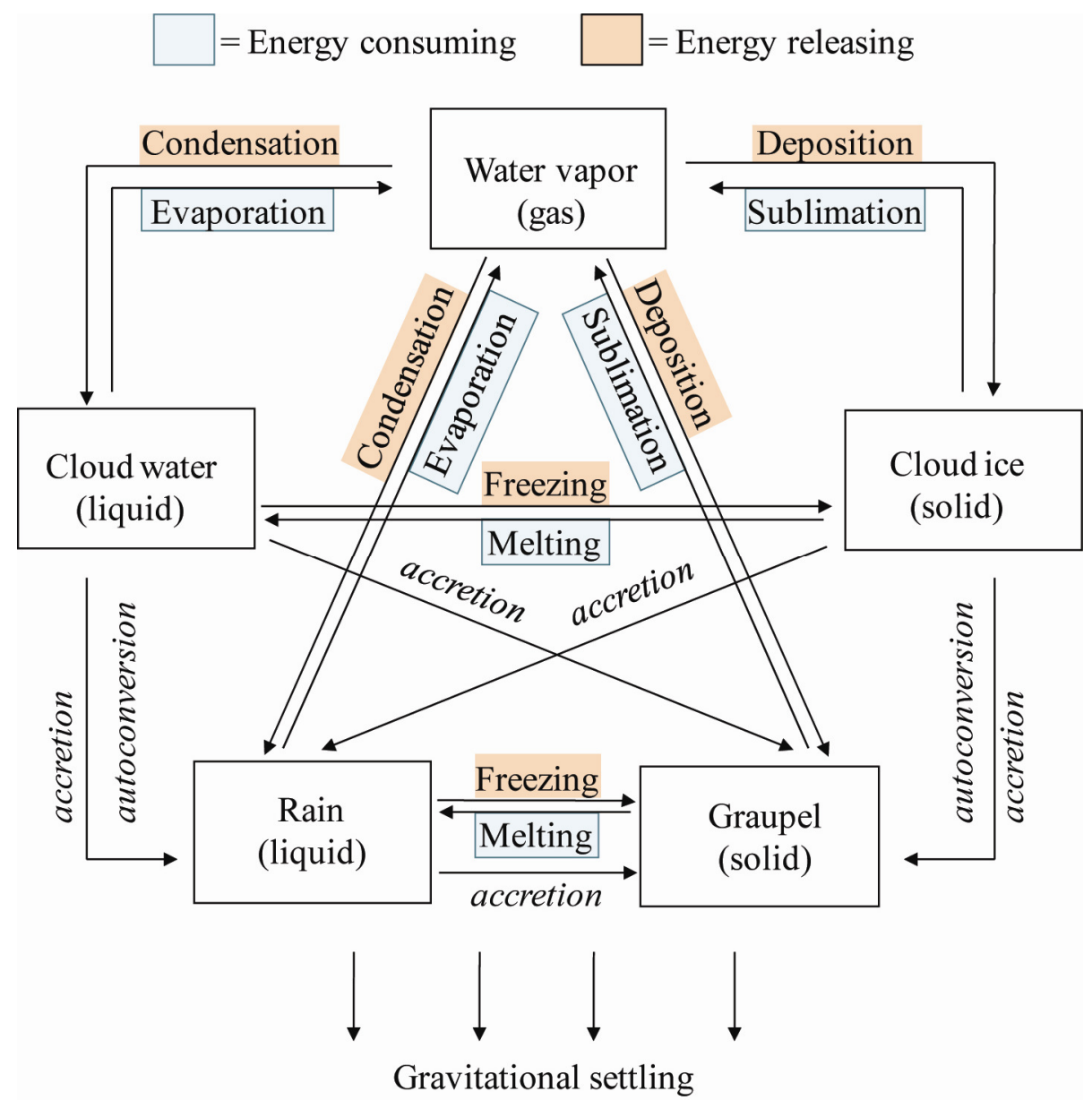

Figure 2.1 Cloud microphysical processes described by ATHAM's bulk parameterization scheme. Phase changes which release latent heat are shown in orange, and those which consume energy from the environment are in blue. Modified from Herzog et al. [1998].

\subsubsection{Volcanological Input}

In ATHAM the state of the eruption is defined by the set of volcanic forcing characteristics shown in Table 2.1. Although this work focuses on the impact of surface water interaction on the dynamics of eruption cloud ascent, the energetic importance of the output rate of hot pyroclasts and magmatic fluids is also recognized. The magma eruption rate (MER), which includes magmatic water, exerts a first-order control on 
eruption style [Wilson et al. 1978] and relates to the area of the plume base (A), density $(\rho)$ and exit velocity $(v)$ of the erupted mixture by:

$$
\operatorname{MER}=A \rho v(\text { Eq. } 1)
$$

To establish a basis of comparison between eruptions with different surface water contents, MER was kept constant within each scenario by adjusting the exit velocity [Koyaguchi and Woods 1996]. This effectively assumes that the addition of surface water alters the density and velocity of the erupted mixture (thereby changing the total eruption rate), but not the plume diameter or MER [Mastin 2007]. Assuming the chilled, erupted particles have a void-free density of $2480 \mathrm{~kg} \mathrm{~m}^{-3}$, the volumetric flow rate of magma can be derived for comparison with empirical height scaling relationships (e.g. Sparks et al. [1997] Eq. 5.1).

\subsubsection{Particle Characteristics}

Three particle classes were used to as tracers in the simulated eruptions: $6 \mu \mathrm{m}$ and 50 $\mu$ m diameter glass (density: $2300 \mathrm{~kg} \mathrm{~m}^{-3}$ ) and $200 \mu \mathrm{m}$ diameter crystals $\left(2900 \mathrm{~kg} \mathrm{~m}^{-3}\right)$. These tracers are taken to comprise $c .35 \%, 35 \%$ and $30 \%$ of the erupted pyroclasts, respectively, based on grain size characteristics of Oruanui unit 3 deposits. An additional aggregate tracer was employed in aggregation simulations, which has a diameter of $1 \mathrm{~cm}$ and density of $1200 \mathrm{~kg} \mathrm{~m}^{3}$.

\subsubsection{Thermodynamic Considerations}

The mixing dynamics of the four magma:water mixing ratios investigated here (ranging from 10 to $40 \%$ liquid water by mass) begins conceptually with magma and $5 \%$ magmatic volatiles [Liu et al. 2006] at the same temperature before eruption. As the mixture exits the conduit, it mingles explosively with cool surface water at constant atmospheric pressure, transferring enthalpy to the water until an equilibrium mixing temperature is attained. The final temperature of the magma-water mixture can be calculated using first principle thermodynamic properties of fuel coolant interaction 
Table 2.1 Volcanic input parameters for simulations with different surface water contents and magma eruption rates (MERs) ${ }^{a}$.

\begin{tabular}{|c|c|c|c|c|}
\hline \multirow[b]{2}{*}{ Volcanic Forcing } & \multicolumn{2}{|c|}{ unsaturated eruptions } & \multicolumn{2}{|c|}{ saturated eruptions } \\
\hline & $d r y$ & $\begin{array}{c}\text { total } \\
\text { vaporization }\end{array}$ & $\begin{array}{c}\text { total } \\
\text { vaporization }\end{array}$ & $\begin{array}{c}\text { partial } \\
\text { vaporization }\end{array}$ \\
\hline \multicolumn{5}{|c|}{ Constant properties } \\
\hline Percent surface water & 0 & 10 & 24 & 40 \\
\hline Mixing temperature $\left({ }^{\circ} \mathrm{C}\right)$ & 767 & 485 & 100 & 100 \\
\hline Percent erupted vapor & 5.00 & 14.50 & 27.80 & 18.10 \\
\hline Percent erupted liquid & 0 & 0 & 0 & 24.90 \\
\hline Percent $6 \mu \mathrm{m}$ glass & 33.00 & 29.90 & 25.27 & 19.95 \\
\hline Percent $50 \mu \mathrm{m}$ glass & 33.00 & 29.90 & 25.27 & 19.95 \\
\hline Percent $200 \mu \mathrm{m}$ crystals & 29.00 & 25.70 & 21.66 & 17.10 \\
\hline Plume bulk density $\left(\mathrm{kg} \mathrm{m}^{-3}\right)$ & 4.23 & 2.00 & 2.12 & 3.26 \\
\hline \multicolumn{5}{|c|}{ Low MER scenarios } \\
\hline Magma eruption rate $\left(\mathrm{kg} \mathrm{s}^{-1}\right)$ & $1.27 \times 10^{8}$ & $1.27 \times 10^{8}$ & $1.27 \times 10^{8}$ & $1.27 \times 10^{8}$ \\
\hline Total eruption rate $\left(\mathrm{kg} \mathrm{s}^{-1}\right)$ & $1.27 \times 10^{8}$ & $1.41 \times 10^{8}$ & $1.67 \times 10^{8}$ & $2.11 \times 10^{8}$ \\
\hline Expanded plume diameter (m) & 675 & 675 & 675 & 675 \\
\hline Initial plume velocity $\left(\mathrm{m} \mathrm{s}^{-1}\right)$ & 84 & 196 & 219 & 181 \\
\hline \multicolumn{5}{|c|}{ High MER scenarios } \\
\hline Magma eruption rate $\left(\mathrm{kg} \mathrm{s}^{-1}\right)$ & $1.13 \times 10^{9}$ & $1.13 \times 10^{9}$ & $1.13 \times 10^{9}$ & $1.13 \times 10^{9}$ \\
\hline Total eruption rate $\left(\mathrm{kg} \mathrm{s}^{-1}\right)$ & $1.13 \times 10^{9}$ & $1.25 \times 10^{9}$ & $1.49 \times 10^{9}$ & $1.88 \times 10^{9}$ \\
\hline Expanded plume diameter $(\mathrm{m})$ & 1275 & 1275 & 1275 & 1275 \\
\hline Initial plume velocity $\left(\mathrm{m} \mathrm{s}^{-1}\right)$ & 209 & 491 & 548 & 452 \\
\hline
\end{tabular}

${ }^{a}$ MER refers to the eruption rate of magma, which includes 5\% magmatic volatiles. Total eruption rate is the output rate of magma plus surface water. Density of the $6 \mu \mathrm{m}$ and $50 \mu \mathrm{m}$-diameter vitric ash is $2300 \mathrm{~kg} \mathrm{~m}^{-3}$ and that of the $200 \mu$ m-diameter ash is $2900 \mathrm{~kg} \mathrm{~m}^{-3}$.

where $x=$ mass fraction magma, $\Delta h_{m}=$ enthalpy change of the magma (in Joules), and $\Delta h_{w}=$ enthalpy change of external water. To determine the enthalpy transfer from magma to water, a first estimate of the final temperature of the eruptive mixture is assumed, giving $\Delta T_{m}\left(=T_{m 0}-T_{m 1}\right)$, where $T_{m 0}$ is taken here as $1040 \mathrm{~K}$. Ascribing two thermodynamic components to the magma - i.e. molten rock and dissolved volatiles in the form of pure water - the resulting change in magma enthalpy is calculated using:

$$
\Delta h_{m}=n C_{p} \Delta T_{m}+(1-n) \Delta h_{v}(\text { Eq. } 2)
$$

where $n=$ mass fraction of volatile-free magma; $C_{p}=$ specific heat capacity of magma (1050 J K ${ }^{-1}$ : Bacon [1977]); and $\Delta h_{v}=$ enthalpy change in magmatic volatiles calculated from the change in temperature. The latter value is read directly off an enthalpy- 
temperature diagram for pure water at $1 \mathrm{~atm}$. Using this value, the relationship of Eq. 2 is used to determine $\Delta h_{w}$, which gives the corresponding temperature of pure water $\left(T_{w}\right.$ final) from steam tables, assuming an initial water temperature of $273 \mathrm{~K}$. The value of $T_{m}$ final is iteratively adjusted until $T_{m \text {-final }}=T_{w \text {-final }}$ within $1 \mathrm{~K}$. Finally, $\Delta h_{w}$ is used to establish the relative proportions of vapor and liquid water from steam tables.

As shown in Table 2.1, surface water contents of $0,10,24$, and $40 \%$ by mass were chosen for sensitivity studies to examine the basic regimes of magma-water interaction. These include: dry ( $0 \%$ surface water); unsaturated (10\%), where total vaporization of the surface water occurs; just saturated $(24 \%)$, characterized by total vaporization and an equilibrium mixing temperature of $100^{\circ} \mathrm{C}$; and fully saturated $(40 \%)$, where only partial vaporization occurs at a mixing temperature of $100^{\circ} \mathrm{C}$ [Koyaguchi and Woods 1996]. Consistent with previous field based estimates of the extent of magma-water mixing in unit 3 of the Oruanui eruption [Wilson 1994], the 24\% surface water scenario is used as a reference to compare with field deposits.

\subsubsection{Meteorological Input}

The Oruanui eruption occurred at $\sim 25.4 \mathrm{ka}$, which was during the last glacial maximum (LGM) in New Zealand [Lowe et al. 2008]. Paleoclimate simulations over New Zealand have indicated that the LGM would have been characterized by cooler, drier conditions, and a slightly warmer lower stratosphere due to higher methane concentrations [Drost 2006, Drost et al. 2007b]. To initialize a reasonable estimate of atmospheric conditions at the time of eruption, the standard midlatitude winter temperature profile of [McClatchey et al. 1972] was modified to account for these general features of local LGM climate. The four atmospheric profiles tested here are shown in Figure 2.2a-d, hereafter referred to as atmospheres A through D. All temperature profiles show a surface temperature of $0{ }^{\circ} \mathrm{C}$ and incorporate a $2-3 \mathrm{~km}$ thick thermal inversion at the tropopause to account for the warmer lower stratosphere. Atmospheres A and B have a tropopause elevation of $15 \mathrm{~km}$ a.s.1., compared to $10 \mathrm{~km}$ for C and D. The two humidity profiles used here represent a humid atmosphere for New Zealand, which decreases from a ground level maximum of $95 \%$ relative humidity, and a dry one, decreasing from a maximum of $55 \%$. Consistent with the general climate characteristics of the LGM, atmosphere $\mathrm{A}$ is used as a reference in this study, consisting of the dry humidity profile 
and $15 \mathrm{~km}$ tropopause elevation. The meteorological input for ATHAM assumes hydrostatic equilibrium conditions, and horizontally homogeneous temperature and humidity profiles throughout the grid.

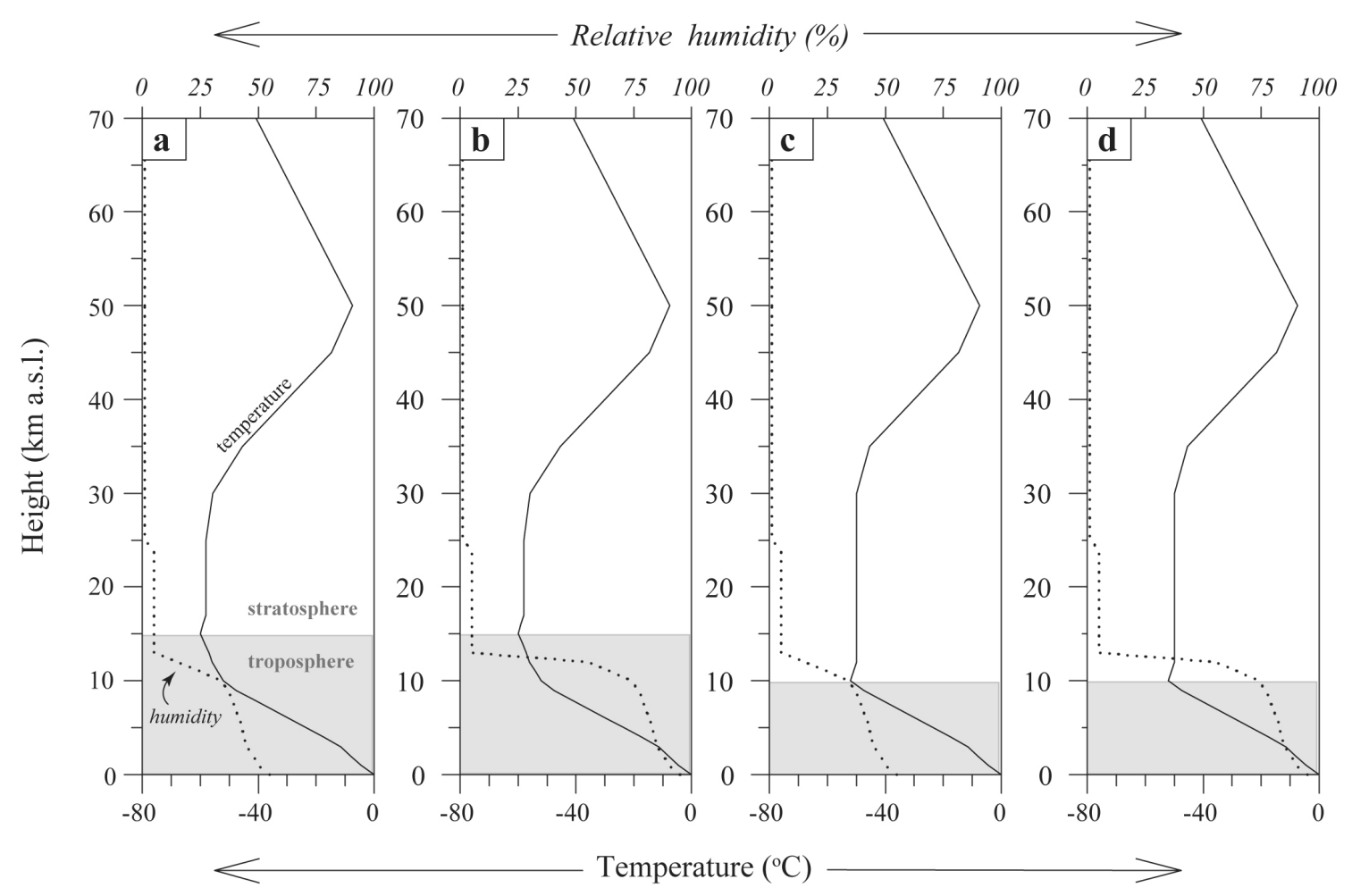

Figure 2.2 Meteorological inputs of background temperature and humidity used in sensitivity studies. Grey boxes designate the troposphere. (a) is used as the reference atmospheric profile, with the tropopause at $15 \mathrm{~km}$ a.s.l. and a relatively dry troposphere.

Table 2.2 lists the combinations of the meteorological and volcanological scenarios used in all the eruption simulations. Those in bold were accompanied by a "nomicrophysics control run' identical in every way except that the microphysical module was disabled. Comparisons with these control runs facilitate examination of the dynamical impact of latent heat exchange on column behavior and ash cloud ascent.

\subsection{Results}

\subsubsection{Overview}

The multiphase large-eddy simulations reveal information about the general structure of the water-rich eruption clouds and their dynamical features. Figure 2.3a-h summarizes broad-scale variations in the ash cloud morphologies after one hour of eruption, given different amounts of magma-water mixing and MER in the reference atmosphere. Ash 
clouds produced by dynamically distinct eruption behaviors are categorized as coignimbrite, formed from a low-level collapsing column and PDCs, and Plinian, from a high sustained column. (Note that the term coignimbrite is used in a general sense here to refer to the material elutriated from PDCs, and not necessarily associated with emplacement of hot ignimbrite deposits sensu stricto.) Also recognized is a third, hybrid, type of ash cloud that is produced by a transitional column fed coevally by Plinian and collapsing eruption mechanisms. This transitional style has been modeled by Neri et al. [2002] and Di Muro et al. [2004], who identified that flow instabilities can induce partial and wholescale collapse even in an eruption with constant vent conditions (e.g. steady MER). The role of this eruption style is explored further in this study by tracking the intrusion dynamics of hybrid volcanic clouds into the upper atmosphere.

Table 2.2 List of numerical simulations used in this study ${ }^{\mathrm{a}}$.

\begin{tabular}{|c|c|c|c|c|c|c|c|c|c|}
\hline \multicolumn{10}{|l|}{ Low MER scenarios } \\
\hline Simulation ID & $0-\mathrm{A}$ & $0-\mathrm{B}$ & $0-\mathrm{C}$ & $0-\mathrm{D}$ & $10-\mathrm{A}$ & $10-\mathrm{B}$ & $10-\mathrm{C}$ & $10-\mathrm{D}$ & $24-\mathrm{A}^{\mathrm{ref}}$ \\
\hline Surface water $(\%)$ & 0 & 0 & 0 & 0 & 10 & 10 & 10 & 10 & 24 \\
\hline Atmosphere $^{\mathrm{b}}$ & $\mathrm{a}$ & $\mathrm{b}$ & $\mathrm{c}$ & $\mathrm{d}$ & $\mathrm{a}$ & $\mathrm{b}$ & $\mathrm{c}$ & $\mathrm{d}$ & $\mathrm{a}$ \\
\hline Trope. elevation ${ }^{\mathrm{c}}$ & 15 & 15 & 10 & 10 & 15 & 15 & 10 & 10 & 15 \\
\hline Rel. hum. & dry & hum. & dry & hum. & dry & hum. & dry & hum. & dry \\
\hline \multicolumn{10}{|c|}{ Low MER scenarios continued } \\
\hline Simulation ID & $\begin{array}{r}24-\mathrm{A}- \\
3 \mathrm{hrs}\end{array}$ & $\begin{array}{r}24-\mathrm{A}- \\
\text { aggs }\end{array}$ & 24-B & $24-\mathrm{C}$ & 24-D & $40-\mathrm{A}$ & $40-\mathrm{B}$ & $40-\mathrm{C}$ & $40-\mathrm{D}$ \\
\hline Surface water $(\%)$ & 24 & 24 & 24 & 24 & 24 & 40 & 40 & 40 & 40 \\
\hline Atmosphere $^{\mathrm{b}}$ & $\mathrm{a}$ & $\mathrm{a}$ & $\mathrm{b}$ & $\mathrm{c}$ & $\mathrm{d}$ & $\mathrm{a}$ & $\mathrm{b}$ & $\mathrm{c}$ & $\mathrm{d}$ \\
\hline Trope. elevation ${ }^{\mathrm{c}}$ & 15 & 15 & 15 & 10 & 10 & 15 & 15 & 10 & 10 \\
\hline Rel. hum. & dry & dry & hum. & dry & hum. & dry & hum. & dry & hum. \\
\hline \multicolumn{10}{|c|}{ High MER scenarios } \\
\hline Simulation ID & $\begin{array}{l}\text { 0-A- } \\
\text { high }\end{array}$ & $\begin{array}{l}10-\mathrm{A} \\
\text { high }\end{array}$ & $\begin{array}{r}24-\mathrm{A} \\
\text { high }\end{array}$ & $\begin{array}{l}\text { 24-A- } \\
\text { high- } \\
\text { aggs }\end{array}$ & $\begin{array}{c}40-\mathrm{A} \\
\text { high }\end{array}$ & & & & \\
\hline Surface water $(\%)$ & 0 & 10 & 24 & 24 & 40 & & & & \\
\hline Atmosphere $^{\mathrm{b}}$ & $\mathrm{a}$ & $\mathrm{a}$ & $\mathrm{a}$ & $\mathrm{a}$ & $\mathrm{a}$ & & & & \\
\hline Trope. elevation ${ }^{\mathrm{c}}$ & 15 & 15 & 15 & 15 & 15 & & & & \\
\hline Rel. hum. & dry & dry & dry & dry & dry & & & & \\
\hline \multicolumn{10}{|c|}{$\begin{array}{l}{ }^{a} \text { Simulations in bold correspond to plumes in Figure } 2.3 \text {, and were run in duplicate: one } \\
\text { with the microphysics module activated and one without. }\end{array}$} \\
\hline \multicolumn{10}{|c|}{${ }^{b}$ Refers to the background atmospheres from Figure 2.2.} \\
\hline${ }^{c}$ Is the tropopaus & elevati & on in $\mathrm{kl}$ & n a.s.l. & & & & & & \\
\hline Indicates the $r$ & ans & neri & & & & & & & \\
\hline
\end{tabular}


Sensitivity of eruption features to variations in magma:water mixing ratios and background atmosphere are examined with respect to changes in two cloud height diagnostics: maximum overshoot and neutral buoyancy level [Herzog and Graf 2010]. Additionally, distinguishing how much of the erupted material punctures the tropopause and emplaces into the stratosphere has direct implications for interaction with long-lived jet stream winds and aircraft thoroughfares, as well as the potential for weather and climate feedbacks. The height of maximum overshoot is defined here as the greatest altitude at which concentrations of total ash are $\geq 0.01 \mathrm{~g} \mathrm{~m}^{-3}$ after 60 minutes of eruption. This threshold is chosen to define the cloud top because it represents the upper c. $0.001 \%$ of the total airborne cloud, yet would still be readily detectable with the naked eye and remote sensing methods. Volcanic ash at this concentration would also be problematic for jet aircraft ( $c f$. the 'no-fly' zone of $0.004 \mathrm{~g} \mathrm{~m}^{-3}$ used by the European Commission [2010]). The neutral buoyancy level (NBL) is distinguished in this study as the height at which the plume buoyancy equals that of the surrounding atmosphere, and generally corresponds to the spreading level of the umbrella cloud [Sparks et al. 1997]. It is quantified here by the altitude containing peak concentrations of the $6 \mu \mathrm{m}$ ash tracer, which is the grain size preferred over coarser, faster-settling particle classes. The NBL is always taken above $500 \mathrm{~m}$ a.s.l. because PDCs may intermittently increase particle concentrations near the ground. In instances where multiple intrusion heights develop in the cloud, the NBL refers to the dominant (farthest traveled) spreading level 60 minutes into eruption.

\subsubsection{Reference Simulation: Saturated Eruption (24\% Surface Water)}

A reference scenario for an eruption similar to Oruanui unit 3 was tested in windless, 2D axisymmetric mode using $24 \%$ surface water in the reference atmosphere (24-A of Table 2.2, Figure 2.3c). The dynamics of this simulated eruption is examined in detail, illustrating the various transport regimes of a transitional phreatoplinian column, which would result in simultaneous emplacement of fall and PDC material.

\section{Dynamics of the transitional column}

With ATHAM's microphysical module deactivated (i.e. ignoring latent heat effects), the reference simulation shows a $6 \mathrm{~km}$ high collapsing column for the duration of eruption 


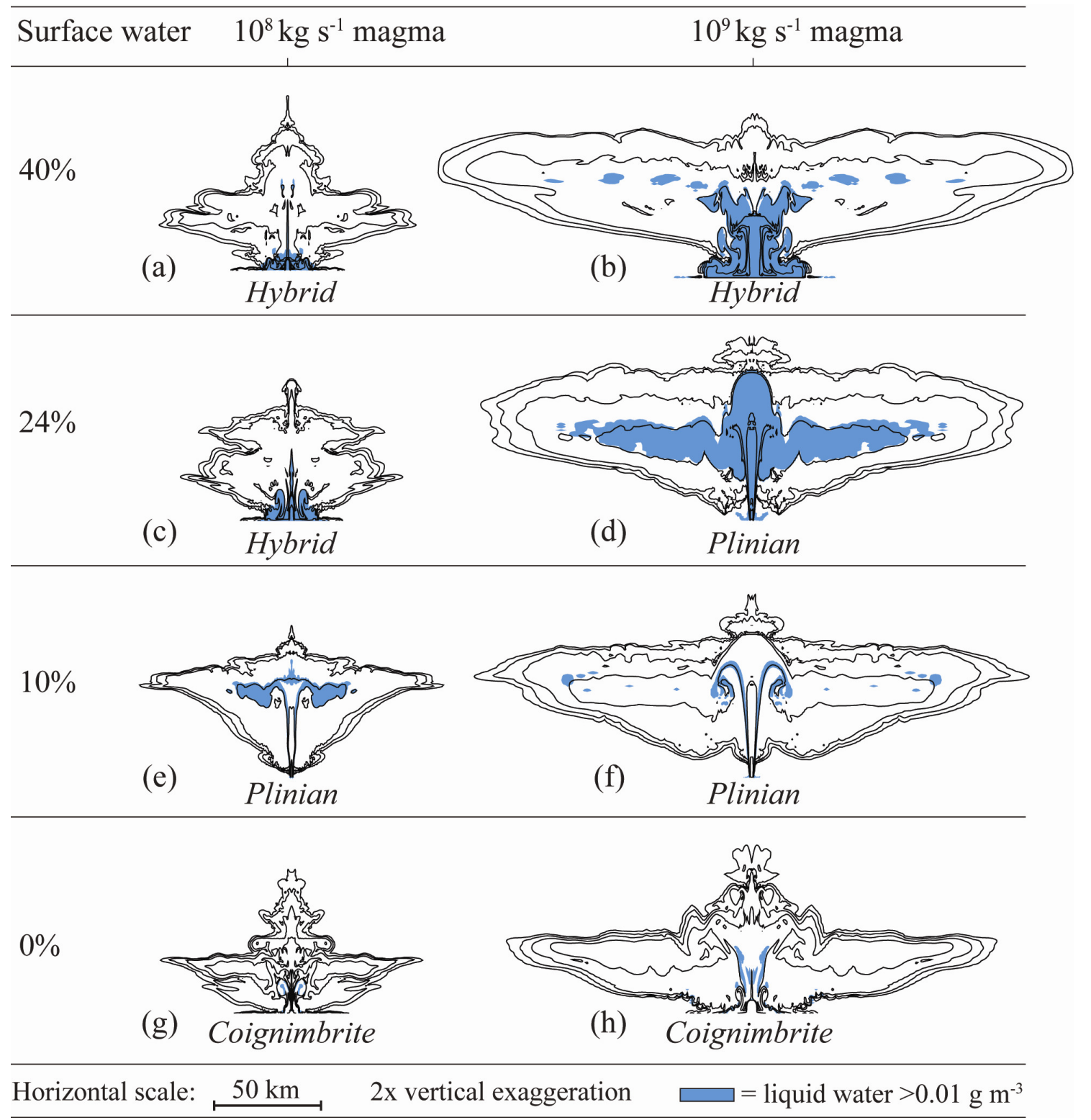

Figure 2.3 Summary of eruption cloud morphologies after 60 minutes of constant eruption in the reference atmosphere. Surface water content and (order of magnitude) magma eruption rate are indicated. Black lines represent contours of total ash concentration in $\mathrm{g} \mathrm{m}^{-3}$, each increasing by one order of magnitude from a minimum of $0.01 \mathrm{~g} \mathrm{~m}^{-3}$ on the outer edge. Blue shading indicates where liquid water content (cloud water and rain) is greater than $0.01 \mathrm{~g} \mathrm{~m}^{-3}$.

(Figure 2.4a). The low-temperature $\left(\leq 100{ }^{\circ} \mathrm{C}\right)$ ash mixture cascades to the ground and spreads to a distance of $7-12 \mathrm{~km}$ from vent. Here, lateral propagation ceases and the PDCs lift off and ascend to a maximum height of c. $5 \mathrm{~km}$ a.s.l. Even after an hour of continuous eruption, $>99 \%$ of the airborne ash has failed to ascend above $5 \mathrm{~km}$.

By incorporating the effects of water phase changes, however, the picture of plume evolution is fundamentally different (Figure 2.4b). The near-vent column development 
in the reference simulation during the first hour is shown in Figure 2.5a-d. During the initial few minutes of eruption (Figure 2.5a), the momentum-driven jet thrust region of the column ascends to $6 \mathrm{~km}$ at an initial, prescribed velocity of $219 \mathrm{~m} \mathrm{~s}^{-1}$, sending up a buoyant cloud of ash that spreads laterally at $20 \mathrm{~km}$ a.s.1. After 10-15 minutes the column destabilizes, undergoes partial collapse, and begins to shed low-temperature dilute density currents $\left(<500 \mathrm{~g} \mathrm{~m}^{-3}\right.$ of ash) that propagate along the ground (Figure $2.5 \mathrm{~b})$. As in the no-microphysics control run, the column continues to generate a $6 \mathrm{~km}$ high collapsing fountain, forming voluminous ground hugging flows. However, these PDCs split into distinct transport regimes: (1) coignimbrite thermals, which become buoyant and feed a high-level, vigorously convective cloud; and (2) very dilute $(<10 \mathrm{~g}$ $\mathrm{m}^{-3}$ ash), fines-dominated PDCs (Figure 2.5d) that continue traveling long distances along the ground (up to $40 \mathrm{~km}$ from vent). Although the meter-scale internal structure of near-vent PDCs is cannot be reliably resolved at this grid resolution, a number of general observations can be made. The currents propagate as a ground hugging cloud 300-500 m thick, and $200 \mu \mathrm{m}$ crystal fraction settles out within $12 \mathrm{~km}$ from source, leaving the farther traveled, non-buoyant PDCs to be dominantly composed of the finer glass fractions.
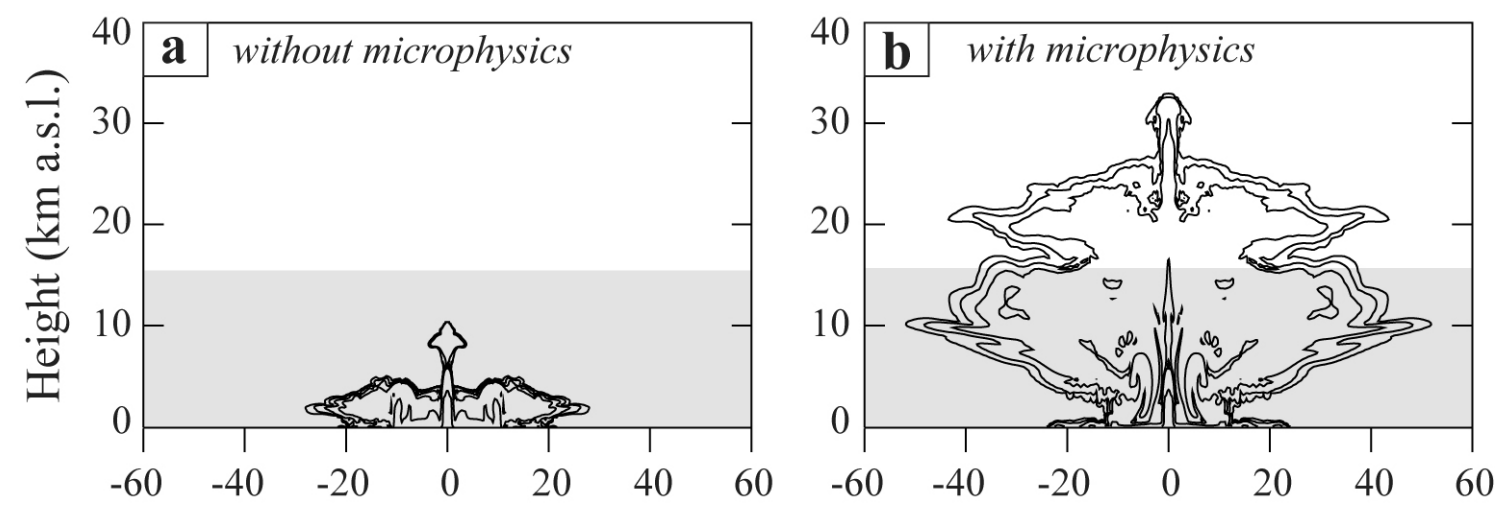

Distance from vent $(\mathrm{km})$

Figure 2.4 Comparison of volcanic cloud development in the reference simulation 24-A after one hour of continuous eruption in (a) the no-microphysics control run, and (b) with the microphysical module activated. Grey boxes designate the troposphere and black contours represent ash concentrations as in Figure 2.3. 


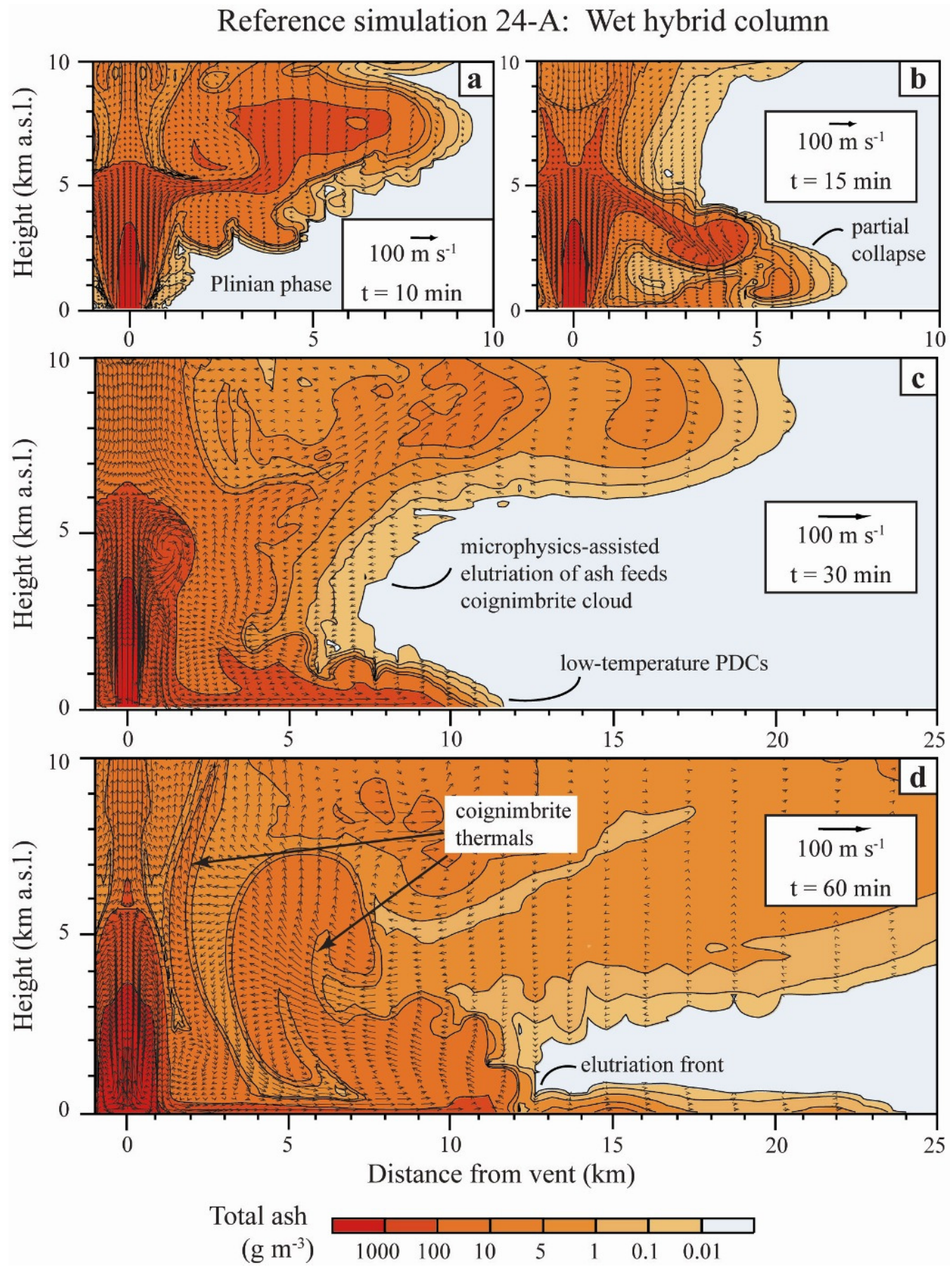

Figure 2.5 Time series of ash concentrations and fluid flow velocities in the lower 10 $\mathrm{km}$ of the atmosphere in the reference simulation (24\% surface water). Note different horizontal scale for the top row of diagrams.

Liftoff of the buoyant thermals occurs at an elutriation front (Figure 2.5d) that shifts between 7 and $12 \mathrm{~km}$ from vent. This behavior is exactly the same with and without microphysics. Therefore, the initial liftoff is attributed in this study to rapidly decreasing velocity of the flows (from a maximum of $80-90 \mathrm{~m} \mathrm{~s}^{-1}$ to negligible values 
by $c .10 \mathrm{~km}$ from vent) and increased buoyancy due to air entrainment and loss of mass to sedimentation.

\section{Convection Dynamics and Influence of Hydrometeors}

The abundance of hydrometeors and role of condensation in lofting the elutriated PDCs to stratospheric heights are illustrated in Figure 2.6. Microphysical impacts begin to take effect as temperatures in the ground-hugging PDCs cool to that of the surroundings within the proximal area $(<12 \mathrm{~km}$ from vent). Triggered by this temperature drop, water vapor in the ash-laden flows converts to liquid water, releasing significant amounts of latent heat. The energy release due to condensation $\left(c .10^{15} \mathrm{~J}\right.$, integrated between $750 \mathrm{~m}$ - $1.5 \mathrm{~km}$ a.s.l. over 60 minutes of eruption) provides the energy boost required to transport significant volumes of material from the ground to upper atmosphere (Figure 2.6b). The lifting PDCs ascend at an initially low velocity (1-5 $\left.\mathrm{m} \mathrm{s}^{-1}\right)$, carrying abundant liquid water and intruding into the atmosphere at an NBL of $c .12 \mathrm{~km}$. Beyond the elutriation front, remnant fines-dominated PDC material propagates along the ground as cold, dry flows to a maximum distance of $c .40 \mathrm{~km} 30$ minutes after the end of the eruption. Assuming a bulk field density of $1200 \mathrm{~kg} \mathrm{~m}^{-3}$, these distal PDCs would leave behind only a thin mm-scale deposit.
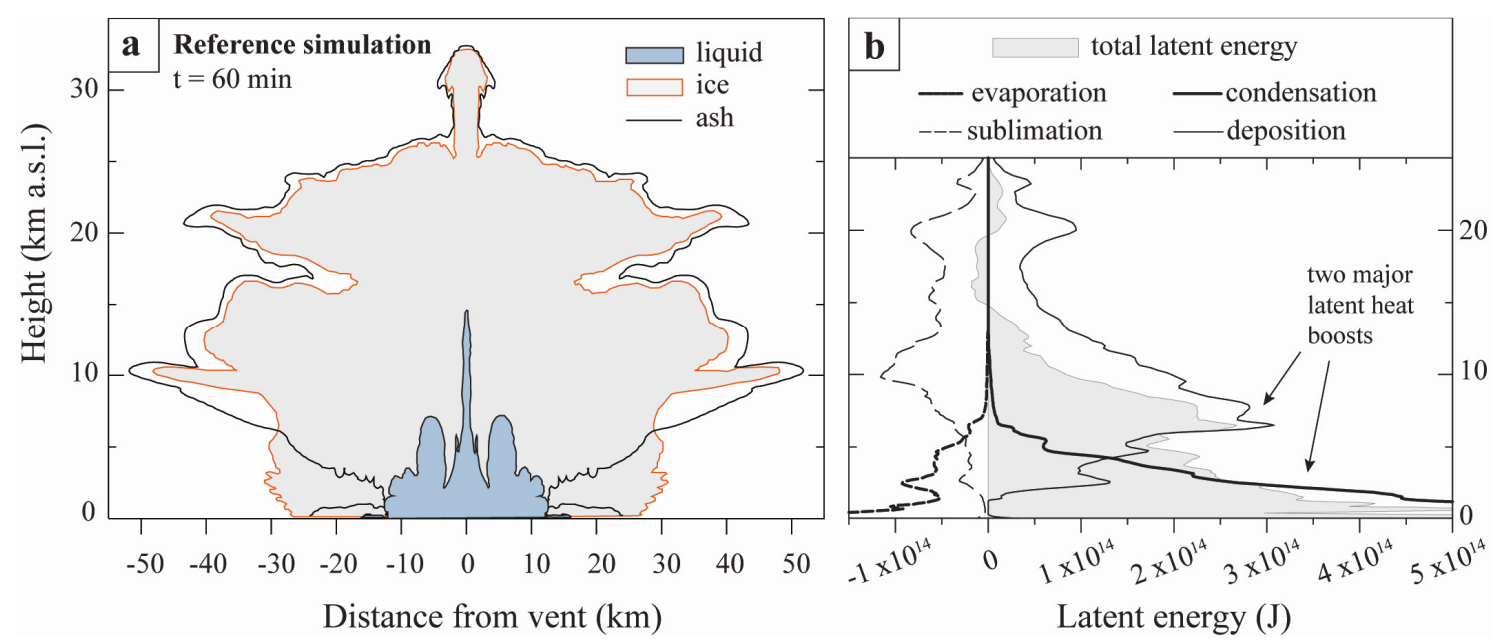

Figure 2.6 Microphysical effects in the reference simulation after one hour of continuous eruption. (a) Shows total ash, liquid hydrometeors (cloud and rain) and frozen hydrometeors (cloud ice and graupel) where they are in concentrations greater than $0.01 \mathrm{~g} \mathrm{~m}^{-3}$. (b) Represents the time-integrated latent energy exchange due to water phase changes over the first hour of eruption. Values are horizontally integrated, showing energy released (positive values) or consumed (negative) at each height in the atmosphere. Energy exchange due to freezing and melting are not shown because the contribution is negligible compared to other phase changes. Note: the peak energy 
release due to condensation occurs off the scale given and is of the order of $10^{15} \mathrm{~J}$ between $750 \mathrm{~m}$ and $1.5 \mathrm{~km}$ a.s.1.

The dominance of condensation and vaporization of water in the lower troposphere indicates an abundance of liquid in this region, and 40-80\% of airborne hydrometeors are in the liquid phase below $2.5 \mathrm{~km}$. Once the ashy currents reach the level of glaciation at $5 \mathrm{~km}$ a.s.1., the cloud is still mixed-phase, and ice coexists with small amounts of liquid (c. 4\%). Between 5 and $10 \mathrm{~km}$ a.s.1., conversion of liquid water and vapor to ice is reflected in the latent heat release (Figure 2.6b), providing another energy boost to the cloud and increasing the vertical velocities to $75-110 \mathrm{~m} \mathrm{~s}^{-1}$. Peak amounts of cloud ice and graupel at $14 \mathrm{~km}$ a.s.1. $\left(9.5 \times 10^{8} \mathrm{~kg}\right)$ are comparable to that of total ash at the same height $\left(5.8 \times 10^{9} \mathrm{~kg}\right)$. This would make the ash difficult to distinguish from meteorological clouds using conventional satellite detection methods [Simpson et al. 2001, Tupper et al. 2004].

Above c. $10 \mathrm{~km}$, the volcanic cloud and surrounding atmosphere contain negligible liquid water as ambient temperatures drop well below $-35{ }^{\circ} \mathrm{C}$, which is the threshold temperature for homogeneous nucleation of ice. Therefore dry, ice-forming processes dominate, generating an extensive cloud of ice and graupel (Figure 2.6a). After an hour of eruption, the total mass of airborne ice associated with the volcanic cloud is $c .48,000$ Mt of ice, more than $20 \%$ of which has been injected into the stratosphere. This is significantly greater than stratospheric ice load estimates from the Rabaul eruption cloud, Papua New Guinea, in 1994 [200-300 Mt: Rose et al. 1995] and from Pinatubo 15 June 1991 [80 Mt: Guo et al. 2004]. Indeed, the amount of airborne ice in the cloud vastly outweighs that of liquid water, which is on the order of 6,300 Mt and is almost entirely limited to the troposphere.

\section{Hybrid Ash Cloud Development}

The developing ash cloud above vent is fed by the continuous formation of coignimbrite thermals from PDCs, which send up episodic pulses of fines-dominated currents with ash concentrations generally $<75 \mathrm{~g} \mathrm{~m}^{-3}$. Consistent with results from the microscale PDC simulations of Neri et al. [2002], there is a delay on the order of 5-10 minutes from the first pulses of PDCs to the point at which the resultant updrafts reach the NBL and begin to spread out laterally. 
Figure 2.7 demonstrates that directly after column collapse, the dominant spreading level of ash (NBL) remains in the lower atmosphere as the collapsing fountain contributes most of its mass into syn-Plinian currents at the ground [Di Muro et al. 2004]. With development of the rising coignimbrite thermals, the spreading umbrella establishes 45 minutes into eruption at $12 \mathrm{~km}$ a.s.l. Fine ash intrudes at this altitude as a gravity-driven current, expanding radially in an episodic manner at velocities of 20-70 $\mathrm{m} \mathrm{s}^{-1}$ as material is supplied from the flows below. The resulting cloud clearly injects into the stratosphere, reaching a maximum overshoot of $33 \mathrm{~km}$ a.s.l. after an hour of eruption. Even when the simulation time is extended to erupt for three hours continuously (24-A-3hrs: Table 2.2), the values of maximum overshoot and spreading level remain relatively constant. After three hours of eruption the umbrella has expanded to a diameter of $c .220 \mathrm{~km}$ (in the assumed crosswind range) and the dilute PDCs reach $60 \mathrm{~km}$ from vent.

It is interesting to note that although the maximum cloud height is well within the stratosphere, in fact $85 \%$ of the total airborne ash is contained within the troposphere below $15 \mathrm{~km}$. Due to the rapid and repeated input of elutriated PDC material converging above the vent some minutes to tens of minutes after column collapse, there is no clear distinction between Plinian and coignimbrite components in the resulting hybrid ash cloud. However, the airborne grain size distribution does respond to changes in eruption style [Neri et al. 2002]. This is most clearly seen in the $200 \mu \mathrm{m}$ crystal tracers, which are less efficiently transported by weak coignimbrite thermals with slow liftoff velocities and fall out of the cloud earlier due to faster settling speeds (Figure 2.7). In contrast, updraft velocities during the Plinian phase are vigorous enough that there is less significant partitioning of size classes. For example, the percent of airborne $200 \mu \mathrm{m}$ ash that rises into the convective cloud above the jet thrust region ( $>6.5 \mathrm{~km}$ a.s.1.) decreases sharply after column collapse from a maximum of $30 \%$ during the Plinian phase to less than $15 \%$ after 45 minutes. Responding to the behavior of the simulated column with time, the resulting hybrid cloud is increasingly fed by fine grained coignimbrite thermals. 


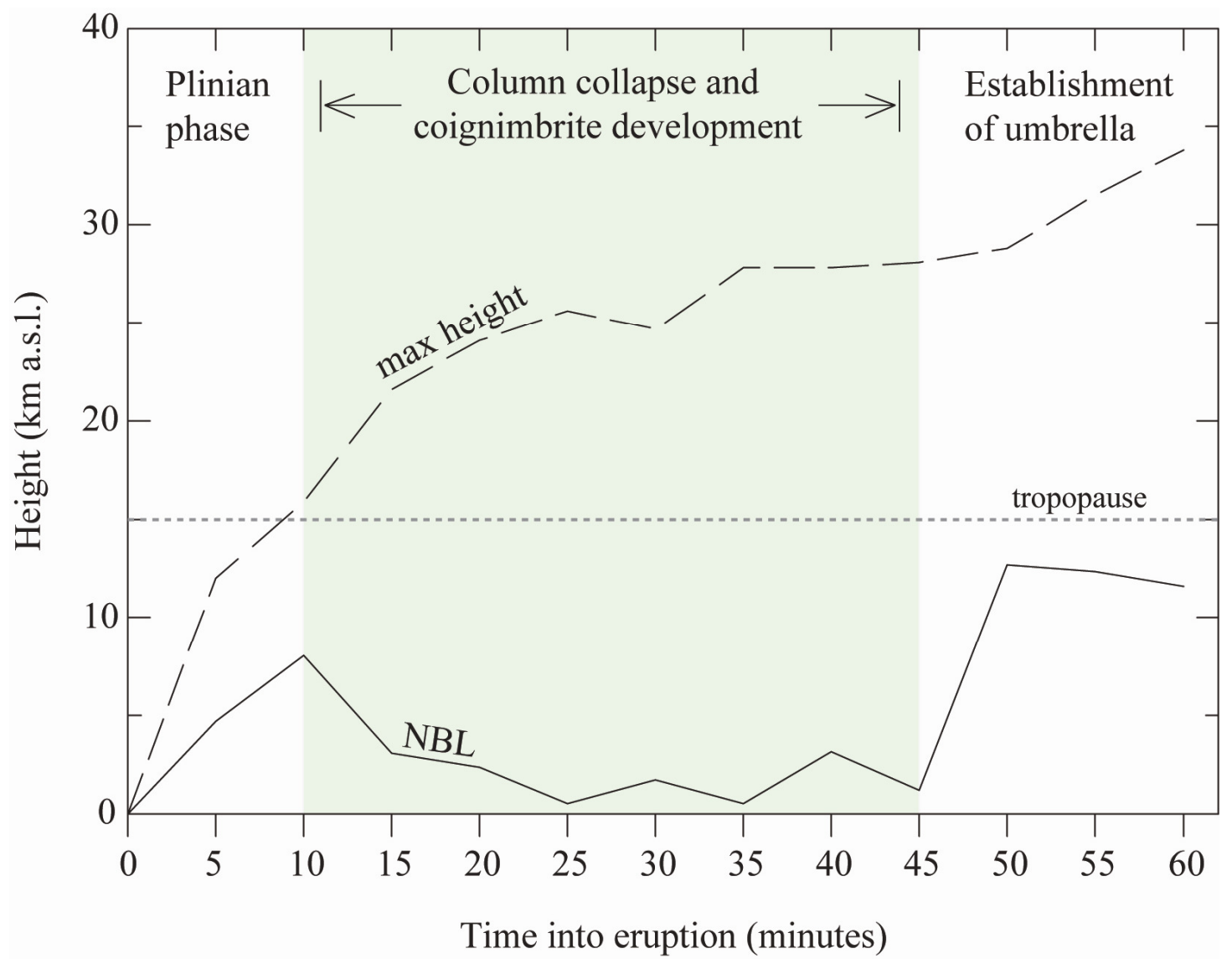

Figure 2.7 Temporal evolution of ash cloud heights (maximum and neutral buoyancy level) in the reference simulation. Note that the maximum height continues to increase even after column collapse, indicating the ability to form fall and flow deposits synchronously throughout most of the eruption.

\section{Influence of Ash Aggregation}

In the absence of a complex ash aggregation module that explicitly solves for the interaction of silicate particles and hydrometeors [e.g. Textor et al. 2006a], a crude approximation is employed in this study to test the impact of abundant aggregation on ascent of the hybrid cloud. Given the presence of abundant liquid water close to the vent (Figure 2.3c), 50\% of the ash particles are assumed to have instantaneously and indiscriminately (i.e. equally across all three size classes) converted into aggregates 1 $\mathrm{cm}$ in diameter with densities of $1200 \mathrm{~kg} \mathrm{~m}^{-3}$. These aggregate dimensions are consistent with field measurements of accretionary lapilli from the Oruanui eruption deposits [Self, 1983, Van Eaton and Wilson in review; Chapter 5 of this thesis] and other field studies of wet eruptions [Schumacher and Schmincke 1991]. However, the assumption of immediate aggregation in the near-vent column is as yet untested with field data. Although direct observations have indicated that aggregates can form in the 
near-vent region [Booth and Walker 1973, Taddeucci et al. 2011], the full spectrum of processes is much more intricate. Additional studies have suggested that ash aggregates can develop in volcanic clouds hours to days after eruption [Guo et al. 2004, Durant et al. 2009], and occur on a variety of timescales [Costa et al. 2010, Folch et al. 2010]. Furthermore, the simplified representation of aggregation used in this investigation does not take account of the removal of hydrometeors (and associated latent energy) with the fallout of aggregated ash, which could have a substantial effect on the latent heatdominated plume dynamics. Given the lack of experimental, theoretical and observational constraints on the locations and timescales of aggregation in very wet eruptions, this approach is an exploratory one, and is generally expected to decrease the height and residence time of the volcanic cloud.

Figure 2.8a-b compares the mass of airborne ash classes at each height with and without aggregation. After an hour of eruption in the aggregation scenario, 98 wt.\% of the airborne aggregates are below $6 \mathrm{~km}$ a.s.l. (Figure 2.8b). A proportion of these aggregate tracers was also carried upward by coignimbrite thermals to heights of $c .27 \mathrm{~km}$ a.s.l. and transported laterally to a maximum distance of $15 \mathrm{~km}$ from vent, indicating the ability to be widely dispersed by the dynamics of the hybrid cloud alone (i.e. in the absence of wind). However, using the current code it is impossible to resolve the stability of the aggregates (due to breakup, for example) during transport in PDCs and buoyant thermals.

As a consequence of half the eruptive material converting to aggregates, about $50 \%$ less volcanic ash is emplaced into the atmosphere compared to the reference simulation in Figure 2.8. This is consistent with the general expectation that aggregation shortens the lifetime of volcanic clouds. However, there was an unanticipated effect on the buoyancy of the transitional column. Remaining particles that were not incorporated into aggregates ascended to significantly higher levels than in the absence of aggregation. This phenomenon is attributed in this study to gravitational separation of aggregates from the column and coignimbrite updrafts, causing the hybrid cloud to become less dense and more buoyant, and resulting in a $27 \%$ increase in the mass of ash in the stratosphere. 

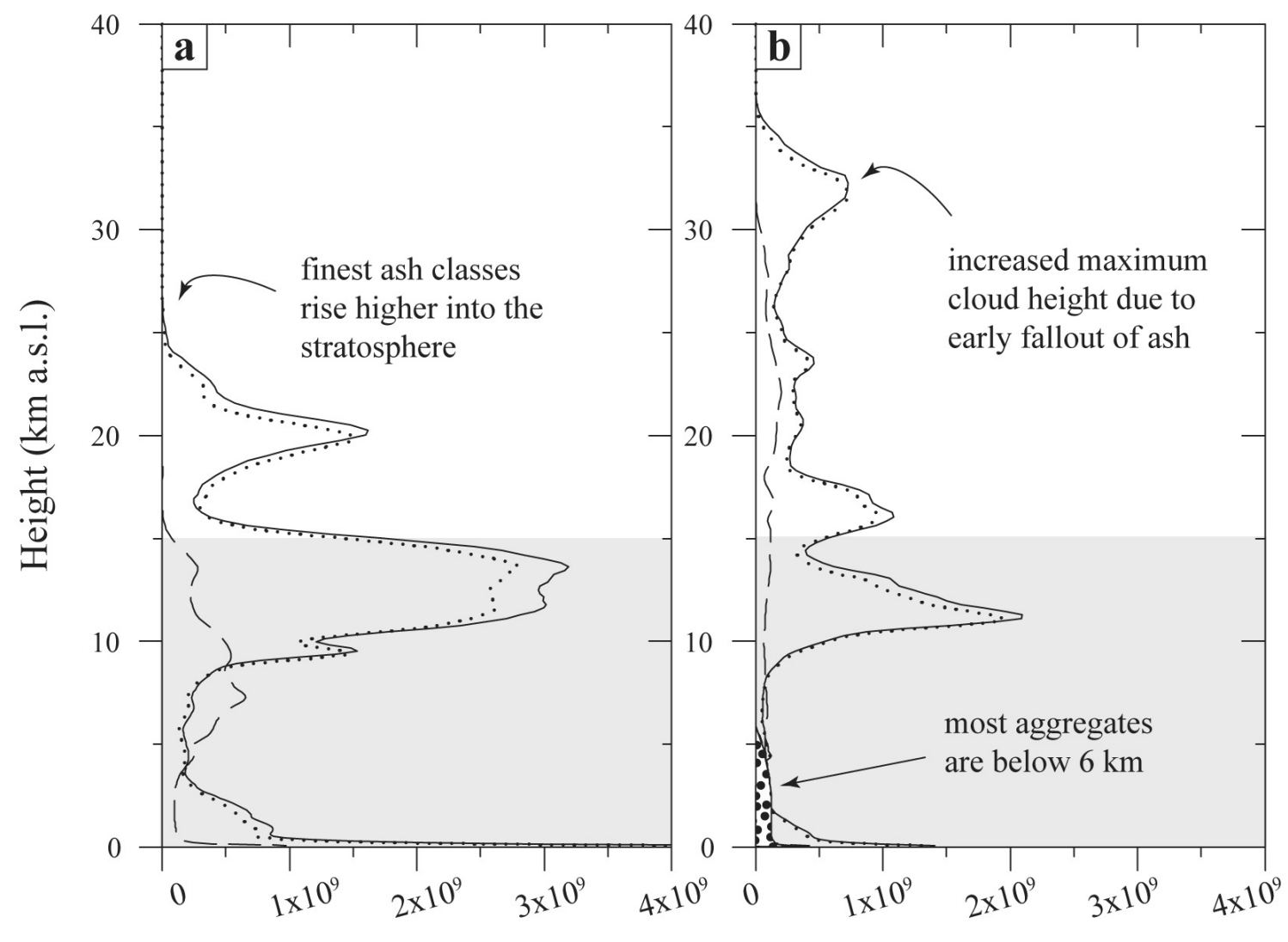

Mass of airborne tracers at each height $(\mathrm{kg})$

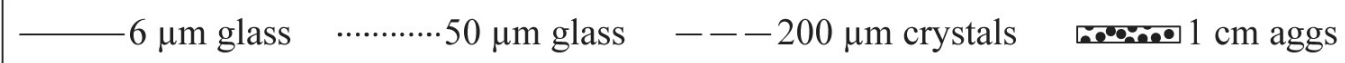

Figure 2.8 Horizontally integrated mass of ash classes at $t=60 \mathrm{~min}$. (a) Shows the reference simulation without aggregation. Grey boxes designate the troposphere. Note how the $6 \mu \mathrm{m}$ and $50 \mu \mathrm{m}$ vitric ash behave similarly, while the coarser, denser crystals are less abundant at higher levels due to inefficient entrainment in coignimbrite updrafts and earlier fallout. (b) Shows the same eruption assuming that $50 \mathrm{wt} . \%$ of the ash instantaneously converts into $1 \mathrm{~cm}$-diameter ash aggregates.

This is an interesting result that deserves attention in future work. The immediate suggestion is that, under certain conditions, aggregation may have the capacity to boost fine ash farther into the stratosphere than previously assumed. ATHAM simulations by Textor et al. [2006b] found a similar buoyancy-promoting effect in their dry, Plinian eruption columns. This is despite using a more complex, aggregation-capable microphysics, and primarily ice-phase plume conditions that led to primarily icy ash aggregates. Their work shows a progressive increase in neutral buoyancy height with progressive increases in the aggregation collection efficiency (their Figure 15). General agreement between the different approaches suggests that the larger and more abundant 
the aggregates, the greater the ability of gravitational separation to boost cloud height. Development of hydrometeor-ash aggregates over time in various regions of the cloud (i.e. in the umbrella vs. column) would exert different controls on plume buoyancy. It is therefore suggested that future modeling efforts in this area compare different timescales and locations of aggregation in volcanic clouds with field data from active eruptions and their deposits.

\section{High MER scenario: Phreatoplinian column}

The reference simulation was modified to examine the effect of an order of magnitude higher MER by increasing the plume diameter to $675 \mathrm{~m}$ and exit velocity to $548 \mathrm{~m} \mathrm{~s}^{-1}$ (24-A-high of Figure 2.3d). As expected from previous 1-D studies on critical thresholds for column stability, the much higher initial velocity pushes the eruption column well into the stability field [Koyaguchi and Woods 1996: Figure 6]. The top of the phreatoplinian plume reaches $35 \mathrm{~km}$ within the first 5 minutes of eruption and forms a spreading umbrella at $27 \mathrm{~km}$. Due to rapid injection of ash from the Plinian column well into the stratosphere, there is significantly less turbulent entrainment of ambient air compared to the case of a collapsing column. Consequently, ash reaching the plume top is still significantly warmer than the surroundings, and liquid water persists at much higher regions of the atmosphere (Figure 2.3d). The total liquid water content in the airborne cloud is $\sim 47,000 \mathrm{Mt}$, more than $75 \%$ of which is stratospheric. This would allow wet microphysical processes, including hydrometeor-ash aggregation, to occur in the stratosphere at distances greater than $80 \mathrm{~km}$ from vent.

In contrast, the no-microphysics control run for this simulation shows comparatively limited capacity for widespread ash dispersal. The eruption column produces a $35 \mathrm{~km}$ high collapsing fountain with a small amount of spreading at $15 \mathrm{~km}$, yet almost all of the erupted material collapses downward in the formation of non-buoyant PDCs. By comparison, it is evident that the ability for the column to form a high level spreading umbrella can be attributed to the energy release associated with condensation and ice formation in the stratosphere (mostly via deposition directly from vapor to ice due to the low temperatures at high altitude). 


\subsection{Modification of the Reference Simulation}

The impact of varying the amount of magma-water interaction for eruptions is examined here with the same MER in the reference atmosphere. As the output rate of magma also exerts a fundamental control on eruption style, the end of each section addresses changes in column behavior due to an order of magnitude increase in MER.

\subsubsection{Dry Collapsing Column (0\% Surface Water)}

Simulation 0-A (Figure 2.9) shows dominantly collapsing behavior due to the low initial velocity of the plume $\left(84 \mathrm{~m} \mathrm{~s}^{-1}\right)$, as the momentum of the erupted material was not sufficient to form a buoyant, convecting column [Woods 1998]. A small proportion of the erupted material $(<10 \mathrm{wt} \%)$ continues rising above the $1 \mathrm{~km}$ high collapsing column and PDCs at $\mathrm{t}=5 \mathrm{~min}$ (Figure 2.9a), but the overwhelming majority of pyroclasts spread laterally away from the vent as hot ground hugging currents. These lift off, forming coignimbrite thermals between 3 and $6 \mathrm{~km}$ from vent (Figure 2.9b-c). The farther traveled, non-buoyant PDCs continue out to $27 \mathrm{~km}$ from vent after an hour of eruption (Figure 2.9d). Lofted thermals form a pulsating coignimbrite column, which punctures the tropopause within 10 minutes and reaches the maximum overshoot after 30 minutes (33 km a.s.1.), forming a spreading umbrella at the base of the stratosphere. The eruption temperature at vent is much higher in this case $\left(767^{\circ} \mathrm{C}\right)$, due to the lack of interaction with surface water, and the sensible heat energy is sufficient to generate significant lofting of the PDCs. Indeed, comparison with the no-microphysics control shows identical column behavior in both simulations, indicating that the entire dynamic of column collapse and elutriation of thermals is driven by the transfer of sensible, rather than latent, energy. The total amount of water released from magmatic volatiles alone during the hour-long eruption is of the order of 23,000 Mt, and entrainment of relatively dry ambient air added $814 \mathrm{Mt}$ of water to the airborne cloud during ascent.

Increasing the initial velocity of the erupted mixture to $209 \mathrm{~m} \mathrm{~s}^{-1}$ increases the MER by approximately an order of magnitude (Figure 2.3h), resulting in greater column stability and generation of an initial Plinian phase. This stable column persists until c. 22 minutes into eruption, followed by wholescale collapse. In the no-microphysics control run, however, collapse never occurs, indicating that, in this case, microphysical effects generated a flow instability partway through eruption. 
Simulation 0-A: Dry collapsing column
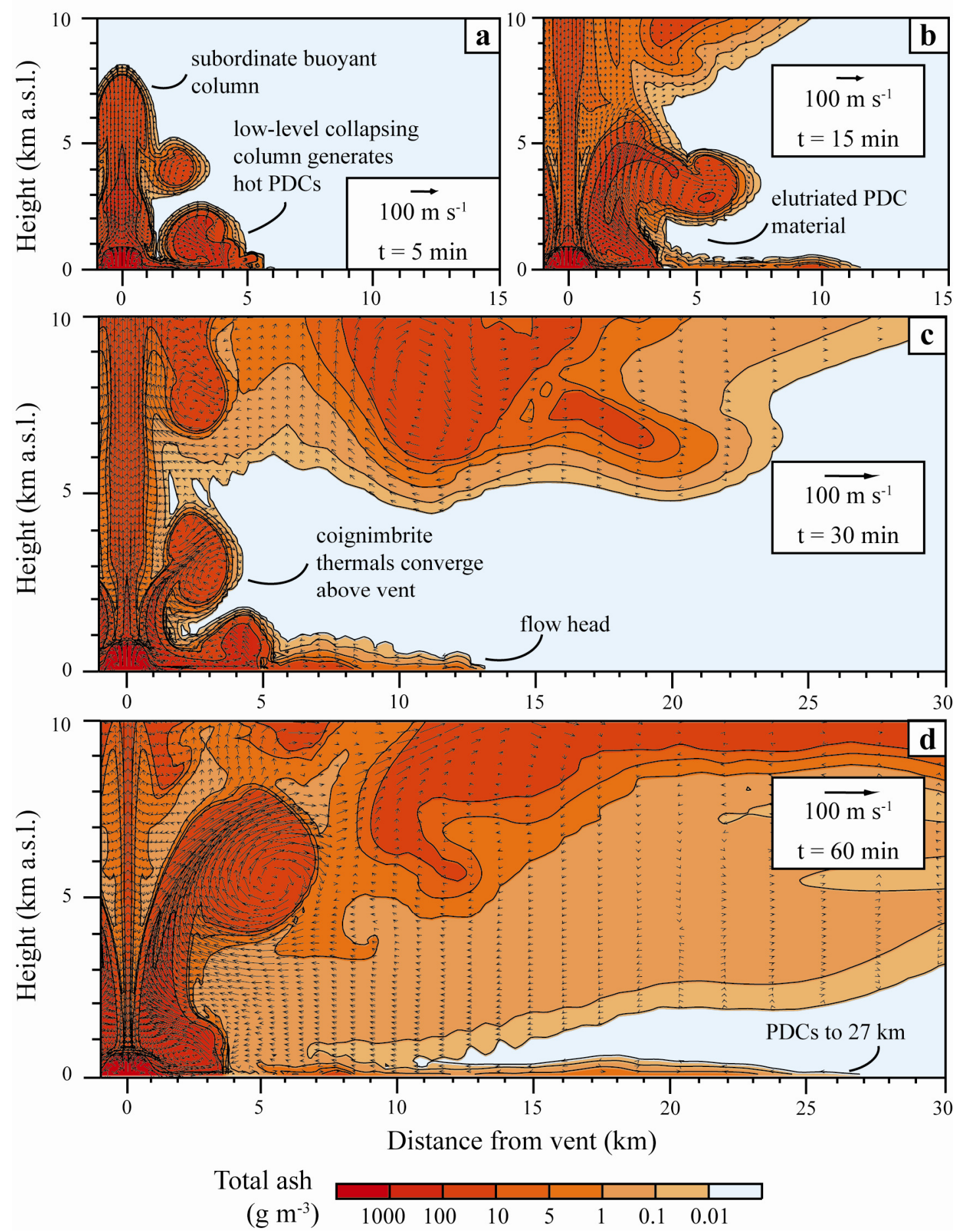

Figure 2.9 Time series of ash concentrations and fluid flow velocities in the lower 10 $\mathrm{km}$ of the atmosphere for the dry collapsing fountain. Note different horizontal scale for the top row of diagrams. 


\subsubsection{Unsaturated Plinian Column (10\% Surface Water)}

Compared to the dry eruption scenario, addition of a small amount of external water at vent (10\%) has a stabilizing effect on the column, consistent with the findings of Koyaguchi and Woods [1996]. Their study indicated that $<15 \%$ surface water decreases the initial density of the erupted mixture (in this case, to $2.0 \mathrm{~kg} \mathrm{~m}^{-3}$, the lowest of all the simulated eruptions in Table 2.1), which promotes the transition from collapsing to convective regimes. Simulation 10-A (Figure 2.10a-c) produces a Plinian-style column with a sustained injection height (NBL) at $23.5 \mathrm{~km}$. During the initial 5 minutes of eruption the ascending flow is unstable, and turbulent currents detach from the central column (Figure 2.10a). This decoupled material continues to rise buoyantly and ascends upward into a convective plume, rather than collapsing downward to form groundhugging currents.

Maximum cloud height is $c .43 \mathrm{~km}$, roughly consistent with the $48 \mathrm{~km}$ cloud height expected from the empirical height scaling relationship of Sparks et al. [1997: their Eq. 5.1]. However it is notable that, in contrast to the coignimbrite or hybrid eruption clouds previously discussed, less than $20 \%$ of the airborne ash is below the tropopause (Figure 2.11). This reflects the much stronger updrafts in the stable central column of a Plinian eruption in comparison with the weaker, but persistent, thermals of a coignimbrite cloud [Herzog and Graf 2010], and suggests that use of height scaling relationships in the Plinian case would lead to a more realistic and useful interpretation of cloud's position in the atmosphere. Figure 2.12 compares the vertical profiles of hydrometeors associated with the volcanic clouds from all of the low MER eruption scenarios and, compared to the reference simulation, it can be seen that the Plinian-style plume from the $10 \%$ water eruption emplaces almost all of its airborne hydrometeors (94 wt.\%) above the tropopause. This results in approximately an order of magnitude greater stratospheric water loading.

With a higher MER, the column retains its Plinian behavior, but increases maximum height and NBL (Figure 2.3f). Like their dry counterparts, both low and high MER eruption scenarios are not appreciably different with respect to the no-microphysics control. 
Simulation 10-A: Wet Plinian column
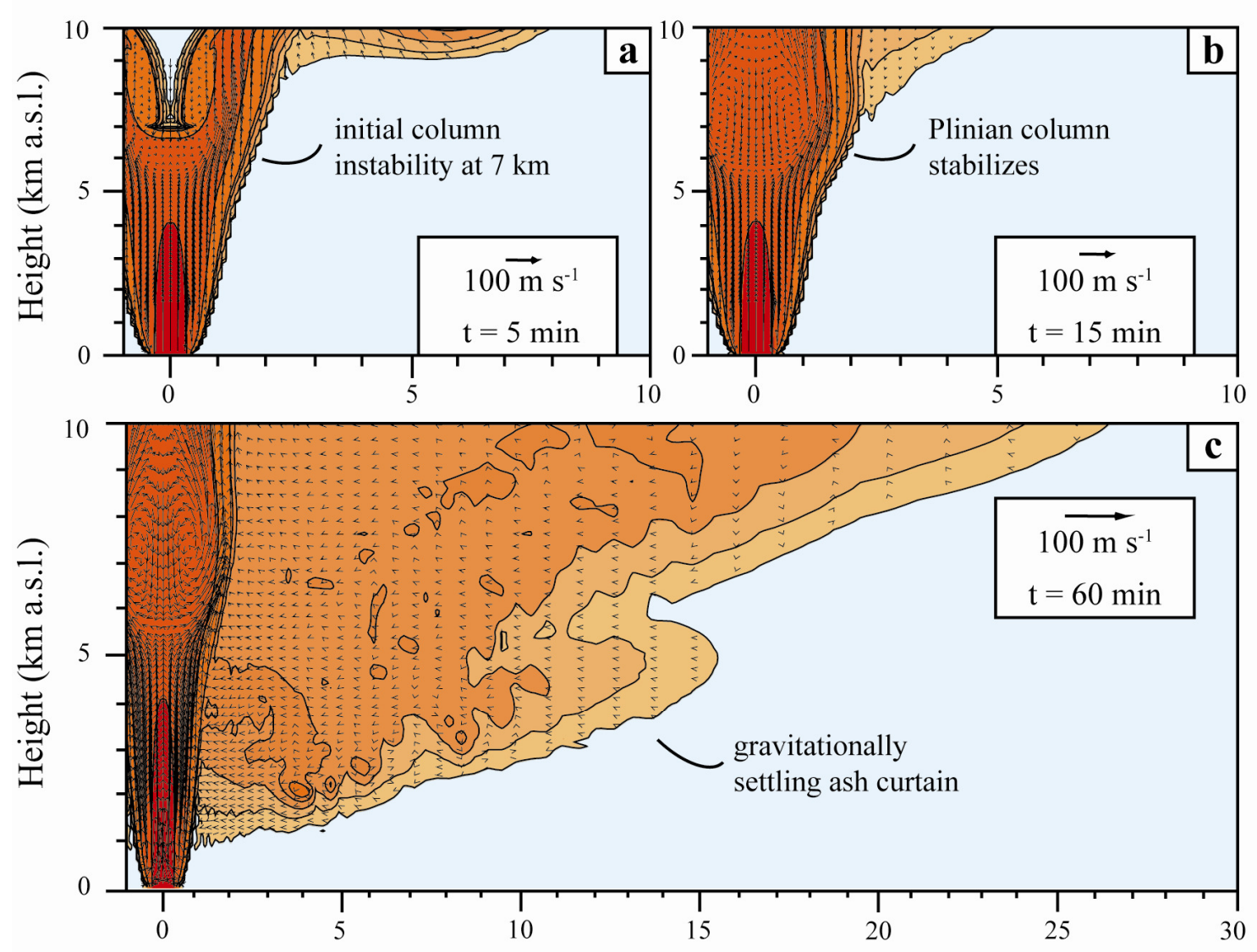

Distance from vent $(\mathrm{km})$

Total ash

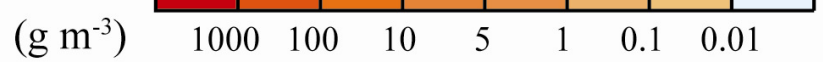

Figure 2.10 Time series of ash concentrations and fluid flow velocities in the lower 10 $\mathrm{km}$ of the atmosphere for the Plinian eruption involving $10 \%$ surface water. Note different horizontal scale for the top row of diagrams. 

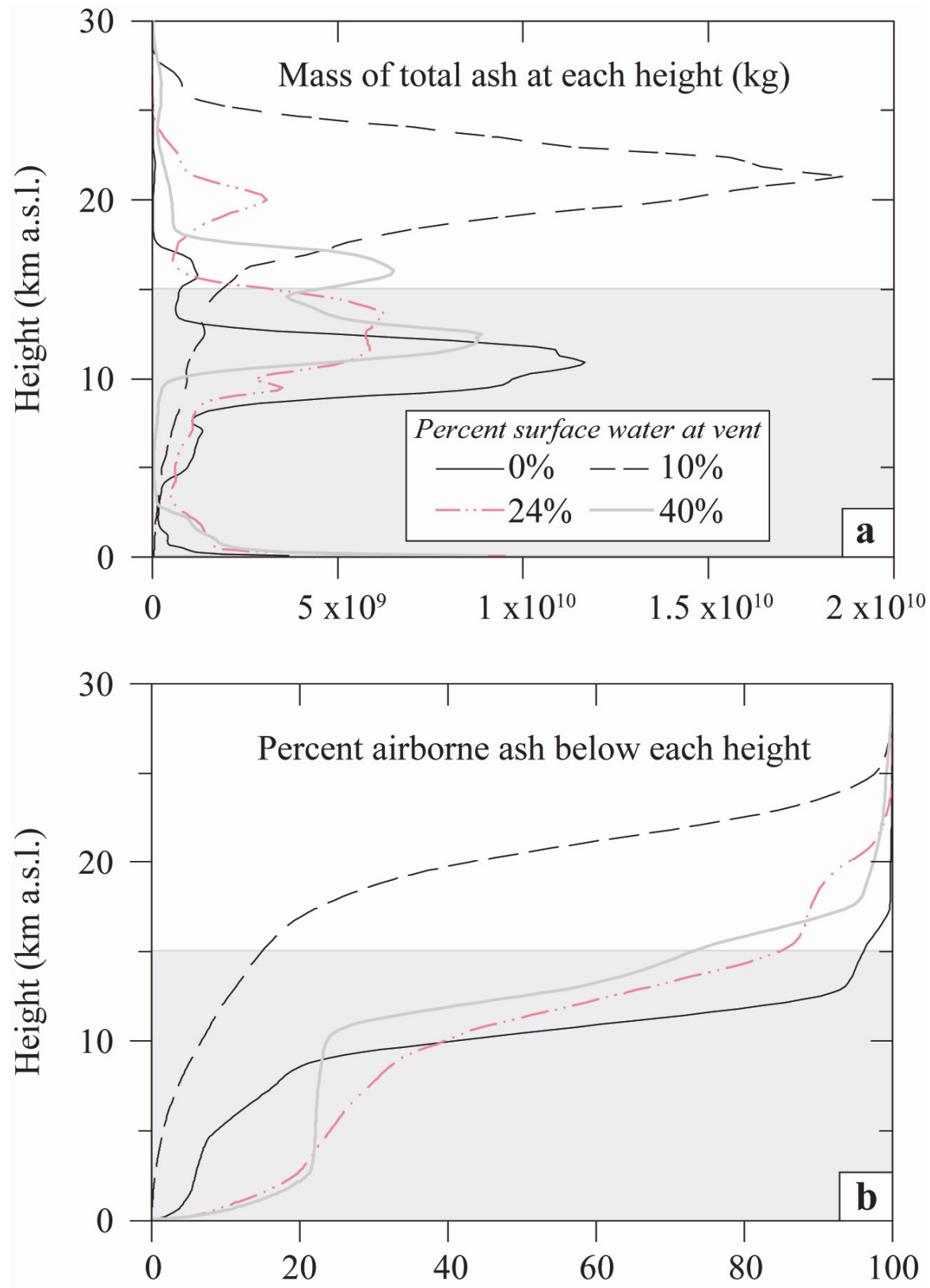

Figure 2.11 Vertical profiles of ash emissions for low MER eruptions in the reference atmosphere at $\mathrm{t}=60 \mathrm{~min}$. (a) shows the horizontally integrated mass of airborne ash at each height, and (b) shows the cumulative percent of airborne ash below a given height. Grey boxes designate the troposphere. 


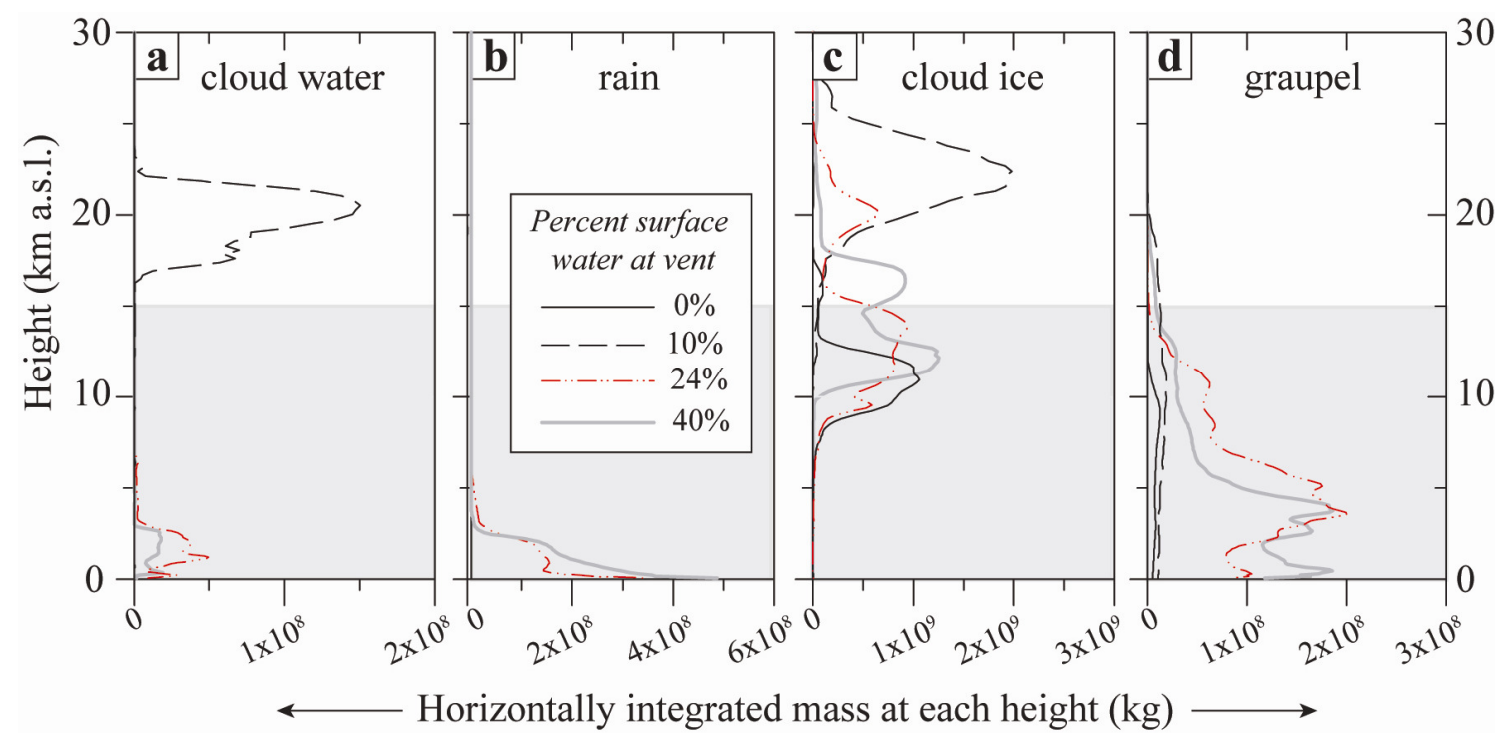

Figure 2.12 Horizontally integrated mass of airborne hydrometeors from each low MER eruption in the reference atmosphere at $\mathrm{t}=60 \mathrm{~min}$, showing (a) cloud water (small liquid drops), (b) rain (large liquid drops), (c) cloud ice (small frozen crystals), and graupel (large frozen clusters). Grey boxes designate the troposphere. Note: eruption clouds with small abundances of a hydrometeor class do not show up.

\subsubsection{Saturated Transitional Column (40\% Surface Water)}

The extreme wet end-member eruption simulation 40-A represents a saturated mixture with respect to water. The magmatic heat is not sufficient to vaporize all the surface water at vent, and erupts as a $100{ }^{\circ} \mathrm{C}$ mixture of pyroclasts, vapor and $c .25 \mathrm{wt} \%$ liquid water (Table 2.1). The simulation yields a transitional column similar to the reference simulation, exhibiting transitional convective and collapsing behavior (Figure 2.13a-d). In contrast, however, there is no initial 'pure' Plinian phase, as both styles of column behavior occur simultaneously throughout the eruption. This combination of intermittent Plinian injection and deep moist convection in the PDCs allows the hybrid cloud to ascend to the highest maximum overshoot of any of the low MER eruptions simulated. The oscillating Plinian column emplaces ash well into the stratosphere (maximum overshoot $40 \mathrm{~km}$, NBL $10.8 \mathrm{~km}$ ), simultaneously shedding voluminous dilute PDCs. As with the reference scenario, the resulting ground hugging currents are partitioned into proximal thermals $(<12 \mathrm{~km}$ from vent) that merge into the Plinian column, and non-buoyant flows that continue propagating along the ground to a maximum distance of $26 \mathrm{~km}$ from vent after 60 minutes, and $42 \mathrm{~km} 30$ minutes after the end of the eruption. 


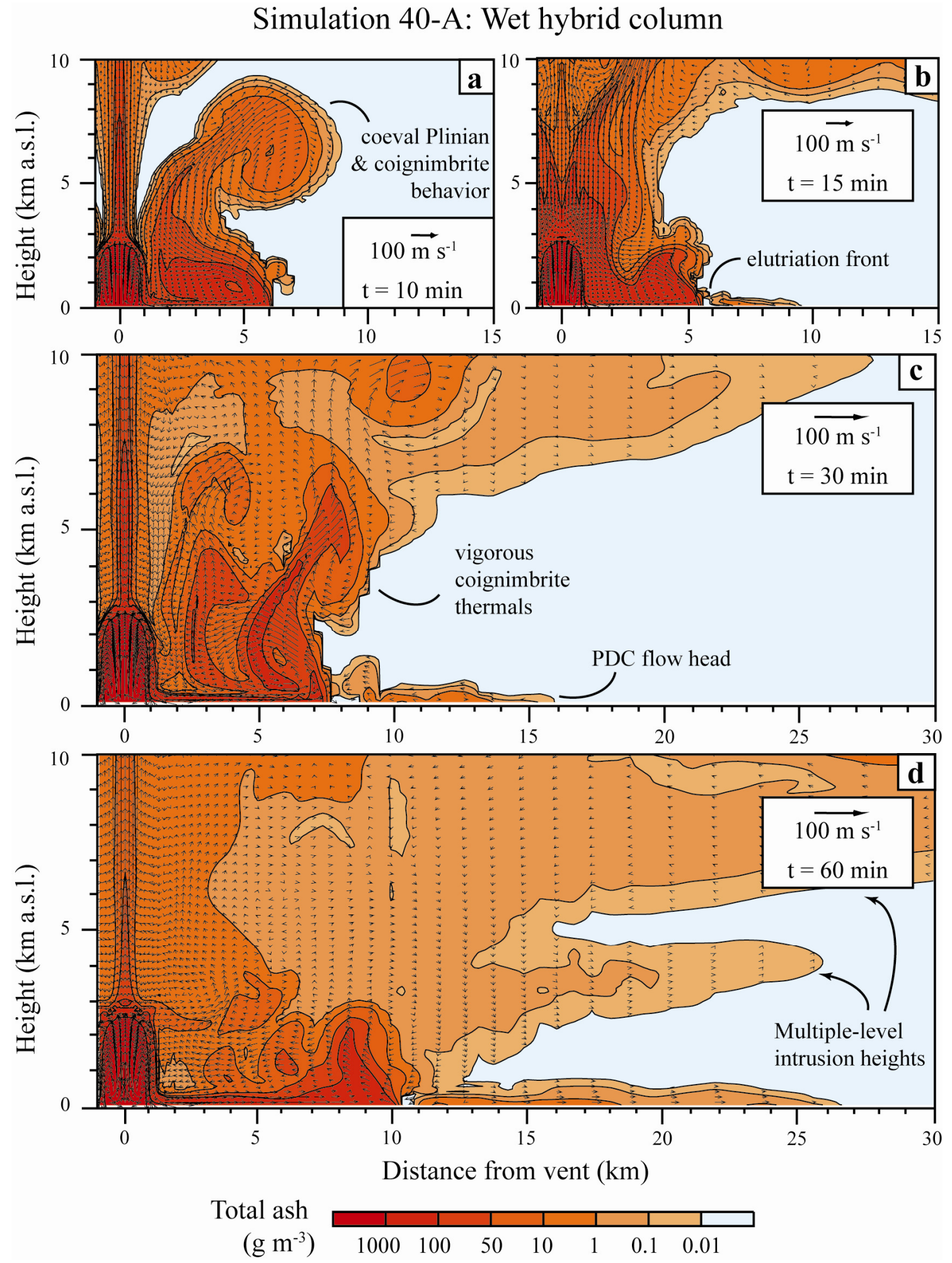

Figure 2.13 Time series of ash concentrations and fluid flow velocities in the lower 10 $\mathrm{km}$ of the atmosphere for the saturated transitional column (40\% surface water). Note different horizontal scale for the top row of diagrams. 
On the contrary, the no-microphysics control generates a $3.5 \mathrm{~km}$ high collapsing column which sends up a small vent-derived cloud to $c .6 \mathrm{~km}$. The majority of material travels as dense PDCs to $45-50 \mathrm{~km}$ from vent and barely ascends above $3 \mathrm{~km}$. This further emphasizes the role of latent heat exchange in producing buoyant, voluminous hybrid cloud from low temperature wet flows. Increasing the MER (Figure 2.3b) does not result in major dynamic changes, except for a notable increase in the NBL to $24 \mathrm{~km}$ a.s.l. and greater injection of liquid water into the stratosphere.

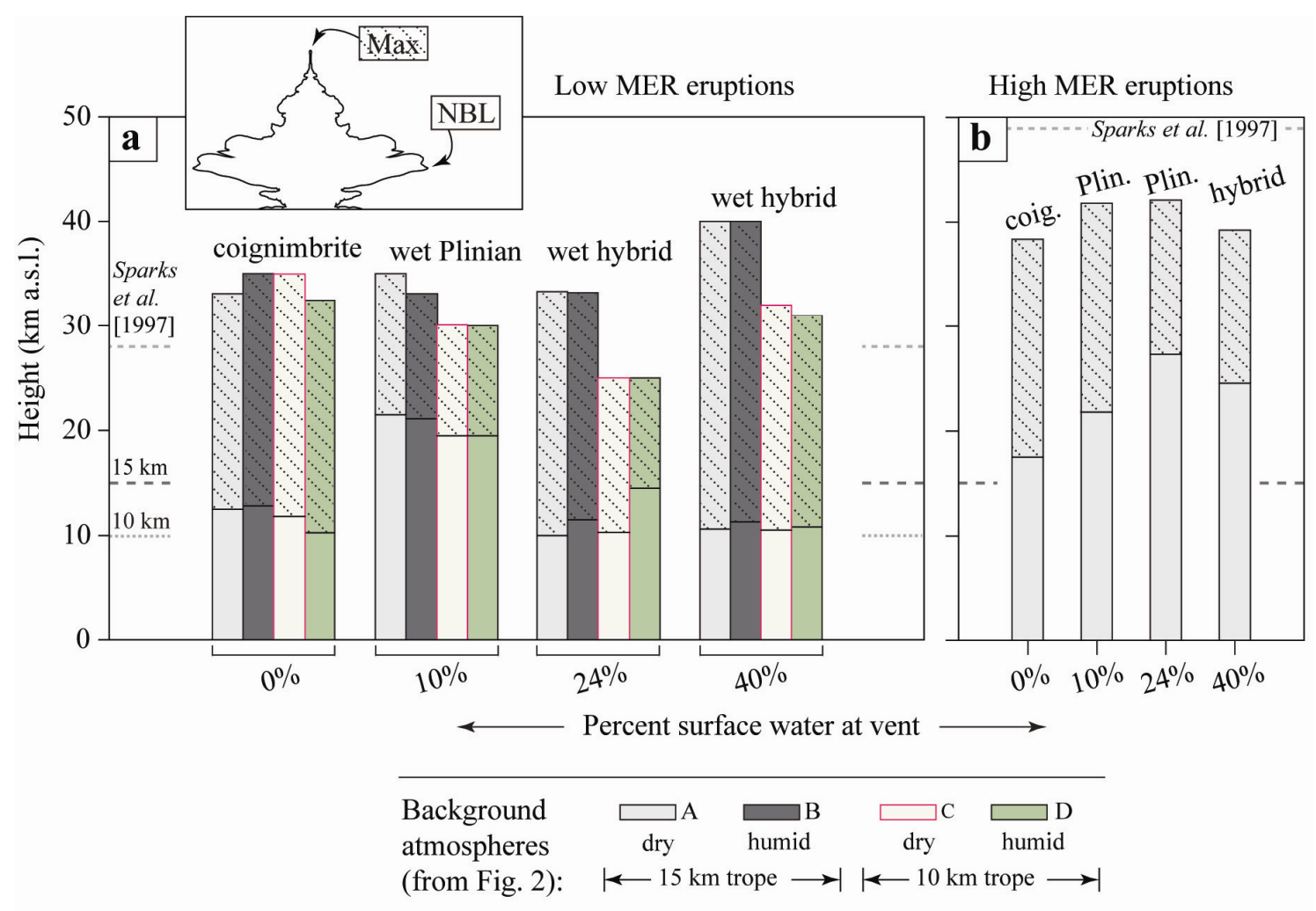

Figure 2.14 Summary of ash cloud heights from low and high MER simulations. Stippled bars indicate maximum overshoot, solid bars indicate neutral buoyancy level (NBL). (a) Low MER eruption cloud heights are shown from all four background atmospheres. (b) Shows high MER eruptions, which were simulated in the reference atmosphere only. For comparison, shaded boxes indicate the $15 \mathrm{~km}$ troposphere (light grey) and $10 \mathrm{~km}$ troposphere (dark grey). In both graphs the dashed line gives the NBL calculated from the scaling relationship of Sparks et al. [1997: their Eq. 5.1].

\subsection{Sensitivity to Surface Water Content and Meteorological Conditions}

Figure 2.14 summarizes volcanic cloud height estimates (maximum height and NBL) from the simulated eruptions at high and low MER. The height summaries indicate that changing the surface water content of an eruption does not systematically increase or 
decrease the injection height of the resulting cloud once microphysics and the dynamics of buoyant PDCs are accounted for. Rather, the maximum overshoot and NBL are strongly controlled by magma output rate and eruption style, which do not vary linearly with surface water content. However, there is a clear relationship between addition of abundant surface water $(>10 \%)$ and the development of column instabilities leading to dynamic collapse and production of buoyant coignimbrite thermals. The unstable columns generally tend to distribute more mass (of ash and hydrometeors) in the troposphere than their stable Plinian counterparts at the same MER. A further important control on ash cloud ascent includes the structure of the atmosphere, as discussed in the following sections. Each of the low MER eruption scenarios was duplicated under the four background atmospheres to examine sensitivity to changes in tropopause height and background humidity (Figure 2.13a).

\subsubsection{Tropopause Height}

The wet eruptions demonstrate a notable sensitivity to tropopause height compared to dry eruptions at the same MER (Figure 2.14a). Although the NBL was not systematically affected, maximum heights for surface water-rich eruptions increased by $10-25 \%$ when tropopause height increased from 10 to $15 \mathrm{~km}$ a.s.l. In contrast, the dry eruptions show a much less pronounced sensitivity to tropopause height, suggesting their dominant ascent mechanism (i.e. sensible heat exchange) is somewhat more vigorous than the deep moist convection in water-rich clouds. With the exception of the stable Plinian eruptions (10\% surface water: Figure 2.14a), all simulations in the low MER suite have a spreading level at or very close to the local tropopause. This is not the case in the high MER examples (Figure 2.14b), which intrude as density currents into the stratosphere regardless of eruption style (coignimbrite, Plinian or hybrid). This indicates that despite the complicating factors of convective process and differences in the thermal structure of the atmosphere, the magma output rate of an eruption still exerts considerable control on the vertical profile of volcanic emissions.

\subsubsection{Background Humidity}

The numerical studies of, for example, Woods [1993], Glaze and Baloga [1996], and Graf et al. [1999] have documented an increase in cloud heights for dry, relatively small eruptions in humid conditions. Similar responses have been indicated for 
pyroconvective (wildfire) clouds [Trentmann et al. 2006]. Each of these studies concludes that incorporation of atmospheric water vapor and the subsequent latent heat boost during condensation results in a greater vertical extent of the cloud. The effect is most pronounced in smaller eruptions with output rates less than $c .10^{6} \mathrm{~kg} \mathrm{~s}^{-1}$ owing to a greater relative amount of entrained water compared to magmatic water already in the plume [Sparks et al. 1997].

In the present simulations of high intensity eruptions with magma output rates $>10^{8} \mathrm{~kg}$ $\mathrm{s}^{-1}$, changes in ambient humidity produced little effect in the heights of eruption clouds. There was a minor increase in the NBL of the wettest eruptions in the more humid atmosphere compared to the dry atmosphere, producing 13-29\% and 3-6 \% increases in NBL for the $24 \%$ and $40 \%$ surface water eruptions, respectively. However, the increase was not systematically observed across all eruptions. A similarly low sensitivity to humidity was found in simulations of large eruptions by Textor et al. [2006b]. A more consistent observation is that atmospheric moisture exerts substantial influence on the total amount of airborne hydrometeors (Figure 2.15a). In all low MER eruptions tested, higher humidity leads to systematically greater production of hydrometeors (atmospheres B and D of Figure 2.15a). The fraction of airborne hydrometeors in the ice phase is given in Figure 2.15b. Even the two wettest eruptions, which produce the greatest mass of airborne liquid water, are still dominated by ice.

\subsection{Implications for Formation of Hydrometeor-Ash Aggregates}

Experimental aggregation studies employing wind tunnel experiments [Gilbert and Lane 1994] and pan agglomeration techniques [Schumacher and Schmincke 1995], have highlighted optimal conditions for the growth of wet aggregates. These conditions include abundant fine ash particles and liquid water (10-30\%), and opportunities for particle collision (in the form of turbulent updrafts and downdrafts, gravitational capture, for example). In the case of the water-rich hybrid clouds from reference simulation 24-A, coignimbrite thermals produce strong updraft velocities in the lower troposphere, coincident with high concentrations of fine ash and liquid. These observations are consistent with ample field data [e.g. Wilson 2001, Brown et al. 2010] in suggesting that conditions for formation of wet aggregates would be readily met in the convective ash clouds following pulses of moist PDCs. Attention should also be 
drawn to the abundance of ice particles in the volcanic cloud, some large enough to gravitationally settle to ground-level (Figure 2.6a). Ice may contribute significantly to aggregation in such cases [Guo et al. 2004], particularly when melting occurs, enveloping ice particles in a sticky liquid film.

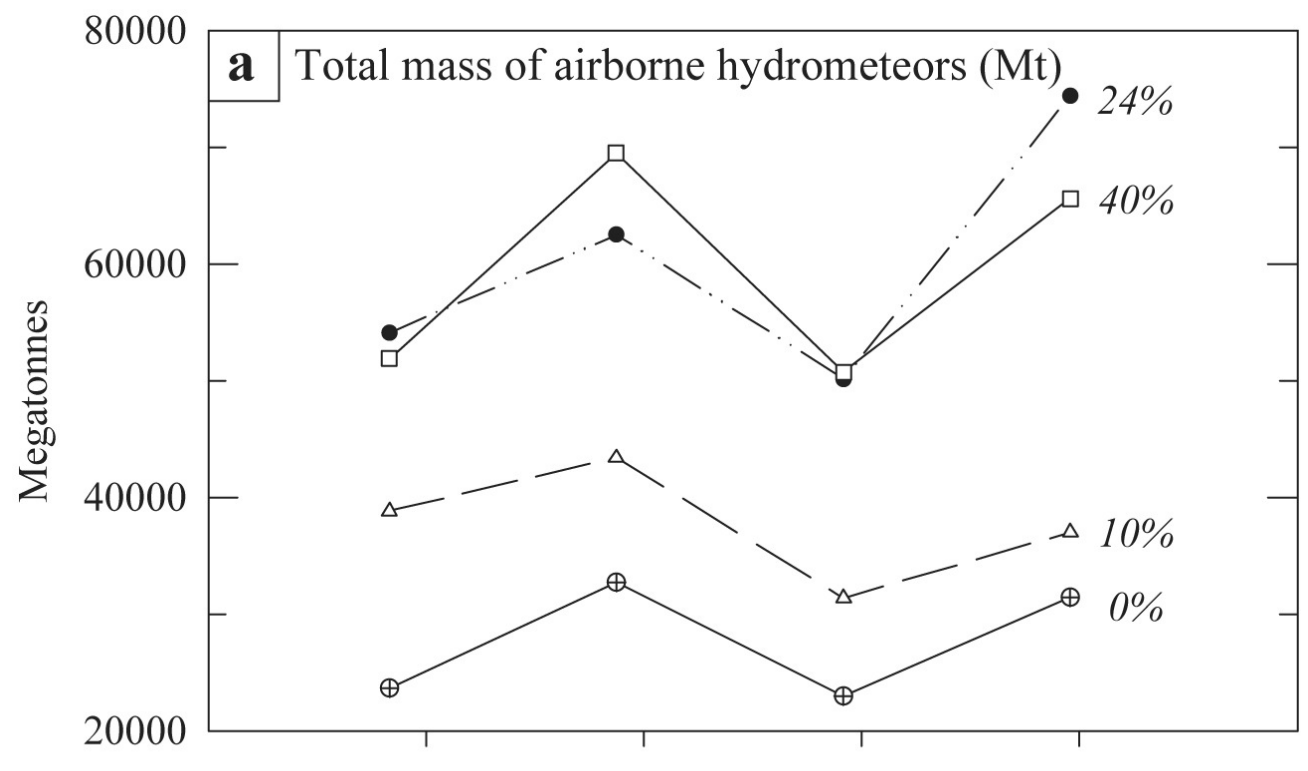
A
B
C
D

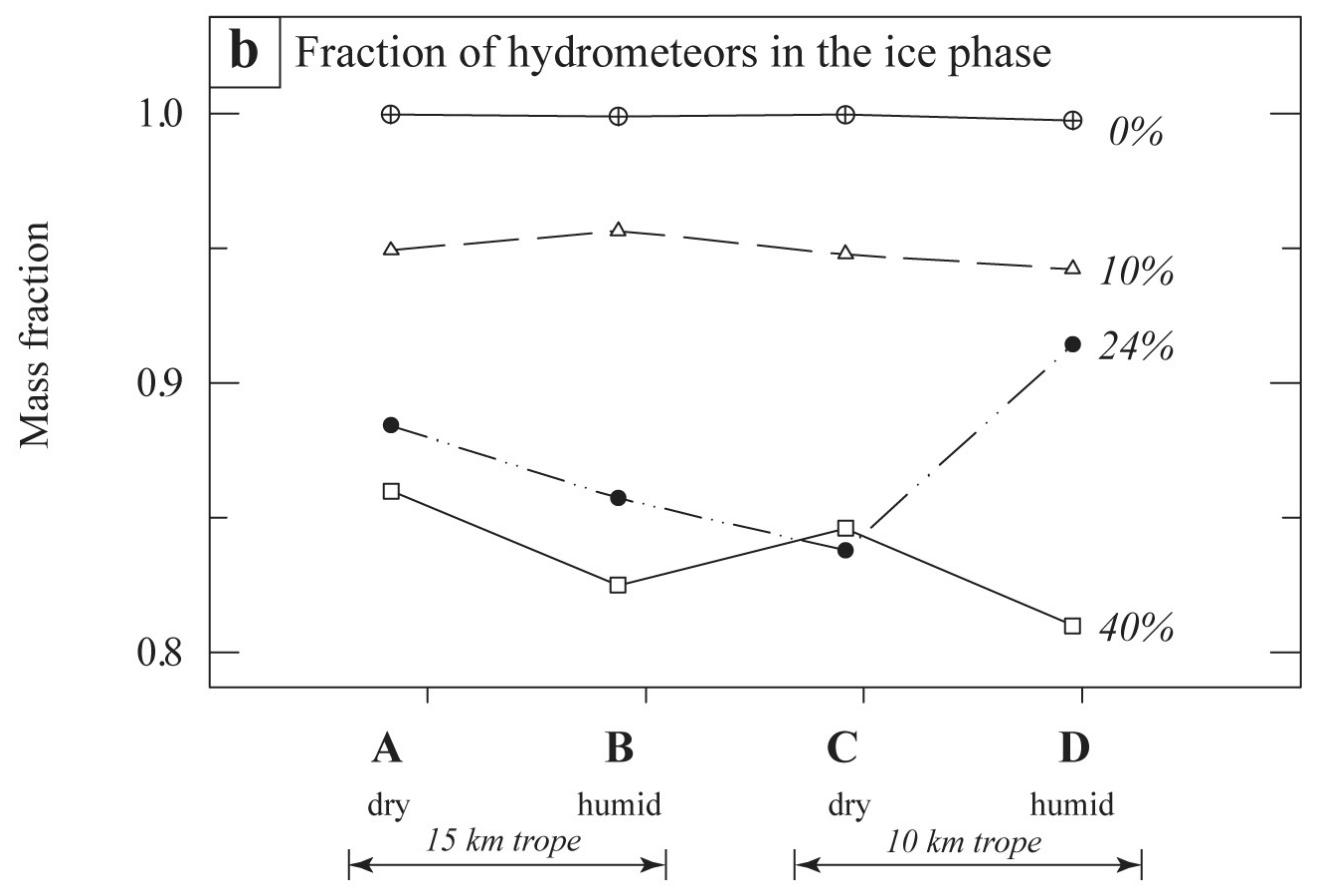

Background atmosphere

Figure 2.15 Sensitivity of volcanic cloud water content to background atmospheres A through D in the low MER simulations after one hour of eruption. Percent surface water characterizing each eruption is indicated in italics. (a) Shows the total mass of airborne hydrometeors (cloud water, rain, cloud ice, graupel) in megatonnes. (b) Gives the fraction of hydrometeors in the solid phase (i.e. in the form of cloud ice or graupel). 
Criteria for wet aggregation are likewise met in the stratospheric reaches of stable phreatoplinian plumes (Figure 2.3d). Simulation 24-A-high illustrates that this style of eruption is capable of injecting enormous amounts of liquid water (well within the 'aggregation window' of 10-30 wt.\% liquid from Schumacher and Schmincke [1995]) along with ash to stratospheric heights, and persisting at those altitudes during transport many tens of kilometers from vent. It is therefore proposed in this study that some degree of wet ash aggregation is very likely to occur in the stratosphere in this case, although the longevity of the interaction due to turbulent mixing with very cold, dry air is unresolved at present, and warrants future work. An aggregation test within this scenario (24-A-high-agg) preliminarily indicates that a plume like that in Figure $2.3 \mathrm{~d}$ is capable of transporting $1 \mathrm{~cm}$ aggregates to stratospheric heights and depositing them at distances greater than $20 \mathrm{~km}$ from vent. The unusual association of ash aggregates with a plinian pumice fall deposit reported by Hayakawa [1983] may thus reflect conditions of a wet but stable plume.

\subsection{Discussion}

\subsubsection{Dynamics of Column Instability: Comparison with Field Deposits}

Field examples of recent and prehistoric eruptions provide evidence for the existence of an unsteady column that either transitioned to a collapsing fountain [e.g. Kos Plateau Tuff: Allen and Cas 1998] or produced both a high, buoyant cloud and ground hugging currents simultaneously, including Vesuvius AD 79 [Carey and Sigurdsson 1987, Cioni et al. 1992], Novarupta 1912 [Houghton et al. 2004, Fierstein and Wilson 2005], 0.76 Ma Bishop Tuff [Wilson and Hildreth 1997] and 25.4 ka Oruanui [Wilson 2001], among others.

From the Oruanui deposits, which represent one of the best documented prehistoric eruption sequences to date, this investigation focuses on unit 3 for comparison with 'wet' column behavior due in part to its excellent preservation near the base of the eruptive stratigraphy. Although unit 3 grain size and dispersal characteristics plot in the phreatoplinian field [Self and Sparks 1978] of the Walker F-D diagram [Walker 1973], proximal stratigraphy indicates this phase of eruption was not characterized solely by a 
stable column. The proximal material (within 30-40 $\mathrm{km}$ from source) reaches thicknesses of greater than $8.5 \mathrm{~m}$ and dominantly comprises multiple, thin $(\mathrm{cm}-\mathrm{dm}$ scale) wedges of normally graded PDC material, inferred to have been deposited from dilute PDCs, interbedded with ultrafine grained vitric ash rich in accretionary lapilli [Wilson 2001]. Beyond this proximal area the deposits landed entirely as fall material from a moisture-flushed ash cloud.

Results from the reference simulation 24-A are consistent with these field observations, indicating spasmodic emplacement of thin, dilute currents with lateral runout distances of 30-40 km. The PDC material thins rapidly away from source, and operates simultaneously with a high, buoyant ash cloud depositing vertically-emplaced fall material. There is abundant liquid water in the lower levels of the hybrid cloud and proximal currents (particularly below $5 \mathrm{~km}$ a.s.l.), which would encourage wet aggregation throughout the eruption. If aggregation were to occur instantaneously at vent (the extreme case), convective dynamics in the elutriated PDCs would still be vigorous enough to transport $1 \mathrm{~cm}$ diameter ash aggregates $15 \mathrm{~km}$ from vent. Analysis of mass partitioning into the convective vs. collapsing regimes after an hour of eruption show that, despite the collapsing nature of the column, microphysics-assisted lofting of PDCs buoys $c .54 \%$ of the total erupted ash well into the convective region (i.e. higher than the top of the collapsing column at $6.5 \mathrm{~km}$ ). This percentage is much greater for the $6 \mu \mathrm{m}$ tracer (74\%) than for the $200 \mu \mathrm{m}$ crystal tracer (17\%).

These results may provide insights into a problematic characteristic of the Oruanui eruption and other large, wet eruption deposits: that is the significant upwind and crosswind deposition compared to dry eruptions [Walker 1981a, Wilson 2001]. Such dimensions are problematic because application of the classic Carey and Sparks [1986] isopleth inversion method to reconstruct column heights would return an unrealistically high column. The spreading of large-scale volcanic clouds has been previously addressed by invoking the influence of Coriolis forces to form a giant, spinning body of ash analogous to a cyclone [Baines and Sparks 2005, Baines et al. 2008]. However, the dynamics of wet eruptions simulated here suggest a number of alternative processes are capable of generating deposits of these dimensions. First, the hybrid ash clouds inject ash into multiple levels in the atmosphere. This vertical spread would provide more opportunities to interact with complex wind trajectories aloft, leading to ash transport in 
directions other than the dominant downwind range. Second, development of coignimbrite updrafts from PDCs that spread tens of kilometers more or less radially from vent may contribute to substantial deposition upwind. And third, even very large ash clouds from unstable columns spread at a dominant neutral buoyancy height, where the material emplaces as a gravity-driven intrusion with significant (if episodic) spreading velocities. When, for example, the reference simulation was extended to erupt for three hours (24-A-3hrs), the main spreading umbrella reached a diameter of $220 \mathrm{~km}$. With expansion velocities between $20-70 \mathrm{~m} \mathrm{~s}^{-1}$, this interaction with the stratified atmosphere may promote 'strong' plume behavior [Carey and Sparks 1986, Bonadonna and Phillips 2003] in coignimbrite and hybrid clouds, transporting ash in all directions at velocities somewhat faster than the ambient wind field.

\subsubsection{Effects of Wind}

Although the effects of wind were not simulated here, the results of this study suggest that water-rich columns would have varying potential to develop complex interactions with the ambient wind field [e.g. Bursik 2001]. In the case of the hybrid or coignimbrite columns resulting from the lower magma eruption rate tested, the plume is fed by slow thermals lifting off the ground-level PDCs (initial velocities generally $<5 \mathrm{~m} \mathrm{~s}^{-1}$ ). These thermals would behave as 'weak plumes' in the lower region of the developing column, before moist convection promotes acceleration to vertical velocities up to $110 \mathrm{~m} \mathrm{~s}^{-1}$ above $5 \mathrm{~km}$ a.s.l. Such highly unstable plumes would be particularly responsive to boundary layer winds. In contrast, the stable phreatoplinian columns generated at higher eruption rate (Figure 2.3d, f) maintain substantial vertical velocities straight up into the stratosphere, and may exhibit less sensitivity to wind. Nonetheless, even these columns are shown to develop local instabilities due to microphysical effects (see section 2.5.1). If strong low-level winds effectively sheared off the top of the column at this stage of development, it is difficult to determine a priori whether a stable column could be become established. A more formal treatment of these potentially complex wind interactions is beyond the scope of the present work, but will form the basis of a followup study using ATHAM. 


\subsubsection{Multiple-Level Intrusion Heights}

An interesting feature in the modeling results is that the coignimbrite and hybrid cloud structures are characterized by multiple intrusions heights (Figure 2.3 and 2.4b). These multi-level flanges are a dynamic aspect of coignimbrite cloud development. As seen in the Figure 2.7 time series, the initial Plinian phase reaches an NBL of $8 \mathrm{~km}$ a.s.l. within the first 10 minutes. After column collapse, the dominant spreading level is much nearer the ground as mass pours into ground-hugging density currents. However, particle-rich coignimbrite thermals reach progressively higher altitudes as the eruption continues. The coignimbrite updrafts appear to build on a thermal pathway of 'failed' intrusions, entraining the warm air that persists in the wake of preceding updrafts to gain buoyancy. Each ascending parcel forms turbulent eddies around the periphery of the updraft, which spall off lower-level intrusions.

This differential buoyancy phenomenon is inferred to be a model analog to the ascent of the 21 April 1990 coignimbrite cloud from Redoubt volcano, Alaska. In that example, the ash cloud originated primarily from a PDC-forming dome collapse, which produced a strongly buoyant thermal ascending to $12 \pm 1 \mathrm{~km}$ a.s.1. within $c .8$ minutes [Woods and Kienle 1994]. The initial, strongest coignimbrite updraft was subsequently fed by weaker, water-rich thermals that continued to rise from the ground-hugging flow, injecting into multiple levels below the spreading umbrella. This stepwise ascent dynamic may therefore provide a mechanism by which non-steady eruption clouds are buoyed to different vertical heights in the atmosphere.

\subsubsection{Defining Ash Cloud Heights: Maximum vs. Spreading Level}

The height attained by volcanic ash clouds relative to cruising levels is critical to the mitigation of jet aircraft hazards, and yet is difficult to measure consistently and accurately during active eruptions [Tupper et al. 2004]. Simulations in this study highlight an important feature of ash cloud heights from large, unstable eruptions: At eruption rates below $c .10^{9} \mathrm{~kg} \mathrm{~s}^{-1}$, there are more significant differences (as much as 27 $\mathrm{km}$ ) between the maximum height and NBL in unstable eruptions compared to their stable Plinian counterparts (Figure 2.14a). This is due in part to the chosen method of defining the NBL by a single 'dominant' umbrella height despite multiple spreading levels, and calls attention to the challenge of how to define (and, therefore, estimate) 
cloud heights from complex, non-steady eruption styles. Similar height differences of up to $17 \mathrm{~km}$ have been documented in large coignimbrite eruptions (MER $>10^{8}-10^{9} \mathrm{~kg}$ $\mathrm{s}^{-1}$ ), including the 17 June 1991 Pinatubo cloud, as discussed by [Mastin et al. 2009]. In their review of historical eruptions it was noted that observations of ash cloud heights are generally reported in terms of the maximum, despite the complicating aspects of lower-level spreading. In this study, a very small proportion of the clouds exists at the maximum height $(<0.001 \%$ by mass); yet simulated ash concentrations at these altitudes would still present a hazard to jet aircraft.

In an operational setting, volcanic ash transport and dispersion models incorporate buoyant plume theory or empirical eruption rate-height scaling relationships to supplement observations of ash clouds during eruptions [Bonadonna et al. 2011a]. Comparison of the simulated maximum cloud heights with those calculated from the scaling relationship of Sparks et al. [1997] gives somewhat close results (generally within 5-10 km: dashed line in Figure 2.14), indicating that the relationship holds for water-rich eruptions. However, it is noted that even a $5 \mathrm{~km}$ difference significantly changes the MER calculated from the height scaling equation. Due to the dynamics of convectively instability outlined here, most of the ash is actually emplaced significantly below the maximum height. A similar result was obtained by Herzog and Graf [2010] when they compared maximum coignimbrite cloud heights from ATHAM to the steadystate 1-D coignimbrite plume model of Baines and Sparks [2005]. This suggests that the simplified models do provide a reasonable estimate of maximum cloud height for unsteady eruptions, but provide very little useful information on the vertical distribution of ash in the atmosphere.

\subsubsection{Improving Parameterization of Wet, Unsteady Eruptions}

Although complex physical processes such as microphysics, hydrometeor-ash aggregation and the formation of turbulent eddies cannot yet be solved explicitly in fastrunning operational dispersion models, general aspects of the behavior of unsteady eruptions can be parameterized to establish more realistic source parameters for forecasting as well as for reconstruction of volcanic clouds from their deposits. Simulations in this investigation indicate that in eruptions with MER $\geq c .10^{9} \mathrm{~kg} \mathrm{~s}^{-1}$, ash is dominantly emplaced into the stratosphere regardless of eruption style (Figure 2.14b). 
However, using an MER c. $10^{8} \mathrm{~kg} \mathrm{~s}^{-1}$, the resulting unsteady eruptions emplaced a greater percentage of the airborne emissions at or below the tropopause (70-80\% by mass: Figure 2.11b) compared to Plinian-style eruptions with the same eruption rate. This feature could be relatively easily parameterized in models used to track the dispersion of coignimbrite or hybrid ash cloud for the purposes of ash cloud forecasting or deposit reconstructions by: (a) using the maximum height derived from empirical scaling relationships or buoyant plume theory, and (b) increasing the low-level mass in the vertical distribution used to initialize the model. Routinely used source term options for the vertical profile of ash in an eruption cloud assume, for example, a linear distribution of mass with height or a Suzuki distribution (giving an umbrella shape) [Table 1 of Bonadonna et al. 2011a]. It is suggested in this study that a coignimbritetype profile could be incorporated as an additional option. For example, an inverse Suzuki distribution [Suzuki 1983] that peaks at the local tropopause as defined by meteorological data, would account for the large observed difference between maximum height and NBL, and concentrate most of the erupted mass below the tropopause. Results such as those presented in Figure 2.11a may be further developed for other (more common) eruption scenarios to help calibrate model outputs with observations.

\subsection{Conclusions}

Using numerical experiments with ATHAM plume dynamics of large-scale eruptions were investigated in this study, varying surface water content ( $0-40 \%$ by mass) and MER (c. $10^{8}-10^{9} \mathrm{~kg}$ magma $\left.\mathrm{s}^{-1}\right)$. Three eruption styles emerged from the simulated columns: wholly collapsing, Plinian, and transitional. The latter produced hybrid volcanic ash clouds generated by simultaneous Plinian behavior and lofted coignimbrite thermals. Dimensions and dynamics of the reference simulation (with 24\% surface water) were consistent with field characteristics of unit 3 from the water-rich $\sim 25.4 \mathrm{ka}$ Oruanui eruption of Taupo volcano, New Zealand, in terms of PDC runout distances and simultaneity of processes that would lead to deposition of fall and flow deposits. Comparison between simulations with different water contents (at constant MER) illustrate that abundant surface water does not systematically lower the maximum height or spreading level attained by the clouds, as previously thought, but does exert significant control on eruption style. In particular, addition of surface water in amounts 
$\geq 24 \%$ in the lower MER scenarios resulted in an unstable pulsating column characterized by voluminous PDCs feeding a high, buoyant ash cloud. Therefore, even water-rich eruptions characterized by cool temperatures and column collapse are capable of ascending to similar heights as Plinian clouds with the same MER purely by moist convective processes. It is further observed that unstable columns distribute more volcanic ash and liquid water in the troposphere, allowing increased availability of wet aggregation processes. In turn, abundant aggregation has the potential to increase gravitational separation of the larger aggregates from individual ash particles, providing a buoyancy boost to the remainder of the volcanic plume. Further experimental, observational and numerical studies of aggregation are necessary to test this result. Compared to stable Plinian plumes, which are largely confined to a single spreading level, the vertical distribution of ash from unstable hybrid or coignimbrite eruptions cannot be easily approximated with 1-D buoyant plume models and height scaling relationships alone. Although ambient humidity was found to be of secondary importance due to the large size of the eruptions tested, tropopause elevation exerted a strong control on the neutral buoyancy level and related injection heights of volcanic ash from eruptions with MER $c .10^{8} \mathrm{~kg} \mathrm{~s}^{-1}$. This work will hopefully stimulate further investigation of volcanic-hydrometeor phenomena to strengthen the links between volcanic research and operational forecasting initiatives. 
Chapter 3 


\section{Methodology: Quantitative size characterization of fine volcanic ash}

\subsection{Introduction}

The primary purpose of this chapter is to establish a consistent and reproducible method of particle size analysis for fine volcanic ash. Although numerous studies have addressed this topic for the analysis of sediment, volcanic ash presents distinct challenges due to the irregular morphologies of ash shards [Heiken and Wohletz 1985] (Figure 3.1), and heterogeneous components (e.g. glass, lithics, crystals and pumice) with widely varying densities [Walker et al. 1971]. These factors make it difficult to compare grain size databases using classic sieve-pipette techniques with the emerging standard of laser diffraction particle size analysis. This study examines four grain size analysis methods for the analysis of sub- $2 \mathrm{~mm}$ volcanic ash, and documents how a bestfit laser diffraction optical model was determined for use in this dissertation.

\subsection{Overview of existing techniques}

Currently, no single existing method is capable of rapidly assessing the full range of coarse lapilli to the finest ash-sized particles in pyroclastic deposits. Sieving can only reliably provide grain size data for particles $\geq 63-31 \mu \mathrm{m}$, requiring a complementary method to provide quantitative information on the finer fraction. Similarly, pipette analysis using the settling method is only appropriate for particles settling under the Stokes' regime $(<63 \mu \mathrm{m})$, and laser diffraction techniques cannot handle particles $>2$ $\mathrm{mm}$. A combination of multiple techniques is required to fully characterize deposits with a wide range of grain sizes. However, each approach employs a unique description of particle diameter and expression of the data in terms of weight percent (sieve and pipette), versus volume percent (laser diffraction, SEM counting). Differences between the various methods become exacerbated by the influence of irregular grain shapes [Blatt et al. 1980] and rough surfaces of volcanic ash particles (Figure 3.1). It is thus critical to examine how particle size is approached and quantified using the various techniques if a useful comparison between methods is to be developed. 

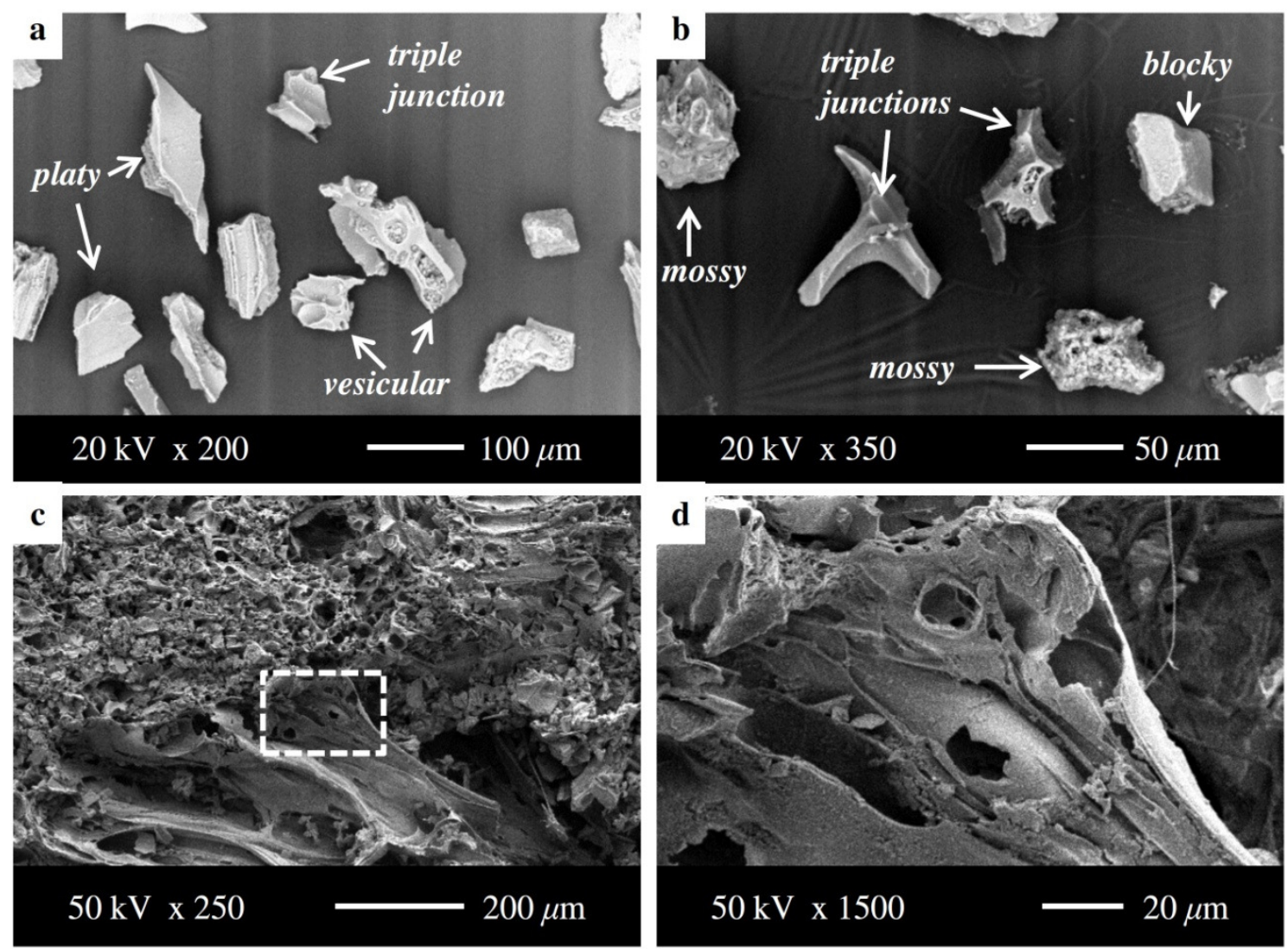

Figure 3.1 SEM images of volcanic ash shard morphologies and pumice textures in Oruanui unit 3. (a-b) Dry sieved ash 45-63 $\mu \mathrm{m}$ from the reference sample, exhibiting vesicular, platy, mossy, blocky and complex morphologies from bubble wall triple junctions. (c-d) Pumice sample (from locality 1293), illustrating how the observed irregularity of bubble wall shards originate from complex vesicle textures: (c) shows tiny vesicles $(<20 \mu \mathrm{m}$ diameter $)$ in the top portion of the image, juxtaposed against elongate vesicles in the lower portion; (d) is an enlargement of the stretched bubble walls.

\subsubsection{Sieving}

The long-established method of grain size analysis by sieving [Udden 1898, Walker 1971] is still the most common method of assessing pyroclasts in the $>63 \mu \mathrm{m}$ size range. Unconsolidated grains are passed through a series of standardized meshes with progressively smaller apertures; the material retained by each sieve is weighed, providing an indirect measure of grain diameter [Folk 1974, Blatt et al. 1980]. The size of particles caught by each mesh depends strongly on their shape and orientation [Rittenhouse 1943, Ludwick and Henderson 1968]. For example, a needle-like particle may pass through the aperture by its smallest cross-sectional diameter, or be retained by its long axis. However, with the large numbers of grains analyzed in sieving (upwards of millions), overall results tend to provide an approximation of intermediate diameter 
[e.g. Komar and Cui 1984], as defined by Griffeths [1967] and Blatt et al. [1980] (Figure 3.2). Owing to the variability induced by the influence of grain shape and orientation, sieve intervals may also contain grains with equivalent spherical diameters that are larger or smaller than the aperture size, and although excellent precision may be reached using standardized techniques [e.g. Folk 1955], user error is generally higher than with automated methods.

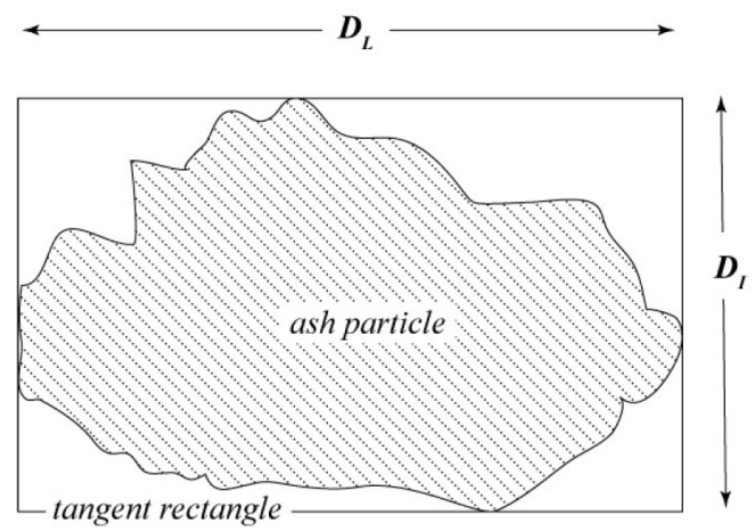

Figure 3.2 Definition sketch of the principle diameters of an irregularly shaped ash particle, with the short axis oriented perpendicular to the page. A tangent rectangle enclosing the grain in this orientation defines the long diameter $\left(D_{L}\right)$ by its longest side and intermediate diameter $\left(\mathrm{D}_{\mathrm{I}}\right)$ by its shortest side. Modified from Blatt et al. [1980].

\subsubsection{Pipette analysis}

Sedimentation techniques employ Stokes' law to estimate particle diameters from their settling velocity through water at a known temperature. Initially well mixed, waterborne fine particles are withdrawn from prescribed depths at standard time intervals [Folk 1974, Blatt et al. 1980]. The maximum settling diameter of particles in a withdrawn aliquot is calculated as the diameter of a sphere of equivalent settling velocity. However, the key distinction is that irregularly shaped and/or vesicular ash particles have a much greater drag force than smooth spheres [Wilson and Huang 1979], and therefore have slower settling velocities than their average cross-sectional area would suggest. Settling techniques thus tend to overestimate the fines content relative to other methods. Before alternative methods such as laser diffraction became widely available in the 1990s, settling techniques were traditionally paired with sieve data to cover the typical range of pyroclastic deposit size distributions [e.g. Walker 1981b]. The merging of the two datasets often require an empirical conversion to match settling diameters with sieve data [e.g. Wilson 1981, Smith 1998]. 


\subsubsection{Laser diffraction}

Laser Diffraction methods utilize the scattering of light from suspended particles to estimate the volume percent of particles within user-defined size ranges [Blott et al. 2004]. For the Beckman-Coulter LS 13 320, as with a number of other commonly used models (e.g. Malvern Mastersizer 2000 [Horwell 2007]), the procedure disperses waterborne particles through the path of a $750 \mathrm{~nm}$ wavelength laser and measures the diffraction pattern. The pattern is converted to an optical particle size distribution using Mie or Fraunhofer-based theories of light scattering, which describe the inverse relationship between diffraction angle and particle diameter. However, the optical model used to describe this transformation can be modified by the user, as discussed in Section 3.3. Laser diffraction methods calculate the dynamic projected area of particles intersecting the laser beam at random orientations, providing a 'mean' optical diameter. Previous studies have found that laser diffraction tends to underestimate the fine fraction in natural samples compared to sieve and pipette data [e.g. McCave et al. 1986, Loizeau et al. 1994, Beuselinck et al. 1998]. This is due in part to the projected area of irregularly-shaped ash particles appearing generally larger than that inferred from settling velocity or intermediate (sieve) diameter. For example, Konert and Vandenberghe [1997] found that particles with pipette settling diameters of $2 \mu \mathrm{m}$ corresponded to optical diameters of $8 \mu \mathrm{m}$. However, laser diffraction presents a number of analytical advantages. Among these are excellent reproducibility, rapidity of measurement and wide range of particle sizes that can be assessed simultaneously (0.4 $\mu \mathrm{m}$ to $2 \mathrm{~mm}$ ) [Blott et al. 2004]. Particles as small as $0.04 \mu \mathrm{m}$ can also be analyzed with a separate PIDS module, but these measurements are generally not required for volcanological studies. The data resolution obtained by laser diffraction is substantially greater than can be realistically achieved with sieving methods, even at high-resolution, $1 / 4 \varphi$ intervals [Eshel et al. 2004]. In contrast to sieving and pipette methods, laser diffraction generates size data in terms of volume, rather than weight, percent. This may be considered an advantage over pipette analysis [Eshel et al. 2004], because it bypasses the assumption of a mean density value (typically $2.65 \mathrm{~g} \mathrm{~cm}^{-3}$ for sedimentation methods). However, it also makes direct comparisons with sieve data more challenging, because the exact density (componentry) of each particle size class cannot be determined a priori. Some workers comparing laser diffraction and sieve data 
[e.g. Wilson 2001] thus choose to sieve down to $63 \mu \mathrm{m}$ before applying laser techniques. This is because the finest fractions of volcanic ash become increasingly glass-dominated, and more readily described by a consistent density.

\subsubsection{Scanning electron microscopy}

'Point counting' the dimensions of individual, magnified grains by hand or using image analysis software is the only technique that employs direct measurements of fine ash particle diameters. The method typically uses scanning electron microscopy (SEM) to obtain 2-dimensional images of fine ash particles. Images are then edited and converted to binary (black and white), before analysis with image processing software such as ImageJ [e.g. Bonadonna et al. 2011b]. The software counts each particle and is capable of generating a wide range of quantitative 2-dimensional size and shape values, as summarized by Riley et al. [2003].

\subsection{Laser diffraction and inter-comparison of techniques}

Several experiments were carried out to calibrate a suitable laser diffraction optical model for grain size analysis of phreatoplinian eruption deposits, and to clarify the variability between the methods described above. The reference volcanic ash material used for comparisons between optical models and different grain size techniques is proximal Oruanui unit 3 ash from locality 1443 of Wilson [2001], sampled 25-30 cm above the base of the deposits. Eight additional samples of unit 3 ash were analyzed using the sieve-pipette technique to assess the correlation with laser diffraction data.

\subsubsection{Determination of an optical model}

The manner in which the light scattering pattern measured by laser diffraction is transformed to an optical particle size distribution is governed by the optical model. For particles between 10-100 $\mu \mathrm{m}$, changes in the optical model generate substantial differences in the resulting size distributions, and have an even more pronounced effect on the sub-10 $\mu \mathrm{m}$ particles [Beckman-Coulter 2003]. The four user-controlled parameters that define the model are: (1) the 'real' refractive index (RI) of the particles, which describes the retardation of light passing through the material [Williams et al. 1982]; (2) the RI of the fluid (taken as a constant 1.333 for water); (3) the absorption 
coefficient or 'imaginary' refractive index (RII), which describes the opacity, or ability of particles to absorb light; and (4) the e-factor, which is an empirical calibration function used to shift the overall data coarser or finer. Due to the variability of components in fine volcanic ash, which include glass, crystals and lithics, the RI and RII must be taken as average values representative of the overall refractive indices of the particle mixture. As discussed previously, this is a limitation of the laser diffraction method in application to volcanic ash samples with multiple components, but becomes less significant with finer grained and more compositionally uniform samples (particularly $<250 \mu \mathrm{m}$ in Oruanui samples). Horwell [2007] determined that representative RI values for explosive volcanic deposits generally range from 1.48-1.66, and an intermediate value of 1.55 was chosen for Oruanui samples. RII values for gray colored material to clear glass range from 1.0 to 0.001 according to the guidelines of Beckman-Coulter [2003; p. 169], and a value of 0.01 was chosen for the Oruanui ash, consistent with 'lightly colored, translucent material' (Appendix B). No correction factor was applied (e-factor $=1$ ). These parameters comprise the Oruanui optical model of RI 1.55, RII 0.01, e-factor 1.0.

To examine the sensitivity of this optical model, the reference ash sample was analyzed by laser diffraction using the parameters described above, and then re-processed in the LS 13320 software (v. 5.01) to systematically tweak each variable. Results plot the vol.\% of volcanic ash binned into each of 92 (central) channel diameters from 0.38 to $2000 \mu \mathrm{m}$ (Figure 3.3a-c). Figure 3.3a demonstrates that incrementally increasing the RI from 1.50-1.65 produces only slight differences in the sub-10 $\mu \mathrm{m}$ fraction, whereas an RI of 1.45 increases the apparent amount of sub-10 $\mu \mathrm{m}$ fines. Variations in the RII (Figure 3.3b) show a slightly more significant impact, with a value of 0.001 producing less fine ash and a noisier signal between 10-70 $\mu \mathrm{m}$. Contrasts between RII values of 0.01-1 are less distinct, but the higher values tend to produce greater amounts of sub-2 $\mu \mathrm{m}$ ash. The e-factor produces the greatest differences in the overall grain size distribution. Values above or below unity shift the entire size distribution coarser or finer, respectively. For example, an e-factor of 1.2 shifts the distribution $20 \%$ coarser, whereas an e-factor of 0.8 shifts it $20 \%$ finer. 

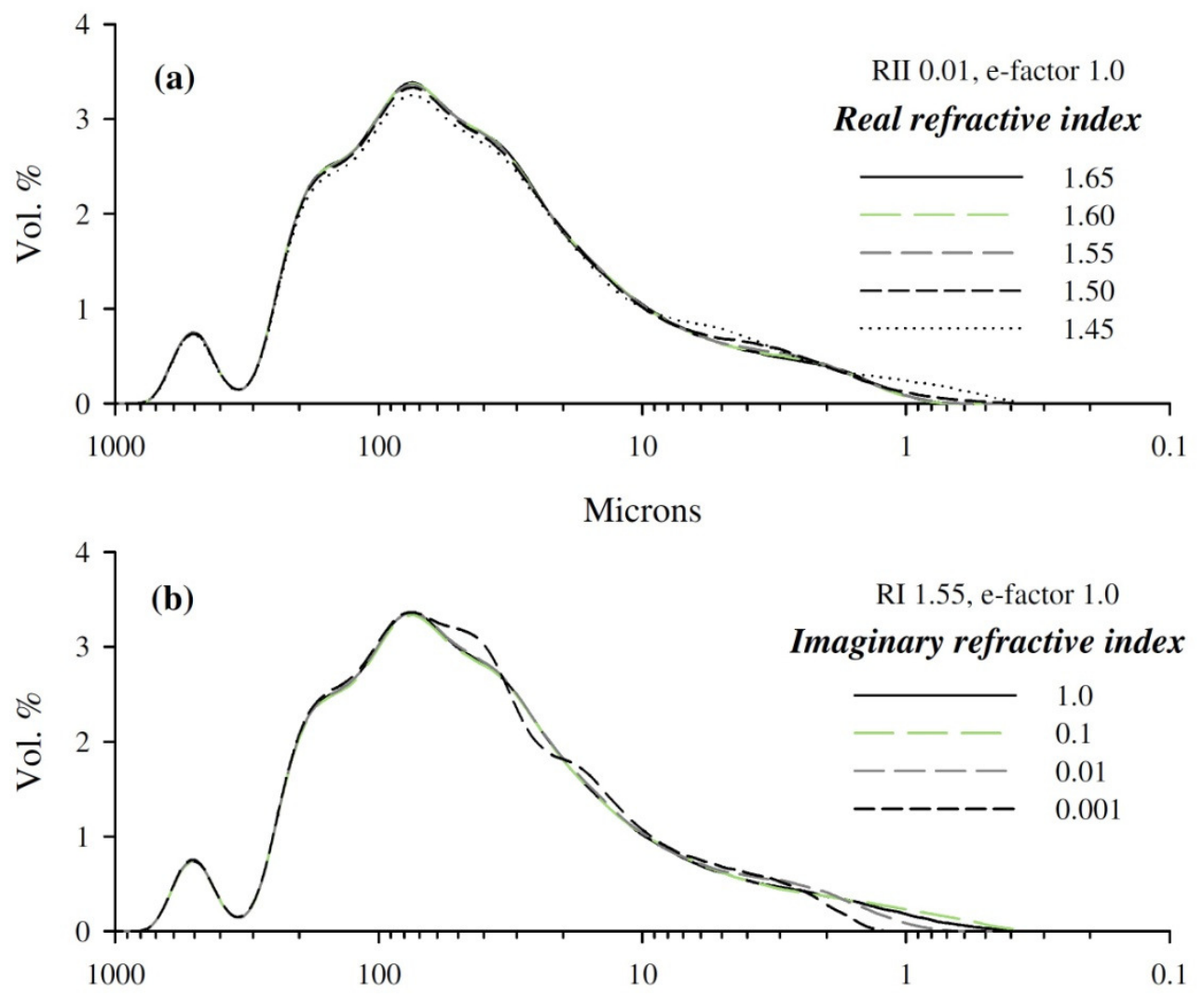

Microns

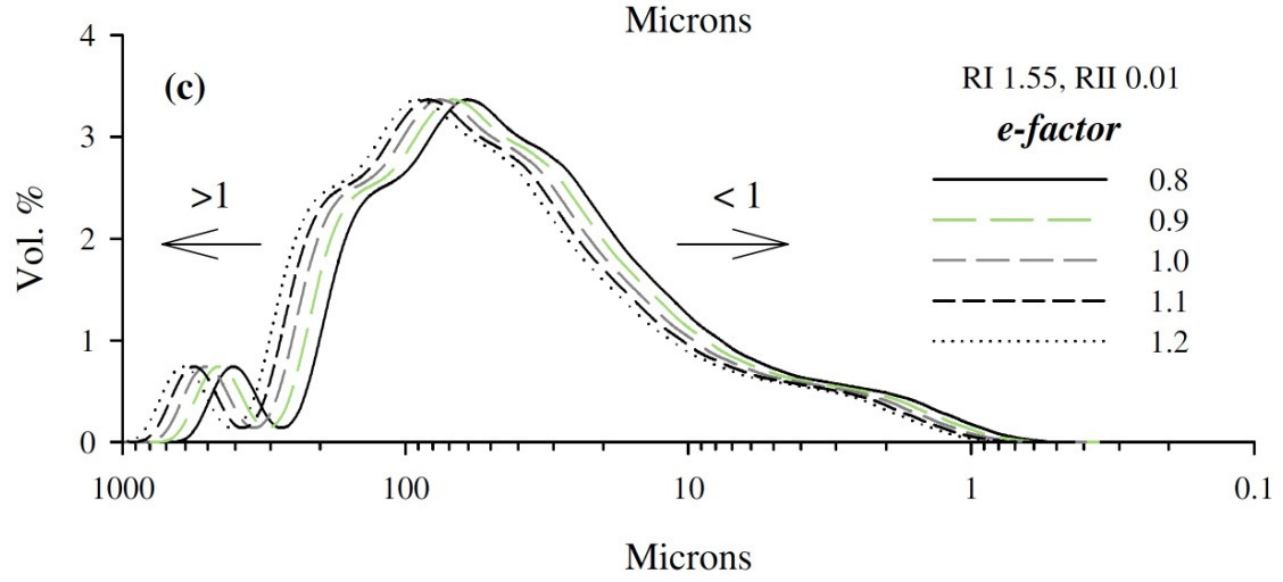

Figure 3.3 Plots illustrating the sensitivity of a grain size distribution to changes the laser diffraction optical model. The parameters that are kept constant are show at the top of each graph. (a) Shows changes in the refractive index (RI); (b) alters the imaginary refractive index (RII), also called the absorption coefficient; and (c) alters the e-factor, previously known as the 'shape factor' on older Beckman-Coulter laser diffraction machines. An e-factor greater than unity shifts the entire size distribution toward the coarser end, whereas an e-factor less than unity shifts it finer. 

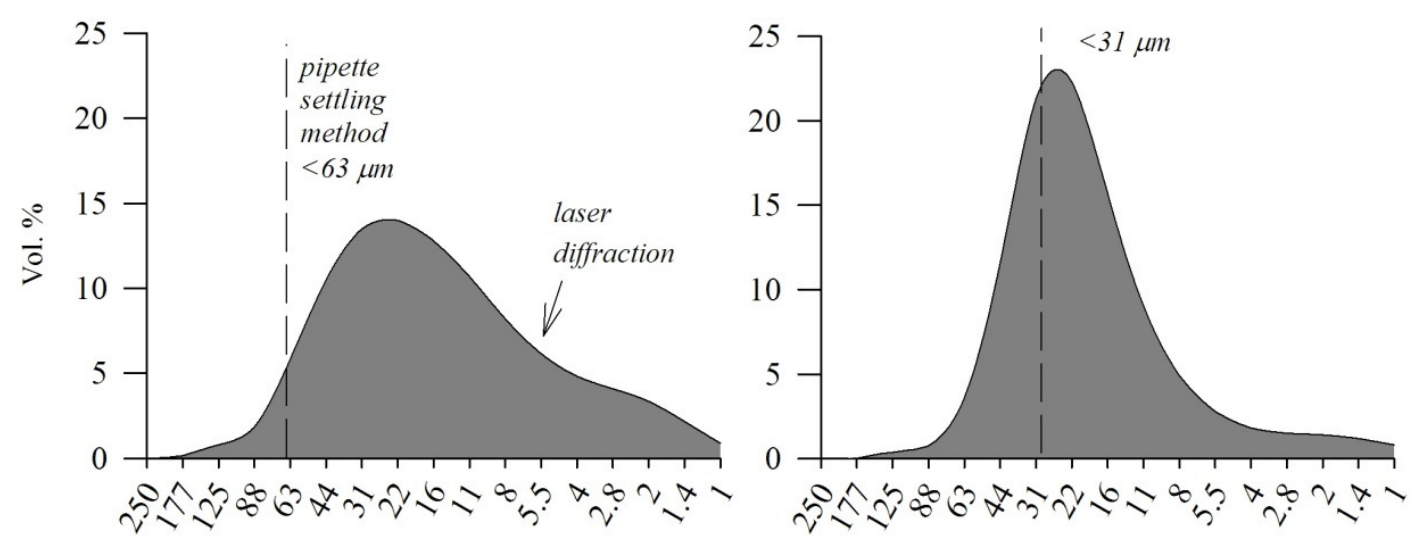

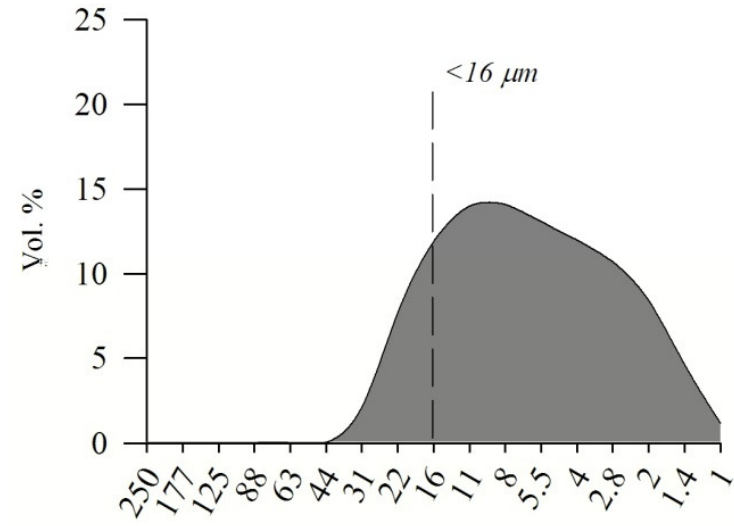

Microns

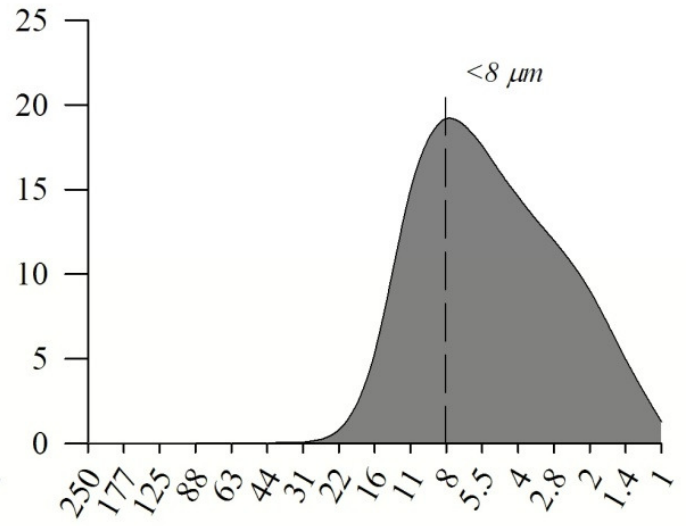

Microns

Figure 3.4 Comparison of grain size distributions obtained from laser diffraction (grey curves) and the pipette settling method (maximum settling diameter indicated by broken line). Laser diffraction detects larger particles than predicted from the settling method. All plots use the reference volcanic ash sample.

\subsubsection{Pipette versus laser diffraction}

For pipette analysis of the reference volcanic ash and additional samples, 1-liter graduated cylinders were kept in a water bath at $25 \pm 0.5^{\circ} \mathrm{C}$ to mitigate the influence of laboratory temperature fluctuations. Weighed, disaggregated samples were passed through a $63 \mu \mathrm{m}$ sieve and suspended in water in the cylinders. Pipette aliquots of 20 $\mathrm{mL}$ were withdrawn and weighed according to the withdrawal table of Haan et al. [1994] (Appendix C). Additional pipette aliquots from the reference material, representing settling diameters of $<63,<31,<16$ and $<8 \mu \mathrm{m}$ were withdrawn and poured directly into the Beckman-Coulter LS 13 320. The pipette aliquots were analyzed using the Oruanui optical model and binned into $1 / 2 \varphi$ intervals for comparison with the upper limit of settling diameters assumed from Stokes' law (Figure 3.4). Comparisons show that the laser diffraction method estimates $14-35$ vol.\% of the sample to be coarser than 
the maximum settling diameter. The poorest match was for the sub-31 $\mu$ m aliquot (Figure 3.4b). These results are consistent with Clifton et al. [1999] and Eshel et al. [2004], who determined similar amounts of fines were 'overestimated' relative to settling techniques.

\subsubsection{Sieving and SEM counting versus laser diffraction}

The reference sample was dry-sieved $<63 \mu \mathrm{m},<45 \mu \mathrm{m}$ and between $45-63 \mu \mathrm{m}$, mounted to SEM stubs and imaged at $75 x$ magnification in backscatter mode using a JOEL JSM5300LV in the School of Chemical and Physical Sciences at Victoria University of Wellington. The images were prepared in Adobe Photoshop and analyzed with imageJ processing software as in Appendix D, and quantitative size and shape parameters were obtained for 2,243 individual ash particles. Measurements of particle area (determined from the area of pixels enclosed within each individual grain) were converted to equivalent circular diameter, and binned into whole- $\varphi$ intervals as percent by area. This 2-dimensional method should provide values comparable to vol.\% obtained by laser diffraction. The sieved samples were also analyzed by laser diffraction using the Oruanui optical model and are plotted in Figure 3.5. Figure 3.5 (a-c) demonstrates that laser diffraction shows reasonably good agreement with the sieve diameters, although it detects both coarser and finer particles than expected from the sieve data. This reflects the complicating factors of elongate grain shapes, some of which may be retained by their long axes or pass through the sieve mesh via their short axes, even if the bulk majority of the sieved sample is described by the intermediate axis. In contrast, SEM counting by image analysis provides significantly coarser particles compared to both sieving and laser diffraction.

\subsubsection{Sieve-pipette versus laser diffraction}

A final comparative experiment assessed the relationship of combined sieve-pipette data and laser diffraction. As previously described, the offset between pipette settling diameters for the sub-63 $\mu \mathrm{m}$ fraction and sieve diameters for the coarser fraction is traditionally handled by applying an empirical correction factor. This typically involves a horizontal transformation of the entire cumulative grain size curve from pipette data until it matches up with the sieve values [Wilson 1981, Smith 1998]. However, due to the inherent variability of the pipette method [Blatt et al. 1980], some analyses may 

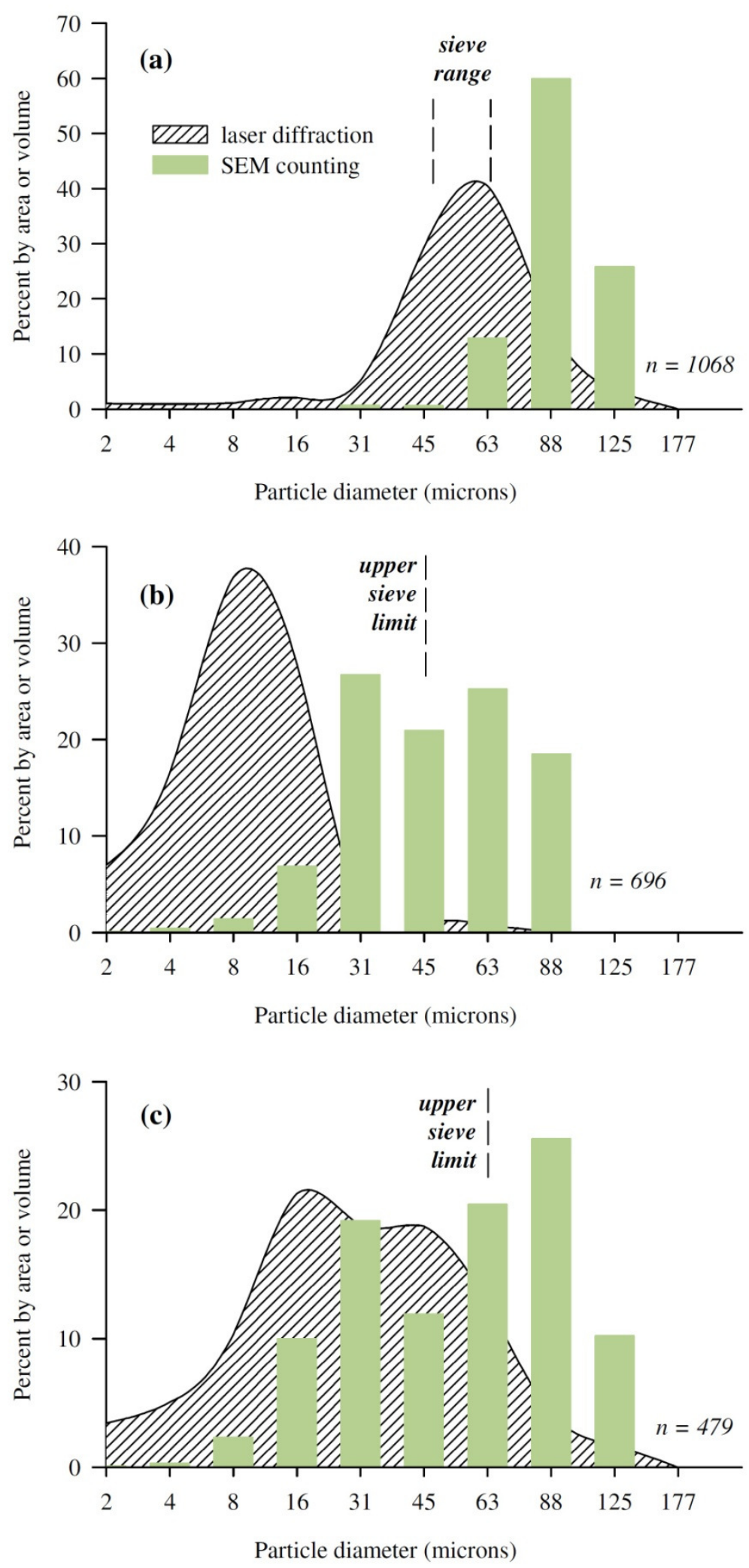

Figure 3.5 Grain size comparison of sieved samples (range indicated by broken line), obtained by laser diffraction as a vol.\% (dashed curve) and SEM counting as an area \% (vertical green bars). Laser diffraction detects larger particles than determined by sieving, whereas SEM counting significantly overestimates the grain sizes relative to both methods. N-values refer to the number of particles counted in SEM image analysis. Sample is the reference material. 
require more or less than the 'average' correction, and in some instances, may require no correction at all. Take for example the reference sample analyzed in triplicate in this study, shown with the corresponding raw sieve values on a cumulative plot (Figure 3.6a). The data require no horizontal correction and can be linearly interpolated between the $4 \varphi(63 \mu \mathrm{m})$ sieve value (shown as a broken line in Figure 3.6b). In contrast, the additional eight Oruanui samples that were analyzed using the same method did require correction factors of $0.5-1.1 \varphi$. Corresponding laser diffraction data for the reference sample was binned into the same size intervals as sieve-pipette analyses and compared in Figures 3.6 (b-c). The data show reasonably good agreement, diverging primarily at $250,63-31 \mu \mathrm{m}$ and in the sub- $8 \mu \mathrm{m}$ fraction, which may be related to

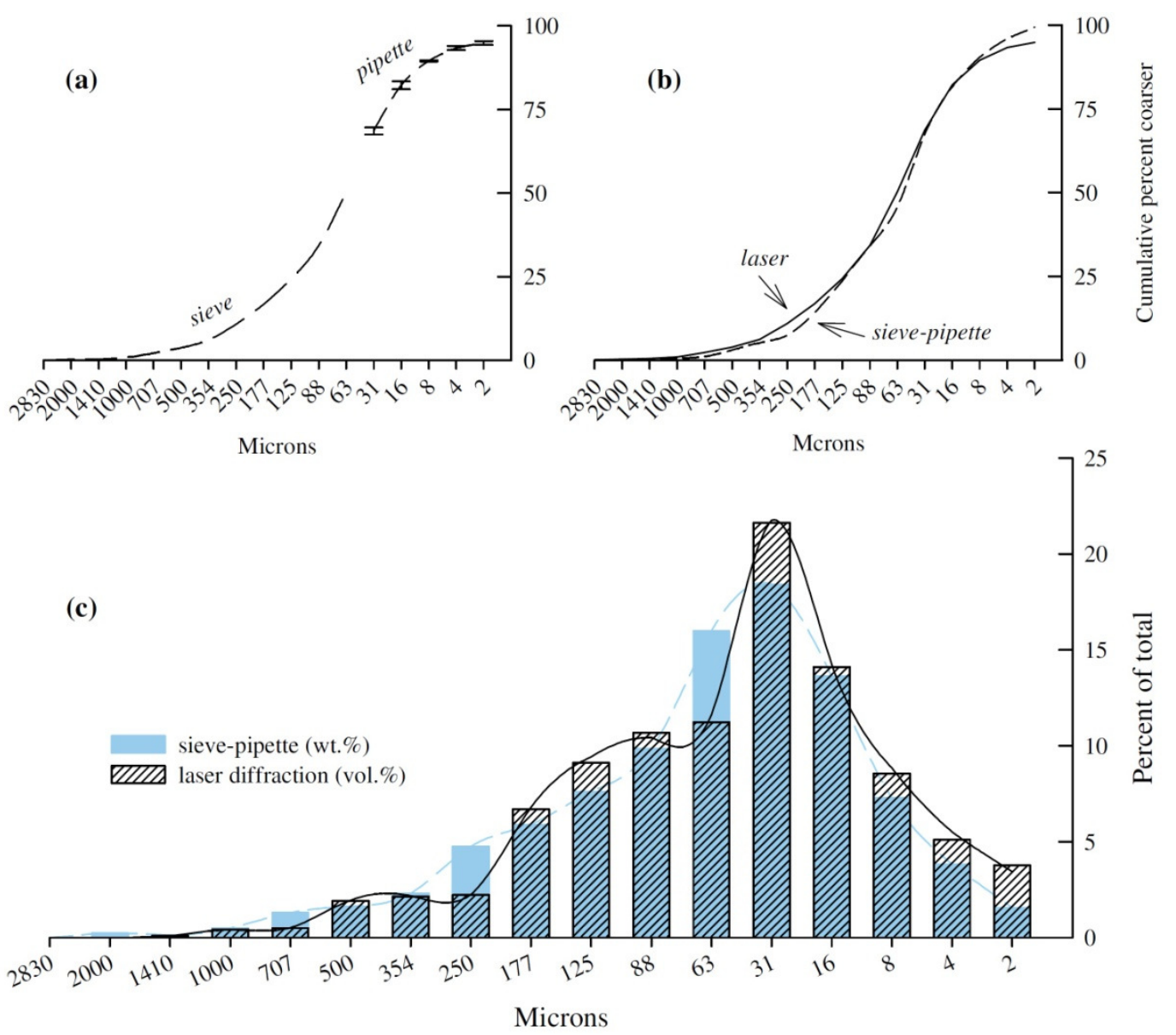

Figure 3.6 Comparison of sieve-pipette and laser diffraction methods. (a) Shows the raw data from sieving at half- $\varphi$ intervals down to $63 \mu \mathrm{m}$, and pipette analysis at whole$\varphi$ intervals for the finer ash, plotted as cumulative wt.\% coarser. Vertical error bars give the minimum and maximum values from triplicate pipette analyses. (b) Shows the combined sieve-pipette data in wt.\% (broken line) and laser diffraction data from the same sample in vol.\%. (c) Re-plots the data from (b) against differential percent in each size class. Note that the two methods show a reasonably close fit, primarily diverging at grain sizes of 250, 63 and $31 \mu \mathrm{m}$. Sample is the reference material. 
differences in crystal content (and thus, density). Results from the nine sieve-pipette analyses (including the reference sample average) were correlated to corresponding laser diffraction values in Figure 3.7. Acceptable agreement between the two methods is given by a positive linear relationship with $r^{2}=0.80$, indicating that the Oruanui optical model provides a sound approach to obtaining reproducible and detailed grain size analyses.

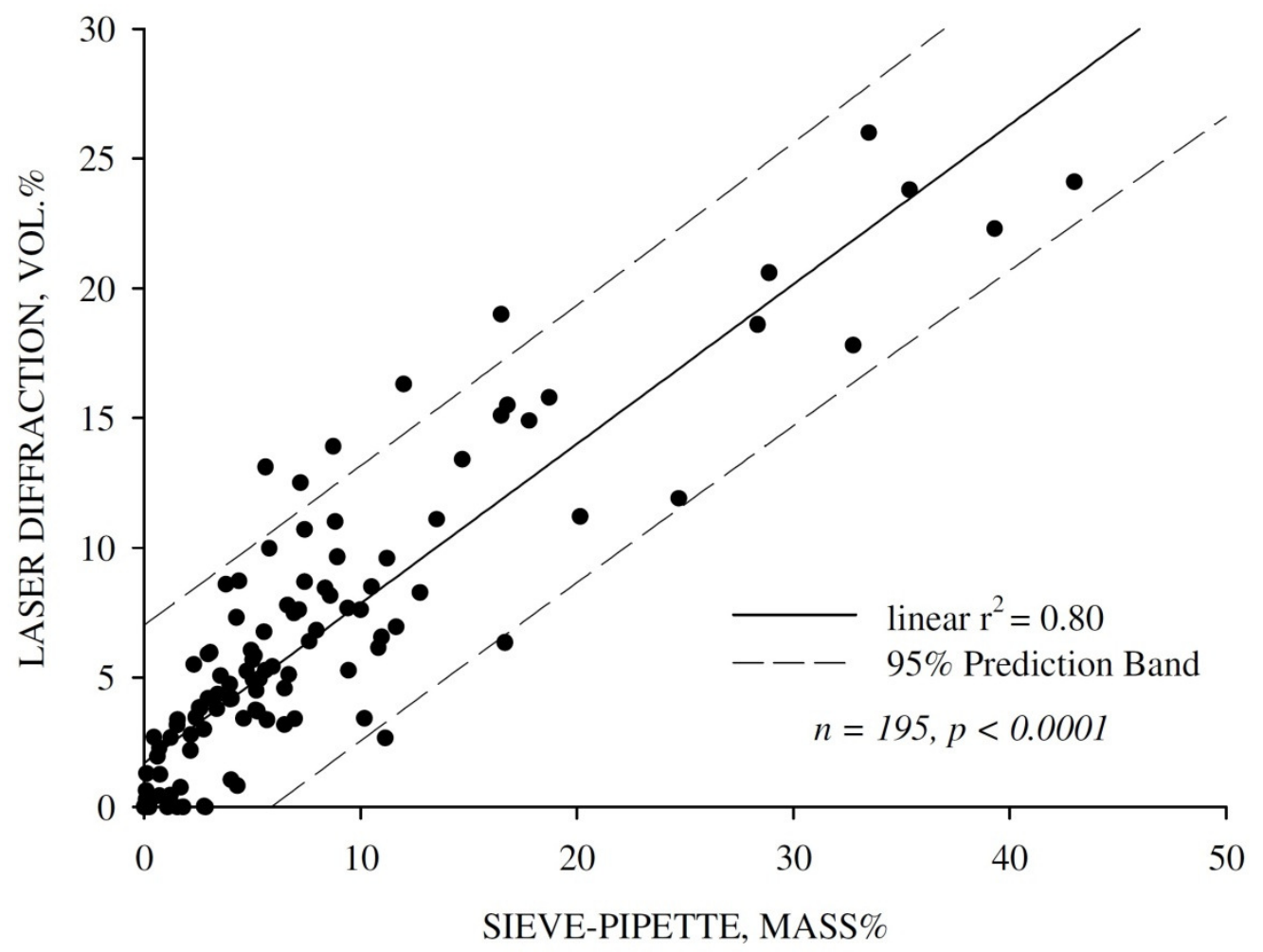

Figure 3.7 Correlation between sieve-pipette and laser diffraction data (using an optical model of RI 1.55, RII 0.01, e-factor 1.0). The plot compares percent in each size classes obtained from both methods on nine unit 3 ash samples, giving a significant positive linear correlation with $\mathrm{r}^{2}=0.80$.

\subsection{Conclusions}

Laser diffraction grain size analyzes applied to nine samples of fine grained Oruanui volcanic ash were compared with traditional grain size methods. Comparisons demonstrate that laser diffraction detects coarser particles than pipette settling and sieving techniques due to shape effects, but detects more fine ash than SEM counting. Optical diameters obtained using an adjusted optical model [refractive index 1.55, imaginary refractive index (absorption coefficient) 0.01 and e-factor of 1 ] are compared 
with changes in the individual parameters, and found to be most sensitive in the sub-10 $\mu \mathrm{m}$ fraction. Overall, the optical model is found to provide a reasonable match to corresponding sieve-pipette data on Oruanui samples. 
Chapter 4 


\title{
4. Growth of volcanic ash aggregates in the presence of liquid water and ice: an experimental approach
}

\begin{abstract}
Key processes influencing the aggregation of volcanic ash and hydrometeors are examined with an experimental method employing vibratory pan aggregation. Mechanisms of aggregation in the presence of hail and ice pellets, liquid water $(\leq 30$ wt.\%), and mixed water phases are investigated at temperatures of 18 and $-20^{\circ} \mathrm{C}$. Experimentally generated aggregates examined in hand samples, impregnated thin sections, SEM images and x-ray microtomography closely match natural examples from phreatomagmatic phases of the $\sim 25.4$ ka Oruanui and 2010 Eyjafjallajökull eruptions. Laser diffraction particle size analysis of parent ash and aggregates is also used to calculate the first experimentally derived aggregation coefficients that account for changing liquid water contents and sub-zero temperatures. These indicate that dry conditions ( $<10 \mathrm{wt} . \%$ liquid) promote strongly size selective collection of sub-63 $\mu \mathrm{m}$ particles into aggregates (given by aggregation coefficients $>1$ ). In contrast, liquidsaturated conditions ( $>15 \mathrm{wt} . \%$ liquid) promote less size selective processes. Crystalline ice was also capable of preferentially selecting volcanic ash $<31 \mu \mathrm{m}$ under liquid-free conditions in a two-stage process of electrostatic attraction followed by ice sintering. However, this did not accumulate more than a monolayer of ash at the ice surface. These quantitative relationships may be used to predict the timescales and characteristics of aggregation, such as aggregate size spectra, densities and constituent particle size characteristics, when the initial size distribution and water content of a volcanic cloud are known. The presence of an irregularly shaped, mm-scale vacuole at the center of aggregates was replicated during interaction of ash and melting ice pellets, followed by sublimation. Fine grained rims were also formed by adding moist aggregates to a dry mixture of sub-31 $\mu \mathrm{m}$ ash, which adhered by electrostatic forces and sparse liquid bridges. From this, it is inferred that the fine grained outer layers of natural aggregates reflect recycled exposure of moist aggregates to regions of volcanic clouds that are relatively dry and dominated by fine ash $<31 \mu \mathrm{m}$.
\end{abstract}




\subsection{Introduction}

Processes of volcanic ash aggregation, by which individual particles adhere to form larger clusters or pellets, have important implications for the dynamics of explosive eruption plumes, and are inferred to occur to some extent in all eruptions that eject abundant fine ash [Sparks et al. 1997]. Previous studies have shown that aggregates preferentially scavenge fine particles from the cloud and then fall out significantly faster than their single-particle constituents [e.g. Carey and Sigurdsson 1982]. Neglecting these first order effects on cloud behavior results in dispersal model outputs that overpredict the extent of aircraft hazards due to airborne fine ash, and under-predict ash loading at the ground closer to source [Rose and Durant 2011]. Refinement of existing field-based numerical models of ash cloud transport thus requires greater understanding of the factors influencing particle aggregation.

In addition to improving model forecasts of eruption cloud behavior, ash aggregates in the geologic record are also used to assess the role of water and infer emplacement mechanisms from deposits of past eruptions. The spectrum of aggregate types is related to the degree of liquid water interaction [Gilbert and Lane 1994, Schumacher and Schmincke 1995]. Relatively dry conditions give rise to loosely bound particle clusters, characterized by low densities and irregular morphologies. These are inferred to be delicately bound by one or more of electrostatic forces [Carey and Sigurdsson 1982], mechanical interlocking of glass shards [Sorem 1982], and small amounts of liquid water [e.g. the melting snowflake-like mechanism of Durant et al. 2009]. Increasing interaction with liquid water results in distinctly different textures. Wetter aggregates form as compact, sub-spherical accretionary lapilli [more generally termed accretionary pellets by Brown et al. 2012], which may be internally massive or concentrically structured. With advances in remote sensing of active eruptions, the role of ice has also emerged as a crucial factor [Rose et al. 1995, 2004]. Field observations from Mount St Helens 1980 [Rosenbaum and Waitt 1981] and satellite data from Pinatubo 1991 [Guo et al. 2004] provide compelling evidence that ice is abundant in volcanic clouds and may be crucial to the nucleation and growth of ash aggregates. However, few studies have described the aggregate textures associated with ice-ash interaction [see review in Brown et al. 2012]. Although extensive observational, experimental and numerical 
studies have addressed these fundamental processes, some key questions about particle aggregation mechanisms remain:

1. Under what conditions does ash interaction with liquid and/or ice play an important role in cloud dynamics, and are the resulting aggregates texturally distinctive?

2. What is the quantitative impact of different aggregation mechanisms on the spatial and temporal evolution of grain size distributions carried by volcanic ash clouds?

3. What do the observed aggregate textures in volcanic deposits reveal about the processes that formed them?

This study examines experimentally the conditions required to reproduce observed textural characteristics of ash-water aggregates, and quantify the preferential selection of fine particles under a range of temperatures and water contents. New empirical data is put in context with published studies of aggregation processes, numerical modelling and field observations of volcanic eruptions and their deposits.

\subsubsection{Conceptual review of wet aggregation}

The attraction between particles in a volcanic cloud can be weak, as in long range electrostatic forces, or strong, as with short range capillary forces from liquid bridges. These binding forces influence the style of aggregation, and govern the size and configuration of particles scavenged into growing clusters [Tardos et al. 1997]. Numerical studies have addressed the processes by which attractive forces overcome the dispersive forces acting on colliding particles in volcanic plumes [e.g. Veitch and Woods 2001, Textor et al. 2006a, Costa et al. 2010]. Here, the conceptual basis for these descriptions is reviewed, focusing on aggregation in the presence of liquid water and drawing on fundamental principles from industrial pharmaceutical applications [e.g. Newitt and Conway-Jones 1958], many of which are relevant to water-particle interactions in moist volcanic ash clouds.

In the top-spray fluidized bed approach to aggregation [e.g. Schumacher and Schmincke 1995, Teunou and Poncelet 2002], liquid drops are sprayed onto a bed of particles and 
mixed, such that the liquid adsorbs to grain surfaces and develops bridges between interacting particles to provide a cohesive force. In an eruption cloud, liquid water may arise from condensation of water vapor directly onto ash particles, or from liquid drops directly ejected with the pyroclasts [e.g. Walker 1981b]. Neglecting gravitational distortion, a static cohesive force is generated from liquid surface tension and capillary suction related to curvature of the liquid meniscus [Rumpf 1962, Mitarai and Nori 2006]. However, in a dynamic system where particles are moving relative to each other, the strength of liquid bonds is also governed by viscous forces and inter-particle friction arising from resistance of the liquid to movement [Ennis et al. 1990, Iveson et al. 2001, Israelachvili 2011]. The growth of wet aggregates depends critically on the ability of these dynamic liquid bridges to dissipate the kinetic energy of particle collisions and promote capture [Ennis et al. 1991, Iveson and Litster 1998].

Experimental observations of wet aggregate growth identify four key stages of behavior (Figure 4.1): (i) wetting and nucleation, where particles develop liquid coatings and collect into initial clusters [Hapgood et al. 2003]; (ii) layering, the accumulation of individual particles or small clusters on to a pre-existing kernel [Sastry et al. 2003]; (iii) coalescence, the capture and assimilation of other soft, deformable aggregates [Iveson and Litster 1998, Liu et al. 2000]; and (iv) slurrying, when aggregates lose their strength and merge into a structureless fluid. A major factor governing the dominant growth behavior is availability of liquid water in the system [Iveson et al. 2001]. For example, a critical change occurs when aggregates become just saturated and water infills nearly all the pore space [Ennis et al. 1991]. Such aggregates develop wet surfaces capable of absorbing additional collisional energy, promoting rapid growth by capture and coalescence [Iveson and Litster 1998]. If the aggregates are soft and deformable, then even at sub-saturated water contents energetic collisions may squeeze water to the impact site and promote particle capture. In contrast, if low-moisture aggregates are surface-dry and kinetic energy dominates the system, collisions are more likely to result in rebound, accompanied by crushing or attrition [Ennis et al. 1991]. In this case, only the smallest particles or clusters are able to be captured by existing aggregates, leading to layering [Sastry et al. 2003]. Once the liquid has dried, mineral precipitates or other remaining residues preserve the structure [Gilbert and Lane 1994, Scolamacchia et al. 2005]. On the other hand, if enough liquid water is present the 
mixture becomes oversaturated, and aggregates collapse into a structureless slurry (cf. volcanic 'mud rain').
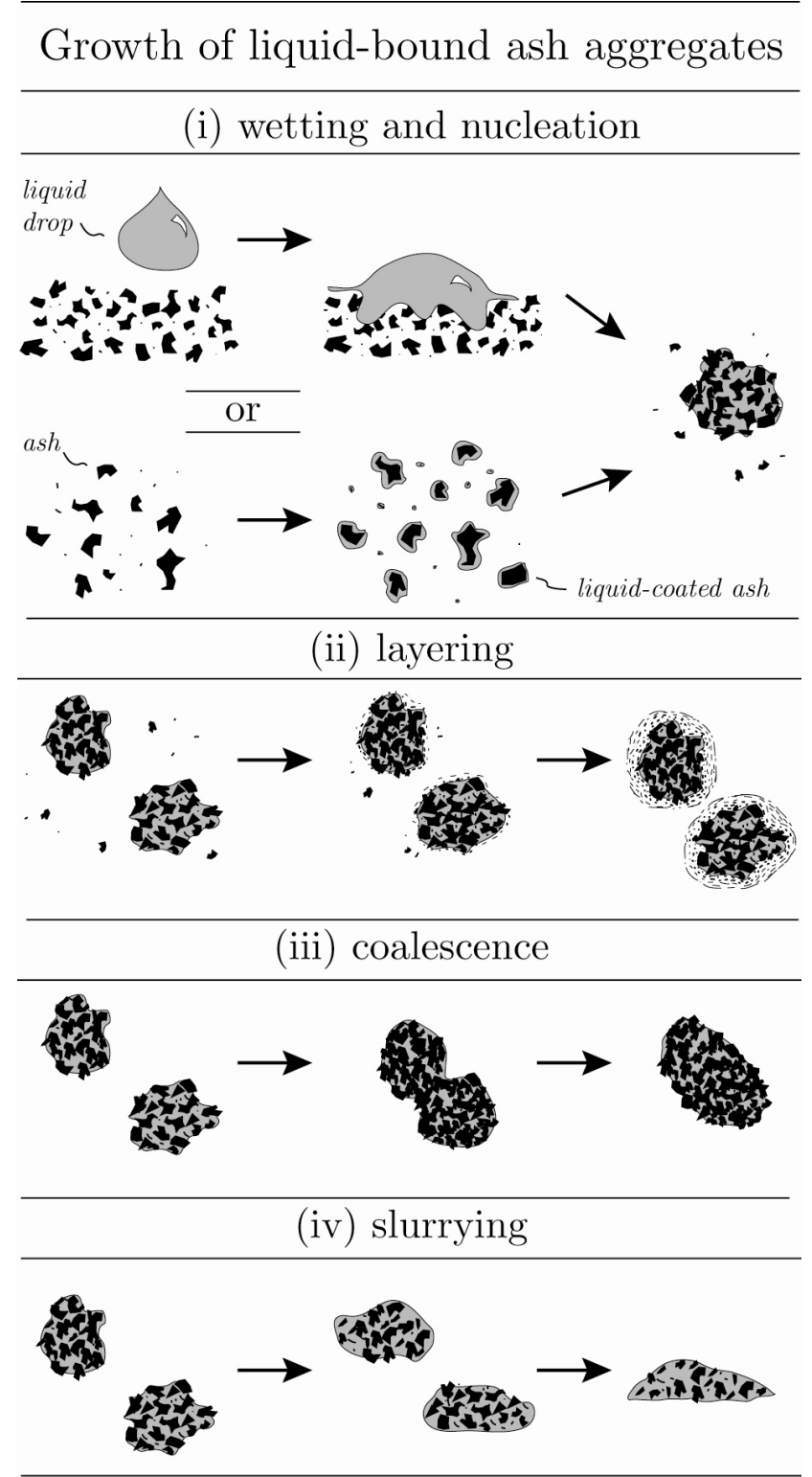

Figure 4.1 Schematic representation of growth mechanisms in liquid-bound ash aggregates. See text for details. Note that in (i) wetting of ash particles by liquid drops (top) or condensation of liquid directly onto ash surfaces (bottom), both lead to nucleation of new clusters during particle collisions. Modified from Iveson et al. [2001].

\subsubsection{Geological context for experiments}

The stages of nucleation, layering, coalescence and slurrying described above are central to the development of ash aggregate textures. Characteristics of ash aggregates in deposits have therefore been used to infer both general information about the availability of liquid water during formation [Sparks et al. 1997], and the specific 
processes governing their development [e.g. Schumacher and Schmincke 1991, Brown et al. 2010].

Although weakly structured aggregates are known to preserve poorly in the geological record, there are numerous accounts of such aggregates falling from historical eruption clouds, such as Sakurajima, Japan [Gilbert and Lane 1994], and Eyjafjallajökull, Iceland [Taddeucci et al. 2011]. Deposits that fell directly from a buoyant plume are also more frequently associated with small $(<5 \mathrm{~mm}$ diameter), internally massive or weakly layered aggregates, termed core-type accretionary lapilli by Schumacher and Schmincke [1991] and AP1 by Brown et al. [2012]. This relationship suggests that simple, vent-derived umbrella clouds alone are not typically capable of generating the complexly laminated aggregate types observed in the geologic record (i.e. rim-types of Schumacher and Schmincke [1991]; accretionary lapilli of Brown et al. [2010)]). Only ash deposits from interactions between pyroclastic density currents (PDCs) and an overriding plume (whether co-PDC or vent-derived) have been documented in association with the most complexly laminated aggregates. Examples include deposits from Tenerife [Brown et al. 2010], Laacher See [Schumacher and Schmincke 1991], Colli Albani [De Rita et al. 2002], and the Oruanui eruption [Figure 4.2a-e; Self 1983; Wilson 2001]. In these concentrically structured aggregates, there is a consistent arrangement of a structureless inner core and layered outer rim dominated by ash $<10$ $\mu \mathrm{m}$ (Figure 4.2a-c), which provides a record of the volcanic and atmospheric conditions experienced by the aggregate during growth. However, to date, there is no unified explanation for how and where these fine grained outer layers form [e.g. Reimer 1983, Gilbert and Lane 1994, Schumacher and Schmincke 1995, Brown et al. 2010].

The textural detail capable of being preserved in aggregated deposits is illustrated in Figure 4.2. From water-rich phases of the $\sim 25.4$ ka Oruanui eruption (Taupo volcano, New Zealand), this includes evidence for coalescence (Figure 4.2c-d) and trapped air bubbles (Figure 4.2e). Furthermore, the irregularly shaped, central cavities at the center of some aggregates (Figure 4.2b), similar to those documented by Kato [1986, Plate I.2] from Okinawa, Japan, are inferred to indicate that $\mathrm{mm}$-scale ice clusters were involved in the initial aggregation process [Van Eaton and Wilson in review; Chapter 5]. In contrast, examples of ice-ash aggregates collected from the 2010 Eyjafjallajökull eruption contain visibly higher ice:ash ratios (Figure $4.2 \mathrm{f}-\mathrm{g}$ ). Although these mm-scale 
aggregates incorporated some ash particles into the icy framework, they lack evidence for having accumulated multiple layers of fine ash as seen in the Oruanui examples. New experimental observations are used here to examine the conditions governing these contrasting modes of ice-ash and liquid-ash interaction, and the specific range of particle sizes that can be aggregated in each case.
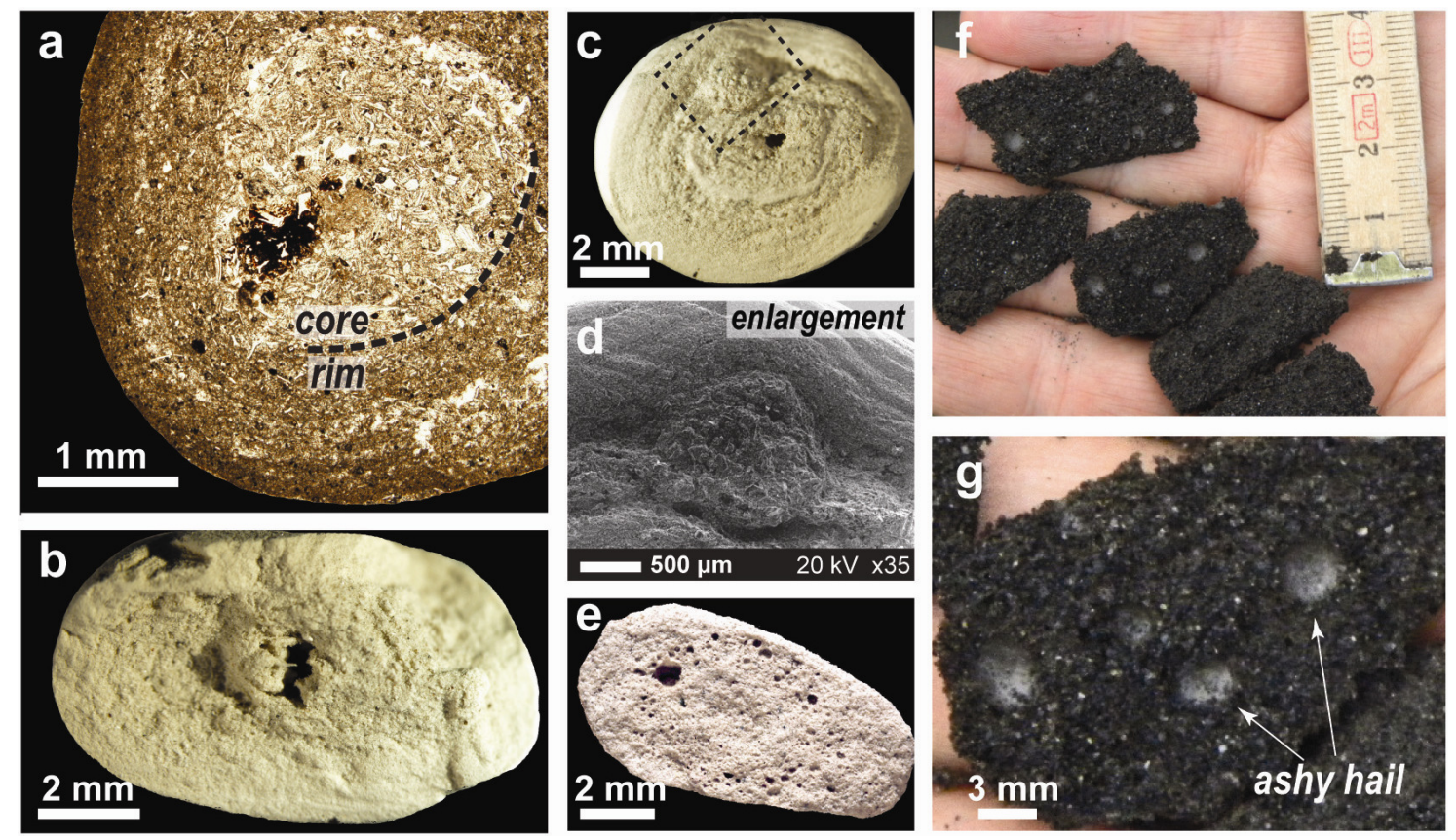

Figure 4.2 Naturally occurring ash aggregates from explosive phreatomagmatic eruptions. The two left-hand columns (a-e) show cross-sectional faces of aggregates from phreatoplinian phases of the $\sim 25.4$ ka Oruanui eruption, New Zealand. (a) Is a thin section photomicrograph (in PPL) of a concentrically laminated aggregate, showing the contrast between the massive internal core with randomly oriented glass shards, and finer grained layered rim. (b) Exhibits an irregular central vacuole, inferred to be the relict of an icy nucleus. (c) Shows evidence for coalescence by capture of a smaller, preexisting aggregate (enlargement in (d) is an SEM image at the core-rim boundary). (e) Is a primarily massive aggregate with a concentric zonation of spherical vesicles. The right-hand column (f-g) shows mm-scale ashy hailstones collected from the basaltic 2010 eruption of Eyjafjallajökull, Iceland. Enlarged area (g) shows that the hail particles have ashy inclusions, but are not enveloped in multiple layers of ash like (b).

\subsubsection{Terminology}

Ash aggregate is used here in the general sense to describe any clusters or pellets of ash and water (regardless of whether the water is liquid, or solid or both). Individual grains of ash are also referred to as particles, regardless of whether they are incorporated into aggregates. Parent ash is used to designate the initial ash mixture used to generate 
aggregates in experiments. In this way, the particle size distributions of parent ash is compared with those of the aggregates (after they have been disaggregated for analysis).

\subsubsection{Previous experimental studies}

Schumacher [1994] and Schumacher and Schmincke [1995] investigated the properties of ash aggregates simulated within an electric field and under liquid water conditions (Table 4.1). They suggested that massive cores likely grow by wet aggregation, while layered, finer grained outer rims may form in dry conditions, bound primarily by electrostatic forces. They also found that increasing the liquid water contents of the parent ash resulted in changes in the aggregate strengths and their constituent particle sizes, and that the particle size distribution of the parent ash was also important. In particular, parent ash $<350 \mu \mathrm{m}$ required less water to promote the development of 'well developed' aggregates (c. 15 wt.\% water, compared to $c .20 \mathrm{wt} . \%$ for parent ash $<2000$ $\mu \mathrm{m})$. Furthermore, they found that experiments using well-sorted parent ash (half-phi splits) could not form compact, subspherical aggregates consistent with the wellpreserved types commonly identified in volcanic deposits.

Gilbert and Lane [1994] focused on the contrast between wet aggregation onto a preexisting nucleus, versus collection of ash by liquid drops. The latter process produced vertically graded, hemispherical aggregates that did not match the massive or concentrically structured field examples, and was therefore ruled out as a useful analogue. They also developed an experimentally derived aggregation coefficient to estimate the particle sizes most likely to be scavenged into liquid-bound aggregates. James et al. [2002] and James et al. [2003] examined properties of fine ash falling vertically through an electric field to establish the apparent densities and fall velocities of loosely bound electrostatic clusters. The delicate aggregates were difficult to extract for examination, limiting the application of particle size analysis. Kueppers [2011] used a sodium metasilicate binder to initiate aggregation in a fluidized bed using very well sorted parent ash. Telling and Dufek [2012] used a similarly narrow size range of volcanic ash in a humidity-controlled tank to quantify the number of particle collisions that resulted in sticking, given different amounts of ambient water vapor. 
Table 4.1 Aggregation experiments using volcanic ash.

\begin{tabular}{|c|c|c|c|}
\hline Authors & Binding agent & Experimental method & $\begin{array}{l}\text { Grain size of parent } \\
\text { ash }\end{array}$ \\
\hline Schumacher (1994) & electrostatic forces & electrostatic powder coater & $<250 \mu \mathrm{m}$ \\
\hline Gilbert and Lane (1994) & liquid water $\pm \mathrm{NaCl}$ & recirculating vertical wind tunnel & $<300 \mu \mathrm{m}^{a}$ \\
\hline $\begin{array}{l}\text { Schumacher and } \\
\text { Schmincke (1995) }\end{array}$ & $\begin{array}{l}\text { (1) electrostatic forces } \\
\text { (2) liquid water }\end{array}$ & $\begin{array}{l}\text { electrostatic powder coater } \\
\text { hand-held spray agglomeration }\end{array}$ & $\begin{array}{l}<250 \mu \mathrm{m} \\
<350 \text { to }<2000 \mu \mathrm{m}\end{array}$ \\
\hline James et al. $(2002,2003)$ & electrostatic forces & pumice-fracturing fall chamber & $<c .200 \mu \mathrm{m}^{b}$ \\
\hline Kueppers et al. (2011) & liquid water $\pm \mathrm{Na}_{2} \mathrm{SiO}_{3}$ & fluidized bed spray aggregation & $90-125 \mu \mathrm{m}^{c}$ \\
\hline Telling and Dufek (2012) & liquid water & humidity-controlled wind tunnel & $1 / 4 \varphi$ splits $106-250 \mu \mathrm{m}$ \\
\hline This study & ice and liquid water & vibratory pan aggregation & $<1414 \mu \mathrm{m}$ \\
\hline
\end{tabular}

${ }^{a}$ Particles in the range of $10-120 \mu \mathrm{m}$ from parent ash and aggregates were size analyzed by Elzone conductivity analyzer.

${ }^{b}$ The full parent ash size distribution was not obtained, but cumulative numbers were provided for the $10-130 \mu \mathrm{m}$ particles.

${ }^{c}$ Parent ash obtained by milling basaltic lapilli.

From these experimental studies, there is sharp contrast between aggregates that are dominated by electrostatic forces, and those which are bound by liquid. Although the weak electrostatic aggregates may be volumetrically dominant in typical volcanic clouds, their poor preservation potential makes it difficult to establish their role in ash transport and dispersal in past eruptions. Furthermore, the interplay between electrostatic forces and water (liquid and/or ice), which is likely to arise in volcanic clouds, has not been experimentally addressed in the context of ash aggregation. The experiments were thus designed to simulate a range of possible interactions between colliding ash particles, ice and varying amounts of liquid under temperature controlled conditions (as suggested by Brown et al. [2012]).

\subsection{Methods}

For the experiments, a vibratory pan aggregation technique was used. This method was modified from the approach of Schumacher and Schmincke [1995], and draws from the top spray fluidized bed principles used in materials engineering [e.g. Tardos et al. 1997]. However, rather than injecting air into the particulate mixture, a sieve shaker is used to produce a consistent and repeatable vibration to initiate particle collisions. The investigation examines (Table 4.2) the effects of adding liquid drops or ice particles to 
ash mixtures in the form of: (1) a fine spray of distilled water; (2) ice particles; and (3) a mixture of liquid water and ice. For each experiment the particle size distributions of parent ash and resulting aggregates were obtained to document the particle sizes that were preferentially scavenged versus excluded under each set of conditions.

Table 4.2 List of experiments in this study using the vibratory pan aggregation method.

\begin{tabular}{llll}
\hline Name & Temperature & Water phases & Experimental description \\
\hline Hail-1 & $-20 \pm 2{ }^{\circ} \mathrm{C}$ & solid & Natural hailstones added to ash \\
Hail-2 & $-20 \pm 2{ }^{\circ} \mathrm{C}$ & liquid + solid & Cyclic wetting and freezing of ashy hail \\
Mix-1 & $-20 \pm 2{ }^{\circ} \mathrm{C}$ & liquid + solid & Distilled liquid drops freezing onto ash \\
Mix-2 & $18 \pm 2{ }^{\circ} \mathrm{C}$ & liquid + solid & Melting ice accumulating ashy layers \\
Liquid-1 & $18 \pm 2{ }^{\circ} \mathrm{C}$ & liquid & Distilled water sprayed onto ash \\
Liquid-2 & $18 \pm 2{ }^{\circ} \mathrm{C}$ & liquid & Liquid-1 aggregates added to ash $<31 \mu \mathrm{m}$ \\
\hline
\end{tabular}

\subsubsection{Experimental scaling and validity}

To reduce the length scale of particle interactions from that of natural plumes [which have particle concentrations from $<<1$ to $10 \mathrm{~g} \mathrm{~m}-3$ above the gas thrust region: Bursik et al. 2004], down to a laboratory scale, a denser particle concentration of $1-10 \mathrm{~kg} \mathrm{~m}^{-3}$ is used. This shortens inter-collisional paths with respect to natural systems, and increases the collision frequencies. The timescales of aggregation are therefore likely to be accelerated in the experiments relative to natural ash clouds. However the mechanisms of aggregate growth related to ash-water interactions are not affected. A weakness of the current approach is the influence of wall effects in the vibratory pan. Impacts with the sides and bottom introduce additional forces to those generated during inter-particle collisions alone, and the walls allow some moist ash to adhere and effectively exit the system. Due to this sticking effect, it was impossible to quantify the percentage of each parent ash mixture that converted into aggregates. Another source of experimental variability arises from the use of natural parent ash material, the particle size distribution of which varies slightly and has to be accounted for in each experiment.

Despite these limitations, the experimental technique provides some advantages. Ash aggregates can be generated without the addition of chemical binding agents [cf. Kueppers et al. 2011] or humicant compounds such as $\mathrm{NaCl}$ solution [Gilbert and Lane 
1994], thereby ensuring simpler conditions for examining post-drying cementation. The liquid experiments also allow observation of wet growth processes from nucleation, to layering, coalescence and slurrying without requiring the use of artificial nuclei to seed growth [Gilbert and Lane 1994]. Moreover, many of the simulated aggregates are strong enough to extract and handle individually, allowing a diversity of measurements to be undertaken.

\subsubsection{Materials and procedures}

The parent ash for all experiments was from fall deposits of the Oruanui eruption. The ash was passed through a $1.4 \mathrm{~mm}$ mesh before use, yielding a particle size distribution fine enough to be analyzed by laser diffraction particle size analyzer, while still allowing for the presence and influence of medium to coarse ash particles. Pre-existing aggregates in the deposits were broken up by brushing through 500 and $250 \mu \mathrm{m}$ mesh sieves. The $<63 \mu \mathrm{m}$ content in the parent ash ranged from 50-75 vol.\% (Figure 4.3) and mean particle sizes were 60-218 $\mu \mathrm{m}$ (Appendix E). These particle size characteristics are consistent with highly explosive eruption clouds [Walker 1981c, Rose and Durant 2009]. The parent ash was polymodal, with modes at c. 3, 30, 70, 180 and $500 \mu \mathrm{m}$. For ice experiments, naturally formed hailstones with diameters of 5-15 mm were collected on two occasions from storms in Wellington, New Zealand. Fine shards of frost were also used to simulate the presence of ice and ice pellets (i.e. graupel).

Prior to each run, $c .0 .5 \mathrm{~g}$ of the parent ash was set aside for particle size analysis. To set up the apparatus, $100 \pm 10 \mathrm{~g}$ of dry parent ash was placed into a flat-bottom steel pan (20 x $4.5 \mathrm{~cm}$ ) on a Fritsch Analysette ${ }^{\circledR}$ sieve shaker. For Liquid-1 and -2 experiments at room temperature (Table 4.2), distilled water was misted onto the ash in c. 2-3 $\mathrm{g}$ increments to promote an even distribution of the liquid onto ash surfaces. Once the liquid was added, a transparent lid was fastened to secure the pan to the shaker and vibration (50 Hz shaking at 2-3 mm amplitude) applied for one minute. It was observed that more energetic vibrations tended to break up the forming aggregates, while lower energies did not sufficiently mobilize the ash particles. One minute of vibration was generally sufficient to attain dynamic equilibrium, whereby no net change in the overall size distributions of the aggregates occurred despite constant breakup and re-forming [cf. Ramaker et al. 1998]. After each one-minute cycle vibration was stopped and a 
brush used to detach and homogenize any ash adhering to the walls and bottom of the pan. This homogenization step also minimized the effects of kinetic stratification before each new cycle. One-minute cycles of spraying, vibration and homogenization were repeated until the desired amount of liquid water was added, allowing progressive saturation of the particles and observation of the corresponding stages of aggregate growth. For experiments involving the addition of ice (Hail-1, Hail-2, Mix-2), all of the ice (c. $30 \mathrm{~g}$ ) was added during the first cycle, followed by one-minute cycles of vibration and homogenization (plus spraying, in Hail-2) as previously described. Cold experiments were conducted inside an ice core storage facility maintained at a temperature of $-20 \pm 2{ }^{\circ} \mathrm{C}$.

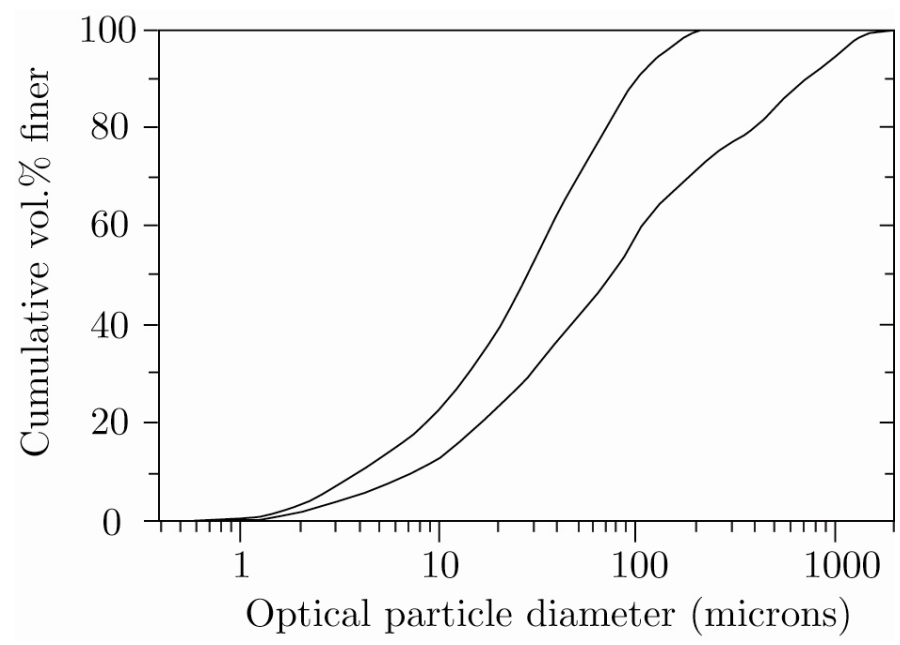

Figure 4.3 Plot showing the full range of particle size distributions in the parent ash used for experiments, given by maximum and minimum values for each half- $\varphi$ particle size and fit with spline curves. See Appendices F and G for further details.

At the end of each Liquid-1 experiment, there were two components left in the pan: (1) ash aggregates and (2) residual material comprising the coarser particles excluded from aggregation plus any ash adhered to the pan walls (Figure 4.4). Aggregates were extracted by gently passing the entire mixture through $8,4,2$, and $1 \mathrm{~mm}$ sieves; the majority were able to survive this step intact. Only aggregates $>1 \mathrm{~mm}$ were included. Although some $<1 \mathrm{~mm}$ aggregates were formed, they could not be reliably separated from coarse individual particles and were therefore counted as residua. Aggregate fractions and residua were weighed wet and dry to obtain water content and the size distributions of the bulk aggregates. The five largest aggregates from each Liquid-1 experiment were weighed individually and the three principal axes measured with 
callipers (or a ruler) to $\pm 0.2 \mathrm{~mm}$. Principal axis dimensions, water content and densities were obtained from 36 individual aggregates from the Mix-2 experiments; selected samples were also mounted in epoxy and imaged by x-ray microtomography at a resolution of $4 \mu \mathrm{m}$ by Corrado Cimarelli at Ludwig-Maximilians-Universitāt, Munich, Germany.

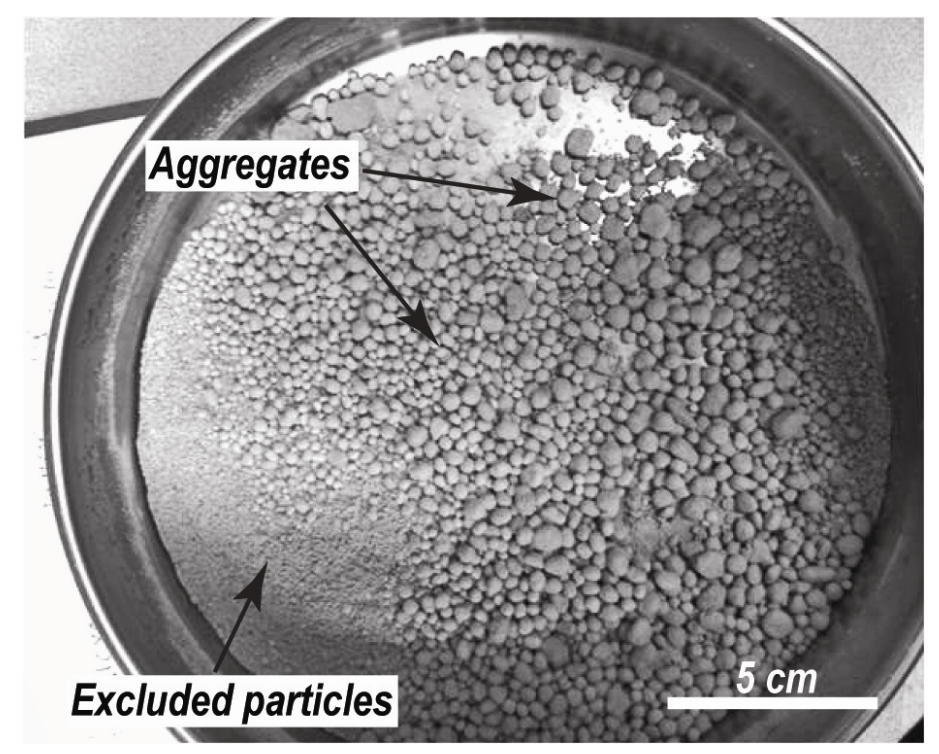

Figure 4.4 Photo from a liquid-1 experiment showing how the vibratory pan aggregation process results in ash aggregates and residual particles that were preferentially excluded from aggregation.

Particle size distributions (Appendix F) of the parent ash and aggregates from 44 Liquid-1 experiments and nine ice experiments were obtained by laser diffraction particle size analyzer (Beckman-Coulter LS13 320) with a $750 \mathrm{~nm}$ laser and software version 5.01. This instrument establishes optical particle diameters in the range of 0.4 to $2000 \mu \mathrm{m}$ (expressed as volume percent of the total) using the scattering of light from individual particles suspended in water. By comparison with other methods of particle size analysis (i.e., sieve-pipette and SEM counting techniques) a best fit optical model for rhyolitic Oruanui ash was obtained using a refractive index of 1.55 and absorption coefficient 0.01 , with no correction factor applied (e-factor $=1$ ). Run times were $60 \mathrm{~s}$, using obscurations of $8-12 \%$ and sonicating during runs to reduce particle flocculation. Representative aggregate particle size distributions were obtained from the $4-8 \mathrm{~mm}$ aggregate fraction of the Liquid-1 experiments. If $>4 \mathrm{~mm}$ aggregates were not produced, the 2-4 $\mathrm{mm}$ fraction was analyzed instead. Each analysis was run in triplicate to obtain estimates of analytical uncertainty. Particle size distributions are plotted as 
maxima and minima, with values binned into half- $\varphi$ intervals from 10 to $0 \varphi(1-2000$ $\mu \mathrm{m})$ and fitted with spline curves.

For comparison with natural deposits, dry densities of $c .880$ individual ash aggregates from the Oruanui deposits were determined using measurements of the three principal axes and dry weight. Aggregates with fine ash rims were stripped of their outer layers, and laser particle size distributions were obtained on the internal cores.

\subsection{Results}

Overlaying the particle size distributions of parent ash and aggregates from individual experiments yields three zones (Figure 4.5). The zone of preferential scavenging indicates the range of particle sizes that is more abundant in the aggregates than the parent ash, whereas the zone of preferential exclusion indicates the coarser sizes that are relatively less abundant. The neutral zone is where the grainsize curves overlap within analytical uncertainty and defines the transition between preferentially scavenged and excluded ash. Recognition of these zones provides a quantitative framework for documenting preferential scavenging effects in each experimental scenario. Experiments yielding equivalent particle size distributions were excluded from further examination because no preferential scavenging could be detected (marked with an $(*)$ in Appendices $\mathrm{E}$ and F). Results are presented below for aggregation experiments in the presence of an ice phase at sub-freezing temperatures (Hail-1, Hail-2, Mix-1), followed by aggregation at room temperature in the presence of melting ice (Mix-2), and then liquid (Liquid-1, Liquid-2). 


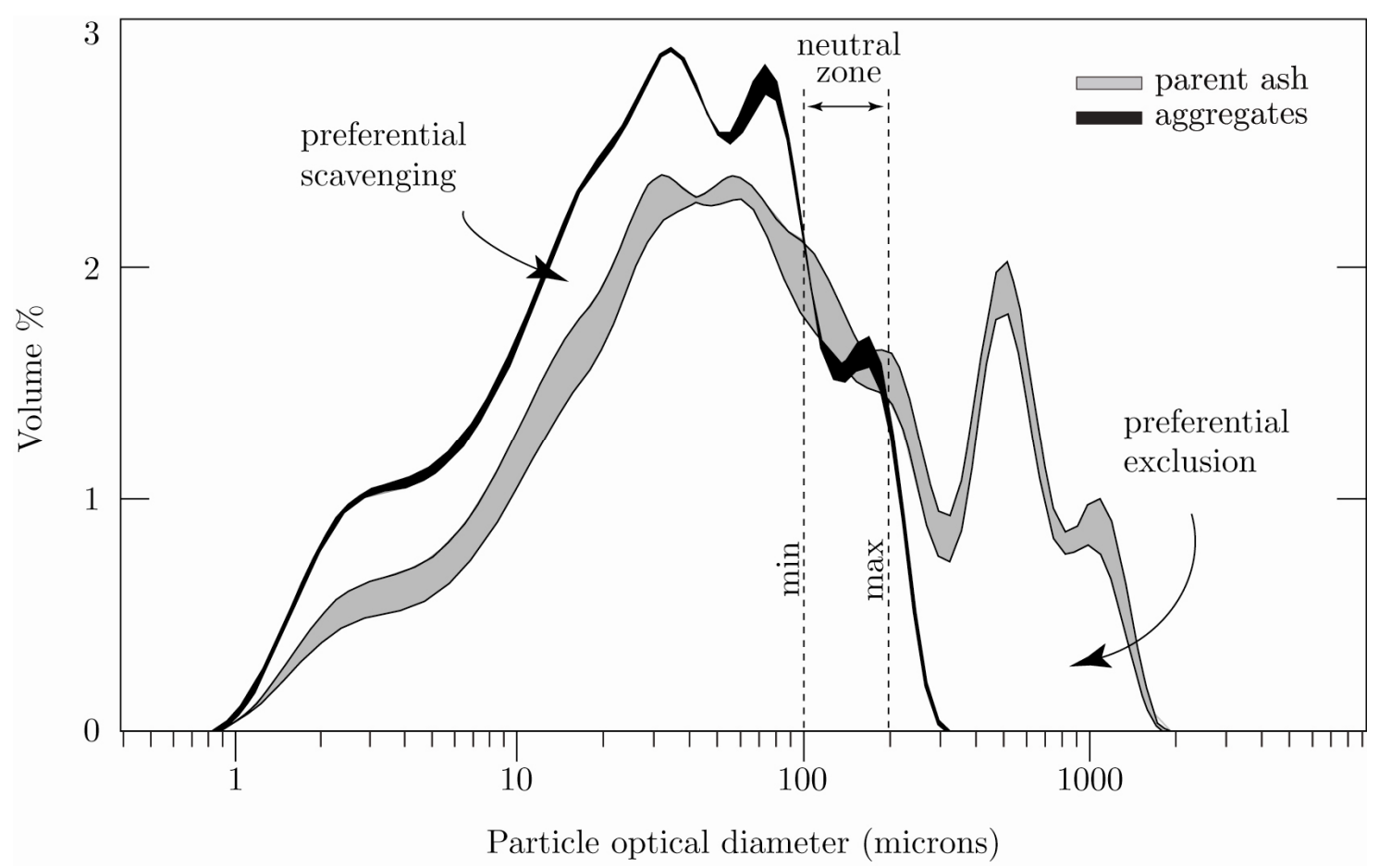

Figure 4.5 Definition sketch identifying key zones in the overlain particle size distributions of parent ash and experimental aggregates. Each curve is given by the maximum and minimum volume \% obtained from triplicate LDPSA analyses (binned into half- $\varphi$ size intervals and fit with spline curves). The thickness of each curve thus represents analytical uncertainty. Arrows indicate the zones defining particle sizes that are preferentially scavenged into aggregates versus excluded relative to the parent ash. The intersection of the curves, bracketed by maximum and minimum intersection points, gives the neutral zone, designating particles that are neither preferentially scavenged nor excluded by aggregates (i.e. aggregation coefficient=1).

\subsubsection{Aggregation at sub-freezing temperatures}

\section{Hail-1: Ice-ash aggregation in the absence of a liquid phase}

For these experiments, dry parent ash was added to the apparatus and cooled to $-20 \pm 2$ ${ }^{\circ} \mathrm{C}$. Approximately $30 \mathrm{~g}$ of natural hailstones (Figure 4.6a) were added to the mixture and vibrated in one-minute cycles. Despite the absence of a liquid phase, the hailstones accumulated a sub-millimeter layer of fine ash after a single cycle (Figure 4.6b). This layer did not change significantly after ten additional cycles. The ash was firmly stuck to the ice, even when individual ashy hailstones were tapped against the edge of the metal pan. Comparison of the grain size distributions in Figure 4.6c shows that only particles finer than $34 \pm 1 \mu \mathrm{m}$ were preferentially adhered to the ice. Furthermore, $99 \%$ of the adhered ash was finer than $173 \mu \mathrm{m}$. Gravimetrically determined ice contents in 
the ashy hailstones were 81-94 wt.\% depending on the volume of the hailstone, indicating very ash-poor aggregates overall.
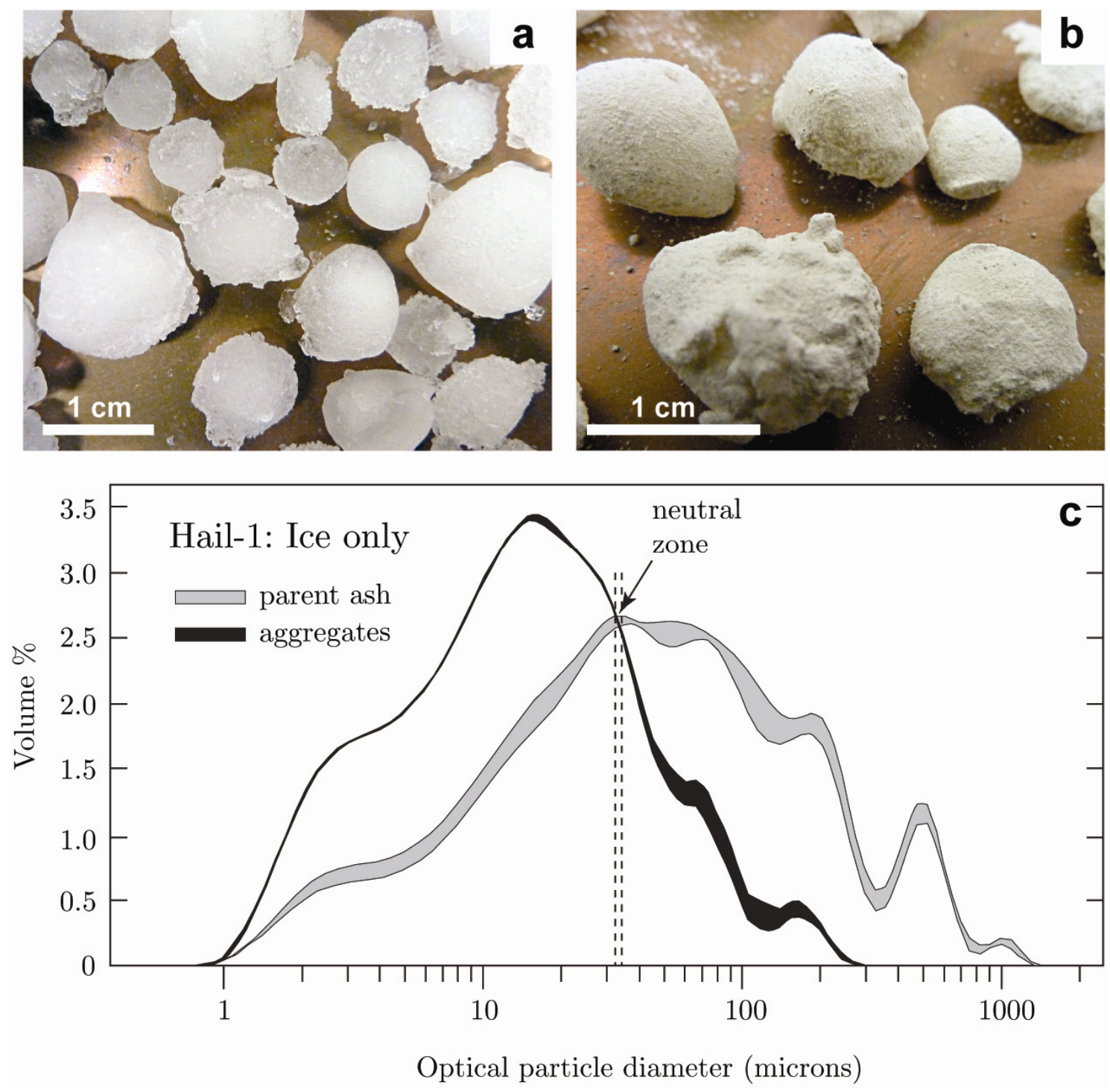

Figure 4.6 Aggregates formed from the Hail-1 experiment: (a) shows natural hailstones before they were added to the ash mixture at $-20^{\circ} \mathrm{C}$; (b) shows the ash-ice aggregates that were formed, exhibiting a thin coating of fine ash firmly adhered to each hailstone; (c) plots particle size distributions of the parent ash (grey) and particles adhering to the ice (black), as in Figure 5. Although some coarser particles did adhere to the ice, only those finer than $\sim 31 \mu \mathrm{m}$ were preferentially scavenged.

\section{Hail-2: Cyclic wetting and freezing of ashy hail}

With the aim of replicating processes of hail growth by accumulation of supercooled liquid drops (riming), followed by wetting [Knight and Knight 1973, Pflaum 1980], samples of ashy hail prepared using the Hail-1 method (Figure 4.6a-b) were subjected to cycles of wetting and freezing at $-20^{\circ} \mathrm{C}$ in the presence of agitated ash. Due to the 
challenge of maintaining liquid water in a supercooled state in the water sprayer, the liquid was kept just above freezing. Following each one-minute vibration cycle, a mist of liquid water was sprayed onto the mixture. The liquid froze onto the ashy hail within a few seconds, enabling individual hailstones to adhere to one another via liquid bridges at contact points, which subsequently froze in place. Slightly more ash adhered to the ice in this case compared to Hail-1, indicated by final ice contents in the range of 70-89 wt.\%. However, even ten cycles of wetting and freezing did not cause significant thickening of the existing fine ash layers on the hail. In contrast, addition of even small amounts of liquid spray appeared to rinse away portions of adhered particles, probably because the liquid was not sufficiently supercooled to freeze immediately on contact.

\section{Mix-1: Aggregation by freezing liquid drops}

The Mix-1 experiments differed from the Hail experiments in that no solid ice was added to the parent ash. Instead, after the parent ash and apparatus were brought to $-20^{\circ} \mathrm{C}$, liquid water was sprayed directly onto the ash particles and then agitated while it froze. As a result, particles were captured by the liquid drops that splashed onto the ash mixture and then rapidly solidified in place. These ice-ash aggregates froze into flakelike morphologies (Figure 4.7a-b) before the liquid could become evenly dispersed throughout the ash mixture. The rapid freezing also inhibited rearrangement of particles into a (sub)spherical configuration. With further additions of liquid water and agitation, icy ash flakes adhered together by liquid bridges that froze, generating irregular, loosely packed morphologies (Figure 4.7b). Aggregates were 15-24 wt.\% water, indicating that greater ash contents could be achieved using an initial liquid phase compared to the liquid-free experiments.

Particle size analyses of parent ash and aggregates (Figure 4.7c) show that differences between the two curves are less distinct than in the Hail experiments, indicating that the freezing drops show significantly less preferential size selection. The coarsest $99^{\text {th }}$ percentile particle size in the aggregates is similar to that of the parent $(600-1000 \mu \mathrm{m})$, suggesting that the rapid freezing of water drops prevented even dispersion of the liquid through the mixture and allowed indiscriminate collection of most particles in immediate contact. 

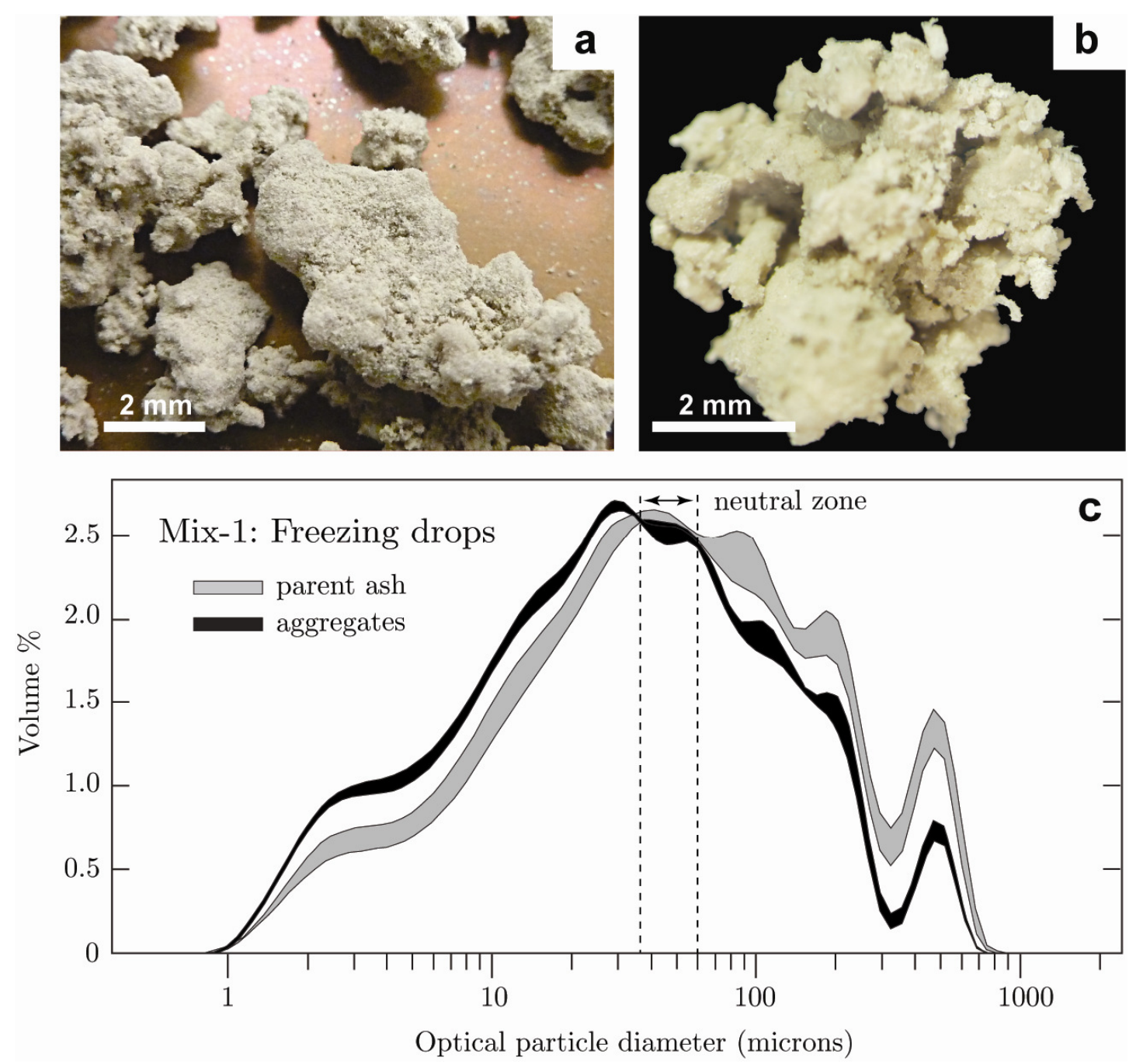

Figure 4.7 Aggregates formed in the Mix-1 experiments, during which distilled liquid water was sprayed onto parent ash at $-20^{\circ} \mathrm{C}$. (a) Shows the irregularly-shaped flakes of ice and ash. (b) Shows an enlargement of an ash-ice cluster. (c) Plots particle size distributions from parent ash and ash-ice aggregates, as in Figure 5. Note the similarity of the two curves compared to previous experiments, indicating less size-selective processes overall.

\subsubsection{Aggregation of ash and melting ice at room temperature (Mix-2)}

The interaction between ash and melting ice pellets [i.e. graupel: Pruppacher and Klett 1978], was simulated by adding mm-scale frost particles to an agitated ash mixture at room temperature $\left(18 \pm 2^{\circ} \mathrm{C}\right)$. During the initial $60 \mathrm{~s}$ of mixing, the ice particles began to melt and develop thin liquid films that refroze during ice-ice collisions. This resulted in the melting ice particles rapidly adhering to one another, forming relatively ash-free clusters of loosely packed irregularly shaped ice pellets, with whisker-like protrusions. This preference of ice-ice binding (over that of ash and ice) during the first minute of the experiments is likely related to the thicker liquid films developing preferentially 
around the ice particles and greater likelihood of solidifying liquid bridges by freezing at ice-ice contact points. Furthermore, the interlocking of dendritic ice shards may have played a role [Jiusto and Weickman 1973]. As the ice pellets formed, small amounts of melt water were shed onto surrounding ash particles, inducing nucleation of tiny (sub$\mathrm{mm}$ ) ash clusters. The ice pellets and nucleated ash clusters continued to behave independently until melting reached a critical point; once ice pellets became nearly saturated with liquid water (evidenced by a liquid sheen developing on their surfaces), they began to collect and accumulate the ash clusters.

The ice-ash aggregates from Mix-2 were dried in two ways to examine their internal textures. Some samples were dried in a $400{ }^{\circ} \mathrm{C}$ oven to rapidly boil constituent water, while the others were dried in a lyophilizer, which reduces pressure and temperature to slowly sublime ice. Internal textures were examined visually in cross-section (Figure 4.8a-b), and imaged using x-ray microtomography (Figure 4.8c) and scanning electron microscopy (Figure 4.8d-f). Two notable features were observed: (1) a central vacuole was preserved in each aggregate (Figure 4.8a-c); and (2) the aggregates had an open framework structure consisting of 100-200 $\mu \mathrm{m}$ clusters of ash (Figure $4.8 \mathrm{~b}-\mathrm{d}$ ). This structure appears to have resulted from the sequence of initially limited moisture supply to the ash, which promoted nucleation of tiny ash clusters (Figure 4.1), followed by binding to the liquid-saturated ice pellets.

Figure 4.8 shows the morphologies of the central vacuoles that resulted from each drying method. The oven-drying method generated a flattened, smooth cavity from melting and boiling at high temperature (Figure 4.8a). This distorted vacuole morphology is inconsistent with most of the natural Oruanui examples, which are usually characterized by sub-spherical shapes and delicate, inward-facing protrusions (Figure 4.2b). The aggregates that were dried by sublimation, however, retained the outline of their irregularly shaped ice pellet cores, and closely resemble natural examples (Figure 4.8b-c). Gravimetrically determined total water contents (i.e. liquid plus ice) in the ice-ash aggregates ranged from 5-30 wt.\% depending on the size of the icy cores. This is supported by a positive correlation between water content and total aggregate diameter $\left(n=36\right.$; linear $\left.r^{2}=0.74\right)$, indicating that larger ice pellets formed larger ice-ash aggregates. By extension, a larger ice pellet core would be expected to form a larger central cavity, thereby decreasing the overall dry density of the aggregate. 

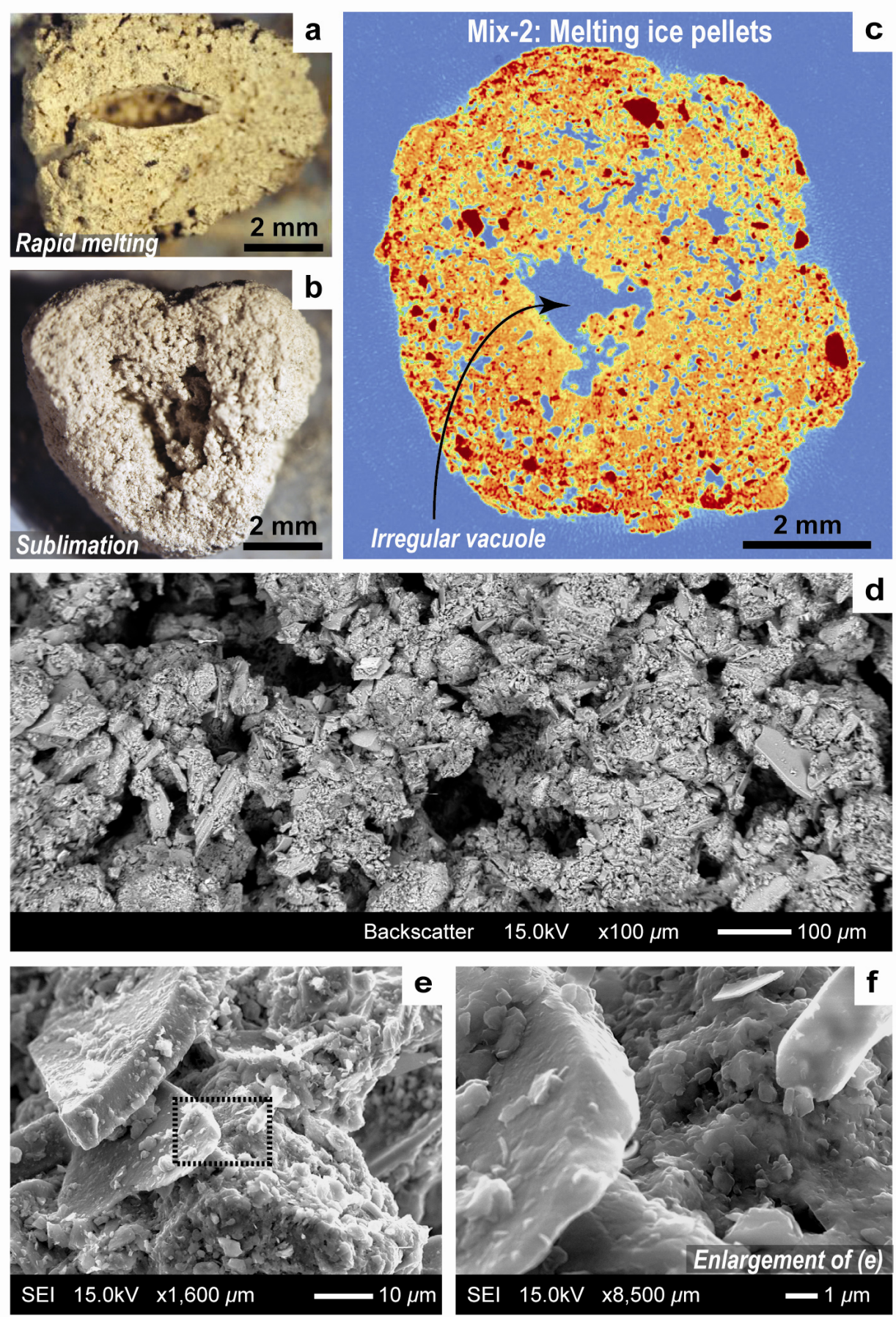

Figure 4.8 Aggregates from Mix-2 experiments (melting ice pellets). (a-c) Emphasize the internal vacuole that results after drying. Contrasting vacuole morphologies formed from rapid melting and boiling (a) versus low-temperature sublimation (b). (c) Is an Xray computed microtomographic image through the center of a freeze-dried Mix-2 aggregate. Note the irregularly shaped central vacuole with inward-facing protrusions, and overall porous structure. Red to yellow color scale indicates higher to lower density, respectively; blue indicates void space. (d) Is an SEM image showing the porous framework of interconnected ash clusters. (e) Shows an enlargement of a face-to-face grain contact, and (f) further enlarges the contact, showing coatings of amorphous silica (confirmed with energy-dispersive spectroscopy using the SEM) that may form during rapid alteration of volcanic glass and clays in the presence of liquid water. 
However, this is not supported by the data. Measurements from 36 individual samples exhibited a wide range in dry densities, from $0.7-1.6 \mathrm{~g} \mathrm{~cm}^{-3}$ (mean of $1.3 \mathrm{~g} \mathrm{~cm}^{-3}$ ), that did not covary with water content. The density of the aggregates may have been governed instead by a variable amount of liquid at the surface of each melting ice pellet. For example, a thicker liquid layer would facilitate greater mobility and consolidation of ash particles during collisions [Iveson et al. 2001], whereas less liquid would promote a more loosely-bound structure. This is supported by the microtomography images (Figure 4.8c), which suggests that ash within a millimeter or so of the center (i.e. the source of liquid) is more tightly bound and dense than the porous outer regions.

\subsubsection{Wet aggregation at room temperature}

\section{Liquid-1: Growth of liquid-bound aggregates}

At room temperature $\left(18 \pm 2^{\circ} \mathrm{C}\right)$, fine sprays of distilled water were added incrementally to a vibrated mixture of parent ash. Increasing the liquid content of the mixture led to a steady progression through phases of wet growth by nucleation, layering, coalescence and slurrying, (Figure 4.1), leading to a variety of aggregates (Figure 4.9a-d). Liquid contents of $<5$ wt.\% resulted in small clusters (generally $<5 \mathrm{~mm}$ ) that had a loosely bound, fluffy appearance. Even at water contents up to 10-15 wt.\%, the aggregates disintegrated easily under handling. Growth at these sub-saturated water contents was dominated by nucleation of clusters, followed by breakage and either re-nucleation or layering onto pre-existing clusters. Evidence for weakly defined layers of similar particle size is preserved in some of the Liquid-1 aggregates (Figure 4.9a); however, most appear massive and ungraded (Figure 4.9c).

Above the critical threshold of 10-15 wt.\% water, the aggregates began to develop a sheen of liquid on their surfaces as void spaces were filling with water and they were nearing saturation. Once this occurred, the majority of the mixture converted into aggregates within seconds to tens of seconds. Furthermore, due to the lubricating effects of the inter-particle fluid, increasing liquid content $>10-15$ wt.\% was accompanied by an increase in aggregate deformability (softness). Growth was dominated by rapid capture and coalescence of other aggregates, resulting in 'irregular clots' [Schumacher and Schmincke 1995] that merged and rounded during continued agitation (Figure 4.9b). The strength of coalescing aggregates is known to increase over time, as constituent 

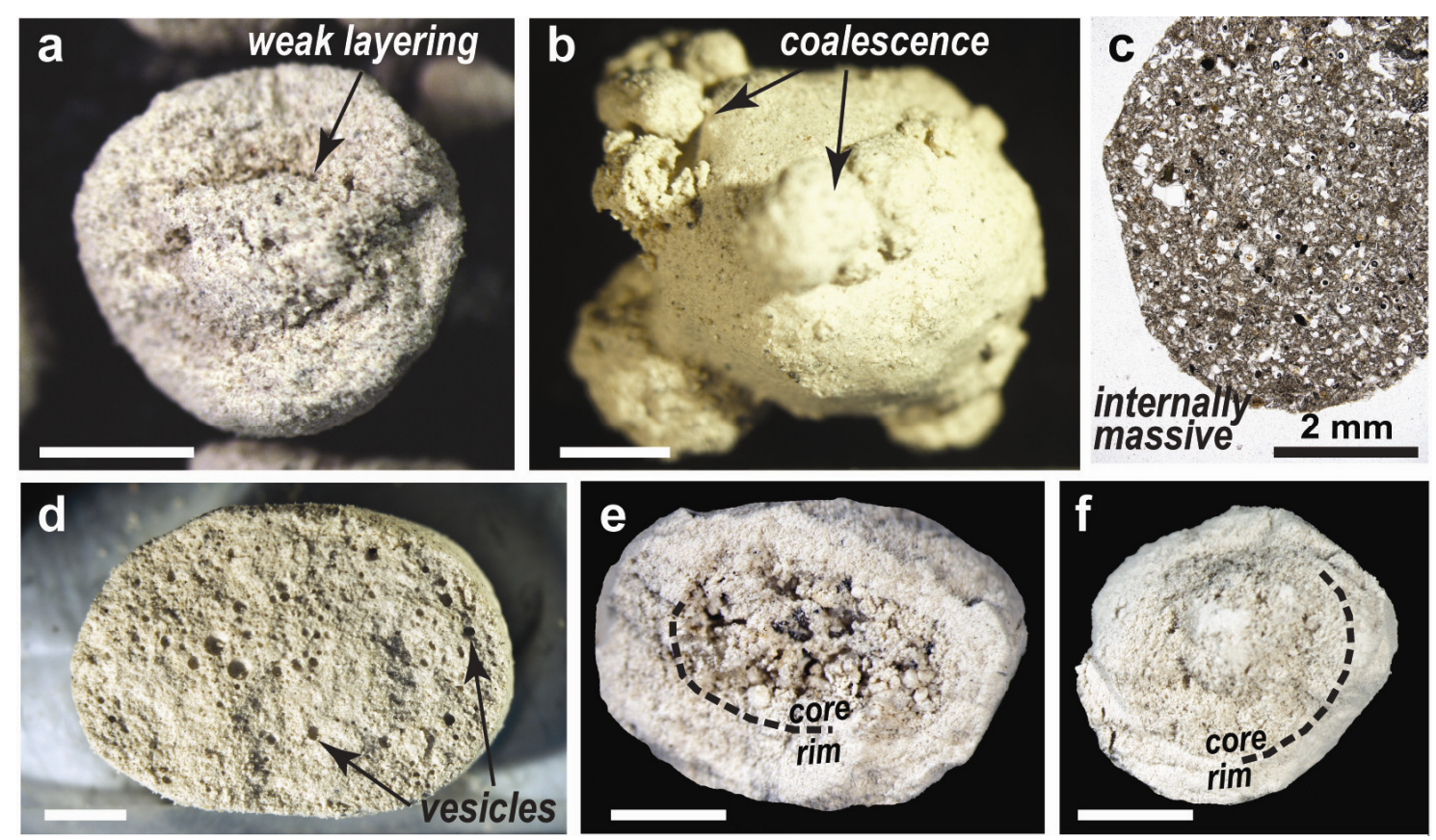

Figure 4.9 Photos illustrating key textural details of aggregates from Liquid-1 (a-d) and Liquid-2 (e-f) experiments. The example in (a) shows weak layering that developed under sub-saturated liquid contents. (b) Is a saturated aggregate ( $>20$ wt. $\%$ liquid) demonstrating capture and coalescence (cf. natural example in Figure 2c). The impregnated thin section in (c) shows the internally massive structure and abundant vesicles typical of saturated aggregates. The freshly broken cross-sectional face in (d) exhibits a weakly concentric zonation of spherical vesicles (cf. Figure 2e). Liquid-2 aggregates have fine grained outer rims (cf. Figure 2a-c) with coarser cores that are massive (e) or weakly layered (f). Scale bar is $2 \mathrm{~mm}$ in all photos.

particles consolidate and decrease pore space during collisions [Iveson et al. 2001]. Aggregates formed in $>10-15$ wt.\% water experiments had noticeably greater strengths, qualitatively observed by an ability to remain intact during handling and after impacts with the ground from $>1 \mathrm{~m}$ height. The occurrence of spherical to subspherical vesicles was also common in these samples (Figure 4.9d), indicating that air was trapped in the saturated (or near-saturated) impermeable ash. With continued addition of liquid, coalescence growth continued until only a few large, aggregates remained, reaching diameters of $c .1 .5 \mathrm{~cm}$ before becoming disc-shaped and unstable, either breaking apart or melding into a slurry at the bottom of the pan. This generally occurred at water contents $>20-25$ wt.\%. Relationships between liquid content and growth behavior are also demonstrated by the sizes of aggregates collected at the end of each experiment (Figure 4.10). The 1-2 mm size fraction dominates at liquid contents $<5 \mathrm{wt} \%$, and aggregates grow exponentially larger with increasing liquid (Figure 4.10a). As the mixture nears saturation $(\sim 10-22$ wt.\% liquid), the smaller clusters are rapidly 
consumed by coalescence growth. The resulting aggregates are primarily massive to very weakly layered with randomly oriented constituent particles (Figure 4.9c). Furthermore, at high water contents (generally $\geq 20$ wt.\%) when the vibration stopped and saturated aggregates came to rest, the constituent particles rearranged under the effects of gravity. Water was squeezed out of pore spaces, transporting the finest ash particles outward and forming a film of liquid on aggregate surfaces. This resulted in a thin $(<100 \mu \mathrm{m})$ outer film of finer ash, which has also been observed in some natural examples of clast-supported aggregates (Figure 4.11) inferred to have emplaced as fallout from a high, wet volcanic plume [Van Eaton and Wilson in review; Chapter 5].
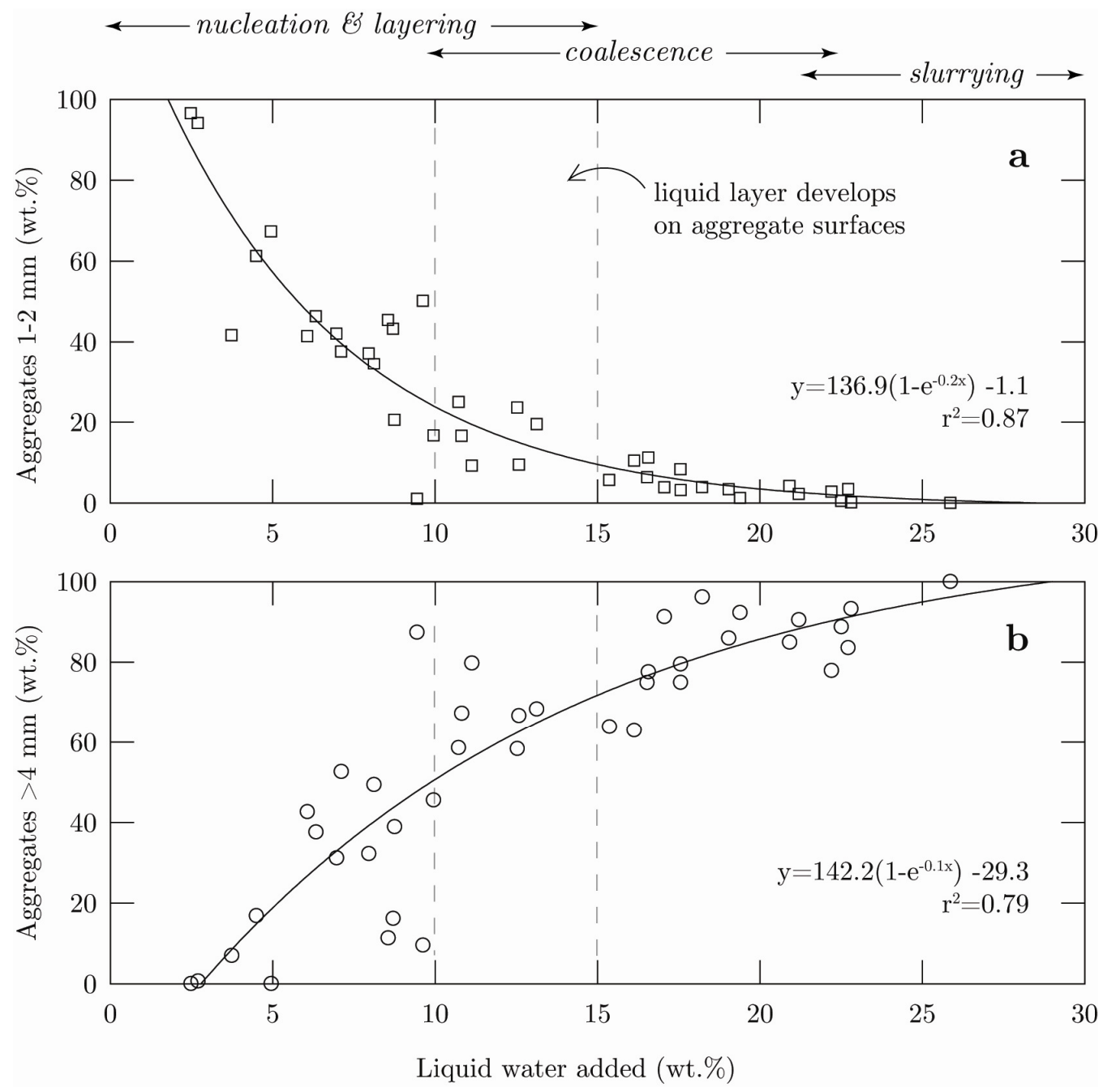

Figure 4.10 Plots the sieve diameters of intact aggregates from Liquid-1 experiments against water content. Liquid water added refers to the average wt.\% water in the system at the end of each experiment. The primary growth mechanism observed at the end of each experiment is indicated at the top of the graph. Least-squares exponential regressions give $p$-values $<0.0001$. 


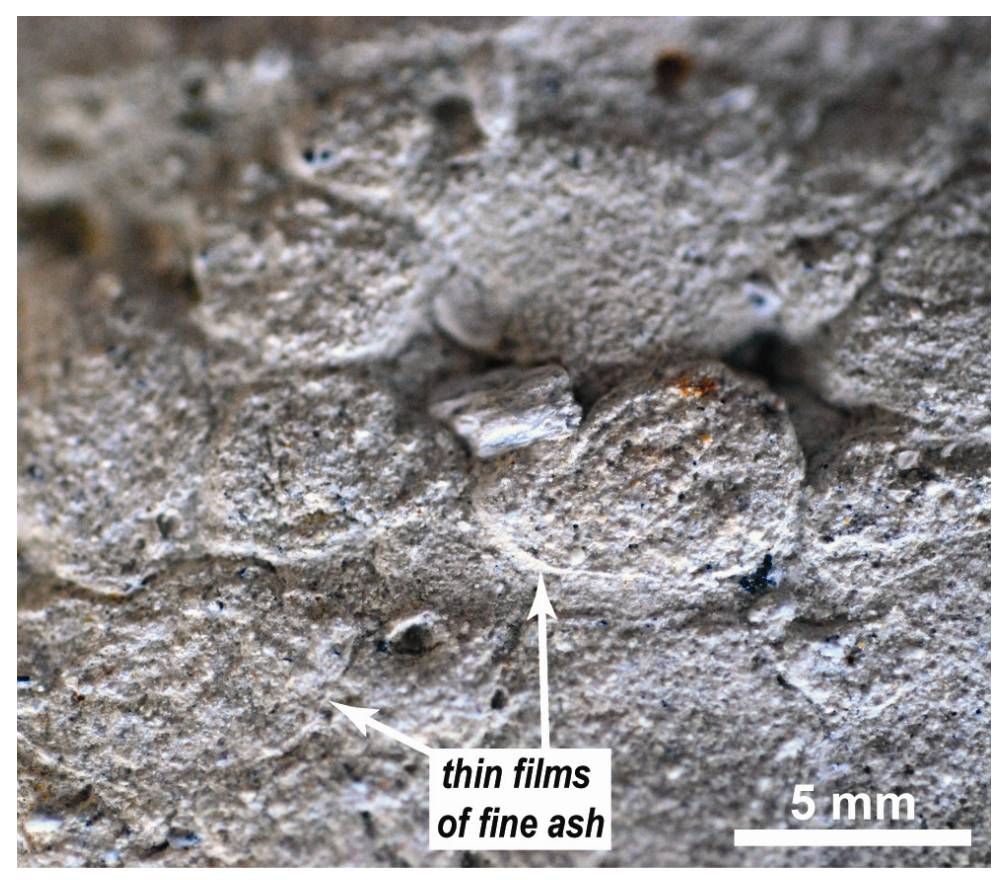

Figure 4.11 Clast-supported aggregates from the Oruanui deposits (locality 1306 of Wilson [2001]). Note the thin $(<100 \mu \mathrm{m})$ outer coatings of fine ash, which closely match textures from the Liquid-1 experimental aggregates involving $>20 \mathrm{wt}$.\% liquid.

\section{Liquid-2: Formation of a fine rim}

Despite evidence of weak layering, textures formed in Liquid-1 aggregates do not match the natural examples of finely laminated, concentrically structured aggregates (e.g. Figure 4.2a-c). An additional set of conditions was therefore examined to test if a concentrically layered rim could be formed through a combination of changes in particle size supply [Gilbert and Lane 1994] and/or liquid water content [Schumacher and Schmincke 1995]. Recently formed Liquid-1 aggregates from experiments with $>15$ wt. $\%$ water were transferred to a separate pan containing $\sim 80 \mathrm{~g}$ of $<31 \mu \mathrm{m}$ ash. The pan was held by hand and tilted gently from side to side, allowing the 'seed' aggregates to roll around in the dry mixture of sub-31 $\mu \mathrm{m}$ ash. No additional water was added. During this process, the moist aggregates accumulated multiple, onion skin-like layers of fine ash with total thicknesses of 1-1.5 mm (Figure 4.9 e-f). The outer layers were somewhat loose and could be easily detached with a fingernail, similar to those observed from the dome collapse elutriation cloud of Soufrière Hills, Montserrat [Bonadonna et al. 2002]. The resultant textures closely match the fine-rimmed aggregates from the Oruanui deposits, with the exception that the Oruanui examples are more tightly packed. Three key factors promoted accumulation of a concentrically layered, fine grained outer rim under these experimental conditions: (1) a supply of very fine ash, (2) moist, pre- 
existing aggregates to 'seed' layering; and (3) restricted availability of liquid water to suppress nucleation of new ash clusters from the surrounding particles.

\subsection{Discussion}

\subsubsection{Comparison of experimental wet aggregates with natural examples}

The particle size characteristics, internal textures, and dry densities of the liquid-1 aggregates are each similar to those of natural aggregate cores, suggesting that aggregation processes described here are a reasonable analogue for the growth of massive to weakly layered aggregates [AP1 types of Brown et al. 2012] in volcanic clouds. Particle size distributions from Liquid-1 aggregates closely overlap with those in cores of natural Oruanui aggregates (Figure 4.12), including showing similar subpopulation modes. Individual dry densities from the five largest aggregates generated in each Liquid-1 experiment are also plotted against equivalent spherical diameter in Figure 4.13. These are overlain with data from: (1) individual aggregates from the Oruanui deposits, (2) loosely-bound particle clusters from the experiments of James et al. [2002, 2003], and (3) aggregates in the May 2010 Eyjafjallajökull plume fallout [Taddeucci et al. 2011]. Experimental aggregates overlap with the lower range of Oruanui examples, and with the higher end of the predominantly electrostatically-bound aggregates from previous work.

These results suggest that aggregate density characteristics move along a continuum from electrostatically-dominated to liquid-dominated conditions. Mean dry densities (Figure 4.14) are shown to increase slightly with water content (linear $r^{2}=0.39 ; p<$ 0.0001), corresponding to the qualitatively observed increase in aggregate strength due to consolidation. However, if a least-squares regression through the data includes the density of electrostatic aggregates from James et al. [2003], a stronger relationship is obtained (exponential $r^{2}=0.68 ; p<0.0001$ ). Although more data is required to test this relationship, preliminary results suggest that at low liquid contents $(<5 \mathrm{wt}$ \% for the parent ash tested here), electrostatic attraction becomes the dominant binding force between particles. In effect, sub-saturated ash aggregates formed in the experiments are likely to be bound both by electrostatic forces and capillary forces between sparse liquid bridges. 


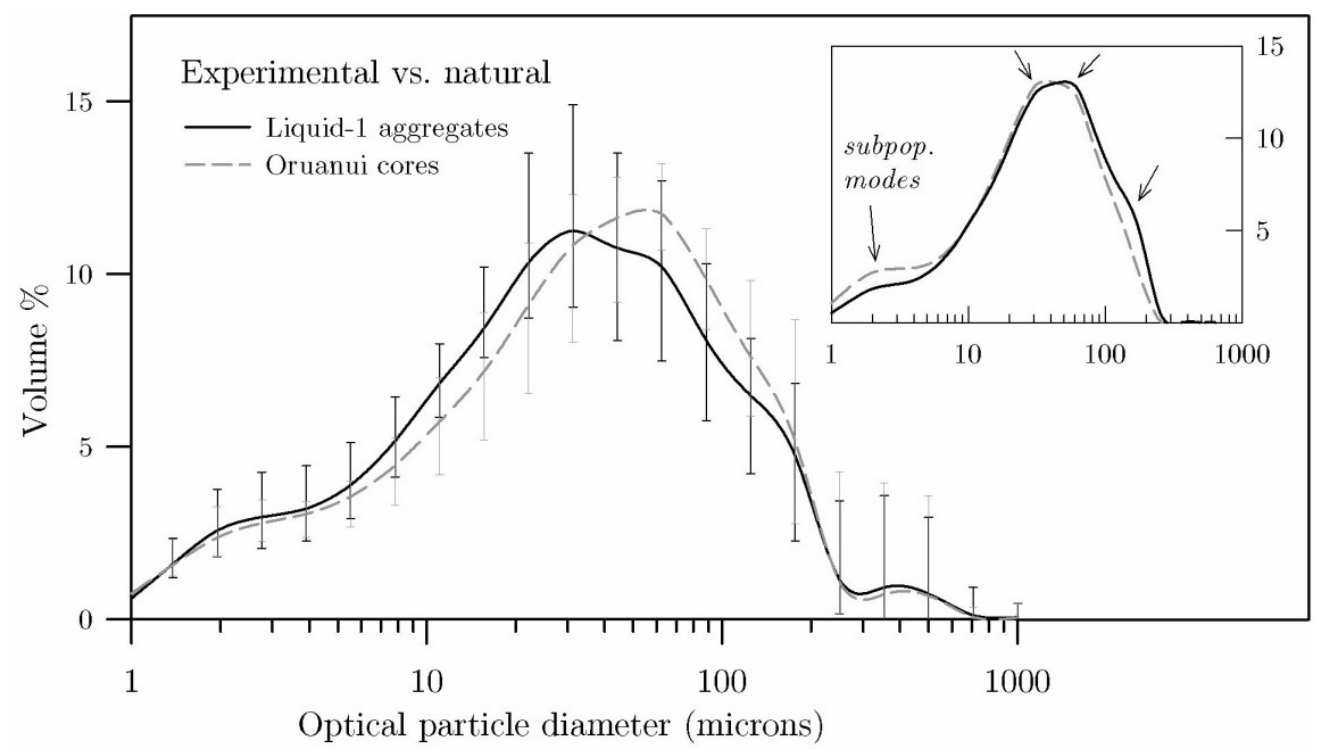

Figure 4.12 Comparison of particle size distributions from fifteen naturally-occurring Oruanui aggregates (cores only), shown as a dashed line, and Liquid-1 aggregates (solid line) from the experiments listed in bold in Appendix E. Curves in the main plot show averaged particle size distributions for the natural and experimental aggregates, with error bars indicating maximum and minimum values for each half- $\varphi$ size class. Inset graph shows representative distributions from both datasets, highlighting their similar subpopulation modes at $c .3,30,17$ and $180 \mu \mathrm{m}$ (indicated by arrows).

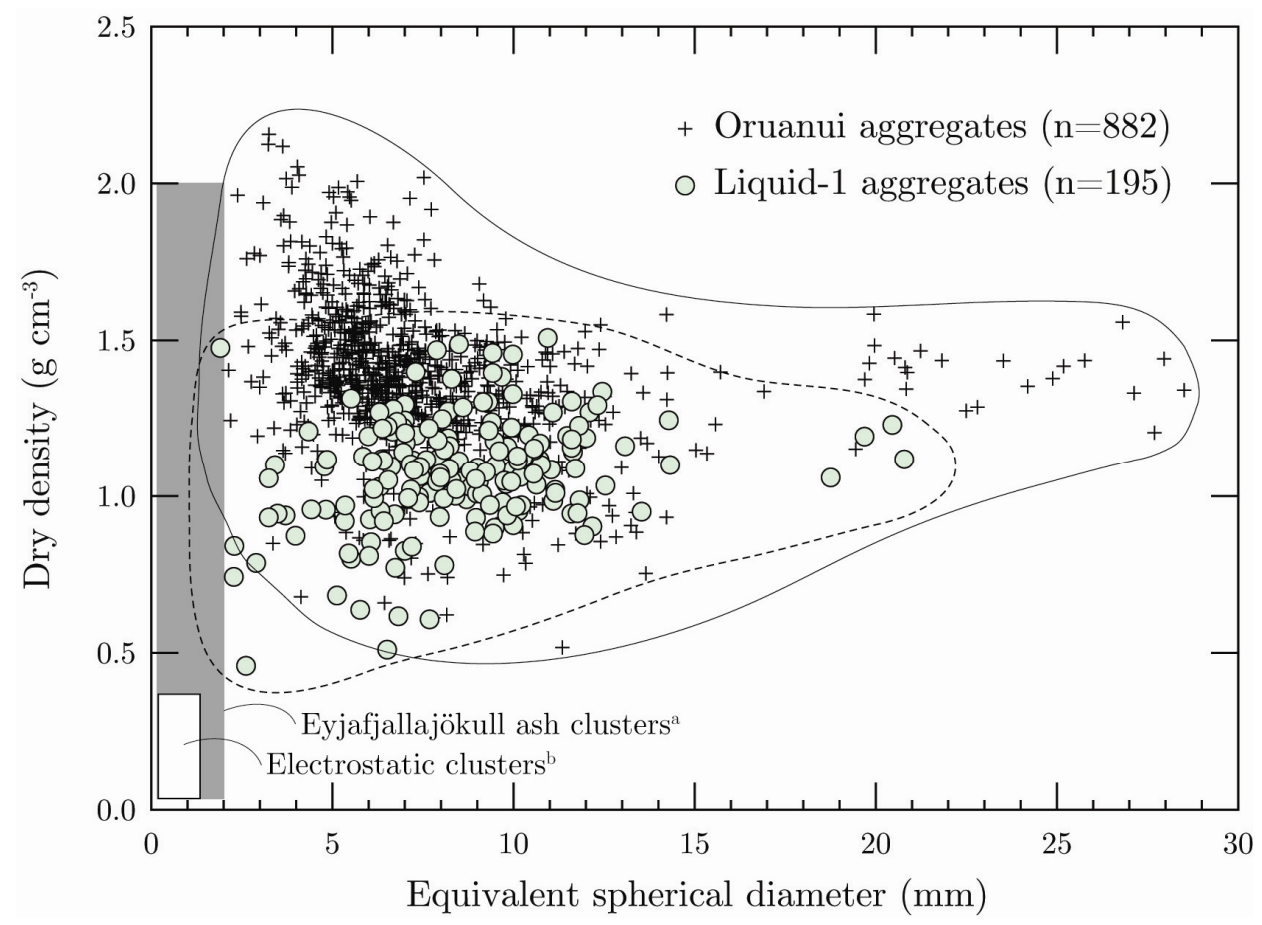

Figure 4.13 Equivalent spherical diameter vs. dry densities of individual aggregates. Circles show measurements from the Liquid-1 experimental aggregates, compared with natural aggregates from Oruanui deposits (crosshairs). ${ }^{a}$ Gives the range of natural ash clusters from the Eyjafjallajökull eruption as reported by Taddeucci et al. [2011]. ${ }^{b}$ Gives the range of experimentally-generated electrostatic clusters from James et al. [2003, their Figure 4a]. 


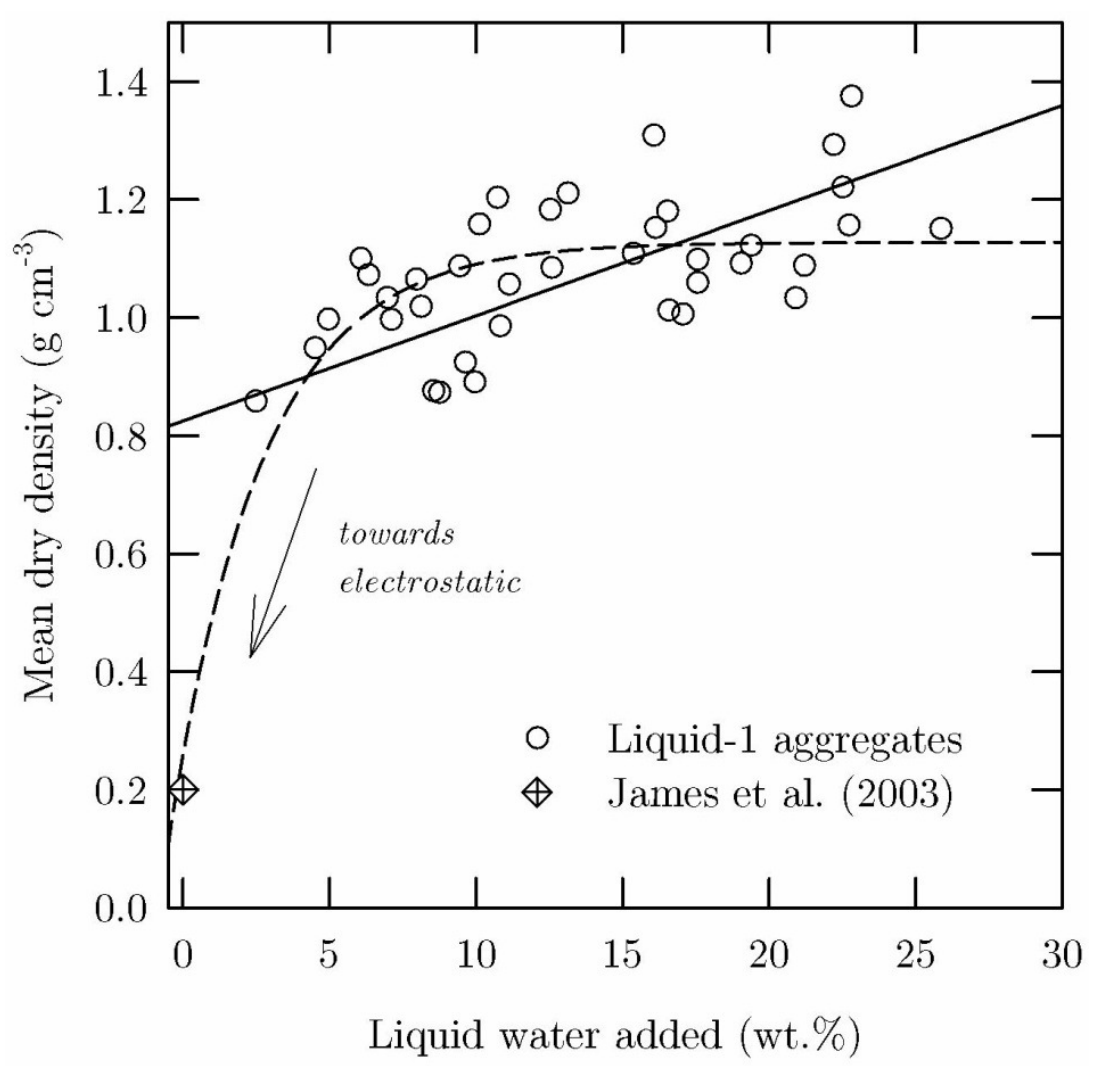

Figure 4.14 Plots of liquid water vs. mean dry density for the Liquid-1 aggregates. Data represent the mean of the five largest aggregates from each experiment. The solid line is a least-squares linear regression through the Liquid-1 data only, given by $y=0.02 \mathrm{x}$ +0.83. Dashed line is an exponential regression including the mean density of electrostatic clusters from James et al. [2003: their Figure 4a], given by $\mathrm{y}=0.26$ $+0.87\left(1-\mathrm{e}^{-0.32 \mathrm{x}}\right)$.

\subsubsection{Analysis of preferential scavenging}

To establish patterns of preferential scavenging with respect to liquid water content, this investigation focuses on the liquid-free (Hail-1) and wet (Liquid-1) end-member experiments where there are clearly defined amounts of liquid water involvement. The experiments that resulted in preferential scavenging were identified, as described previously (Figure 4.5). Two factors contributing to an experiment failing to demonstrate preferential scavenging within analytical uncertainty include: (1) exceedingly fine parent ash, and (2) high water content, particularly to the point of oversaturation or slurrying. Furthermore, due to the dependence of saturation state on particle size as well as water content, only experiments using parent ash with a dominant mode between 30-70 $\mu \mathrm{m}$ were used for the following discussion (Refer to Appendix F for details on which experiments meet these criteria). 


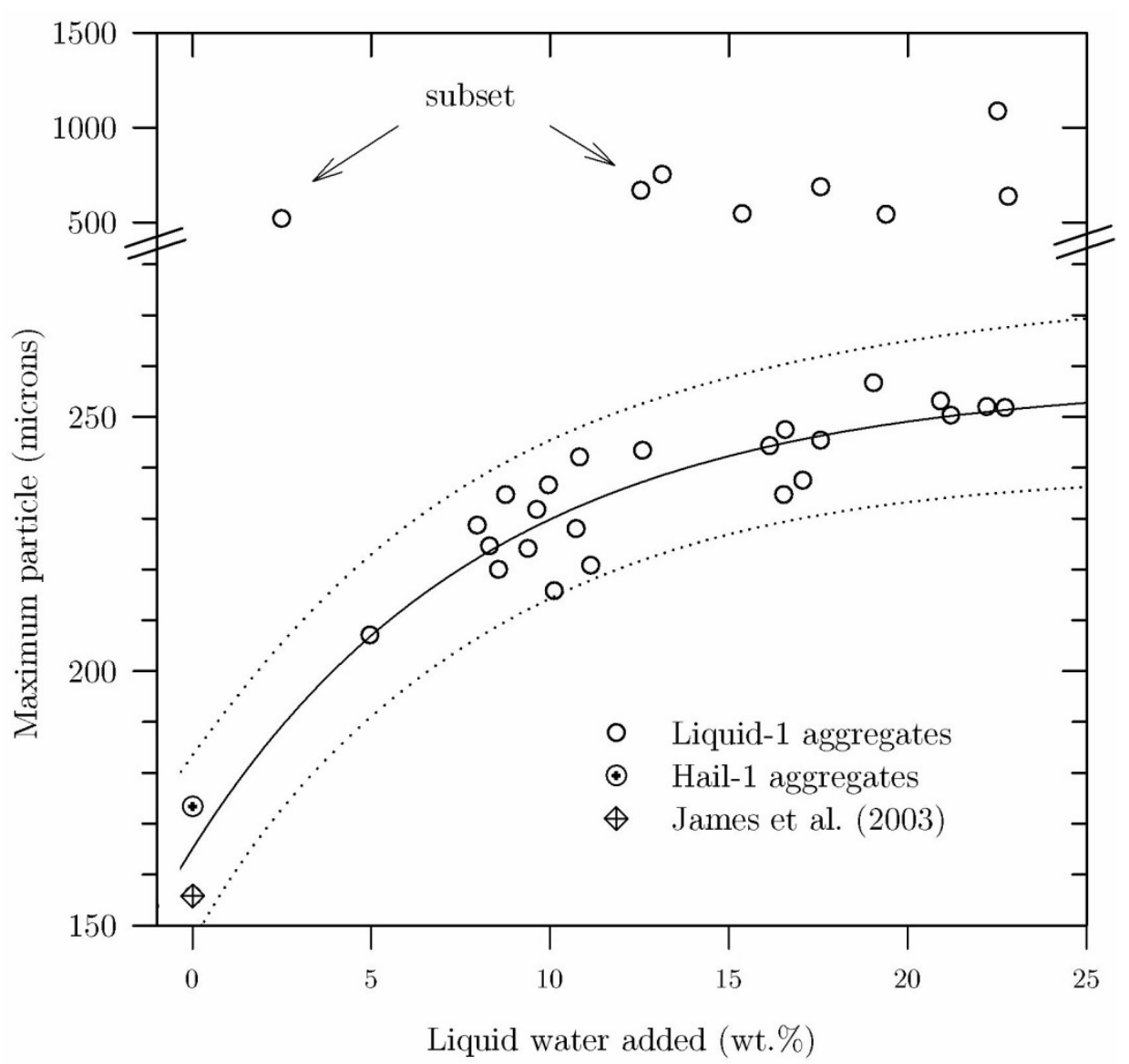

Figure 4.15 Plot of liquid water added vs. maximum particle size incorporated into Liquid-1 and Hail-1 aggregates (represented by the coarsest $99^{\text {th }}$ percentile). Mean data from the electrostatic clusters $>600 \mu \mathrm{m}$ from James et al. [2003, their Figure 3c] are also shown. Most of the data follow an exponential relationship given by $y=68.2$ $+8.06 \mathrm{x}-0.20 \mathrm{x}^{2}$ (solid line), indicating that greater availability of liquid water results in the collection of larger particles. Dotted line gives the $95 \%$ prediction band. Data falling into the subset category may represent localized pockets of oversaturated ash in the apparatus. Note break in scale above $280 \mu \mathrm{m}$.

\section{Maximum particle size}

Figure 4.15 shows the 'maximum' particle size $\left(99^{\text {th }}\right.$ percentile) incorporated into the 4$8 \mathrm{~mm}$ aggregates at different water contents. Including the maximum particle incorporated into the electrostatic aggregates of James et al. [2003], these data plot along an exponential trend $\left(\mathrm{n}=25 ; r^{2}=0.90 ; p<0.0001\right)$, indicating that increased availability of liquid results in an exponential increase in the largest particle size that can be scavenged into aggregates. However, eight experiments diverge from this trend, giving maximum particles that are 2-10 times greater than expected and do not covary with water content (see subset in Figure 4.15). A plausible explanation for this variability is that uneven dispersion of liquid in the apparatus (due to heterogeneous 
drop sizes in the spray, or energy variations in the pan) resulted in localized pockets of oversaturated ash. It is also possible that during coalescence growth (at water contents $>10-15$ wt.\%), some larger particles became trapped and incorporated between colliding wet, deformable aggregates - however, this is likely to be of second order importance. Due to this observed variability of maximum particle size, it is suggested that the preferential scavenging relationships presented here are only applicable to systems in which liquid water is evenly dispersed (on mm-cm scales).

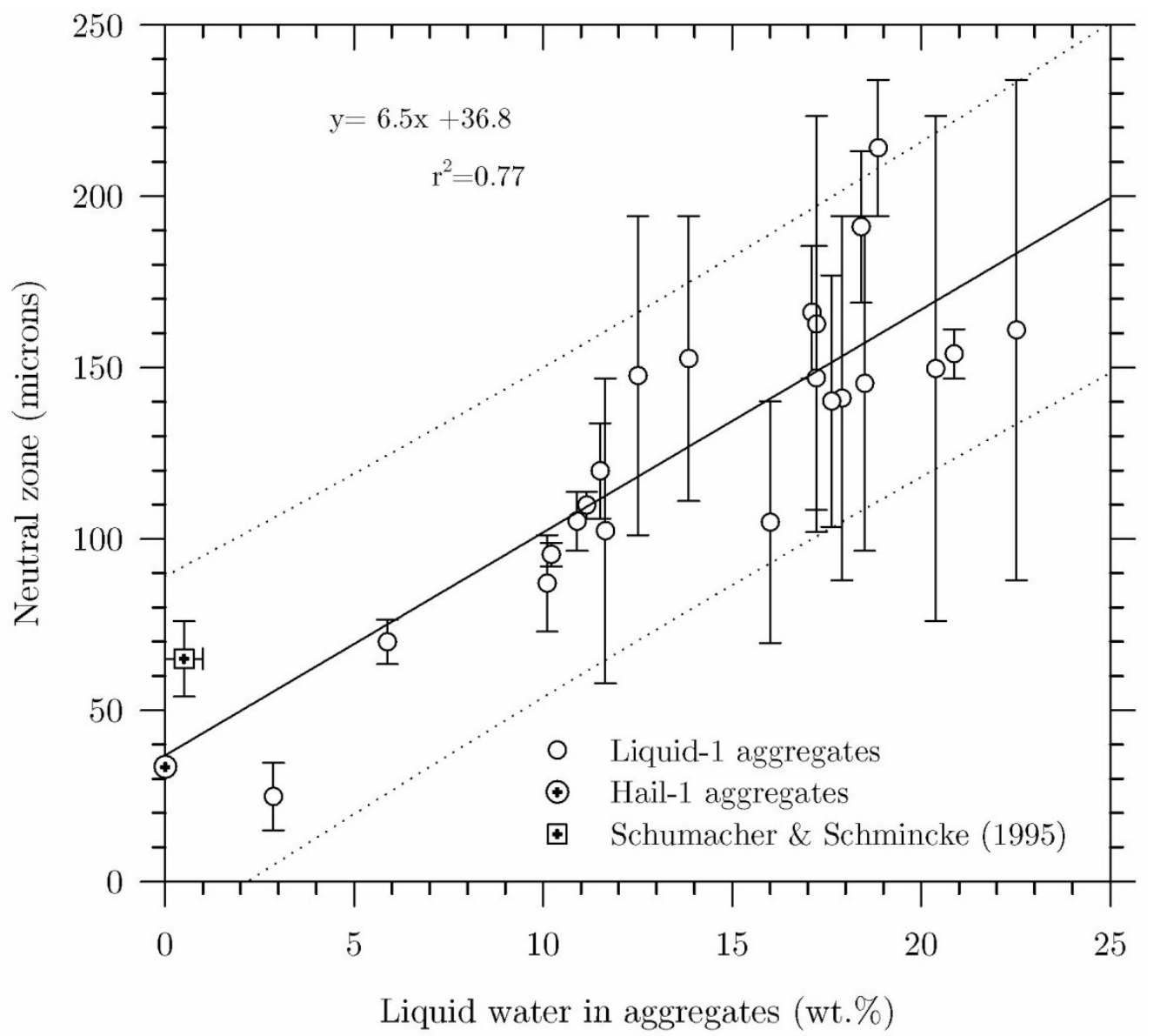

Figure 4.16 Plot of liquid water in aggregates* vs. neutral zone (in microns) from Liquid-1 and Hail-1 experiments. Error bars represent the maximum and minimum intersection points of the parent and aggregate particle size distributions, as in Figure 5. Data derived from the electrostatic aggregates of Schumacher and Schmincke [1995: their Table 3] are also shown. Linear regression gives a $p$-value $<0.0001$. Dotted line gives the $95 \%$ prediction band. *Liquid water in aggregates refers to the wt. $\%$ measured in the 4-8 $\mathrm{mm}$ aggregates from each experiment.

\section{Neutral zone}

The neutral zone, or region of overlap between parent and aggregate particle size distributions (Figure 4.5) marks the transition from preferentially scavenged to excluded 
particle sizes. The mean neutral zone increases with increasing water content (Figure 4.16), including data from the electrostatic aggregates of Schumacher and Schmincke [1995] (linear $r^{2}=0.77 ; p<0.0001$ ). The range of neutral zone values (Figure 4.16) becomes greater in wetter conditions as the particle size curves overlap and eventually merge at higher water contents. However, the relationship shows that even at $>15 \mathrm{wt} . \%$ liquid, particles $>200 \mu \mathrm{m}$ are rarely preferentially incorporated with respect to the parent. This provides a testable upper limit on the ability of aggregates to collect coarser particles under limited liquid water conditions. Furthermore, the similarity of the liquidfree Hail-1 aggregates to those from the electrostatic experiments of James et al. [2002, 2003] and Schumacher and Schmincke [1995] in terms of maximum particle size and neutral zone, suggests that ice and electrostatic forces have comparable scavenging effects. This relationship is discussed further below.

\section{Aggregation coefficients}

Gilbert and Lane [1994] introduced an experimentally derived aggregation coefficient for volcanic ash [Veitch and Woods 2001, Costa et al. 2010], which estimates the probability that a particle of a certain size will be incorporated into aggregates (i.e. maximum value is unity). Their normalized aggregation coefficient for each particle size class was obtained using the equation: $\left(N_{\text {agg }} / N_{\text {parent }}\right) / R_{\max }$, where $N_{\text {agg }}$ is the absolute number of particles in the aggregate, $N_{\text {parent }}$ is the number of particles in the parent ash and $R_{\max }$ is the maximum ratio of $N_{\text {agg }}$ to $N_{\text {parent }}$ determined for all particle sizes in the experiment. Here, the aggregation coefficient is used as a means of transforming the particle size distribution of the parent ash into aggregates. We calculate a raw, un-nomalized aggregation coefficient for each half-phi particle size using $V_{\text {agg }} / V_{\text {parent }}$, where $V_{\text {agg }}$ is the relative vol.\% in the aggregates from LDPSA data and $V_{\text {parent }}$ is the vol.\% in the parent ash. A value of unity for a given particle size indicates that the particle is neither preferentially scavenged nor excluded (i.e. neutral zone of Figure 4.5). Values greater than or less than unity indicate preferential scavenging and exclusion, respectively. As the amount of liquid water (or ice) involved in the system is clearly an important factor, this study determines aggregation coefficients specific to experiments involving different amounts of added water (Figure 4.17). 


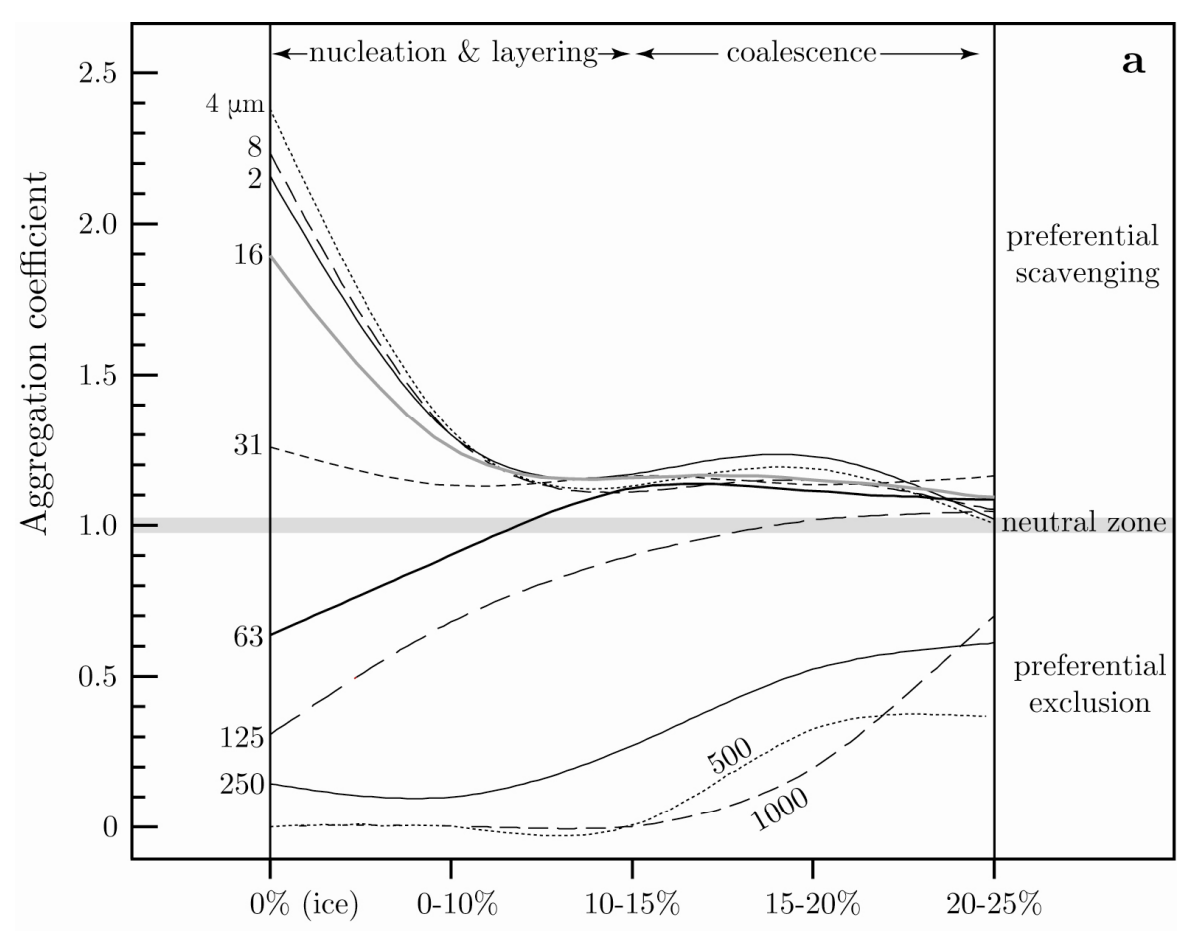

Liquid water in aggregates (wt.\%)

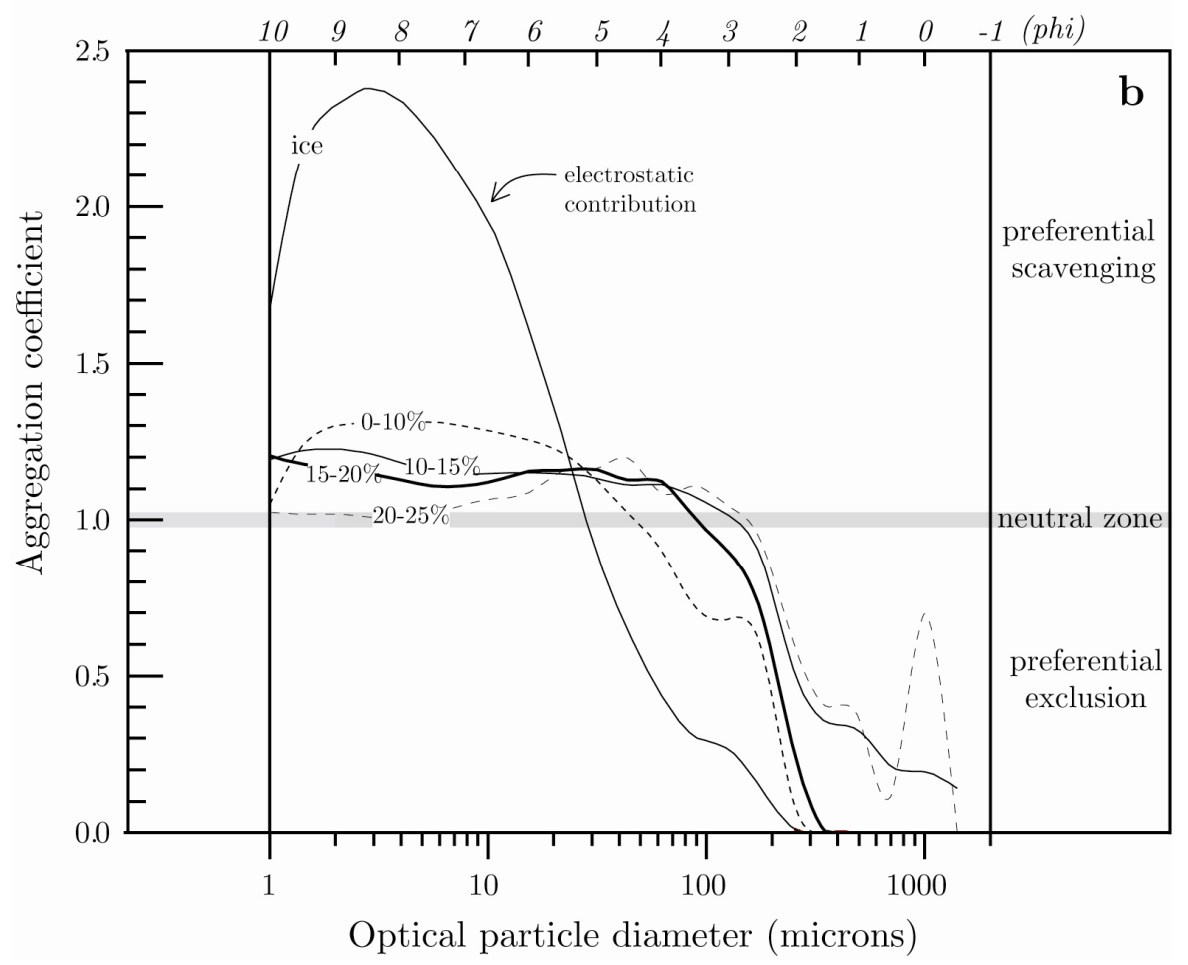

Figure 4.17 Aggregation coefficients at varying liquid water contents*, determined from Liquid-1 and Hail-1 experiments (see Appendix G for details). Data are fit with spline curves. (a) Shows liquid water in aggregates vs. aggregation coefficient for each whole- $\varphi$ particle size class. Dominant growth mechanisms are provided at the top of the graph. (b) Shows particle diameter vs. aggregation coefficient, grouped by liquid water content. Corresponding $\varphi$ classes are shown along the upper axis. Note in (b) that the curves intersect at roughly $31 \mu \mathrm{m}$, suggesting this grain size is scavenged equally over the range of liquid contents shown. *Liquid water in aggregates derived as in Figure 5.16 . 
Experiments used in these calculations are listed in bold in Appendices E-F and aggregation coefficient values are in Appendix G. Figure 4.17a illustrates how the scavenging of each particle size class changes with water content, while Figure $4.17 \mathrm{~b}$ represents the scavenging of different particle sizes within fixed ranges of water content. These trends reflect a progressive evolution of the particle sizes incorporated into aggregates under different conditions. At low liquid contents $(<10$ wt.\%), during which nucleation and layering mechanisms dominate, fine particles are strongly preferred, and coarser particles are excluded. At near-saturated liquid contents $>10-15$ wt.\% aggregation coefficients of all size classes approach unity, indicating a diminishingly size selective process. However, these observations are consistent with the neutral zone data from Figure 4.16 in suggesting that particles $>125-250 \mu \mathrm{m}$ are preferentially excluded at all liquid contents below 20-25 wt.\%. Indeed, above this point, the curves merge and no preferential scavenging occurs; particles are collected indiscriminately by slurried droplets. Note that the curves in Figure $4.17 \mathrm{~b}$ appear to intersect at $c .31 \mu \mathrm{m}$. This suggests that particles around $31 \mu \mathrm{m}$ are scavenged relatively equally, whether under ice- or liquid-dominated conditions. It is suggested here that this reflects an interplay between electrostatic attraction and the forces arising from films of liquid water and/or ice. It may be that electrostatic forces are only effective at attracting and adhering particles $<63 \mu \mathrm{m}$ and keeping them in contact long enough to become adhered by liquid or ice bridges.

\subsubsection{Insights into aggregation mechanisms}

\section{Ice-initiated aggregation below freezing}

Relatively liquid-poor, ice rich conditions may occur in volcanic clouds by freezing of liquid-coated particles and ash-laden liquid drops, or by deposition of ice directly onto ash particles from the vapor phase [Shaw et al. 2005, Durant et al. 2008]. The Hail-1 experiments here provide insights into the growth of ash aggregates in such situations. It was observed that solid ice at $-20^{\circ} \mathrm{C}$ could preferentially collect $<63 \mu \mathrm{m}$ ash from a wider particle size population. The preferential scavenging characteristics of these icebound aggregates are comparable to the electrostatic aggregates of Schumacher and Schmincke [1995] and James et al. [2003] (Figures 4.15-16). It is therefore suggested that ash particles in the Hail-1 experiments were initially attracted and adhered to the ice by electrostatic forces generated by particle collisions. This mechanism is inferred to be 
similar to the formation of 'single grain clusters' from Schumacher [1994]. Particles that were electrostatically adhered to the ice over tens of seconds to minutes subsequently generated more permanent bonds by a process known as ice sintering [Hobbs 1974]. Sintering occurs at sub-freezing temperatures due to a gradient in chemical potential energy arising from different radii of curvature at the contact points between ice and solid particles [Blackford 2007]. In order to minimize surface-free energy, ice diffuses to the contact site, primarily via vapor phase diffusion, and redeposits to form an ice neck [Hobbs and Mason 1964]. At $-20^{\circ} \mathrm{C}$, this process has been shown to form necks of appreciable radius $(\sim 1 \mu \mathrm{m})$ between ice spheres on timescales as short as tens of seconds [Hobbs 1965]. Involvement of impurities such as hygroscopic compounds would accelerate sintering by promoting development of thin surface layers of liquid on the ice, even at sub-freezing temperatures [Blackford 2007]. This two-step mechanism of electrostatic attraction followed by sintering explains why ash particles in the ash-ice aggregates closely match the size characteristics of electrostatic aggregates from previous work. It also explains why portions of the adhered ash were 'rinsed' away during subsequent addition of a liquid spray in the Hail-2 experiments; the liquid would have conducted and dissipated the electrostatic charge in addition to melting any sintered contacts. However, because the electric field in the experimental setup was not measured, it is unclear how changes in the magnitude of electrostatic charging would affect the grain size of the ice-ash aggregates. Furthermore, due to the temperaturedependent sticking properties of ice [Lin et al. 1983], aggregation coefficients obtained from the experiments only strictly apply to temperatures in the range of $-20^{\circ} \mathrm{C}$.

An important implication of aggregation under these conditions is that the ability to bind particles to the ice is limited by the diffusion timescale of ice sintering. Particle collisions alone, which last only milliseconds in the absence of electrostatic or capillary forces, are insufficient to keep ice-coated particles in contact long enough to sinter. Observations of ash-poor volcanic hailstones from the Eyjafjallajökull deposits (Figure 4.2 f-g) and the 1963-64 Surtsey eruption [Thorarinsson 1967] support the idea that solid ice particles at sub-freezing temperatures are unlikely to accumulate substantial ash layers (tens of microns or more) on their outer surfaces. In the case of these natural volcanic hail, the presence of ash at their centers suggests that ice nucleated on the ash particles, but adhered very little ash thereafter. Note, however, that microphysical simulations of volcanic eruptions [Textor et al. 2006b] suggest that even small degrees 
of ice-ash interaction under liquid-poor conditions could yet have measurable impacts on plume height and transport.

\section{Ash aggregation under mixed phase conditions}

In contrast to the liquid-free scenarios, ice would provide an effective vehicle for wet aggregation once melting occurs. The melting ice pellet experiments (Mix-2) displayed limited interaction between ice and ash during initial stages of melting, but rapid collection of ash once ice pellets became saturated with liquid. This is consistent with observations of mm-scale thicknesses of ash enveloping inferred ice pellet cores from the Oruanui deposits (Figure 4.2b) and Okinawa deposits [Kato 1986]. Durant et al. [2009] similarly implicated the role of melting ice (in a process analogous to snowflake formation) to generate the loosely bound aggregates in the Mount St Helens 1980 eruption plumes. Despite the influence of ice in mixed phase scenarios, the results imply that the preferential scavenging characteristics of resulting aggregates will be controlled by the liquid phase and its dominant binding forces. As mixed-phase aggregates grow, they should therefore scavenge particles according to the relationships obtained under pure liquid water conditions (Figs. 4.15-17). Collection of ash-laden ice onto preexisting aggregates would also provide a growth mechanism, and this is expected to leave behind a porous structure in the aggregate once the water is driven off.

\section{Growth of liquid-bound aggregates: nucleation, layering and coalescence}

Under liquid-dominated conditions, the growth of aggregates was strongly dependent on the water content. At $<10-15$ wt.\% water, nucleation and layering mechanisms dominated, while above this growth was primarily by coalescence. This critical threshold is consistent with the minimum of 14-15 wt.\% water to form well developed aggregates reported by Schumacher and Schmincke [1995]. A steady increase in aggregate sizes during coalescence (Figure 4.10) reflects the high deformability of wet ash aggregates, which inhibits rebound [Iveson and Litster 1998]. Accompanying these shifts in growth behavior, the particle sizes incorporated into aggregates also depend on liquid availability. Assuming even dispersion of the liquid phase, increased water contents result in less and less preferential selection of particles (Figure 4.17), eventually yielding an oversaturated slurry that indiscriminately collects all particles $<1.4 \mathrm{~mm}$. However, at sub-saturated liquid contents, the interplay between sparse liquid 
bridges and electrostatic forces become more important, and it is inferred that ash aggregates are predominately electrostatically bound at water contents $<5 \mathrm{wt}$.\%.

\subsubsection{Origin of aggregate textures}

\section{Massive versus concentric structure}

The particle size distributions (Figure 4.12) and densities (Figure 4.13) of the liquidbound experimental aggregates are very similar to the internally massive examples from the Oruanui deposits. It is therefore suggested that massive aggregates likely form in natural volcanic clouds predominantly by nucleation and coalescence in the presence of liquid water. Aggregates with randomly oriented particles are unlikely to have undergone extensive periods in the layering regime, suggesting that liquid water contents were high enough for the ash to be nearly saturated and promote coalescence. It is also possible that growth of massive aggregates above the freezing level is supplemented by collection of ice-coated particles by ice sintering [Supulver et al. 1997, Sirono 1999]. However, it is unlikely that aggregates capable of preservation in the geologic record are generated in the absence of liquid water, due to the slow sintering rates with respect to timescales of particle collisions. The implication is that conditions for formation of internally massive or weakly layered aggregates that can survive impact with the ground are only readily met where liquid water is present in ash clouds.

The formation of concentrically layered, finer grained rims occurred under distinct laboratory conditions, when moist, Liquid-1 aggregates were added to a mixture of dry fines. It is inferred here that liquid contents were low at the contact points between the pre-existing aggregates and surrounding finer particles, leading to the formation of sparse liquid bridges. Therefore, it was likely by a combination of capillary forces and electrostatic attraction that the ash particles were adhered. Note that this layering process, although size selective (see 0-10 wt.\% regions of Figure 4.17 a-b), was unable to collect purely fine ash (i.e. predominantly $<31 \mu \mathrm{m}$ ) from a wider population. This is important because it suggests that the finest outer layers of natural aggregates could not have been culled from a poorly sorted mixture; instead they require a source of dominantly fine ash. The potential for the fine rims to form by electrostatic forces followed by ice sintering (i.e. at high levels of a volcanic plume) is unlikely because the presence of ice would be expected to leave behind a porous texture, or evidence of 
slurrying after melting, whereas naturally formed examples lack vesicles in the finely laminated outer rims.

It is inferred that the rims could not be formed due to electrostatic attraction alone, without the presence of liquid water, because of the lack of a binding mechanism after the electric charge had dissipated. It is further suggested that the combination of longrange electrostatic attraction and short range capillary forces from liquid bridges are both crucial to the layering of ultrafine rims. Therefore, the observed, consistent structure of natural concentrically structured aggregates (i.e. loosely-packed, massive and often vesicular core, followed by a fine grained outer rim) is consistent with transport of saturated, liquid-bound aggregates through a relatively dry, dilute and dominantly fine grained region of a volcanic cloud.

\section{Internal voids}

Two kinds of internal voids occur in natural aggregates. Near-spherical, internally smooth vesicles (e.g. Figure 4.2e) are inferred to represent air bubbles that were trapped in an impermeable ash matrix, implying that the aggregate was liquid saturated at some stage. In contrast, the occurrence of a sub-mm to mm-scale, irregularly shaped void with whisker-like protrusions (and generally at the center of the aggregate) is likely to represent an ice particle or ice pellet nucleus that underwent melting. Comparison of the boiled versus sublimed textures from the Mix-2 experiments suggest that ice-cored aggregates, such as those inferred from the Oruanui and Okinawa deposits [Kato 1986], may lose some or all of their ice by sublimation to vapor, which then diffuses out of the aggregate during fallout from the volcanic cloud. Sublimation processes in volcanic ash clouds have been inferred from observations of mammatus clouds in historic eruptions [Durant et al. 2009], and reproduced numerically in cloud-resolving simulations of explosive eruption columns [Textor et al. 2006b, Van Eaton et al. 2012a, Chapter 2]. Alternative explanations, such as slow melting of the ice and draining out of the aggregate after landing cannot necessarily be ruled out. However, the process of ice removal requires that the delicate protrusions of ash in the vacuole be preserved, which is not likely to occur during rapid melting. 


\subsubsection{Secondary binding mechanisms}

When natural wet or icy aggregates lose their water, some form of solid bridges must be left behind for them to preserve in the geologic record. Furthermore, these bridges must be able to form on timescales that allow the aggregates to remain intact on landing. Based on the occurrence of gypsum crystals in some accretionary lapilli from Sakurajima [Tomita et al. 1985, Gilbert and Lane 1994] it was thought that hygroscopic species in volcanic clouds may provide cohesion via salts precipitated during evaporation [Sparks et al. 1997, Scolamacchia et al. 2005]. However, there are many observations of fresh ash aggregates that lack visible traces of salts or other solid precipitates [Bonadonna et al. 2002, 2011b]. In the experiments, the ease with which wet aggregates could be generated with distilled water provides a compelling case that hygroscopic species (e.g. sulphates) are not universally required in abundance to keep aggregates together. This would help explain the common occurrence of mm-cm scale aggregates observed in phreatomagmatic eruption deposits worldwide, regardless of the chemistry of the eruptive mixture or the source of external water (i.e. seawater vs. freshwater). Therefore, it is suggested here that rapid alteration of reactive volcanic glass or clays in the presence of liquid water may be capable of leaving behind amorphous silica coatings that bind close-range particles in place after drying (Figure $4.8 \mathrm{~d}-\mathrm{f})$.

\subsection{Suggestions for future work}

The results of this study suggest that analogue experiments can illuminate aspects of the ash aggregation mechanisms in volcanic clouds. Further work is, however, needed to strengthen the links between experimental relationships and natural processes, including the following.

- There is a need to quantify relationships between atmospheric conditions (e.g. pressure), volcanic gas chemistry and the resulting ratios of ash and water in volcanic clouds. This may be explored, for example, by using a humiditycontrolled vessel and investigating the effects of relative humidity, pressure, temperature and the presence of various hygroscopic species on the amounts of liquid water and/or ice that develop on ash surfaces. 
- Experiments using different particle size distributions, such as those characteristic of basaltic phreatomagmatic eruptions, would be useful to establish the liquid contents required to reach different stages of wet growth. Use of consistent grain size distributions using ballotini, for example, may also help simplify the experimental parameters.

- It would be valuable to quantify ice-dominated aggregation processes at temperatures from -20 to $-40{ }^{\circ} \mathrm{C}$, covering a range of atmospheric temperatures encountered by volcanic clouds. Does the temperature-dependent efficiency of ice sintering result in different preferential scavenging characteristics?

- The change in dynamic yield strengths of experimental aggregates [e.g. Pietsch et al. 1969, Iveson and Litster 1998, Verkoejin et al. 2002] with respect to water content and collisional energies may help identify natural scenarios capable of generating 'strong' aggregates that would survive environmental stress such as impacts with the ground or settling through bodies of water.

- The observation that aggregates were unstable at vibrations greater than $\sim 50 \mathrm{~Hz}$ at 2-3 mm amplitude suggests there is an upper limit of collisional energy for formation of aggregates. Conditions related to this upper limit could be quantified to constrain the likelihood of forming intact aggregates in regions of a volcanic eruption (i.e. ground-hugging density currents vs umbrella clouds).

- Alternative mechanisms could be explored to account for the preservation of aggregate structures after drying. Does alteration of volcanic glass (and/or adhering clays) in presence of water or hygroscopic compounds occur rapidly enough to generate solid bridges before the aggregates hit the ground?

\subsection{Conclusions}

Using vibratory pan aggregation, the growth patterns and preferential scavenging mechanisms of volcanic ash aggregation under a range of temperatures and water influences has been experimentally investigated. The approach is unique in two ways: (1) it includes the first trials of ice aggregation below freezing and at room temperature, and (2) it quantifies the influence of water content (liquid and ice) on aggregation coefficients. The patterns described here provide a general template for the formation of 
aggregates, and a novel approach to quantifying the effects of particle scavenging. Key findings include:

1. Aggregate characteristics such as bulk diameter, density, and constituent particle sizes fall along a more or less continuous spectrum, implying that the attractive forces governing their formation also operate along a continuum. At low liquid contents $(<5 \mathrm{wt} . \%$ for the experiments presented here), electrostatic attraction dominates. However, if liquid bridges develop a more continuous network through the aggregate (>5 wt.\% liquid), capillary forces dominate.

2. For conditions in which volcanic ash is not oversaturated with respect to liquid, size-selective aggregation depends on liquid content. At $<10 \mathrm{wt}$ \% liquid, only particles $<63 \mu \mathrm{m}$ are preferentially scavenged into aggregates from a wider population. At higher water contents ( $>20 \mathrm{wt} . \%$ ) particles up to $200 \mu \mathrm{m}$ may be preferentially incorporated. However, once slurrying occurs ( $>20-25 \%)$, there is no preferential scavenging and particles up to $1.4 \mathrm{~mm}$ (the maximum size in the experiments) could be incorporated into aggregates.

3. Aggregation of ash particles to crystalline ice at $-20{ }^{\circ} \mathrm{C}$ is highly size-selective, preferentially collecting $\leq 31 \mu \mathrm{m}$ particles from a wider size population. The mechanism is explained by a two-step process of electrostatic attraction, followed by ice sintering. However, the sintering process is limited by the timescale of diffusion, requiring ice particles to remain in continuous contact with ice for tens of seconds to minutes.

4. Melting ice is shown to be capable of accumulating mm-scale thicknesses of fine ash, forming aggregates that preserve an irregularly shaped central vacuole after slow drying.

5. Liquid-dominated aggregation follows a growth pattern of nucleation and layering to coalescence and slurrying, linked directly to the liquid saturation state of the volcanic ash. Liquid contents $<10-15 \mathrm{wt} \% \%$ resulted in nucleation (formation of new aggregates) or layering onto pre-existing aggregates. Coalescence dominated at greater liquid contents until oversaturation occurred (>20-25 wt.\% water), resulting in slurrying and loss of aggregate structure.

6. Timescales of wet aggregation are dependent on particle concentration and liquid water availability. At the particle concentrations tested here $\left(1-10 \mathrm{~kg} \mathrm{~m}^{-3}\right)$, 
saturated or near-saturated ash was capable of forming aggregates within a matter of seconds.

7. The particle size distribution of the parent ash influences the amount of liquid water required to reach different amounts of saturation, and a poorly sorted size distribution is required to generate stable aggregates.

8. The ability of experimental aggregates to maintain their structure after drying, without addition of hygroscopic species suggests that formation of secondary salts may not be as important to the formation of solid bridges as previously thought. Other mechanisms, such as rapid alteration of volcanic glass, may provide an alternative explanation.

9. Natural ash aggregates that are internally massive, with smooth vesicles and no preferential grain alignment, are likely to have formed by nucleation and coalescence in the presence of abundant liquid water (>10-15 wt.\%).

10. The formation of fine outer rims may be explained by electrostatic forces and sparse liquid bridges at low water contents. However, decreasing liquid content alone cannot explain the shift from coarser grained cores to extremely fine (dominantly $<10 \mu \mathrm{m}$ ) rims observed in the geologic record. This is because even at liquid contents $<5$ wt. $\%$, particles up to $\sim 63 \mu \mathrm{m}$ can be preferentially scavenged from a wider population. Therefore, the combination of decreased liquid content and a supply of fine grained ash are both key factors. Ice is unlikely to play an important role in this process because it would be expected to leave behind a porous texture. 
Chapter 5 


\title{
5. The nature, origins and distribution of ash aggregates in a large-scale wet
} eruption deposit: Oruanui, New Zealand

\begin{abstract}
This study documents the processes and products of volcanic ash aggregation in the $\sim 25.4$ ka Oruanui supereruption from Taupo volcano, New Zealand. The eruption is divided into ten phases on the basis of ten units in the fall deposit stratigraphy, with coeval pyroclastic density current (PDC) deposits erupted in all phases. Aggregate textures and distributions are considered for seven of the units, ranging from relatively dry (fall units 2,5 ) to mixed (units $6,7,8$ ) to dominantly wet (unit 3). Despite a macroscopically uniform appearance in the Oruanui deposits, aggregate textures shift abruptly over vertical scales of $\mathrm{cm}$ to $\mathrm{dm}$ within individual units. Six categories of ash aggregates are identified in the deposits, and are used to infer distinct volcanic and meteorological interactions in the eruption cloud using dispersal characteristics and mode of emplacement (i.e. fall versus PDC deposition). Field observations presented here support the notion of Brown et al. [2010, Origin of accretionary in ground-hugging density currents: evidence from pyroclastic couplets on Tenerife, Geol. Soc. Am. Bull. 122, 305-320] that deposits bearing matrix-supported accretionary lapilli with ultrafine rims (i.e. outer layers dominated by ash $<10 \mu \mathrm{m}$ ) and abundant rim fragments are associated with emplacement of PDCs. However, on the basis of grain size distributions and field relationships, it is inferred that these types of ash aggregates formed their outer layers in the overriding fine ash cloud elutriated from PDCs, not during transport within the dense lower level of ground-hugging currents. The propagation of PDCs beneath an overriding, buoyant plume (whether coignimbrite or vent-derived in origin) generates concentrically layered accretionary lapilli in the ash cloud by producing updrafts of convectively unstable ash-laden air. The apparent coarsening of mean grain size observed in aggregate-bearing fall deposits reflects a combination of multi-level plume transport and preferential scavenging of fines during proximal aggregation. The role of ice fallout (and melting) within $\sim 100 \mathrm{~km}$ from vent was likely to have played an important role in providing a source of liquid water for wet aggregation in the ash clouds. Inferences from this work may be extended to interpreting aggregation
\end{abstract}


signatures from fall deposit grain-size distributions in cases where intact aggregate structures are no longer preserved.

\subsection{Introduction}

Aggregation processes play a central role in the transport, dispersal and deposition of ash from explosive eruption clouds [Carey and Sigurdsson 1982, Brown et al. 2012]. Although some degree of aggregation occurs in all eruptions generating fine ash, it is a particularly important process in volcanic clouds incorporating abundant water. As a result, the deposits and fallout patterns of water-influenced ('wet') eruptions exhibit distinctive differences to their dry counterparts [Walker 1971, Self and Sparks 1978, Walker 1981b, Houghton et al. 2000]. Defining the conditions under which varying spatial and temporal scales of aggregation occur in eruption clouds has practical implications for understanding the dispersal behavior of volcanic ash, and is central to interpreting deposits from 'wet' eruptions.

While the signatures of aggregation in deposits (e.g. inferred or preserved aggregate structures) are frequently used to interpret the dynamics of plume development in past eruptions [Brown et al. 2012], the range of processes leading to the observed spectrum of aggregate types poses a number of challenges. One key complication is that the various styles of aggregation affect fallout patterns of volcanic ash at different stages of plume development. For example, particle clustering can be triggered immediately after eruption due to incorporation of surface water at the vent [Tomita et al. 1985], or many hours afterwards (100s to 1000s of kilometers downwind) by entrainment of tropospheric moisture or melting of ice carried in the plume [Durant et al. 2009]. These spatiotemporally variable sources of liquid water and ice also respond to changes in eruption style (e.g. buoyant rise versus column collapse), resulting in overprinting of multiple processes. The purpose of this study is to document the delicate balance and feedbacks between plume dynamics and availability of water on the behavior of eruption clouds, as recorded in the resulting deposits. Distinctive styles of ash aggregation are defined and classified in deposits from one of the largest water-rich eruptions yet documented (the $\sim 25.4$ ka Oruanui eruption of Taupo volcano, New Zealand) as a means to investigate these dynamic feedbacks. 
The term ash aggregate is used here in a broad sense to refer to all clusters of volcanic ash particles. These clusters are bound together by cohesive forces related to one or more of: electrostatic attraction [Schumacher 1994, James et al. 2002, 2003], liquid water [Gilbert and Lane 1994, Schumacher and Schmincke 1995] and ice [Brown et al. 2012, Van Eaton et al. 2012b, Chapter 4 of this thesis], with cementation potentially acting to develop permanent solid bridges between grains. A number of previous fieldfocused studies have mapped aggregate-bearing layers with distance from vent in the deposits of past eruptions [e.g. Self 1983, Schumacher and Schmincke 1991, Scolamacchia et al. 2005, Brown et al. 2010], suggesting that the characteristics of aggregate types can be directly linked to specific emplacement processes. In particular, the model of Brown et al. [2010] infers that ash aggregates with concentric structure are generally formed within ground-hugging density currents, rather than by direct fallout from a high, buoyant plume.

The nature and origins of ash aggregate types in the Oruanui eruption are explored in context with their end-member modes of emplacement, i.e. whether the aggregates landed vertically as 'fall' or with a lateral component from emplacement in PDCs. (Note: PDC is used here as a general term for deposits of gravity-controlled, groundhugging currents, whether dilute or concentrated.) Selected localities and fall units from this eruption (units 2, 3, 5, 6, 7 and 8) are used to illustrate the spectrum of aggregate types and commensurate complexity of processes that may have given rise to the observed textures. This study focuses on the evolution of aggregation signatures with distance from vent and vertically through the eruption sequence. Interpretations are drawn from numerical modeling of wet plume dynamics [Chapter 2, Van Eaton et al. 2012a] and laboratory experiments of ash aggregation [Chapter 4, Van Eaton et al. 2012b] to address two key aspects of wet eruption plumes in general and the Oruanui eruption in particular: (1) controls on the nature of aggregation in volcanic clouds, and (2) resulting impacts on the dispersal and grain size distribution of volcanic products.

\subsection{The $\sim 25.4$ ka Oruanui eruption}

The Oruanui eruption is an archetype for the largest scale of water-rich eruption styles, occurring when silicic magma mingles explosively with surface water [phreatoplinian: Self and Sparks 1978]. It represents one of the most widely dispersed examples of wet 
volcanism yet documented [Self 1983], producing an estimated $430 \mathrm{~km}^{3}$ of fall and 320 $\mathrm{km}^{3}$ of PDC deposits (mostly ignimbrite), together with $\sim 420 \mathrm{~km}^{3}$ of intracaldera fill, equating to a total of $\sim 530 \mathrm{~km}^{3}$ of magma [Wilson 2001]. The eruption occurred at $22,590 \pm 230{ }^{14} \mathrm{C}$ years BP [Wilson et al. 1988], equivalent to $27 \pm 1$ calendar ka (Lowe et al., 2008), just prior to the last glacial maximum (LGM). At the time of the eruption, mean sea level was $~ 120$ m lower [e.g., Newnham et al. 1999; their Figure 3], and an elongate paleo-lake [Lake Huka: Grindley 1965, Self and Healy 1987, Manville and Wilson 2004] resided directly over what became the Oruanui vent areas (Figure 5.1). Paleoenvironmental reconstructions [Pillans et al. 1993, Shulmeister et al. 2004, Alloway et al. 2007, Lorrey et al. 2012] and climate simulations [Drost et al. 2007a, Rojas et al. 2009] have indicated overall colder, drier conditions and stronger westerly winds in New Zealand during the LGM, with weather patterns that were likely to have been significantly influenced by the axial ranges (Figure 5.1) [Salinger 1980b, a]. This information provides context for discussion of the role of paleocirculation patterns on dynamics and dispersal of the Oruanui eruption products.

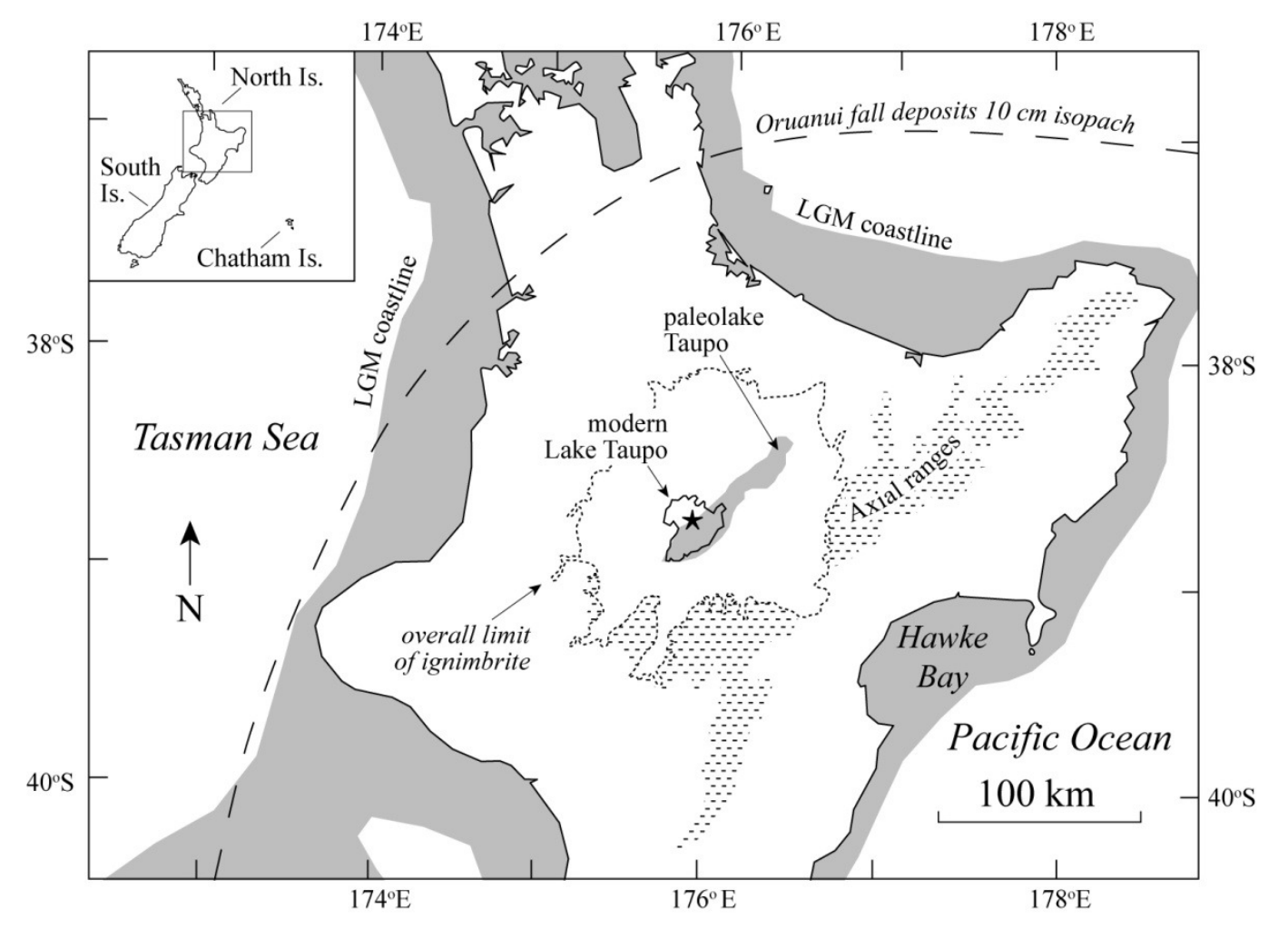

Figure 5.1 Location map and key paleogeographic features relevant to the $\sim 25.4 \mathrm{ka}$ Oruanui eruption in the North Island of New Zealand. The axial ranges (stippled fill, shown where topography is $\geq 750 \mathrm{~m}$ above modern sea level) would have provided a significant barrier to circulation patterns during the Last Glacial Maximum (LGM). Star indicating the virtual source adopted for the Oruanui eruption products, and extents of the overall fall and ignimbrite deposits are from Wilson [2001]. The LGM 
paleoshoreline is taken as $-120 \mathrm{~m}$ with respect to modern sea level. Modified from Manville and Wilson [2004].

The Oruanui eruption is divided by Wilson [2001] into ten phases based on fall deposit stratigraphy. Each phase of eruption interacted to some extent with overlying Lake Huka, and each is associated with the generation of PDCs. Evidence for extensive magma-water interaction includes abundant ash aggregates, the exceptionally fine grain size of the overall deposits [Self and Sparks 1978, Wilson 2001] when compared to typical dry fall deposits compiled by Walker [1971], and the entirely non-welded nature of the ignimbrite, with emplacement temperatures of $150-250{ }^{\circ} \mathrm{C}$ [Wilson 2001].

The spectrum of aggregate types preserved in the Oruanui deposits ranges from mud rain that 'splashed' on impact with the ground [Walker 1981b], to material inferred to represent delicate, snowflake-like ash clusters [e.g. Sorem 1982]. Previous work linking aggregate textures with distinct depositional styles in the eruption [Self 1983, Wilson 2001], has led to fundamental questions about the role of aggregation in transport and dispersal of volcanic emissions. In particular, grain size analyses of the Oruanui unit 3 deposits have demonstrated that they show an increase in mean grain size downwind of vent [Figure 13 of Wilson 2001]. This peculiar characteristic has also been observed in aggregate-bearing deposits of the $1.8 \mathrm{ka}$ Taupo eruption [Hatepe ash: Smith 1998] and co-PDC deposits from a 1991 Unzen block-and-ash flow [Watanabe et al. 1999]. What are the mechanisms responsible for this coarsening with distance, and might it be more common than previously thought? Given the entirely non-welded nature of the deposit, the Oruanui case study enables the integration of grain size data and aggregate facies analysis within a well-established stratigraphy to address these issues.

The dynamics of wet eruption columns are challenging to model numerically due to the complex partitioning of energy into solid, liquid and gaseous phases of water. Numerical studies of explosive eruption plumes have shown that column stability depends critically on eruption rate and magma temperature [Wilson et al. 1978] as well as the amount of water incorporated into the plume from surface water [Koyaguchi and Woods 1996] or atmospheric moisture [Woods 1993, Glaze and Baloga 1996, Graf et al. 1999]. Microphysics-capable simulations by Van Eaton et al. [2012a, Chapter 2] have further indicated that abundant magma-water mixing in large-scale eruptions $\left(\geq 10^{8}\right.$ 


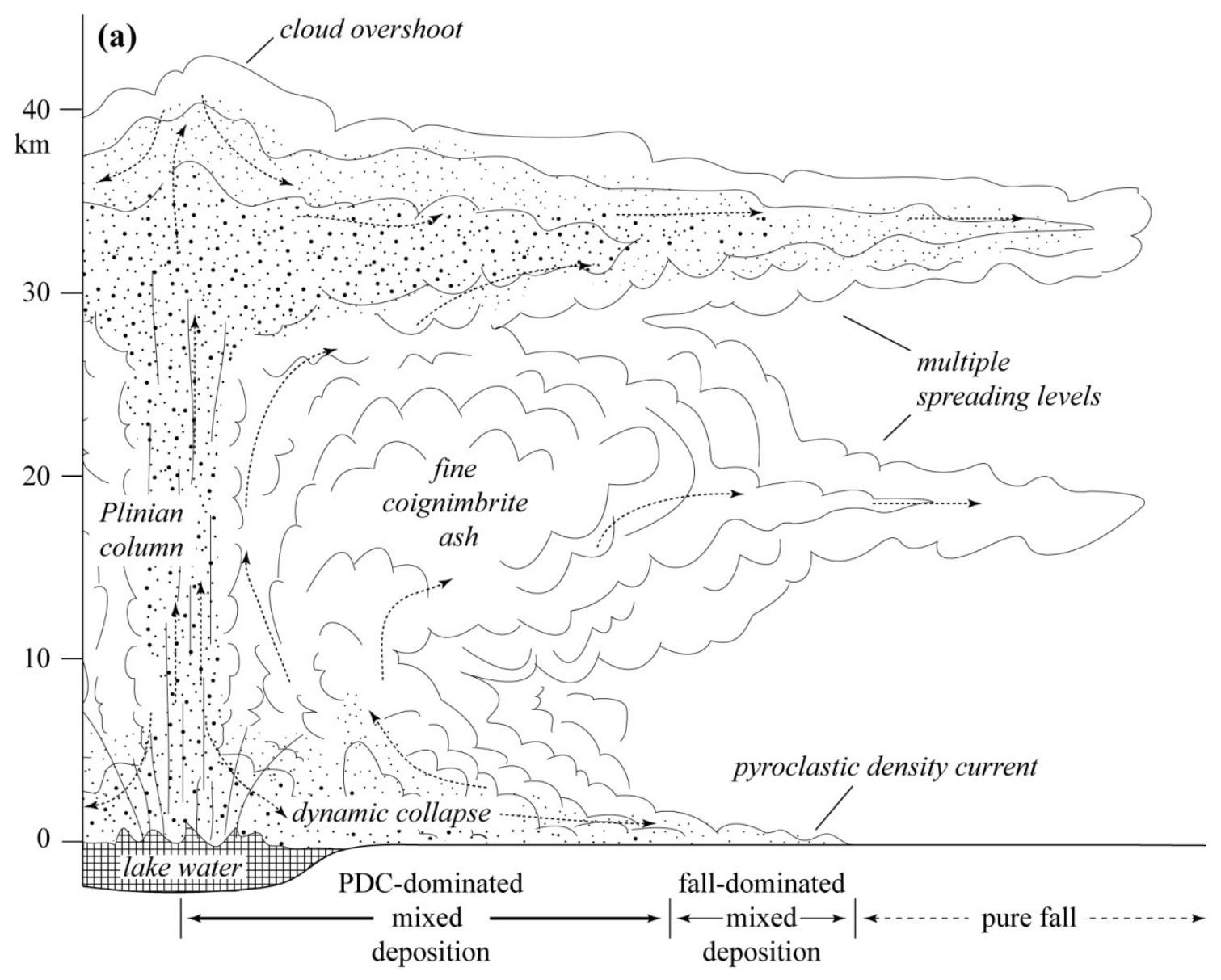

(b) Depositional regimes in Oruanui units, shown as distance from virtual source.

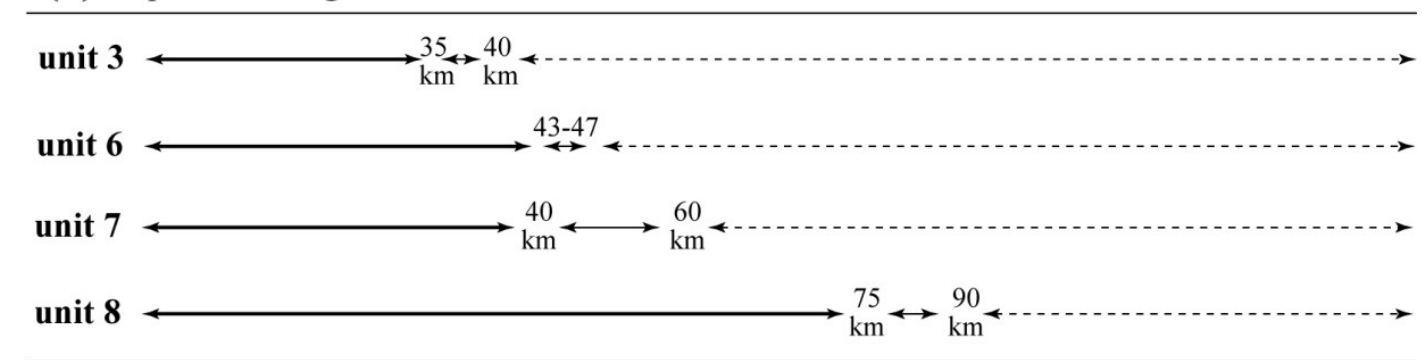

Figure 5.2 (a) Definition sketch of a dynamically unstable phreatoplinian eruption column, illustrating processes that result in a hybrid volcanic cloud, scaled and modified from Van Eaton et al. [2012a]. Ascent of a vent-derived Plinian column and shedding of PDCs occur simultaneously or closely spaced in time, generating ash clouds at multiple levels in the atmosphere. The resulting depositional regimes are labelled at the bottom of the diagram. (b) Lateral extent of the three depositional regimes inferred from Oruanui units 3, 6, 7 and 8, with distance being approximately downwind of virtual source.

$\mathrm{kg} \mathrm{s}^{-1}$ magma) results in transitional column behavior. Even very wet, unstable eruption columns are simulated to have the capacity to rise to stratospheric levels due to energetic boosts from latent heat release. These processes give rise to a dynamically unstable column (Figure 5.2a), characterized by Plinian-style (sustained buoyant) 
ascent, simultaneous shedding of ground-hugging currents and lofting of PDC-derived 'coignimbrite' ash. Following Chapter 2 and Van Eaton et al. [2012a], three concentric zones of deposition are distinguished from the hybrid cloud system (Figure 5.2): (1) a proximal

zone of chaotically mixed, laterally- and vertically-transported currents, dominated by PDCs; (2) a fall-dominated transitional zone, in which a laterally persistent fall stratigraphy emerges, interlayered with PDCs; and (3) a distal zone that is beyond the limits of PDCs, and characterized by pure fall deposition.

One key aspect of this hybrid cloud system is that selective transport of pyroclasts and hydrometeors (i.e. airborne water phases: hail, rain, graupel, snow) occurs in different parts of the cloud. The dense, inner (Plinian) 'core' [Woods 1988] is relatively insulated from turbulent air entrainment, and able to maintain higher temperatures and vertical velocities. This core transports larger pyroclasts into the upper atmosphere [e.g. Neri et al. 2002], and may, under some circumstances, be capable of sustaining water in a liquid state at high altitudes [Figure 2.3 of this thesis, Van Eaton et al. 2012a]. In contrast, the coignimbrite updrafts ascend at initially slow vertical velocities, leaving behind coarser particles and lofting only the finest ash [Woods and Wohletz 1991]. Interaction between the inner core and coignimbrite thermals results in multiple injection heights in the atmosphere. The hybrid cloud thus represents a multi-level transport system: coarser particles are carried aloft by the high plume and laterally by ground-hugging currents, whereas dilute fractions lofted from PDC form a lower-level cloud of preferentially finer ash.

From both field-based and numerical studies of the Oruanui eruption it is inferred that PDCs and fall deposit were generated largely simultaneously during each eruptive phase, with the proximal, PDC-dominated zones varying in distance from source [Wilson 2001]. This study focuses on key sites at varying stages of PDC run-out and beyond (Figure 5.3) to examine how the processes and products of aggregation change with respect to each depositional regime. 


\subsection{Field and laboratory methods}

Exposures of the Oruanui deposits were logged and samples obtained by carving out intact monoliths of ash. The deposits are unlithified, but are generally dense and compact enough to extract intact samples. To reveal the detail of aggregate outlines and void space in the matrix, the monoliths were scraped flat and air-dried before being dusted with a soft brush and etched with a compressed air jet. This enabled more accurate modal volume estimates of whole versus fragmented aggregates than was

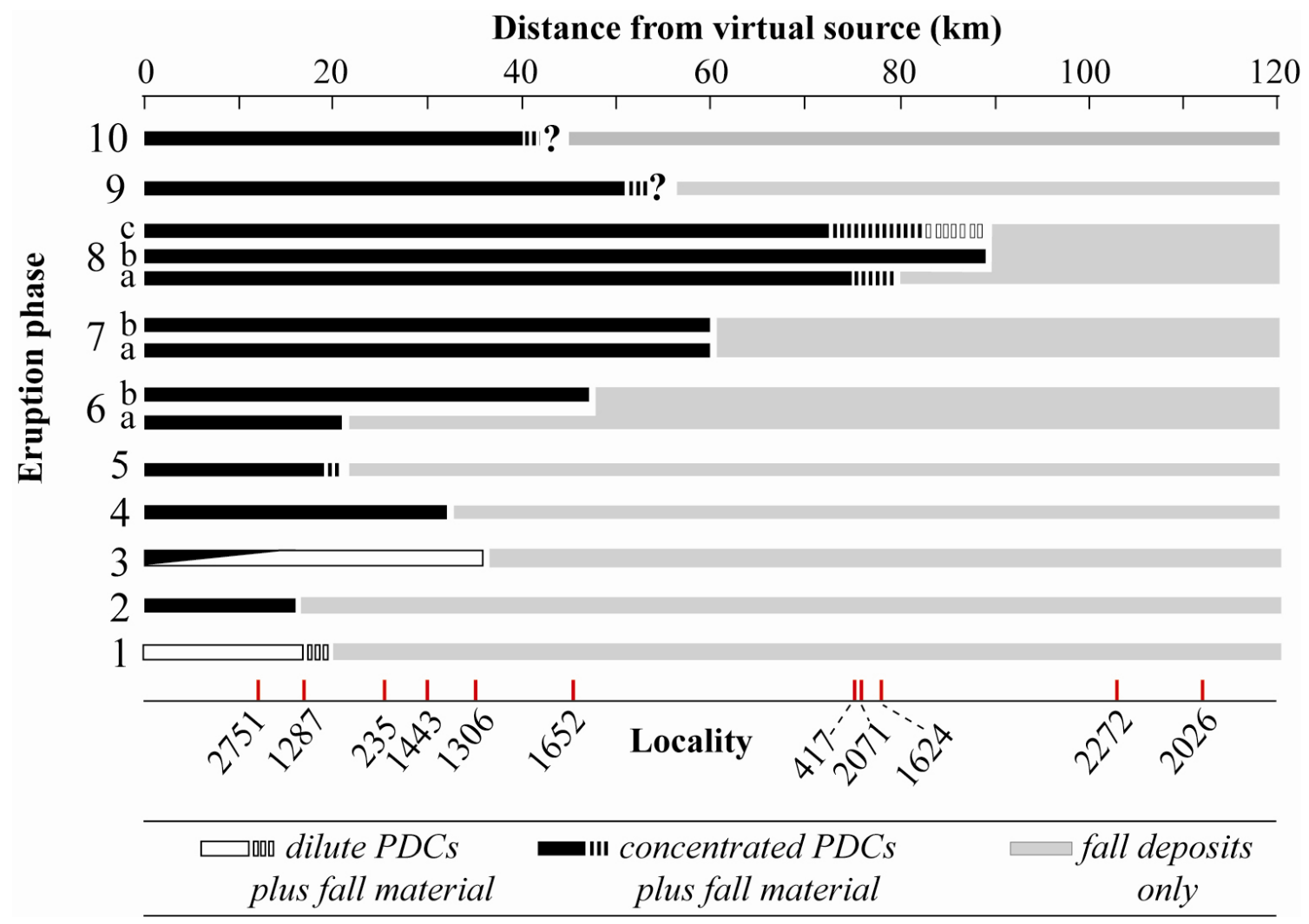

Figure 5.3 Field localities described in this study, shown with respect to the lateral extents of dilute and concentrated PDCs and fall deposits from each phase of eruption. Locality numbers are the same as in Wilson [2001]. Broken bars indicate irregular run out distances or patchy preservation. Modified from Wilson [2001].

possible in the field. The support framework of aggregates in situ was defined as matrix supported (where aggregates did not touch each other) or clast supported. The term 'just' matrix-supported is used where $\sim 50 \%$ of the aggregates are touching, but the others are not. Concavo-convex contacts were noted where aggregates showed soft deformation and 'point' contacts where they met at tangent points. Epoxy-impregnated thin sections were used for examining internal microstratigraphy (e.g. orientation of 
individual particles, number of internal laminae) and for indentifying cryptic or poorlydefined aggregates that could not be readily distinguished in the field. X-ray computed microtomography was also employed to visualize 3-dimensional structures, and was particularly useful for weak samples that collapsed during the impregnation stage of thin sectioning.

Where possible, individual aggregates were extracted intact from the deposit and their three principal axes and dry masses measured. The aggregates were halved and characterized with respect to the morphology and dimensions of any internal layering, void space (e.g. ovoid vesicles, loosely-packed grains or large cavities), and grain size. Splits of the ash deposit samples and the representative individual aggregates from each interval were gently and thoroughly disaggregated, sonicated in $1 \%$ calgon solution and passed through a $1.4 \mathrm{~mm}$ mesh before laser diffraction analysis (to avoid blockages in the machine). A Beckman Coulter LS 13320 laser diffraction particle size analyzer was used to obtain grain size distributions. The optical model assumed a refractive index of 1.55 and absorption coefficient (imaginary refractive index) of 0.01 , with no correction factor applied, as in Van Eaton et al. [2012b] (see Chapter 3 of this thesis for details). For concentrically structured aggregates, additional analyses were performed on cores and rims. Optical diameters were binned into half-phi size intervals and grain size parameters were calculated as in [Folk and Ward 1957].

\subsection{Aggregates in the Oruanui deposits}

\subsubsection{Aggregate types - classification and terminology}

The growing number of detailed studies of ash aggregates has led to diverse classification schemes. Although the general term 'ash aggregates' is used to encompass all types, this phrase has also been used interchangeably with pisolites [Lacroix 1904], chalazoidites [Berry 1928, Self and Healy 1987], accretionary lapilli [Moore and Peck 1962], volcanic hailstones [Kato 1986] and mud balls [Schumacher and MuesSchumacher 1997]. Where it is necessary to discriminate between subtle aggregate characteristics, however, a more specific nomenclature is required. Therefore the basic themes reviewed by Brown et al. [2012] are adopted here in distinguishing between the end-member types comprised of loosely-bound individual particles (particle clusters) 
Table 5.1 Categories of aggregate types identified in the Oruanui deposits, with field descriptions, typical range of diameters and inferred mechanism of formation. Inferred water contents refer to the weight percent in aggregates based on the laboratory experiments of Chapter 4 [Van Eaton et al. 2012b].

\begin{tabular}{|c|c|c|c|c|}
\hline Appearance & Name & Symbol & Field description & General interpretation \\
\hline 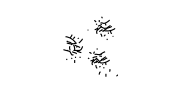 & particle clusters & $\mathrm{Pc}$ & $\begin{array}{l}\text { Occur as loosely-packed, 'porous' ash deposits with } \\
\text { variable preservation of irregularly-shaped fine ash } \\
\text { clusters. Diameters }<1 \mathrm{~mm} \text {. (ash } \text { clusters }^{c}, P C 1^{e} \text { ) }\end{array}$ & $\begin{array}{l}\text { Individual ash particles which adhered under } \\
\text { predominantly dry conditions ( }<5 \text { wt. } \% \\
\text { liquid) }\end{array}$ \\
\hline \multirow[t]{4}{*}{ 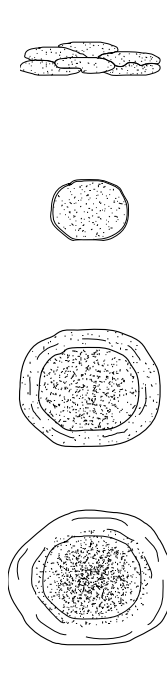 } & $\begin{array}{l}\text { coalesced ash } \\
\text { pellets }\end{array}$ & Cpel & $\begin{array}{l}\text { Occur as dense, vesicular ash beds with wholly to } \\
\text { partially coalesced, internally massive aggregates with } \\
\text { diameters }<5 \mathrm{~mm} \text {. }\left(A P 3^{e}\right)\end{array}$ & $\begin{array}{l}\text { Massive ash pellets that were oversaturated } \\
(>20-25 \text { wt. } \% \text { water) and splashed, deformed } \\
\text { or melted after landing. }\end{array}$ \\
\hline & massive ash pellets & Mpel & $\begin{array}{l}\text { Subspherical aggregates that are internally massive, } \\
\text { although some have a single thin }(<200 \mu \mathrm{m}) \text { outer film } \\
\text { of finer ash. Usually clast-supported, with concavo- } \\
\text { convex contacts. Diameters }<1 \mathrm{~mm} \text { to } 1 \mathrm{~cm} \text {. (type } A^{a} \text {, } \\
\text { core type }^{b} \text {, ash pellets }{ }^{c, d}, A P 1^{e} \text { ) }\end{array}$ & $\begin{array}{l}\text { Growth by wet nucleation and rapid } \\
\text { coalescence under saturated conditions }(\sim 15- \\
20 \text { wt.\% liquid), followed by freezing during } \\
\text { transport. Deformation occurred from } \\
\text { landing wet or melting after deposition. }\end{array}$ \\
\hline & $\begin{array}{l}\text { layered accretionary } \\
\text { lapilli }\end{array}$ & Lacc & 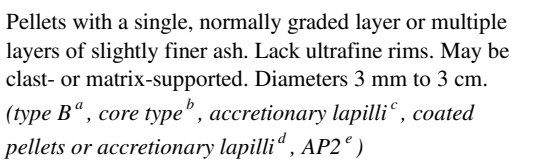 & $\begin{array}{l}\text { Massive ash pellets that accreted outer layers } \\
\text { by cycling through regions with varying grain } \\
\text { size populations and/or sub-saturated } \\
\text { conditions (< } 15 \text { wt. } \% \text { liquid). }\end{array}$ \\
\hline & $\begin{array}{l}\text { ultrafine rim-type } \\
\text { accretionary lapili }\end{array}$ & Ufacc* & $\begin{array}{l}\text { One or more layers of ultrafine ash (mode } 4 \mu \mathrm{m} \text { ) around } \\
\text { a coarser core. Distinct core-rim boundary; usually } \\
\text { matrix-supported and fragmented; may have complex } \\
\text { morphologies. Diameters } 2 \mathrm{~mm} \text { to } 3 \mathrm{~cm} \text {. (type } B^{a} \text {, rim } \\
\text { type }^{b} \text {, accretionary lapilli }{ }^{c} \text {, coated pellets or } \\
\text { accretionary lapilli }^{d}, A P 2^{e} \text { ) }\end{array}$ & $\begin{array}{l}\text { Massive ash pellets or layered accretionary } \\
\text { lapilli that circulated through moist, but sub- } \\
\text { saturated }(<10-15 \text { wt.\% liquid), regions of } \\
\text { predominantly ultrafine ash. }\end{array}$ \\
\hline & $\begin{array}{l}\text { complexly layered } \\
\text { accretionary lapilli }\end{array}$ & cLacc* & $\begin{array}{l}\text { Ten or more outer laminations; truncations and lenses } \\
\text { common. Outermost layer characterized by fine (mode } \\
16 \mu \mathrm{m} \text { ), but not ultrafine, ash. Generally lack a distinct } \\
\text { core-rim boundary. Diameters } 1-4 \mathrm{~cm} \text {. (type } B^{a} \text {, rim } \\
\text { type }^{b} \text {, accretionary lapilli, }{ }^{c, d}, A P 2^{e} \text { ) }\end{array}$ & $\begin{array}{l}\text { Massive ash pellets or other nuclei that were } \\
\text { vigorously re-entrained through abrasive, } \\
\text { grain size-stratified regimes under sub- } \\
\text { saturated conditions ( }<10-15 \text { wt. } \% \text { liquid) }\end{array}$ \\
\hline
\end{tabular}

Italics provide corresponding terminology from the studies of: ${ }^{a}$ Reimer [1983], ${ }^{\mathrm{b}}$ Schumacher and Schmincke [1991],

${ }^{c}$ Thordarsson [2004], ${ }^{d}$ Brown et al. [2010] and ${ }^{\mathrm{e}}$ Brown et al. [2012].

*Designates aggregate types that are only found in PDC deposits. 
versus very water-rich aggregates (akin to mud rain). A further distinction is made between subspherical, internally structureless simple aggregates (ash pellets) versus the more compact, internally layered complex varieties, which are referred to here as accretionary lapilli, in rough equivalence to the core-type vs. rim-type classification of Schumacher and Schmincke [1991]. Note, however, that although 'accretionary lapilli' as a general term does include all ash aggregates with diameters $2 \mathrm{~mm}$ to $2 \mathrm{~cm}$ [Moore and Peck 1962, Schumacher and Schmincke 1991], the more specific usage has also been applied as a means to differentiate between the simple vs. complex end-members [Gilbert and Lane 1994, Smith 1998, Wilson 2001, Brown et al. 2010]. Here the specific usage of the term 'accretionary lapilli' is employed to refer to ash aggregates with a concentrically layered internal structure, regardless of their size.

The spectrum of aggregate types in the Oruanui deposits is divisible by distinctive characteristics defining six categories (Table 5.1), three of which are types of accretionary lapilli. Each aggregate type is categorized on the basis of internal structure, grain size and relationship to the host deposit, and each has variants with a larger pumice, lithic, or crystal fragment at the center (referred to as 'armoured lapilli' by White and Houghton [2000]). The dry end-member type is particle clusters, irregularlyshaped linkages of fine ash that are loosely bound by electrostatic forces and/or small amounts of liquid water ( $<5$ wt.\%), akin to snowflake clusters [Sorem 1982, James et al. 2002]. This type is inferred to comprise fall layers in the Oruanui deposits with low bulk densities $\left(<900 \mathrm{~kg} \mathrm{~m}^{-3}\right)$ and a loosely-packed appearance with no subspherical pellets visible [Wilson 2001]. The most water-rich example, coalesced ash pellets, must also be inferred from the deposits due to the partial or complete destruction of aggregate structures after landing. These aggregate types occur as near-homogeneous layers of dense, cohesive fine ash with a scalloped base (Figure 5.4a) or as individual lumps 'caked' onto surrounding structures (Figure 5.4b). Although the constituent aggregates cannot be readily distinguished in the field, these deposits contain abundant ovoid vesicles, indicative of air bubbles trapped in a saturated matrix [Lorenz 1974]. Rosi [1992] inferred that this texture forms from beds of ash pellets that were wet enough to splash or coalesce on landing, and are akin to mud rain. It is likely that during the Oruanui eruption, many of these examples may also have landed frozen and melted after impact [cf. 1969 Ruapehu eruption: Healy et al. 1978]. Experimental studies have 
shown that the coalescence and slurrying behavior in fine ash occurs at water contents approaching 20-25 wt.\% [Walker 1981b, Schumacher and Schmincke 1995, Chapter 4, Van Eaton et al. 2012b]. However, the lack of large-scale slurrying in the Oruanui deposits, [cf.
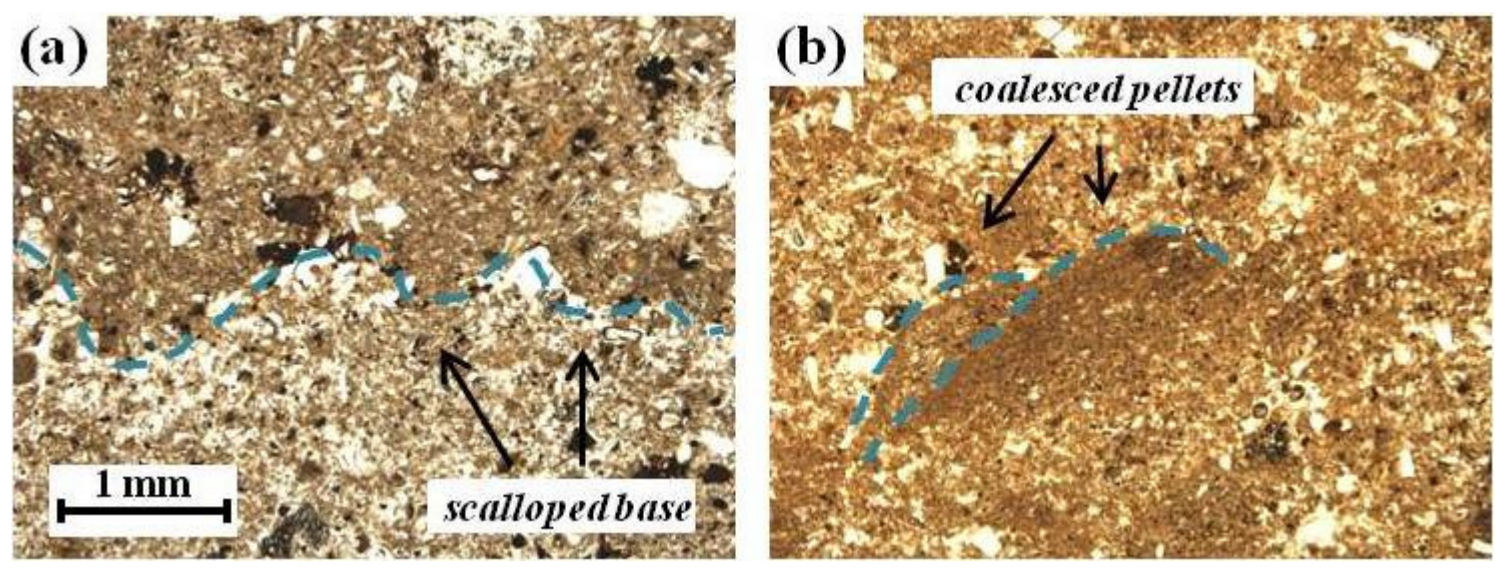

Figure 5.4 Impregnated thin sections of coalesced ash pellets, cut perpendicular to bedding. These are inferred to have deposited wet (as mud rain) or melted after landing. (a) Scalloped basal outline of coalesced pellets that impacted onto previously deposited material. (b) Two partially disintegrated ash pellets that appear to have splashed or melted onto one another. Both images are from the basal $10 \mathrm{~cm}$ of unit 3 at locality 2751.

erosional gullies in the 1.8 ka Hatepe and Rotongaio deposits: Walker 1981b], suggests that water contents did not exceed these amounts on deposition. Massive ash pellets are characterized by more coherent, spherical to subspherical morphologies, although they frequently show evidence for soft deformation on landing. They are commonly 2-6 mm, but almost always $\leq 1 \mathrm{~cm}$ in diameter (Figure 5.5a-f). They lack internal stratigraphy, with the exception that some exhibit a very thin $(\leq 0.1 \mathrm{~mm})$ outer layer of finer-grained ash (Figure 5.5b). This outer film has been replicated experimentally in saturated ash pellets (15-20 wt.\% liquid) that compacted after coming to rest, squeezing water and the finest ash particles to their surfaces [Van Eaton et al. 2012b]. Consequently, dried and etched natural samples of massive pellets in situ often show a honeycomb-like network of inverse aggregate structures, in which the concavo-convex contacts between individual aggregates are delineated by the more resistant outer films. In cases where the aggregates did not deform as much on landing, point contacts between individual pellets leave behind an open framework with vermiform or cuspate voids in between (Figure 5.5c). The smallest pellets (diameters 100-300 $\mu \mathrm{m}$ ) become more common $>100$ $\mathrm{km}$ from vent, and are associated with similarly-sized crystals, glass shards and ash- 

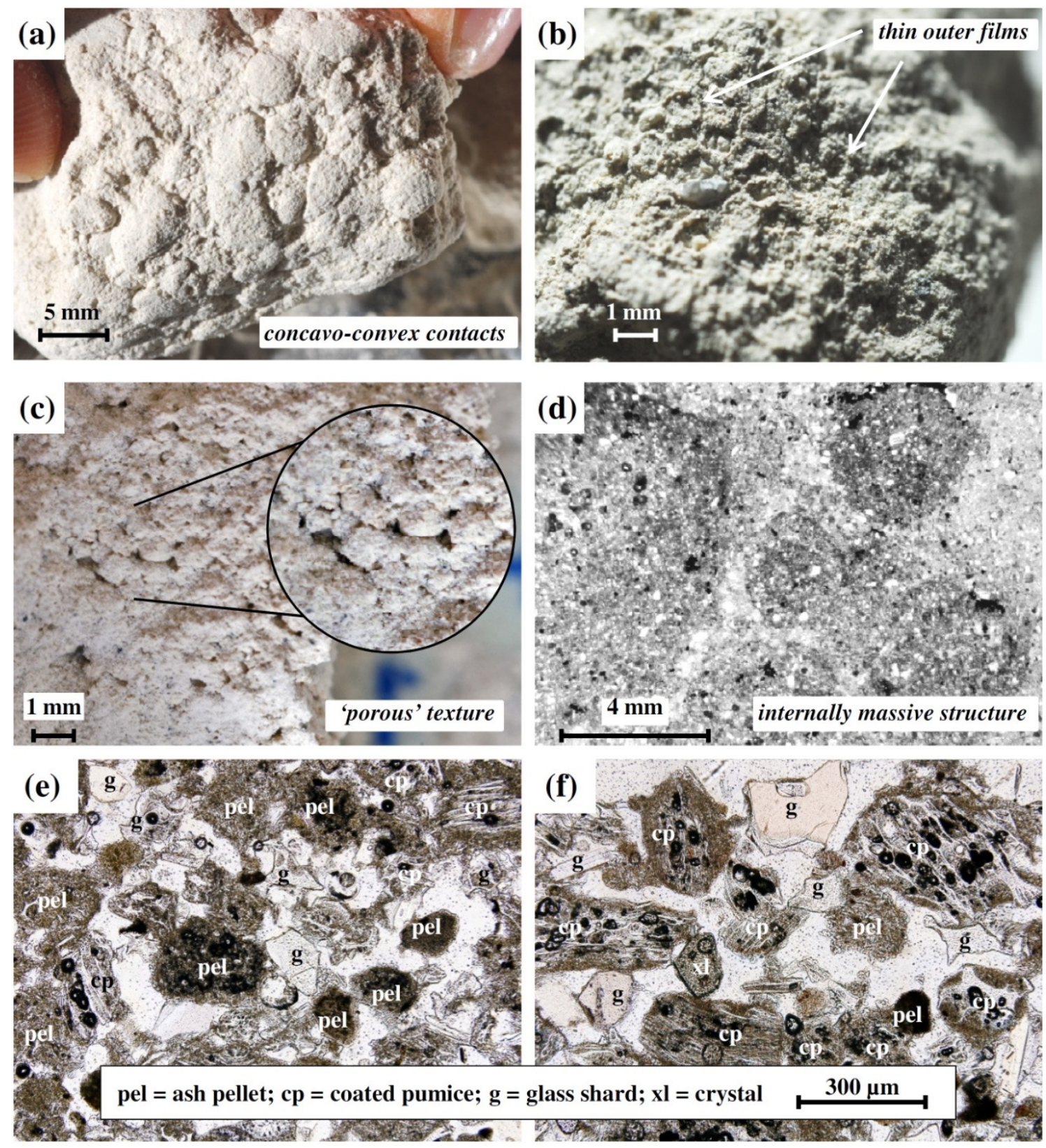

Figure 5.5 In situ examples of massive ash pellets, demonstrating textural features described in the text, as identified in dried, etched monoliths (a-c) and thin section microphotographs (d-f). (a) Soft, concavo-convex contacts between pellets in unit 3 $(\sim 100 \mathrm{~cm}$ from the base of the unit) at proximal locality 235. (b) Pellets with thin outer films, which suggest water saturation, $\sim 30 \mathrm{~cm}$ above the base of unit 3 at locality 235 . (c) Porous texture in pelletized unit 2 at distal locality 2266, $\sim 117 \mathrm{~km}$ from source; diameter of enlargement is $6 \mathrm{~cm}$. (d) Internally massive pellets from basal unit 3 at proximal locality 2751. (e, f) Small ash pellets, of similar size to surrounding ash-coated particles, from distal unit 3 at locality 2272, 102 km from source . 

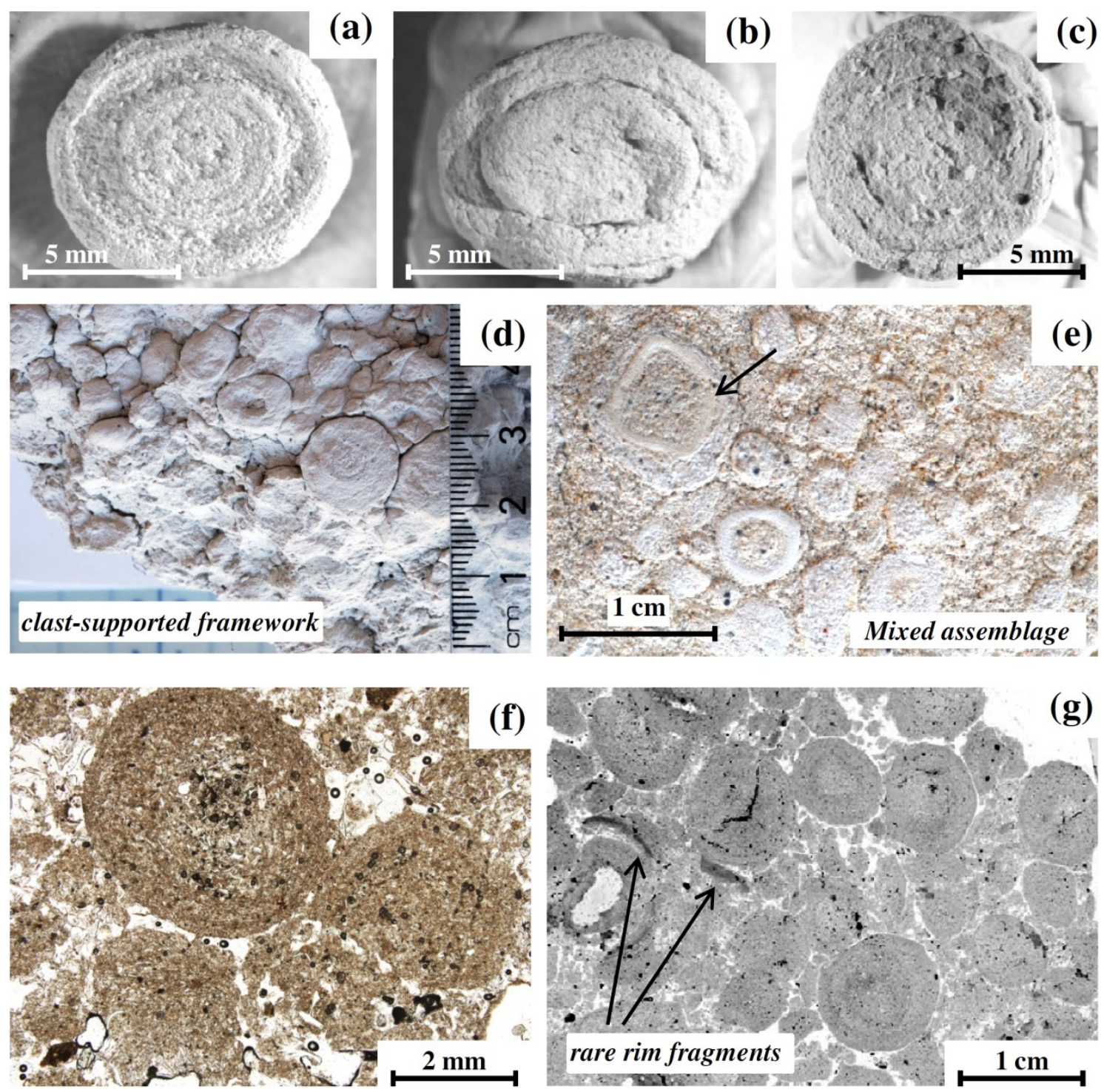

Figure 5.6 Examples of layered accretionary lapilli, which lack ultrafine rims, but exhibit concentric structure in the form of normal grading or internal laminations of similarly-sized ash. Top row shows photographs of individual aggregate cross sections from (a, b) unit 3 at locality 235, 98 cm from the base, and (c) unit 8 fall deposits at locality 1624. (d) Clast-supported framework of layered accretionary lapilli from unit 8 fall deposits at locality 1624. (e) Mixed assemblage of 'just' matrix supported pellets and layered accretionary lapilli (some with internal ultrafine layers, indicated by arrow) from $\sim 80 \mathrm{~cm}$ above base in unit 3 at locality 1306. (f) Thin section photomicrograph of layered accretionary lapilli from unit 8 at locality 2267 . Note the concentric orientation of glass shards comprising the aggregates, and matrix of broken aggregates. (g) Scanned thin section of the unit 8 fall layer from (d), emphasizing the matrix of broken aggregates and rare fragments of ultrafine rims inferred to have been elutriated from PDCs and redeposited as fall.

coated pumices (Figure 5.5e-f). Massive ash pellets occur most frequently in a clastsupported (Figure 5.5a-c) or 'just' clast-supported network (Figure 5.5d) of aggregates, indicating that they were emplaced as fallout from an overlying cloud. The individual 
constituent particles exhibit random orientations, suggesting the pellets grew by rapid, chaotic coalescence in a single event. Massive pellets may represent a range of conditions; examples with a porous, low-density structure indicate sub-saturated water contents ( $<15$ wt.\% water), while dense, vesicular aggregates with evidence for soft deformation and outer films form within saturated conditions (15-20 wt.\%) via wet nucleation and coalescence [Van Eaton et al. 2012b].

Layered accretionary lapilli (Figure 5.6a-g) are characterized by distinct internal grain size layering and spherical to subspherical morphologies. These aggregates are found wholly clast-supported (Figure 5.6d, f-g) or 'just' matrix-supported (Figure 5.6e) in the Oruanui deposits, and occur well beyond the limits of PDCs. They are generally larger than massive pellets (typically 5-20 mm diameter) and consist of one to five laminae of similarly-sized or slightly finer ash. These layers may comprise alternating bands with sharp contacts, or a single normally graded structure that fines outward from the central core. Constituent particles are almost always concentrically-oriented, indicating that they experienced a period of layering. This 'layering' regime of aggregate growth [Sastry et al. 2003] is also indicated by individual particles adhering to a larger nucleus, rather than adhering in clumps. In laboratory experiments [Van Eaton et al. 2012b], this regime was attained by lowering the availability of liquid water. It is therefore inferred that the presence of concentric layering signifies that the aggregate experienced (cyclic) passage through relatively drier regions of the eruptive cloud.

Ultrafine rim-type accretionary lapilli are distinct from layered accretionary lapilli in two ways. First, ultrafine rim-types have one or more sharply defined outer layer(s) of 'ultrafine' ash (defined here as $<10 \mu \mathrm{m}$ ) which has a dominant mode of $4 \mu \mathrm{m}$ (Figure 5.7a-j). The presence of ultrafine ash can be determined in the field with a fingernail scratch test: ultrafine ash feels soft rather than sandy. Secondly these types are nearly always matrix-supported in PDC deposits, floating in a matrix of loose, fine ash and abundant aggregate fragments, particularly cuspate rim fragments (Figure $5.7 \mathrm{~g}-\mathrm{h}, \mathrm{j}$ ). Ultrafine rim-types range from $\sim 2-30 \mathrm{~mm}$ across and show complex morphologies, including multiple cores (Figure 5.7c), as also observed by [Raza et al. 2001]. Cauliform or kidney bean-like morphologies are also common (Figure 5.7d, f). In all cases, particles comprising the ultrafine outer rim are concentrically oriented, indicating a shift to drier conditions as described above for layered accretionary lapilli. However, 

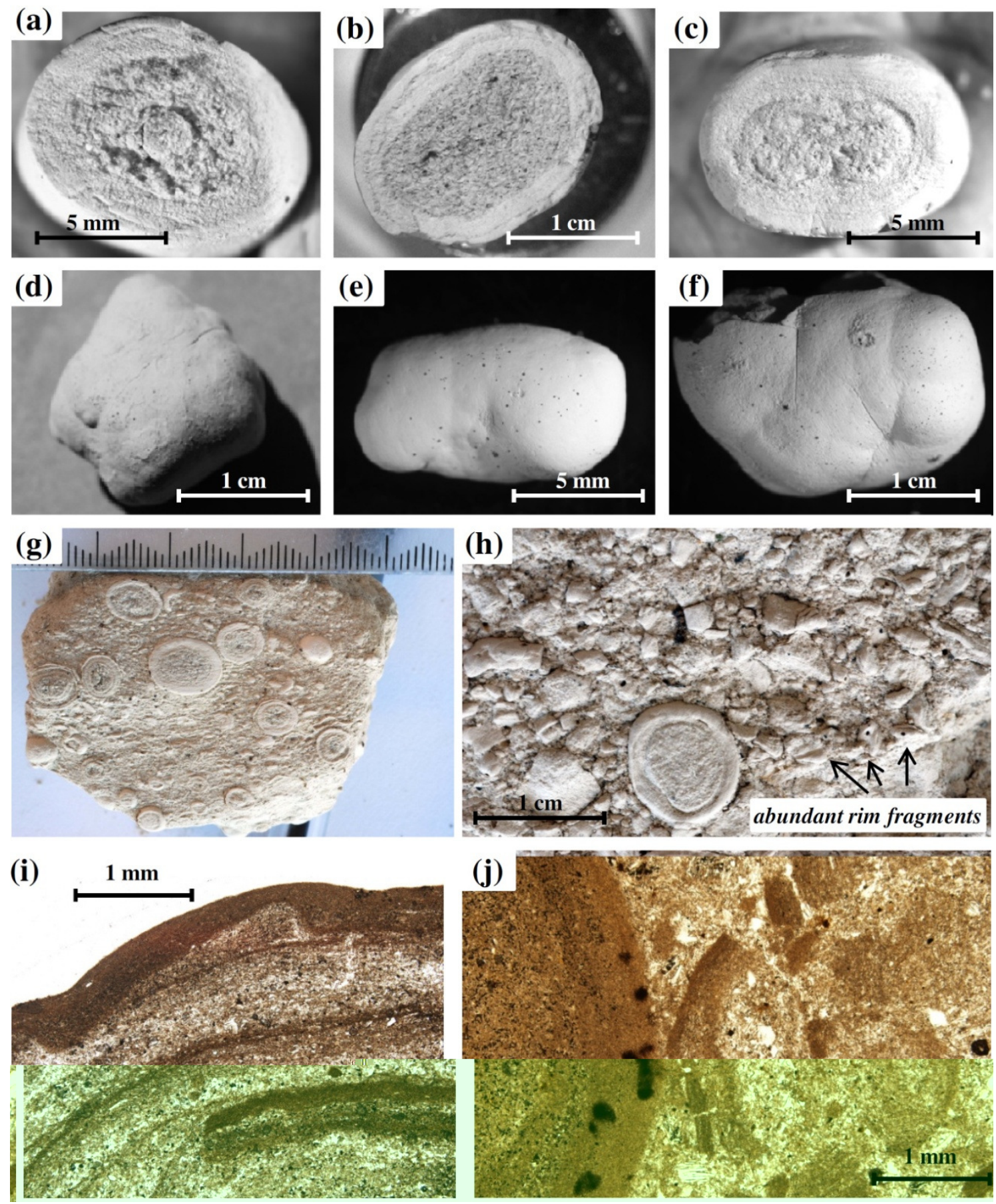

Figure 5.7 Examples of ultrafine rim-type accretionary lapilli. (a-c) Individual aggregate cross-sections from proximal unit 3, emphasizing the sharp boundary between ultrafine outer rim and coarser, vesicular core. Notice the internal pellet in (a), vesicular core in (b) and double core (coalesced pellets side by side) in (c). (d-f) Examples of complex aggregate morphologies from proximal unit 3 at locality 235, including the cauliform base (d, f) and 'kidney bean' shape (e). (g, h) In situ examples from dried, etched monoliths: ( $\mathrm{g}$ ) aggregates floating in a matrix of individual particles and fragmented aggregates, proximal locality 1287, $\sim 30 \mathrm{~cm}$ above the base of unit 3; (h) aggregates immersed in abundant ultrafine rim fragments, from unit 8 at locality 1622 . $(i, j)$ Impregnated thin sections showing (i) detail in a complex outer rim from unit 8 at locality 1622 and (j) relationship with surrounding aggregate fragments from unit 3 at $1306, \sim 60 \mathrm{~cm}$ above the base. 
note that in the experiments of Van Eaton et al. [2012b], fine ash rims $(<31 \mu \mathrm{m})$ with appreciable, mm-scale thicknesses could only be formed by cycling moist pellets through a supply of sub-31 $\mu \mathrm{m}$ ash at temperatures above freezing. In contrast, attempts to layer mm-thick rims of preferentially finer ash from a poorly sorted mixture were unsuccessful. Therefore, the ultrafine rims are more likely to form when moist ash pellets circulate through a drier, ultrafine ash-dominated region of the volcanic cloud immediately before landing (see also Section 5.6.3).

Complexly layered accretionary lapilli. These are composed of tens of fine concentric laminae and lenses with sharp truncations (Figure 5.9). They are unusually large (sometimes $>3 \mathrm{~cm}$ in diameter), always matrix-supported and are not found beyond the extent of PDC deposits. Although they do generally fine outward, with the outermost layer being the finest, complexly layered accretionary lapilli differ from ultrafine rimtypes in that these outer layers are not generally dominated by a $4 \mu \mathrm{m}$ mode. Furthermore, they lack the sharp textural and grain size demarcation between core and rim. Conditions leading to the formation of these accretionary lapilli are envisioned as an extreme version of the two previous types, involving vigorous, cyclic passage through stratified regions of a volcanic cloud.
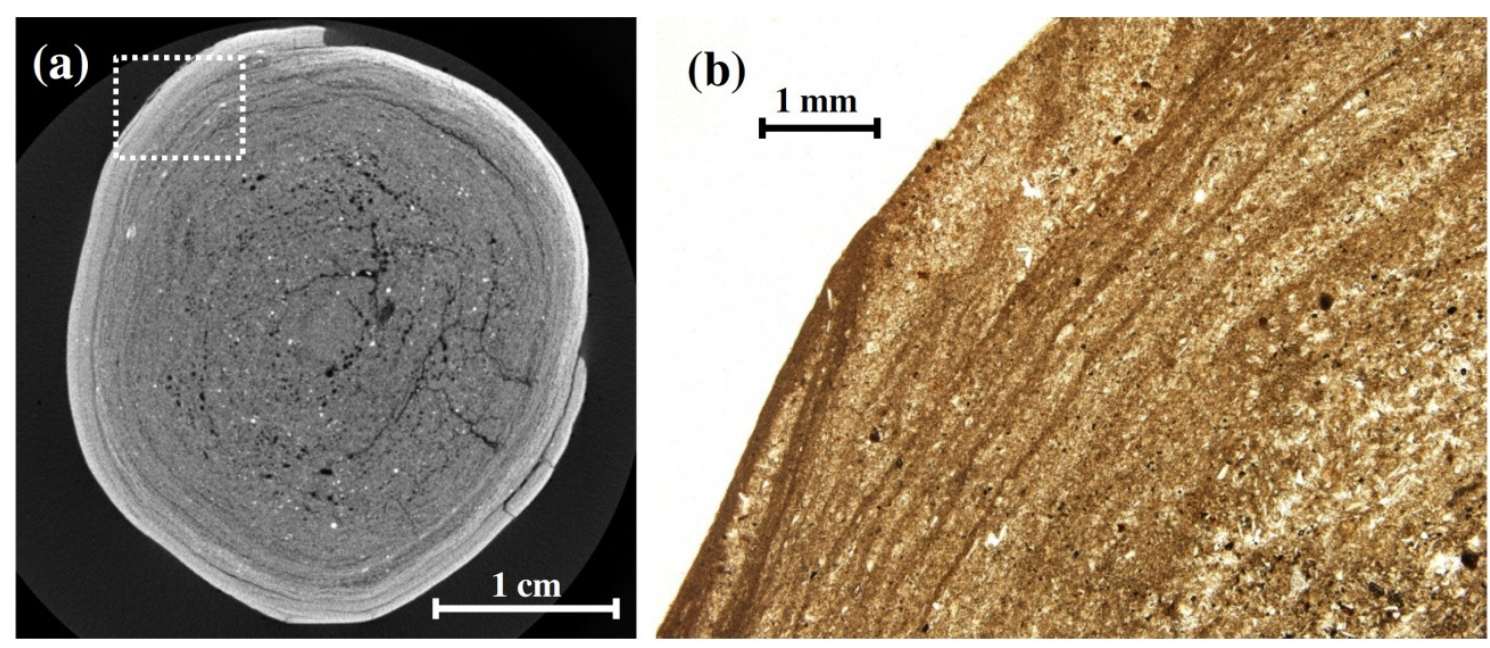

Figure 5.8 Examples of complexly layered accretionary lapilli. (a) X-ray computed microtomography slice through the center of one example from unit 10 at locality 1640 . Lighter shades indicate denser material. Note the massive, vesicular internal core and finely laminated outer layers. (b) Detail of the outer portion of a complexly layered accretionary lapillus from unit 7 at locality 1652, consisting of multiple, very thin laminations of fine to ultrafine ash. 


\subsubsection{Aggregate types - Quantitative classification}

The texturally distinctive pellets and accretionary lapilli described above can also be delineated on the basis of grain size. The characteristic grain size distributions of each type are shown in Figure 5.9a-d. Particle clusters and coalesced ash pellets were not analyzed because they could not be extracted from the deposits reliably intact. However, coalesced ash pellets examined in thin section were found to be texturally similar to massive pellets, and are assumed to have comparable grain size characteristics. Grain size characteristics of the aggregates (Figure 5.10a) are similar to the finest fraction of fall deposit data from the Oruanui eruption [Wilson 2001]. Further comparison of the cores and rims of accretionary lapilli (Figure 5.10b) shows that rims are demonstrably finer and better sorted than the cores. Each aggregate type can be additionally grouped on the basis of grain size (Figure 5.11). All but the ultrafine rim-type accretionary lapilli plot along a linear trend, increasing in fine ash $(63-4 \mu \mathrm{m})$ content with increasingly complex internal structure. The ultrafine rim-types diverge from this trend by showing a distinctive increase in material $<4 \mu \mathrm{m}$. In each category of accretionary lapilli, the internal core is texturally indistinguishable from massive ash pellets. It is therefore inferred that the ash pellets represent an earlier stage of accretionary lapilli growth, which is then variably followed by rim growth under suitable conditions [Brown et al. 2010], as described in detail in Section 5.6.3. 

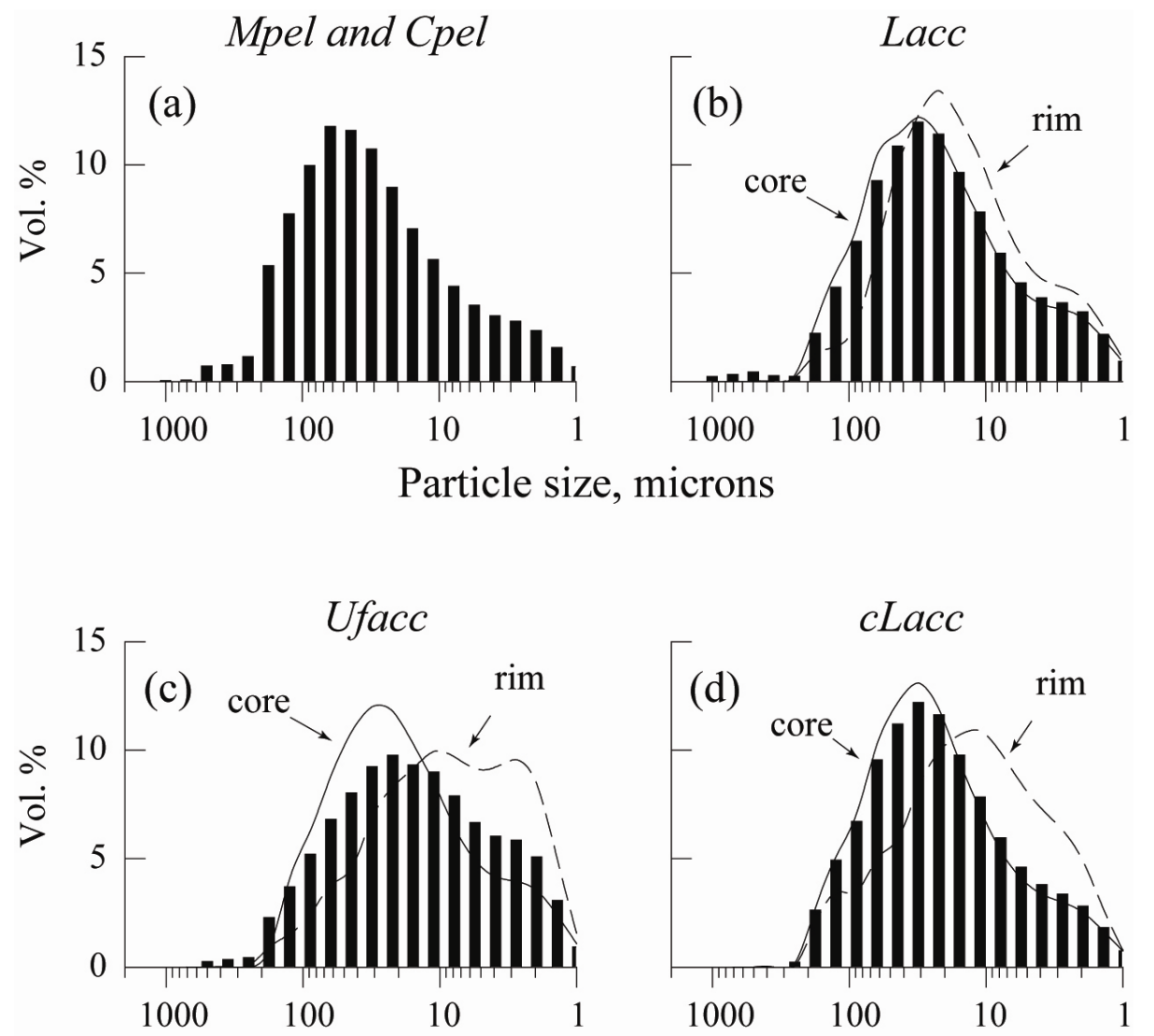

Particle size, microns

Figure 5.9 Representative optical particle size distributions, plotted at half phi intervals, from the aggregate types that could be extracted intact from deposits. Distributions from whole, crushed aggregates are shown as black vertical bars, and cores (solid line) and rims (dashed line) of the accretionary lapilli are fitted with spline curves. (a) Massive and coalesced ash pellets, which are texturally similar; (b) layered accretionary lapilli; (c) ultrafine rim-type accretionary lapilli. Note the grain size of the rim material, which has prominent modes at 4 and $10 \mu \mathrm{m}$. (d) Complexly layered accretionary lapilli. The rim shown in (d) is the outermost layer, which is clearly finer grained than the core but lacks abundant $4 \mu \mathrm{m}$ ash. 

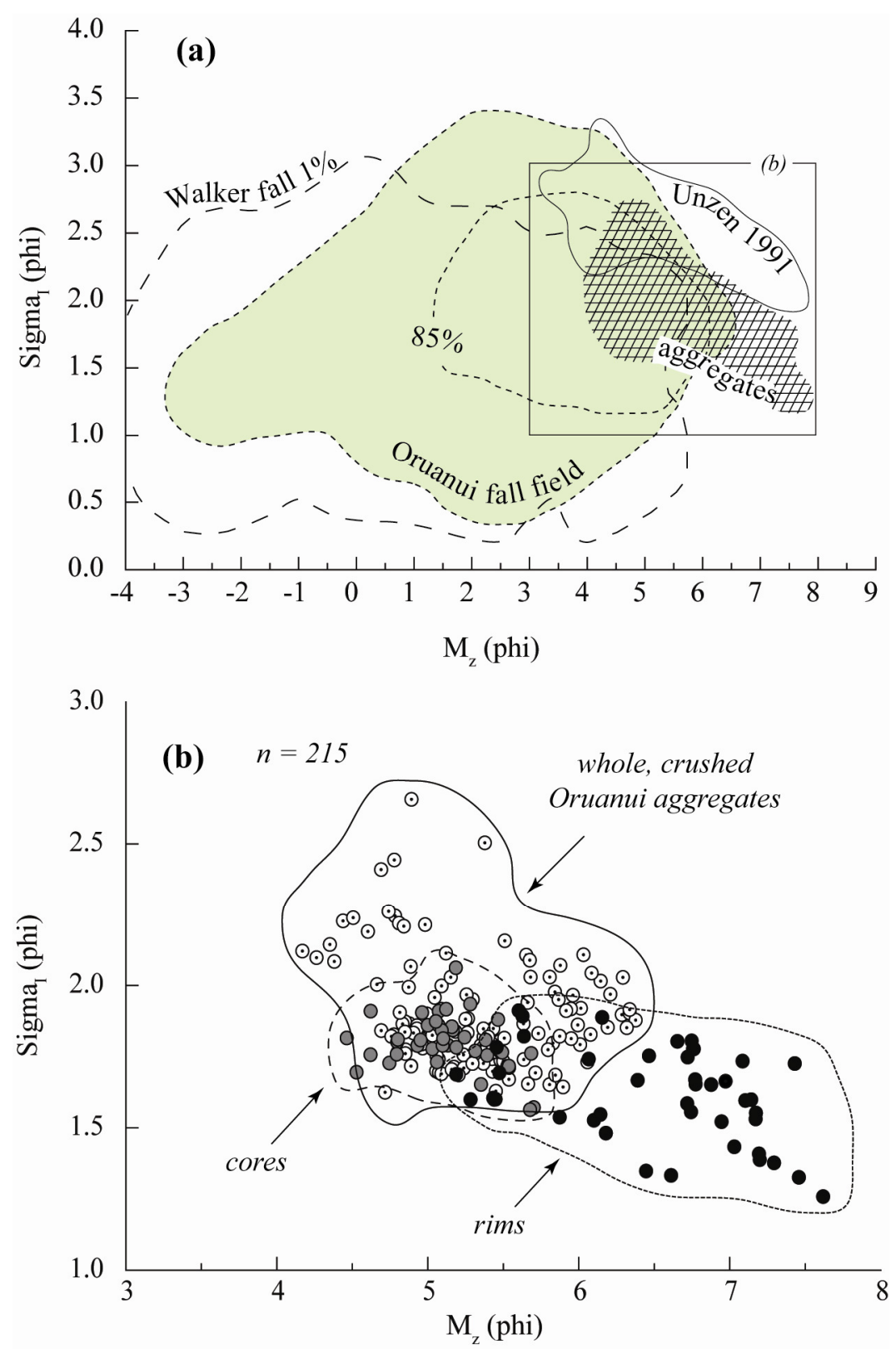

Figure 5.10 Mean grain size $\left(M_{z}\right)$ and inclusive graphic standard deviation $\left(\sigma_{I}\right)$ parameters [Folk and Ward 1957] for Oruanui ash aggregates. (a) Compares the aggregate field to: total Oruanui fall deposit data from Wilson [2001] ( $\mathrm{n}=965$, note inner contour encloses $85 \%$ of the data); fall deposits from Walker [1971], showing the $1 \%$ contour which encloses $\sim 99 \%$ of the data $(\mathrm{n}=\sim 1300)$; and the co-PDC fall field from the 1991 block-and-ash flow of Mount Unzen from Watanabe [1999] ( $n=14)$. (b) Detail of the aggregate data, with symbols denoting whole aggregates (white circles), cores (gray circles) and rims (black circles). 


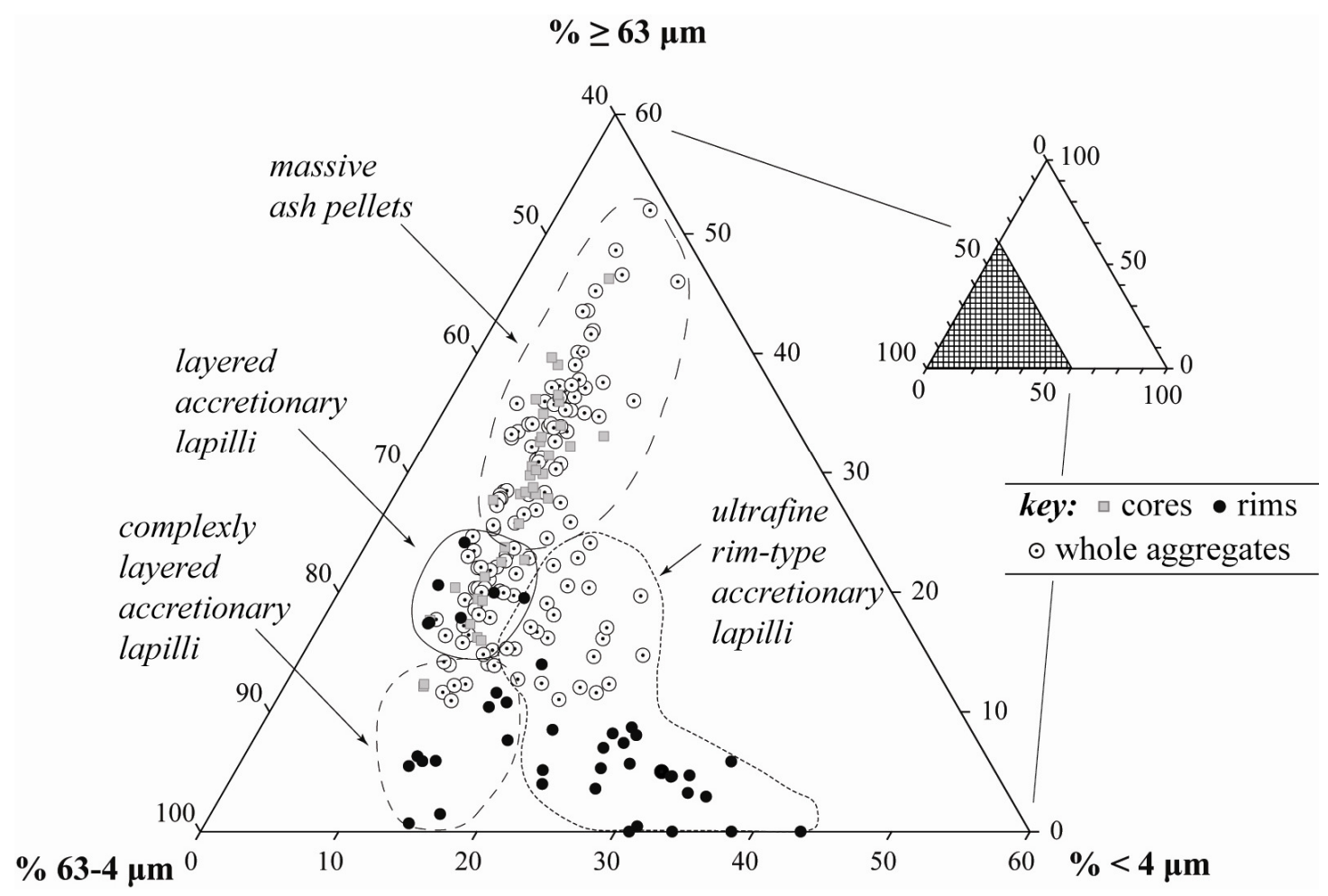

Figure 5.11 Oruanui aggregate grain size data (symbols as in Figure 5.10). Dashed fields indicate the region dominated by each type of aggregate (massive or coalesced ash pellets, layered accretionary lapilli, complexly layered and ultrafine rim-type accretionary lapilli). Note that pellets are coarser than layered and complexly layered accretionary lapilli, while ultrafine rim-types are distinguished on the basis of their $<4$ $\mu \mathrm{m}$ ash contents.

The thickness of rims from accretionary lapilli, collected from a site in the proximal area of fall unit 3 (locality 235), are compared with total diameter expressed as equivalent spherical diameter, or ESD (Figure 5.12a-d). Data from ultrafine rim-types and layered accretionary lapilli indicate that the larger aggregates generally have thicker rims, as also observed in the Laacher See tephra [Schumacher and Schmincke 1991, their Figure 5.10a-b]. There is also a weak positive relationship between rim volume and core diameter (Figure 5.12b), suggesting that the larger cores are more effective at scavenging ultrafine ash. Measurements of bulk density, mass, aspect ratio and calculated terminal fall velocity are plotted with respect to aggregate ESD in Figure 5.13. Density does not show a significant dependence on aggregate size (Figure 5.13a), and best-fit regressions from plots of ESD vs. mass give average dry and wet aggregate densities of 1.36 and $1.63 \mathrm{~g} \mathrm{~cm}^{-3}$, respectively (Figure 5.13b). Figure 5.13c shows that the largest aggregates $(>1.5 \mathrm{~cm})$ have generally smaller aspect ratios, indicating greater sphericity, and very few show elongate morphologies like those observed from El 


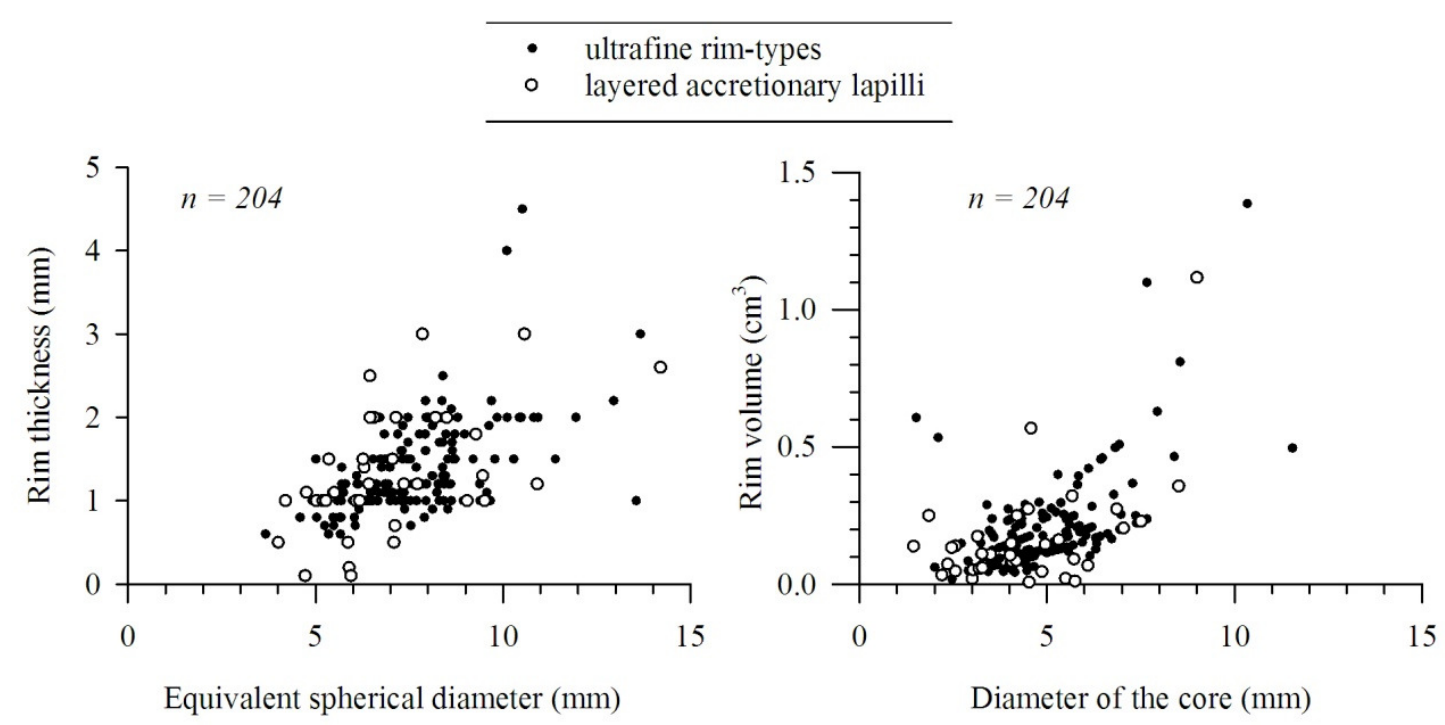

Figure 5.12 Core-rim relationships in ultrafine rim-type and layered accretionary lapilli from proximal unit 3 at locality 235. (a) Rim thickness versus total aggregate diameter (of an equivalent sphere). (b) Rim volume versus the core diameter.

Chichón by Scolamacchia et al. [2005]. Fall velocities are estimated for wet and dry aggregates in Figure 5.13d, exhibiting roughly parallel, linear relationships with ESD.

\subsection{Stratigraphic context}

One of the key challenges in studies of wet-erupted deposits is determining the role of water involvement at each stage of eruption, transport and deposition [Houghton et al. 2000]. Although explosive mixing of magma and water is inferred to generate abundant fine ash, the quantitative relationships between fine ash content, magma:water ratio and eruption energetics are poorly defined [Zimanowski et al. 2003]. A number of processes can alter the 'initial' vent-derived grain size distribution, including milling in the column or PDCs during transport, and wet aggregation during sedimentation. Although all phases of the Oruanui eruption saw some magma-water interaction at vent (as evidenced by the wholly non-welded nature of the ignimbrite), each unit exhibits different degrees of 'wet' deposition in terms of fine ash content and aggregate types. For this study, the Oruanui deposits are considered within a framework of three intergradational styles of wet eruption and deposition: (1) wet throughout; (2) alternating wet and dry; and (3) 'drier' plinian-like, focusing on units 2, 3, 5, 6, 7 and 8. Table 5.2 summarizes the main aggregate-bearing facies in the Oruanui eruption 
deposits, as well as their occurrence and inferred processes involved in transport and deposition.
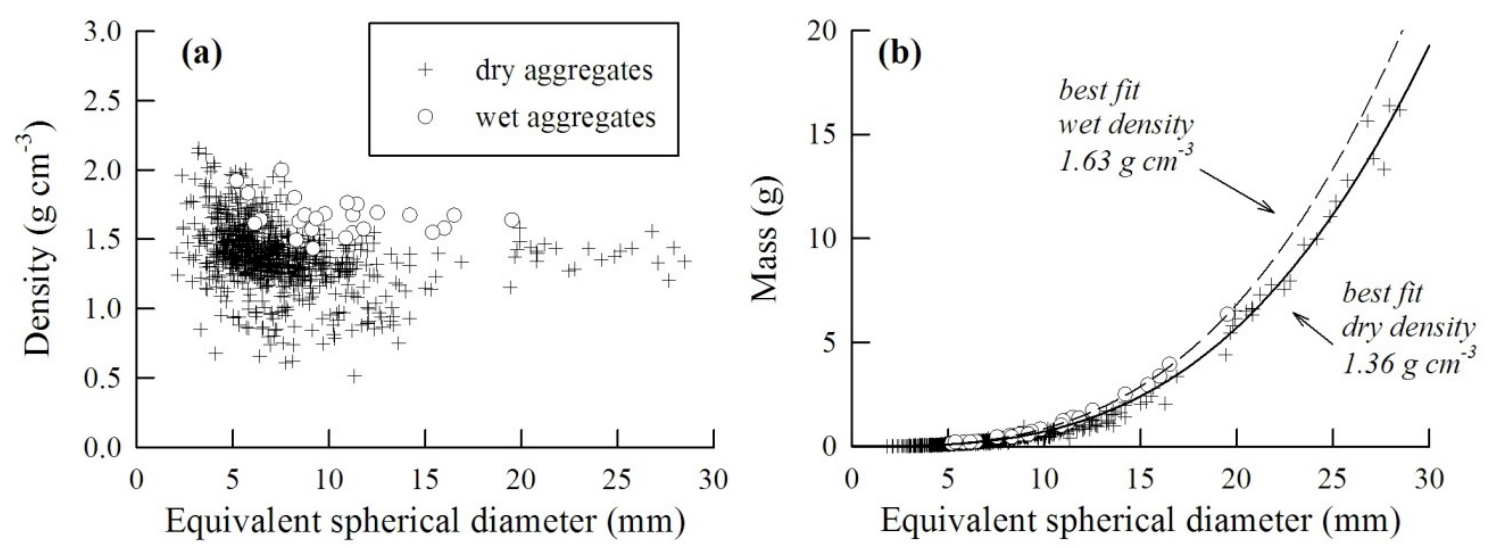

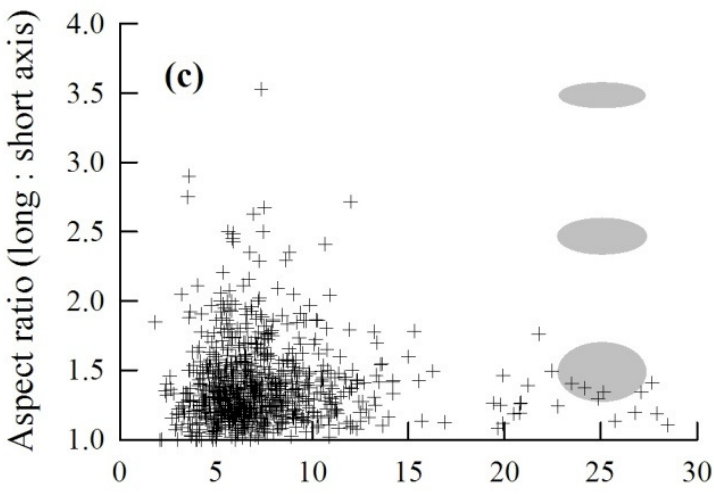

Equivalent spherical diameter $(\mathrm{mm})$

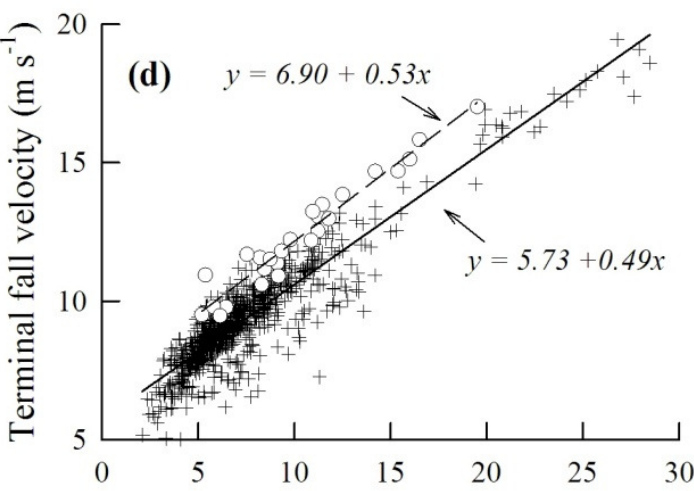

Equivalent spherical diameter $(\mathrm{mm})$

Figure 5.13 Compiled morphological and dimensional data from 886 individual aggregates from units 3, 6, 7 and 8 at multiple localities. (a) Densities obtained by measuring the three principal axes and mass of air-dried (open circles) and undried aggregates (crosses). (b) Mass versus equivalent spherical diameter, showing densities derived from least squares cubic regressions $\left(y=7.14 \times 10^{-4} \mathrm{x}^{3}\right.$ for dried aggregates; $\mathrm{y}=$ $8.54 \times 10^{-4} \mathrm{x}^{3}$ for wet aggregates). Both equations yield $\mathrm{r}^{2}$ values of 0.99 and $\mathrm{p}<0.0001$. (c) Aspect ratio of longest to shortest axis for dried aggregates, with grey ovals illustrating the shape of spheres with aspect ratios of 1.5, 2.5 and 3.5. (d) Terminal fall velocity (w) for 2-30 mm diameter aggregates at mean sea level, approximated as in Carey and Sparks (1986) using $\mathrm{w}=\mathrm{Cd} \cdot \sqrt{ }(\mathrm{d} \cdot \mathrm{g} \cdot \rho / \beta)$, where $\mathrm{Cd}=\mathrm{drag}$ coefficient (taken as 1.054), $\mathrm{d}=$ equivalent spherical diameter, $\mathrm{g}=$ acceleration due to gravity, $\rho=$ measured aggregate density in $\mathrm{kg} \mathrm{m}^{-3}$, and $\beta=$ air density at sea level $\left(1.2 \mathrm{~kg} \mathrm{~m}^{-3}\right)$. Linear least squares regression curves are shown for dry and wet terminal fall velocities (with $r^{2}$ values of 0.84 and 0.94 , respectively; $p<0.0001$ for both). 
Table 5.2 Aggregate facies in the Oruanui eruption deposits.

\begin{tabular}{|c|c|c|}
\hline Aggregates & Facies description and occurrence & Inferred processes \\
\hline $\mathrm{pc}$ & $\begin{array}{l}\text { Low-density fine ash beds with vesicular texture; } \\
\text { occur in distal exposures (e.g. Chathams) }\end{array}$ & $\begin{array}{l}\text { Primarily dry or 'moisture- } \\
\text { flushed' fallout }\end{array}$ \\
\hline $\begin{array}{l}\text { Mpel } \pm \\
\text { Cpel }\end{array}$ & $\begin{array}{l}\text { Dense, clast-supported beds of massive ash pellets } \\
\text { and/or variably coalesced ash pellets. Forms the } \\
\text { basal sequence of unit } 3 \text {; also found interlayered } \\
\text { with PDC deposits and in the distal area. }\end{array}$ & $\begin{array}{l}\text { Fallout from a simple, } \\
\text { water-rich plume }\end{array}$ \\
\hline Lacc & $\begin{array}{l}\text { Clast-supported or 'just' clast supported, layered } \\
\text { accretionary lapilli. Examples with matrix between } \\
\text { aggregates is generally larger clasts or broken } \\
\text { aggregates Occur in cm-dm thick beds interlayered } \\
\text { with PDC deposits and well beyond the reaches of } \\
\text { PDCs. }\end{array}$ & $\begin{array}{l}\text { Fallout from a complex, } \\
\text { turbulent cloud system }\end{array}$ \\
\hline $\begin{array}{l}\text { Mpel + } \\
\text { Lacc }\end{array}$ & $\begin{array}{l}\text { Whole and broken massive pellets and layered } \\
\text { accretionary lapilli; matrix-supported or 'just' } \\
\text { matrix-supported in a weakly cross-bedded matrix. } \\
\text { Directly underlies the first appearance of ultrafine } \\
\text { rim-types in unit } 3 \text {. }\end{array}$ & $\begin{array}{l}\text { Laterally-emplaced; early } \\
\text { PDCs interacting with a } \\
\text { high plume; limited } \\
\text { elutriation and segregation } \\
\text { of fines from the ground- } \\
\text { hugging flows. }\end{array}$ \\
\hline Ufacc & $\begin{array}{l}\text { Whole and broken ultrafine rim-type accretionary } \\
\text { lapilli; Occur scattered or in pulses throughout } \\
\text { weakly cross-stratified, normally graded to massive } \\
\text { beds with scoured bases. }\end{array}$ & $\begin{array}{l}\text { Laterally-emplaced; PDCs } \\
\text { interacting with a high } \\
\text { plume; indicates the } \\
\text { presence of a low-altitude } \\
\text { cloud of elutriated ultrafine } \\
\text { ash and condensed } \\
\text { moisture. }\end{array}$ \\
\hline cLacc & $\begin{array}{l}\text { Matrix-suppported, complexly layered accretionary } \\
\text { lapilli. Scattered occurrences in concentrated flows } \\
\text { (s.s.), gas escape pipes, and in overlying vitric } \\
\text { coignimbrite ash. }\end{array}$ & $\begin{array}{l}\text { Laterally-emplaced; } \\
\text { vigorous interaction of } \\
\text { PDCs with an overriding } \\
\text { cloud system. }\end{array}$ \\
\hline $\begin{array}{l}\mathrm{Lacc}+ \\
\text { Ufacc }\end{array}$ & $\begin{array}{l}\text { 'Just' matrix-supported, mixed assemblage of } \\
\text { layered and ultrafine accretionary lapilli found at the } \\
\text { distal fringes of PDC deposits. }\end{array}$ & $\begin{array}{l}\text { Primarily fallout; waning } \\
\text { interaction between PDCs, } \\
\text { elutriated ash and a higher } \\
\text { plume. }\end{array}$ \\
\hline
\end{tabular}

\subsubsection{Wet throughout: unit 3}

Unit 3 deposits provide the most detailed record of transitions between aggregate types in time (vertically in the sections) and space (laterally) due to excellent preservation near the base of the Oruanui eruptive stratigraphy. Unit 3 is also the most uniformly fine-grained part of the Oruanui; even the near-vent PDC deposits have $>50$ wt. $\%$ finer than $63 \mu \mathrm{m}$, suggesting that this eruptive phase saw the most extensive magma-water fragmentation and 'moisture-flushed' fallout [Wilson 2001]. As shown in stratigraphic logs (Figure 5.14a-j), the overall size and complexity of ash aggregates diminish with 

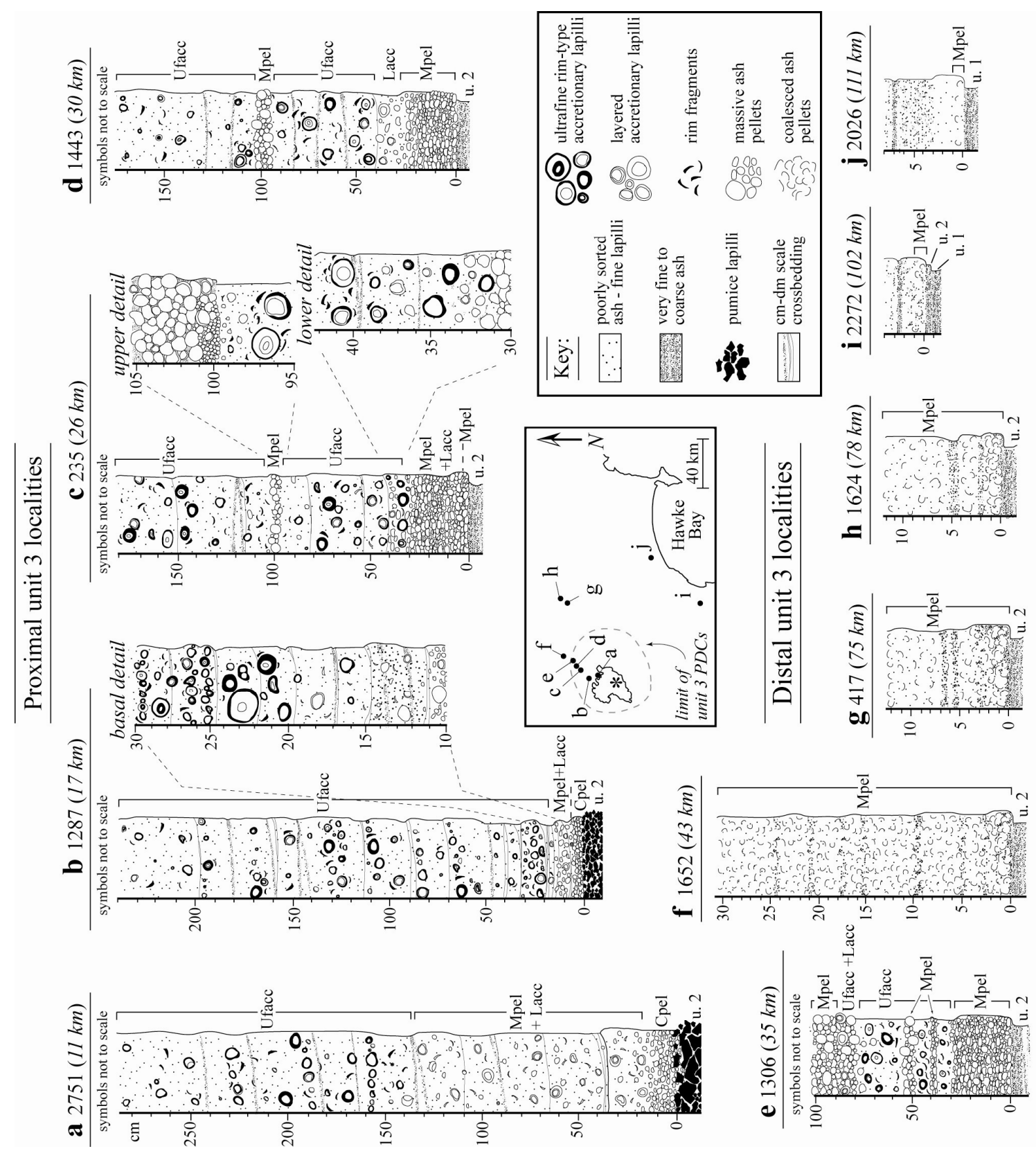

Figure 5.14 Stratigraphic logs showing the erosional profile and dominant aggregate types in unit 3 deposits at localities (Appendix A for grid references) with varying distance from virtual source (indicated in italics). Vertical scale is in $\mathrm{cm}$ and ' $\mathrm{u} .2$ ' $=$ unit 2 as the basal reference unit. Where indicated, symbols are not to scale but represent the observed textural characteristics of the given interval. Proximal localities (a-d) are dominated by laterally emplaced material with fragmented, ultrafine rim-type accretionary lapilli. At the transitional locality in (e), fall material dominates in the form of clast-supported aggregates, with intercalated PDC deposits. Distal localities (f-j) are pure fall and include only massive to weakly structured pellets. 
distance from vent, and are closely related to the emplacement of PDCs. These aggregation characteristics are described with respect to the proximal, transitional and distal depositional regimes shown in Figure 5.2. Features of the unit 3 deposits are thus used to introduce the primary mechanisms of aggregate development, and to provide a template against which the other aggregate-bearing Oruanui units are compared.

\section{Proximal regime: PDC-dominated, mixed deposition}

The PDC-dominated proximal regime in unit 3 extends to $\sim 30 \mathrm{~km}$ from vent (Figure 5.14a-d). Here, the majority of the deposits were laterally emplaced by PDCs, recorded by the occurrence of fragmented accretionary lapilli in a poorly sorted matrix with scoured contacts and pinch and swell bedding. However, even in the proximal area, distinctive phases of fall deposition can be identified from the aggregate facies and grain size characteristics. For example, the base of unit 3 is everywhere a denselypacked sequence of clast-supported, wholly to partially coalesced, massive ash pellets that is recognizable outwards into the distal area. These pellets commonly exhibit thin outer films, suggesting they were moist upon landing, or landed frozen but melted shortly afterwards (see Section 5.4.1). This facies is inferred to represent fallout from a buoyant, water-rich cloud, illustrated as stage (i) in Figure 5.15.

Upwards in the sequence, deposits grade into a mixed assemblage of massive pellets and layered accretionary lapilli that are fragmented and matrix-supported, reflecting the earliest emplacement of PDCs related to partial column collapse. Coeval inputs of both massive pellets and layered accretionary lapilli are inferred to indicate increasing complexity in the buoyant plume, driven by interaction with ground-hugging currents (stage (ii) of Figure 5.15). This behavior would promote recycling of aggregates through an increasingly complex, multi-level cloud system. Occurrence of massive ash pellets in unit 3 deposits well beyond the limit of its PDCs (Figures 5.14f-j) suggests that conditions in the buoyant cloud must be suitable for their formation. It is therefore envisaged that some of the simple, massive pellets falling out of the high plume from phase 3 experienced additional growth during re-entrainment and/or passage through stratified levels of the buoyant cloud. During this prolonged transport, the outer layers characteristic of layered accretionary lapilli were formed. Increasing cloud complexity at this stage of the eruption was likely triggered by generation of PDCs which acted as 
wedges to generate warm, moisture-laden updrafts beneath the higher, vent-derived plume (e.g., Figure 2.5 of this thesis and Van Eaton et al. [2012a]). However, a notable lack of ultrafine rims in the mixed assemblage of aggregates indicates that conditions amenable to their formation were not consistently met during early development of the hybrid cloud system.

(i) Simple, buoyant plume: Cpel-Mpel

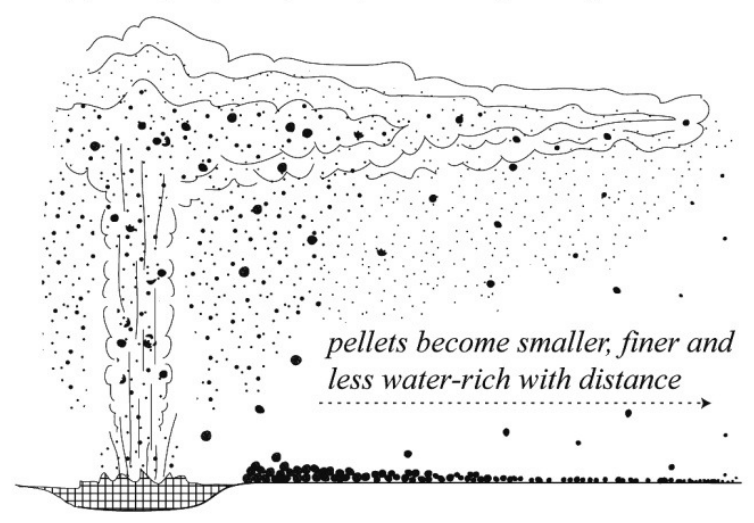

(ii) Early-stage interaction with PDCs: Lacc

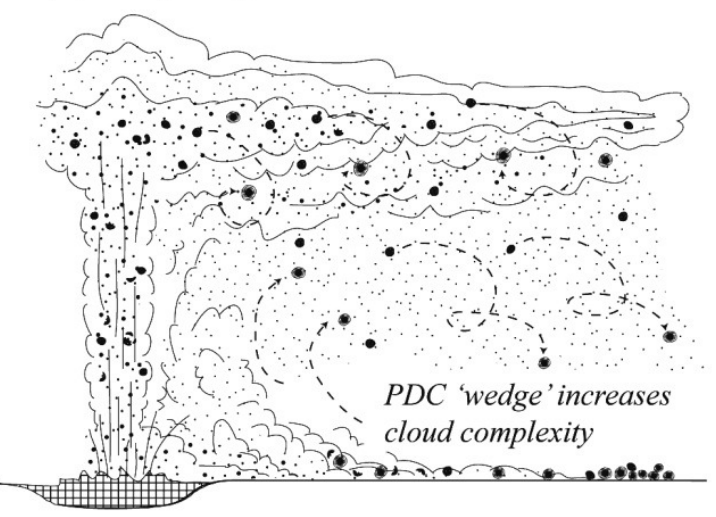

(iii) Late-stage interaction with PDCs: Ufacc

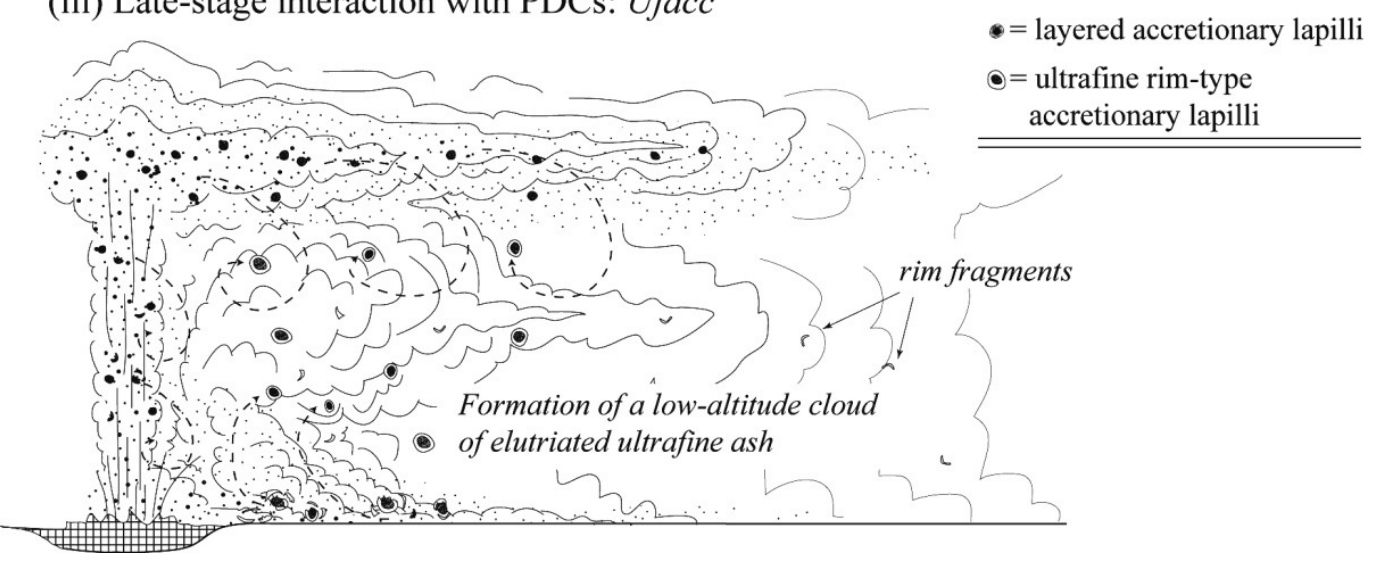

Figure 5.15 Schematic model for the formation of observed aggregate textures in unit 3 deposits. Stage (i) is characterized by fallout from a high, stable plume, depositing clastsupported massive (Mpel) and coalesced ash pellets (Cpel) (see text for definitions of terms). In stage (ii), the early generation of PDCs forces vigorous thermals of moistureand ash-laden air upwards, which interact with the high plume to form a hybrid volcanic cloud. Increased recycling promotes formation of layered accretionary lapilli (Lacc), which fragment as they drop into the ground-hugging PDCs. Stage (iii) represents the multiple pulses of PDCs elutriating preferentially finer ash as they weaken with distance from vent. The lower atmosphere becomes choked with ultrafine ash, providing the final stage of growth for ultrafine rim-type accretionary lapilli (Ufacc) before they drop onto the flows and breakup during transport. Some rim fragments generated in the PDCs behave as coherent particles and are re-entrained into the buoyant, airborne cloud. 
The ultrafine rim-type accretionary lapilli appear in abundance directly above this mixed assemblage. They are accompanied by abundant fragments of cuspate, ultrafine rims in a relatively loose, sandy matrix. This material comes in as multiple, $\mathrm{cm}-\mathrm{dm}$ scale beds, characterized by normal grading and tops rich in ultrafine rim-type accretionary lapilli (Figure 5.16a). The beds represent episodic emplacement of turbulent, dilute PDCs [Wilson 2001], followed by accretionary lapilli fallout from the overlying hybrid plinian-coignimbrite cloud. As depicted in stage (iii) of Figure 5.15, this situation may have arisen in the later stage of hybrid cloud development, during which ultrafine ash was preferentially elutriated from the ground-hugging currents [Neri et al. 2002, Van Eaton et al. 2012a]. As massive pellets (or layered accretionary lapilli) dropped out of the buoyant plume, they passed through the low-level coignimbrite cloud. There, the availability of condensed moisture and multiple, turbulent updrafts in the ascending thermals would provide optimum conditions for accretion of additional (ultrafine ash) layers. The growing accretionary lapilli would then drop out of the suspended cloud into the laterally-moving undercurrent, where abrasion and breakage occurred before deposition.

The temporal shift from fall to PDC-dominated deposition described above is reflected in the unit 3 aggregate characteristics (see 'basal detail' of Figure 5.14b). The distinction between material that was emplaced primarily as vertical fallout or PDCs is also reflected in grain size characteristics. Figure 5.17 shows laser diffraction grain size data from a proximal exposure (locality 235: $26 \mathrm{~km}$ from vent). The proximal PDC material is typified by matrix-supported accretionary lapilli with abundant ultrafine ash (>10-15 vol.\% $<4 \mu \mathrm{m}$ ). In contrast, the clast-supported massive ash pellets or layered accretionary lapilli characterizing fall deposits generally have a coarser mode ( $>60 \mu \mathrm{m})$ and $<4 \mu \mathrm{m}$ ash contents less than $10 \mathrm{vol} . \%$.

\section{Transitional regime: fall-dominated, mixed deposition}

The region $\sim 30-40 \mathrm{~km}$ downwind of vent (Figure 5.14e) is defined by fall-dominated, mixed deposition ( $>50 \%$ fall material by thickness), and includes more distinctly interbedded fall and PDC deposits. Similar to the proximal region, the basal layer here comprises partially to wholly coalesced ash pellets. However, rather than grading upward into a matrix-supported, fragmented assemblage of massive ash pellets and 

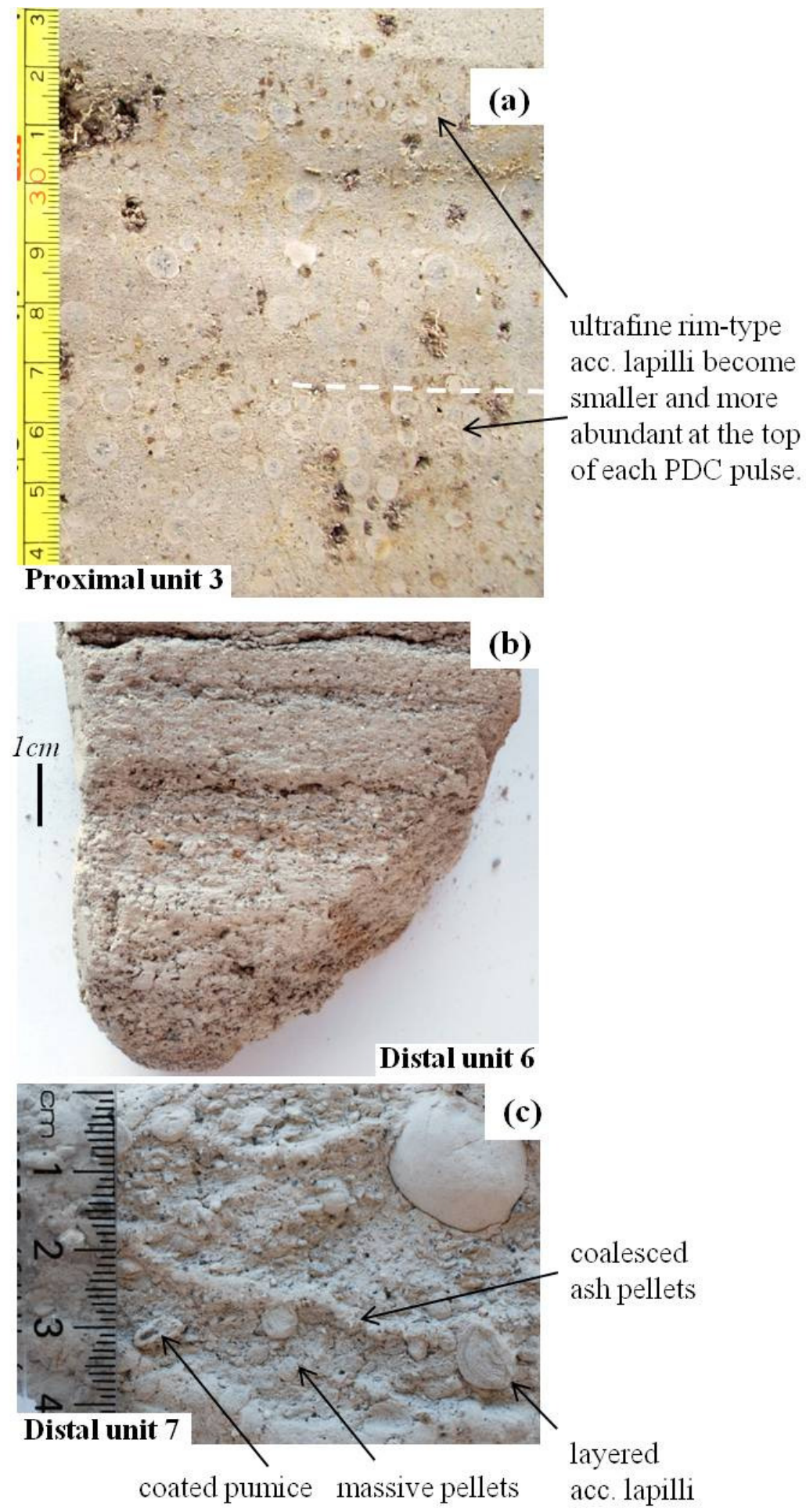

Figure 5.16 Ash aggregate textures in Oruanui units 3, 6 and 7. (a) Field photo of a scraped face from proximal unit 3 at locality 1287, $17 \mathrm{~km}$ from source, showing cm$\mathrm{dm}$ scale pulses of PDC deposits with tops rich in smaller, ultrafine rim-type accretionary lapilli. Scale in $\mathrm{cm}$. (b) Dried, etched monolith of distal unit 6 ash from locality 417, showing the aggregate-bearing coarse ash facies. Note the whitish, partially coalesced ash pellets at the base of the sample. (c) Complex aggregate deposition in distal unit 7 from locality 417. Contact between the finer base and coarse top of unit 7 occurs at the $3.2 \mathrm{~cm}$ mark. Notice the matrix-supported massive ash pellets, irregular lenses of coalesced ash pellets and larger layered accretionary lapilli deposited in close succession and mixed with coarse ash to fine lapilli throughout. 


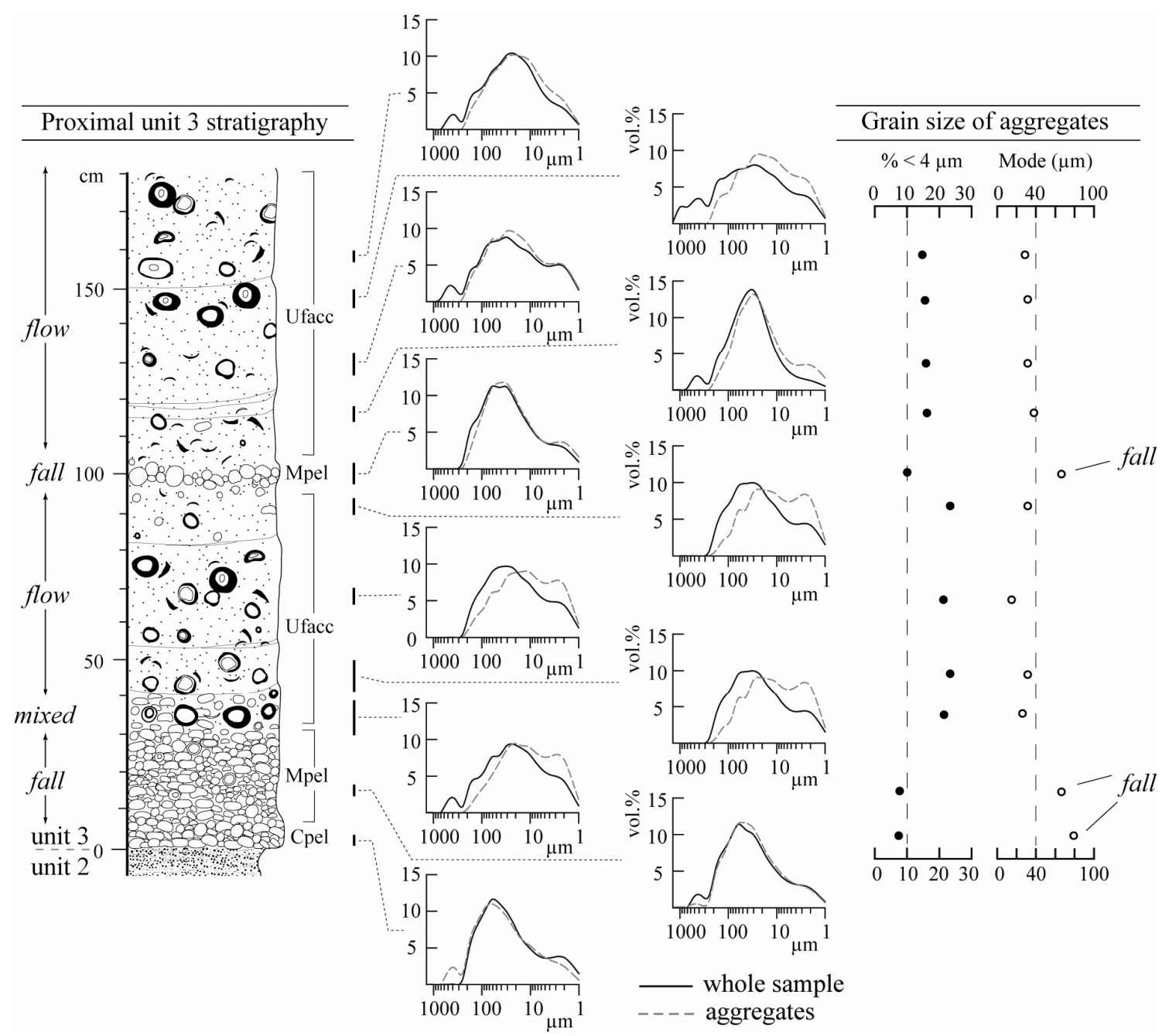

Figure 5.17 Proximal unit 3 stratigraphy at locality 235, showing grain size distributions of bulk samples (solid lines) and extracted ash aggregates from each interval. LDPSA data are shown as optical particle diameters from $1 \mu \mathrm{m}$ to $1.4 \mathrm{~mm}$, binned into half phi intervals and fitted with spline curves. Grain size statistics of the aggregates are shown as vol. $\%<4 \mu \mathrm{m}$ and the dominant mode (data points are horizontally aligned with the midpoint of each stratigraphic interval). Vertical dashed lines represent the cutoff point between characteristics of fall and PDC deposits (proximal and transitional regions only).

layered accretionary lapilli, the medial equivalents are clast-supported and inferred to have been emplaced as fall. The finer grain size of these massive ash pellets, combined with their comparatively loosely-packed internal structure and less deformed contacts (e.g. Figure 5.5a) indicate less overall water involvement [Section 4.3 .3 of this thesis] when compared to the underlying sequence of coalesced ash pellets. This shift is consistent with a cloud system that was stratified with respect to water content. Pulses of the farthest-reaching dilute PDCs are marked by a sharp basal contact against the fall 
material, with ultrafine rim-type accretionary lapilli and rim fragments abundant in the matrix. This stratigraphic succession is inferred to result from an overriding coignimbrite ash cloud interacting with fallout from a higher wet plume.

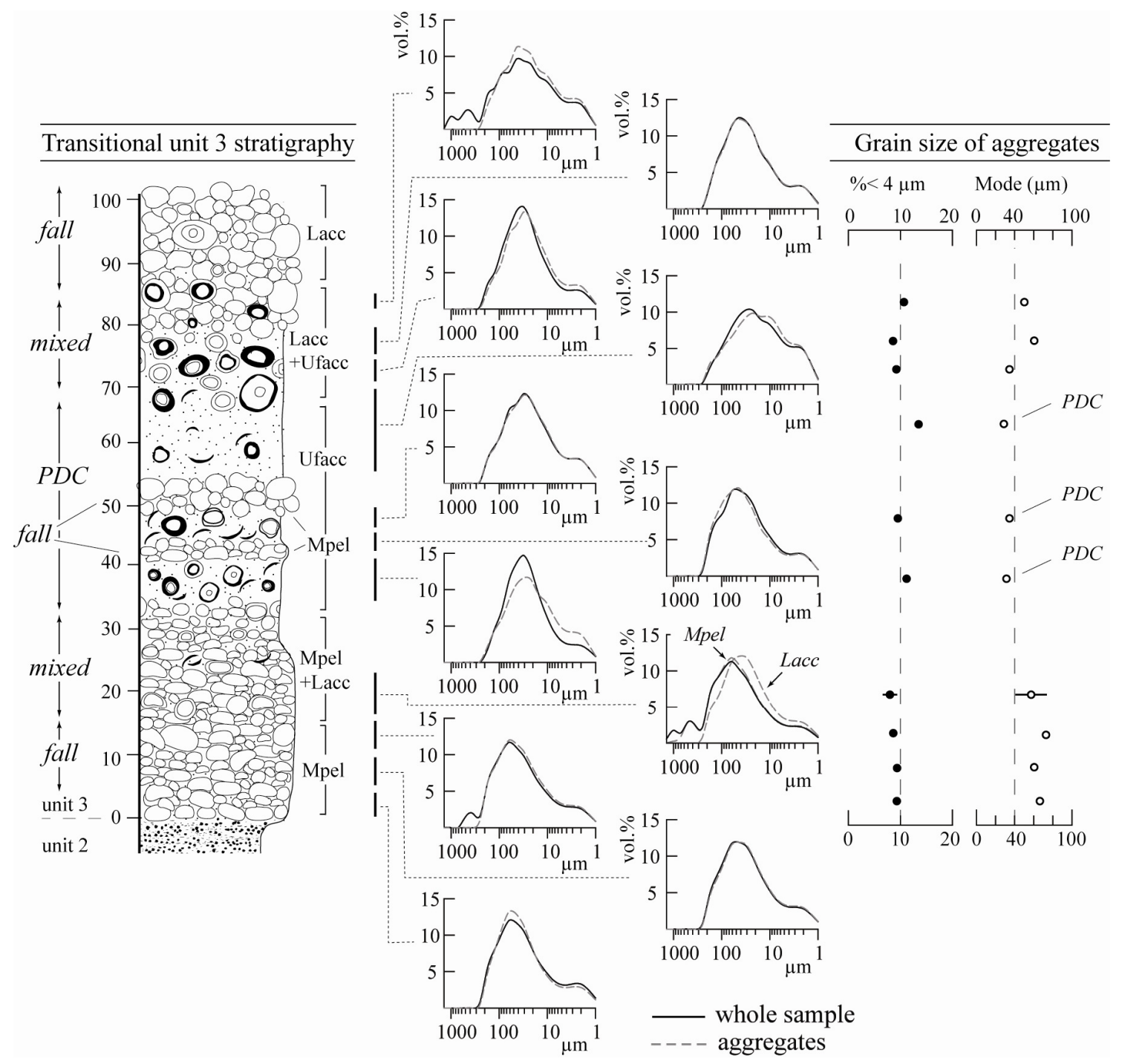

Figure 5.18 Transitional unit 3 stratigraphy at locality 1306 (U17/830941), showing grain size data from bulk samples and enclosed aggregates, as in Figure 5.17. Note that the aggregate data from $15-22 \mathrm{~cm}$ include both massive pellets (Mpel) and layered accretionary lapilli (Lacc).

Grain size data from a representative exposure in the transitional area (locality 1306: $\sim 35 \mathrm{~km}$ from vent) are given in Figure 5.18. The basal $30 \mathrm{~cm}$ of unit 3 fall material at this site shows a gradual shift from partially coalesced, massive pellets to clast supported pellets plus layered accretionary lapilli. Sparse fragments $(<1$ modal vol.\%) of ultrafine rims are also present above $\sim 15 \mathrm{~cm}$. These fragments are clearly foreign to the enclosed aggregates (which do not have ultrafine layers themselves), and it is 
inferred that some of the ultrafine rim fragments were generated in PDCs closer to source, and re-entrained into a buoyant, farther travelled portion of the plume (stage (iii) of Figure 5.15). Samples from the basal $30 \mathrm{~cm}$ show aggregates with $<4 \mu \mathrm{m}$ ash contents $<10$ vol.\%, and grain size modes of generally 60 to $80 \mu \mathrm{m}$. Above this, deposition shifted to alternating beds of laterally-and vertical-emplaced material. Grain size analysis of aggregates from the laterally-emplaced beds show ultrafine ash contents generally $\geq 10 \mathrm{vol} . \%$ and a finer grain size mode of $\sim 40 \mu \mathrm{m}$. Where the final input of PDC material fades out above $65 \mathrm{~cm}$, the deposits contain 'just' matrix-supported, ultrafine and layered accretionary lapilli, indicating the waning influence of PDCderived, elutriated ash and a return to the stage (ii) scenario shown in Figure 5.15. As the hybrid cloud continued to weaken, the deposit grades into partially coalesced massive ash pellets, representing a return to pure fall from a simple, buoyant plume (above $\sim 90 \mathrm{~cm}$ in Figure 5.18).

\section{Distal regime - pure fall}

The limits of PDCs in unit 3 are signified by a lateral transition to pure fall material $>40$ $\mathrm{km}$ from vent (Figure 5.14f-j). The dominant, accreted portions of the fall material are characterized by clast-supported, massive ash pellets, diminishing in size and cohesiveness into the distal area. At distances $>100 \mathrm{~km}$, aggregates occur as very small pellets $(<1 \mathrm{~mm}$ diameter $)$ or particle clusters, and are found in association with similarly-sized, ash-coated pyroclasts (see thin sections in Figure 5.5e-f). Grain size analyses from locality 417 ( $75 \mathrm{~km}$ from vent) are shown in Figure 5.19. Due to the structural weakness of unit 3 aggregates in the distal area, individual pellets could not be reliably extracted; therefore, only the grain size data from whole ash deposit samples are shown. Grain size modes vary from $40-70 \mu \mathrm{m}$ and generally $10 \mathrm{vol} \%$ or less is composed of sub-4 $\mu \mathrm{m}$ ash. Considering that the sampled intervals include aggregates as well non-aggregated coarser particles, it is reasonable to infer that the enclosed massive ash pellets are themselves even finer. Therefore, by comparison with grain size analyses from ash pellets in the proximal and transitional regions, this pellet fall material exhibits an overall fining trend downwind into the distal area. 


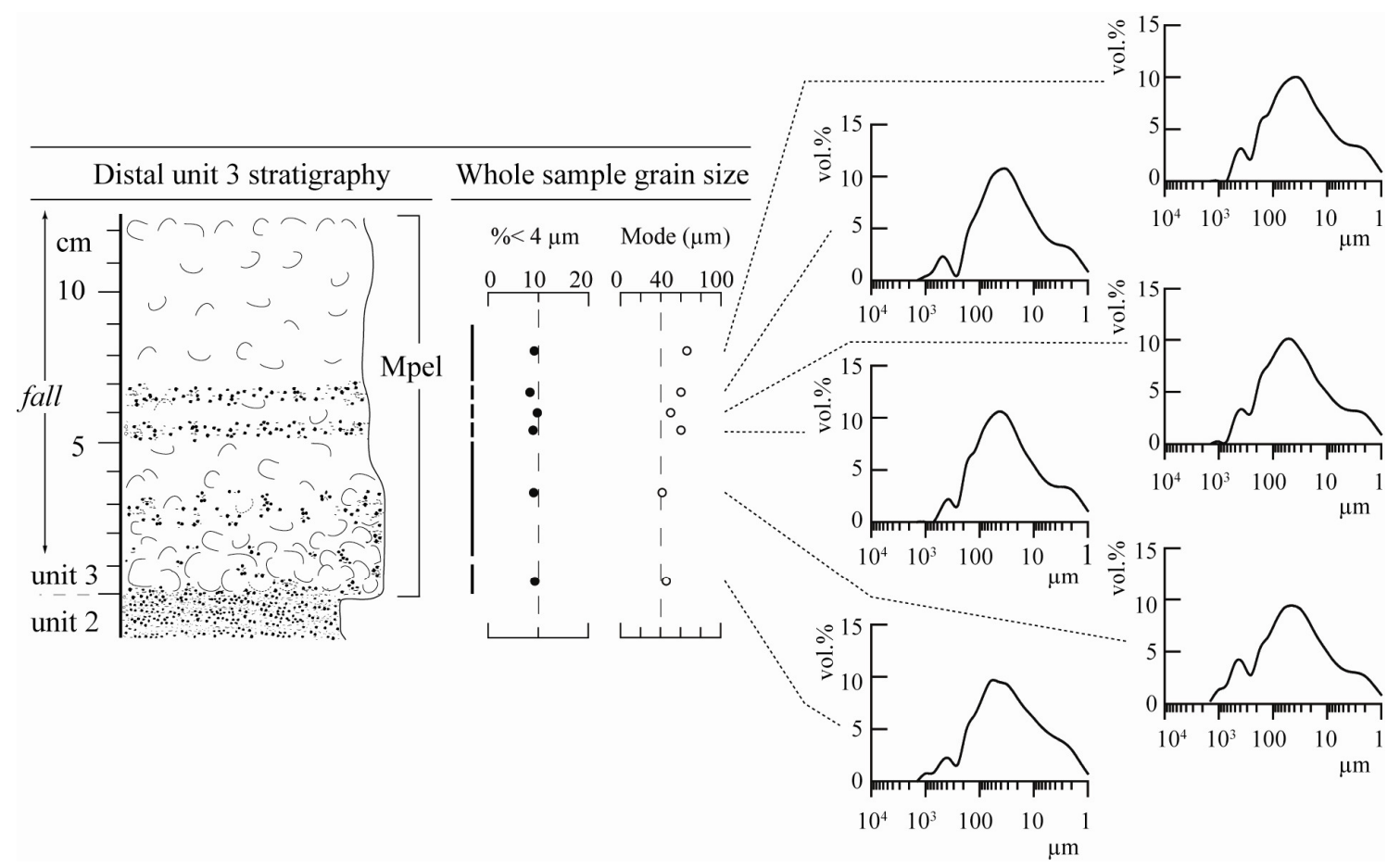

Figure 5.19 Distal unit 3 stratigraphy at locality 417 showing the size statistics and grain size distributions of whole samples as in Figure 5.17 (note that individual aggregates could not be extracted for analysis). Notice the overall finer grain size compared to the fall material from proximal and transitional regimes.

\section{Lateral coarsening of unit 3}

Despite evidence described above indicating that massive ash pellets dropping out of the buoyant plume become finer with distance from source, Wilson [2001] identified a coarsening signature in the overall unit 3 deposits. Samples collected over the full deposit thickness at each site show that the mean grain size coarsens up to a distance $\sim 110 \mathrm{~km}$ from vent and fines beyond [Figure 13 of Wilson 2001]. The fractionation of each whole-phi grain size interval is shown in Figure 5.20 (a). At the time of writing, this coarsening signature has only been previously documented in the aggregate-bearing facies of the $1.8 \mathrm{ka}$ Taupo eruption [Smith 1998] and in the Unzen co-PDC deposits [Figure 13b of Watanabe et al. 1999], although it may be a more common feature of aggregated eruption deposits than previously assumed. This observation is related to two mechanisms: 
(a)
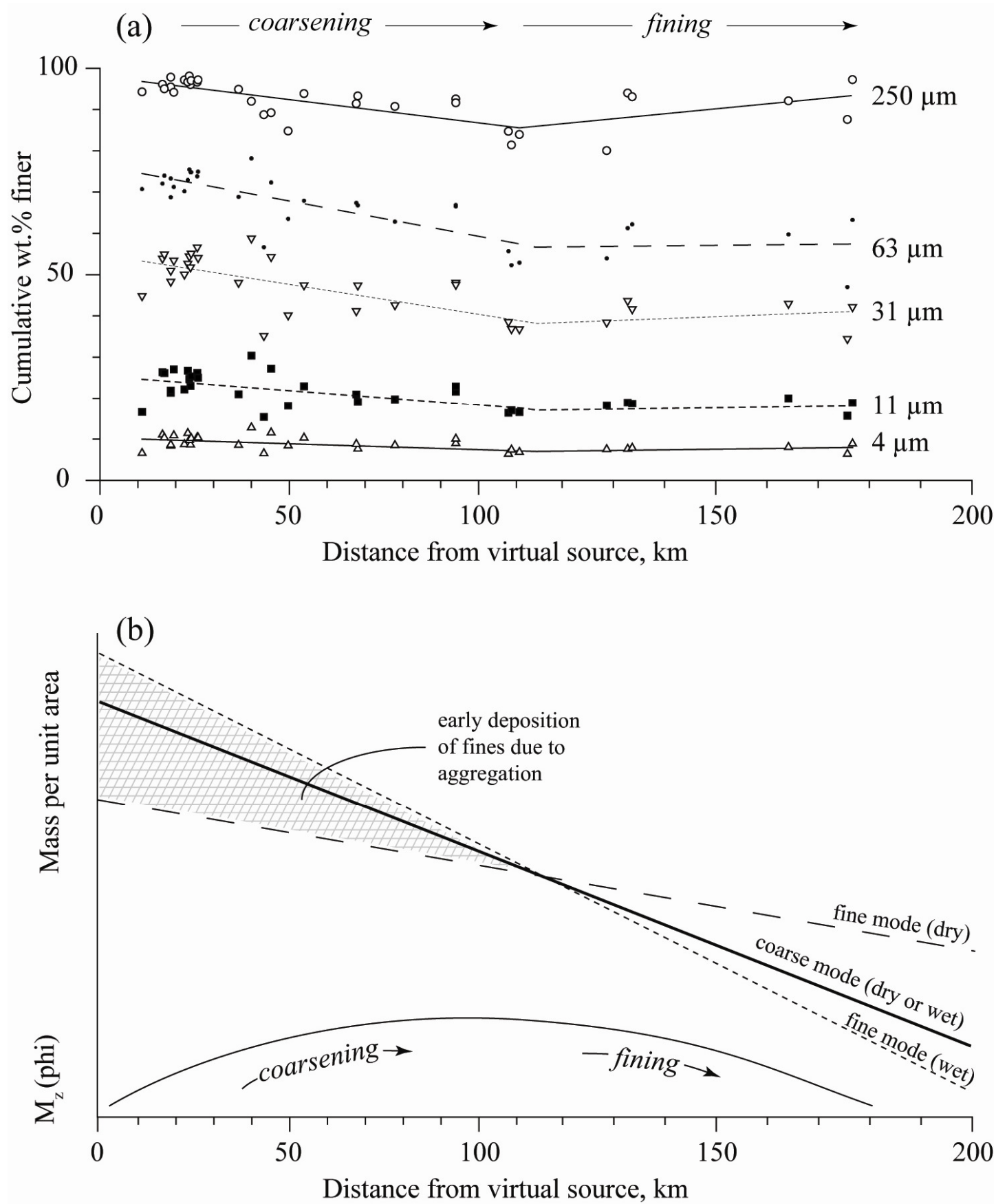

Figure 5.20 (a) Relative abundances of size fractions in unit 3 channel sample versus distance from source (from Wilson [2001]). (b) Conceptual model of the unit 3 coarsening signature from panel (a), showing qualitative mass fallout from the cloud (above) and corresponding mean grain size measured in the deposits (below). Deposition of $\geq 250 \mu \mathrm{m}$ ash from the volcanic cloud decreases at effectively the same rate regardless of the available of liquid water. In contrast, finer particles deposit significantly more mass close to source due to aggregation under wet conditions, lowering the mean grain size. 
Aggregation-enhanced fallout: Wet aggregation preferentially selects particles finer than $\sim 250 \mu \mathrm{m}$ from a wider grain size population [Schumacher and Schmincke 1995, Figure 4.17 of this thesis] because coarser particles are too large to be efficiently captured by liquid bridges. Consider the effect this has on a moist volcanic plume, in terms of mass fallout (per unit area) as the cloud drifts away from vent (Figure 5.20b). Particles $>250 \mu \mathrm{m}$ will have a similar fallout pattern whether conditions are wet or dry; that is, the larger particles preferentially sediment close to source, with less mass and finer sizes depositing at greater distances, as in normal dry fall deposits [Walker 1971]. The coarser component in unit 3 is expressed as two or three normally graded horizons in the distal area. In contrast, $<250 \mu \mathrm{m}$ ash deviates from this pattern as the clumping of fine ash into heavier pellets and accretionary lapilli during wet conditions significantly enhances fallout closer to source. In cases where liquid water does not become readily available until the cloud has drifted some tens to hundreds of kilometers from source, this results in a secondary thickness maximum [e.g. Mount St Helens 1980: Carey and Sigurdsson 1982]. However, during phreatomagmatic eruptions vaporizing abundant surface water, liquid water condenses in the volcanic cloud and is available almost immediately after eruption (Figure 2.6 of this thesis, Van Eaton et al. [2012a]). Under these conditions, the mass of finer ash that deposits as aggregates in the near-source area outweighs coarser particles that settle individually, up to a certain distance from vent. In unit 3, this distance is $\sim 110 \mathrm{~km}$, beyond which much of the finest ash is depleted, and downwind fining resumes (Figure 5.20b).

Multi-level transport: The transport heights of volcanic ash clouds are also likely to contribute to an outwards coarsening signature in the deposits. In modeling of wet plumes [Chapter 2, Van Eaton et al. 2012a], Plinian-like injection from vent combined with lofting of material from PDCs produces a multi-level, hybrid volcanic cloud (Figure 5.2) that is stratified with respect to grain size. Whereas the Plinian column transports a poorly sorted particle population to the maximum cloud height, PDCs preferentially elutriate finer particles, yielding a bi-level transport system with a higher level, coarser component and a lower level cloud of fine ash. Because higher level transport generally deposits particles farther from vent [Carey and Sparks 1986], especially if there is interaction with stronger winds aloft [e.g. the jetstream: Bursik et al. 2009], this would accentuate deposition of coarser particles farther from vent and finer particles closer to vent. 


\subsubsection{Alternating wet and dry: units 6, 7, and 8}

Two characteristics of units 6,7 and 8 are striking in comparison with unit 3 . First is the evidence for exceedingly powerful, episodic outbursts that are more widely dispersed that the products of unit 3. Second, there is substantially greater deposition of medium ash to lapilli-sized clasts throughout these phases of eruption, despite abundant evidence for wet depositional processes. The combined influences of non-steady eruption column heights [Walker 1981a], variable degrees of magma-water mixing at vent [Kokelaar 1983], and changing availability of liquid water on deposition [Walker 1981b] are all likely to have promoted the mixed fallout of fine ash and coarser clasts. In the following section grain size and aggregation characteristics from these units are used to examine the eruption, transport and dispersal patterns distinctive to each eruptive phase. Representative stratigraphic logs and grain size distributions of units 6,7 and 8 are shown in Figures 5.21 and 5.22.

\section{Unit 6}

Phase 6 represents extensive generation and deposition of fine ash, overprinted by a coarser mode $(>250 \mu \mathrm{m})$. Normal grading in the fall deposits imply that phase 6 comprised three major outbursts, of which the third was the most powerful and its deposits the most widely dispersed. Unit 6 is rich in layered accretionary lapilli in the transitional regime, 43-47 km from virtual source (as defined in Figure 1.5b after Wilson [2001]), with lateral equivalents weakening to small, partially coalesced ash pellets downwind into the distal area (Figure 5.16c). Although most of the PDCs are inferred to have been concentrated flows (s.s.), sites directly northeast of Lake Taupo show evidence for local emplacement of multiple dilute PDCs with overlying layers rich in ultrafine accretionary lapilli. Just beyond the limits of the most widespread unit 6 PDCs (Figure 5.21a, $43 \mathrm{~km}$ from vent), fall deposits are a clast-supported mixture incorporating massive pellets and layered accretionary lapilli. Some of the latter include one or more internal layers of ultrafine ash (cf. Figure 5.6e) which are inferred to record earlier stage(s) of growth during which the aggregates passed through suspended ultrafine ash from waning PDCs, perhaps before being re-entrained into a more waterrich, poorly sorted region of the cloud. The unit 6 transitional regime shows similar aggregate characteristics to the corresponding unit 3 regime, with the shift from mixed 


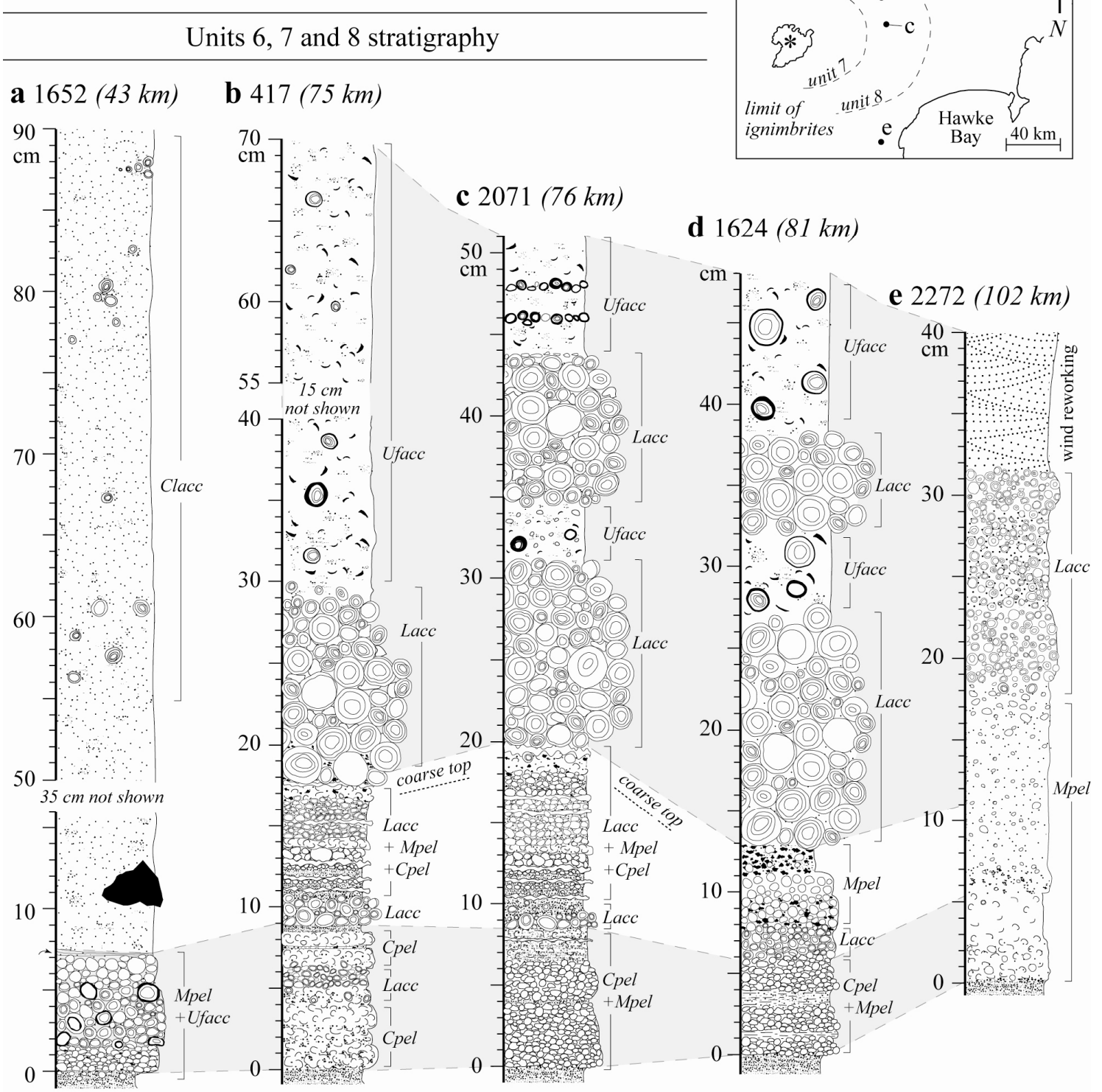

Figure 5.21 Erosional profile stratigraphic logs showing units 6, 7 and 8 at localities with varying distances from virtual source (Appendix A), as in Figure 5.14. Symbols are to scale. The lower shaded region indicates unit 6; unit 7 is unshaded and the upper shading indicates unit 8 . The top of unit 5 is also shown at the base of each section for reference. Note that unit 8 is not exposed at locality 1652 in (a). The dominant aggregate types are shown at the right of each stratigraphic section. The 'coarse top' of unit 7 west of the ranges (b-d) is also labeled. Photographs of the aggregate facies in units 6 and 7 in (b) are in Figure 5.16. 


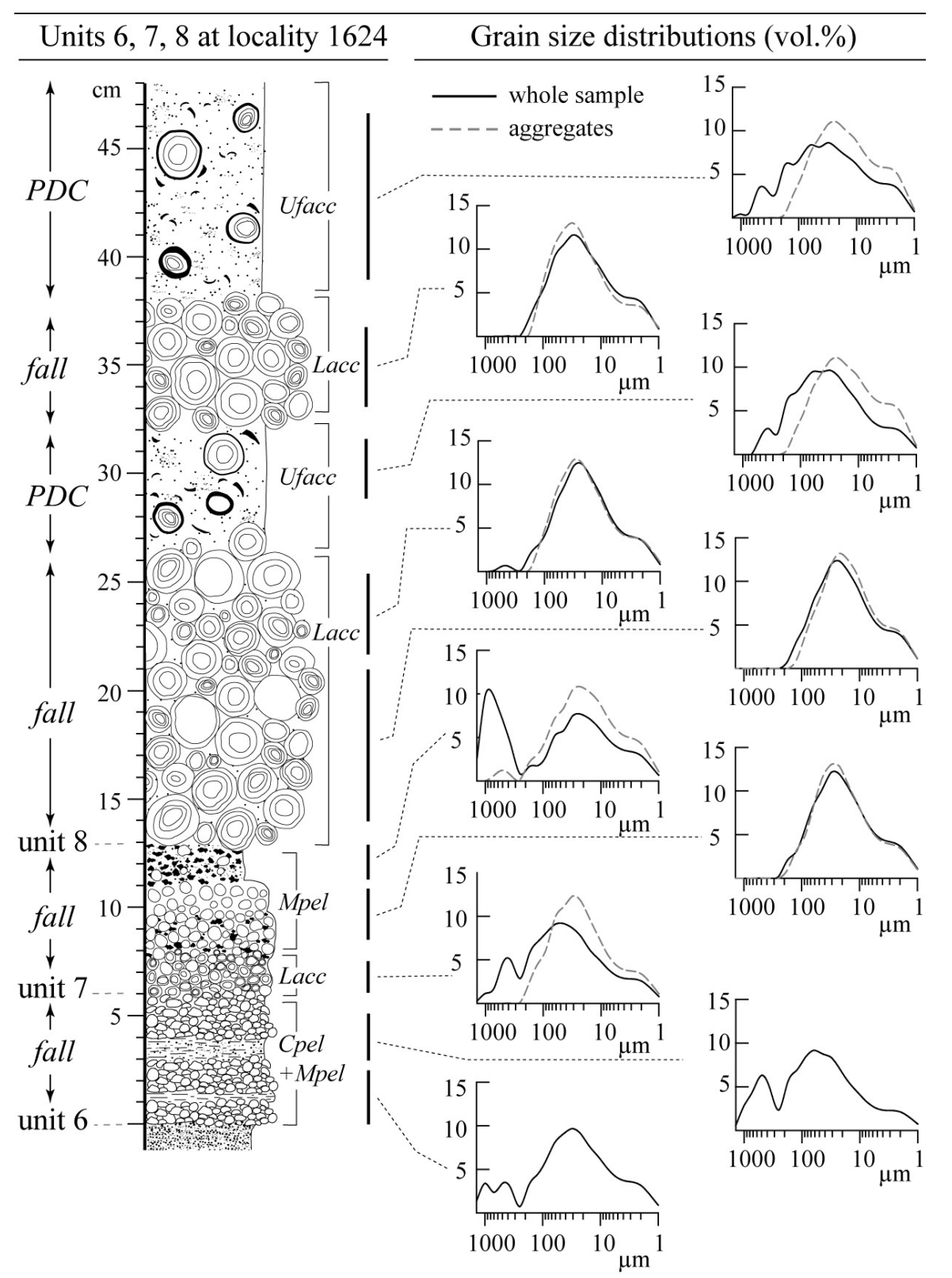

Figure 5.22 Grain size distributions of bulk samples (solid curve) and enclosed aggregates (dashed curve) from units 6, 7 and 8 at locality 1624 (as in Figure 5.17).

aggregate populations (e.g. Figure $5.14 \mathrm{e}$, between $70-85 \mathrm{~cm}$ ) to primarily massive (or coalesced) ash pellets. However, the size of the unit 6 aggregates decrease more slowly beyond $\sim 75 \mathrm{~km}$ from source. This implies overall similar wet aggregation mechanisms for the two eruptive phases. However, a more powerfully dispersed cloud system operated during phase 6 that was capable of sustaining aggregates farther from vent. Unit 6 persists to $>150 \mathrm{~km}$ to the south and becomes a single normally graded bed with no visible aggregate structure and only sparse fine ash matrix beyond 100-120 km from source. 


\section{Unit 7}

This eruptive phase produced multiple PDCs (mostly concentrated flows, s.s.) and exceptionally widely dispersed fall deposits (bed B of Wilson [1994]). The proximal area of unit 7 (within 40-50 km of vent) is dominated by emplacement of PDCs, with sparse intermittent contribution from fall material. In the transitional area, for example locality 1652, 43 km from vent (Figure 15 of Wilson [2001]), complexly layered accretionary lapilli are dispersed in the ignimbrite flow units and form clast-supported lenses. The accretionary lapilli are associated with fluidization segregation pods and pipes, and are interpreted to have landed, along with liquid water, into hot $\left(>100{ }^{\circ} \mathrm{C}\right)$ flow material which then boiled the water to steam. It is inferred that interaction occurred between two emplacement systems in the transitional unit 7 area: hot, dense ground-hugging currents and overlying accretionary lapilli-generating clouds. The passage of PDCs is inferred to have triggered aggregation in this case by wedging ashladen, moisture-rich air upwards, and perhaps also by interacting with a buoyant plume above. Just beyond this area and out of reach of the earliest unit 7 PDCs, the large accretionary lapilli (some $>3 \mathrm{~cm}$ ) fell directly to the ground as fall material (e.g. locality 1629).

The distal fall equivalent of this sequence exhibits two distinctive layers [Wilson 2001] west of the axial ranges (Figure 5.1) Here, the basal part is a poorly sorted, aggregatebearing layer, whereas the upper part is somewhat better sorted and predominantly aggregate-poor (see coarse bed in logs b-d; also referred to as 'bed B' in Wilson [1994]). The aggregate-bearing basal component reflects the lateral equivalent of PDC-triggered aggregation in lower-altitude clouds elutriated during the passage of several fartravelled PDCs. The sizes of aggregates in basal unit 7 diminish rapidly beyond the proximal area. For example, maximum diameters decrease from 3.1 to $1.0 \mathrm{~cm}$ between 43 and $75 \mathrm{~km}$ from virtual source (Figure 5.23). Based on the distribution of aggregate facies and grain size characteristics beyond the proximal regime in unit 7, two key processes are distinguished, which independently influenced the distal transport of the volcanic cloud in space and time. 


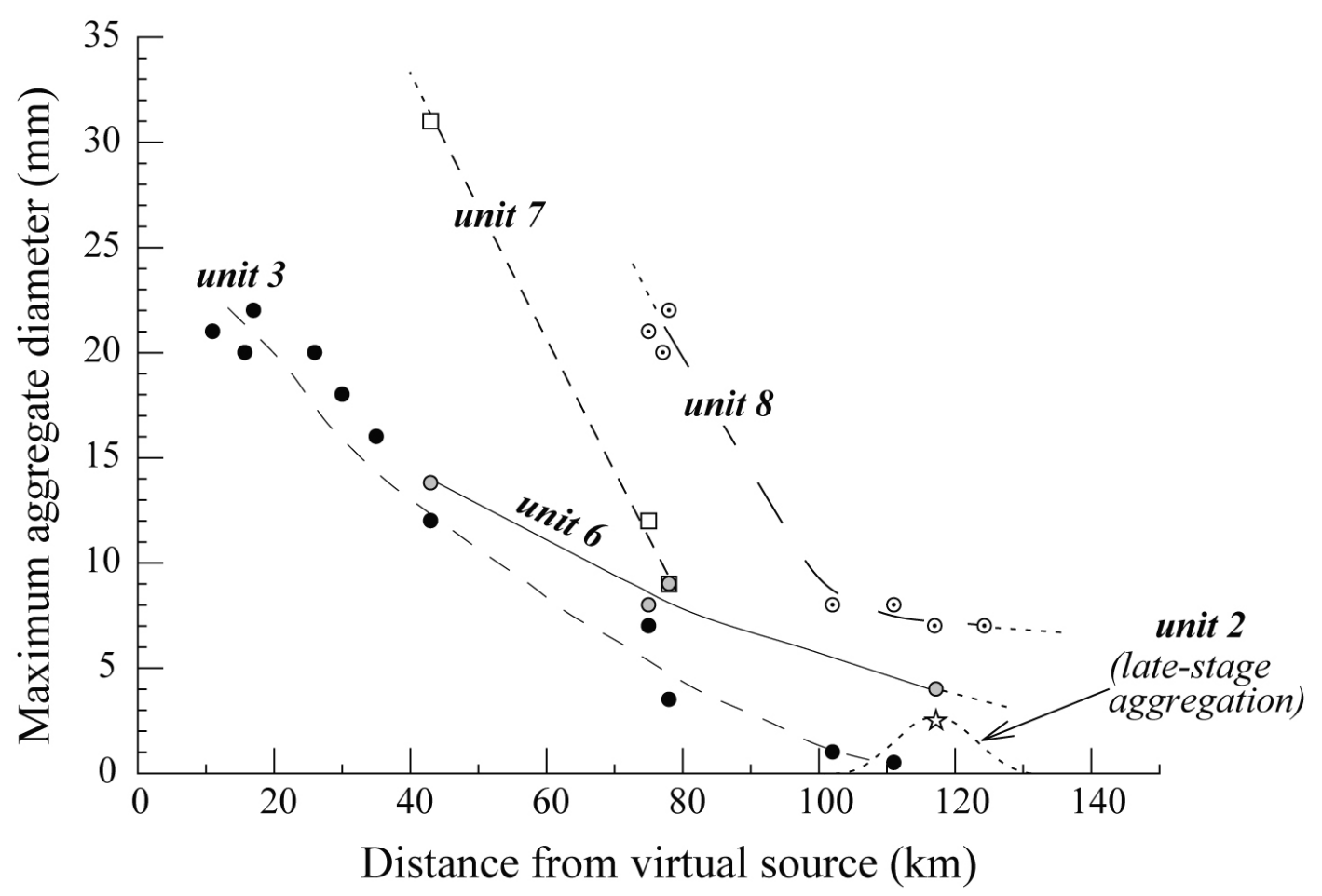

Figure 5.23 Maximum aggregate sizes (average of 3-5 largest maximum diameters) from units 2, 3, 6, 7 and 8. Note the rapid decrease in aggregate sizes in unit 7 (due to the influence of the axial ranges), and the distal pelletization of unit $2 \sim 120 \mathrm{~km}$ from source, attributed to atmospheric moisture and/or cloud subsidence and melting.

Orographic drying: It is notable that the aggregated, matrix-rich base of the unit 7 fall deposits only persists between the source region and the axial ranges, and not farther east (Figure 5.1). It is inferred from the overall SE-ward distribution of fall unit 7 (Wilson [1994] and C.J.N. Wilson unpublished data) that northwesterly winds pushed the hybrid eruption cloud against the axial ranges. This would have caused rapid ascent of the moist, lower-level co-ignimbrite material, promoting extensive condensation and precipitation in the cloud, as occurs in modern weather patterns [Salinger 1980a]. At representative sites $\sim 75 \mathrm{~km}$ from vent (Figure 5.21b-c), just upwind of the ranges, the aggregated basal portion of unit 7 is composed of massive ash pellets, locally irregular layers of coalesced pellets and layered accretionary lapilli deposited in close succession (Figure 5.16b). This complex deposition style is envisioned to occur when wet aggregation occurs simultaneously at multiple plume levels, accompanied by local downbursts of ash-laden rain [cf. Smith 1998]. In contrast, unit 7 fall deposits east of the ranges consist of a single, ungraded bed of aggregate-poor, fine ash to fine lapilli, implying that the volcanic cloud lost much of its water during this process and progressed to relatively dry deposition from a uniformly sustained plume. 
'Drying out' at vent: Contrasting sharply with the aggregate-bearing base, the upper portion of the unit 7 fall sequence west of the ranges $(2-2.5 \mathrm{~cm}$ thick in Figure $5.21 \mathrm{~b}-\mathrm{d})$ is a matrix-poor bed of medium ash to medium lapilli, poor in macroscopic aggregates. This suggests that the phase 7 eruption transitioned abruptly to drier depositional processes with time. Grain size characteristics of the coarser ('drier') top are consistent with single particle settling from a buoyant cloud (with limited influence of aggregation), reflected in a decreasing mean grain size of the entire unit with distance from source (Figure 5.13 of Wilson [2001]). It is likely that the drier depositional processes in part reflect decreased magma-water interaction at vent. An increase in eruption rate is precluded as an influence because of the lack of grading in the distal fall deposits, and it is inferred that the voluminous coeval generation of ignimbrite effectively reduced the amount of magma-water interaction at vent by blocking water access [e.g. Walker 1981b, Kokelaar 1986]. An implication of this shift is that while less fine ash may have been generated overall, the paucity of wet aggregation would have allowed the fine ash to be transported farther, i.e. over the Pacific Ocean.

\section{Unit 8}

Phase 8 saw generation of the most powerfully emplaced and widespread PDCs, depositing voluminous, non-welded ignimbrite in the proximal area within $90 \mathrm{~km}$ from the virtual source. However, phase 8 differs from preceding phases in that it lacked a distinctive Plinian component. Instead, grain size and dispersal characteristics suggest that the eruption produced a short-lived, collapsing column with extremely high mass eruption rates. Most of the material was laterally emplaced or lofted from PDCs. Numerical simulations of a collapsing fountain with mass eruption rate of $\sim 10^{9} \mathrm{~kg} \mathrm{~s}^{-1}$ (Figure 2.3h) imply that even in the absence of a sustained Plinian-like column, nearvent lofting of the ground-hugging density currents is capable of transporting ash-laden updrafts to stratospheric heights. These near-vent updrafts are generally hotter and more vigorous (i.e. able to transport coarser particles) than those lofting from PDCs many kilometers away from source. Therefore, although phase 8 did not likely have a ventderived, central column like that depicted in Figure 5.2, multi-level transport is envisaged due to higher-altitude injection of vigorous near vent updrafts, and lower injection of more passively elutriated, fine coignimbrite ash. 
In the proximal area, the earliest unit 8 deposit is widespread ignimbrite. Generation of PDCs early in this phase triggered the aggregation and fallout of the large $(1-2.5 \mathrm{~cm})$, layered accretionary lapilli found in the PDC deposit. In stratigraphic logs from sites $75-81 \mathrm{~km}$ from source (Figure 5.21b-d), which are within or just beyond the extent of the basal ignimbrite, layered accretionary lapilli immediately follow the ignimbrite as a 12-14 cm clast-supported fall bed. This is overlain by a repeated sequence of PDCs, incorporating abundant, matrix-supported, whole and fragmented ultrafine rim-type accretionary lapilli. Each pulse of PDC deposits is followed by a clast-supported bed of layered accretionary lapilli, and the transition is recorded in the aggregate textures. This pattern is superficially similar to the 'pyroclastic couplets' identified by Brown et al. [2010], except that the overlying clast-supported aggregates have concentric structure. At the contact between clast-supported beds of layered accretionary lapilli and poorly sorted ignimbrite, aggregates appear to have been ripped up and fragmented (Figure 5.24). The aggregates that follow have thin outer layers of ultrafine ash rims. This demonstrates that the passage of ground-hugging currents promotes development of ultrafine rims on aggregates, and are intimately linked to the preferential elutriation of fine ash from PDCs. Furthermore, since the cores of the ultrafine rim-types are texturally identical to the layered accretionary lapilli in intervening fall layers, it is inferred that layered accretionary lapilli form via selective scavenging of finer ash [Chapter 4, Van Eaton et al. 2012b] in the multi-level plumes generated from the Plinian column or vigorous lofting of PDCs closer to vent. Continuous fallout of these aggregates thus provide nuclei for ultrafine rim accretion during passage through the low-altitude fine ash cloud more directly associated with the ground-hugging density currents.

Beyond the limits of the unit 8 PDCs, ultrafine rim-types do not occur, although by comparison with units 3 and 6 there may be a previously unrecorded layer (potentially destroyed by erosion) just beyond the flows, recording simultaneous deposition of ultrafine rim-types with layered pellets. However, as far as the modern east coast of the North Island in Hawkes Bay [e.g. Berry 1928] the unit 8 fall deposits are characterized by layered accretionary lapilli that become smaller and less complex with distance from source. An additional observation from the unit 8 aggregates preserved in the Hawkes Bay region is that many appear to be 'just' matrix supported, i.e., just touching each 
other, with some matrix in between. From thin sections, the intervening matrix is shown to be composed of broken aggregates and larger individual particles (Figure 5.6f), supporting the assertion that the material landed as a combination of aggregate fallout (some of which broke on landing) and single-particle sedimentation.
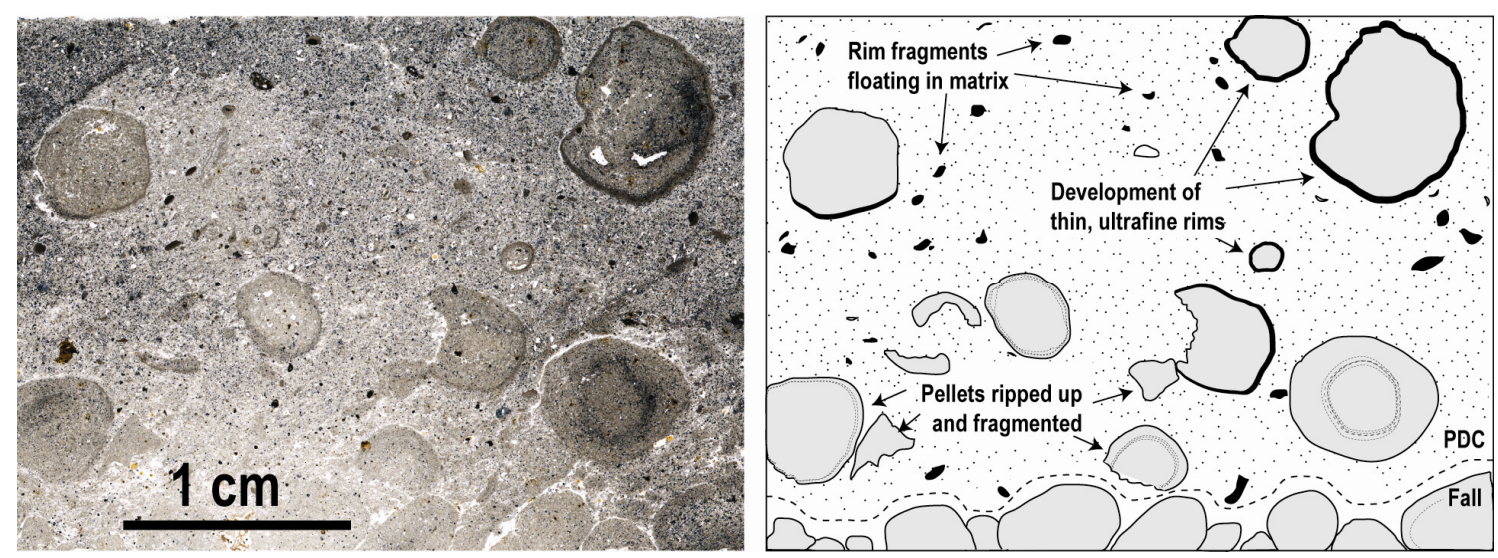

Figure 5.24 Impregnated thin section (left) and interpretation (right) from unit 8 at locality 1624 (81 km from source), showing the transition between 'fall-related clastsupported massive pellets and 'PDC-related' matrix-supported accretionary lapilli with ultrafine rims.

One important distinguishing feature between aggregation in unit 8 versus that in unit 3 is the nature of the intervening fall layers. In both phases of eruption these are composed of clast-supported, massive pellets (unit 3) or layered accretionary lapilli (unit 8). However, the clast-supported aggregates are much larger and more complex in unit 8, and cm-sized accretionary lapilli were evidently capable of forming and depositing to $>100 \mathrm{~km}$ from vent. This suggests that plume processes were much more vigorous during phase 8 , possibly reflecting the inferred greater eruption rate, and associated stronger updraft velocities.

\subsection{3. 'Drier' plinian-like: units 1, 2, 4 and 5}

Units 1, 2, 4 and 5 of the Oruanui eruption show grain size characteristics more similar to those of conventional plinian fall deposits, although of moderate to exceptional dispersal (particularly unit 5 = bed A of Wilson [1994]). Most parts of these deposits are relatively well sorted, become systematically finer with distance from vent and lack visible ash aggregates and are thus inferred to have seen only moderate amounts of interaction with water at vent [Wilson 2001]. However, evidence for localized water- 
flushing is present, and ash aggregation occurs farther downwind over regional extents, exemplified here with field observations from units 2 and 5.

Unit 2 generated fall deposits with plinian dispersal which accompanied localized PDC deposits (to only $<20 \mathrm{~km}$ from vent), and was coupled with sporadic outbursts from vents that became vigorously active in phase 3 of the eruption. The fall deposits were emplaced primarily by single-particle settling, and lack visible ash aggregates within 60-70 km from vent. Beyond this, however, the deposit has a loosely-packed, microvesicular texture which, at the best preserved sites, demonstrates that ash fell dominantly as mm-scale, massive spherical pellets (Figure 5.5c). This suggests that the phase 2 ash cloud experienced an increase in liquid water content in distal regions, leading to formation of pellets. In contrast, unit 5 is a normally graded bed that is mostly well sorted at all vertical levels, but east of the axial ranges has developed a loose, low-density, moderately to poorly sorted top portion forming up to $50 \%$ of the total unit thickness. There is a lack of visible aggregate textures, and this upper part is inferred to have been deposited by moist aggregation, in the form of snowflake-like particle clusters, as has been observed or inferred in recent eruptions including Mount St. Helens 1980 and Crater Peak 1992 [e.g. Durant and Rose 2009, Durant et al. 2009].

Several factors may have contributed to the distal aggregation in these units: (1) downwind fining increases the availability of sub-250 $\mu \mathrm{m}$ particles, which are more likely to become aggregated; (2) an influx of moist coastal air on the east side of the axial ranges, adding water to the cloud [Wilson 2001]; and (3) fallout and melting of ice particles carried downwind in the volcanic cloud [Durant et al. 2009]. Enhanced aggregation has generated secondary thickness maxima in recent Plinian eruptions [e.g. Carey and Sigurdsson 1982], but in the Oruanui, varying degrees of compaction and preservation have precluded identification of a secondary thickness maximum in any of the eruptive phases.

An interesting aspect of the drier eruption and dispersal of units 1, 24 and 5 is that, despite generation of both a Plinian column and PDCs in all cases, there is no evidence to suggest that interaction between the two led to large-scale aggregation in the relevant proximal areas. Two possible reasons for this are: (1) that there may not have been enough liquid water available in the buoyant cloud and PDCs, and (2) that the PDCs 
may have been too small (relative to the volume and dispersal power of the Plinian plume) to produce significant updrafts capable of destabilizing the buoyant cloud and inducing substantial aggregation. The relative partitioning of erupted material into fall versus PDC [e.g. Neri et al. 2002] may thus be a governing factor in the development of large and complex aggregates.

\subsection{Discussion}

It is clear from the variety of eruptive styles and associated aggregate textures in the Oruanui eruption that aggregation processes have a significant impact on the fallout of volcanic ash at different stages of eruption. The key stages at which aggregates are inferred to have developed in the cloud system are summarized here, and the more detailed processes and implications are considered as a series of questions.

\subsubsection{Summary model: Impact of aggregation on cloud development}

Figure 5.25 provides a summary sketch of aggregate-forming processes in the Oruanui eruption plumes. An important aspect of the proposed model is recognition of multilevel transport of particles from a column that is generally unstable, generating simultaneously buoyant and non-buoyant material. Medium ash to lapilli-sized clasts $(>250 \mu \mathrm{m})$ are partitioned into ground-hugging PDCs and a higher-level plume, with the latter fed by a Plinian column and vigorous near-vent updrafts from proximal PDCgeneration. In contrast, preferentially finer material is lofted into a lower-level (coignimbrite) cloud from PDCs as they travel farther away from vent. Aggregation in the high plume occurs primarily where liquid is available through condensation on ash surfaces or melting of ice, promoting growth of massive ash pellets by nucleation and coalescence. However, regions of the plume at altitudes significantly colder than $c$. $15^{\circ} \mathrm{C}$ (the nucleation freezing temperature of silicic ash [Durant and Shaw 2005]) are less likely to contain abundant and persistent concentrations of liquid water for wet aggregation. Therefore, cloud transport at these levels is relatively drier and less influenced by aggregate formation and fallout. Assuming, for example, sea level temperatures between 0 and $5{ }^{\circ} \mathrm{C}$ and a tropospheric lapse rate of $-5{ }^{\circ} \mathrm{C} / \mathrm{km}$ for the North Island during the last glacial period [cf. Drost et al. 2007a], this 'higher, drier' transport level would be above $\sim 3-4 \mathrm{~km}$ elevation. 


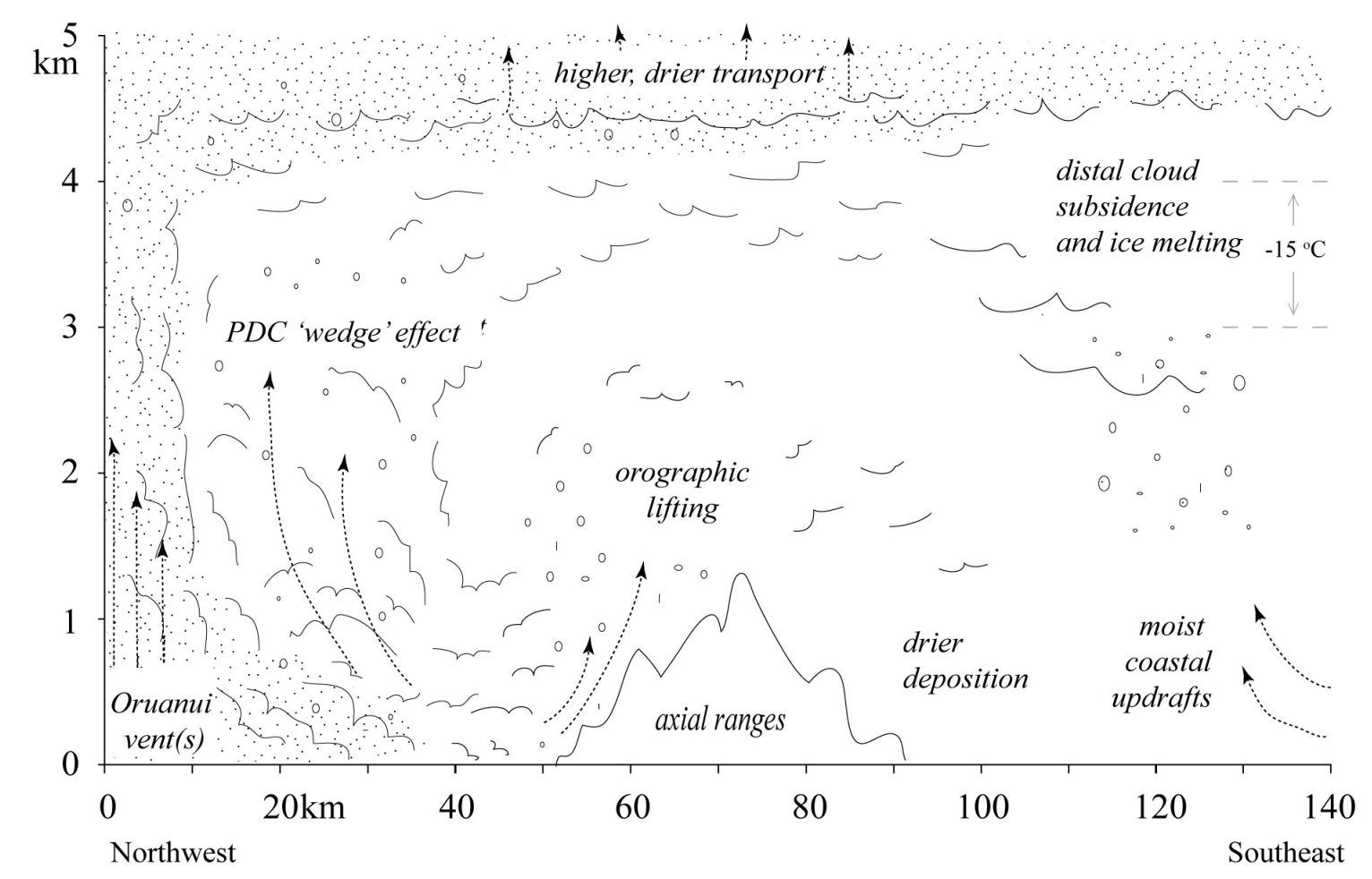

Figure 5.25 Cartoon illustrating the primary locations and mechanisms of ash aggregation in the Oruanui clouds in the lower atmosphere.

Where the PDCs generated from column collapse are of comparable or greater volumes than the coeval high plume, interaction between the two components is inferred to lead to formation of complex aggregate types. This is illustrated by Oruanui unit 3 deposits, which contain abundant, concentrically layered accretionary lapilli only during or after the deposition of PDCs. This is attributed to a wedge effect, whereby warm updrafts of fine ash rising from PDCs converge with the high plume to induce convective instabilities in the cloud system and inject more water vapor from the lower atmosphere, which then condenses to promote rapid aggregation. The turbulent updrafts from the PDCs also provide stronger vertical velocities capable of sustaining larger aggregates, and allow re-entrainment through vertically stratified layers of the cloud system. This recycling is inferred to generate the concentric laminations of layered accretionary lapilli. A further effect comes from each ground-hugging PDC generating an overriding cloud of fine ash and condensing moisture. As massive ash pellets and layered accretionary lapilli fall out from higher levels, the final stage of growth occurs in this preferentially finer-grained, low-level cloud to produce the ultrafine rims of accretionary lapilli that drop into laterally moving PDCs. 
As the hybrid cloud system moves 50-80 km downwind from vent under the dominant westerly or northwesterly wind flow, the low level portion of the cloud is forced upward by air currents on the west side of the axial ranges. Orographic lifting causes rapid cooling and condensation of moisture, accompanied by heavy precipitation and wet aggregation, while on the downwind side, the material is relatively drier. This effect is best shown in unit 7 deposits, which exhibit a contrast between heavily aggregated basal deposits west of the ranges and relatively matrix-poor equivalents to the east. Considering its exceptionally wide dispersal characteristics, unit 7 may have been more strongly influenced by these topographic effects owing to a vertical plume structure that was more distinctly bimodal. For example, the water-rich portion of the cloud may have been constrained to lower levels and separated from the high, drier component that injected into the jet stream. This contrasts with deposits from units 3 and 6, during which the eruptions were more likely to have intruded water-rich ejecta relatively homogenously throughout the atmosphere. In this case, water-rich portions of the clouds may have ascended higher and been capable of passing above the axial ranges unaffected by orographic precipitation (because water was in the ice phase). Beyond the axial ranges, > 100 km from source, other Oruanui eruption phases (2 and 5) experienced late-stage aggregation in the finer grained, downwind cloud, possibly triggered by: (1) cloud subsidence and melting of carried ice, and/or (2) incorporation of moist, oceanic air as the cloud approached the Pacific coastline.

\subsubsection{Do ultrafine rim-type accretionary lapilli form in pyroclastic density currents?}

Ultrafine rim-type accretionary lapilli are only found in PDC deposits in the Oruanui products, in agreement with the Brown et al. [2010] hypothesis that their formation is closely linked with the passage of PDCs. However, the Brown et al. [2010] model differs from that presented here in two respects.

(1) Brown et al. [2010] propose that concentrically layered aggregates with ultrafine ash rims are confined to PDC deposits in most circumstances. This is inconsistent with observations from the Oruanui deposits. For example, cm-sized layered accretionary lapilli (with normally graded, fine ash rims) exist in the unit 8 fall deposits to over 120 $\mathrm{km}$ from source. The persistence of layered accretionary lapilli fallout beds between 
pulses of PDCs, as well as beyond the flows, indicates that there are processes in buoyant plumes capable of generating concentrically structured aggregates without direct interaction with (i.e. deposition within) a passing ground-hugging PDC. It is therefore concluded that the presence of concentrically layered aggregates is not sufficient to identify a deposit as laterally emplaced [cf. Brown et al. 2010], but does indicate an association with coeval PDC emplacement.

(2) Brown et al. [2010] infer that massive ash pellets, generated in the higher, lofted coignimbrite cloud, subsequently drop into the underlying PDC where they accrete fine ash layers in the hot, denser lower portion of the ground-hugging current. They propose that higher temperatures in the PDCs limit the availability of liquid water, promoting preferential selection of fine ash from the broader particle population carried in the current. However, Van Eaton et al. [2012b] could not reproduce such an extreme preferential selection of $<31 \mu \mathrm{m}$ ash, even with very low liquid water contents $(\sim 2-5$ wt.\%). Furthermore, the ultrafine rim-type accretionary lapilli found in the PDC deposits are, without exception, associated with abundant rim fragments. This indicates that conditions in the lower region of the ground-hugging currents promote attrition and fragmentation of aggregates, rather than growth and layering of additional material. It is therefore more likely that the ultrafine rims on these types of aggregates form in the dilute, turbulent cloud lofted from PDCs, rather than actually within the more concentrated lower regions of the ground-hugging current.

\subsubsection{What is the mechanism of ultrafine rim formation?}

Three styles of aggregation of the ultrafine ash rims have been proposed to occur in volcanic clouds: electrostatic attraction [Sorem 1982], hailstone-like growth [Kato 1986] and liquid-dominated, wet aggregation [Gilbert and Lane 1994]. Here the potential for each of these mechanisms to contribute to ultrafine aggregate rims in elutriated co-PDC clouds is considered.

Electrostatic attraction: Schumacher [1994] showed that electrostatic forces can select sub-45 $\mu \mathrm{m}$ grains from a poorly sorted mixture, but these bonds are weak and particles are loosely adhered. In contrast, the ultrafine coatings of natural accretionary lapilli are tightly packed, concentrically layered and sufficiently strong to partially survive 
transport in concentrated PDCs. Therefore, in the absence of some unidentified cementation process (such as alteration of volcanic glass, proposed in Section 4.4.5), this mechanism alone cannot account for the ultrafine ash rims. In addition, highaltitude, dry, electrostatic attraction would require the aggregate to not accumulate any more ash during fallout through the lower, water-rich levels of the cloud where aggregation would be expected to be active.

Hailstone-like growth: Clustering of icy particles by a combination of wet and liquidfree mechanisms [Knight 1968, Prodi et al. 1986] is another method by which volcanic ash particles may become aggregated [Textor et al. 2006a, b]. However, the involvement of ice particles is likely to leave behind a porous texture after drying (Figure 4.8 of this thesis, Van Eaton et al. [2012a]). Although loosely-packed frameworks are common in the cores of accretionary lapilli, this mechanism is not compatible with the tightly-packed textures observed in the ultrafine ash outer rims.

Liquid-dominated, wet accretion: Liquid water alone is not sufficiently size selective to collect ultrafines from a poorly sorted mixture (Section 4.4.4). To promote aggregation of ultrafine ash under wet conditions requires sub-31 $\mu \mathrm{m}$ ash to dominate the size population in the parent cloud. With these conditions met (liquid water and a supply dominated by ultrafine ash) a coherent, concentrically layered rim of ultrafines could be collected onto moist pellets in laboratory experiments (Section 4.3.3). However, the experimental rims were not as compact and brittle as those observed in the deposits, suggesting that cementation process operating in the natural cloud were missing in the lab experiments. For example, in the experiments, the use of distilled water may not have accounted for precipitates from hygroscopic compounds [cf. Gilbert and Lane 1994], or the use of $\sim 25.4 \mathrm{ka}$ ash did not provide glass surfaces that were fresh and reactive enough to undergo rapid alteration.

Experimental evidence therefore supports the formation of the ultrafine outer layers in the presence of (limited) liquid water and with a preferential supply of ultrafine ash. Coarser pellet cores likely form under liquid-saturated conditions in the poorly sorted regions of the cloud (i.e. the Plinian column or most vigorously lofted currents from PDCs). The pellets likely grow by nucleation and coalescence from a combination of wet aggregation, electrostatic attraction and ice sintering (Section 4.4.3) as the cloud 
cools during ascent and entrains freezing ambient air. Pellets may cycle multiple times through stratified regions of the cloud (particularly after wedging of ground-hugging PDCs) to add additional concentric layers. Once the ground-hugging currents slow or wane, they will leave a low-level cloud of ultrafines, which is then the last volume that the massive pellets (or layered accretionary lapilli) pass through during fallout. As a result, accretionary lapilli-rich layers directly associated with the passage of PDCs always contain ultrafine rims.

\subsubsection{How and where do ultrafine rim fragments form?}

The outer rims of ultrafine rim-type accretionary lapilli behaved in a brittle manner during the eruption, breaking off in angular cuspate or shell-like fragments. These shapes, along with field relationships showing that whole aggregates and fragments were co-deposited, indicate that the rims were brittle before landing (i.e. their brittleness cannot be attributed to post-emplacement cementation). Gilbert and Lane [1994] emphasized the potential role of mineral precipitates in cementing aggregates, although this study has found no evidence for such precipitates in Oruanui examples (Chapter 4, Van Eaton et al. [2012b]). Brown et al. [2010] addressed the possibility of heat in the PDCs providing a mechanism to essentially sinter the finest ash, but this is precluded by the low temperatures of the Oruanui deposits. It is inferred that the reactivity of freshly generated ultrafine glass fragments may have caused cementation through rapid alteration, potentially aided by warmth after falling within the vicinity of a groundhugging density current. Regardless of the processes involved, it is evident that the process was rapid enough to allow rim fragments to be transported as individual particles to $100-120 \mathrm{~km}$ from source.

The abundance of rim fragments in the Oruanui PDC deposits indicates that aggregates broke up during transport and emplacement. The layered and ultrafine rim-type accretionary lapilli have the potential to collide and break whilst airborne, in the same fashion as normal hail. However, it is noticeable that the layered accretionary lapilli in clast-supported fall layers may be slightly deformed on impact, but are overwhelmingly intact. In contrast, the abundance of rim fragments in laterally-emplaced material, some samples of which are $>75 \%$ rim fragments by volume, have clearly experienced significant fragmentation during lateral emplacement. The presence of abundant rim 
fragments in unit 8 fall deposits $\sim 120 \mathrm{~km}$ from source at Napier, tens of kilometers beyond the limits of the farthest-travelled flows, indicates that such fragmentation can yield particles that can be re-entrained and transported in the plume to land as fall material. Thus, cementation before landing in PDCs plays a key role in the preservation of rim fragments and whole aggregates.

\subsubsection{How are $\mathrm{mm}$ to $\mathrm{cm}$-sized aggregates transported $>100-150 \mathrm{~km}$ from vent?}

The occurrence of layered accretionary lapilli with diameters sometimes $>1 \mathrm{~cm}$ in the Hawkes Bay region in unit 8, 100-120 km from vent [Berry 1928], represents one of the farthest traveled examples of accretionary lapilli yet documented, with the exception of the $\sim 1$ Ma Kidnappers eruption, New Zealand [Alloway et al. 2004]. Aggregates of this size have terminal fall velocities of $\sim 10 \mathrm{~m} \mathrm{~s}^{-1}$ (Figure 5.13d), indicating that the volcanic cloud must have been capable of sustaining updrafts of similar magnitude $>100 \mathrm{~km}$ away, assuming that the aggregates were formed closer to vent and carried downwind. The sustained velocities are likely to stem from a combination of (1) distal updrafts related to near-vent lofting of PDCs and (2) transport by strong winds (e.g., in the jet stream). The alternative, that growth occurred primarily during transport in the distal cloud (cf., distal pelletization in unit 2), is considered less likely due to a consistent, recognizable stratigraphy in the deposition of layered accretionary lapilli from proximal to distal areas (Figure 5.21) and the observation that aggregates become systematically smaller away from source (Figure 5.23). Although there is likely to have been some aggregate growth during dispersal in the ash cloud, field observations are consistent with a near-source origin, followed by simple fallout during distal transport. It may be that the exceptionally large mass flux of volcanic ash associated with phase 8 of the eruption, and the far-travelled PDCs (maximum of $~ 90 \mathrm{~km}$ from vent), would have contributed warm, buoyant updrafts to considerable distances, and interacted with the prevailing westerly-norwesterly winds.

\subsubsection{How does the formation of ice in the volcanic cloud affect aggregate formation?}

Central vacuoles are preserved in both layered and ultrafine rim-type accretionary lapilli (see Figure 4.2b in Chapter 4), although they are comparatively less common in massive ash pellets and complexly layered accretionary lapilli. They are present in aggregates from all units of the Oruanui eruption deposits, and in some layers up to $30 \%$ of the 
aggregates contain vacuoles. Their presence strongly supports the existence of mmscale snow pellet or coherent ice particle providing nuclei during wet aggregation [Van Eaton et al. 2012b]. It is likely that ashy ice particles were falling into regions of the cloud where liquid water was abundant and wet aggregation could take place. Microphysical simulations [Chapter 2 of this thesis, Van Eaton et al. 2012a] indicate that during large-scale wet eruptions, the area within tens to hundreds of kilometers from source is likely to be showered by a curtain of graupel. These icy particles would therefore be falling into the ground-hugging PDCs and the areas beyond, providing effective nuclei for wet aggregation once they began to melt. In the Oruanui unit 3 deposits $\sim 100 \mathrm{~km}$ from vent, the aggregates are reduced to weak, massive ash pellets 1$5 \mathrm{~mm}$ in diameter and smaller ash clusters, which are difficult to identify in the field. This distance roughly coincides with the distance at which the overall deposit starts to become finer with distance (Figure 5.20a), that is, normal 'dry' processes take over, and the change in aggregate style is also likely related to relatively lower water content in the cloud. The decrease in fallout of ice particles beyond $\sim 100 \mathrm{~km}$ from vent in the microphysical simulations (after 3 hours of simulated eruption), support the idea of an overall decrease in the availability of water for wet aggregation, and aggregates deposited at distances in excess of $\sim 150 \mathrm{~km}$ are inferred to be particle clusters formed in the plume under conditions of limited liquid water contents.

\subsubsection{Comparison with phreatoplinian phases of the $1.8 \mathrm{ka}$ Taupo eruption}

What promotes the formation of complex accretionary lapilli in some water-rich eruptions and not others? Although the Hatepe and Rotongaio units of the $1.8 \mathrm{ka}$ Taupo eruption [Self and Sparks 1978, Walker 1981b, Smith and Houghton 1995a, Smith 1998] incorporated abundant lake water and generated PDC deposits (albeit on a much smaller scale than the Oruanui), only massive ash pellets, most of which are $<3-4 \mathrm{~mm}$ diameter, are preserved in the deposits. One of the primary differences between these examples and the Oruanui is the relative abundance of PDC versus fall deposits. The Oruanui eruption phases which saw production of abundant aggregates (units 3,6,7 and 8) are each associated with volumetrically comparable or dominant PDC deposits relative to material that was emplaced as fallout. The implication is that the formation of complex aggregates is promoted by high-energy interaction between PDCs and an overriding plume (whether co-PDC or vent-derived in origin). The PDCs in the Hatepe and 
Rotongaio eruption phases were simply too weak and/or small relative to the buoyant cloud to generate turbulent currents capable of producing and supporting larger aggregates.

Another key comparison between the Oruanui and Taupo eruptions is the nature of coarsening of some deposits away from vent. Overall, the Oruanui unit 3 and $1.8 \mathrm{ka}$ Hatepe ash deposits both become coarser grained with distance. However, Oruanui unit 3 coarsens up to $\sim 110 \mathrm{~km}$ downwind of source, while the Hatepe only coarsens out to $\sim 20 \mathrm{~km}$ [Smith 1998]. It is likely that this difference relates to eruptive dispersal power, as the Hatepe ash is approximately an order of magnitude smaller volume compared to Oruanui unit 3.

\subsection{Conclusions}

The Oruanui eruption represents one of the largest examples of wet volcanism yet documented, with ten episodic eruption phases generating ten fall units and numerous, contemporaneous PDCs. Fueled by extensive magma-water interaction, the eruptions generated complex volcanic plumes, generalized by the concept of an unstable Plinian column feeding a higher-level cloud and periodically augmented by thermals rising with elutriated ash from ground-hugging density currents. Coarser material ( $>250 \mu \mathrm{m}$ ) was partitioned into the high plume by Plinian-style ascent, and into non-buoyant portions of PDCs. Finer material was preferentially lofted to lower atmospheric levels by elutriation from medial to distal PDCs.

The Oruanui deposits contain a range of aggregate types spanning the entire, previously documented spectrum, from mud rain through concentrically layered accretionary lapilli and massive pellets to ash 'snowflakes' (particle clusters). The style of accretion is related to the presence or absence of liquid water versus ice, the water: ash ratio, and geographic position with respect to contemporaneous PDCs. Liquid water, ice and electrostatic accretion all contribute to growth of aggregates, preferentially scavenging finer $(<250 \mu \mathrm{m})$ particles from the more poorly-sorted mixtures in the eruption cloud. However, the presence of rims rich in ultrafine $(<10 \mu \mathrm{m})$ ash on layered accretionary lapilli is inferred from experimental observations to require sub-saturated liquid water conditions in a cloud dominated by very fine ash $(<31 \mu \mathrm{m})$. Such conditions are only 
met at relatively low levels $(<3-5 \mathrm{~km})$ in parts of the cloud dominated by preferentially elutriated co-PDC clouds (and not in the PDCs themselves, which are coarser grained). Ultrafine-rim accretionary lapilli are therefore indicative of the presence of PDCs, but deposition of broken rim fragments (derived by collisional attrition in the PDCs) occurs to distances of 100-120 km from source in fall deposits, tens of kilometers beyond the limits of the PDCs themselves.

The absolute abundances and relative proportions of the different types of accreted particles vary greatly in the Oruanui deposits, in response to several factors. First, PDC deposits are associated with matrix-supported, whole and fragmented accretionary lapilli, particularly the ultrafine rim-type accretionary lapilli. In contrast, fall deposits are mostly associated with clast-supported massive ash pellets or layered accretionary lapilli. Second, there are vertical changes in the types and proportions of accreted particles in the deposits from single phases, reflecting changes in ash:water ratio in the plume and in the abundance of PDC deposits. There is also a concomitant lateral change from near-source areas (the proximal zone) where deposition is dominated by PDCs and their associated layered and ultrafine-rim-type accretionary lapilli, to the distal zone where deposition is purely by fallout and massive ash pellets dominate. Third, within the eruption sequence, the abundance of accreted particles reflects to some degree the amount of magma-water interaction at vent and PDC generation. Deposits of drier phases (e.g. units 2 and 5) have smaller proportions of PDC deposits and only subordinate amounts of accreted particles generated by lower water:ash ratios, such as ash 'snowflakes' and small pellets. Deposits of wetter phases (particularly unit 3) are dominated by PDC deposits and have abundant quantities of massive ash pellets, layered accretionary lapilli and ultrafine-ash-rim lapilli.

The presence of PDCs is critical in controlling the generation and nature of accreted particles. The passage of PDCs that are large compared to the overriding plume is proposed to generate a wedge effect in the overlying cloud, forcing warm, moist air and fine, elutriated ash upward. This is inferred to generate layered aggregates by recycled passage through grain size- and water-stratified regions of the cloud. Such a wedging effect is reflected in the changing nature of the dominant accretionary particle types as the importance of PDC deposition changes vertically with time and laterally with 
distance from source within the deposits of individual eruption phases, and can be replicated in numerical modeling experiments.

Within the eruptive cloud there is a balance between outwards transport of material and the steady preferential deposition of coarser particles by single-grain settling (as is the norm in dry, Plinian plumes) versus the premature flushing out of fine components by accretion into ash aggregates. In fall material from drier phases of the Oruanui eruption (particularly units 5 and 7), the deposits show gradual outwards fining at rates that are not matched by the most powerful dry eruptions [Wilson 1994]. This suggests that outwards expansion of the plume is boosted by limited amounts of water interaction [Koyaguchi and Woods 1996]. In fall material from the wettest phase (unit 3), the premature flushing of preferentially finer ash in the proximal area leads to the mean grain size of unit 3 coarsening outward to $\sim 110 \mathrm{~km}$ from source before resuming outwards fining. Such outwards coarsening has only been documented elsewhere in the $1.8 \mathrm{ka}$ Hatepe ash (Taupo) and the 1991 co-PDC deposits at Unzen (Japan), and is similarly attributed to a combination of multi-level plume transport and preferential selection of $<250 \mu \mathrm{m}$ particles by aggregation-enhanced fallout.

The extent of aggregate formation and premature deposition of fine ash within the Oruanui ash cloud for various eruption phases investigated here falls into two endmember situations. For a majority of the deposits, variations in grain size of whole-unit samples and in the sizes of the largest grains (pumice and lithic fragments) and accreted particles with distance from vent are only consistent with the water in the plume being sourced in the vent area itself, from the pre-eruptive Lake Huka. There is then a complex pattern in the degrees of water-induced flushing which is dependent on (1) the amount and distance travelled of PDC generation and (2) orographic forcing as the eruption cloud traverses the axial ranges of the North Island under the influence of the dominantly west or northwest winds. For the drier phases of the eruption (2 and 5), which also have lesser proportions of PDC deposits, more of the eruption plume was carried downwind over the axial ranges without premature flushing of fines. However, fallout of ice and snow pellets from the volcanic cloud and/or an influx of low-level moisture from the Pacific Ocean caused accretion in areas $>100 \mathrm{~km}$ from source to form either small pellets or ash 'snowflakes' and generate distal fall material that has a lowdensity, microvesicular texture, and is poorly sorted and richer and fines. 
The Oruanui deposits exemplify the complexity of plume dynamics, cloud transport and depositional styles that result from large-scale wet explosive volcanism. The role of water is not simply in inducing premature aggregation and flushing of fine ash, but is also expressed in its control on stability of the overall plume (i.e., buoyant versus nonbuoyant) and, via the generation of PDCs and on the levels in the atmosphere at which aggregation occurs (e.g. ice versus water). The grain size of the cloud is influenced also not only by the primary eruptive jet at source, but also by atmospheric injection of elutriated fine ash from PDCs, such that there is a layering in ash size and water content reflected in the size and nature of the aggregation processes and products. 
Chapter 6 


\title{
6. High-flying diatoms: widespread dispersal of microorganisms in a volcanic super-eruption
}

\begin{abstract}
This study documents the unusual occurrence of diatom remains in widely dispersed deposits from a large volcanic eruption. Sixty-two freshwater diatom species have been identified in deposits of the $\sim 25.4$ ka Oruanui phreatomagmatic super-eruption (Taupo volcano, New Zealand). Three lines of evidence indicate that the diatoms originated from ancestral Lake Huka, an extensive Pleistocene lake which overlay the eruption vent: (1) the extraordinary abundance of whole and fragmented diatoms throughout the pyroclastic deposits (mean of 11 per $\mu \mathrm{g}$ of volcanic ash, amounting to $\sim 0.6 \mathrm{~km}^{3}$ of erupted diatom remains); (2) the occurrence of large ( $\mathrm{cm}-\mathrm{dm}$ sized), diatom-bearing lithic clasts in the Oruanui ignimbrite; and (3) diatom assemblages dominated by planktic species, consistent with a large lake source. A novel mechanism is proposed for long distance dispersal of diatoms and associated microorganisms in the form of largescale volcanic plumes, which transport material hundreds to thousands of kilometers from source. Wider implications for this newly recognized process include: (1) a means of characterizing the source of external water in phreatomagmatic deposits (i.e. seawater versus freshwater); (2) providing a biogenic 'fingerprint' of country rock lithologies excavated and dispersed during eruptions; (3) the possibility that some viable diatoms may survive transport from lake to atmosphere, seeding new microorganism populations over large areas; and (4) establishing a new mechanism of global biogenic particle transport, with the potential to contaminate exposed surfaces with foreign microfossils. By extension, volcanically dispersed diatoms are a possible source for anomalous microfossils found in Antarctic ice cores and elsewhere.
\end{abstract}

\subsection{Introduction}

Microfossil occurrences and assemblages provide a rich archive of the environmental conditions in which they lived, forming an integral component of understanding local to global changes in the ocean and climate. In particular, the remains of diatoms (siliceous 
microalgae) in sediments and ice cores are frequently employed to establish local changes in $\mathrm{pH}$ and salinity levels, among other factors [Baker 2001]. Contamination of primary sediments by diachronous populations of 'foreign' diatoms thus poses considerable complications for the interpretation of paleoenvironmental factors [e.g. Burckle and Potter 1996]. To date, the primary mechanisms invoked for long-distance transport of biogenic particles (e.g., diatoms, foraminifera and pollen) include bird migration [Atkinson 1980], wind entrainment from dusty environments [Harper and McKay 2010], globally interconnected ocean currents [Cermeño and Falkowski 2009] and ejection during infrequent meteorite impacts [McKay et al. 2008].

In situ aquatic microorganisms and their fossil remains are also known to be disrupted by small-scale phreatic or phreatomagmatic eruptions, which can excavate deep-seated lithologies and churn modern sediments (e.g. the 1886 Rotomahana 'mud', New Zealand [Nairn 1979, Pearson et al. 2010]). Early observations of diatom remains in the Oruanui super-eruption deposits by Colin Vucetich and Prof. Steve Self (Victoria University of Wellington) in the 1970s suggested microorganisms may also be involved in larger scale events (Steve Self, Personal Communication). More recently, a case study of diatom remains from a single, distal outcrop of the Lava Creek B volcanic ash from Yellowstone, USA, by Winsborough [2000] has lent further support to this idea. Although the wall rock contents and lithologies erupted by volcanic events contain a detailed record of eruption mechanisms, the potential information contained in the biogenic signatures of volcanic deposits have never been documented in detail.

This study introduces and investigates the occurrence of aquatic microorganism remains in subaerial volcanic deposits of the world's youngest super-eruption, the $\sim 25.4 \mathrm{ka}$ Oruanui eruption from Taupo volcano, New Zealand. The deposits are extremely widespread in dispersal and recognizable to $1000 \mathrm{~km}$ from source [Carter et al. 1995, Wilson 2001]. Ten eruptive phases are recognized, based on the stratigraphy of ten corresponding units in the fall deposits [Wilson 2001]. Evidence in the eruption deposits for extensive magma-water interaction is attributed to a large paleo-lake overlying the vent area during the Pleistocene (i.e. Lake Huka), which left behind the Huka Falls Formation, a $\sim 350 \mathrm{~m}$ package of intercalated volcaniclastic and diatomaceous lacustrine sediments [Grindley 1965, Manville and Wilson 2004]. Physical evidence of interaction between the Oruanui eruption vents and Lake Huka includes water-rich deposition [Self 
and Sparks 1978] and cm-dm scale rip-up clasts (lithics) of the Huka Falls Formation incorporated in the proximal ignimbrite deposits. Preliminary examination of the proximal and distal pyroclastic deposits have also shown whole and fragmented diatoms to be present in the volcanic ash. The primary aim of this work is to establish the quantities and species of diatomaceous remnants associated with this eruption, and to determine the possible mechanisms by which they were incorporated and dispersed.

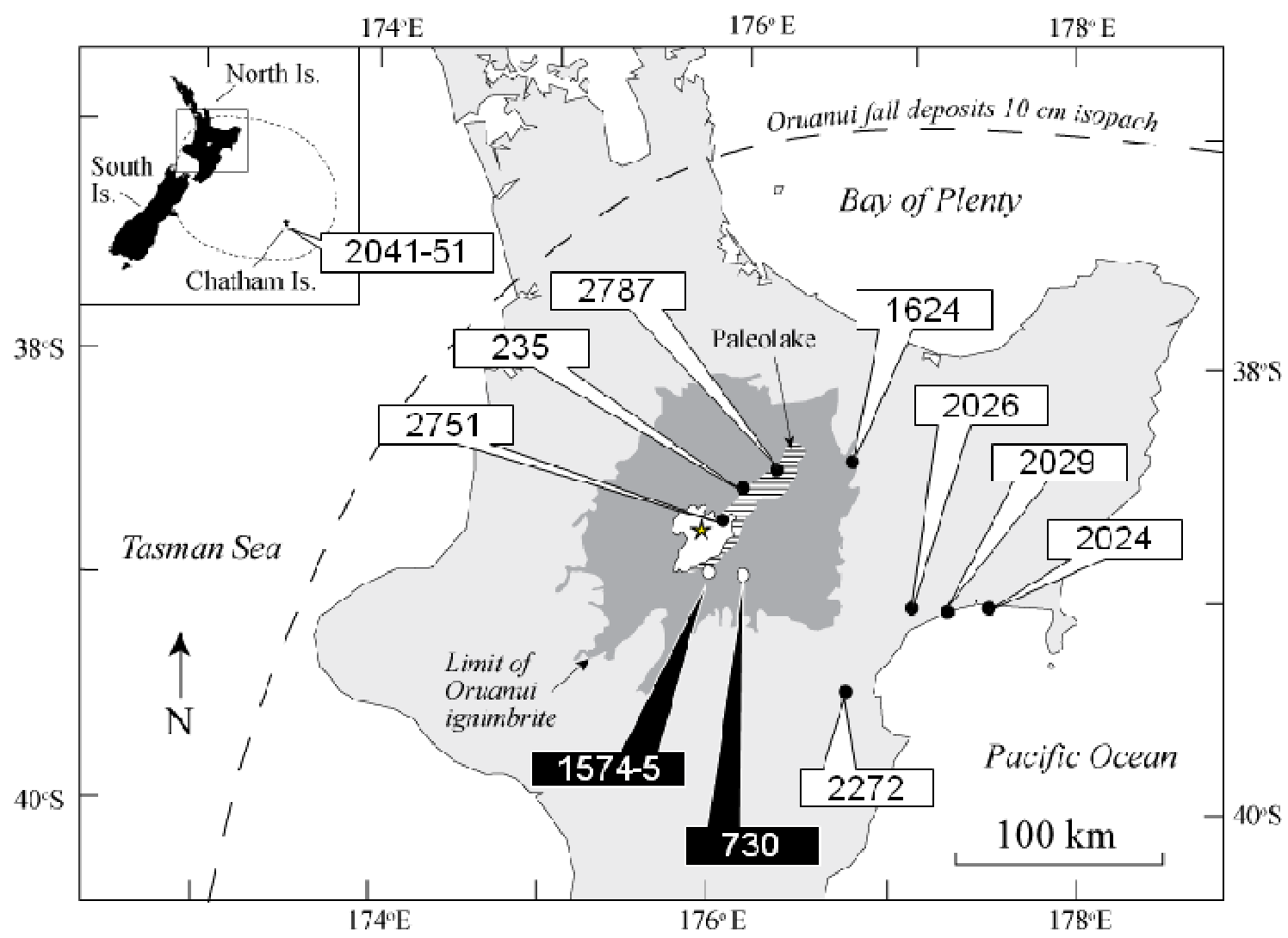

Figure 6.1 Map showing the sampling locations of Oruanui ash deposits (white labels) and lacustrine lithic clasts from the ignimbrite (black labels), collected from the North Island and Chatham Islands (which include Pitt Is.). Locality numbers refer to those in Table 6.1. Modern Lake Taupo is shown with the virtual source of Oruanui volcanism (star) at its center Wilson [2001]. The extent of the paleolake (cross-hatched area) at the time of eruption $\sim 25.4 \mathrm{ka}$ is inferred from the presence of pre-eruptive lake sediments [Grindley 1965, Manville and Wilson 2004]. The limit of the Oruanui ignimbrite and extent of the $10 \mathrm{~cm}$ isopach for fall deposits (shown as a dashed line in the main map and inset) are from Wilson [2001].

\subsection{Methods}

Twenty-two volcanic ash samples from the Oruanui eruption deposits were collected from eleven sites up to $850 \mathrm{~km}$ from the volcanic source (Table 6.1, Figure 6.1). Two fresh sites were sub-sampled in detail to examine individual eruptive units that 
interacted with abundant lake water at vent (units 3, 6 and 8), compared to those which saw relatively less water (units 5 and 7), following Wilson [2001] and Chapter 5 of this thesis [Van Eaton and Wilson, in review]. For selected units, the basal portion and upper portion (labeled a and b, respectively) were sampled separately to establish variability within individual units. A sample of loessic paleosol directly underlying the Oruanui fall deposits was also collected at one distal site to examine the local background assemblages prevailing in the cold, arid climate at the time of eruption [Newnham et al. 1999]. Additional samples of fist-sized ( $10 \mathrm{~cm}$ diameter), accidental lithic clasts of lake sediment were collected from the Oruanui ignimbrite within $22 \mathrm{~km}$ from source. These represent rip-up clasts excavated from lake sediments overlying the vent area during eruption.

Slides containing known quantities of volcanic ash were prepared for analysis of absolute abundances of microfossils. Although the lacustrine lithic samples were prepared using standard techniques [i.e. Patrick and Reimer 1966], the paucity of organic material and carbonate in the volcanic ash rendered pre-treatment with $\mathrm{H}_{2} \mathrm{O}_{2}$ and $\mathrm{HCl}$ unnecessary. Volcanic ash and paleosol samples were dry sieved through a 63 $\mu \mathrm{m}$ mesh to remove coarser material, and a suspension was created by adding distilled water to measured amounts of the sub-63 $\mu \mathrm{m}$ fines. Measured aliquots of well-mixed suspensions of each sample (generally $0.5 \mathrm{~mL}$ ) were pipetted onto round, $22 \mathrm{~mm}$ diameter glass cover slips, gently dried on a $60{ }^{\circ} \mathrm{C}$ hotplate and mounted to slides using Naphrax mountant with a refractive index of 1.65. Controls of filtered water were prepared alongside each step to assess laboratory contamination during preparation. The permanently mounted slides were evaluated to determine absolute abundances of fragmented and whole diatom remains by examination under a Leitz Diaplan differential interference contrast microscope at 1000x magnification. Only fragments $>2$ $\mu \mathrm{m}$ that were positively identifiable as diatomaceous were included in the calculations, even if they could not be identified at species level. At least 150 fields of view across diameter transects of the glass coverslips were analyzed for each sample, totalling $>4,000$ fragmented and whole diatom remains counted in the volcanic ash preparations. These counts provided the number of diatom fragments per mass of sub- $63 \mu \mathrm{m}$ ash. To obtain estimates of abundances in the entire deposits, these values were normalized to the fine fraction measured by laser diffraction (using the method described in Chapter 3 of this thesis). 
Table 6.1 Locations and descriptions of samples used in this study.

\begin{tabular}{|c|c|c|c|c|}
\hline Locality $^{a}$ & Sample ID $^{b}$ & $\begin{array}{l}\text { Distance } \\
(\mathrm{km})^{c}\end{array}$ & $\begin{array}{l}\text { NZTM }^{d} \\
\text { Easting }\end{array}$ & $\begin{array}{l}\text { NZTM } \\
\text { Northing }\end{array}$ \\
\hline \multicolumn{5}{|c|}{ Primary Oruanui volcanic ash deposits } \\
\hline 2751 & unit 3 & 11 & 1830291 & 5523694 \\
\hline 235 & unit 3 & 26 & 1865602 & 5726716 \\
\hline 2787 & unit 3 & 39 & 1881527 & 5759321 \\
\hline 1624 & units $3,6,7 \mathrm{a}, 7 \mathrm{~b}, 8 \mathrm{a}$ & 81 & 1923932 & 5743294 \\
\hline 2272 & units $3,5 \mathrm{a}, 5 \mathrm{~b}, 6,7 \mathrm{a}, 8 \mathrm{a}, 8 \mathrm{~b}$ & 102 & 1920321 & 5627278 \\
\hline 2026 & unit 8a & 111 & 1957639 & 5667841 \\
\hline 2029 & units $3,8 \mathrm{a}$ & 124 & 1970657 & 5664044 \\
\hline 2024 & unit $8 \mathrm{a}$ & 137 & 1985074 & 5668159 \\
\hline 2041 & Chatham Is. Sample No. 2552 & 850 & C.I. 339.0 & C.I. 676.0 \\
\hline 2048 & Chatham Is. Sample No. 2570 & 850 & C.I. 364.3 & C.I. 676.0 \\
\hline 2051 & Chatham Is. Sample No. 2590 & 850 & C.I. 357.2 & C.I. 649.5 \\
\hline Pitt Is. & Locality $14 \mathrm{a}$ of Holt et al. [2010] & 900 & C.I. 373.2 & C.I. 616.8 \\
\hline \multicolumn{5}{|c|}{ Lacustrine lithic clasts in proximal unit 10 Oruanui ignimbrite } \\
\hline 730 & Sample No. R843 & 22 & 1864139 & 5685888 \\
\hline 1574 & Sample No. R845 & 21 & 1861438 & 5685785 \\
\hline 1575 & Sample No. R844 & 15 & 1856231 & 5689683 \\
\hline \multicolumn{5}{|c|}{ Underlying, pre-eruptive paleosol } \\
\hline 2272 & Paleosol & 102 & 1920321 & 5627278 \\
\hline
\end{tabular}

${ }^{a}$ Locality numbers are equivalent to those used in Wilson [2001] unless otherwise noted, and are labeled on the location map in Figure 6.1.

${ }^{b}$ Sample ID distinguishes eruptive units where possible. For units 5, 7 and 8 the basal portion and upper portion (labeled a and $b$, respectively) were sub-sampled to examine variability within individual units. Deposits from Chatham Island and Pitt Island were sampled in bulk, but are inferred to include units 5, 7 and 9 [Wilson 2001].

${ }^{c}$ Distance gives approximate kilometers from the virtual source of Oruanui volcanism from Wilson [2001], shown as a star in Figure 6.1.

${ }^{d}$ NZTM denotes New Zealand Transverse Mercator 2000 projection. Grid references with a C.I. prefix use the Chatham Islands Transverse Mercator 1979 projection. 
In addition, diatom species were identified in the volcanic deposits, lacustrine lithic clasts and paleosol sample by Dr. Margaret Harper (Victoria University of Wellington). In total, 62 diatom taxa were identified in the Oruanui ash (Appendix H) from $>2,500$ specimen identifications. Samples of processed ash were also examined under scanning electron microscope (SEM) to assess the structural preservation of the diatom remains. Finally, a control sample of diatomite from Pukaki crater (Auckland Volcanic Field, New Zealand) was heated to $800{ }^{\circ} \mathrm{C}$ for one hour in a muffle furnace and examined under SEM to investigate what (if any) physical effects may be recorded in the diatom fragments after they experience maintained, high temperatures.

\subsection{Results}

\subsubsection{Abundances and preservation}

Absolute concentrations of whole and fragmented diatom particles in Oruanui deposits and from mainland New Zealand and Chatham Island sites range from 1-51 fragmented and whole diatom remains per microgram of volcanic ash, with an average of 11 per microgram (Table 6.2). These extraordinarily high abundances are of a similar order of magnitude to (whole) valve contents in lacustrine [e.g. Parker and Edgington 1979] or marine sediments [e.g. Koç et al. 1993]. The blank slides prepared alongside each sample suite did exhibit a small number of glass shards and diatom fragments 1-3 $\mu \mathrm{m}$ across (generally fewer than 5 remains per coverslip), indicating that some laboratory contamination occurred during preparation. However, diatom fragments contributed by contamination were negligible (equating to $<0.01$ fragments per microgram) in comparison to the large numbers counted in the Oruanui samples.

There are no systematic changes in abundances with distance from the Oruanui volcanic vent, nor between individual units at each site (Figure 6.2), indicating that concentrations are distributed more or less uniformly between the different phases of eruption. The heavily fragmented nature of the diatom remains is reflected in the high abundance of $2-5 \mu \mathrm{m}$ particles (25-63\%) in each sample. Furthermore, only $6-32 \%$ of the valve fragments were $>25 \%$ intact. Examples of significant fragments from the 
Oruanui deposits imaged with the light microscope and SEM (Figures 6.3-6.4) exhibit extraordinary preservation compared to many of the more poorly preserved examples

Table 6.2 Absolute abundances of diatom remains (whole and fragmented valves) in the Oruanui volcanic ash deposits. The mean and range (maxima and minima) are reported for multiple transect counts. Single-transect counts are denoted by n.a.

\begin{tabular}{|c|c|c|c|c|c|c|c|c|}
\hline Locality & $\begin{array}{l}\text { kms } \\
\text { from } \\
\text { vent }\end{array}$ & Unit & $\begin{array}{l}\text { Total mass } \\
\text { analyzed, } \\
\text { in } \mathrm{mg}\end{array}$ & $\begin{array}{l}\text { Total } \\
\text { specimens } \\
\text { counted }\end{array}$ & $\begin{array}{l}\text { Percent } \\
\text { deposit } \\
<63 \mu \mathrm{m}\end{array}$ & $\begin{array}{l}\text { Normalized } \\
\text { abundance, } \\
\text { per } \mu \mathrm{g}\end{array}$ & $\begin{array}{l}\text { Norm. } \\
\text { min }\end{array}$ & $\begin{array}{l}\text { Norm } \\
\max \end{array}$ \\
\hline 2751 & 11 & 3 & 0.0281 & 339 & 54.6 & 6.65 & 6.35 & 7.00 \\
\hline 235 & 26 & 3 & 0.0173 & 142 & 64.6 & 5.31 & n.a. & n.a. \\
\hline 2787 & 39 & 3 & 0.0174 & 321 & 63.9 & 11.80 & n.a. & n.a. \\
\hline 1624 & 81 & 3 & 0.0173 & 258 & 59.4 & 8.87 & n.a. & n.a. \\
\hline 1624 & 81 & 6 & 0.0175 & 400 & 49.2 & 11.26 & n.a. & n.a. \\
\hline 1624 & 81 & $7 \mathrm{a}$ & 0.0024 & 104 & 81.0 & 35.25 & n.a. & n.a. \\
\hline 1624 & 81 & $7 \mathrm{~b}$ & 0.0043 & 223 & 39.8 & 20.80 & 18.09 & 23.50 \\
\hline 1624 & 81 & $8 \mathrm{a}$ & 0.0024 & 195 & 62.2 & 50.93 & n.a. & n.a. \\
\hline 2272 & 102 & 3 & 0.0038 & 33 & 64.9 & 3.70 & 2.28 & 5.70 \\
\hline 2272 & 102 & $5 a$ & 0.0040 & 52 & 15.1 & 1.06 & 0.50 & 1.98 \\
\hline 2272 & 102 & $5 b$ & 0.0039 & 54 & 54.9 & 3.98 & 1.83 & 7.61 \\
\hline 2272 & 102 & 6 & 0.0042 & 67 & 44.8 & 3.45 & 1.38 & 7.08 \\
\hline 2272 & 102 & $7 \mathrm{a}$ & 0.0039 & 34 & 10.5 & 0.57 & 0.34 & 0.91 \\
\hline 2272 & 102 & $8 \mathrm{a}$ & 0.0039 & 36 & 59.4 & 3.42 & 2.02 & 5.48 \\
\hline 2272 & 102 & $8 b$ & 0.0038 & 22 & 31.9 & 1.49 & 1.11 & 1.86 \\
\hline 2026 & 111 & $8 \mathrm{a}$ & 0.0094 & 360 & 46.3 & 17.77 & n.a. & n.a. \\
\hline 2029 & 124 & 3 & 0.0169 & 201 & 53.9 & 6.41 & n.a. & n.a. \\
\hline 2029 & 124 & $8 \mathrm{a}$ & 0.0073 & 390 & 56.4 & 30.01 & n.a. & n.a. \\
\hline 2024 & 137 & $8 \mathrm{a}$ & 0.0345 & 464 & 60.8 & 8.18 & n.a. & n.a. \\
\hline 2048 & 850 & $\geq 5$ & 0.0631 & 114 & 49.4 & 0.89 & 1.45 & 0.63 \\
\hline 2041 & 850 & $\geq 5$ & 0.0118 & 129 & 62.7 & 2.75 & 2.57 & 2.93 \\
\hline 2051 & 850 & $\geq 5$ & 0.0406 & 141 & 60.1 & 1.38 & 1.72 & 1.05 \\
\hline \multicolumn{8}{|c|}{ Total diatomaceous fragments counted } & 4,079 \\
\hline \multicolumn{8}{|c|}{ Combined mean abundance (fragments per microgram volcanic deposit) } & 11 \\
\hline
\end{tabular}

from the underlying paleosol (Figure $6.4 \mathrm{a}-\mathrm{b}$ ). Well preserved features include rare remains of pigmented cell contents (Figure 6.3a,c), alveoli (Figure $6.4 \mathrm{c}-\mathrm{d}$ ), and interlocked valves (Figure 6.4e-f). Aside from being heavily fragmented, there was little evidence for alteration processes such as dissolution or recrystallization [e.g. Rodgers et 
al. 2002]. The exception to this generally excellent preservation is Oruanui ash collected from the Pitt Island locality, in which no diatom fragments could be identified. The presence of pitted (etched) sponge spicules and pollen grains in this sample indicate

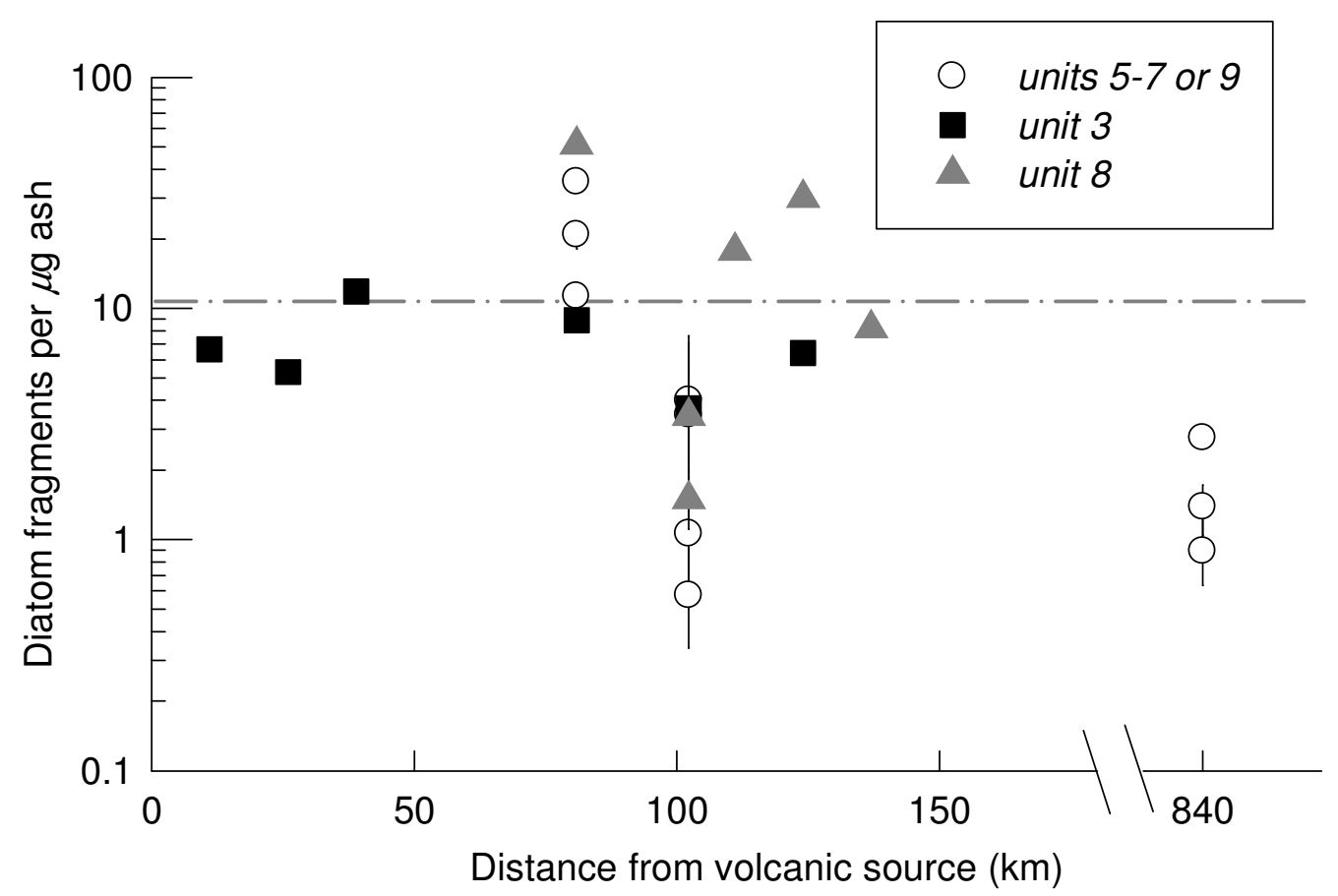

Figure 6.2 Absolute abundances of whole and fragmented diatom remains per microgram of Oruanui volcanic ash, at varying distances from the virtual source. Note break in scale on the $\mathrm{x}$-axis. Black squares represent unit 3, grey triangles are unit 8 , and white circles are undifferentiated units 5-7 or 9. Error bars provide minimum and maximum values (about the mean) for multiple transect counts. Dashed line gives the combined mean of 11 diatom fragments per microgram from all samples (excluding the Pitt Is. locality).

poor preservation conditions and likely reworking, suggesting that any diatomaceous fragments originally present in the deposits at this site are likely to have undergone partial to complete dissolution.

To estimate the volume of erupted diatom remains contained in the Oruanui volcanic deposits $\left(V_{\text {erupt }}\right)$ the total volume of the eruption was multiplied by the volume percent of constituent diatoms. This was calculated using the following equation and associated assumptions:

$$
\mathrm{V}_{\text {erupt }}=\mathrm{V}_{\text {tot }} \cdot\left[\left(\text { Ndiatom } \cdot \mathrm{Vdiatom} / \mathrm{V}_{\text {volc }}\right) \cdot 100\right]
$$

Where $V_{\text {tot }}$ is the bulk volume of eruption deposits (taken as $750 \mathrm{~km}^{3}$ from Wilson [2001], which includes fall and PDC deposits, but not intracaldera fill); $N_{\text {diatom }}$ is the 
number of diatom remains in the volcanic deposits (taken as the mean value of 11 fragments per microgram from Table 6.2); $V_{\text {diatom }}$ is the volume per unit mass of diatom remains (taken as uniform spheres of $5 \mu \mathrm{m}$ diameter); and $V_{\text {volc }}$ is the volume per unit mass of Oruanui deposits (derived from the bulk field density of $1.2 \mathrm{~g} \mathrm{~cm}^{-3}$ [Wilson 2001]). Although the actual shapes and size distributions of the whole and fragmented diatom valves were not explicitly quantified in this study, they were noted to be highly variable (e.g., long axes ranged from $\sim 2$ to $50 \mu \mathrm{m}$ ). Roughly $60 \%$ of the counted fragments were larger than $5 \mu \mathrm{m}$. We therefore take the $5 \mu \mathrm{m}$ spherical diameter value as a reasonable, conservative approximation for this calculation, resulting in a total volume of erupted diatom remains of $\sim 0.6 \mathrm{~km}^{3}$.

\subsubsection{Volcanic ash assemblages and comparison with sediments}

Diatom assemblages in the Oruanui deposits are compared with the Huka Falls Formation lithic clasts recovered from the ignimbrite, and with the underlying paleosol in Figure 6.5. The Oruanui ash and lacustrine sediment samples show a close match, sharing 39 species (Appendix H). The Oruanui assemblages are dominated by freshwater flora (marine diatoms are absent), with each mainland sample containing roughly similar quantities of the dominant species Aulacoseira ambigua, Cyclostephanos novaezeelandiae and Discostella stelligera. Dominance of these open water planktic species is consistent with a large lake source. The overall, mean diatom assemblage averaged from all samples of Oruanui ash ('Oruanui mean' in Figure 6.5) is strikingly consistent with the lacustrine lithic clasts. However, diatoms dispersed in the ash do not exhibit a single, dominant species (like Sample No. R845 in Figure 6.5), as would be expected if the assemblages reflected a single 'snapshot' of the depositional environment. The Oruanui ash samples and two of the Huka Falls Formation lithic clasts thus provide a 'mixed' depositional signal.

The paleosol material sampled directly beneath the Oruanui fall deposits at locality 2272 (Table 6.1), although diatomaceous, contains an assemblage that is markedly different from both the Oruanui deposits and the lacustrine lithics. More than $80 \%$ of the paleosol species are soil-dwelling diatoms, which are rare in the Oruanui ash and lithics $(<1 \%)$. Furthermore, planktic diatoms are poorly preserved (Figure 6.4a-b) in the 
paleosol, suggesting they may have been reworked or mobilized downward from the overlying Oruanui sequence.
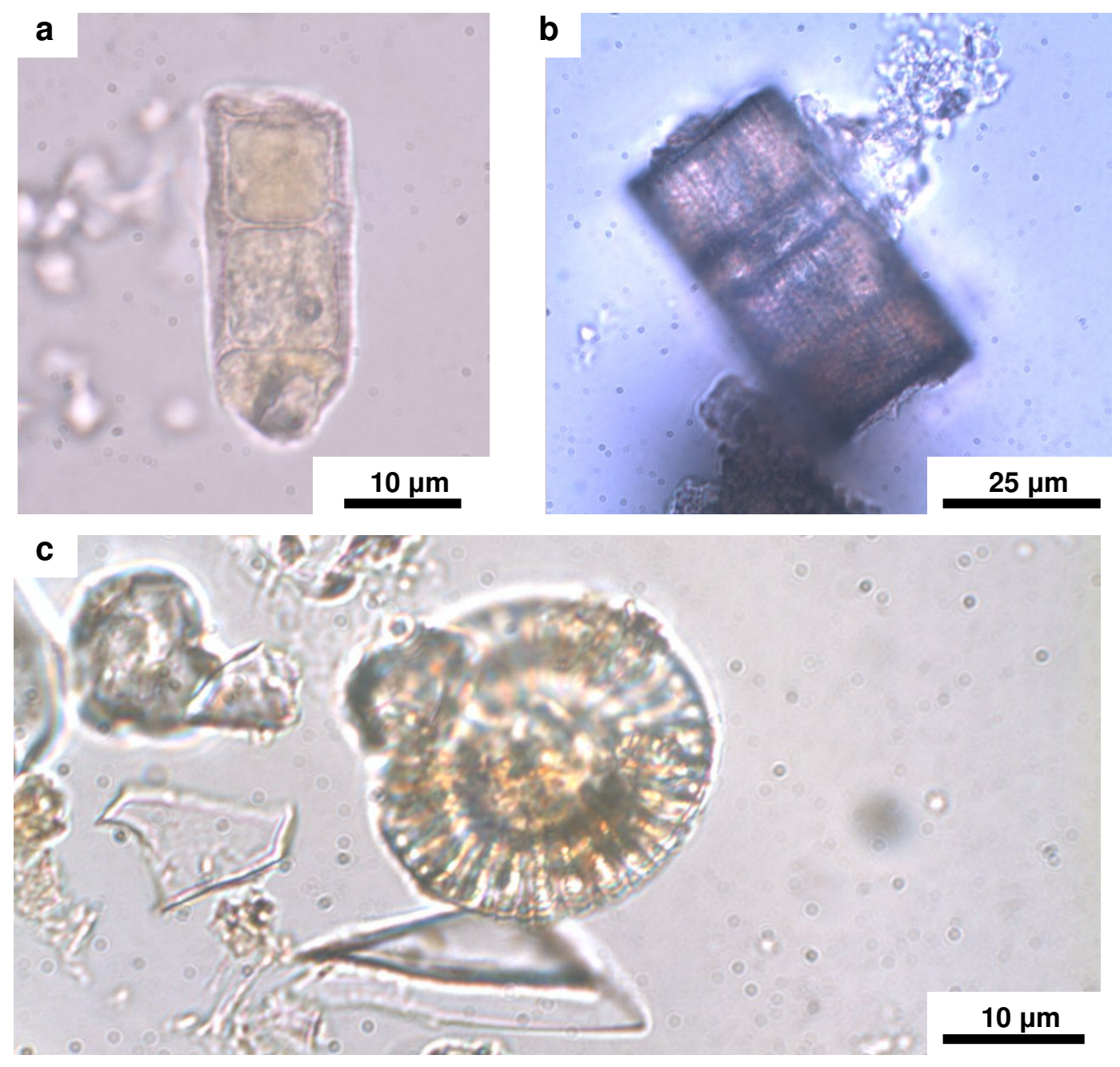

Figure 6.3 Light microscope images of diatom specimens (at $\sim 1000 x$ magnification) showing preserved organic matter. The translucent, golden-brown pigmented regions are indicative of plastid remains. (a) Aulacoseira ambigua from unit 3 Oruanui volcanic ash at locality 2787. (b) Aulacoseira ambigua from a lacustrine lithic clast at locality 730 (Sample No. R843). (c) Cyclostephanos novaezeelandiae from volcanic ash at Chatham Island locality 2041. 

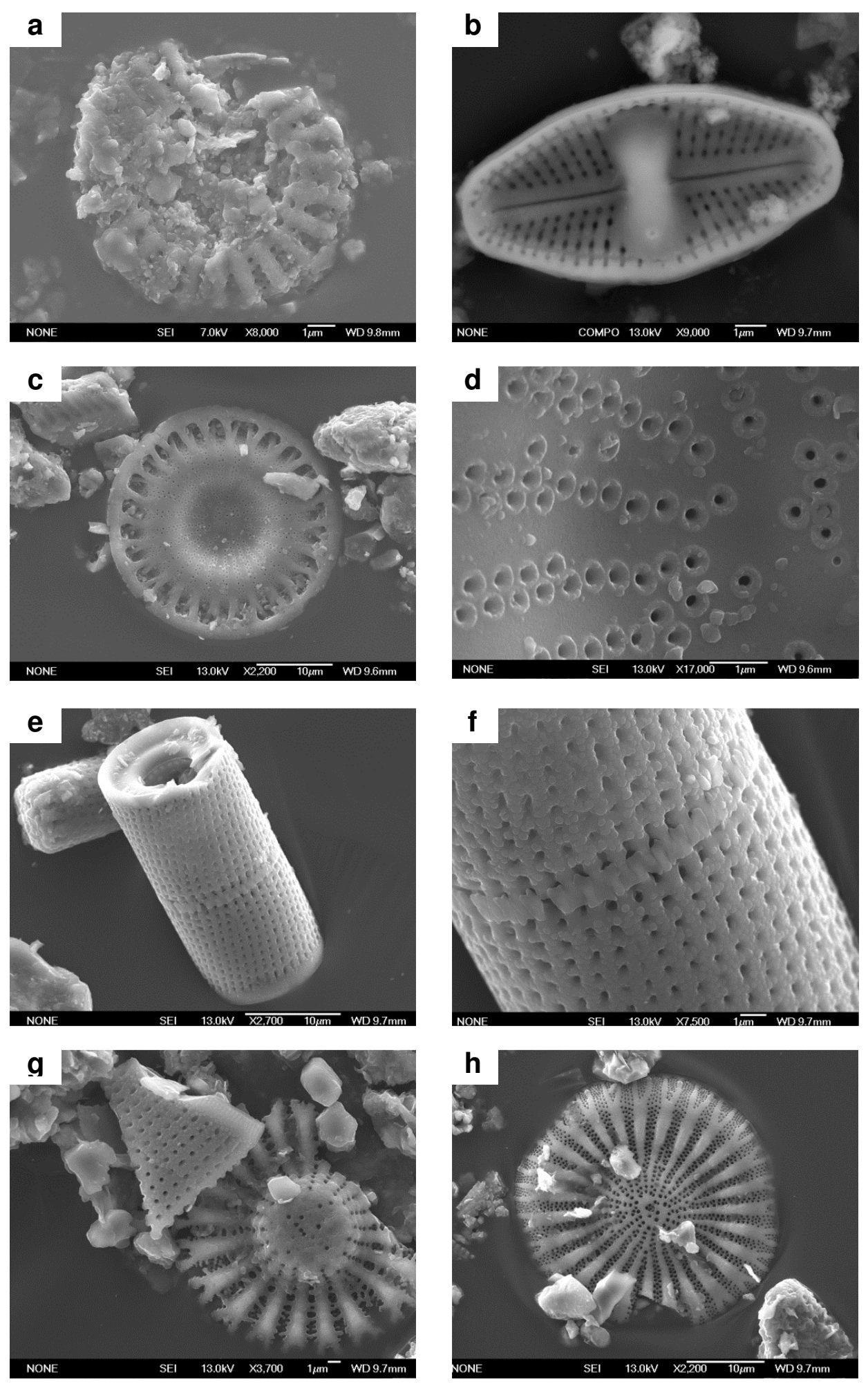

Figure 6.4 SEM images of diatoms

from the Oruanui volcanic ash and underlying paleosol. (a-b) Examples from the underlying paleosol at locality 2272, showing in (a) an external view of a poorly preserved planktonic Stephanodiscoid sp. and in (b) a soil-dwelling Luticola mutica. (c-f) Well-preserved specimens from Oruanui unit 8a volcanic ash deposits at locality 1624: (c) internal view of a Cyclostephanos novaezeelandiae and detail of the alveoli pore walls in (d); (e) interlocked valves of Aulacoseira ambigua and detail in (f). (g-h) Diatoms from 
(Continued from previous page) Oruanui ash on Chatham Island at locality 2570: Specimen in the upper left of (g) is a fragment of Aulacoseira ambigua and in the center-right is an example of the New Zealand endemic Cyclostephanos novaezeelandiae; (h) shows a nearly intact specimen of Cyclostephanos novaezeelandiae.
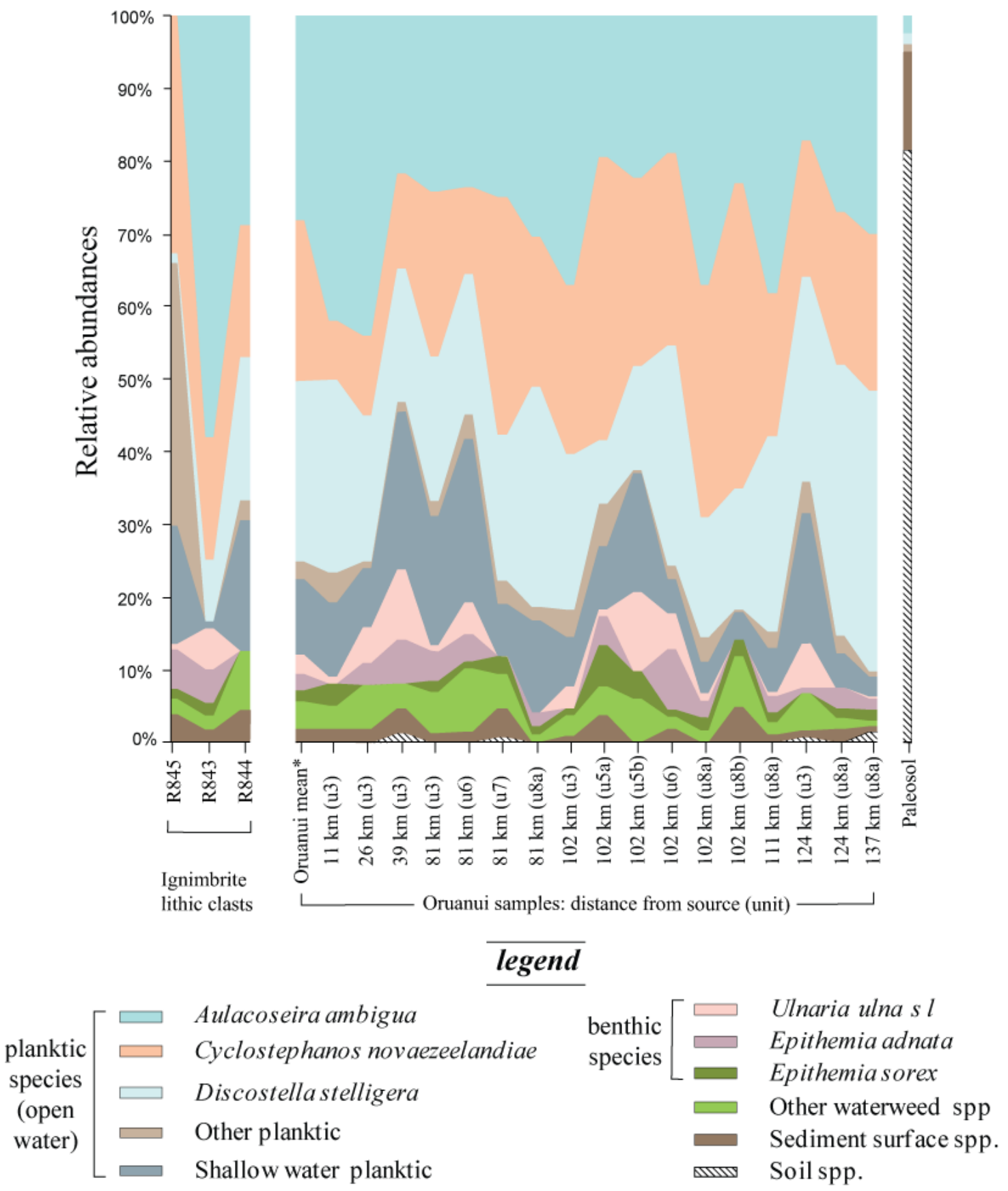

Figure 6.3 Diatom assemblages in the ignimbrite lithic clasts (left panel), Oruanui ash deposits (middle) and underlying pre-eruptive paleosol (right panel). The three most abundant species in the Oruanui ash and lithics are all planktic. *Oruanui mean gives mean species abundances from all the volcanic ash samples. Note that the paleosol (from locality 2272, $102 \mathrm{~km}$ from the Oruanui virtual source) has a distinctive assemblage dominated by soil diatoms, which are primarily absent $(<1 \%)$ in the volcanic ash and lacustrine lithics. 


\subsection{Discussion}

\subsubsection{How were diatoms incorporated?}

The abundance of well preserved, freshwater diatoms and diatomaceous fragments in the Oruanui eruption deposits could be explained by three distinct mechanisms (Figure 6.6): (i) post-depositional reworking and mixing of younger diatoms into the Oruanui deposits from wind-blown transport and rain; (ii) incorporation of airborne diatoms during eruption and transport of the Oruanui ash clouds; and/or (iii) syn-eruptive disturbance and excavation of lacustrine sediments surrounding and overlying the volcanic vent. Although each of these processes are expected to occur to some degree during volcanic eruptions, the following discussion focuses on isolating the mechanism primarily responsible for such unusual proportions of diatomaceous material within the deposit. Each of the hypotheses are examined in light of the data presented to establish the most plausible explanation.

i. post-depositional

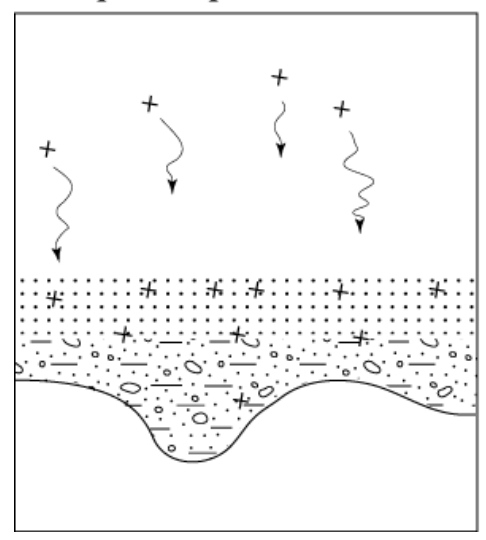

ii. during airborne transport

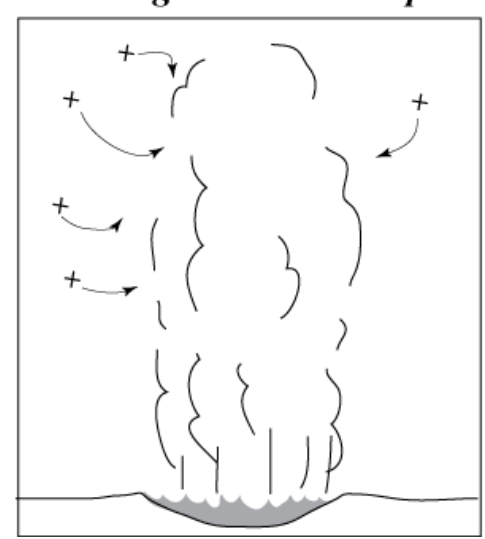

iii. syn-eruptive

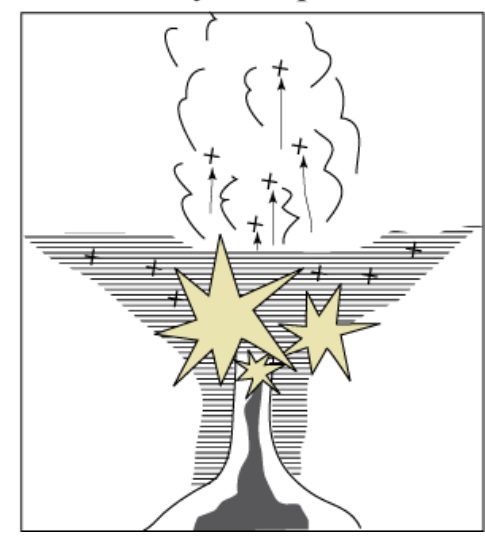

Figure 6.4 Depictions of the three hypotheses critically examined in this study to explain the observed abundances and species of diatomaceous fragments in Oruanui deposits: (i) post-depositional downmixing; (ii) entrainment of airborne diatoms by the eruption clouds; and (iii) syn-eruptive evacuation of underlying lacustrine sediments and unconsolidated lake bed material.

Post-depositional reworking would be expected to leave behind a number of signatures in the deposits. For example, a proportion of local windborne diatoms would be expected to settle to the surface of the Oruanui layers after the eruption. The diatomaceous particles could then percolate downward with rain water, following permeable pathways in the volcanic ash. In the absence of whole-scale physical mixing, 
which can be comprehensively ruled out from the well preserved stratigraphic detail at mainland sites used in this study, the downmixed assemblages would be expected to include additional biogenic particles. For example, pollen, phytoliths and marine diatoms would be expected to be present in subequal abundances with the freshwater diatoms, given the proximity to the oceans and associated sea spray (Figure 6.1). This appears to be the case in the Pitt Island sample, which contained abundant sponge spicules, pollen grains and phytoliths (siliceous plant remains), but the Oruanui deposits (from mainland New Zealand and the Chatham Islands) contain remarkably consistent freshwater diatom assemblages (Figure 6.5). Downmixing would also be expected to transport a similar assemblage of biogenic particles from the uppermost soil layers into soil below. The underlying pre-Oruanui paleosol, however, contains a markedly distinct flora compared to the Huka and Oruanui samples: in particular, less than $20 \%$ of the paleosol diatom species are shared. Furthermore, downmixing should result in higher biogenic particle concentrations in the upper portions of the deposit, followed by a systematic decrease into lower units. Of the three localities from which Oruanui layers were sampled in stratigraphic succession, only locality 1624 (81 km from vent) shows such a trend (Figure 6.2). Post-depositional reworking can thus be ruled out as a governing factor in diatom emplacement in this case.

An alternative possibility is that a large supply of airborne diatoms, lofted from exposed lacustrine sediments around the North Island, existed in the background atmosphere at the time of the Oruanui eruption. In this scenario, diatoms could be entrained and incorporated from the ambient atmosphere into the voluminous eruption clouds during transport. However, the mechanism is considered untenable here because the preeruptive loessic palaeosol sample contains such a low proportion of lacustrine diatoms.

The inference in this study is that syn-eruptive mechanisms are primarily responsible for the abundant microfossils measured in the Oruanui deposits. Phreatomagmatic eruptions are known to incorporate abundant wall rock lithics by mechanical erosion of the conduit and slumping of wet, unconsolidated sediments into the vent [White and Houghton 2000]. A similar scenario is highly likely in the Oruanui setting, supported by evidence for abundant magma-water interaction and the thick sequences of Huka Falls Formation sediments inferred to be actively depositing in Lake Huka in the lead-up to the Oruanui eruption [Grindley 1965, Manville and Wilson 2004]. Requirements for an 
extensive source of freshwater microorganisms are thus readily met in the region directly surrounding the volcanic vent. By comparison with assemblage data, the Oruanui diatom species are closely matched by those in the lacustrine sediments (Figure 6.5), further consistent with a Lake Huka origin. A syn-eruptive, volcanogenic origin therefore provides the simplest mechanism consistent with data presented in this study.

However, one aspect of this finding is particularly intriguing. If the lacustrine sediments of Lake Huka were disrupted and excavated by phreatomagmatic eruptions at the vent, it would be expected that volcanic deposits showing the most evidence for magmawater interaction would contain the highest concentrations of diatomaceous material. As demonstrated in Figure 6.2, this is not the case. For example, units 3, 6 and 8 show more 'water-rich' depositional characteristics than units 5 (a-b) and unit 7 (particularly the 'plinian-style' lower portion, 7b), as described in detail in Chapter 5 of this thesis. Yet there are no significant or systematic differences between the microfossil contents of these units. The observation suggests that roughly similar diatom scavenging processes were operative during all the eruption phases investigated here, regardless of the amount of inferred magma-water interaction.

\subsubsection{Could some microorganisms survive the eruption process?}

The extraordinary preservation state of some of the diatoms, evidenced by the presence of plastids with other cell contents (Figure 6.3a-c), suggests the intriguing possibility that some live specimens may have survived volcanic excavation and transport. Although the species of diatoms identified here are not regularly found in hightemperature environments (i.e. water temperatures $>45^{\circ} \mathrm{C}$ ), it is currently unknown exactly how much environmental stress these varieties of microalgae can withstand, and over what timescales. Given the observation that diatom tests do not appear to produce alteration features even at sustained temperatures of $800{ }^{\circ} \mathrm{C}$ (Figure 6.7), it is challenging to estimate how many of the specimens may have experienced temperatures too extreme to survive. However, a peculiarity of phreatomagmatic eruptions is that the temperature of the erupted mixture can be drastically reduced compared to their dry counterparts. This was certainly the case in the Oruanui eruption. Magma-water mixing cooled the pyroclastic mixture to bulk emplacement temperature $<250{ }^{\circ} \mathrm{C}$ [Wilson 2001], and perhaps locally, to temperatures near ambient (Chapter 2 of this thesis and 
Van Eaton et al. [2012b]). The heterogeneous nature of magma-water interaction leading to strong gradients in temperature and mechanical energy in the vent region is summarized in Figure 6.8. Rapid ascent of actively vesiculating and fragmenting Oruanui magma through the overlying Lake Huka sediments would trigger a number of processes. Mechanical erosion of the weakened vent walls would be induced by phreatomagmatic explosions and uprushing gases [White and Houghton 2000, Lorenz and Kurszlaukis 2007], while slumping and churning of poorly consolidated lake bed sediments would add to a slurried mixture of pyroclasts, water, steam and sediment at the edges of the vent [Kokelaar 1983]. The highest energy processes would likely fracture and fragment the diatomaceous tests by violent expansion of heated steam [Wohletz 1986]. However, due to the chaotic nature of the mixing process, some of the microorganisms would be locally insulated from these high-energy, high-temperature processes. For example, churned lake water and lake bed sediments at the peripheries of the vent areas may 'passively' liberate individual specimens, rather than fracturing them, before they became entrained into the rising eruption column (Figure 6.8). Even relatively unstable, wet eruptions are capable of ascending into the stratosphere (Chapter 2 and Van Eaton et al. [2012b]), and it is envisioned that the eruption clouds would be capable of transporting (possibly live) microorganisms and their fossilized remains hundreds to thousands of $\mathrm{kms}$ from source in a variety of atmospheric levels, including the jet stream.
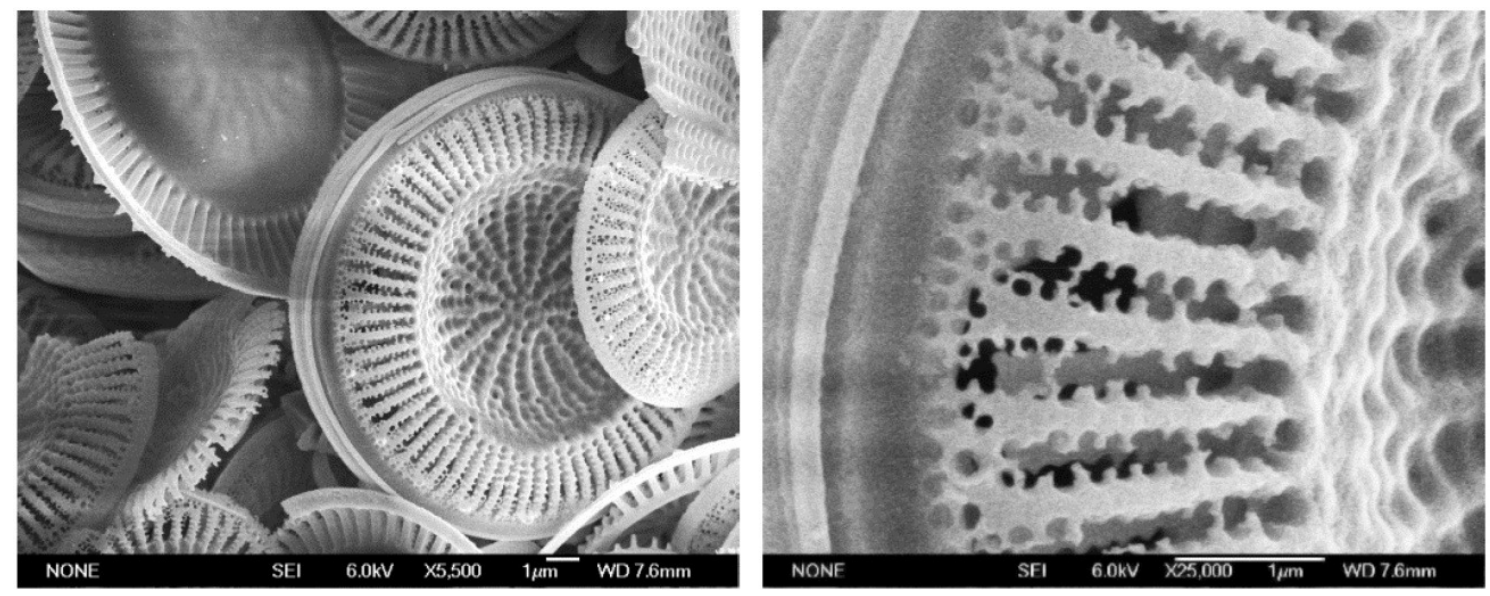

Figure 6.5 SEM images of Discostella stelligera from Pukaki crater, Auckland Volcanic Field, New Zealand, show no obvious physical effects (such as recrystallization or annealing) after heating to $800{ }^{\circ} \mathrm{C}$ for one hour, with the exception that all organic matter (i.e. cell contents) have been volatilized. 


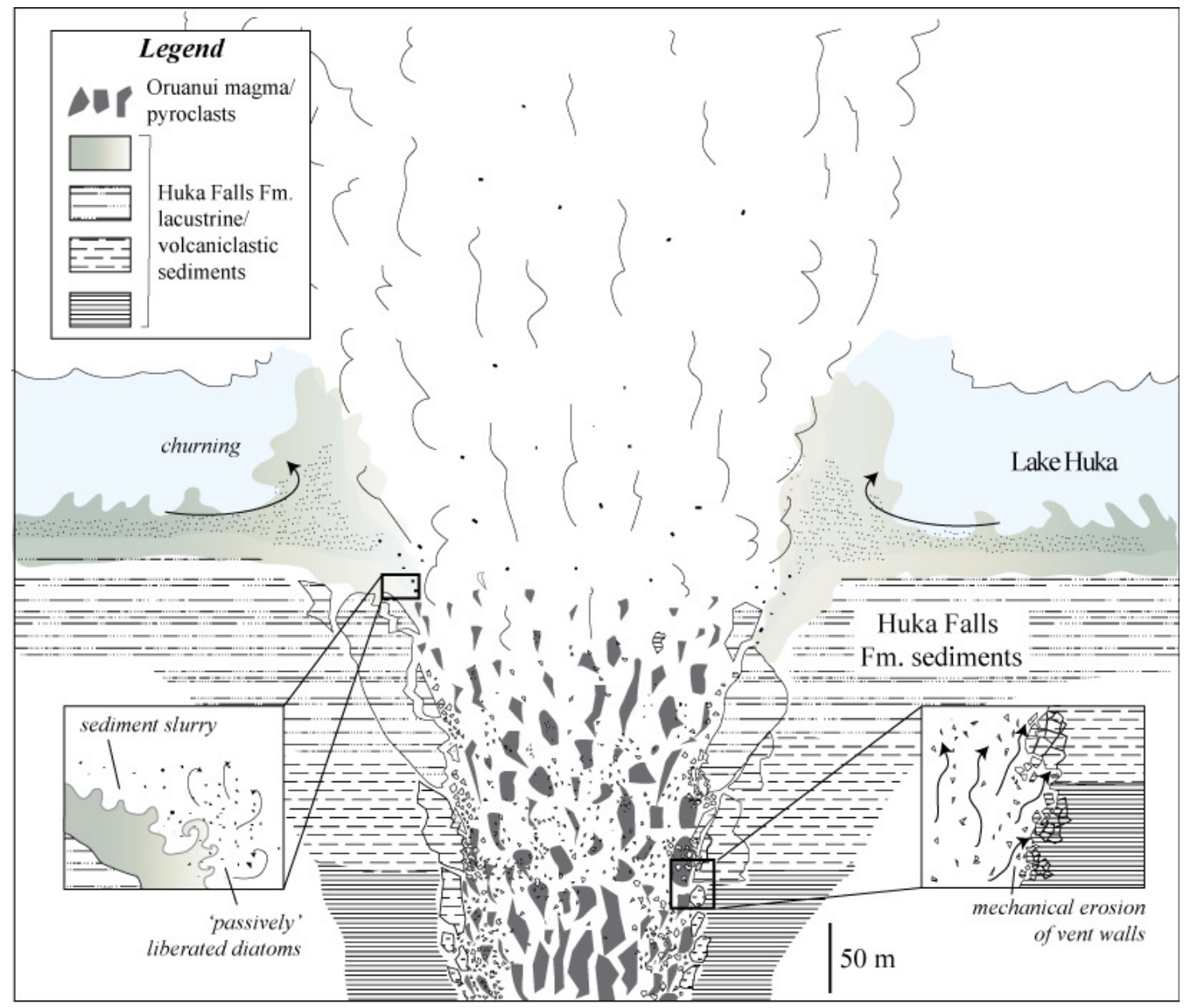

Figure 6.6 Schematic diagram of the proposed model for syn-eruptive disturbance and transport of freshwater diatoms during the Oruanui eruption, with approximate vertical scale in meters. Each of the subsurface lithologies represents one of the four units in the Huka Falls Formation described by Grindley [1965], showing increasing compaction with depth. The uppermost (greenish) unit represents unconsolidated lake bed sediments actively depositing at the time of the eruption. Vesiculating, fragmenting Oruanui magma interacts explosively with wet lacustrine sediments and lake water during ascent, resulting in mechanical erosion and slumping of the vent walls [White and Houghton 2000]. On the periphery of the vent and eruption column, extensive churning and disturbance of the unconsolidated lake bed sediments [Kokelaar 1983] would also have resulted in lower energy, lower temperature liberation of microorganisms into the margins of the eruption column.

\subsubsection{Broader implications}

The volume of whole and fragmented diatoms dispersed from the Oruanui supereruption is estimated to be $\sim 0.6 \mathrm{~km}^{3}$, roughly half the volume of volcanic products generated by the climactic eruption of Mount St. Helens on 18 May 1980 [SarnaWojcicki 1981b]. The potential for widespread dispersal of such extraordinary quantities of biogenic material by volcanic eruptions, primarily diatoms in this case, has global 
implications for studies of explosive volcanism and paleoenvironmental reconstructions. The new mechanism augments existing models for the transport of airborne diatoms worldwide [Harper and McKay 2010], extending beyond currently accepted modes of, for example, wind entrainment, sea spray liberation [Cipriano and Blanchard 1981] and rare meteorite impacts [McKay et al. 2008]. In comparison with arid desert source regions invoked in the wind model [Harper and McKay 2010], the greater likelihood of volcanically erupted microorganisms being sourced from active lakes increases the potential for global transport of live specimens, and subsequent seeding of new populations. Furthermore, the ability for volcanic eruptions to excavate and transport significant quantities of microfossils from subsurface sediments into the stratosphere suggests that the bioaerosols would be capable of encircling the earth, perhaps multiple times before settling to the ground, as observed during the June 2011 eruption of Puyehue-Cordón Caulle, Chile [Gutro 2011]. The small size of the whole and fragmented diatom remains (generally $<50 \mu \mathrm{m}$ ) allows aerodynamic behavior that is similar to fine volcanic glass shards during transport and dispersal. Widespread contamination of exposed surfaces may therefore be facilitated in part by small quantities of 'foreign' microorganisms settling from volcanic fallout (e.g. Sirius Group, Antarctica [Burckle and Potter 1996]). It may thus be more important than previously considered for studies of paleoenvironmental reconstructions, which frequently use volcanic layers as time markers, to take care in considering that the products of explosive volcanism may carry their own biological assemblages.

In studies of explosive volcanism, the potential for eruption deposits to preserve the biological imprints of sediments surrounding the source vent may provide an additional tool for identifying eruption characteristics. In the case of diatomaceous volcanic deposits, the source of water (e.g. lacustrine or marine) involved in phreatomagmatism would be readily identified by analysis of species assemblages. Assemblage data may also provide information about the depths of wall rock excavation if the underlying stratigraphy is well defined. Furthermore, eruptions known to have interacted with sediments (e.g. Vesuvius 79 AD [Cioni et al. 1992]), are likely to contain a biogenic 'fingerprint' unique to the source region, capable of aiding distal ash deposit identification and correlation. Microorganisms like diatoms have also been shown to be effective ice nuclei due to the ordered structure of their organic matrix and siliceous tests [Knopf et al. 2010, Mohler and Hoose 2010]. This suggests that incorporation of 
abundant live and/or fossilized specimens may alter the ice formation mechanisms in high-altitude volcanic clouds [Rose et al. 1995, Durant et al. 2008]. Although likely to have only second-order effects in the overall dynamics of ash clouds, unusually high ratios of diatom to ash particles in the system may yet generate previously unconsidered nonlinear effects in the cloud microphysics.

\subsection{Conclusions}

This study has demonstrated that large quantities of whole and fragmented diatoms are present in primary pyroclastic deposits from the $\sim 25.4$ ka Oruanui eruption of Taupo volcano, New Zealand (mean abundance 11 per microgram of volcanic ash), totalling $\sim 0.6 \mathrm{~km}^{3}$ erupted diatom remains from this eruption. The microalgae remains are linked to syn-eruptive excavation of lacustrine sediments from Lake Huka (i.e., paleo-Lake Taupo) at the volcanic vent site, based on consistent species assemblages that match units from the Huka Falls Formation sediments, the presence of diatom-bearing lithic blocks recovered in near-vent ignimbrite, and the paucity of evidence for reworking or downmixing in the volcanic deposits. It is therefore proposed that explosive volcanic events through water bodies and sedimentary strata are capable of transporting fossilized and, potentially, live microorganisms from the source region to the atmosphere during eruption. Global transport of ancient and modern assemblages of biogenic particles (some of which may have remained viable) may therefore occur more frequently and on a larger scale than previously assumed. Future work testing the occurrence of these observations in other explosive eruption deposits, particularly those erupting through water or aquatic sediment, are required to assess how common the mechanism may be, and to test the applicability of this new tool. 
Chapter 7 


\section{Synthesis and conclusions}

7.1. Key findings

The work in this thesis represents the first time that numerical simulations, analogue physical experiments and field measurements have been synergistically applied to a single pre-historic eruption deposit. Dynamics of the $\sim 25.4 \mathrm{ka}$ Oruanui eruption from Taupo volcano, New Zealand, have been investigated in detail to develop a new contextual framework applicable to the description and analysis of large-scale wet volcanic eruptions worldwide. Each of the four central aims of this thesis, set out in Chapter 1, are summarized here in light of the findings presented in the preceding chapters.

Aim 1: Examine the microphysical and dynamic impact of abundant magmawater interaction on eruption style and injection of volcanic ash into the atmosphere, providing a new conceptual model of explosive, water-rich eruptions.

Numerical simulations of eruption columns using the cloud-resolving Active Tracer High resolution Atmospheric Model (ATHAM) [Oberhuber et al. 1998] were initialized with eruption rates, temperatures and magma-water mixing ratios consistent with the broad field constraints available from the Oruanui eruptive products. Changes in the amount of water involved in idealized eruption columns (0-40 wt.\%) were simulated with respect to magma eruption rates $\left(10^{8}-10^{9} \mathrm{~kg} \mathrm{~s}^{-1}\right)$. This work has established that the involvement of abundant magma-water mixing does not have a simple, predictable effect on eruption style. In other words, increasing or decreasing the water content by even amounts ( $\sim 5 \mathrm{wt} . \%)$ produces non-linear effects in the dynamic system that cannot be pigeon-holed into, for example, buoyant versus non-buoyant (collapsing) behavior [cf. Koyaguchi and Woods 1996]. However, with few exceptions, each of the wet eruptions simulated in this study exhibited some degree of column instability, and were prone to rapid oscillations between Plinian-style ascent and partial to wholesale collapse. This dynamic behavior produced hybrid volcanic plumes that were 
periodically or simultaneously fed by a vent-derived plume and/or lofted thermals from voluminous ground-hugging pyroclastic density currents (PDCs). Although a greater percentage of erupted mass was intruded into lower levels (i.e. below the simulated cold point tropopause) by the hybrid system, the plumes were clearly capable of ascending to equivalent maximum heights and levels of neutral buoyancy as their stable, Plinian counterparts. The fundamental differences between these simulation results and the traditional approaches using one-dimensional top-hat models indicates that plume dynamics resulting from phreatomagmatism can only be accurately depicted with a complete microphysical package resolved in at least two spatial dimensions.

A particularly interesting finding is that these numerical plume simulations are the first to spontaneously generate multiple-level intrusion heights into the atmosphere, a dynamic behavior consistent with historical observations (e.g. Figure 1.2), and observed in laboratory analogue experiments by Carazzo and Jellinek [2012], published after Van Eaton et al. [2012a]. The vertical height with the 'active' intrusion level (i.e. with the fastest spreading rate) was found to change with time depending on the periodicity and vigor of lofted PDCs from below. This result exposes limitations in the present convention of defining ash cloud positions by a single height in the atmosphere. A further key feature is that the simulated water-rich columns demonstrating stable, Plinian behavior injected liquid water into the stratosphere. This implicates the previously unconsidered possibility that wet aggregation may be capable of occurring at these levels. Abundant aggregation in the column was also found boost cloud heights (maximum and level of neutral buoyancy) due to gravitational separation, suggesting that the early deposition of fine ash may actually have the effect of increasing the capacity for widespread dispersal of a higher (potentially coarser), partitioned fraction.

A new generalized model for phreatoplinian eruptions thus consists of a hybrid volcanic column with multiple transport levels: coarser particles are injected to higher altitudes by the plinian 'core', whereas pyroclastic density currents elutriate preferentially finer ash into the lower atmosphere.

Aim 2: Investigate the mechanisms of volcanic ash aggregation, linking field observations of aggregate textures with the occurrence of specific processes and water phases in volcanic ash clouds. 
Employing a new semi-automated method of vibratory pan aggregation, analogue laboratory experiments have demonstrated the dominant role of liquid aggregation in rapid and widespread aggregation of pellets capable of surviving in the geologic record. The work has identified a previously undocumented continuum between the forces of electrostatic attraction, liquid tension and (the proposed mechanism of) ice sintering. This finding provides a unified framework by which complex processes of aggregation may be simplified using continuous relationships, rather than separate, stepwise numerical treatments in volcanic ash transport and dispersion models. The new experimental approach provides a systematic and reproducible method of generating experimentally-determined aggregation coefficients, based on systematic, laser diffraction-based particle size analysis, which provides a means of estimating the effective grain size of particles in aggregating volcanic clouds. The experiments have also emphasized the key roles of liquid water and parental grain size in generating: (1) layered, concentric structure in aggregates, and (2) the anomalously ultrafine-grained rims of a characteristic class of aggregates observed in the field. Melting snow pellets were found to accrete ash very effectively (with closely matched analogues in natural samples), in contrast to the liquid-free mechanism of electrostatic attraction plus ice sintering inferred to be at work in high, dry levels of volcanic clouds. This observed contrast in the physical experiments suggests that conditions required for formation of coherent ash pellet structures are only readily met in regions of the ash cloud that contain liquid water. The novel approach also provides an improved framework for testing and developing experimental aggregation work in the future.

Aim 3: Clarify the roles of magma-water interaction and ash aggregation in eruption, transport and depositional characteristics of large-scale wet eruptions.

Field observations and measurements of the Oruanui eruption deposits have emphasized two end-member styles of volcanic ash aggregation: proximal PDC-influenced, and distal 'late-stage' aggregation. The former style, which dominates in water-rich portions of units 3, 6, 7 and 8, generates a wide range of aggregate types from coalesced ash pellets to complexly layered accretionary lapilli. Stratigraphic relationships using these types provide a demonstrable link with the passage of PDCs and an overriding cloud. 
The relative volume of PDCs versus buoyant plume material is thought to play an important role in this process: only coeval timing and sub-equal mass partitioning into the two components appear to support formation of the most complex aggregate types. It is thus inferred that complexly layered and ultrafine rim-type accretionary lapilli are intimately linked to the passage of PDCs (i.e. through their role in creating a destabilizing 'wedge' effect below the overlying cloud), but are not formed directly in the lower, ground-hugging poorly sorted and more concentrated portion of PDCs. This fundamental distinction allows aggregates with complex structure to be linked to the passage of PDCs even if they were not themselves deposited in laterally emplaced material. The proposed phreatoplinian model of a hybrid coarser, upper level cloud and lower-level ultrafine ash cloud invoked in formation of the PDC-influenced Oruanui aggregates may prove a useful, testable concept in other field areas. Evidence for recycling of the aggregate components is documented, for example, in: (1) aggregates with repeated and cyclic internal microstratigraphy (Figure 5.6e) and (2) ultrafine rim fragments found in deposits tens of kilometers from their point of origin (Figure 5.6g), indicating complex re-entrainment processes. In contrast, the later-stage aggregation processes are relatively lower energy and produce correspondingly less complex aggregate types. Orographic effects and subsidence/melting of ice particles transported by the cloud (best expressed in Oruanui units 2 and 5) are proposed as key factors in the initiation of weak ash pellets (which are only preserved at exceptionally fresh sites in unit 2) and snowflake-like particle clusters (in the fine top of unit 5) some $80-120 \mathrm{~km}$ from source. These preserved structures are perhaps the best documented examples of a pre-historic analogue to the 'sorem clusters' of Mount St Helens 1980 [Sorem 1982].

Aim 4: Explore the conditions under which distal volcanic deposits may uniquely record evidence for magma-water interaction in a lake environment.

The unanticipated discovery that diatoms are abundant in the Oruanui deposits (estimated total diatom volume of $\sim 2.6 \mathrm{~km}^{3}$ ), and that they can be positively matched with a logical source (the Huka Falls Formation sediments directly surrounding and overlying the vent area), provides a novel mechanism of widespread microorganism transport. The capacity for ancient and fossilized biogenic remains, as well as possibly viable organisms, also indicates a previously unconsidered capacity for eruptions to 
'seed' biological material between locations separated by hundreds to thousands of kilometers. The proposed mechanism by which they are incorporated into the eruption plumes (by vent wall erosion and churning/slumping of lake sediments in the vent during magma evacuation) further suggests that phreatoplinian eruptions may not be the only volcanic events capable of excavating microfossil remains. It may be that the majority of explosive eruptions interacting with sediments contain a geographically and temporally unique biogenic fingerprint.

\subsection{Future perspectives}

In the process of addressing the specific aims highlighted above, a number of new questions and possibilities have arisen for future work. Although it is clear that unsteady phreatoplinian columns are capable of stratospheric ascent in the windless 2-d simulations employed here, the impacts of a cross-wind have never been tested. There is likely to be an additional layer of complexity to phreatoplinian ascent dynamics that was neglected using ATHAM's cylindrical mode. For example, the initially weak thermals lofted off PDCs may be sheared off by strong winds, potentially inducing wholescale collapse in a plume that exhibited powerful ascent in the windless case. Therefore, how does the influence of wind impact the entrainment characteristics and ascent dynamics of what may be described as 'dirty thunderstorms'? This topic would form a rewarding follow-up study using ATHAM.

Another important topic for future research is the incorporation of an explicit description of ash-hydrometeor aggregation. The version of the ATHAM code used in this work did not include the aggregation module of Textor et al. [2006a,b], leaving ample scope for an entirely new facet of water-rich eruptions to be simulated for the first time. This would have the potential to address the role of wet versus dry aggregate growth in different regions of the plume under various eruption styles (stable versus transitional), and would be of great value for calibrating the faster-running parameterizations of these processes in an operational context.

Ice was found to play a central role in the microphysics of phreatoplinian eruptions, with both field observations and numerical simulations indicating enhanced ability for 
ice and liquid scavenging processes out to $80-100 \mathrm{~km}$ or more from vent. However, neither the Oruanui field deposits nor modeling indicates a role for downbursts, or powerful downdrafts of rain [cf. Walker 1981b, Smith 1998]. Therefore, the question still remains: what are the contrasting conditions that lead to rain gullying and intraformational erosion like that seen in abundance in the $1.8 \mathrm{ka}$ Hatepe and Rotongaio ashes? One possible way to address this issue using the approach developed in this thesis would be to investigate phreatoplinian ATHAM simulations in a warmer atmosphere. Due to the slightly cooler climate prevailing during the Oruanui eruption $\sim 25.4 \mathrm{ka}$, the simulated atmosphere was prescribed a ground-level temperature of $0^{\circ} \mathrm{C}$. Thus, the observed 'curtain' of graupel and ice particles falling out of the simulated Oruanui clouds was not given the opportunity to melt before landing. New simulations examining a warmer scenario, perhaps with ground-level temperatures of $20^{\circ} \mathrm{C}$ may show these effects more strongly.

A further intriguing observation is the lack of hurricane-like supercell behavior [c.f. Baines and Sparks 2005, Chakraborty et al. 2009] inferred from the Oruanui deposits. Taking the 'dirty thunderstorm' analogue to its logical conclusion suggests that the high heat flux and abundant water may have provided sufficient conditions for mesocyclone formation. However, Oruanui deposits lack the regional-scale wind-reworking that would be expected to occur during hurricane-force winds, and no rotational effects were noted in the ATHAM simulations performed here. The latter is attributed to the short simulated eruption times $(\leq 3 \mathrm{~h})$, which is likely insufficient for Coriolis forces to take effect, as well as the 2-d framework and simplified wind field. However, an excellent approach for future study would be to couple high-resolution ATHAM output from an idealized phreatoplinian eruption with an aerosol-tracing weather model (such as WRFChem). This coupled approach may provide insights into the factors governing the observed presence or absence of Mount Pinatubo-like rotational effects.

A number of key questions also exist in terms of volcanic ash aggregation. In particular, it is still unclear what mechanism provides the integrity of aggregates that survive landing (without obvious evidence for precipitated salts). Must secondary precipitates always provide the permanent binding forces, or is there some other process at work? Further aggregation experiments testing the processes of aggregation in high temperature, pyroclastic flow-like conditions would be interesting to discover if there 
was something missing in the room-temperature and below-freezing experimental systems employed here. Furthermore, the coarsening mean grain size with distance from vent observed in the Oruanui unit 3 deposits may be examined more quantitatively in the future using a tephra fallout model such as ASHFALL [Hurst and Turner 1999]. For example, prescribing a total grain size distribution consistent with unit 3 and subsequently imposing 'aggregation-induced' fall velocities during transport would help define the extent to which aggregation must occur (and in which size classes) to reproduce the observed coarsening.

Overall, the work presented in this thesis has demonstrated that magma-water interaction at vent governs column stability and intrusion dynamics, which in turn governs aggregation processes and depositional facies. The complexity of ash aggregates preserved in the geologic record are related to a suite of temporally and spatially-dependent processes, and the observations highlighted here need to be compared against other aggregate-bearing eruption deposits to fine-tune a general model. Furthermore, it is clear that evidence from the geological record can both inform and expand the capacity of current numerical models to handle complex, non-linear volcanic plume phenomena. Thus combined, the strength of this coupled volcanological and meteorological approach has great potential as a research tool for calibrating and fine-tuning the physics of operational models needed for better response to modern volcanic eruption clouds with respect to aviation safety and other issues. The further development of fast computing capabilities allied with the more rigorous approaches to plume modeling applied here may be key to more accurate responses to future volcanic ash cloud events. 


\section{References}

ALLEN, S. R., and R. A. F. CAS (1998), Rhyolitic fallout and pyroclastic density current deposits from a phreatoplinian eruption in the eastern Aegean Sea, Greece, Journal of Volcanology and Geothermal Research, 86, 219-251.

Alloway, B., J. Westgate, B. Pillans, N. Pearce, R. M. Newnham, M. Byrami, and S. AARBURG (2004), Stratigraphy, age and correlation of middle Pleistocene silicic tephras in the Auckland region, New Zealand: a prolific distal record of Taupo Volcanic Zone volcanism, New Zealand Journal of Geology and Geophysics, 47, 447-479.

Alloway, B. V., D. J. Lowe, D. J. A. Barrell, R. M. Newnham, P. C. Almond, P. C. Augustinus, N. A. N. Bertler, L. Carter, N. J. Litchfield, M. S. McGlone, J. Shulmeister, M. J. Vandergoes, P. W. Williams, and N. I. Members (2007), Towards a climate event stratigraphy for New Zealand over the past 30000 years (NZINTIMATE project), Journal of Quaternary Science, 22, 9-35.

ARAMAKI, S., and Y. HAYAKAWA (1984), Sequence and mode of eruption of the October 3-4, 1983 eruption of Miyakejima, Bulletin of the Volcanological Society of Japan, 29, S24-S35.

ARMienti, P., G. MACEdonio, and M. PAReschi (1988), A numerical model for the simulation of tephra transport and depostion: applications to May 18, 1980 Mount St Helens eruption, Journal of Geophysical Research, 93, 6463-6476.

Arvidson, R. E., S. W. Ruff, R. V. Morris, D. W. Ming, L. S. Crumpler, A. S. Yen, S. W. Squyres, R. J. Sullivan, J. F. Bell, N. A. Cabrol, B. C. Clark, W. H. Farrand, R. Gellert, R. Greenberger, J. A. Grant, E. A. Guinness, K. E. Herkenhoff, J. A. Hurowitz, J. R. Johnson, G. Klingelhofer, K. W. LEwis, R. Li, T. J. McCoy, J. Moersch, H. Y. McSween, S. L. Murchie, M. Schmidt, C. Schroder, A. Wang, S. Wiseman, M. B. Madsen, W. Goetz, and S. M. McLennan (2008), Spirit Mars Rover Mission to the Columbia Hills, Gusev Crater: Mission overview and selected results from the Cumberland Ridge to Home Plate, Journal of Geophysical Research, 113, E12S33, doi: 10.1029/2008JE003183.

AtKinson, K. M. (1980), Experiments in dispersal of phytoplankton by ducks, British Phycological Journal, 15, 49-58.

BACON, C. R. (1977), High temperature heat content and heat capacity of silicate glasses: experimental determination and a model for calculation, American Journal of Science, 277, 109-135.

BAines, P. G., and R. S. J. Sparks (2005), Dynamics of giant volcanic ash clouds from supervolcanic eruptions, Geophysical Research Letters, 32, L24808, doi: 10.1029/2005GL024597.

Baines, P. G., M. T. Jones, and R. S. J. Sparks (2008), The variation of largemagnitude volcanic ash cloud formation with source latitude, Journal of Geophysical Research, 113, D21204, doi: 10.1029/2007JD009568. 
BAKER, P. A. (2001), The history of South American tropical precipitation for the past 25,000 years, Science, 291, 640-643.

BARKER, A. (1999), Independent palaeomagnetic investigation into the emplacement temperature of the Oruanui ignimbrite, B.Sc. Honors thesis, University of Oxford, Oxford, U.K.

Beckman-Coulter (2003), LS 13320 Laser Diffraction Particle Size Analyzer Instrument Manual, 183 pp., Beckman Coulter, Inc., Miami.

BERRY, J. A. (1928), The volcanic deposits of Scinde Island. With special reference to the pumice bodies called chalazoidites, Transactions and Proceedings of the New Zealand Institute, 59, 571-608.

Beuselinck, L., G. Govers, J. Poesen, G. Degraer, and L. Froyen (1998), Grainsize analysis by laser diffractometry: comparison with the sieve-pipette method, Catena, 32, 193-208.

BLACKFORD, J. R. (2007), Sintering and microstructure of ice: a review, Journal of Physics, 40, R355-R385.

Blatt, H., G. Middleton, and R. MurRay (1980), Origin of Sedimentary Rocks, 2nd ed., 782 pp., Prentice-Hall, Englewood Cliffs, New Jersey.

Blott, S. J., D. J. Croft, K. Pye, S. E. Saye, and H. E. Wilson (2004), Particle size analysis by laser diffraction, Geological Society, London, Special Publications, 232, 6373.

Bonadonna, C., and J. C. Phillips (2003), Sedimentation from strong volcanic plumes, Journal of Geophysical Research, 108, 2340, doi: 10.1029/2002JB002034.

Bonadonna, C., G. C. Mayberry, E. S. Calder, R. S. J. Sparks, C. Choux, P. Jackson, A. M. Lejeune, S. C. Loughlin, G. E. Norton, W. I. Rose, G. Ryan, and S. R. Young (2002), Tephra fallout in the eruption of Soufriere Hills volcano, Monserrat, in The Eruption of Soufriere Hills Volcano, Montserrat from 1995-1999, edited by T. H. Druit and B. Kokelaar, pp. 483-516, Geological Society of London Memoirs 21.

Bonadonna, C., C. B. Connor, B. F. Houghton, L. Connor, M. Byrne, A. Laing, and T. K. HiNCKS (2005), Probabilistic modeling of tephra dispersal: Hazard assessment of a multiphase rhyolitic eruption at Tarawera, New Zealand, Journal of Geophysical Research, 110, B03203, doi: 10.1029/2003JB002896.

Bonadonna, C., A. Folch, S. Loughlin, and H. Puempel (2011a), Future developments in modelling and monitoring of volcanic ash clouds: outcomes from the first IAVCEI-WMO workshop on Ash Dispersal Forecast and Civil Aviation, Bulletin of Volcanology, published online, doi: 10.1007/s00445-011-0508-6.

Bonadonna, C., R. Genco, M. Gouhier, M. Pistolesi, R. Cioni, F. Alfano, A. HOSKULDSSON, and M. RIPEPE (2011b), Tephra sedimentation during the 2010 Eyjafjallajökull eruption (Iceland) from deposit, radar, and satellite observations, Journal of Geophysical Research, 116, B12202, doi: 10.1029/2011JB008462. 
Bond, A., and R. S. J. Sparks (1976), The Minoan eruption of Santorini, Greece, Journal of the Geological Society of London, 132, 1-16.

Booth, B., and G. P. L. WALKer (1973), Ash deposits from the new explosion crater, Etna 1971, Philosophical Transactions of the Royal Society of London, A274, 147-151.

BRANNEY, M. J. (1991), Eruption and depositional facies of the Whorneyside Tuff Formation, English Lake District - an exceptionally large-magnitude phreatoplinian eruption, Geological Society of America Bulletin, 103, 886-897.

BRANNEY, M. J., and R. J. BROWN (2011), Impactoclastic density current emplacement of terrestrial meteorite-impact ejecta and the formation of dust pellets and accretionary lapilli: evidence from Stac Fada, Scotland, Journal of Geology, 119, 275-292.

BRATHWAite, R. L. (2003), Geological and mineralogical characterization of zeolites in lacustrine tuffs, Ngakuru, Taupo Volcanic Zone, New Zealand, Clays and Clay Minerals, 51, 589-598.

Brathwaite, R. L., D. HiLl, and R. J. Merchant (2006), Zeolite deposits in lacustrine tuffs, Ngakuru, Taupo Volcanic Zone, in Geology and Exploitation of New Zealand Mineral Deposits, edited by A. B. Christie and R. L. Brathwaite, pp. 211-216, Australasian Institute of Mining and Metallurgy Monograph 25.

Brazier, S., A. N. Davis, H. Sigurdsson, and R. S. J. Sparks (1982), Fall-out and deposition of volcanic ash during the 1979 explosive eruption of the Soufriere of St. Vincent, Journal of Volcanology and Geothermal Research, 14, 335-359.

Brazier, S., R. S. J. Sparks, S. N. Carey, H. Sigurdsson, and J. A. Westgate (1983), Bimodal grain size distribution and secondary thickening in air-fall ash layers, Nature, 301, 115-119.

Brown, R. J., B. P. KoKelaAR, and M. J. BranNeY (2007), Widespread transport of pyroclastic density currents from a large silicic tuff ring: the Glaramara tuff, Scafell caldera, English Lake District, UK, Sedimentology, 54, 1163-1189.

Brown, R. J., M. J. Branney, C. MAHER, and P. D. HARris (2010), Origin of accretionary lapilli within ground-hugging density currents: evidence from pyroclastic couplets on Tenerife, Geological Society of America Bulletin, 122, 305-320.

Brown, R. J., C. Bonadonna, and A. J. Durant (2012), A review of volcanic ash aggregation, Physics and Chemistry of the Earth, in press, doi: 10.1016/j.pce.2011.11.001.

Burckle, L. H., and N. PotTER (1996), Pliocene-Pleistocene diatoms in Paleozoic and Mesozoic sedimentary and igneous rocks from Antarctica: a Sirius problem solved, Geology, 24, 235-238.

BursiK, M. (1998), Tephra dispersal, in The Physics of Explosive Volcanic Eruptions, edited by J. S. Gilbert and R. S. J. Sparks, pp. 115-144, Geological Society of London Special Publication 145. 
BURSIK, M. (2001), Effect of wind on the rise height of volcanic plumes, Geophysical Research Letters, 28, 3621-3624.

BursiK, M. I., and A. W. Woods (1991), Buoyant, superbuoyant and collapsing eruption columns, Journal of Volcanology and Geothermal Research, 45, 347-350.

Bursik, M. I., R. S. J. Sparks, S. N. Carey, and J. N. Gilbert (2004), The concentration of ash in volcanic plumes, inferred from dispersal data, United States Geological Survey Bulletin, 2047, 19-29.

Bursik, M., S. Kobs, A. Burns, O. Braitseva, L. Bazanova, I. Melekestsev, A. Kurbatov, and D. PIERI (2009), Volcanic plumes and wind: Jetstream interaction examples and implications for air traffic, Journal of Volcanology and Geothermal Research, 186, 60-67.

CAlder, E. S., R. S. J. Sparks, and A. W. Woods (1997), Dynamics of co-ignimbrite plumes generated from pyroclastic flows of Mount St. Helens (7 August 1980), Bulletin of Volcanology, 58, 432-440.

Carazzo, G., and A. M. JellineK (2012), A new view of the dynamics, stability and longevity of volcanic clouds, Earth and Planetary Science Letters, 325-326, 39-51.

Carey, R. J., B. F. Houghton, and T. Thordarson (2010), Tephra dispersal and eruption dynamics of wet and dry phases of the 1875 eruption of Askja Volcano, Iceland, Bulletin of Volcanology, 72, 259-278.

CAREY, S., and H. SigurdSSON (1982), Influence of particle aggradation on deposition of distal tephra from the May 18, 1980, eruption of Mount St. Helens volcano, Journal of Geophysical Research, 87, 7061-7072.

CAREY, S., and H. Sigurdsson (1987), Temporal variations in column height and magma discharge rate during the A.D. 79 eruption of Vesuvius, Geological Society of America Bulletin, 99, 303-314.

Carey, S., and H. Sigurdsson (1989), The intensity of plinian eruptions, Bulletin of Volcanology, 51, 28-40.

CAREY, S., and R. S. J. SPARKS (1986), Quantitative models of the fallout and dispersal of tephra from volcanic eruption columns, Bulletin of Volcanology, 48, 109-125.

CARroll, M. R., and J. R. Holloway (Eds.) (1994), Volatiles in magmas, Mineralogical Society of America, Reviews in Mineralogy 30, 517 pp.

Carter, L., C. S. Nelson, H. L. Neil, and P. C. Froggatt (1995), Correlation, dispersal, and preservation of the Kawakawa Tephra and other late Quaternary tephra layers in the Southwest Pacific Ocean, New Zealand Journal of Geology and Geophysics, 38, 29-46.

Carter, L., B. Alloway, P. Shane, and J. Westgate (2004), Deep-ocean record of major late Cenozoic rhyolitic eruptions from New Zealand, New Zealand Journal of Geology and Geophysics, 47, 481-500. 
Casadevall, T. J. (1994), The 1989-1990 eruption of Redoubt volcano, Alaska: impacts on aircraft operations, Journal of Volcanology and Geothermal Research, 62, 301-316.

Cashman, K. V., M. T. Mangan, and S. Newman (1994), Surface degassing and modifications to vesicle size distributions in active basalt flows, Journal of Volcanology and Geothermal Research, 61, 45-68.

Cashman, K. V., B. Sturtevant, P. Papale, and O. Navon (2000), Magmatic fragmentation, in Encyclopedia of volcanoes, edited by H. Sigurdsson, B. F. Houghton, S. R. McNutt, H. Rymer and J. Stix, pp. 421-430, Academic Press San Diego.

CERmeño, P., AND P. G. FAlKowski (2009), Controls on diatom biogeography in the ocean, Science, 325, 1539-1541.

Chakraborty, P., G. Giola, and S. W. KiefFer (2009), Volcanic mesocyclones, Nature, 458, 497-500.

Cioni, R., P. Marianelli, and A. SbRana (1992), Dynamics of the AD 79 eruption: Stratigraphic, sedimentological and geochemical data on the successions from the Somma-Vesuvius southern and eastern sectors, Acta Vulcanologica, 2, 109-123.

CIPRIANO, R. J., and D. C. BLANChARD (1981), Bubble and aerosol spectra produced by a laboratory breaking wave, Journal of Geophysical Research, 86, 8085-8092.

Clifton, J., P. McDonald, A. Plater, and F. Oldfield (1999), An investigation into the efficiency of particle size separation using Stokes' law, Earth Surface Processes and Landforms, 24, 725-730.

Connor, L. J., and C. B. ConNOR (2006), Inversion is the key to dispersion: understanding eruption dynamics by inverting tephra fallout, in Statistics in Volcanology, edited by H. M. Mader, S. G. Cole, C. B. Connor and L. J. Connor, pp. 231-242, Special Publications of IAVCEI 1.

Cooper, G. F., C. J. N. Wilson, M.-A. Millet, J. A. Baker, and E. G. C. Smith (2012), Systematic tapping of independent magma chambers during the $1 \mathrm{Ma}$ Kidnappers supereruption, Earth and Planetary Science Letters, 313-314, 23-33.

Costa, A., A. FolCh, and G. MACEDONiO (2010), A model for wet aggregation of ash particles in volcanic plumes and clouds: 1. Theoretical formulation, Journal of Geophysical Research, 115, B09201, doi: 10.1029/2009JB007175.

Cotton, W. R., and R. A. Anthes (1989), Storm and Cloud Dynamics, 883 pp., Academic Press, San Diego.

DARBY, D. J., and C. M. MEERTENS (1995), Terrestrial and GPS measurements of deformation across the Taupo back-arc and Hikurangi fore-arc regions in New Zealand, Journal of Geophysical Research, 100, 8221-8232.

De Rita, D., G. Giordano, A. Esposito, M. Fabbri, and S. Rodani (2002), Large volume phreatomagmatic ignimbrites from the Colli Albani volcano (Middle Pleistocene, Italy), Journal of Volcanology and Geothermal Research, 118, 77-98. 
Di Muro, A., A. Neri, and M. Rosi (2004), Contemporaneous convective and collapsing eruptive dynamics: The transitional regime of explosive eruptions, Geophysical Research Letters, 31, L10607, doi: 10.1029/2004GL019709.

Dobran, F., A. Neri, and G. MACEDONIO (1993), Numerical simulation of collapsing volcanic columns, Journal of Geophysical Research, 98, 4231-4259.

Doswell, C. A. (2001), Severe convective storms, Meteorological Monographs, 28, 126.

Drost, F. (2006), An investigation into New Zealand's climate during the last glacial maximum: A climate modelling approach, Ph.D. thesis, $343 \mathrm{pp}$, Victoria University of Wellington, Wellington, New Zealand.

Drost, F., J. Renwick, B. Bhaskaran, H. Oliver, and J. McGregor (2007a), Variability of the atmospheric circulation in the southern hemisphere during the last glacial maximum, Journal of Geophysical Research, 112, D10101, doi: 10.1029/2005JD006970.

Drost, F., J. Renwick, B. Bhaskaran, H. Oliver, and J. McGregor (2007b), A simulation of New Zealand's climate during the Last Glacial Maximum, Quaternary Science Reviews, 26, 2505-2525.

DunN, M. G., and D. P. WADE (1994), Influence of volcanic ash clouds on gas turbine engines, U.S. Geological Survey Bulletin, 2047, 107-117.

Durant, A. J., and R. A. ShaW (2005), Evaporation freezing by contact nucleation inside-out, Geophysical Research Letters, 32, L20814, doi: 10.1029/2005GL024175.

Durant, A. J., and W. I. Rose (2009), Sedimentological constraints on hydrometeorenhanced particle deposition: 1992 eruptions of Crater Peak, Alaska, Journal of Volcanology and Geothermal Research, 186, 40-59.

Durant, A. J., R. A. Shaw, W. I. Rose, Y. Mi, and G. G. J. Ernst (2008), Ice nucleation and overseeding of ice in volcanic clouds, Journal of Geophysical Research, 113, D09206, doi: 10.1029/2007JD009064.

Durant, A. J., W. I. Rose, A. M. SARnA-Wojcicki, S. CAReY, and A. C. M. Volentik (2009), Hydrometeor-enhanced tephra sedimentation: Constraints from the 18 May 1980 eruption of Mount St. Helens, Journal of Geophysical Research, 114, B03204, doi: 10.1029/2008JB005756.

ENNIS, B. J., J. L. Li, G. I. TARDOS, and R. PfeFFER (1990), The influence of viscosity on the strength of an axially strained pendular liquid bridge, Chemical Engineering Science, 45, 3071-3088.

ENNIS, B. J., G. TARDOS, and R. PFEFFER (1991), A microlevel-based characterization of granulation phenomena, Powder Technology, 65, 257-272.

Eshel, G., G. J. LeVy, U. Mingelgrin, and M. J. Singer (2004), Critical evaluation of the use of laser diffraction for particle-size distribution analysis, Soil Science Society of America Journal, 68, 736-743. 
European Commission (2010), Volcano Crisis Report: Report on the actions undertaken in the context of the impact of the volcanic ash cloud crisis on the air transport industry, available online at: http://ec.europa.eu/transport/doc/ash-cloud-

crisis/2010_06_30_volcano-crisis-report.pdf, last accessed: 26 April 2012.

FIERSTEIN, J., and C. J. N. WILSON (2005), Assembling an ignimbrite: compositionally defined packages in the 1912 Valley of Ten Thousand Smokes ignimbrite, Alaska, Geological Society of America Bulletin, 117, 1094-1107.

Fisher, R. V., and H. U. SCHMINCKe (1984), Pyroclastic Rocks, 472 pp., Springer, Berlin.

Folch, A., A. Costa, A. Durant, and G. Macedonio (2010), A model for wet aggregation of ash particles in volcanic plumes and clouds: 2. Model application, Journal of Geophysical Research, 115, B09202, doi: 10.1029/2009jb007176.

Folch, A., A. Costa, and S. BASART (2012), Validation of the FALL3D ash dispersion model using observations of the 2010 Eyjafjallajokull volcanic ash clouds, Atmospheric Environment, 48, 165-183.

FOLK, R. L. (1955), Student operator error in determination of roundness, sphericity and grain size, Journal of Sedimentary Petrology, 25, 297-301.

FolK, R. L. (1974), Petrology of Sedimentary Rocks, 182 pp., Hemphill Publishing Company, Austin, Texas.

FOLK, R. L., and W. C. WARD (1957), Brazos River bar: a study in the significance of grain size parameters, Journal of Sedimentary Petrology, 27, 3-26.

FrogGatT, P. C., and D. J. Lowe (1990), A review of late Quaternary silicic and some other tephra formations from New Zealand: their stratigraphy, nomenclature, distribution, volume, and age, New Zealand Journal of Geology and Geophysics, 33, 89-109.

Froggatt, P. C., R. Howorth, C. Vucetich, J. Healy, C. J. N. Wilson, and S. SelF (1988), Wairakei Formation, New Zealand: stratigraphy and correlation, New Zealand Journal of Geology and Geophysics, 31, 391-396.

Gamble, J., J. WoOdhEAd, I. WRight, and I. E. M. SMith (1989), Basalt and sediment geochemistry and magma petrogenesis in a transect from oceanic island arc to rifted continental margin arc: the Kermadec-Hikurangi Margin, SW Pacific, Journal of Petrology, 37, 1523-1546.

GILBERT, J. S., and S. J. LANE (1994), The origin of accretionary lapilli, Bulletin of Volcanology, 56, 398-411.

GILBERT, J. S., and R. S. J. SpARKS (Eds.) (1998), The physics of explosive volcanic eruptions, 178 pp., Geological Society of London Special Publication 145.

Glaze, L. S., and S. M. BAloga (1996), Sensitivity of buoyant plume heights to ambient atmospheric conditions: Implications for volcanic eruption columns, Journal of Geophysical Research, 101, 1529-1540. 
GLAZE, L. S., and S. SELF (1991), Ashfall dispersal for the 16 September 1986 eruption of Lascar, Chile, calculated by a turbulent-diffusion model, Geophysical Research Letters, 18, 1237-1240.

Glaze, L. S., S. M. BALOGA, and L. WiLSON (1997), Transport of atmospheric water vapor by volanic eruption columns, Journal of Geophysical Research, 102, 6099-6108.

GoKhale, N. R. (1975), Hailstorms and Hailstone Growth, State University of New York Press, Albany.

Graf, H.-F., M. Herzog, J. M. Oberhuber, and C. TeXtor (1999), Effect of environmental conditions on volcanic plume rise, Journal of Geophysical Research, 104, 24309-24320.

Graham, I. J., J. W. Cole, R. M. Briggs, J. A. Gamble, and I. E. M. Smith (1995), Petrology and petrogenesis of volcanic rocks from the Taupo Volcanic Zone: a review, Journal of Volcanology and Geothermal Research, 68, 59-87.

GRIFFETHS, J. C. (1967), Scientific Method in Analysis of Sediments, McGraw-Hill, New York.

GRINDLEY, G. W. (1965), The geology, structure and exploitation of the Wairakei Geothermal Field, Taupo, New Zealand 131 pp., New Zealand Geological Survey Bulletin, 75 .

Guo, H., J. E. Penner, M. Herzog, and H. Pawlowska (2007), Examination of the aerosol indirect effect under contrasting environments during the ACE-2 experiment, Atmospheric Chemistry and Physics, 7, 535-548.

Guo, S., W. I. Rose, G. J. S. Bluth, and I. M. WAtson (2004), Particles in the great Pinatubo volcanic cloud of June 1991: the role of ice, Geochemistry Geophysics Geosystems, 5, Q05003, doi: 10.1029/2003GC000655.

Gutro, R. (2011) NASA satellite gallery shows Chilean volcano plume moving around the world, available online at: http://www.nasa.gov/topics/earth/features/20110615volcano.html, last accessed: 20 July 2012.

HaAn, C. T., B. J. BARfiEld, and J. C. HAYeS (1994), Design Hydrology and Sedimentology for Small Catchments, Academic Press, San Diego.

HAnstrum, B. N., and A. S. WATson (1983), A case study of two eruptions of Mount Galunggung and an investigation of volcanic eruption cloud characteristics using remote sensing techniques, Australian Meteorological Magazine, 31, 171-177.

HAPGOOD, K. P., J. D. LitsteR, and R. SMITH (2003), Nucleation regime map for liquid bound granules, Aiche Journal, 49, 350-361.

HARPER, M. A. (1994), Did Europeans introduce Asterionella formosa Hassall to New Zealand? in Proceedings of the Eleventh Symposium on Fossil and Living Diatoms, edited by J. P. Kociolek, pp. 479-484, Memoirs of the Californian Academy of Sciences 17. 
HARPER, M. A., and R. M. MCKAY (2010), Diatoms as markers of atmospheric transport, in The Diatoms: Applications for the Environmental and Earth Sciences, edited by J. P. Smol and E. F. Stoermer, pp. 552-557, Cambridge University Press Cambridge.

HAYAKAWA, Y. (1983), The Hachinohe Ash: an example of an accretionary lapilli-fall deposit from Towada volcano, Japan, Bulletin of the Volcanological Society of Japan, $28,25-40$.

HAYAKAWA, Y. (1990), Mode of eruption and deposition of the Hachinohe phreatoplinian ash from the Towada volcano, Japan, Geographical Reports of Tokyo Metropolitan University, 25, 167-182.

Healy, J., E. F. Lloyd, D. E. H. Rishworth, C. P. Wood, R. B. Glover, and R. R. DibBLE (Eds.) (1978), The Eruption of Ruapehu, New Zealand, on 22 June 1969, Department of Science and Industrial Research Bulletin 224, 80 pp., Wellington, New Zealand.

HeIKEN, G., and K. WoHLETZ (1985), Volcanic ash, 258 pp., University of California Press, Berkeley.

HeIKEN, G., and K. Wohletz (1991), Fragmentation processes in explosive volcanic eruptions, in Sedimentation in Volcanic Settings, edited by R. Fisher and G. Smith, pp. 19-26, Society for Sedimentary Geology Special Publication 45, Tulsa, Oklahoma.

Heise, W., H. M. Bibby, T. G. Caldwell, S. C. Bannister, Y. Ogawa, S. TAKAKURA, and T. UCHIDA (2007), Melt distribution beneath a young continental rift: the Taupo Volcanic Zone, New Zealand, Geophysical Research Letters, 37, L14313, doi: 10.1029/2007GL029629.

HERzOG, M., and H.-F. GRAF (2010), Applying the three-dimensional model ATHAM to volcanic plumes: Dynamics of large co-ignimbrite eruptions and associated injection heights for volcanic gases, Geophysical Research Letters, 37, L19807, doi: 10.1029/2010GL044986.

Herzog, M., H.-F. Graf, C. Textor, and J. M. Oberhuber (1998), The effect of phase changes of water on the development of volcanic plumes, Journal of Volcanology and Geothermal Research, 87, 55-74.

Herzog, M., J. M. Oberhuber, and H.-F. Graf (2003), A prognostic turbulence scheme for the nonhydrostatic plume model ATHAM, Journal of the Atmospheric Sciences, 60, 2783-2796.

HobBs, P. V. (1965), The aggregation of ice particles in clouds and fogs at low temperatures, Journal of the Atmospheric Sciences, 22, 296-300.

HobBs, P. V. (1974), Ice Physics, Oxford University Press, Oxford, UK.

HobBs, P. V., and B. J. MASON (1964), Sintering and adhesion of ice, Philosophical Magazine, 9, 181-197. 
HoblitT, R. P. (1986), Observations of the eruption of July 22 and August 7, 1980, at Mount St. Helens, Washington, US Geological Survey Professional Paper, 1335, 1-44.

Holt, K. A., R. C. Wallace, V. E. Neall, B. P. Kohn, and D. J. Lowe (2010), Quaternary tephra marker beds and their potential for palaeoenvironmental reconstruction on Chatham Island, east of New Zealand, southwest Pacific Ocean, Journal of Quaternary Science, 25, 1169-1178.

HORWELL, C. J. (2007), Grain-size analysis of volcanic ash for the rapid assessment of respitory health hazard, Journal of Environmental Monitoring, 9, 1107-1115.

Houghton, B. F., and C. J. N. WiLsOn (1989), A vesicularity index for pyroclastic deposits, Bulletin of Volcanology, 51, 451-462.

Houghton, B. F., and C. J. N. WiLsON (1998), Fire and water: physical roles of water in large eruptions at Taupo and Okataina calderas, in Water-Rock Interaction: Proceedings of the 9th International Symposium on Water-Rock Interaction - WRI-9, Taupo, New Zealand, edited by G. B. Arehart and J. R. Hulston, pp. 25-30, Rotterdam: A.A. Balkema 2.

Houghton, B. F., C. J. N. Wilson, R. T. Smith, and J. S. Gilbert (2000), Phreatoplinian eruptions, in Encyclopedia of Volcanoes, edited by H. Sigurdsson, B. F. Houghton, S. McNutt, H. Rymer and J. Stix, pp. 513-525, Academic Press San Diego, California.

Houghton, B. F., C. J. N. Wilson, J. Fierstein, and W. Hildreth (2004), Complex proximal deposition during the Plinian eruptions of 1912 at Novarupta, Alaska, Bulletin of Volcanology, 66, 95-133.

Houze, R. A. (1993), Cloud Dynamics, 573 pp., Academic Press, San Diego.

Hurst, A. W., and R. TURneR (1999), Performance of the program ASHFALL for forecasting ashfall during the 1995 and 1996 eruptions of Ruapehu volcano, New Zealand Journal of Geology and Geophysics, 42, 615-622.

ICAO (2004), Handbook on the International Airways Volcano Watch (IAVW): operational procedures and contact list, 117, 2nd edition, Doc 9766-AN/968, available online at: http://www.skybrary.aero/bookshelf/books/450.pdf, last accessed: 26 April 2012.

ISRAELACHVILI, J. N. (2011), Intermolecular and Surface Forces, 3rd ed., 661 pp., Academic Press, Waltham, Massachusetts.

IVESON, S. M., and J. D. LITSTER (1998), Growth regime map for liquid-bound granules, Aiche Journal, 44, 1510-1518.

IVEson, S. M., J. D. LiTster, K. HAPGOOD, and B. J. EnNIS (2001), Nucleation, growth and breakage phenomena in agitated wet granulation processes: a review, Powder Technology, 117, 3-39. 
James, M. R., J. S. Gilbert, and S. J. LANE (2002), Experimental investigation of volcanic particle aggregation in the absence of a liquid phase, Journal of Geophysical Research, 107, 2191, doi: 10.1029/2001JB000950.

James, M. R., S. J. LANE, and J. S. Gilbert (2003), Density, construction, and drag coefficient of electrostatic volcanic ash aggregates, Journal of Geophysical Research, 108, 2435, doi: 10.1029/2002JB002011.

JiUsto, J. E., and H. K. WeICKMAN (1973), Types of snowfall, Bulletin of the American Meteorological Society, 54, 1148-1162.

KATO, Y. (1986), Accretionary lapilli originated from hailstone, Journal of the Geological Society of Japan, 92, 429-437.

Kessler, E. (Ed.) (1992), Thunderstorm Morphology and Dynamics, 2nd ed., Thunderstorms: A Social, Scientific, and Technological Documentary 2, 411 pp., University of Oklahoma Press, Norman, Oklahoma.

KNAUth, L. P., D. M. Burt, and K. H. Wohletz (2005), Impact origin of sediments at the opportunity landing site on Mars, Nature, 438, 1123-1128.

KNIGHT, C. A. (1968), On the mechanism of spongy hailstone growth, Journal of the Atmospheric Sciences, 25, 440-444.

KNight, N. C., and J. Heymsfield (1983), Measurements and interpretation of hailstone density and terminal velocity, Journal of the Atmospheric Sciences, 40, 15101516.

Knight, C. A., and N. C. KNIGHT (1968), Spongy hailstone growth criteria II: microstructures, Journal of the Atmospheric Sciences, 25, 453-459.

Knight, C. A., and N. C. KNIGHT (1973), Quenched, spongy hail, Journal of the Atmospheric Sciences, 30, 1665-1671.

Knopf, D. A., P. A. Alpert, B. WAng, and J. Y. Aller (2010), Stimulation of ice nucleation by marine diatoms, Nature Geoscience, 4, 88-90.

Koç, N., E. JANSEN, and H. HAFLIDASON (1993), Paleoceanographic reconstructions of surface ocean conditions in the Greenland, Iceland and Norwegian Seas through the last 14 ka based on diatoms, Quaternary Science Reviews, 12, 115-140.

KoKelaAR, B. P. (1983), The mechanism of Surtseyan volcanism, Journal of the Geological Society of London, 140, 939-944.

KOKELAAR, B. P. (1986), Magma-water interactions in subaqueous and emergent basaltic volcanism, Bulletin of Volcanology, 48, 275-289.

Komar, P. D., and B. CuI (1984), The analysis of grain-size measurements by sieving and settling-tube techniques, Journal of Sedimentary Petrology, 54, 603-614. 
Konert, M., and J. VAndenberghe (1997), Comparison of laser grain size analysis with pipette and sieve analysis: a solution for the underestimation of the clay fraction, Sedimentology, 44, 523-535.

KoYAGUCHI, T., and A. W. Woods (1996), On the formation of eruption columns following explosive mixing of magma and surface water, Journal of Geophysical Research, 101, 5561-5574.

Koyama, M., M. Kawashima, T. Takamatsu, G. P. Glasby, and P. Stoppers (1989), Mineralogy and geochemistry of sediments from Lakes Taupo and Waikaremoana, New Zealand, New Zealand Journal of Marine and Freshwater Research, 23, 121-130.

Kueppers, U., B. Auer, C. Cimarelli, T. Scolamacchia, and D. B. Dingwell (2011), Experimentally constraining the boundary conditions for volcanic ash aggregation, Geophysical Research Abstracts, 13, EGU2011-11999.

LaCroix, A. (1904), La Montagne Pelee et ses eruptions, 662 pp., Masson et Cie, Paris.

LAHEY, K. (2012), No volcanic ash clouds to rain on Australia's parade, The Australian Journal of Emergency Management, 27, 16-19.

Langmann, B., A. Folch, M. Hensch, and V. Matthias (2012), Volcanic ash over Europe during the eruption of Eyjafjallajokull on Iceland, April-May 2010, Atmospheric Environment, 48, 1-8.

Lathem, T. L., P. Kumar, A. Nenes, J. Dufek, I. N. Sokolik, M. Trail, and A. RUSSELL (2011), Hygroscopic properties of volcanic ash, Geophysical Research Letters, 38, L11802, doi: 10.1029/2011GL047298.

LIN, Y.-L., R. D. FARLEY, and H. D. ORVILlE (1983), Bulk parameterization of the snow field in a cloud model, Journal of Climate and Applied Meteorology, 22, 10651092.

LipMan, P. W., and D. R. Mullineaux (Eds.) (1981), The 1980 eruptions of Mount St. Helens, Washington 1250, 844 pp., United States Geological Survey Professional Paper 1250 .

LiU, L. X., J. D. Litster, S. M. Iveson, and B. J. EnNis (2000), Coalescence of deformable granules in wet granulation processes, Aiche Journal, 46, 529-539.

LiU, Y., A. T. Anderson, C. J. N. Wilson, A. M. Davis, and I. M. Steele (2006), Mixing and differentiation in the Oruanui rhyolitic magma, Taupo, New Zealand; evidence from volatiles and trace elements in melt inclusions, Contributions to Mineralogy and Petrology, 151, 71-87.

Loizeau, J. L., D. Arbouille, S. Santiago, and J. P. Vernet (1994), Evaluation of a wide range laser diffraction size analyzer for use with sediments, Sedimentology, 41, 353-361.

LoRENZ, V. (1974), Vesiculated tuffs and associated features, Sedimentology, 21, 273291. 
LORENZ, V., and S. KURSZLAUKIS (2007), Root zone processes in the phreatomagmatic pipe emplacement model and consequences for the evolution of maar-diatreme volcanoes, Journal of Volcanology and Geothermal Research, 159, 4-32.

Lorrey, A. M., M. Vandergoes, P. Almond, J. Renwick, T. Stephens, H. Bostock, A. Mackintosh, R. M. Newnham, P. W. Williams, D. Ackerley, H. Neil, and A. M. Fowler (2012), Palaeocirculation across New Zealand during the last glacial maximum at $21 \mathrm{ka}$, Quaternary Science Reviews, 36, 189-213.

Lowe, D. J., P. A. R. Shane, B. V. Alloway, and R. M. Newnham (2008), Fingerprints and age models for widespread New Zealand tephra marker beds erupted since 30,000 years ago: a framework for NZ-INTIMATE, Quaternary Science Reviews, 27, 95-126.

LudLAM, F. H. (1950), The composition of coagulation elements in cumulonimbus, Quarterly Journal of the Royal Meteorological Society, 88, 30-50.

LudWICK, J. C., and P. L. HENDERSON (1968), Particle shape and inference of size from sieving, Sedimentology, 11, 197-235.

Macedonio, G., M. Pareschi, and R. SAntacroce (1988), A numerical simulation of the Plinian fall phase of the 79 AD eruption of Vesuvius, Journal of Geophysical Research, 93, 14817-14827.

ManviLle, V., and C. J. N. WiLSON (2004), The 26.5 ka Oruanui eruption, New Zealand: a review of the roles of volcanism and climate in the post-eruptive sedimentary response, New Zealand Journal of Geology and Geophysics, 47, 525-547.

Marshall, J. S., and W. M. PALMER (1948), The distribuion of raindrops with size, Journal of Meteorology, 5, 165-166.

MASTIN, L. G. (2007), A user-friendly one-dimensional model for wet volcanic plumes, Geochemistry Geophysics Geosystems, 8, Q03014, doi: 10.1029/2006GC001455.

Mastin, L. G., M. Guffanti, R. Servranckx, P. Webley, S. Barsotti, K. Dean, A. Durant, J. W. Ewert, A. Neri, W. I. Rose, D. Schneider, L. Siebert, B. Stunder, G. Swanson, A. Tupper, A. Volentik, and C. F. Waythomas (2009), A multidisciplinary effort to assign realistic source parameters to models of volcanic ashcloud transport and dispersion during eruptions, Journal of Volcanology and Geothermal Research, 186, 10-21.

McCave, I. N., R. J. Bryant, H. F. Cook, and C. A. Coughanowr (1986), Evaluation of a laser diffraction size analyzer for use with natural sediments, Journal of Sedimentary Petrology, 56 561-564.

McClatchey, R. A., R. W. Fenn, J. E. A. Selby, F. E. Volz, and J. S. Garing (1972), Optical properties of the atmosphere (3rd Ed.) Report. AFCRL-72-0497, Air Force Cambridge Research Laboratories, Bedford, Massachusetts.

McKay, R. M., P. J. BARrett, M. A. HARPER, and M. J. HANnAh (2008), Atmospheric transport and concentation of diatoms in surficial and glacial sediments of the Allan 
Hills, Transantarctic Mountains, Palaeogeography, Palaeoclimatology, Palaeoecology, 260, 168-183.

Mitarai, N., and F. Nori (2006), Wet granular materials, Advances in Physics, 55, 145.

Mohler, O., and C. Hoose (2010), Ocean algae and atmospheric ice, Nature Geoscience, 4, 76-77.

Moore, J. G., and D. L. PeCK (1962), Accretionary lapilli in volcanic rocks of the western continental United States, Journal of Geology, 70, 182-193.

Moore, J. G., K. Nakamura, and A. Alcaraz (1966), The 1965 eruption of Taal volcano, Science, 151, 955-960.

Morton, B. R., G. I. TAYloR, and J. S. TURnER (1956), Turbulent gravitational convection from maintained and instantaneous sources, Proceedings of the Royal Society of London, A234, 1-23.

NAIRN, I. A. (1979), Rotomahana-Waimangu eruption, 1886: base surge and basalt magma, New Zealand Journal of Geology and Geophysics, 22, 363-378.

Neal, C., R. G. McGimsey, and M. F. Diggles (2001), Volcanoes of the Wrangell Mountains and Cook Inlet Region, Alaska - selected photographs, edited, United States Geological Survey digital data series DDE-39.

NERI, A., and G. MACEDONIO (1996), Numerical simulation of collapsing volcanic columns with particles of two sizes, Journal of Geophysical Research, 101, 8153-8174.

Neri, A., A. Di Muro, and M. Rosi (2002), Mass partition during collapsing and transitional columns by using numerical simulations, Journal of Volcanology and Geothermal Research, 115, 1-18.

Neri, A., T. E. Ongaro, G. Macedonio, and D. Gidaspow (2003), Multiparticle simulation of collapsing volcanic columns and pyroclastic flows, Journal of Geophysical Research, 108, 2202, doi: 10.1029/2001JB000508.

Newhall, C. G., and R. S. Punongbayan (Eds.) (1996), Fire and Mud: Eruptions and lahars of Mount Pinatubo, Phillipines, 1126 pp., University of Washington Press, Seattle.

NewitT, D. M., and J. M. Conway-Jones (1958), A contribution to the theory and practice of granulation, Transactions of the Institution of Chemical Engineers, 36, 422441.

Newnham, R. M., D. J. Lowe, and P. W. WiLliams (1999), Quaternary environmental change in New Zealand: a review, Progress in Physical Geography, 23, 567-610.

Newnham, R. M., D. J. Lowe, T. Giles, and B. V. Alloway (2007), Vegetation and climate of Auckland, New Zealand, since ca. 32000 cal. yr ago: support for an extended LGM, Journal of Quaternary Science, 22, 517-534. 
Oberhuber, J. M., M. Herzog, H. F. Graf, and K. Schwanke (1998), Volcanic plume simulation on large scales, Journal of Volcanology and Geothermal Research, $87,29-53$.

Orsi, G., M. Dantonio, S. Devita, and G. Gallo (1992), The Neopolitan Yellow Tuff, a large-magnitude trachytic phreatoplinian eruptions - eruptive dynamics, magma withdrawal and caldera collapse, Journal of Volcanology and Geothermal Research, 53, 275-287.

Oswalt, J. S., W. Nichols, and J. F. O'HARA (1996), Meteorological observations of the 1991 Mount Pinatubo eruption, in Fire and Mud: Eruptions and lahars of Mount Pinatubo, Phillipines, edited by C. G. Newhall and R. S. Punongbayan, pp. 197-212 University of Washington Press Seattle.

PARKER, J. I., and D. N. EdGINGTON (1979), Concentration of diatom frustules in Lake Michigan sediment cores, Limnology and Oceanography, 21, 887-893.

PAtrick, A., and C. W. ReIMER (1966), The diatoms of the United States, 688 pp., Monographs of the Academy of Natural Sciences No. 13, 1, Philadelphia.

Pearson, L. K., C. H. Hendy, D. P. Hamilton, and R. C. Pickett (2010), Natural and anthropogenic lead in sediments of the Rotorua lakes, New Zealand, Earth and Planetary Science Letters, 297, 536-544.

PETLAB Database (2012), managed by GNS Science, New Zealand, available online at: http://pet.gns.cri.nz last accessed: 26 April 2012.

Pflaum, J. C. (1980), Hail formation via microphysical recycling, Journal of the Atmospheric Sciences, 37, 160-173.

Pflaum, J. C., and H. R. PrupPaCher (1978), A wind tunnel study of the growth of graupel initiated from frozen drops, Journal of the Atmospheric Sciences, 36, 680-689.

PIETSCH, W., E. HofFMAN, and H. RUMPF (1969), Tensile strength of moist aggregates, Industrial and Engineering Chemistry: Process Design and Development, 8, 58-62.

Pillans, B., M. McGlone, A. Palmer, D. Mildenhall, B. Alloway, and G. BERGER (1993), The Last Glacial Maximum in central and southern North Island, New Zealand: a paleoenvironmental reconstruction using the Kawakawa Tephra Formation as a chronostratigraphic marker, Paleoceanography, Palaeoclimatology, Palaeoecology, $101,283-304$.

PRANDTL, L. (1954), Essentials of fluid dynamics, 452 pp., Blackie and Son, Ltd., London.

PRATA, A. J., and I. F. GRANT (2001), Retrieval of microphysical and morphological properties of volcanic ash plumes from satellite data: Application to Mt Ruapehu, New Zealand, Quarterly Journal of the Royal Meteorological Society, 127, 2153-2179.

PRODI, F. (1970), Measurements of local density in artificial and natural hailstones, Journal of Applied Meteorology, 9, 903-910. 
Prodi, F., G. Santachiara, and A. Franzini (1986), Properties of ice accreted in twostage growth, Quarterly Journal of the Royal Meteorological Society, 112, 1057-1080.

PRUPPACHER, H. R., and J. D. KLETT (1978), Microphysics of Clouds and Precipitation, 714 pp., D. Reidel Publishing Company, Dordrecht, Holland.

Pufahl, P. K., E. E. Hiatt, C. R. Stanley, J. R. Morrow, G. J. Nelson, and C. T. EDWARDS (2007), Physical and chemical evidence of the 1850 Ma Sudbury impact event in the Baraga Group, Michigan, Geology, 35, 827-830.

Ramaker, J. S., M. A. Jelgersma, P. Vonk, and N. W. F. Kossen (1998), Scale-down of a high-shear pelletisation process: Flow profile and growth kinetics, International Journal of Pharmaceutics, 166, 89-97.

RAZA, M., S. M. CASShYAP, and A. KHAN (2001), Accretionary lapilli from the basal Vindhyan volcanic sequence, south of Chittaurgarh, Rajasthan and their implication, Journal of the Geological Society of India, 57, 77-82.

REIMER, T. O. (1983), Accretionary lapilli in volcanic ash falls: physical factors governing their formation, in Coated grains, edited by T. M. Peryt, pp. 56-68, Springer Berlin.

RILEY, C. M., W. I. Rose, and G. J. S. BLUTH (2003), Quantitative shape measurements of distal volcanic ash, Journal of Geophysical Research, 108, B2504, doi: 10.1029/2001JB000818.

RitTEnHouse, G. (1943), Relation of shape to the passage of grains through sieves, Industrial and Engineering Chemistry, 15, 153-155.

Rodgers, K. A., K. L. Cook, P. R. L. Browne, and K. A. Campbell (2002), The mineralogy, texture and significance of silica derived from alteration by steam condensate in three New Zealand geothermal fields, Clay Minerals, 37, 299-322.

Rojas, M., P. Moreno, M. Kageyama, M. Crucifix, C. Hewitt, A. Abe-Ouchi, R. Ohgaito, E. C. BRADY, and P. Hope (2009), The southern westerlies during the last glacial maximum in PMIP2 simulations, Climate Dynamics, 32, 525-548.

Rose, W. I., and A. J. DuRAnT (2009), Fine ash content of explosive eruptions, Journal of Volcanology and Geothermal Research, 186, 32-39.

Rose, W. I., and A. J. Durant (2011), Fate of volcanic ash: Aggregation and fallout, Geology, 39, 895-896.

Rose, W. I., D. J. Delene, D. J. Schneider, G. J. S. Bluth, A. J. Krueger, I. Sprod, C. McKeE, H. L. DAVles, and G. G. J. ERnst (1995), Ice in the 1994 Rabaul eruption cloud: Implications for volcano hazard and atmospheric effects, Nature, 375, 477-479.

Rose, W. I., G. J. S. Bluth, D. J. Schneider, G. G. J. Ernst, C. M. Riley, L. J. HENDERSON, and R. G. MCGIMSEY (2001), Observations of volcanic clouds in their first few days of atmospheric residence: the 1992 eruptions of Crater Peak, Mount Spurr volcano, Alaska, Journal of Geology, 109, 677-694. 
Rose, W. I., G. J. S. Bluth, and I. M. WAtson (2004), Ice in volcanic clouds: when and where?, Proceedings of the Second International Conference on Volcanic Ash and Aviation Safety, OFCM Washington DC, Session 3:61.

Rosenbaum, J. G., and R. B. WaitT (1981), Summary of eyewitness accounts of the May 18 eruptions, United States Geological Survey Professional Paper, 1250, 53-67.

Rosi, M. (1992), A model for the formation of vesiculated tuff by the coalescence of accretionary lapilli, Bulletin of Volcanology, 54, 429-434.

RUMPF, H. (1962), The strength of granules and agglomerates, in Agglomeration, edited by W. A. Knepper, pp. 379-418, American Institute of Mechanical Engineers New York, NY.

Salge, T., R. TAgle, and P. Claeys (2000), Accretionary lapilli from the CretaceousTertiary boundary site of Guayal, Mexico: Preliminary insights into expansion plume formation, Meteoritics \& Planetary Science, 35, A140-A141.

SAlinger, M. J. (1980a), New Zealand climate: II. Temperature patterns, Monthly Weather Review, 108, 1905-1912.

SAlinger, M. J. (1980b), New Zealand climate: I. Precipitation patterns, Monthly Weather Review, 108, 1892-1904.

SARnA-Wojcicki, A. M., R. B. WAitT, M. J. Woodward, S. Shipley, and J. RiverA (1981a), Premagmatic ash erupted from March 27 through May 14, 1980 - extent, mass, volume, and composition, United States Geological Survey Professional Paper, 1250, $569-575$.

SARna-Wojcicki, A. M., S. Shipley, R. B. WaitT, D. Dzurisin, and S. H. Wood (1981b), Areal distribution, thickness, mass, volume and grain size of air-fall ash from the six major eruptions of 1980, United States Geological Survey Professional Paper, $1250,577-600$.

SAstry, K. V. S., P. Dontula, and C. Hosten (2003), Investigation of the layering mechanism of agglomerate growth during drum pelletization, Powder Technology, 130, 231-237.

Scasso, R. A., H. Corbella, and P. Tiberi (1994), Sedimentological analysis of the tephra from the 12-15 August 1991 eruption of Hudson volcano, Bulletin of Volcanology, 56, 121-132.

Schneider, D. P., C. M. Ammann, B. L. Otto-Bliesner, and D. S. KAUfMAn (2009), Climate response to large, high-latitude and low-latitude volcanic eruptions in the Community Climate System Model, Journal of Geophysical Research, 114, D15101, doi: 10.1029/2008JD011222.

Schultz, D. M., K. M. Kanak, J. M. Straka, R. J. Trapp, B. A. Gordon, D. S. Zrnic, G. H. Bryan, A. J. Durant, T. J. Garrett, P. M. Klein, and D. K. Lilly (2006), The mysteries of mammatus clouds: Observations and formation mechanisms, Journal of the Atmospheric Sciences, 63, 2409-2435. 
SCHUMACHER, R. (1994), A reappraisal of Mount St. Helens ash clusters - depositional model from experimental observation, Journal of Volcanology and Geothermal Research, 59, 253-260.

SCHUMACHER, R., and U. MUES-SCHUMACHER (1997), The pre-ignimbrite (phreato) plinian and phreatomagmatic phases of the Akdag-Zelve ignimbrite eruption in Central Anatolia, Turkey, Journal of Volcanology and Geothermal Research, 78, 139-153.

SCHUMACHER, R., and H.-U. SCHMINCKE (1991), Internal structure and occurrence of accretionary lapilli - a case study at Laacher See volcano., Bulletin of Volcanology, 53, 612-634.

SCHUMACHER, R., and H.-U. SCHMINCKE (1995), Models for the origin of accretionary lapilli, Bulletin of Volcanology, 56, 626-639.

Scolamacchia, T., J. L. Macias, M. F. Sheridan, and S. R. Hughes (2005), Morphology of ash aggregates from wet pyroclastic surges of the 1982 eruption of El Chichon Volcano, Mexico, Bulletin of Volcanology, 68, 171-200.

SEARCy, C., K. Dean, and W. STRINGER (1998), PUFF: A high-resolution volcanic ash tracking model, Journal of Volcanology and Geothermal Research, 80, 1-16.

SELF, S. (1983), Large-scale phreatomagmatic silicic volcanism: a case study from New Zealand, Journal of Volcanology and Geothermal Research, 17, 433-469.

SELF, S. (2006), The effects and consequences of very large explosive volcanic eruptions, Philosophical Transactionsof the Royal Society of London A364, 2073-2097.

SELF, S., and S. BlaKe (2008), Consequences of explosive super-eruptions, Elements, 4, 41-46.

SElf, S., and J. HeAly (1987), Wairakei Formation, New Zealand: stratigraphy and correlation, New Zealand Journal of Geology and Geophysics, 30, 73-86.

SELF, S., and R. S. J. SPARKS (1978), Characteristics of widespread pyroclastic deposits formed by the interaction of silicic magma and water, Bulletin Volcanologique, 41, 196212.

SELF, S., and R. S. J. SPARKS (1979), Oldest marine carbonate ooids reinterpreted as volcanic accretionary lapilli, Onverwacht Group, South Africa, Journal of Sedimentary Petrology, 49, 661-663.

Shaw, R. A., A. J. Durant, and Y. Mi (2005), Heterogeneous surface crystallization observed in undercooled water, Journal of Physical Chemistry, 109, 9865-9868.

Shulmeister J., I. Goodwin, J. Renwick, K. Harle, L. Armand, M. S. McGlone, E. Cook, J. Dodson, P. P. Hesse, P. Mayewski, M. Curran (2004), The Southern Hemisphere westerlies in the Australasian sector over the last glacial cycle: a synthesis, Quaternary International, 118-119, 23-53.

SHERIDAN, M. F., and K. H. Wohletz (1983), Hydrovolcanism - Basic considerations and review, Journal of Volcanology and Geothermal Research, 17, 1-29. 
Simpson, J. J., G. L. HufFord, D. PIERI, and J. S. BERG (2001), Failures in detecting volcanic ash from a satellite-based technique - Response, Remote Sensing of Environment, 78, 347-357.

SIRONO, S. (1999), Effects by sintering on the energy dissipation efficiency in collisions of grain aggregates, Astronomy and Astrophysics, 347, 720-723.

SMITH, E. G. C., T. STERN, and M. REYNERS (1989), Subduction and back-arc activity at the Hikurangi convergent margin, New Zealand, Pure and Applied Geophysics, 129, 203-231.

SMITH, R. T. (1998), Eruptive and depositional models for units 3 and 4 of the $1.85 \mathrm{ka}$ Taupo eruption: Implications for the nature of large-scale 'wet' eruptions, Ph.D. thesis, 309 pp, University of Canterbury, Christchurch, New Zealand.

SMITH, R. T., and B. F. Houghton (1995a), Vent migration and changing eruptive style during the 1800a Taupo eruption: New evidence from the Hatepe and Rotongaio phreatoplinian ashes, Bulletin of Volcanology, 57, 432-439.

Smith, R. T., and B. F. Houghton (1995b), Delayed deposition of plinian pumice during phreatoplinian volcanism - the 1800-year-BP Taupo eruption, New Zealand, Journal of Volcanology and Geothermal Research, 67, 221-226.

SOREM, R. K. (1982), Volcanic ash clusters: tephra rafts and scavengers, Journal of Volcanology and Geothermal Research, 13, 63-71.

SPARKS, R. S. J. (1978), Dynamics of bubble formation and growth in magmas: a review and analysis, Journal of Volcanology and Geothermal Research, 3, 1-37.

SPARKS, R. S. J. (1986), The dimensions and dynamics of volcanic eruption columns, Bulletin of Volcanology, 48, 3-15.

SpARKS, R. S. J., and L. WILSON (1976), A model for the formation of ignimbrite by gravitational column collapse, Journal of the Geological Society of London, 132, 441451.

Sparks, R. S. J., L. WiLSON, and H. SigurdSSON (1981), The pyroclastic deposits of the 1875 eruption of Askja, Iceland, Philosophical Transactions of the Royal Society of London, A299, 241-273.

Sparks, R. S. J., J. Barclay, C. Jaupart, H. M. Mader, and J. C. Phillips (1994), Physical aspects of magmatic degassing. 1. Experimental and theoretical constraints on vesiculation, Reviews in Mineralogy, 30, 413-445.

Sparks, R. S. J., M. I. Bursik, S. N. Carey, J. E. Gilbert, L. Glaze, H. Sigurdsson, and A. W. Woods (1997), Volcanic Plumes, 574 pp., John Wiley, Hoboken, N.J., New York.

Stohl, A., A. J. Prata, S. Eckhardt, L. Clarisse, A. Durant, S. Henne, N. I. Kristiansen, A. Minikin, U. Schumann, P. Seibert, K. Stebel, H. E. Thomas, T. T. K. TøRSETH, and B. WEINSIERL (2011), Determination of time- and height-resolved 
volcanic ash emissions and their use for quantitative ash dispersion modeling: the 2010 Eyjafjallajökull eruption, Atmospheric Chemistry and Physics, 11, 4333-4351.

Supulver, K. D., F. G. Bridges, S. Tiscareno, J. Lievore, and D. N. C. Lin (1997), The sticking properties of water frost produced under various ambient conditions, Icarus, 129, 539-554.

SuZUKI, T. (1983), A theoretical model for dispersion of tephra, in Arc Volcanism: Physics and Tectonics, edited by D. Shimozuru and I. Yokoyama, pp. 93-113, Terra Scientific Publishing Company (TERRAPUB) Tokyo.

Taddeucci, J., P. Scarlato, C. Montanaro, C. Cimarelli, E. D. Bello, C. Freda, D. Andronico, M. T. Gudmundsson, and D. B. Dingwell (2011), Aggregationdominated ash settling from the Eyafjallajökull volcanic cloud illuminated by field and laboratory high-speed imaging, Geology, 39, 891-894.

TANAKA, H. L. (1994), Development of a prediction scheme for volcanic ash fall from Redoubt volcano, Alaska, U.S. Geological Survey Bulletin, 2047, 283-291.

TARDOS, G. I., M. I. KHAN, and P. R. MORT (1997), Critical parameters and limiting conditions in binder granulation of fine powders, Powder Technology, 94, 245-258.

TELLING, J., and J. DUFEK (2012), An experimental evaluation of ash aggregation in explosive volcanic eruptions, Journal of Volcanology and Geothermal Research, 209$210,1-8$.

TEunou, E., and D. Poncelet (2002), Batch and continuous fluid bed coating - review and state of the art, Journal of Food Engineering, 53, 325-340.

Textor, C., and G. G. J. ERNST (2004), Comment on "Particle aggregation in volcanic eruption columns" by Graham Veitch and Andrew W. Woods, Journal of Geophysical Research, 109, B05202, doi: 10.1029/2002JB002291.

Textor, C., H.-F. Graf, M. Herzog, and J. M. Oberhuber (2003), Injection of gases into the stratosphere by explosive volcanic eruptions, Journal of Geophysical Research, 108, 4606, doi: 10.1029/2002JD002987.

Textor, C., H.-F. Graf, M. Herzog, J. M. Oberhuber, W. I. Rose, and G. G. J. ERNST (2006a), Volcanic particle aggregation in explosive eruption columns. Part I: Parameterization of the microphysics of hydrometeors and ash, Journal of Volcanology and Geothermal Research, 150, 359-377.

Textor, C., H.-F. Graf, M. Herzog, J. M. Oberhuber, W. I. Rose, and G. G. J. ERNST (2006b), Volcanic particle aggregation in explosive eruption columns. Part II: Numerical experiments, Journal of Volcanology and Geothermal Research, 150, 378394.

Thorarinsson, S. (1967), Surtsey - The New Island in the North Atlantic, 47 pp., Viking Press, New York. 
THORDARSON, T. (2004), Accretionary-lapilli-bearing pyroclastic rocks at ODP Leg 192 Site 1184: a record of subaerial phreatomagmatic eruptions on the Ontong Java Plateau, Geological Society of London, Special Publications, 229, 275-306.

TIMMRECK, C., and H.-F. GRAF (2006), The initial dispersal and radiative forcing of a Northern Hemisphere mid-latitude super volcano: a model study, Atmospheric Chemistry and Physics, 6, 35-49.

TOMiTA, K., T. KANAI, T. KoBAyASHI, and N. OBA (1985), Accretionary lapilli formed by the eruption of Sakurajima volcano, Journal of the Japanese Association of Mineralogists, Petrologists and Economic Geologists, 80, 49-54.

Trentmann, J., M. O. Andreae, and H.-F. Graf (2003a), Chemical processes in a young biomass-burning plume, Journal of Geophysical Research, 108, 4705, doi: 10.1029/2003JD003732.

Trentmann, J., B. Früh, B. Boucher, O. Trautmann, and M. O. Andreae (2003b), Three-dimensional solar radiation effects on the actinic flux field in a biomass-burning plume, Journal of Geophysical Research, 108, 4558, doi: 10.1029/2003JD003422.

Trentmann, J., G. Luderer, T. Winterrath, M. D. Fromm, R. ServranckX, C. Textor, M. Herzog, H. F. Graf, and M. O. Andreae (2006), Modeling of biomass smoke injection into the lower stratosphere by a large forest fire (Part I): reference simulation, Atmospheric Chemistry and Physics, 6, 5247-5260.

TuPPER, A., and K. KinOSHITA (2003), Satellite, air and ground observations of volcanic clouds over islands of the Southwest Pacific, South Pacific Study, 23, 21-46.

Tupper, A., G. G. J. Ernst, C. Textor, K. Kinoshita, J. S. Oswalt, and D. ROSENFELD (2004), Volcanic cloud conceptual models for volcanic ash advisory centre operations, paper presented at Second International Conference on Volcanic Ash and Aviation Safety, Alexandria, Virginia, USA, 21-24 June.

TupPer, A., J. S. Oswalt, and D. RosenfELD (2005), Satellite and radar analysis of the volcanic-cumulonimbi at Mount Pinatubo, Philippines, 1991, Journal of Geophysical Research, 110, D09204, doi: 10.1029/2004JD005499.

Tupper, A., I. Itikarai, M. S. Richards, F. Prata, S. CARn, and D. Rosenfield (2007), Facing the challenges of the International Airways Volcano Watch: the 2004/05 eruptions of Manam, Papua New Guinea, Weather Forcasting, 22, 175-191.

Tupper, A., C. TeXtor, M. Herzog, H.-F. Graf, and M. S. Richards (2009), Tall clouds from small eruptions: the sensitivity of eruption height and fine ash content to tropospheric instability, Natural Hazards, 51, 375-401.

TURner, J. S. (1979), Buoyancy Effects in Fluids, 368 pp., Cambridge University Press, Cambridge, England.

TURNER, R., and T. HURST (2001), Factors influencing volcanic ash dispersal from the 1995 and 1996 eruptions of Mount Ruapehu, New Zealand, Journal of Applied Meteorology, 40, 56-69. 
UdDen, J. A. (1898), Mechanical Composition of Wind Deposits, 69 pp., Augustana Library Publications, 1, Rock Island, Illinois.

VAlentine, G. A., and K. H. Wohletz (1989), Numerical-models of Plinian eruption columns and pyroclastic flows, Journal of Geophysical Research, 94, 1867-1887.

Vandergoes, M. J., A. G. Hogg, D. J. Lowe, R. M. Newnham, G. H. Denton, J. Southon, F. Petchey, K. Dabell, D. J. A. Barrell, P. C. Almond, A. Allan, C. J. N. Wilson, M. S. McGlone, and M. BlaAuw (2011), Refining the age of the Kawakawa/Oruanui tephra of New Zealand, in Abstract volume, Geosciences 2011 Conference, Nelson, New Zealand, edited by N. J. Litchfield and K. Clark, pp. 113-114, Geoscience Society of New Zealand Miscellaneous Publication 130A.

VAn EAton, A. R., AND C. J. N. Wilson (in review), The nature, origins and distribution of ash aggregates in a large-scale wet eruption deposit: Oruanui, New Zealand, Journal of Volcanology and Geothermal Research.

Van Eaton, A. R., M. Herzog, C. J. N. Wilson, and J. McGregor (2012a), Ascent dynamics of large phreatomagmatic eruption clouds: The role of microphysics, Journal of Geophysical Research, 117, B03203, doi: 10.1029/2011JB008892.

Van Eaton, A. R., J. D. Muirhead, C. J. N. Wilson, and C. Cimarelli (2012b), Growth of volcanic ash aggregates in the presence of liquid water and ice: and experimental approach, accepted for publication in Bulletin of Volcanology.

VeITCH, G., and A. W. Woods (2001), Particle aggregation in volcanic eruption columns, Journal of Geophysical Research, 106, 26,425-426,441.

Veitch, G., and A. W. Woods (2004), Particle aggregation in volcanic eruption columns; reply, Journal of Geophysical Research, 109, B05203, doi: 10.1029/2003JB002388.

Verkoejin, D., G. M. H. Meesten, P. H. W. Vercoulen, and B. Scarlett (2002), Determining granule strength as a function of moisture content, Powder Technology, $124,195-200$.

Vucetich, C. G., and R. Howorth (1976), Proposed definition of the Kawakawa Tephra, the c. 20 000-years-B.P. marker horizon in the New Zealand region, New Zealand Journal of Geology and Geophysics, 19, 43-50.

WALKER, G. P. L. (1971), Grain-size characteristics of pyroclastic deposits, Journal of Geology, 79, 696-714.

WALKER, G. P. L. (1973), Explosive volcanic eruptions - a new classification scheme, Geologische Rundschau, 62, 431-446.

WALKeR, G. P. L. (1981a), Generation and dispersal of fine ash and dust by volcanic eruptions, Journal of Volcanology and Geothermal Research, 11, 81-92.

WALKeR, G. P. L. (1981b), Plinian eruptions and their products, Bulletin Volcanologique, 44, 223-240. 
WALKER, G. P. L. (1981c), Characteristics of two phreatoplinian ashes and their waterflushed origin, Journal of Volcanology and Geothermal Research, 9, 395-407.

Walker, G. P. L., and R. Croasdale (1972), Characteristics of some basaltic pyroclasts, Bulletin Volcanologique, 35, 303-317.

Walker, G. P. L., L. Wilson, and E. L. G. Bowell (1971), Explosive volcanic eruptions - I. The rate of fall of pyroclasts, Geophysical Journal of the Royal Astronomical Society, 22, 377-383.

Watanabe, K., K. Ono, K. Sakaguchi, A. Takada, and H. Hoshizumi (1999), Coignimbrite ash-fall deposits of the 1991 eruptions of Fugen-dake, Unzen Volcano, Japan, Journal of Volcanology and Geothermal Research, 89, 95-112.

WAters, A. C., and R. V. Fisher (1971), Base surges and their deposits: Capelinhos and Taal volcanoes, Journal of Geophysical Research, 76, 5596-5614.

WEBLEY, P., and L. MASTIN (2009), Improved prediction and tracking of volcanic ash clouds, Journal of Volcanology and Geothermal Research, 186, 1-9.

White, J. D. L., and B. F. Houghton (2000), Surtseyan and related phreatomagmatic eruptions, in Encyclopedia of Volcanoes, edited by H. Sigurdsson, B. F. Houghton, S. R. McNutt, H. Rymer and J. Stix, pp. 495-511, Academic Press San Diego.

Williams, H., F. J. Turner, and C. M. GILbert (1982), Petrography: An Introduction to the Study of Rocks in Thin Sections, 2nd ed., 626 pp., Freeman, San Francisco.

WiLson, C. J. N. (1981), Studies on the origins and emplacement of pyroclastic flows, 402 pp, Imperial College, University of London, London, UK.

Wilson, C. J. N. (1993), Stratigraphy, chronology, styles and dynamics of late Quaternary eruptions from Taupo volcano, New Zealand, Philosophical Transactions of the Royal Society of London, A343, 205-306.

WILSON, C. J. N. (1994), Ash-fall deposits from large-scale phreatomagmatic volcanism: limitations of available eruption column models, U.S. Geological Survey Bulletin, 2047, 93-99.

Wilson, C. J. N. (2001), The 26.5 ka Oruanui eruption, New Zealand: an introduction and overview, Journal of Volcanology and Geothermal Research, 112, 133-174.

WiLsON, C. J. N. (2008), Supereruptions and supervolcanoes: processes and products, Elements, 4, 29-34.

Wilson, C. J. N., and W. HiLdreth (1997), The Bishop Tuff: new insights from eruptive stratigraphy, Journal of Geology, 105, 407-439.

Wilson, C. J. N., V. R. Switsur, and A. P. WARD (1988), A new ${ }^{14}$ C age for the Oruanui (Wairakei) eruption, New Zealand, Geological Magazine, 125, 297-300. 
Wilson, C. J. N., B. F. Houghton, P. J. J. KamP, and M. O. McWilliams (1995a), An exceptionally widespread ignimbrite with implications for pyroclastic flow emplacement, Nature, 378, 605-607.

Wilson, C. J. N., B. F. Houghton, M. O. McWilliams, M. A. Lanphere, S. D. WEAVER, and R. M. BRIGGS (1995b), Volcanic and structural evolution of Taupo Volcanic Zone, New Zealand: a review, Journal of Volcanology and Geothermal Research, 68, 1-28.

Wilson, C. J. N., S. Blake, B. L. A. Charlier, and A. N. Sutton (2006), The 26.5 ka Oruanui eruption, Taupo volcano, New Zealand: development, characteristics and evacuation of a large rhyolitic magma body, Journal of Petrology, 47, 35-69.

WILSON, L. (1972), Explosive volcanic eruptions - II. The atmospheric trajectories of pyroclasts, Geophysical Journal of the Royal Astronomical Society, 30, 381-392.

WILSON, L. (1976), Explosive volcanic eruptions - III. Plinian eruption columns, Geophysical Journal of the Royal Astronomical Society, 45, 543-556.

Wilson, L., and T. C. HuANG (1979), The influence of particle shape on the atmospheric settling velocity of volcanic ash particles, Earth and Planetary Science Letters, 44, 311-324.

WILSON, L., and J. W. HEAD (2007), Explosive volcanic eruptions on Mars: Tephra and accretionary lapilli formation, dispersal and recognition in the geologic record, Journal of Volcanology and Geothermal Research, 163, 83-97.

Wilson, L., R. S. J. Sparks, T. C. HuAng, and N. D. WATKIns (1978), The control of volcanic eruption column heights by eruption energetics and dynamics, Journal of Geophysical Research, 83, 1829-1836.

Wilson, L., R. S. J. Sparks, and G. P. L. WALKeR (1980), Explosive volcanic eruptions -IV. The control of magma properties and conduit geometry on eruption column behaviour, Geophysical Journal of the Royal Astronomical Society, 63, 117148.

Winsborough, B. M. (2000), Pleistocene diatoms from the 602,00 yr B.P. Lava Creek B Ash, Diatom Research, 15, 159-166.

WoHLETZ, K. H. (1983), Mechanisms of hydrovolcanic pyroclast formation: grain-size, scanning electron microscopy, and experimental studies, Journal of Volcanology and Geothermal Research, 17, 31-63.

WoHLETZ, K. H. (1986), Explosive magma-water interactions: thermodynamics, explosion mechanisms, and field studies, Bulletin of Volcanology, 48, 245-264.

Woods, A. W. (1988), The fluid dynamics and thermodynamics of eruption columns, Bulletin of Volcanology, 50, 169-193.

Woods, A. W. (1993), Moist convection and the injection of volcanic ash into the atmosphere, Journal of Geophysical Research, 98, 17627-17636. 
Woods, A. W. (1998), Observations and models of volcanic eruption columns, in The Physics of Explosive Volcanic Eruptions, edited by J. S. Gilbert and R. S. J. Sparks, pp. 91-114, Geological Society of London, Special Publications 145.

Woods, A. W., and S. M. Bower (1995), The decompression of volcanic jets in a crater during explosive volcanic eruptions, Earth and Planetary Science Letters, 131, 189205.

Woods, A. W., and J. KIENLE (1994), The dynamics and thermodynamics of volcanic clouds; theory and observations from the April 15 and April 21, 1990 eruptions of Redoubt Volcano, Alaska, Journal of Volcanology and Geothermal Research, 62, 273299.

Woods, A. W., and K. H. WoHLETZ (1991), Dimensions and dynamics of co-ignimbrite eruption columns, Nature, 350, 225-227.

Zimanowski, B., K. H. Wohletz, P. Dellino, and R. Buttner (2003), The volcanic ash problem, Journal of Volcanology and Geothermal Research, 122, 1-5. 
Appendices 
Appendix A. Key localities of Oruanui exposures referred to in this thesis (from Wilson [2001])

\begin{tabular}{llllllll}
\hline Locality & Sheet & $\begin{array}{l}\text { NZMG } \\
\text { Northing } \\
(\mathrm{m})\end{array}$ & $\begin{array}{l}\text { NZMG } \\
\text { Easting } \\
(\mathrm{m})\end{array}$ & $\begin{array}{l}\text { Elevation } \\
(\mathrm{m})\end{array}$ & $\begin{array}{l}\text { Distance } \\
\text { from virtual } \\
\text { source }(\mathrm{km})\end{array}$ & $\begin{array}{l}\text { NZTM } \\
\text { Northing }\end{array}$ & $\begin{array}{l}\text { NZTM } \\
\text { Easting }\end{array}$ \\
\hline 235 & U17 & 6288300 & 2775700 & 560 & 25.7 & 5726716 & 1865602 \\
417 & V17 & 6299200 & 2830600 & n/a & 75.3 & 5737685 & 1920535 \\
1287 & T17 & 6283000 & 2761900 & 508 & 17.0 & 5721398 & 1851799 \\
1293 & T17 & 6281100 & 2767400 & 455 & 15.7 & 5719502 & 1857304 \\
1306 & U17 & 6294100 & 2783000 & 350 & 34.5 & 5732527 & 1872900 \\
1443 & U17 & 6291300 & 2778200 & 500 & 29.5 & 5729720 & 1868100 \\
1622 & V17 & 6303000 & 2830600 & 290 & 77.1 & 5741489 & 1920530 \\
1624 & V17 & 6304800 & 2834000 & 190 & 80.9 & 5743294 & 1923932 \\
1629 & U16 & 6316400 & 2782000 & 360 & 53.9 & 5754842 & 1871874 \\
1640 & U16 & 6314100 & 2798700 & 367 & 59.9 & 5752561 & 1888589 \\
1652 & U17 & 6302200 & 2786800 & 385 & 43.3 & 5740637 & 1876694 \\
2024 & X19 & 6229700 & 2895000 & 40 & 136.9 & 5668159 & 1985074 \\
2026 & W19 & 6229400 & 2867600 & 140 & 110.8 & 5667841 & 1957639 \\
2266 & V21 & 6184200 & 2846900 & 90 & 117.2 & 5622580 & 1936939 \\
2267 & V21 & 6184400 & 2846900 & 100 & 117.0 & 5622780 & 1936939 \\
2272 & V21 & 6188900 & 2830300 & 115 & 102.3 & 5627278 & 1920321 \\
2751 & U18 & 6273900 & 2770800 & 541 & 11.1 & 5712301 & 1860713 \\
2787 & U17 & 6294600 & 2789500 & 330 & 39.0 & 5733035 & 1879405 \\
3078 & V16 & 6319523 & 2829404 & n/a & 85.0 & 5758026 & 1919313 \\
\hline
\end{tabular}




\section{Appendix B. Imaginary refractive index (R.I.I.) guidelines ${ }^{a}$}

\begin{tabular}{ll}
\hline clear materials, glass & $<0.001$ \\
translucent materials, quartz & $<0.01$ \\
lightly colored, translucent material & $0.01-0.1$ \\
$\begin{array}{l}\text { white or transparent powders } \\
\text { gray or lightly pigmented materials, metal oxides, } \\
\text { highly colored materials }\end{array}$ & $0-0.1$ \\
$\begin{array}{l}\text { Black or highly pigmented materials, metallic } \\
\text { particles, carbon }\end{array}$ & $1-10$ \\
\hline
\end{tabular}

${ }^{a}$ From Beckman-Coulter [2003]

Appendix C. Time of pipette withdrawal for given temperature, depth, and particle diameter (from Haan et al. [1994]) ${ }^{a}$

\begin{tabular}{|c|c|c|c|c|c|c|}
\hline $\begin{array}{l}\text { Diameter of } \\
\text { particle (mm) }\end{array}$ & 0.063 & 0.031 & 0.016 & 0.008 & 0.004 & 0.002 \\
\hline Phi size class & $4 \varnothing$ & $5 \varnothing$ & $6 \varnothing$ & $7 \varnothing$ & $8 \varnothing$ & $9 \varnothing$ \\
\hline \multirow[t]{3}{*}{$\begin{array}{l}\text { Depth of } \\
\text { withdrawal (cm) }\end{array}$} & 15 & 15 & 10 & 10 & 5 & 5 \\
\hline & \multicolumn{6}{|c|}{ Time of withdrawal } \\
\hline & & \multicolumn{4}{|c|}{ Min:sec } & \\
\hline Temp $\left({ }^{\circ} \mathrm{C}\right)$ & Sec & & & & & Hr:min \\
\hline 20 & 44 & $2: 52$ & $7: 40$ & $30: 40$ & $61: 19$ & $4: 5$ \\
\hline 21 & 42 & $2: 48$ & $7: 29$ & $29: 58$ & $59: 50$ & 4:0 \\
\hline 22 & 41 & $2: 45$ & $7: 18$ & 29:13 & $58: 22$ & $3: 54$ \\
\hline 23 & 40 & $2: 41$ & 7:08 & 28:34 & $57: 5$ & $3: 48$ \\
\hline 24 & 39 & $2: 38$ & $6: 58$ & $27: 52$ & $55: 41$ & $3: 43$ \\
\hline 25 & 38 & $2: 34$ & $6: 48$ & $27: 14$ & $54: 25$ & $3: 38$ \\
\hline 26 & 37 & $2: 30$ & $6: 39$ & $26: 38$ & $52: 2$ & $3: 33$ \\
\hline 27 & 36 & $2: 27$ & $6: 31$ & 26:02 & $52: 2$ & $3: 28$ \\
\hline 28 & 36 & $2: 23$ & $6: 22$ & $25: 28$ & $50: 52$ & $3: 24$ \\
\hline 29 & 35 & $2: 19$ & $6: 13$ & $24: 53$ & $49: 42$ & 3:19 \\
\hline 30 & 34 & $2: 16$ & 6:06 & $24: 22$ & $48: 42$ & 3:15 \\
\hline
\end{tabular}

${ }^{a}$ Values in this table are based on particles of assumed spherical shape with an average specific gravity of 2.65, the constant of acceleration due to gravity $=9.80 \mathrm{~m} \mathrm{~s}^{-2}$, and viscosity varying from $0.010087 \mathrm{~cm}^{2} \mathrm{sec}^{-1}$ at $20^{\circ} \mathrm{C}$ to $0.008004 \mathrm{~cm}^{2} \mathrm{sec}^{-1}$ at $30^{\circ} \mathrm{C}$. 
Appendix D. Procedure for SEM image analysis of fine volcanic ash

Procedure summary: (1) Dry sieving; (2) Preparation of SEM stubs; (3) SEM imaging; (4) Photoshop editing; (5) Image analysis using ImageJ; (6) Export to Excel. The detailed procedure is as follows:

> Dry-sieve the dry, disaggregated sample of volcanic ash between, for example, $63 \mu \mathrm{m}(4 \varphi)$ and $45 \mu \mathrm{m}(4.5 \varphi)$.

$>$ Prepare SEM stubs by adding small squares of double-sided sticky tape to the stubs, and lightly dusting the sieved sample onto them. One successful approach was to add a small amount of ash to a piece of paper, fold up the sides and tap the edge to administer a light dusting of grains. The aim is to add enough grains to fill the view at $75 \mathrm{x}$ magnification, but not so many that they are touching.

$>$ Obtain backscatter images of the samples at $75 x$ magnification in backscatter mode.

$>$ Open an SEM image in Photoshop. Click image $\rightarrow$ mode $\rightarrow$ greyscale. Then save as a copy. Increase brightness and contrast, use a filter such as filter $\rightarrow$ blur $\rightarrow$ smart blur to remove noise.

Now use the magic wand tool (turn off anti-alias option, noncontiguous, and put threshold at something high, like 100) to highlight the white parts of the grains. This should get most of them, but there will be grain edges that need to be included. Lower the threshold to 3050 and hold shift while using the magic wand to add grain facets to the selection until they are all reasonably represented.

> While this is still highlighted, hit $\mathrm{Ctrl}+\mathrm{C}$, make a new layer, and $\mathrm{Ctrl}+\mathrm{V}$ the selected grains into the new layer. Now the new layer can be clicked on and off to compare with the original image. In this new layer, magic wand the background, then click image $\rightarrow$ select inverse to activate the grains again, and hit edit $\rightarrow$ fill $\rightarrow$ white to make them all white. Save.

> Next, the grains need to be cleaned up and separated (if touching) using the pencil tool (size ca. 4 pixels for separations). If grains cannot be separated without sacrificing the integrity of one of them (i.e., $<90$ $95 \%$ intact), save the one on top and erase remnants of the loser by 
pencilling over it in black. Also, in the analysis process any grains touching the edge of the frame won't be counted, so if there are any decent clasts $90-95 \%$ visible that you want to count, separate them from the frame edge with a black pencil edge (at least 1 pixel wide).

$>$ Once all the grains all accounted for, SAVE, then select a white patch using magic wand, click select $\rightarrow$ inverse, and fill in the background with black. Make sure the processed image compares well with the original image, making sure there are not any tiny white artefacts from smashed grains or pixelated imaging. Then crop off the scale bar, Save and move on to the next step.

> ImageJ. Open both the original image and the photoshopped one. Use the scale bar on the original one to set the scale by zooming in on it, drawing a straight line on it (shift+drag), and hitting measurements $\rightarrow$ set scale $\rightarrow$ actual size (insert scale here $\mu \mathrm{m}$ ) and check 'global'.

$>$ Now return to the photoshopped image and click process $\rightarrow$ binary $\rightarrow$ make binary and binary $\rightarrow$ fill holes. Now save. Set Measurements to include perimeter, area, bounding rectangle, and feret diameter. To measure grains, go to Process $\rightarrow$ analyze particles $\rightarrow$ include 1-infinity pixels, 0 -infinity roundness, draw outlines, show results and proceed. Double-check that the grain outlines look realistic.

Finally, copy the results into Excel using edit $\rightarrow$ select all $\rightarrow$ ctrl $+\mathrm{C}$. 
Appendix E. Volume-weighted statistics (arithmetic) of particle size distributions from parent ash used in the experiments for this study. Calculations are based on laser diffraction particle size analyser channel diameters from 0.375 to $2000 \mu \mathrm{m}$. Statistics calculated using the Beckman-Coulter LS 13320 software v.5.01

\begin{tabular}{|c|c|c|c|c|c|c|}
\hline \multirow{2}{*}{ Experiment ID } & \multicolumn{2}{|c|}{ Liquid water (wt.\%) } & \multicolumn{4}{|c|}{ Parent ash size statistics } \\
\hline & Added $^{\mathrm{a}}$ & in $\mathrm{Aggs}^{\mathrm{b}}$ & Mean & Median & Mode & $\%<63 \mu \mathrm{m}$ \\
\hline \multicolumn{7}{|c|}{$\overline{\text { Liquid only experimental series (Liquid-1) }}$} \\
\hline Test 4 & 18.6 & 17.2 & 224.0 & 83.4 & 55.1 & 42.7 \\
\hline Test 5 & 6.8 & 9.4 & 90.0 & 32.7 & 28.7 & 65.1 \\
\hline Test 6 & 11.1 & 11.1 & 72.5 & 40.4 & 41.7 & 64.3 \\
\hline Test 7 & 16.5 & 17.6 & 81.8 & 46.1 & 66.4 & 59.9 \\
\hline Test 8 & 16.1 & 17.2 & 80.1 & 43.1 & 60.5 & 61.7 \\
\hline Test $9 *$ & 22.2 & 22.9 & 62.5 & 41.1 & 72.9 & 63.5 \\
\hline Test 10 & 8.6 & 10.2 & 82.0 & 43.7 & 45.8 & 61.3 \\
\hline Test 11 & 9.6 & 10.9 & 78.1 & 44.1 & 55.1 & 61.2 \\
\hline Test 12 & 16.6 & 18.4 & 79.1 & 41.9 & 55.1 & 62.6 \\
\hline Test $13^{*}$ & 22.7 & 22.9 & 68.4 & 42.8 & 66.4 & 62.7 \\
\hline Test 14 & 2.5 & 2.9 & 77.6 & 43.0 & 66.4 & 61.9 \\
\hline Test 15 & 8.8 & 11.5 & 91.8 & 43.4 & 66.4 & 61.4 \\
\hline Test $16^{*}$ & 12.6 & 15.0 & 59.7 & 40.5 & 72.9 & 64.9 \\
\hline Test 17 & 10.0 & 11.6 & 81.8 & 47.8 & 66.4 & 59.6 \\
\hline Test $18^{*}$ & 17.6 & 18.7 & 81.3 & 46.5 & 60.5 & 60.3 \\
\hline Test $19 *$ & 21.2 & 22.1 & 79.8 & 46.5 & 60.5 & 60.6 \\
\hline Test 20 & 10.8 & 13.9 & 83.4 & 44.5 & 55.1 & 61.4 \\
\hline Test 21 & 5.0 & 5.9 & 87.0 & 48.0 & 60.5 & 59.1 \\
\hline Test $22 *$ & 17.1 & 17.0 & 64.1 & 44.0 & 45.8 & 62.9 \\
\hline Test $23 *$ & 20.9 & 21.6 & 87.0 & 48.3 & 60.5 & 59.0 \\
\hline Test $24^{*}$ & 25.9 & 25.1 & 128.0 & 51.1 & 45.8 & 56.3 \\
\hline Test $25^{*}$ & 22.5 & 23.1 & 125.0 & 52.6 & 66.4 & 55.4 \\
\hline Test 26 & 19.0 & 20.4 & 92.4 & 35.3 & 31.5 & 63.8 \\
\hline Test 27 & 17.6 & 18.9 & 152.0 & 47.5 & 31.5 & 55.7 \\
\hline Test 28 & 19.4 & 20.9 & 120.0 & 43.0 & 31.5 & 58.2 \\
\hline Test 29 & 22.8 & 22.5 & 117.0 & 41.8 & 31.5 & 59.0 \\
\hline Test 30 & 15.4 & 17.1 & 125.0 & 44.1 & 31.5 & 57.8 \\
\hline Test $31^{*}$ & 9.4 & 14.7 & 42.6 & 28.4 & 34.6 & 76.5 \\
\hline Test 38 & 12.5 & 17.9 & 197.0 & 58.7 & 34.6 & 51.6 \\
\hline Test $39^{*}$ & 16.1 & 19.7 & 177.0 & 53.4 & 34.6 & 54.0 \\
\hline Test 40 & 13.1 & 18.5 & 219.0 & 64.5 & 34.6 & 49.5 \\
\hline Test 41 & 8.0 & 12.5 & 180.0 & 56.6 & 55.1 & 52.7 \\
\hline Test 42 & 10.7 & 16.0 & 132.0 & 50.2 & 34.6 & 55.7 \\
\hline Test 43 & 10.1 & 10.1 & 104.0 & 43.4 & 38.0 & 60.0 \\
\hline Test 47 & 8.3 & 1.8 & 127.0 & 51.4 & 80.1 & 56.0 \\
\hline Test 48 & 8.7 & 9.2 & 218.0 & 75.1 & 87.9 & 45.8 \\
\hline Test 49 & 3.7 & 0.6 & 96.9 & 50.8 & 72.9 & 56.5 \\
\hline Test 50 & 4.5 & 4.2 & 147.0 & 62.6 & 80.1 & 50.2 \\
\hline Test 51 & 7.0 & 7.5 & 167.0 & 63.7 & 80.1 & 49.7 \\
\hline Test 52 & 6.3 & 8.0 & 136.0 & 57.7 & 87.9 & 52.3 \\
\hline Test $53^{*}$ & 18.2 & 14.6 & 168.0 & 64.0 & 87.9 & 49.6 \\
\hline Test 54 & 8.1 & 11.6 & 192.8 & 67.7 & 87.9 & 48.3 \\
\hline Test 55 & 7.1 & 8.0 & 128.0 & 63.5 & 87.9 & 49.8 \\
\hline Test 56 & 6.1 & 8.7 & 119.0 & 56.5 & 87.9 & 53.1 \\
\hline \multicolumn{7}{|c|}{ Mixed-phase or ice-only experimental series } \\
\hline Hail-1 & 0.0 & 0.0 & 99.1 & 42.6 & 34.6 & 60.7 \\
\hline Hail-2 & $\mathrm{n} / \mathrm{a}$ & $\mathrm{n} / \mathrm{a}$ & 88.4 & 41.4 & 50.2 & 62.1 \\
\hline Mix-1 & $\mathrm{n} / \mathrm{a}$ & $\mathrm{n} / \mathrm{a}$ & 94.8 & 44.0 & 41.7 & 59.9 \\
\hline $\operatorname{Mix}-2^{\mathrm{c}}$ & $\mathrm{n} / \mathrm{a}$ & $\mathrm{n} / \mathrm{a}$ & 87.6 & 36.9 & 34.4 & 65.7 \\
\hline
\end{tabular}

Bold font designates experiments that were used to derive aggregation coefficients (Appendix G) and the experimental curve of Figure 4.12.

*Indicates that preferential scavenging could not be satisfactorily detected within uncertainty.

${ }^{a_{1}}$ Liquid water added' is the average wt.\% water measured gravimetrically from all fractions of the experiment (including aggregates and residua).

${ }^{b_{1}}$ Liquid water in aggregates' is the wt.\% water in the aggregate fraction used for particle size analysis (usually the 4-8 $\mathrm{mm}$ size fraction where possible).

${ }^{\mathrm{c}}$ Mix-2 (Melting ice) data are reported as an average of six experiments. Aggregates were not analyzed for particle size distribution.

$\mathrm{n} / \mathrm{a}$ indicates that the liquid water content is unknown due to the presence of mixed water phases during the experiment. 


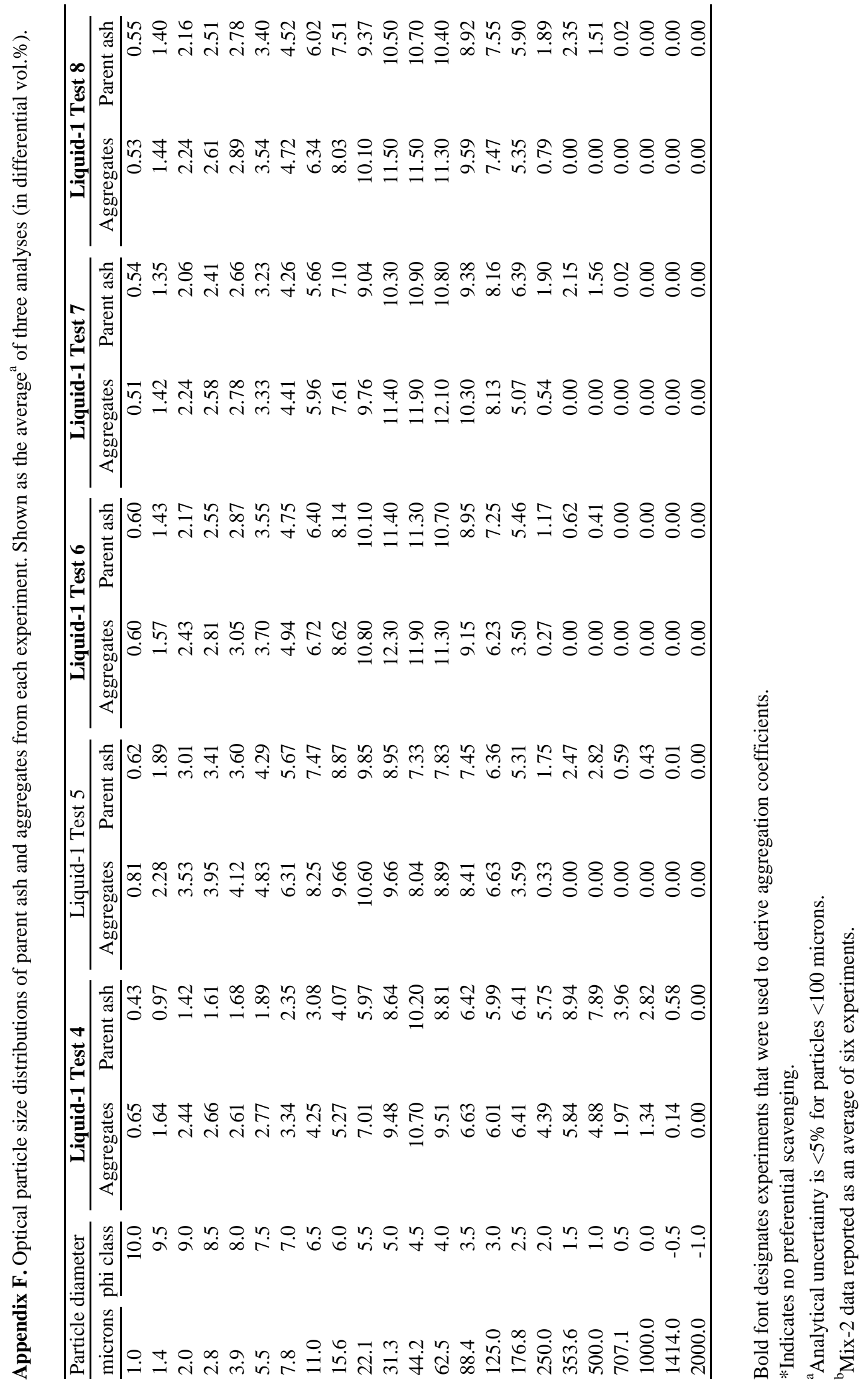




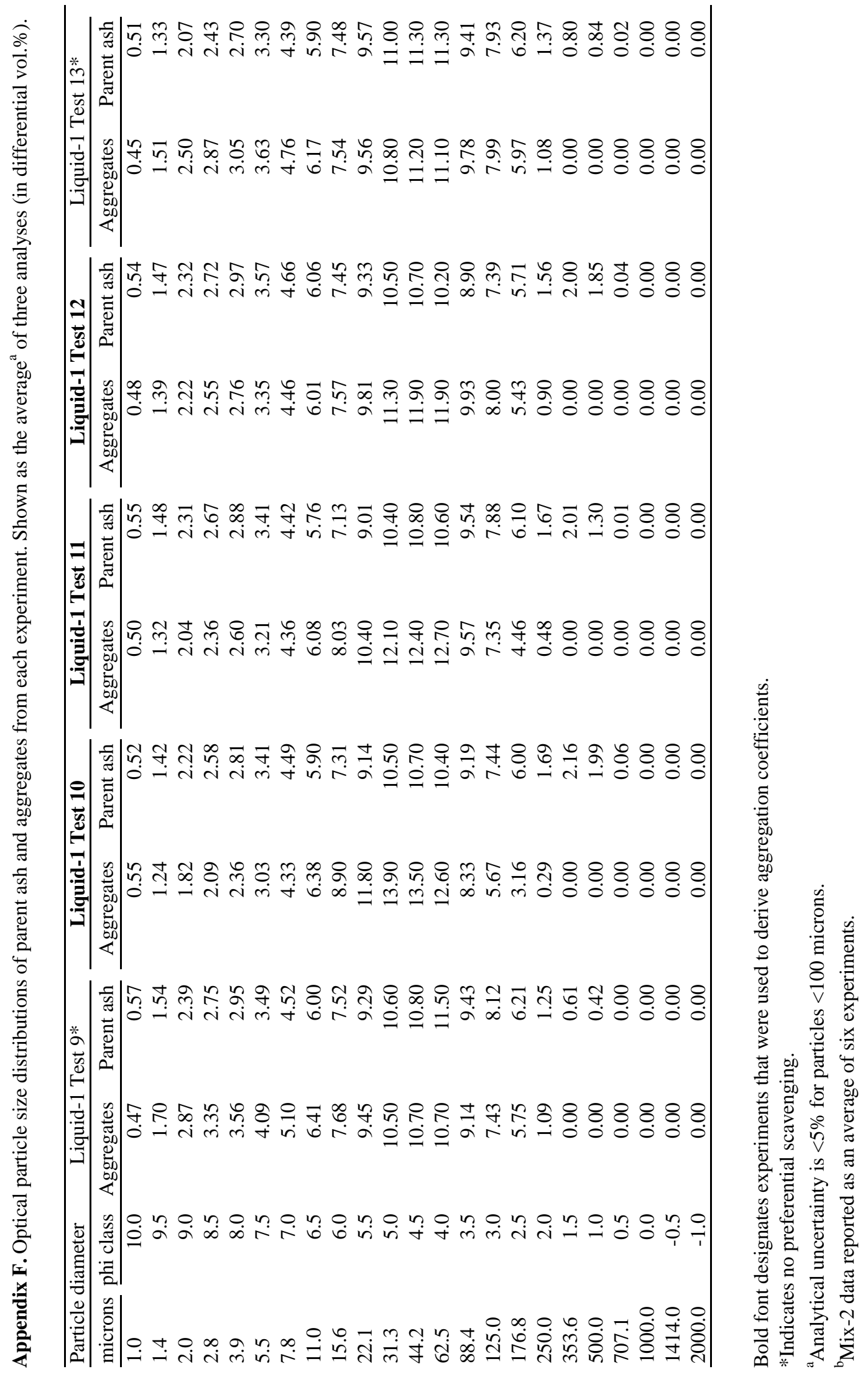




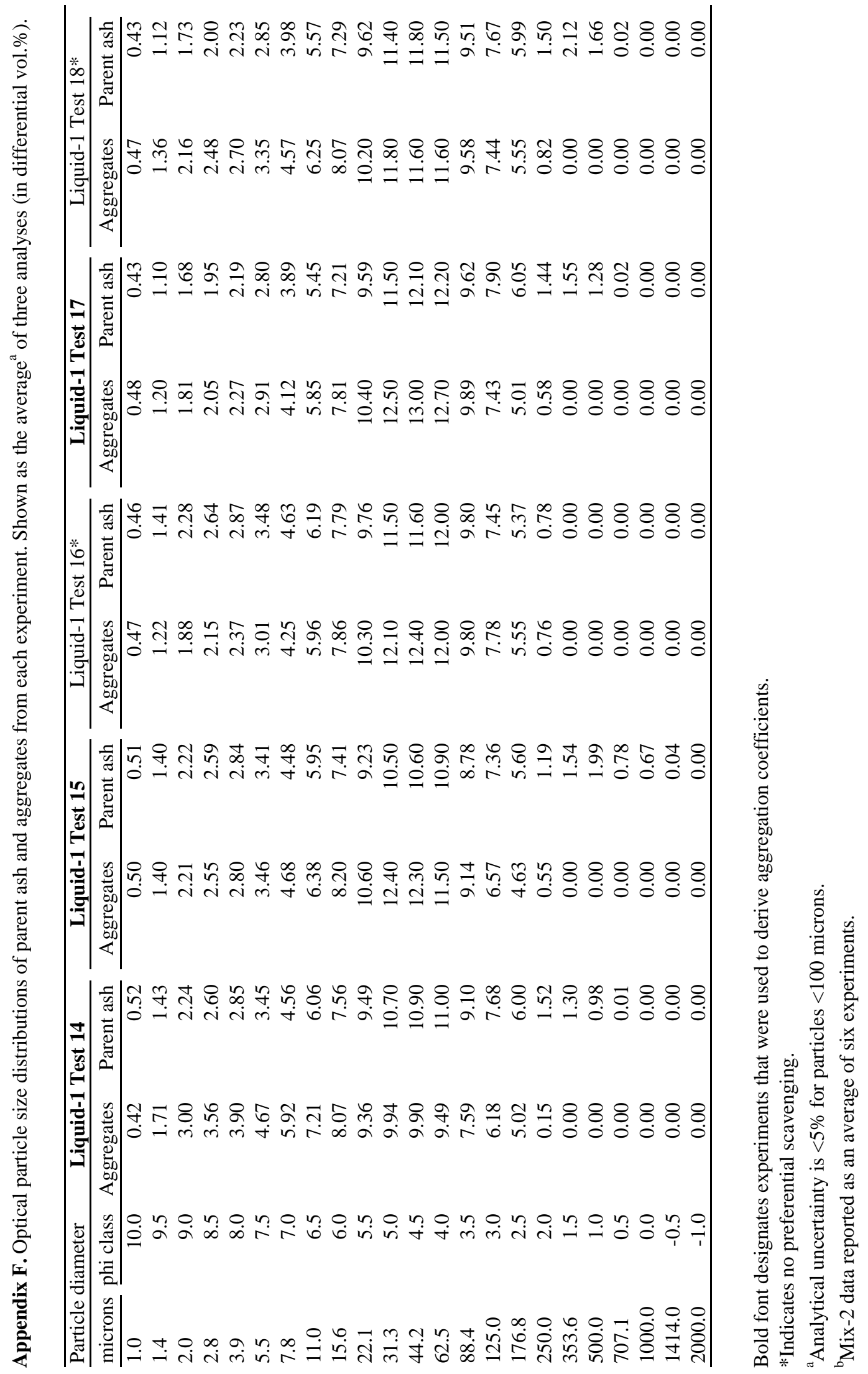




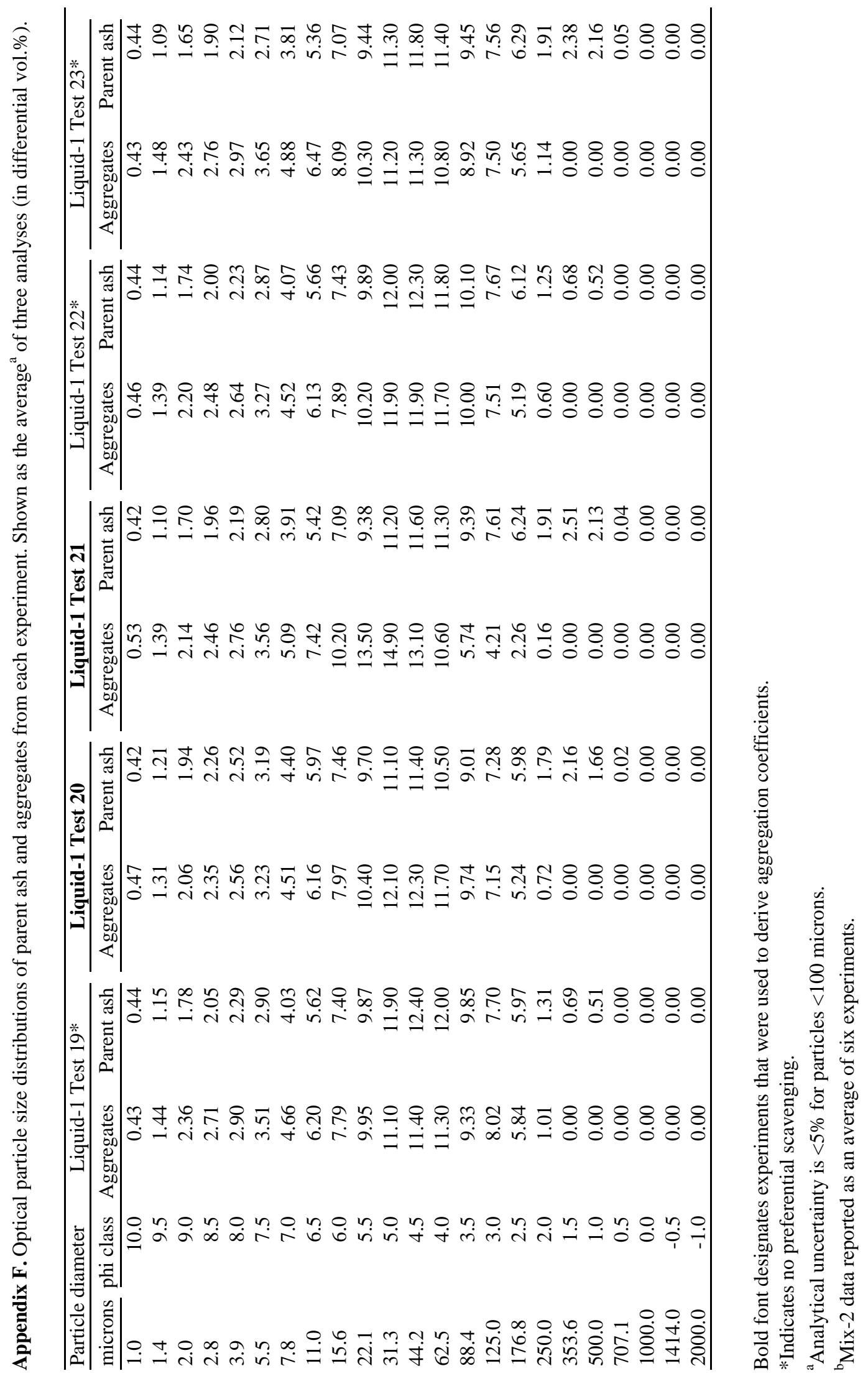




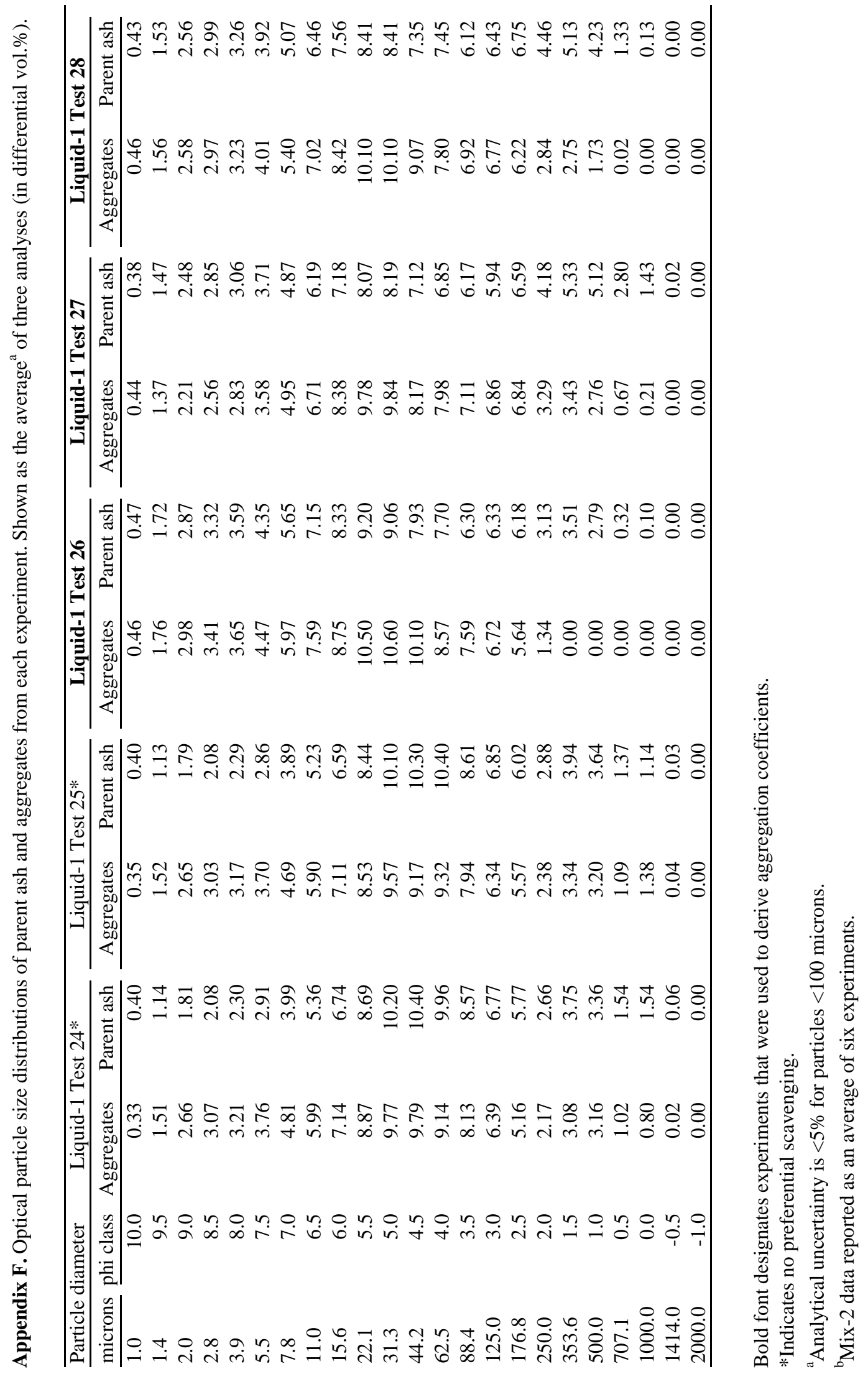




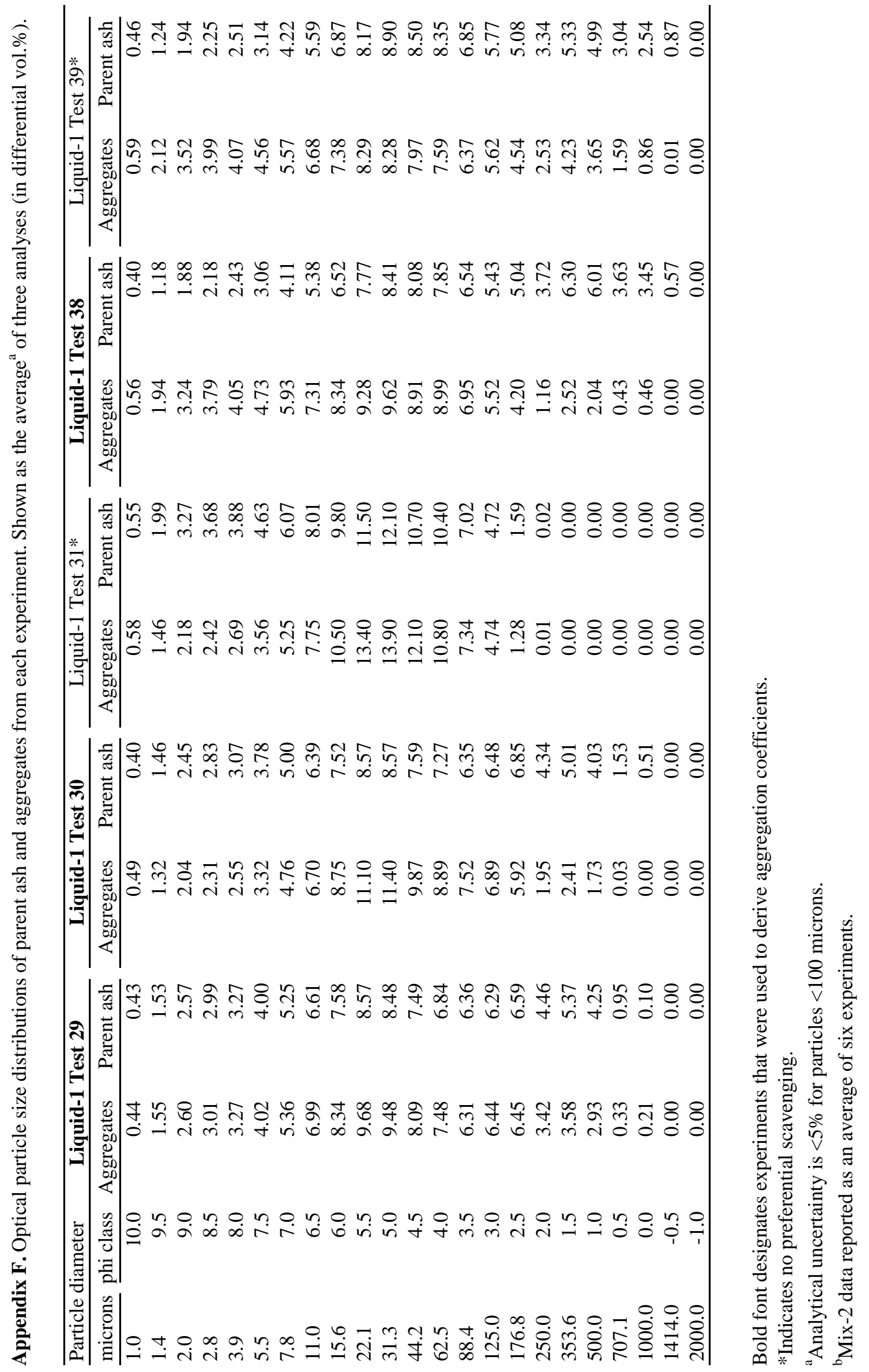




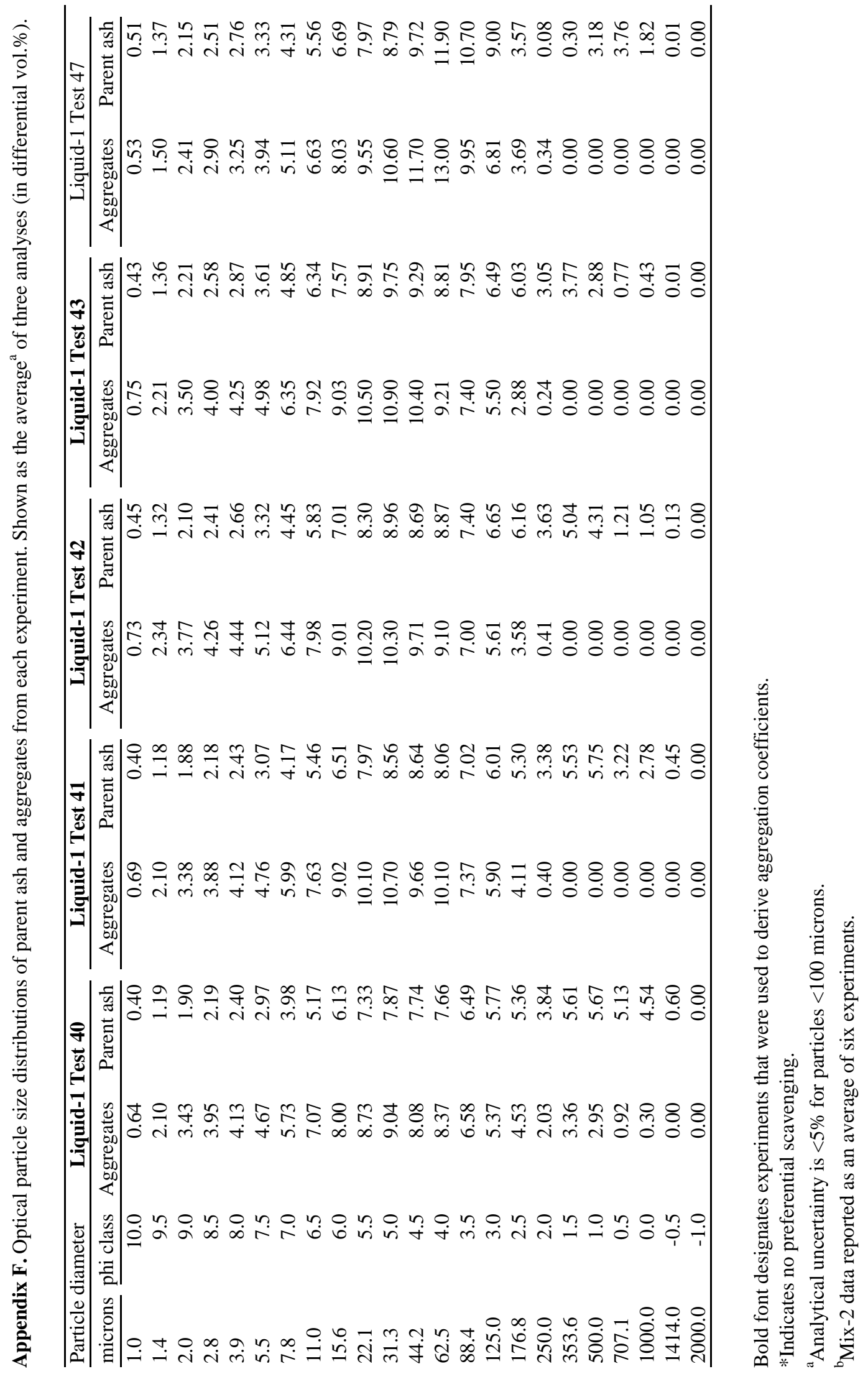




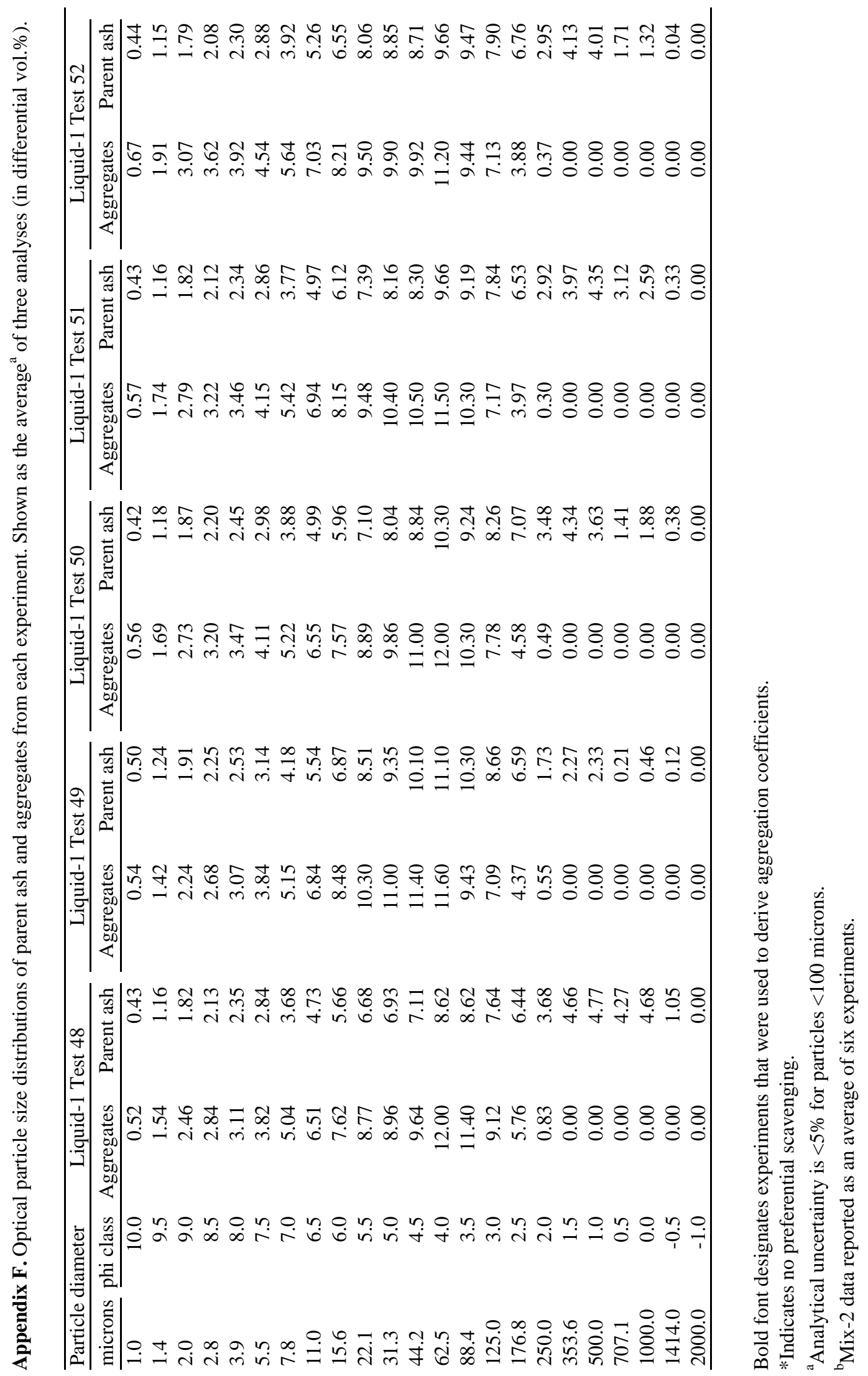




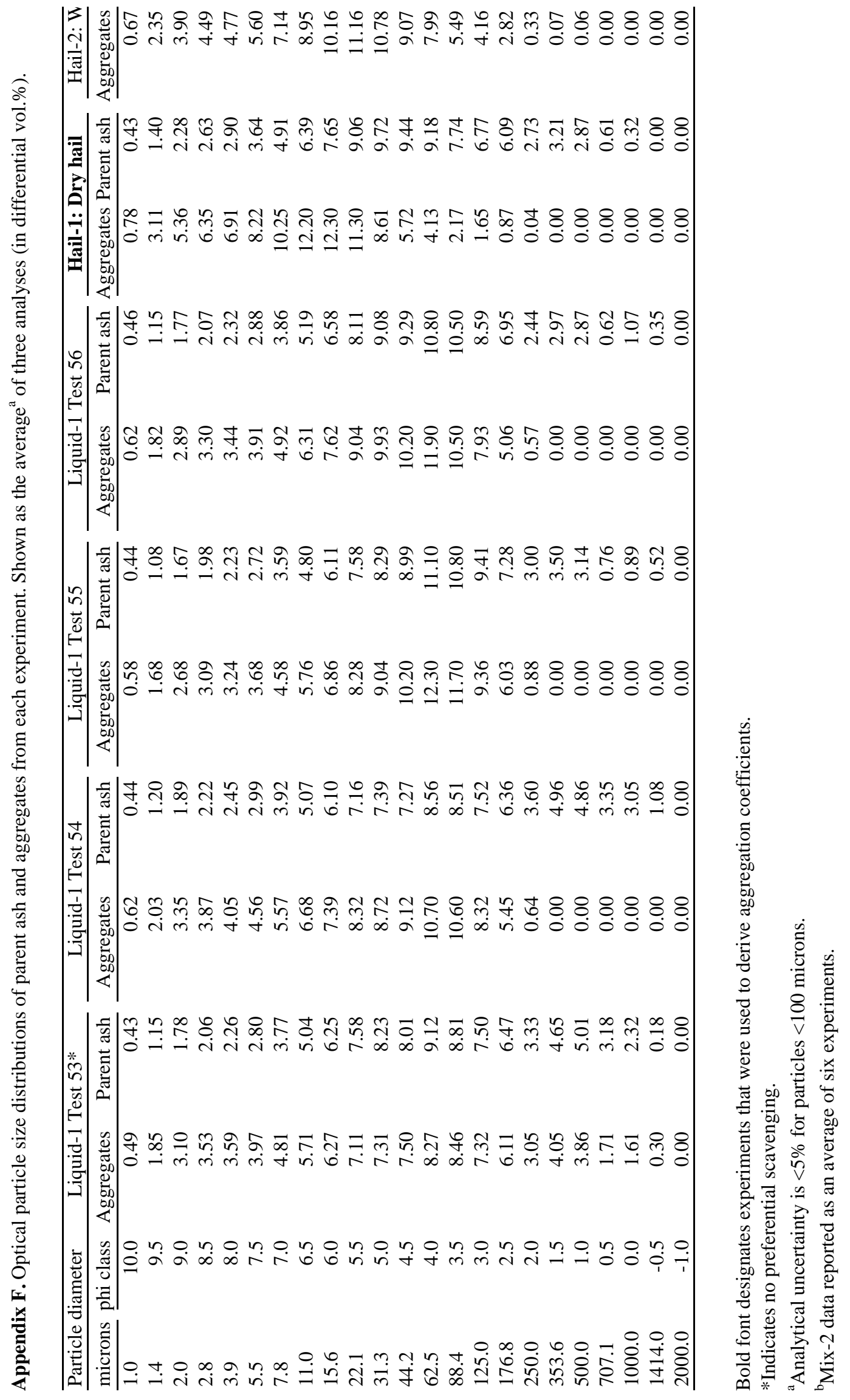




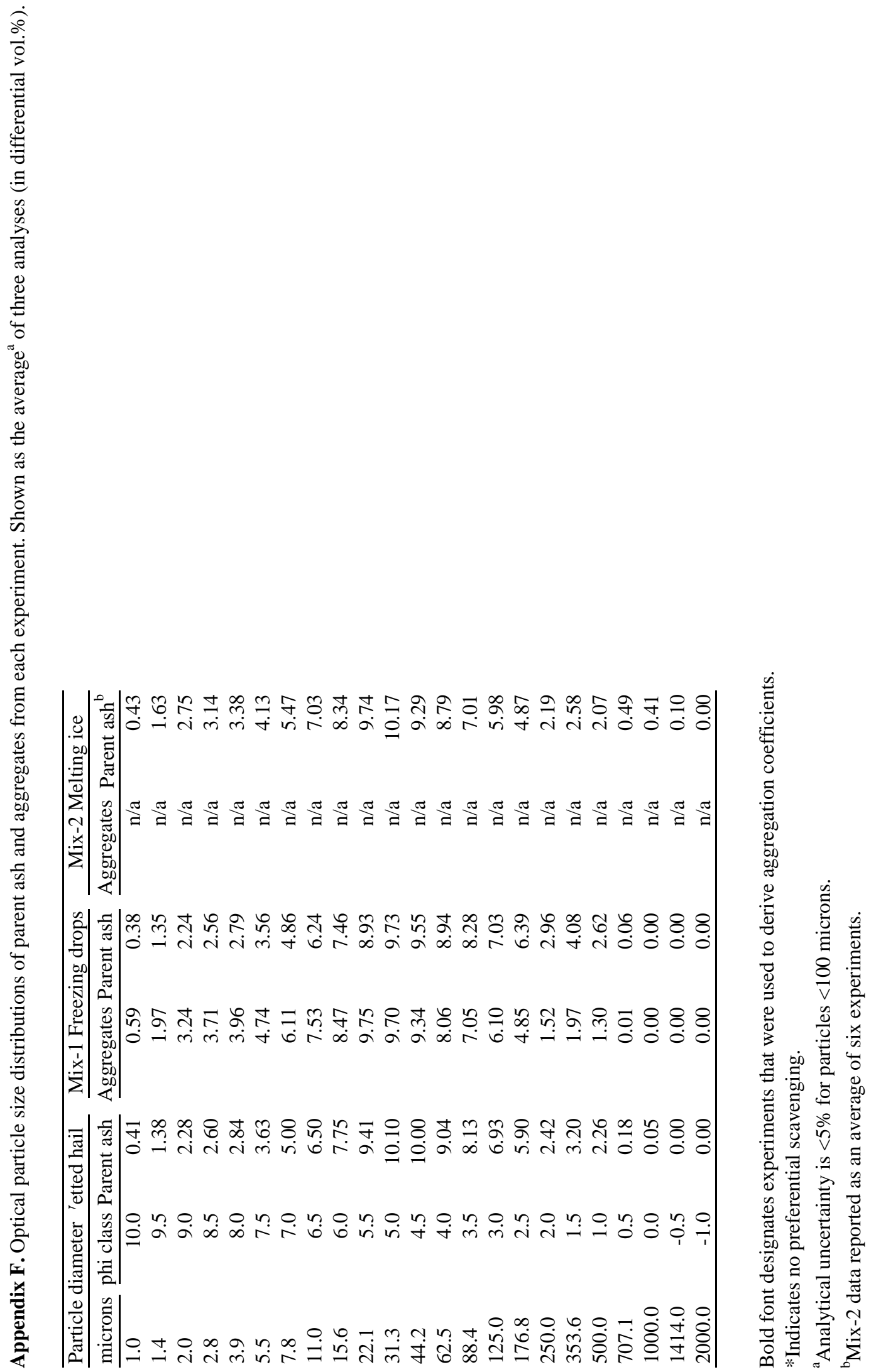


Appendix G. Aggregation coefficients from (vol.\% in aggregates / vol.\% in parent ash). Values averaged from experiments that showed preferential scavenging (from Tests 4-43 of Appendix F).

\begin{tabular}{|c|c|c|c|c|c|c|}
\hline \multirow{2}{*}{\multicolumn{2}{|c|}{$\begin{array}{l}\text { Particle diameter } \\
\text { microns phi class }\end{array}$}} & \multicolumn{5}{|c|}{ Aggregation coefficients (by wt.\% liquid in aggregates) } \\
\hline & & $0 \%$ (ice) & $0-10 \%$ & $10-15 \%$ & $15-20 \%$ & $20-25 \%$ \\
\hline 1.0 & 10.0 & 1.63 & 1.03 & 1.21 & 1.19 & 1.02 \\
\hline 1.4 & 9.5 & 2.16 & 1.23 & 1.18 & 1.22 & 1.02 \\
\hline 2.0 & 9.0 & 2.32 & 1.30 & 1.17 & 1.23 & 1.02 \\
\hline 2.8 & 8.5 & 2.38 & 1.31 & 1.15 & 1.21 & 1.01 \\
\hline 3.9 & 8.0 & 2.34 & 1.31 & 1.13 & 1.18 & 1.00 \\
\hline 5.5 & 7.5 & 2.23 & 1.31 & 1.11 & 1.16 & 1.02 \\
\hline 7.8 & 7.0 & 2.08 & 1.30 & 1.11 & 1.15 & 1.05 \\
\hline 11.0 & 6.5 & 1.89 & 1.28 & 1.13 & 1.15 & 1.07 \\
\hline 15.6 & 6.0 & 1.59 & 1.25 & 1.15 & 1.15 & 1.09 \\
\hline 22.1 & 5.5 & 1.26 & 1.21 & 1.16 & 1.15 & 1.16 \\
\hline 31.3 & 5.0 & 0.90 & 1.13 & 1.16 & 1.13 & 1.16 \\
\hline 44.2 & 4.5 & 0.64 & 1.02 & 1.13 & 1.11 & 1.20 \\
\hline 62.5 & 4.0 & 0.43 & 0.90 & 1.12 & 1.11 & 1.08 \\
\hline 88.4 & 3.5 & 0.31 & 0.72 & 1.01 & 1.07 & 1.11 \\
\hline 125.0 & 3.0 & 0.27 & 0.68 & 0.90 & 1.02 & 1.05 \\
\hline 176.8 & 2.5 & 0.14 & 0.60 & 0.71 & 0.89 & 0.94 \\
\hline 250.0 & 2.0 & 0.01 & 0.10 & 0.27 & 0.52 & 0.61 \\
\hline 353.6 & 1.5 & 0.00 & 0.00 & 0.00 & 0.35 & 0.40 \\
\hline 500.0 & 1.0 & 0.00 & 0.00 & 0.00 & 0.33 & 0.37 \\
\hline 707.1 & 0.5 & 0.00 & 0.00 & 0.00 & 0.21 & 0.12 \\
\hline 1000.0 & 0.0 & 0.00 & 0.00 & 0.00 & 0.20 & 0.70 \\
\hline 1414.0 & -0.5 & 0.00 & 0.00 & 0.00 & 0.14 & 0.00 \\
\hline 2000.0 & -1.0 & 0.00 & 0.00 & 0.00 & 0.00 & 0.00 \\
\hline
\end{tabular}


Appendix H. Diatom species in the Oruanui and lacustrine sedimentary samples of Chapter 6.

\begin{tabular}{|c|c|c|c|c|c|c|}
\hline Taxa identified & \%psol & \%Seds & \% Oruanui & \%Seds+Ou & Grouped & No. \\
\hline Achnanthidium cf. minutissimum (Kütz.) Czarnecki & & & $\mathrm{v}$ & & Shallow waterweed spp. & 9 \\
\hline Achnanthidium cf. exiguum (Grunow) Czarnecki & & & $\mathrm{v}$ & & Shallow waterweed spp. & 9 \\
\hline Amphicampa mirabilis Ehrenberg & & & $\mathrm{v}$ & & Shallow waterweed spp. & 9 \\
\hline Amphora cf. libyca Ehr. & & $\mathrm{r}$ & r & both & Sediment surface spp. & 10 \\
\hline Aulacoseira ambigua (Grunow) Simonsen & 2.4 & 41.57 & 28.06 & in three & Aulacoseira ambigua & 1 \\
\hline $\begin{array}{l}\text { Aulacoseira cf. granulata var.angustissima (O.Mueller) } \\
\text { Simonsen }\end{array}$ & & $\mathrm{f}$ & $\mathrm{s}$ & both & Other planktic & 4 \\
\hline Aulacoseira crenulata (Ehrenberg) Thwaites & & r & $\mathrm{s}$ & both & Other planktic & 4 \\
\hline Caloneis cf. bacillum (Grunow) Cleve & $\mathrm{v}$ & & & & Sediment surface spp. & 10 \\
\hline Cavinula scutelloides (W. Smith) Lange-Bertalot & & $\mathrm{v}$ & & & Sediment surface spp. & 10 \\
\hline Cocconeis neodiminuta Krammer & & $\mathrm{v}$ & $\mathrm{v}$ & both & Shallow waterweed spp. & 9 \\
\hline Cocconeis placentula var. lineata (Ehrenberg) Van Heurck & & & $\mathrm{v}$ & & Shallow waterweed spp. & 9 \\
\hline Cocconeis placentula var. placentula Ehrenberg & & & $\mathrm{v}$ & & Shallow waterweed spp. & 9 \\
\hline Cyclostephanos cf. dubius (Fricke) Round & & & $\mathrm{v}$ & & Other planktic & 4 \\
\hline Cyclostephanos novaezeelandiae (Cleve) Round & & 25.86 & 22.07 & both & $\begin{array}{l}\text { Cyclostephanos } \\
\text { novaezeelandiae }\end{array}$ & 2 \\
\hline Cyclotella atomus Hustedt & & $\mathrm{v} ?$ & $\mathrm{v}$ & both & Other planktic & 4 \\
\hline Cyclotella striata (Kuetzing) Grunow & & & v & & Other planktic & 4 \\
\hline Cymbella aspera (Ehrenberg) H.Peragallo & & & v & & Shallow waterweed spp. & 9 \\
\hline Cymbella cf. novazeelandiana Krammer & & $\mathrm{r}$ & $\mathrm{v}$ & both & Shallow waterweed spp. & 9 \\
\hline Cymatopleura solea (Brebisson) W.Smith & & $\mathrm{v}$ & $\mathrm{v}$ & both & Sediment surface spp. & 10 \\
\hline Diploneis interrupta (Kuetzing) Cleve & & r & r & both & Sediment surface spp. & 10 \\
\hline Diploneis subovalis Cleve & & r & r & both & Sediment surface spp. & 10 \\
\hline Discostella cf. elentarii (Alfi et Tagliaventi) Houk et Klee & & & $\mathrm{v}$ ? & & Other planktic & 4 \\
\hline Discostella stelligera (Cleve \& Grunow) Houk \& Klee & 1.6 & 10.82 & 24.83 & in three & Discostella stelligera & 3 \\
\hline Discostella stelligera var. robusta (Hustedt) Houk et Klee & & & $\mathrm{v}$ & & Other planktic & 4 \\
\hline Encyonema cf. neogracile Krammer & & $\mathrm{v}$ & $\mathrm{v}$ & both & Shallow waterweed spp. & 9 \\
\hline Epithemia adnata (Kuetzing) Brebisson & & 1.82 & 2.58 & both & Epithemia adnata & 7 \\
\hline Epithemia sorex Kuetzing & & 0.57 & 1.51 & both & Epithemia sorex & 8 \\
\hline Eunotia exigua ( Brebisson) Rabenhorst & & $\mathrm{v}$ & & & Shallow waterweed spp. & 9 \\
\hline Eunotia minor (Kuetzing) Grunow & & & $\mathrm{v}$ & & Shallow waterweed spp. & 9 \\
\hline $\begin{array}{l}\text { Fragilaria capucina var. vaucheriae (Kuetzing) Lange- } \\
\text { Bertalot }\end{array}$ & & $\mathrm{r}$ & $\mathrm{v}$ & both & Other planktic & 4 \\
\hline Fragilaria cf. crotonensis Kitton & & & $\mathrm{r}$ & & Other planktic & 4 \\
\hline Fragilariforma cf. virescens (Ralfs) Williams \& Round & & & $\mathrm{r}$ & & Shallow water planktic & 5 \\
\hline Geissleria cf. decussis (Ostrup) Lange-Bertalot \& Metzeltin & & $\mathrm{v}$ & & & Sediment surface spp. & 10 \\
\hline Gomphonema angustum Agardh & & $\mathrm{v}$ & $\mathrm{v}$ & both & Shallow waterweed spp. & 9 \\
\hline $\begin{array}{l}\text { Hantzschia cf. amphioxys (Ehr.) Grunow in Cleve et } \\
\text { Grunow }\end{array}$ & 54.4 & $\mathrm{v}$ & $\mathrm{s}$ & in three & Soil spp. & 11 \\
\hline $\begin{array}{l}\text { Karayevia cf clevei (Grun. in Cl. \& Grun.) Round \& } \\
\text { Bukhtiyarova }\end{array}$ & & $\mathrm{v}$ & $\mathrm{v}$ & both & Shallow waterweed spp. & 9 \\
\hline Luticola mutica (Kützing) D.G. Mann & $\mathrm{s}$ & & $\mathrm{v}$ & & Soil spp. & 11 \\
\hline $\begin{array}{l}\text { Martyana martyi (Héribaud) Round in Round Crawford \& } \\
\text { Mann }\end{array}$ & & $\mathrm{v}$ & $\mathrm{v}$ & both & Shallow waterweed spp. & 9 \\
\hline Melosira nummuloides (Dillwyn) C.A. Agardh & & & $\mathrm{v}$ & & Other planktic & 4 \\
\hline Melosira varians Agardh & & $\mathrm{v}$ & & & Sediment surface spp. & 10 \\
\hline Navicula cincta (Ehr.) Ralfs in Pritchard & & $\mathrm{v}$ & & & Sediment surface spp. & 10 \\
\hline Navicula cf. gregaria Donkin & $\mathrm{v}$ & & & & Sediment surface spp. & 10 \\
\hline Navicula cf. subrhynchocephala Hustedt & $\mathrm{v}$ ? & & & & Sediment surface spp. & 10 \\
\hline Navicula cryptocephala Kuetzing & s & & r & odd & Sediment surface spp. & 10 \\
\hline Navicula radiosa Kützing & s & & & & Sediment surface spp. & 10 \\
\hline Nitzschia amphibia Grunow & $\mathrm{s}$ & & $\mathrm{r}$ & odd & Sediment surface spp. & 10 \\
\hline Nitzschia cf. paleaeformis Hustedt & & $\mathrm{v}$ & & & Sediment surface spp. & 10 \\
\hline Nitzschia cf. vermicularis (Kutzing) Hantzsch & & $\mathrm{V}$ & & & Other planktic & 4 \\
\hline Nitzschia vitrea Norman & & $\mathrm{v}$ ? & $\mathrm{v}$ & both & Other planktic & 4 \\
\hline Pinnularia borealis Ehrenberg & 23.2 & $\mathrm{v}$ & r & in three & Soil spp. & 11 \\
\hline Pinnularia brebissonii (Kutz.) Rabenhorst & & $\mathrm{v} ?$ & & & Sediment surface spp. & 10 \\
\hline Pinnularia divergentissima & $\mathrm{r}$ & & $\mathrm{v}$ & odd & Sediment surface spp. & 10 \\
\hline Pinnularia gibba Ehrenberg & & $\mathrm{v}$ & $\mathrm{v}$ & both & Sediment surface spp. & 10 \\
\hline Pinnularia gibba cf. var. mesogongyla (Ehrenberg) Hustedt & & $\mathrm{v}$ ? & $\mathrm{v}$ & both & Sediment surface spp. & 10 \\
\hline Pinnularia cf.maior (Kutzing) Rabenhorst & & $\mathrm{r}$ & $\mathrm{v}$ & both & Sediment surface spp. & 10 \\
\hline Pinnularia subcapitata Gregory & & $\mathrm{r}$ & $\mathrm{v}$ & both & Sediment surface spp. & 10 \\
\hline Pinnularia cf. viridis (Nitzsch) Ehrenberg & & & $\mathrm{v}$ & & Sediment surface spp. & 10 \\
\hline Pinnularia cf. vasta Hustedt in A. Schmidt & & $\mathrm{v}$ ? & & & Sediment surface spp. & 10 \\
\hline Placoneis placentula (Ehr.) Heinzerling & & $\mathrm{v}$ & & & Sediment surface spp. & 10 \\
\hline $\begin{array}{l}\text { Planothidium cf. granum (Hohn \& Hellerman) Lange- } \\
\text { Bertalot }\end{array}$ & & $\mathrm{v}$ & $\mathrm{v}$ & both & Shallow waterweed spp. & 9 \\
\hline Planothidium delicatulum (Kutz.) Round \& Bukhtiyarova & & $\mathrm{v}$ & $\mathrm{v}$ & both & Shallow waterweed spp. & 9 \\
\hline $\begin{array}{l}\text { Planothidium cf. lanceolatum (Brebisson ex Kützing) } \\
\text { Lange-Bertalot }\end{array}$ & & $\mathrm{s}$ & $\mathrm{v}$ & both & Shallow waterweed spp. & 9 \\
\hline $\begin{array}{l}\text { Pseudostaurosira brevistriata (Grunow) Williams \& } \\
\text { Round } 1987\end{array}$ & & $\mathrm{r}$ & $\mathrm{s}$ & both & Shallow water planktic & 5 \\
\hline Rhopalodia cf. gibba (Ehr.) O.Muller & & $\mathrm{r}$ & r & both & Shallow waterweed spp. & 9 \\
\hline Rhopalodia gibberula (Ehrenberg) O. Mueller & & & $\mathrm{v}$ & & Shallow waterweed spp. & 9 \\
\hline Rossithidium linearis (W.Sm.) Round \& Bukhtiyarova & & & $\mathrm{v}$ & & Shallow waterweed spp. & 9 \\
\hline Sellaphora bacillum (Ehrenberg) D.G.Mann & & $\mathrm{r}$ & & & Sediment surface spp. & 10 \\
\hline Stauroneis cf. anceps Ehrenberg & & & $\mathrm{v}$ & & Sediment surface spp. & 10 \\
\hline
\end{tabular}

Key: f, frequent $=>5-10 \%$, s, sparse $=>0.5-5 \%, \mathrm{r}$, rare $>0.09-0.5 \%$, v, very rare $<0.09 \%$ 
Appendix H. Diatom species in the Oruanui and lacustrine sedimentary samples of Chapter 6.

\begin{tabular}{|c|c|c|c|c|c|c|}
\hline Taxa identified & \%psol & \%Seds & $\%$ Oruanui & \%Seds+Ou & Grouped & No. \\
\hline Stauroneis phoenicenteron (Nitzsch) Ehrenberg & & & $\mathrm{v}$ & & Sediment surface spp. & 10 \\
\hline Staurosira cf venter (Ehr.) Cleve \& Moeller & & $\mathrm{s}$ & f & both & Shallow water planktic & 5 \\
\hline Staurosira cf. construens Ehrenberg & & $\mathrm{s}$ & $\mathrm{r}$ & both & Shallow water planktic & 5 \\
\hline Staurosira elliptica (Schumann) Cleve \& Möller & & $\mathrm{v}$ & $\mathrm{v}$ & both & Shallow water planktic & 5 \\
\hline Staurosirella cf. pinnata (Ehr.) Williams \& Round & & s & s & both & Shallow water planktic & 5 \\
\hline Staurosirella cf. leptostauron (Ehr.) Williams \& Round & & & $\mathrm{s}$ & & Shallow water planktic & 5 \\
\hline Stephanodiscus alpinus Hustedt & $\mathrm{v}$ & & & & Other planktic & 4 \\
\hline Stephanodiscus hantzschii Grunow in Cl. \& Grun. & & $\mathrm{v}$ & & & Other planktic & 4 \\
\hline Stephanodiscus cf. minutulus (Kützing) Cleve \& Moeller & & s & s & both & Other planktic & 4 \\
\hline Synedrella cf. parasitica (W.Sm.) Round \& Maidana & & $\mathrm{v}$ & $\mathrm{v}$ & both & Shallow waterweed spp. & 9 \\
\hline Tryblionella cf. hungarica (Grunow) D.G. Mann & & $\mathrm{v}$ & & & Sediment surface spp. & 10 \\
\hline Ulnaria acus (Kützing) Aboal 2003 & & $\mathrm{v}$ & $\mathrm{v}$ & both & Ulnaria ulna s.l. & 6 \\
\hline Ulnaria cf. ulna (Nitzsch.) Compère & & 1.53 & 2.41 & both & Ulnaria ulna s.l. & 6 \\
\hline total number of taxa identified & 13 & 52 & 62 & & & \\
\hline total number of species identified & 13 & 52 & 59 & & & \\
\hline \multicolumn{7}{|c|}{ 81total taxa recorded, 39 in both the sediment samples and Oruanui samples, 4 in Paleosol as well. } \\
\hline Only 3 occur in just paleosol and Oruanui samples. & & & & & & \\
\hline$>2500$ diatoms identified in Oruanui samples and $>1000 \mathrm{frc}$ & sedimentar & amples. & & & & \\
\hline
\end{tabular}

